Prepared in cooperation with the

Place-Based Studies,

Recoverability and Vulnerability of Desert Ecosystems Program of the U.S. Geological Survey

\title{
Perennial Vegetation Data from Permanent Plots on the Nevada Test Site, Nye County, Nevada
}


COVER PHOTOGRAPH

(1999). Skull Mountain from Rock Valley, Nevada Test Site (Phil Medica) 


\section{Perennial Vegetation Data From Permanent Plots on The Nevada Test Site, Nye County, Nevada}

By Robert H. Webb, Marilyn B. Murov, Todd C. Esque, Diane E. Boyer, Lesley A.

DeFalco, Dustin F. Haines, Dominic Oldershaw, Sara J. Scoles, Kathryn A. Thomas, Joan B. Blainey, and Philip A. Medica

U.S. GEOLOGICAL SURVEY

Open-Flle Report 03-336

Prepared as part of the

Recoverability and Vulnerability of Desert Ecosystems Project

of the U.S. Geological Survey 


\section{U.S. DEPARTMENT OF THE INTERIOR}

GALE A. NORTON, Secretary

\section{U.S. GEOLOGICAL SURVEY}

Charles G. Groat, Director

Any use of trade, product, or firm names in this publication is for descriptive purposes only and does not imply endorsement by the U.S. Government.

For additional information write to:

Regional Research Hydrologist

U.S. Geological Survey

345 Middlefield Road

Menlo Park, CA 94025

http://water.usgs.gov/nrp
Copies of this report can be purchased from:

U.S. Geological Survey

Information Services

Building 810

Box 25286, Federal Center

Denver, CO 80225-0286 


\section{CONTENTS}

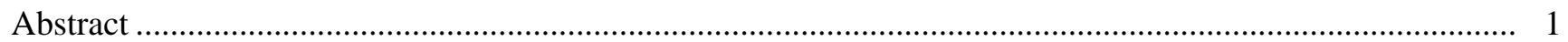

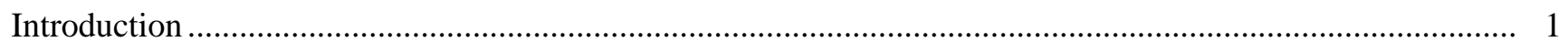

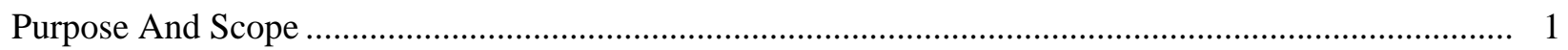

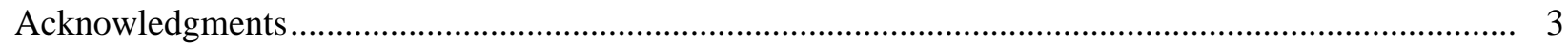

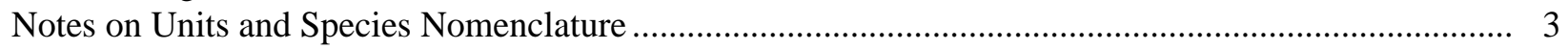

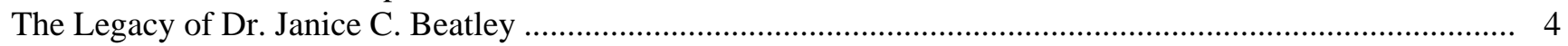

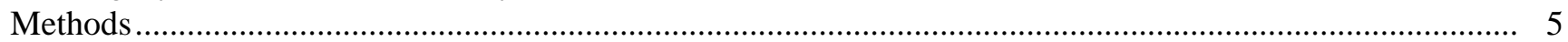

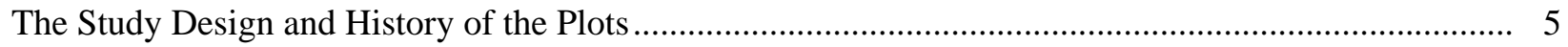

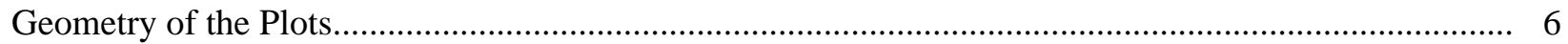

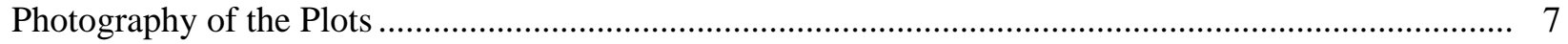

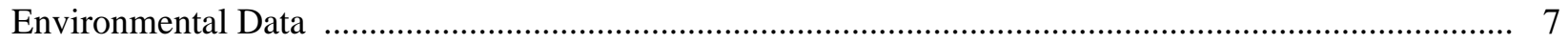

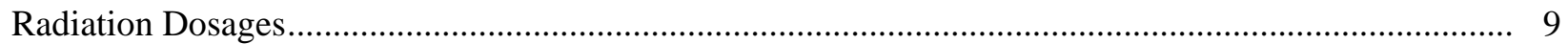

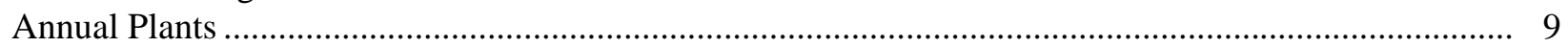

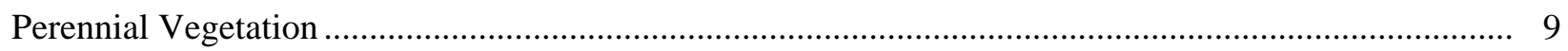

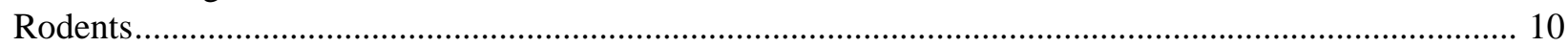

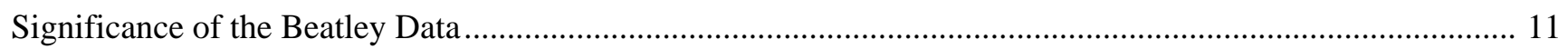

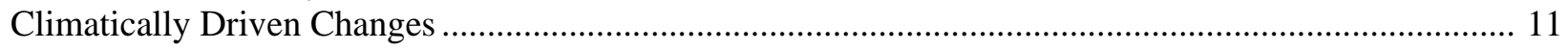

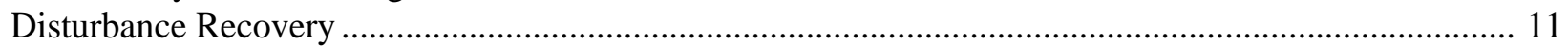

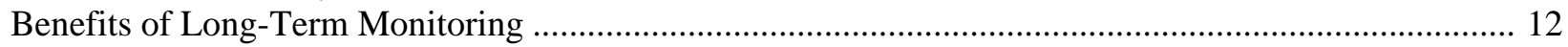

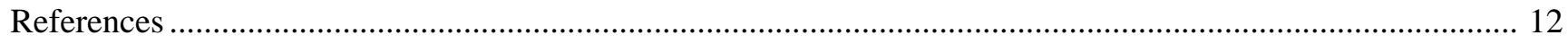

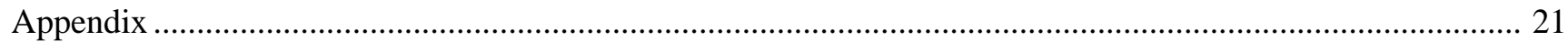

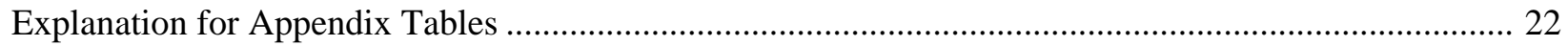




\section{FIGURES}

Figure 1. Map of the Nevada Test Site showing the locations of the Beatley permanent plots.........................2

Figure 2. Photograph of Dr. Janice C. Beatley on Plot 54 in March 1965 ....................................................... 3

Figure 3. Graph showing elevation and annual precipitation for the permanent plots ..................................5

Figure 4. Graph showing time series of modeled precipitation for selected plots, 1960-200.......................... 8

\section{TABLES}

Table 1. Summary characteristics of the permanent plots on the Nevada Test Site ........................................ 14

Table 2. Climate stations in the vicinity of the Nevada Test Site ................................................................ 16

\section{CONVERSION FACTORS AND DATUMS}

CONVERSION FACTORS

\begin{tabular}{rll}
\hline Multiply & By & To obtain \\
\hline inch (in) & 25.41 & millimeter \\
foot $(\mathrm{ft})$ & 0.3048 & meter \\
mile $(\mathrm{mi})$ & 1.609 & kilometer \\
square foot $\left(\mathrm{ft}{ }^{2}\right)$ & 0.09290 & square meter \\
square mile $\left(\mathrm{mi}^{2}\right)$ & 2.590 & square kilometer \\
\hline
\end{tabular}

\section{VERTICAL DATUM}

Vertical coordinate information is referenced to the National Geodetic Vertical Datum of 1929 (NGVD 29).

HORIZONTAL DATUM

Horizontal coordinate information is referenced to the North American Datum of 1983 (NAD 83), unless otherwise noted. UTM coordinates are given as zone12. 


\title{
Perennial Vegetation Data From Permanent Plots on The Nevada Test Site, Nye County, Nevada
}

\author{
By Robert H. Webb, Marilyn B. Murov, Todd C. Esque, Diane E. Boyer, Lesley A. DeFalco, \\ Dustin F. Haines, Dominic Oldershaw, Sara J. Scoles, Kathryn A. Thomas, Joan B. Blainey, \\ and Philip A. Medica
}

\section{ABSTRACT}

Perennial vegetation data from 68 permanent plots on the Nevada Test Site, Nye County, Nevada, are given for the period of 1963 through 2002. Dr. Janice C. Beatley established the plots in 1962 and then measured them periodically from 1963 through 1975 . We remeasured 67 of these plots between 2000 and 2003; the remaining plot was destroyed at some time between 1975 and 1993. The plots ranged from 935 to $2,274 \mathrm{~m}$ in elevation and are representative of common plant associations of the Mojave Desert, the transition to Great Basin Desert, and pinyon-juniper woodlands. The purpose of this report is to describe the complete set of ecological data that Beatley collected from the Nevada Test Site from 1963 through 1975 and to present the data for perennial vegetation collected from 2000 through 2003.

\section{INTRODUCTION}

Agencies responsible for land management in the Mojave Desert have a need to understand the rate of changes to ecosystems, particularly the recovery that follows severe disturbances. Two major elements of the Recoverability and Vulnerability of Desert Ecosystems Program of the U.S. Geological Survey involve the study of perennial vegetation recovery following severe disturbances and ecosystem changes in response to climatic fluctuations. The Nevada Test Site (NTS; fig. 1) has high security, which controls the amount of disturbance to the landscape (approximately $8 \%$, Hunter and Medica, 1992). This protection makes NTS an ideal place to conduct long-term ecosystem research. Established in 1951 for the purpose of testing nuclear weapons (Fehner and Gosling, 2000), managers eliminated grazing by domestic animals and strictly controlled access to the $1,375 \mathrm{mi}^{2}$ NTS.

In 1962, Dr. Janice C. Beatley, a principal investigator with the University of California, Laboratory of Nuclear Medicine and Radiation Biology, was funded by the U.S. Atomic Energy Commission to study the effects of ionizing radiation on various aspects of the Mojave and Great Basin Desert ecosystems. She established permanent plots on NTS for this purpose and began measurements in 1963. The focus of her research changed after the Limited Test Ban Treaty of 1963, when she initiated a characterization of the common plant associations of the northern Mojave and transition Great Basin Desert and to document long-term changes in these ecosystems. This report discusses the types of data that Beatley collected and presents data on perennial vegetation that Beatley collected from 1963 through 1975 and that we collected from the plots from 2000 through 2003.

\section{Purpose And Scope}

The purpose of this report is to document all of the methods used by Dr. Beatley to measure ecosystem attributes on NTS and to present data on perennial vegetation on the plots collected from 1963 through 2003. This report contains a synthesis of methods used to collect data from these plots as well as a limited discussion of other available data. These methods were 


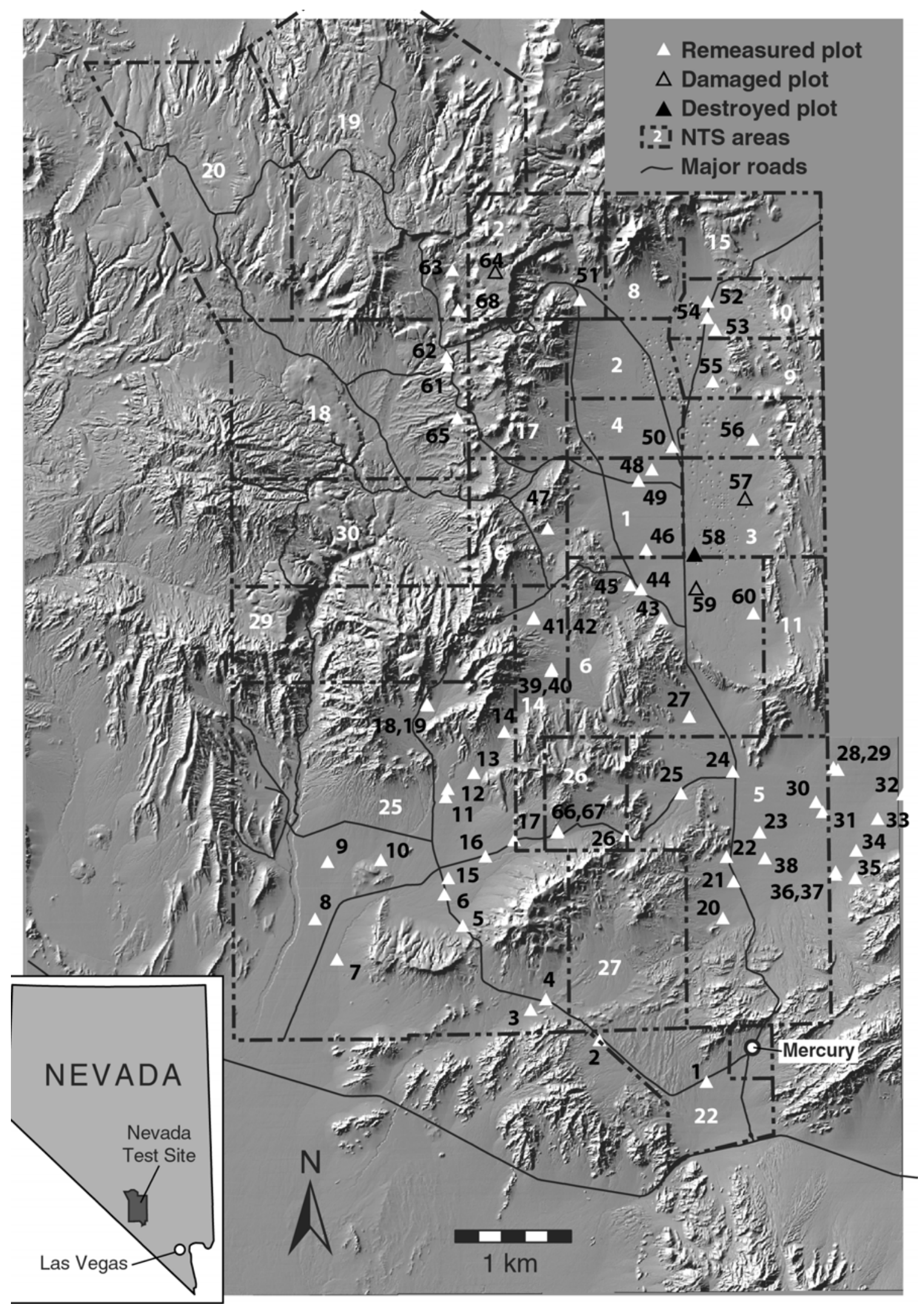

Figure 1. Map of the Nevada Test Site showing the locations of the Beatley permanent plots. The map is based on a 10-m digital elevation model. 


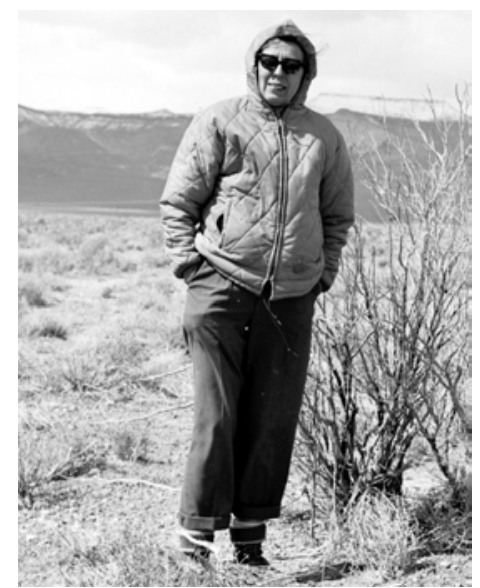

either previously published by Beatley, unpublished but determined from her notes and (or) data, or determined to have been a necessary component of her study design during our remeasurement of the plots from 2000 through 2003.

\section{Acknowledgments}

We wish to thank the U.S. Department of Energy, Nevada Operations Office, and Bechtel Nevada for their logistical and technical support. We especially thank Bob Furlow and Fred Penrod of the Department of Energy for their help with all aspects of work on NTS. Jerry Magner of the U.S. Geological Survey and Kathleen Matson of Bechtel Nevada Corporation, colocated at the Nevada Test Site USGS Field Office/Core Library, provided extensive logistical support. Scientists who helped with the field work on this project include Jonathan R. Abu-Saba, Lisa M. Barnhill, Dorothy A. Beals, Janice E. Bowers, Catherine J. Bukowski, Elizabeth A. Burguieres, Julie M. Day, Elizabeth S. Deliso, Sara E. Eckert, Ann G. Frazier, Kimberley A. Goodwin, Kelly J. Goward, Jessica L.Grandas, Sharleen P. Johnson, Megan E. Kelley, Adam Malisch, Sharilyn L. Meyers, David M. Miller, W. Kent Ostler, Robyn M. Powers, Helen A. Raichle, Elizabeth M. Ralston, Kristin K. Riebeling, Heather L. Robinson, Renée J. Rondeau, Mary B. Saethre, Nira Salant, Caimee A. Schoenbaechler, Andrew K. Thorpe, and Jason A. Williams. John Freudenstein of Ohio State University and John Caruso of the University of Cincinnati provided information on Beatley's career and legacy in Ohio. We especially thank Mary Killeem of the University of Cincinnati for supplying us with Dr. Beatley's final curriculum vitae. Alan Flint of the U.S. Geological Survey supplied the
Figure 2. Dr. Janice C. Beatley on Plot 54 in March

10-m digital elevation model depicted in Figure 1.

Peter G. Griffiths produced the geographical information system graphics used in this report. The report was critically reviewed by W. Kent Ostler of Bechtel Nevada, Richard Hunter of Salisbury University, and Debra Hughson of Mojave National Preserve (National Park Service). We especially thank Dr. Beatley for her foresight in setting up these ecological study plots.

\section{Notes on Units and Species Nomenclature}

A mixture of types of units are used in this report, reflecting how Beatley originally made her measurements and the standard usage of metric units in scientific reports. Beatley mostly used English units for her measurements; some exceptions include quadrat frames, which were $0.1 \mathrm{~m}^{2}$ in area. We remeasured her plots in English units to allow direct comparisons, although our remeasurements of plant heights were generally in centimeters. We converted her temperature and precipitation measurements, originally collected in English units, to metric units. As an aid to any future researchers who may want to remeasure these plots, we report some values in the original English units as this more easily facilitates reoccupation and remeasurement of these plots.

We have chosen to retain many of the species names that Beatley used in her original analysis. Taxonomic revisions are constantly made, and the choice of whether to accept a change to the name of a species often depends on widespread acceptance in the scientific community, which may require up to a decade after the proposed change. In this report, we opt for a conservative approach to changing Beatley's original species names. We change her Haplopappus 
cooperi to Ericameria cooperi because that revision has been long accepted. Beatley herself chose to use Psorothamnus instead of Dalea for that genus of the Fabaceae. We retain usage of Stipa speciosa and Oryzopsis hymenoides (both are proposed for the genus Achnatherum); Ceratoides lanata (instead of Krashinninikovia lanata); and the genus Hilaria (instead of Pleuraphis).

\section{THE LEGACY OF DR. JANICE C. BEATLEY}

Dr. Janice C. Beatley (fig. 2) was born in Columbus, Ohio, on March 18, 1919, and she received her B.S. (1940), M.S. (1948), and Ph.D (1953) degrees in botany from Ohio State University (Stuckey, 1990). After holding several non-tenured teaching positions in the East, she moved west to New Mexico Highlands University in 1959 and to the University of California at Los Angeles (UCLA), Laboratory of Nuclear Medicine and Radiation Biology, in 1960. She was part of a larger team of UCLA researchers who contracted with the U.S. Atomic Energy Commission (USAEC) to do ecological work on NTS between June 1960 and March 1973. From 1973 until her death in November 1987, she was an Associate Professor in the Department of Biological Sciences at the University of Cincinnati in Ohio.

The Laboratory of Nuclear Medicine and Radiation Biology was primarily interested in the effects of ionizing radiation released during aboveground nuclear testing on the desert environment of NTS. In 1962, Beatley established a network of 68 permanent ecological plots on NTS with the intent of addressing a number of long-term ecological questions primarily related to the effects of ionizing radiation. With establishment of the Limited Test Ban Treaty of 1963 (Fehner and Gosling, 2000), Beatley's interests shifted from the effects of ionizing radiation to vegetation mapping and ecosystem processes. The plots are within the major vegetation assemblages of the Mojave Desert, and their siting was due in part to her interest in describing and mapping plant communities on NTS (Beatley, 1976c), particularly the spatial distribution of creosote bush (Larrea tridentata).

During her career (Stuckey, 1990), Janice Beatley wrote 42 publications, including two books (e.g., Beatley, 1976c). Thirty of her publications are described in a recent annotated bibliography (Wills and Ostler, 2001). Beatley only published some of the data collected from her plots between the mid-1960s and early 1980s (Beatley, 1969a, 1969b, 1979, 1980). Because her plots were designed for repeated measurements to assess long-term change, most of the data are unpublished, particularly for her plots that document disturbance recovery. Beatley spent much of her time in the 1970s putting all of her data into computer files for final analysis. After Dr. Beatley died on November 14, 1987, her estate provided an endowment to Ohio State University, which was used to create the Janice Carson Beatley Herbarium Award in 1988. This award annually funds 2-5 students with up to $\$ 1,500$ each for field studies in plant systematics and ecology. The first award was given in 1990 by the Department of Botany at Ohio State University, which later was reorganized into the Department of Evolution, Ecology, and Organismal Biology.

As part of her last requests, Beatley gave her data to the U.S. Geological Survey (USGS) after her death in 1987. The contents of her office that were related to work on NTS were not well described. No overall description of the permanent plots or the exact nature or purpose of several measurements was found. The contents of Beatley's office were packed and shipped to the USGS Project Office, located at the Desert Laboratory in Tucson, Arizona. Data were available in computer printouts and 9-track tapes that were translated onto modern computer media; computer punch cards, which Beatley used to create the 9-track tapes, were discarded. All the materials from Beatley's office have recently been moved to long-term storage at the USGS facility in Henderson, Nevada. The purpose of this publication is to provide access to part of her extensive dataset, which appeared to be her intention when she gave her data to USGS.

Reoccupation of the Beatley ecological study plots provides invaluable data on long-term changes in undisturbed Mojave Desert ecosystems. In addition, Beatley's study design allows an opportunity for assessing long-term recovery from several types of disturbances. This report describes the Beatley permanent plots, depicts the data for perennial vegetation, and discusses their usefulness for addressing several important ecological questions. Our remeasurement of the Beatley permanent plots between 1999 and 2003 validates her long-term vision of what would be needed to understand ecosystem change in the Mojave Desert. 


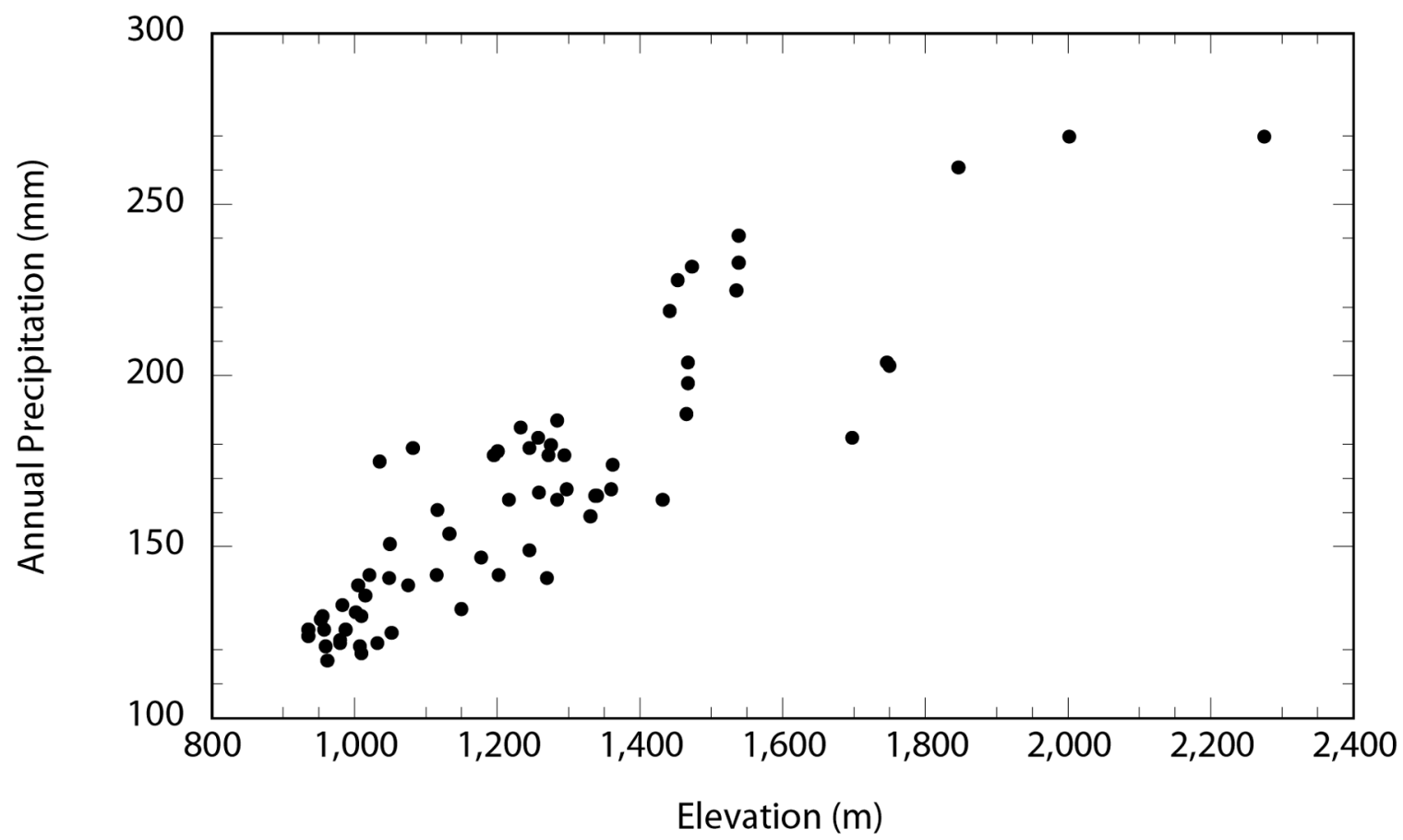

Figure 3. Graph showing the relation between elevation and annual precipitation for the 68 permanent plots on the Nevada Test Site. Precipitation data were collected between 1963 and 1972 (Beatley, 1976c).

\section{METHODS}

As previously noted, Beatley did not leave an overall description of her plots, or the design of her studies, except to the extent of what appears in her publications. In particular, those interested in Beatley's descriptions of her work are directed to several key publications that she wrote (Beatley, 1965, 1976c, 1979). The following descriptions were developed by both reading her generalized descriptions and knowing the specifics of her plots and their locations as determined during our remeasurements.

\section{The Study Design and History of the Plots}

Beatley established 68 study plots beginning in the fall of 1962. Of the 68 plots, 59 plots are in undisturbed settings selected to document long-term ecological changes in "climax" vegetation (Beatley, 1980). Nine plots were established to document disturbance response; some of the disturbed plots were paired with nearby undisturbed plots while others represent disturbance gradients. Some of Beatley's plots coincided with plots originally established in the late 1950s by Rickard (Rickard and Beatley, 1965). The plots ranged in elevation from 935 to $2,274 \mathrm{~m}$ in elevation (fig. 3 ) and are representative of the climate and elevational range of the Mojave Desert.

Beatley (1979) provided only a vague description of why her plots were located where they are without describing in detail the exact procedure for choosing her corner posts. From our visitation of the plots and reading her papers, we can assume that she had several overall hypotheses that she was testing. One of those concerned the spatial distribution, and patchiness, of creosote bush on the landscape, because many of her plots (e.g., plots $44,48,49,54)$ were in isolated patches of creosote bush within a larger plant assemblage with no creosote bush. The publication of Beatley (1974b) implies this study design. Another concerned changes with respect to elevation above closed playa basins, again indicated by the publication of Beatley (1975). Finally, it is apparent that she sought replicate plots for the major plant assemblages of NTS. Whether the exact locations of the plots were determined randomly or because of some nowunapparent factor (e.g., road access) cannot be known. A listing of the plots, their dominant vegetation, and their locations appears in Table 1. 
Several plots $(48,52-54)$ are sited to assess longterm recovery from the effects of atmospheric nuclear testing with distance away from ground zero (Beatley, 1965). Three plots $(19,40,42)$ were established in blackbrush (Coleogyne ramosissima) assemblages that were burned in the 1950s. One plot (67) was located in the Wahmonie townsite, which was abandoned in 1928; this townsite has been repeatedly measured by other researchers as part of on-going studies of disturbance recovery in the Mojave Desert (Wells, 1961; Webb and Wilshire, 1980; Webb, 2002; Webb and Thomas, 2003).

Several of Beatley's plots were damaged or threatened by construction projects during her tenure on NTS. Plots 24, 61, and 62 had road or other construction near or adjacent to the plots at known times in the 1960s. In the case of plot 24, our remeasurements suggest that plants on one side of the plot were enhanced by runoff from a nearby road, and therefore data from this plot must be interpreted carefully. About one half of another plot (57) was damaged by construction in 1965, and Beatley established a new plot 57N nearby in 1966; both plots were remeasured, with the original plot 57 , which we refer to in Appendix 1 as plot 57A, now partially representing disturbance recovery. Three plots $(9,29$, and 31) were either damaged by gophers between 1963 and 1975 or had "questionable history" (Beatley, 1980); our remeasurements indicate that these plots did not have significant problems between 2000 and 2003 and were retained in the set. Between 1975 and 1992, one plot (58) was destroyed by an underground nuclear test. Another plot (59) was damaged during construction of an NTS facility, and although about one-third of the plot was destroyed, this plot was remeasured for its undisturbed section. One plot (64) was damaged by road construction at an unknown time after 1975, but its undisturbed sections were remeasured.

Two additional studies that post-date Beatley's work should be noted. Ostler and others (2000) provide a new vegetation map of NTS that supercedes Beatley's map (Beatley, 1976c). Between 1987 and 1994, the Basic Environmental Compliance And Monitoring Program (BECAMP) measured climate, annual and perennial vegetation, and rodent populations at many sites (Hunter, 1994), including within or adjacent to several Beatley plots. None of Beatley's plots were replicated using her methods, however, and the relation between the BECAMP measurements and the data presented in Appendix 1 is unclear. We are remeasuring the BECAMP plots to determine this relation and will provide those results in a subsequent report.

\section{Geometry of the Plots}

Each plot was established with a primary goal of measurement of perennial vegetation within a 100 foot by 100 foot square area, referred to here as the central area. At least three plots varied in dimensions from the $100 \mathrm{ft}$ by $100 \mathrm{ft}$ design owing to site-specific objectives or geometric constraints. Plots 66 and 67 (Wahmonie townsite) were set up as $75 \mathrm{ft}$ by $300 \mathrm{ft}$ plots, joined along a center $300 \mathrm{ft}$ line. Plot $57 \mathrm{~N}$, set up after the original plot 57 was damaged, was $75 \mathrm{ft}$ by $150 \mathrm{ft}$; Beatley left no explanation as to why she deviated from her original $100 \mathrm{ft}$ by $100 \mathrm{ft}$ design for this plot. The types of corner posts varied considerably from $1 \mathrm{in}$. angle iron (the most stable) to 4 in. by 4 in. wooden stakes to $1 \mathrm{in}$. by $1 \mathrm{in}$. stakes to wood lath (least stable). Measurements of perennial vegetation were made from steel tapes stretched across the plot (see below), and additional stakes marked the locations of where these lines were placed. In all cases, the original study design called for the lines to be $10.0 \mathrm{ft}$ apart, indicating that the typical plot had 11 marks on two sides, including 9 markers between the cornerposts. Most of these markers were 1/4 in. steel stakes with hooked ends, although on some plots (e.g., plots 66 and 67) wooden lath was used instead.

In order to remeasure the plots, we had to determine how accurately Beatley measured these dimensions, as well as whether the plots were consistently square. For most plots, the dimensions between cornerposts on the sides with additional stakes varied between 99.9 and $100.1 \mathrm{ft}$; the dimension along the lines varied between about 100.5 and $101.0 \mathrm{ft}$. We suspect that the longer distance occurred because of the leaders used on the steel tapes that Beatley used, and we adjusted the length along these lines to $100.0 \mathrm{ft}$ to be consistent with her measurements. By stretching steel tapes between the cornerposts, we determined that the intermediate stakes were generally accurate to \pm 0.1 $\mathrm{ft}$, both in distance along the line and distance to one side or the other from the line. This is the same magnitude of error that we believe to be inherent in our measurements with the same equipment. 
We encountered five possibilities of plot reestablishment, depending upon how many cornerposts remained standing. In the best-case scenario of all four cornerposts standing, the plots were relatively easy to reoccupy. In the case of no cornerposts standing, we carefully measured the distances between the existing intermediate stakes and re-established the cornerposts. All stakes were replaced with $3 / 8$ in. steel reinforcing bar (rebar) from 2000 through 2003.

For all plots, a perimeter fence cordoned off the central area from vehicular use and extraneous trampling, and a sign stating the project name faced the nearest access point. In general, the perimeter fence consisted of light fence posts driven outside the four corners of the central area and connected with 1/16 in. light wire. Few of these fences were intact when we relocated the plots, owing to a combination of wind, weathering of the metal, occasional visitation by NTS security, and (or) passage of pronghorn antelope or feral horses. As described below, rainfall, air temperature, soil temperature, and soil moisture measurements were made at one point within the central area. Annual plants were sampled outside of the central area, and rodents were trapped at 8 points around the perimeter of the plot with one trap station in the center of the plot. Some plots were measured as part of the BECAMP studies of the late 1980s and early 1990s (e.g., Hunter, 1994), and these transects were marked with a combination of wood lath painted white on top and other markers, including white PVC pipe encasing metal fence posts. As a result, many different types of stakes with different geometric configurations are adjacent to these plots. In addition, an array of closely spaced metal stakes appear within the central area of some of the plots; the purpose of this array is unknown.

\section{Photography of the Plots}

Beatley had most of her plots photographed from the bed of a truck in April and May, 1964, as part of her long-term documentation of these plots. Camera stations were established on opposing corners of the plots just outside of the perimeter fence. Two professional photographers - either Richard (Doc) Collier or Richard Borden - took the photographs in 1964; Beatley took photographs in 1974 and 1975. The professional photographers worked for Pan Am, the primary contractor with the USAEC at the time. We have no way of knowing which photographer took which photograph, so all of the photographs are attributed to Beatley in this report. The photographers mounted a $4 \times 5$ Crown Graphic camera equipped with a $135 \mathrm{~mm}$ lens and 4 in. by 5 in. Plus-X film in cut-film holders, on a tripod in the bed of a truck to take downward-looking photographs of the plots. Two photographs were taken from each camera station, generally showing a corner of the plot near the edge of the photograph.

Some plots (e.g., Plots 39, 41, and 64) were either not photographed in 1964 or the negatives were lost; for other photographs, one or more of the exposed negatives was lost before transfer to the U.S. Geological Survey; and for one plot (Plot 58), the camera stations were destroyed by subsequent land use. Some plots (e.g., Plot 18) were apparently not photographed in 1964 or 1965 , but in 1974 and 1975 , Beatley photographed these plots using a hand-held 35 $\mathrm{mm}$ camera with a lens of 70 to $100 \mathrm{~mm}$ focal length from standing positions within the plot. Like the professional photographers, Beatley used black-andwhite negative film.

We replicated all available photographs of the permanent plots using a Crown Graphic 4x5 camera with a $135 \mathrm{~mm}$ lens and modern color and black-andwhite film. A total of 351 views of the permanent plots were matched from 1999 through 2003 as part of this study, and examples of the original and matches are given in Appendix 1. A listing of the relevant data for the plot photography appears in Table A-69.

\section{Environmental Data}

Environmental data were collected from all plots every two weeks for the 10 years spanning 1963-1972. The data include: 1) soil temperature; 2) soil moisture potential, using gypsum blocks, at 3, 6, and $10 \mathrm{in}$. depths; 3) soil $\mathrm{pH}$ and particle-size distribution; 4) radiation exposure on dosimeter film badges; 5) maximum-minimum air temperatures; and 6) precipitation in storage gages. These data were collected for all plots at roughly two-week intervals between 1963 and 1972. The log books for these measurements were retained after Beatley died, providing the exact dates of environmental measurements. Although there are some missing data, as a whole the data are remarkably complete. 
Soil temperature was recorded using thermistors embedded in gypsum blocks; these temperature readings are instantaneous and only reflect conditions at the time of measurement. All gypsum blocks were calibrated on pressure-plate apparatus; the calibration curves were developed and applied to the raw data by Dr. Beatley and her staff. These data have not been published and are not included in this report. Particlesize distribution and soil $\mathrm{pH}$ were measured for soil pits; the particle-size distribution, measured using a coarse set of engineering screens with openings in English units, were deemed unusable for subsequent work. Soil pH was measured using a soil slurry and a standard $\mathrm{pH}$ meter. Beatley did not publish these data and they are not included in this report. Radiation exposure is discussed in the following section.

Air temperatures were measured using recording maximum-minimum mercury thermometers placed in a makeshift shelter at a height of $1 \mathrm{ft}$ above the ground surface. These thermometers only record the maximum and minimum temperatures between readings and therefore do not provide a continuous record of temperature on a daily basis. Precipitation (mostly rainfall) was measured in a storage gage placed below ground surface and equipped with a small amount of oil to minimize evaporation. Precipitation collected in an above-ground funnel flowed into the storage gage, which periodically was measured and emptied. Rainfall data are valuable for monthly and annual precipitation estimates only. These data are only included in this report as mean-annual precipitation (Table 1).

Other climate data are available for NTS (Table

2). In addition to data from Yucca Mountain and vicinity (Flint and Davies, 1997), storage rainfall gages were placed near several Beatley plots during the period of BECAMP studies, 1989-1995 (e.g., Hunter, 1994). Climatic data also are routinely collected near major DOE facilities on NTS, such as at Mercury, as well as in surrounding communities and other locations (Table 2). These data were used to model precipitation for the Beatley plots to determine the 1963-1972 average, measured on the plots, with a modeled average predicted for 1958-2000.

Monthly precipitation data were spatially distributed over NTS using an interpolation scheme developed as a combination of multivariate regression using geospatial position, elevation, and inverse distance weighting. The weighting scheme reverts to only inverse distance if the regression fit becomes statistically insignificant. Three sources of monthly precipitation data, representing 35 stations over an area of approximately $15,000 \mathrm{~km}^{2}$, were used (see Table 2).

Monthly precipitation was calculated using the gradient plus inverse distance-squared method (GIDS) (Nalder and Wein, 1998). Multiple linear-regression coefficients define precipitation along north-south, east-west, and elevation gradients for each month. These gradients are used to weight inverse distancesquared interpolation as follows:

$$
Z=\frac{\left[\sum_{i=1}^{N} \frac{Z_{i}+\left(X-X_{i}\right) \times C_{x}+\left(Y-Y_{i}\right) \times C_{y}+\left(E-E_{i}\right) \times C_{e}}{d_{i}^{2}}\right]}{\left[\sum_{i=1}^{N} \frac{1}{d_{i}^{2}}\right]}
$$

where $X$ and $X_{i}$ are the easting of a grid cell and climate station $i, Y$ and $Y_{i}$ are the northing of a grid cell and precipitation station $i$, and $E$ and $E_{i}$ are the elevation of a grid cell and a precipitation station. $C_{x}, C_{y}$, and $C_{e}$ are the multiple linear regression coefficients for easting, northing, and elevation, respectively. $N$ is the number of precipitation stations and $d_{i}$ is the distance between a grid cell and precipitation station $i$. Monthly precipitation was summed to annual totals and averaged over the period of record; those modeled annual precipitation totals appear in Appendix 1. For selected plots, a time series of modeled annual precipitation over the period of perennial plant measurements appears in Figure 4.

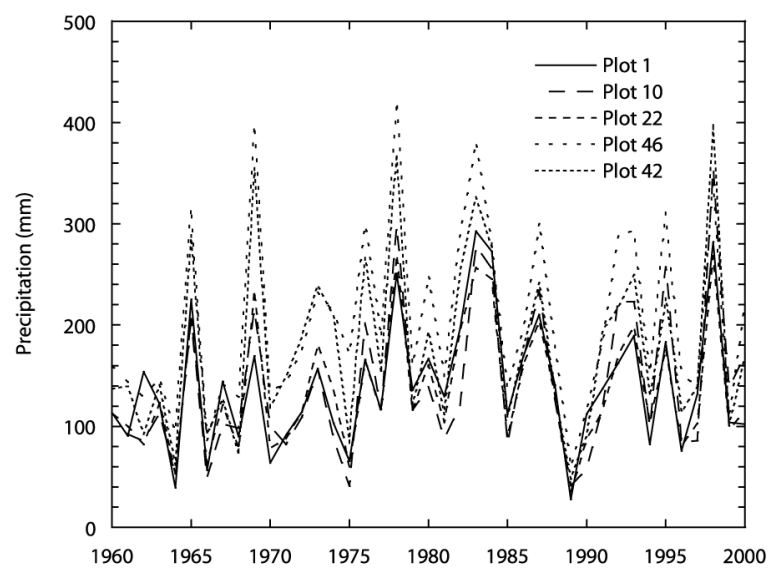

Figure 4. Time series of modeled precipitation for selected Beatley plots on the Nevada Test Site, 1960-2000. 


\section{Radiation Dosages}

All plots were monitored with film badges (Dupont Type 556, component film 508 and 834, containing a filter for beta and low-energy gamma) suspended from the under side of a plywood cover for a maximum-minimum thermometer installation at 12 in. height above the soil surface. These data were collected concurrently with environmental data from 1963 through 1972. The installations were on the northeast sides of shrub-clumps at the periphery of the shrub canopy. Film badges were exposed to gamma-radiation levels intermediate between those of the open soil surface in one direction and the accumulated debris around the bases of the shrubs in the other direction. Badges were collected and replaced weekly or monthly, as desirable with changing seasonal air temperatures.

Among the papers left by Beatley was a description by REECO Dosimetry of the types of film badges used by Beatley on NTS. From November 1962 until 1969, the E.I. Dupont type 556 film packet consisted of a U-shaped strip of lead ( 0.028 in. by 0.5 in.) pressed about one end of the packet and sealed within a 4-mm thick plastic bag. The type 556 film contained two components: 519 low range and 834 high range. The film was pre-numbered at the factory with impression dies so that the film was readily identified before and after processing and was routinely calibrated to radium and ${ }^{60} \mathrm{Co}$ gamma sources. Beta calibrations were made with ${ }^{90} \mathrm{Sr}-{ }^{90} \mathrm{Yt}$ point sources and uranium slab sources. Under field conditions the minimum reportable gamma dose was 30 mrem and the minimum reportable beta dose was $50 \mathrm{mrad}$. Film were read on an Eberline transmission densitometer using a ${ }^{137} \mathrm{Cs}$ calibration curve that best represents the mixed radiation fields at NTS. ${ }^{60} \mathrm{Co}$ exposures are approximately $15 \%$ higher than ${ }^{137} \mathrm{Cs}$. In late 1969 and early 1970, the type 556 film was phased out because the manufacturer discontinued its production, and it was replaced with Eastman Kodak type 3 film. Kodak type 3 film packets contain two components similar to Dupont 556 components. Minimum reportable doses are the same; however, Kodak type 3 film is more sensitive so the initial base fog is greater than Dupont 556 film. The film sensitivity varies with the type and energy of exposure but occurs generally at energies below $0.2 \mathrm{mev}$; the film is also very sensitive to heat, light, and humidity.

\section{Annual Plants}

Beatley measured annual plants at peak production during the spring months on all plots for 6 years and on 7 selected plots for 10 years. She was particularly interested in invasive annuals, both Mediterranean annual grasses (Bromus rubens; Beatley, 1966b) and summer weedy herbs (Salsola iberica; Beatley, 1973).

Winter annuals were measured during their reproductive peak in April-June from 1963-1968 for 61 plots and 1963-1972 for plots 3, 4, 48, and 52-55. Cover-class, average height, and density were measured for each species in quadrat frames placed along a line 10 feet outside of the central area and parallel to one of the sides. Each line was 100 -ft long, and the $0.1 \mathrm{~m}^{2}$ frames were placed every $2 \mathrm{ft}$ for a total of 50 estimates per plot (Beatley, 1969a). Beginning in 2000 , the ends of lines on the remeasured plots were marked with $3 / 8$ in. rebar.

In addition, biomass of all winter annual species combined was measured destructively for each of the 68 plots between 1964 and 1966 by harvesting plants within $0.1 \mathrm{~m}^{2}$ frames placed every $5 \mathrm{ft}$ (20 measurements per plot and adjacent to the 100-ft line (Beatley, 1969a). The side of the plot chosen varied to minimize cumulative-disturbance effects in subsequent years. Summer annuals were measured on all plots for 5 years and on 5 plots annually for an additional 5 years using the same techniques. Beatley published some of these results (Beatley, 1966a, 1966b, 1967, 1969a), mostly of a qualitative nature, and Hunter (1991) reported density data collected from Rock Valley plots between 1963 and 1972. We remeasured winter annuals on a subset of the Beatley plots between 2000 and 2003 and will report the data in subsequent publications. Extensive additional work is warranted in relating the annuals to environmental data.

\section{Perennial Vegetation}

Measurement of perennial vegetation was the key component of Beatley's sampling design, and she reported her results for perennial vegetation in undisturbed plots primarily in Beatley $(1979,1980)$. She differentiated "perennial vegetation" into woody perennials and herbaceous perennials and measured the second type more frequently. We discontinue this approach and lump the two groups together in this 
report. Because Beatley measured both types of vegetation using the same method, we refer to both as perennial vegetation in the following methods description.

Perennial vegetation was measured in the central area using the line-intercept method on eleven 100-foot lines spaced 10-feet apart. As a result, most plots represent intercepts over an 1,100 ft distance. As noted above, the original lines were marked with a variable combination of large nails, hooked stakes, or wooden stakes at both ends of the transects; these markers were replaced with 3/8 in. rebar between 2000 and 2003. The intercept start and end were measured for each plant; and overlapping canopies were recorded separately, making the sum of the individual plant covers greater than the total ground cover. The maximum height of individuals, as differentiated from the maximum height encountered along the intercept, and their phenological status were noted (Beatley, 1980). We multiplied the maximum height by the intercept distance to create a two-dimensional index of biomass, in units of $\mathrm{m}^{2}$, and report this information in the B tables included in Appendix 1.

Perennial vegetation was measured in the summer and fall of 1963 and the spring of 1975 and is presented in summary form for all plots in Appendix 1. This dataset was also presented in Beatley (1979, 1980), although many plots were combined to provide a vegetation-assemblage average in those reports. Herbaceous perennials were measured annually between 1963 and 1967 and again in 1975, but we only present the data collected simultaneously with the woody perennials in Appendix 1. Most disturbed plots were measured in 1963, 1964, 1965, 1966, 1967, 1970, and 1975, and these additional years of data for these plots (e.g., plots 52-54) are presented in Appendix 1 as well. As previously noted, plot 57 is a special case where the original plot, which we refer to as plot 57A, was partially destroyed in 1965; we present the 1963 and 2002 data for this plot. The new plot, which Beatley called 57N, has data from 1966, 1975, and 2002.

Beatley designed her interpretations around a set of codes for each plant. For condition of whole plant, the codes are: $\mathrm{L}=$ living (dormant or barely alive); $\mathrm{D}=$ dead; $\mathrm{V}=$ vegetative (with leaves or bracts); $\mathrm{F}=$ flowering, fruiting, or reproductive buds; $\mathrm{S}=$ living sprouts present; and $\mathrm{N}=$ no condition was noted (but the plant was alive). For condition of portion of plant intercepted by the line, she also used the following codes: $\mathrm{L}=$ living, $\mathrm{D}=$ dead, $\mathrm{B}=\mathrm{a}$ combination of live and dead parts, and $\mathrm{S}=$ sprouts. Our interpretation of her codes is straight forward for $\mathrm{L}, \mathrm{D}, \mathrm{V}$, and F, and we added another code ( $\mathrm{dblp}=$ dead branch on a live plant) to correctly separate dead from live cover while maintaining the statistics on number of live plants and their average height. N, S, and B are calculated as live plants in our calculations, although we did not change her codes in the data files. Beatley double-coded these notations for some species on some plots. We interpreted her double-coded entries as: $\mathrm{LB}=\mathrm{L}, \mathrm{NB}=$ $\mathrm{L}, \mathrm{SB}=\mathrm{L}, \mathrm{LD}=\mathrm{dblp}, \mathrm{ND}=\mathrm{dblp}$, and $\mathrm{SD}=\mathrm{dblp}$.

Our interpretation of Beatley's codes introduces an unknown amount of error into our calculations. We strictly separated dead and live portions of plants in our intercept data, and it is not apparent that Beatley did the same. It is also not apparent that she needed to do this, because much of the dead plant material we encountered resulted from a severe drought in 19891991 and that may have produced different vegetative conditions than what Beatley encountered. This interpretation is supported by Beatley's photographs, which do not show large amounts of dead parts on plants on her plots in 1964. To ensure quality control in our re-analysis of Beatley's data, we compared our summary data with the data that she calculated and reported in Beatley (1979), whenever she reported data that were not averaged over more than one plot.

We do not report all of the data for perennial vegetation that Beatley collected, in part because she did not necessarily leave an unambiguous method for us to replicate. For example, perennial seedlings were measured annually on all plots for 5 years; these data have not been published or remeasured. Definition of "seedling" is problematic and requires an interpretation that we cannot make in a manner reproducible with Beatley's measurements. We do not provide any analysis of Beatley's phenological information, which she used to construct an elaborate model of plant phenology in the Mojave Desert (Beatley, 1974a), because much of the data she used in this analysis was obtained in the bi-weekly visits to the plots to capture environmental data.

\section{Rodents}

Rodents were censused on all plots once annually for 5 years (Beatley, 1976a, 1976b). These data are probably inadequate for proper population 
studies of rodents but can be used as an index of species composition. Small mammal transect lines were superimposed over the central area to form an effective trapping area of 1.14 ha. Seven lines with 7 traps - making a total of 49 trap stations with one trap per station - spaced at 15-m intervals formed the 91 by $91 \mathrm{~m}$ grid. Each plot was sampled after the end of the reproductive season in July-September using Sherman live traps baited with rolled barley. Sampling occurred for 2 consecutive nights; this sampling protocol of only 2 nights per year precludes any assessment of absolute density of all rodents, but instead yields species composition information primarily on animals that are nocturnally active in July. We have remeasured rodents on several Beatley plots and will report the data in subsequent reports.

\section{SIGNIFICANCE OF THE BEATLEY DATA}

The Beatley plot data, combined with our remeasurements from 2000-2003, represent the longest ecological dataset from the Mojave Desert. Because of these data, long-term changes in annual plants, perennial vegetation, and rodent populations can be detected and compared with climatic fluctuations or other variables that create conditions for environmental change. Here we provide some generalized discussion of the implications of the perennial vegetation data (Appendix 1) in determining the causes for change in the Mojave Desert.

\section{Climatically Driven Changes}

Beatley (1980) concluded that most of the living plants in 1963 were still present when she remeasured her plots in 1975. An additional 20-30 percent of the plants measured in 1975 were new, and total cover had increased as a result of high rainfall in the late 1960s and early 1970s (fig. 4). Beatley (1980) concluded that the size and cover of woody perennial plants in the Mojave Desert are strongly correlated with precipitation.

The period between 1975, when Beatley last measured the plots, and 2000 had several climatic extremes. The period of 1977-1984 was one of the wettest periods of the 20th century; an extreme drought occurred in 1989-1991 (Hunter, 1994; Flint and Davies, 1997); and lesser albeit significant droughts occurred in 1996 and 1999-2002 (fig. 4). Many shrubs died during the 1989-1991 drought, suggesting that droughts are a major mechanism for change in Mojave Desert ecosystems. Certain frost-sensitive species, such as spiny menodora (Menodora spinescens), suffered major damage during a severe frost on December 2425,1990 . Another possible factor is the gradually rising levels of carbon dioxide in the atmosphere, which would tend to promote increased biomass.

Despite the droughts and frosts, the increase in biomass between 1963 and 2000 is striking (Appendix 1). Associations dominated by creosote bush had large increases in the heights of individual plants as well as increases in total cover, whereas assemblages dominated by Chenopod species (saltbushes) had large decreases in cover. Some blackbrush assemblages, in contrast, had small decreases in perennial plant cover, probably as a result of the droughts (Appendix 1). Whether precipitation increases alone caused increased plant growth, or whether carbon dioxide increases played a significant role, will be the subject of future research.

\section{Disturbance Recovery}

Part of Beatley's experimental design for plot location emphasized recovery from a variety of different disturbances. In particular, she emphasized recovery from fires that have occurred naturally (lightning strikes) or as a result of human activities on NTS. As part of her larger study design, she established three replicate sets of paired burned-undisturbed plots in blackbrush assemblages in Topopah and Mid Valleys (plots 18-19, 39-42; fig. 1). Two of these plot pairs were placed within a larger area that burned after a lightning strike in 1959, and the third was in an area that burned about 1950. Repeat photography of these sites (Appendix 1) shows several important changes in 36 years. First, Joshua trees (Yucca brevifolia) have increased dramatically in the background unburned areas, supporting the interpretation of Joshua trees as a relatively fast-growing and medium-lived species (Comanor and Clark, 2000). Second, mortality is so high on the disturbed plots, and growth rates are so large, that identifying common individuals in replicate photographs is difficult. In the burned plot, the cover and biomass of perennial vegetation - particularly 
woody shrubs - is much higher after 36 years of recovery, indicating that recovery is occurring relatively quickly (Appendix 1).

Reoccupation of the permanent plots verifies what is visibly obvious in the photographs. One important caveat about the disturbance monitoring plots is that they are always paired by straddling them at the formerly "hard edge" of disturbances. This emphasizes recovery at disturbance edges adjacent to intact vegetation stands. We have considered recovery on the plots and made observations in the larger study area. One important insight is that there may have been as few as one blackbrush establishment event on burned sites during the past 36-50 years. The pattern of germination also seems to be in a thin band along the edge of the disturbance, and no more than $20 \mathrm{~m}$ from the edge of the intact blackbrush stand. We hypothesize that this line may be linked to granivore activities.

In the 1960s and 1970s, grasses (notably Stipa speciosa) dominated the perennial vegetation on many of the plots in Yucca Flat and on plots recovering from disturbances (Hunter, 1994). The drought in 1989, one of the most severe in the history of southern Nevada, greatly reduced the grasses on the burned plots, and now the proportions of long-lived and short-lived shrubs are about equal to grasses in terms of vegetation cover (Appendix 1). Total cover on the burned plots is approaching the total cover on nearby undisturbed plots after 50 years of recovery, in accord with what is known of recovery in other types of disturbed areas (Webb and Thomas, 2003). Despite the increases in woody vegetation, the species composition is not even close to the undisturbed plots, and extrapolation suggests that as long as a millennium will be required for recovery of species composition.

\section{Benefits of Long-Term Monitoring}

The Beatley permanent plots represent long-term monitoring that is beneficial to researchers and land managers. They provide critical ecological information that can be collected in no other way. The history and data of the Beatley plots illustrate the opportunities to generate and test hypotheses about environmental change far outside the normal time-frame of 3-5 year funding cycles. From these plots, we are generating information about disturbance-recovery regimes, climate change, non-native plant invasions, and plant/animal interactions through synthesis of data collected over a half century. What we learn from the Beatley plots is stimulating discovery and insight for a geographic area much greater than that occupied by the sites, and of immediate use to managers, researchers, and the general public. The data from these plots are also important for generating significant ecological hypotheses that will be tested using field-scale experiments on and off of NTS. The continued preservation and periodic monitoring of the Beatley permanent plots will continue to provide an irreplaceable record of vegetation change over time in the Mojave Desert.

\section{REFERENCES}

Beatley, J.C., 1965, Ecology of the Nevada Test Site IV. Effects of the Sedan detonation on desert shrub vegetation in northeastern Yucca Flat, 1962-1965: Los Angeles, California, University of California at Los Angeles, Laboratory of Nuclear Medicine and Radiation Biology Report UCLA 12-571, 55 p.

Beatley, J.C., 1966a, Winter annual vegetation following a nuclear detonation in the northern Mojave Desert (Nevada Test site): Radiation Botany, v. 6, p. 69-82.

Beatley, J.C., 1966b, Ecological status of introduced brome grasses (Bromus spp.) in desert vegetation of southern Nevada: Ecology, v. 47, 548-554.

Beatley, J.C., 1967, Survival of winter annuals in the northern Mojave Desert: Ecology, v. 48, p. 745-750.

Beatley, J.C., 1969a, Biomass of desert winter annual plant populations in southern Nevada: Oikos, v. 20, p. 261273.

Beatley, J.C., 1969b, Dependence of desert rodents on winter annuals and precipitation: Ecology, v. 50, p. 721-724.

Beatley, J.C., 1973, Russian-thistle (Salsola) species in western United States: Journal of Range Management, v. 26, p. 225-226.

Beatley, J.C., 1974a, Phenological events and their environmental triggers in Mojave Desert ecosystems: Ecology, v. 55, p. 856-863.

Beatley, J.C., 1974b, Effects of rainfall and temperature on the distribution and behavior of Larrea tridentata (creosote-bush) in the Mojave Desert of Nevada: Ecology, v. 55, p. 245-261.

Beatley, J.C., 1975, Climates and vegetation pattern across the Mojave/Great Basin Desert transition of southern Nevada: American Midland Naturalist, v. 93, p. 53-70.

Beatley, J.C., 1976a, Environments of kangaroo rats (Dipodomys) and effects of environmental change on populations in southern Nevada: Journal of Mammalogy, v. 57, p. 67-93. 
Beatley, J.C., 1976b, Rainfall and fluctuating plant populations in relation to distributions and numbers of desert rodents in southern Nevada: Oecologia, v. 24, p. 21-42.

Beatley, J.C., 1976c, Vascular plants of the Nevada Test Site and central-southern Nevada: Springfield, Virginia, National Technical Information Service, TID-26881, $308 \mathrm{p}$.

Beatley, J.C., 1979, Shrub and tree data for plant associations across the Mojave/Great Basin desert transition of the Nevada Test Site, 1963-1975: Springfield, Virginia, National Technical Information Service, DOE/EV/ 2307-15 U-48, 52 p.

Beatley, J.C., 1980, Fluctuations and stability in climax shrub and woodland vegetation of the Mojave, Great Basin and Transition Deserts of southern Nevada: Israel Journal of Botany, v. 28, p. 149-168.

Comanor, P.O., and Clark, W.H., 2000, Preliminary growth rates and a proposed age-form classification for the Joshua tree, Yucca brevifolia (Agavaceae): Haseltonia, no. 7, p. 37-46.

Fehner, T.R., and Gosling, F.G., 2000, Origins of the Nevada Test Site: U.S. Department of Energy, DOE/MA-0518, $95 \mathrm{p}$.

Flint, A.L., and Davies, W.J., 1997, Meteorological data for water years 1988-94 from five weather stations at Yucca Mountain, Nevada: U.S. Geological Survey Open-File Report 96-462, $10 \mathrm{p}$.

Hunter, R.B.,1991, Bromus invasions on the Nevada Test Site: present status of $B$. rubens and B. tectorum with notes on their relationship to disturbance and altitude: Great Basin Naturalist, v. 51, p. 176-182.

Hunter, R.B., 1994, Status of the flora and fauna on the Nevada Test Site, 1989-1991: Las Vegas, U.S. Department of Energy, Nevada Operations Office, Report DOE/ NV/11432-57, 377 p.

Hunter, R.B., and Medica, P.A., 1992, Extent of land disturbance on the Nevada Test site, in Hunter, R.B. (ed.), Status of the flora and fauna on the Nevada Test Site, 1988: Las Vegas, Nevada, U.S. Department of Energy Report DOE/NV/10630-29, p. 3-7.

Nalder, I.A., and Wein, R.W., 1998, Spatial interpolation of climatic normals: Test of a new method in the Canadian boreal forest: Agricultural and Forest Meteorology, v. 92, p. 211-225.

Ostler, W.K., Hansen, D.J., Anderson, D.C., and Hall, D.B., 2000, Classification of vegetation on the Nevada Test Site: Las Vegas, Department of Energy, Nevada Operations Office, Report DOE/NV/11718-477.

Rickard, W.H., and Beatley, J.C., 1965, Canopy-coverage of the desert shrub vegetation mosaic of the Nevada Test Site: Ecology, v. 46, p. 524-529.
Slate, J.L., Berry, M.E., Rowley, P.D., Fridrich, C.J., Morgan, K.S., Workman, J.B., Young, O.D., Dixon, G.L., Williams, V.S., McKee, E.H., Ponce, D.A., Hildenbrand, T.G., Swadley, W.C., Lundstrom, S.C., Ekren, E.B., Warren, R.G., Cole, J.C., Fleck, R.J., Lanphere, M.A., Sawyer, D.A., Grunwald, D.J., Laczniak, R.J., Menges, C.M., Yount, J.C., and Jayko, A.S., 1999, Digital geologic map of the Nevada Test Site and vicinity, Nye, Lincoln, and Clark counties, Nevada, and Inyo County, California, revision 4, OpenFile Report, OF 99-0554-A, p. (1 sheet).

Stuckey, R.L., 1990, Janice Carson Beatley (1919-1987): Taxonomist and ecologist: Taxon, v. 39, p. 212-217.

Webb, R.H., 2002, Recovery of severely compacted soils in the Mojave Desert, California, USA: Arid Lands Research and Management, v. 16, p. 291-305.

Webb, R.H., and Wilshire, H.G., 1980, Recovery of soils and vegetation in a Mojave Desert ghost town, Nevada, U.S.A.: Journal of Arid Environments, v. 3, no. 6, p. 291-303.

Webb, R.H., and Thomas, K.A., 2003, Recoverability of severely disturbed soils and vegetation in the Mojave Desert, California, USA: Proceedings of the Dubai International Conference on Desertification, A.A. Balkema, in press.

Wells, P.V., 1961, Succession in desert vegetation on streets of a Nevada ghost town: Science, v. 134, p.670-671.

Wethermax, M. (managing editor), 2002, The Jepson desert manual, vascular plants of southeastern California: Berkeley, University of California Press, $624 \mathrm{p}$.

Wills, C.A., and Ostler, W.K., 2001, Ecology of the Nevada Test Site: An annotated bibliography: Las Vegas, Nevada, U.S. Department of Energy, DOE/NV/11718594, 386 p. 
Table 1A. Summary characteristics of the 68 Beatley permanent plots on the Nevada Test Site, Nye County, Nevada.

\begin{tabular}{|c|c|c|c|}
\hline $\begin{array}{c}\text { Plot } \\
\text { Number }\end{array}$ & $\begin{array}{c}\text { Nevada Quadrangle Sheet } \\
\left(7.5^{\prime}\right)\end{array}$ & Plant assemblage (1963-1975) ${ }^{1}$ & Plant assemblage $(2000-2001)^{1}$ \\
\hline 1 & Camp Desert Rock & Larrea-Ambrosia & Larrea-Krameria-Ambrosia \\
\hline 2 & Specter Range NW & Larrea-Grayia-Lycium & Larrea-Ambrosia-Ephedra \\
\hline 3 & Specter Range NW & Larrea-Ambrosia/Larrea-Lycium-Grayia & Ambrosia-Larrea-Lycium \\
\hline 4 & Specter Range NW & Larrea-Ambrosia/Larrea-Lycium-Grayia & Ambrosia-Oryzopsis-Larrea \\
\hline 5 & Specter Range NW & Larrea-Lycium-Grayia & Larrea-Krameria-Ambrosia \\
\hline 6 & Jackass Flats & Larrea-Ambrosia/Larrea-Lycium-Grayia & Ambrosia-Larrea-Ephedra \\
\hline 7 & Jackass Flats & Larrea-Ambrosia & Menodora-Larrea-Ambrosia \\
\hline 8 & Busted Butte & Larrea-Ambrosia & Ambrosia-Larrea-Oryzopsis \\
\hline 9 & Jackass Flats & Menodora-Ephedra & Menodora-Ephedra-Krameria \\
\hline 10 & Jackass Flats & Larrea-Ambrosia/Larrea-Lycium-Grayia & Ambrosia-Larrea-Lycium \\
\hline 11 & Jackass Flats & Larrea-Lycium-Grayia & Larrea-Ephedra-Krameria \\
\hline 12 & Jackass Flats & Larrea-Lycium-Grayia & Larrea-Ephedra-Krameria \\
\hline 13 & Skull Mountain & Coleogyne/Larrea-Grayia-Lycium & Coleogyne-Lycium-Ephedra \\
\hline 14 & Mine Mountain & Coleogyne/Grayia-Lycium & Menodora-Coleogyne-Lycium \\
\hline 15 & Jackass Flats & Larrea-Ambrosia/Larrea-Lycium-Grayia & Larrea-Lycium-Ambrosia \\
\hline 16 & Skull Mountain & Larrea-Lycium-Grayia & Larrea-Ambrosia-Lycium \\
\hline 17 & Skull Mountain & Larrea-Lycium-Grayia & Larrea-Lycium-Ephedra \\
\hline 18 & Topopah Spring & Coleogyne & Coleogyne-Ephedra \\
\hline 19 & Topopah Spring & Ephedra & Ephedra-Encelia \\
\hline 20 & Cane Spring & Larrea-Ambrosia/Larrea-Lycium-Grayia & Larrea-Ambrosia-Ceratoides \\
\hline 21 & Cane Spring & Larrea-Lycium-Grayia & Larrea-Oryzopsis \\
\hline 22 & Cane Spring & Larrea-Ambrosia & Larrea-Ambrosia-Psorothamnus \\
\hline 23 & Frenchman Lake & Larrea-Ambrosia/Larrea-Lycium-Grayia & Larrea-Ambrosia \\
\hline 24 & Cane Spring & Larrea-Ambrosia/Larrea-Lycium-Grayia & Larrea-Ambrosia-Ephedra \\
\hline 25 & Cane Spring & Larrea-Ambrosia/Larrea-Lycium-Grayia & Ambrosia-Larrea-Ephedra \\
\hline 26 & Cane Spring & Grayia-Lycium & Ephedra-Hymenoclea-Lycium \\
\hline 27 & Yucca Lake & Larrea-Lycium-Grayia & Larrea-Krameria-Ambrosia \\
\hline 28 & Frenchman Lake & Larrea-Ambrosia & Larrea-Ambrosia \\
\hline 29 & Frenchman Lake & Larrea-Atriplex & Ephedra-Atriplex confertifolia-Krameria \\
\hline 30 & Frenchman Lake & Atriplex canescens & Atriplex canescens \\
\hline 31 & Frenchman Lake & Atriplex confertifolia & Atriplex confertifolia \\
\hline 32 & Frenchman Lake SE & Larrea-Ambrosia & Ambrosia-Menodora-Krameria \\
\hline 33 & Frenchman Lake & Larrea-Psorothamnus & Larrea-Krameria \\
\hline 34 & Frenchman Lake & Larrea-Lycium shockleyi-Atriplex & Lycium shockleyi-Larrea \\
\hline 35 & Frenchman Lake & Larrea-Lycium shockleyi-Atriplex & Atriplex confertifolia \\
\hline 36 & Frenchman Lake & Larrea-Lycium shockleyi-Atriplex & Larrea-Lycium shockleyi-Ambrosia \\
\hline 37 & Frenchman Lake & Lycium shockleyi-Atriplex & Lycium shockleyi \\
\hline 38 & Frenchman Lake & Lycium pallidum-Grayia & Lycium pallidum-Grayia \\
\hline
\end{tabular}


Table 1A. Summary characteristics of the 68 Beatley permanent plots on the Nevada Test Site, Nye County, Nevada.

\begin{tabular}{|c|c|c|c|}
\hline $\begin{array}{c}\text { Plot } \\
\text { Number }\end{array}$ & $\begin{array}{c}\text { Nevada Quadrangle Sheet } \\
\left(7.5^{\prime}\right)\end{array}$ & Plant assemblage (1963-1975) ${ }^{1}$ & Plant assemblage (2000-2001) \\
\hline 39 & Mine Mountain & Coleogyne/Grayia-Lycium & Coleogyne-Ephedra-Lycium \\
\hline 40 & Mine Mountain & Ephedra-Lycium & Achnatherum-Ephedra-Hymenoclea \\
\hline 41 & Mine Mountain & Coleogyne & Coleogyne-Ephedra \\
\hline 42 & Mine Mountain & Ephedra & Ephedra \\
\hline 43 & Yucca Lake & Larrea-Atriplex/Coleogyne & Larrea-Ephedra-Coleogyne \\
\hline 44 & Yucca Lake & Coleogyne/Larrea-Grayia-Lycium & Coleogyne-Larrea-Ephedra \\
\hline 45 & Yucca Lake & Coleogyne/Grayia-Lycium & Coleogyne-Ephedra-Lycium \\
\hline 46 & Yucca Flat & Grayia-Lycium & Ephedra-Lycium-Grayia \\
\hline 47 & Tippipah Spring & Larrea-Grayia-Lycium & Coleogyne-Ericameria-Artemisia tridentata \\
\hline 48 & Yucca Flat & Grayia-Larrea & Larrea-Hymenoclea \\
\hline 49 & Yucca Flat & Larrea-Grayia-Lycium & Larrea-Ephedra-Lycium \\
\hline 50 & Yucca Flat & Grayia-Lycium & Oryzopsis-Atriplex-Lycium \\
\hline 51 & Rainier Mesa & Coleogyne & Coleogyne-Ephedra \\
\hline 52 & Oak Spring & Hymenoclea-Lycium & Oryzopsis-Hymenoclea \\
\hline 53 & Oak Spring & Ceratoides-Hymenoclea & Oryzopsis-Stipa \\
\hline 54 & Oak Spring & Lycium-Larrea-Coleogyne & Larrea-Oryzopsis-Lycium \\
\hline 55 & Yucca Flat & Larrea-Lycium-Grayia & Larrea-Lycium \\
\hline 56 & Paiute Ridge & Coleogyne/Larrea-Grayia-Lycium & Coleogyne-Larrea \\
\hline $57 \mathrm{~A}$ & Paiute Ridge & Larrea-Grayia-Lycium & Mixed disturbed-undisturbed \\
\hline $57 \mathrm{~N}$ & Paiute Ridge & Larrea-Grayia-Lycium & Larrea-Lycium-Ceratoides \\
\hline 58 & Yucca Flat & Atriplex-Ceratoides & Destroyed \\
\hline 59 & Yucca Lake & Atriplex-Kochia & Mixed disturbed-undisturbed \\
\hline 60 & Plutonium Valley & Grayia-Lycium & Chrysothamnus viscidiflorus-Lycium-Grayia \\
\hline 61 & Ammonia Tanks & Artemisia nova & Artemisia nova \\
\hline 62 & Ammonia Tanks & Artemisia tridentata & Artemisia tridentata \\
\hline 63 & Ammonia Tanks & Artemisia nova-Pinyon-Juniper & Juniper-Pinyon-Artemisia nova \\
\hline 64 & Rainier Mesa & Artemisia nova-Pinyon-Juniper & Purshia-Pinyon-Artemisia nova \\
\hline 65 & Tippipah Spring & Atriplex canescens & Oryzopsis-Eriogonum kearneyi \\
\hline 66 & Skull Mountain & Larrea-Grayia-Lycium & Larrea-Ephedra-Thamnosma \\
\hline 67 & Skull Mountain & Ephedra-Lycium & Ephedra-Thamnosma-Larrea \\
\hline 68 & Rainier Mesa & Artemisia tridentata-Pinyon-Juniper & Artemisia tridentata-Pinyon-Juniper \\
\hline
\end{tabular}

1. Beatley's plant assemblage (1963-1975) is based on her interpretation of the mappable vegetation unit, which may or may not reflect dominant species by cover. Her use of ' $/$ reflects her identification of low-cover but important subdominants. Our plant assemblage type is based on the top three or less species in terms of cover. Therefore, the latter two columns of this table cannot be directly compared. 
Table 1B. Summary characteristics of the 68 Beatley permanent plots on the Nevada Test Site, Nye County, Nevada.

\begin{tabular}{|c|c|c|c|c|c|c|}
\hline $\begin{array}{c}\text { Plot } \\
\text { Number }\end{array}$ & $\begin{array}{l}\text { 1st Corner } \\
\text { Easting }\end{array}$ & $\begin{array}{l}\text { 1st Corner² } \\
\text { Northing }\end{array}$ & $\begin{array}{l}\text { Elevation } \\
\qquad(\mathrm{m})^{2}\end{array}$ & $\begin{array}{l}\text { Annual Precipitation } \\
(\mathrm{mm})\end{array}$ & $\begin{array}{l}\text { Original Purpose of } \\
\text { Plot }\end{array}$ & Plot Condition \\
\hline 1 & 586082 & 4055318 & 1020 & 142 & Undisturbed & Good \\
\hline 2 & 577798 & 4058539 & 1082 & 179 & Undisturbed & Good \\
\hline 3 & 572425 & 4060844 & 1035 & 175 & Undisturbed, IBP & Good \\
\hline 4 & 573610 & 4061656 & 1049 & 151 & Undisturbed, IBP & Good \\
\hline 5 & 567150 & 4067288 & 1114 & 142 & Undisturbed & Good \\
\hline 6 & 565733 & 4069659 & 1075 & 139 & Undisturbed & Good \\
\hline 7 & 557383 & 4064717 & 979 & 122 & Undisturbed & Good \\
\hline 8 & 555685 & 4067788 & 961 & 117 & Undisturbed & Good \\
\hline 9 & 556660 & 4072190 & 1009 & 130 & Undisturbed & Good \\
\hline 10 & 560790 & 4072331 & 1051 & 125 & Undisturbed & Good \\
\hline 11 & 565838 & 4077127 & 1202 & 142 & Undisturbed & Good \\
\hline 12 & 565996 & 4077753 & 1245 & 149 & Undisturbed & Good \\
\hline 13 & 568006 & 4078978 & 1297 & 167 & Undisturbed & Good \\
\hline 14 & 570374 & 4082145 & 1465 & 189 & Undisturbed & Good \\
\hline 15 & 566051 & 4070942 & 1149 & 132 & Undisturbed & Good \\
\hline 16 & 568895 & 4072581 & 1177 & 147 & Undisturbed & Good \\
\hline 17 & 571147 & 4073855 & 1269 & 141 & Undisturbed & Good \\
\hline 18 & 564458 & 4084205 & 1452 & 228 & Control for 19 & Good \\
\hline 19 & 564392 & 4084216 & 1442 & 219 & Burned in 1959 & Good \\
\hline 20 & 587360 & 4067886 & 1005 & 139 & Undisturbed & Good \\
\hline 21 & 588207 & 4070688 & 982 & 133 & Undisturbed & Good \\
\hline 22 & 587651 & 4072554 & 1001 & 131 & Undisturbed & Good \\
\hline 23 & 590196 & 4074435 & 979 & 123 & Undisturbed & Good \\
\hline 24 & 588148 & 4079095 & 1048 & 141 & Undisturbed & $\begin{array}{c}\text { Minor } \\
\text { damage }\end{array}$ \\
\hline 25 & 584095 & 4077437 & 1116 & 161 & Undisturbed & Good \\
\hline 26 & 579842 & 4074196 & 1232 & 185 & Undisturbed & Good \\
\hline 27 & 584780 & 4083308 & 1132 & 154 & Undisturbed & Good \\
\hline 28 & 596296 & 4079223 & 1007 & 121 & Undisturbed & Good \\
\hline 29 & 595950 & 4079392 & 987 & 126 & Undisturbed & Good \\
\hline 30 & 594597 & 4076762 & 957 & 126 & Undisturbed & Good \\
\hline 31 & 595126 & 4075982 & 954 & 130 & Undisturbed & Good \\
\hline 32 & 601358 & 4077357 & 1031 & 122 & Undisturbed & Good \\
\hline 33 & 599387 & 4075488 & 1009 & 119 & Undisturbed & Good \\
\hline 34 & 597722 & 4073049 & 935 & 124 & Undisturbed & Good \\
\hline 35 & 597639 & 4070916 & 1015 & 136 & Undisturbed & Good \\
\hline 36 & 596075 & 4071221 & 935 & 126 & Undisturbed & Good \\
\hline 37 & 596126 & 4071396 & 953 & 129 & Undisturbed & Good \\
\hline 38 & 590648 & 4072451 & 959 & 121 & Undisturbed & Good \\
\hline
\end{tabular}


Table 1B. Summary characteristics of the 68 Beatley permanent plots on the Nevada Test Site, Nye County, Nevada.

\begin{tabular}{|c|c|c|c|c|c|c|}
\hline $\begin{array}{l}\text { Plot } \\
\text { Number }\end{array}$ & $\begin{array}{l}\text { 1st Corner² } \\
\text { Easting }\end{array}$ & $\begin{array}{l}\text { 1st Corner² } \\
\text { Northing }\end{array}$ & $\begin{array}{l}\text { Elevation } \\
\qquad(\mathrm{m})^{2}\end{array}$ & $\begin{array}{l}\text { Annual Precipitation } \\
\qquad(\mathrm{mm})\end{array}$ & $\begin{array}{c}\text { Original Purpose of } \\
\text { Plot }\end{array}$ & Plot Condition \\
\hline 39 & 574064 & 4086900 & 1467 & 204 & Control for 39 & Good \\
\hline 40 & 574031 & 4086764 & 1467 & 198 & Burned $\sim 1950$ & Good \\
\hline 41 & 572680 & 4090850 & 1538 & 241 & Control for 42 & Good \\
\hline 42 & 572661 & 4090761 & 1538 & 233 & Burned 1959 & Good \\
\hline 43 & 582621 & 4090807 & 1274 & 180 & Undisturbed & Good \\
\hline 44 & 580985 & 4092999 & 1257 & 182 & Undisturbed & Good \\
\hline 45 & 580147 & 4093321 & 1284 & 187 & Undisturbed & Good \\
\hline 46 & 581411 & 4096052 & 1245 & 179 & Undisturbed & Good \\
\hline 47 & 573772 & 4097654 & 1472 & 232 & Undisturbed & Good \\
\hline 48 & 580825 & 4101300 & 1283 & 164 & Tower (T-1) shots & Good \\
\hline 49 & 581867 & 4102183 & 1258 & 166 & Control for 48 & Good \\
\hline 50 & 583431 & 4103937 & 1337 & 165 & Undisturbed & Good \\
\hline 51 & 576238 & 4115181 & 1535 & 225 & Undisturbed & Good \\
\hline 52 & 586207 & 4115020 & 1359 & 167 & Sedan 1962 & Good \\
\hline 53 & 586167 & 4113778 & 1431 & 164 & Sedan 1962 & Good \\
\hline 54 & 586774 & 4112900 & 1362 & 174 & Sedan 1962 & Good \\
\hline 55 & 586582 & 4108939 & 1330 & 159 & Balloon Tests & Good \\
\hline 56 & 589693 & 4104467 & 1339 & 165 & Undisturbed & Good \\
\hline $57 \mathrm{~A}$ & 589579 & 4100067 & 1271 & 177 & Undisturbed & Damaged \\
\hline $57 \mathrm{~N}$ & 589579 & 4100067 & 1271 & 177 & Undisturbed & Good \\
\hline 58 & 37.002 & 116.042 & 1200 & 178 & Undisturbed & Destroyed \\
\hline 59 & 585288 & 4093008 & 1195 & 177 & Undisturbed & Damaged \\
\hline 60 & 589723 & 4091158 & 1216 & 164 & Undisturbed & Good \\
\hline 61 & 565988 & 4110149 & 1746 & 204 & Undisturbed & Good \\
\hline 62 & 565902 & 4110810 & 1749 & 203 & Undisturbed & Good \\
\hline 63 & 566361 & 4117467 & 2001 & 270 & Undisturbed & Good \\
\hline 64 & 570051 & 4117144 & 2274 & 270 & Undisturbed & Damaged \\
\hline 65 & 566710 & 4106154 & 1697 & 182 & Undisturbed & Good \\
\hline 66 & 574534 & 4074529 & 1294 & 177 & Control for 67 & Good \\
\hline 67 & 574486 & 4074440 & 1294 & 177 & Ghost town & Good \\
\hline 68 & 566807 & 4114414 & 1846 & 261 & Undisturbed & Good \\
\hline
\end{tabular}

2. UTM (zone 12S) and elevation data are from hand-held GPS units, which reported an accuracy of between \pm 3 and $\pm 5 \mathrm{~m}$. 
Table 1C. Summary characteristics of the 68 Beatley permanent plots on the Nevada Test Site, Nye County, Nevada.

\begin{tabular}{|c|c|c|c|c|c|c|}
\hline $\begin{array}{c}\text { Plot } \\
\text { Number }\end{array}$ & $\begin{array}{l}\text { NTS }^{3} \\
\text { Area }\end{array}$ & $\begin{array}{l}\text { NTS }^{3} \\
\text { Grid }\end{array}$ & $\begin{array}{l}\text { Geomorphic } \\
\text { Surface }\end{array}$ & $\begin{array}{c}\text { Slope }^{4} \\
\text { (degrees) }\end{array}$ & $\begin{array}{c}\text { Aspect }^{4} \\
\text { (degrees) }^{\text {deges }}\end{array}$ & $\begin{array}{c}\text { Elevation }^{5} \text { Above } \\
\text { Playa (m) }\end{array}$ \\
\hline 1 & 22 & S-7 & alluvial fan & 16.8 & 308.3 & NA \\
\hline 2 & 22 & $\mathrm{~N}-8$ & alluvial fan & 2.3 & 161.6 & NA \\
\hline 3 & 25 & $\mathrm{~J}-10$ & alluvial fan & 11.7 & 346.0 & NA \\
\hline 4 & 25 & $\mathrm{~J}-11$ & alluvial fan & 6.4 & 63.4 & NA \\
\hline 5 & 25 & G-13 & alluvial fan & 6.1 & 144.5 & NA \\
\hline 6 & 25 & E-15 & alluvial fan & 5.9 & 194.0 & NA \\
\hline 7 & 25 & ZZ-15 & alluvial fan & 5.1 & 98.1 & NA \\
\hline 8 & 25 & $X X-14$ & alluvial fan & 1.4 & 0.0 & NA \\
\hline 9 & 25 & ZZ-16 & alluvial fan & 3.0 & 135.0 & NA \\
\hline 10 & 25 & B-16 & alluvial fan & 1.4 & 270.0 & NA \\
\hline 11 & 25 & F-19 & alluvial fan & 11.7 & 104.0 & NA \\
\hline 12 & 25 & F-19 & alluvial fan & 12.0 & 229.8 & NA \\
\hline 13 & 25 & G-19 & alluvial fan & 5.9 & 104.0 & NA \\
\hline 14 & 25 & $\mathrm{I}-21$ & broken terrain & 11.2 & 18.4 & NA \\
\hline 15 & 25 & F-15 & alluvial fan & 5.1 & 135.0 & NA \\
\hline 16 & 25 & $\mathrm{H}-17$ & alluvial fan & 3.0 & 135.0 & NA \\
\hline 17 & 25 & $\mathrm{I}-17$ & alluvial fan & 10.0 & 315.0 & NA \\
\hline 18 & 25 & F-23 & alluvial fan & 20.0 & 275.9 & NA \\
\hline 19 & 25 & $\mathrm{~F}-23$ & alluvial fan & 9.0 & 135.0 & NA \\
\hline 20 & 5 & $\mathrm{~T}-14$ & alluvial fan & 6.1 & 315.0 & 58 Frenchman \\
\hline 21 & 5 & U-15 & alluvial fan & 2.9 & 0.0 & 40 Frenchman \\
\hline 22 & 5 & $\mathrm{~T}-16$ & alluvial fan & 5.1 & 278.1 & 24 Frenchman \\
\hline 23 & 5 & $\mathrm{~V}-17$ & alluvial fan & 9.9 & 90.0 & 80 Frenchman \\
\hline 24 & 5 & $\mathrm{U}-20$ & alluvial fan & 10.2 & 326.3 & 88 Frenchman \\
\hline 25 & 5 & R-19 & alluvial fan & 7.9 & 84.8 & 271 Frenchman \\
\hline 26 & 5 & O-17 & alluvial fan & 10.1 & 219.3 & 890 Frenchman \\
\hline 27 & 6 & $\mathrm{R}-22$ & alluvial fan & 2.9 & 0.0 & 183 Frenchman \\
\hline 28 & 5 & Z-19 & alluvial fan & 8.1 & 344.8 & 49 Frenchman \\
\hline 29 & 5 & Z-19 & alluvial fan & 12.9 & 135.0 & 49 Frenchman \\
\hline 30 & 5 & Y-18 & alluvial fan & 4.0 & 45.0 & 7 Frenchman \\
\hline 31 & 5 & Y-18 & playa & 10.1 & 129.3 & 0.7 Frenchman \\
\hline 32 & Nellis & W64C & alluvial fan & no data & no data & 70 Frenchman \\
\hline 33 & Nellis & W64B & alluvial fan & 5.9 & 104.0 & 52 Frenchman \\
\hline 34 & 5 & Z-16 & playa & 11.7 & 115.0 & 2 Frenchman \\
\hline 35 & 5 & Z-15 & broken terrain & 31.3 & 189.5 & 67 Frenchman \\
\hline 36 & 5 & Z-15 & playa & 2.3 & 108.4 & 3 Frenchman \\
\hline 37 & 5 & Z-15 & playa & 6.1 & 315.0 & 3 Frenchman \\
\hline 38 & 5 & W-16 & alluvial fan & 3.2 & 153.4 & 7 Frenchman \\
\hline 39 & 14 & L-24 & alluvial fan & 8.3 & 329.0 & NA \\
\hline 40 & 14 & L-24 & alluvial fan & 14.0 & 180.0 & NA \\
\hline 41 & 14 & $\mathrm{~K}-26$ & alluvial fan & 15.6 & 26.6 & NA \\
\hline 42 & 14 & $\mathrm{~K}-26$ & alluvial fan & 6.4 & 296.6 & NA \\
\hline 43 & 6 & Q-26 & alluvial fan & 11.4 & 60.3 & 70 Yucca \\
\hline 44 & 6 & $\mathrm{P}-27$ & alluvial fan & 4.5 & 251.6 & 61 Yucca \\
\hline 45 & 6 & $\mathrm{P}-27$ & alluvial fan & 3.7 & 168.7 & 82 Yucca \\
\hline 46 & 1 & P-29 & alluvial fan & 13.8 & 14.7 & 12 Yucca \\
\hline
\end{tabular}


Table 1C. Summary characteristics of the 68 Beatley permanent plots on the Nevada Test Site, Nye County, Nevada.

\begin{tabular}{|c|c|c|c|c|c|c|}
\hline $\begin{array}{c}\text { Plot } \\
\text { Number }\end{array}$ & $\begin{array}{l}\text { NTS }^{3} \\
\text { Area }\end{array}$ & $\begin{array}{l}\text { NTS }^{3} \\
\text { Grid }\end{array}$ & $\begin{array}{l}\text { Geomorphic } \\
\text { Surface }\end{array}$ & $\begin{array}{c}\text { Slope }^{4} \\
\text { (degrees) }\end{array}$ & $\begin{array}{c}\text { Aspect }^{4} \\
\text { (degrees) }^{\text {degres }}\end{array}$ & $\begin{array}{c}\text { Elevation }^{5} \text { Above } \\
\text { Playa (m) }\end{array}$ \\
\hline 47 & 16 & $\mathrm{~K}-30$ & alluvial fan & 13.5 & 351.0 & $\mathrm{NA}$ \\
\hline 48 & 1 & P-32 & alluvial fan & 7.7 & 111.8 & 82 Yucca \\
\hline 49 & 1 & P-32 & alluvial fan & 3.0 & 135.0 & 67 Yucca \\
\hline 50 & 2 & P-37 & alluvial fan & 1.0 & 135.0 & 61 Yucca \\
\hline 51 & 12 & M-39 & alluvial fan & 2.3 & 161.6 & 317 Yucca \\
\hline 52 & 10 & $\mathrm{~T}-39$ & alluvial fan & 8.3 & 121.0 & 140 Yucca \\
\hline 53 & 10 & $\mathrm{~T}-38$ & alluvial fan & 6.4 & 333.4 & 149 Yucca \\
\hline 54 & 10 & $\mathrm{~T}-38$ & alluvial fan & 5.2 & 326.3 & 152 Yucca \\
\hline 55 & 9 & U-36 & alluvial fan & 13.9 & 315.0 & 119 Yucca \\
\hline 56 & 7 & $\mathrm{~V}-33$ & alluvial fan & 15.4 & 329.9 & 131 Yucca \\
\hline 57 & 3 & $\mathrm{~V}-31$ & alluvial fan & 11.7 & 335.0 & 61 Yucca \\
\hline 58 & 3 & $S-29$ & alluvial fan & 4.5 & 288.4 & 14 Yucca \\
\hline 59 & 6 & S-27 & playa & 9.1 & 141.3 & 3 Yucca \\
\hline 60 & 6 & $\mathrm{~V}-26$ & alluvial fan & 6.4 & 206.6 & 10 Yucca \\
\hline 61 & 18 & F-37 & broken terrain & 58.8 & 328.0 & NA \\
\hline 62 & 18 & F-37 & broken terrain & 3.2 & 296.6 & NA \\
\hline 63 & 19 & F-40 & alluvial fan & 12.2 & 170.0 & NA \\
\hline 64 & 12 & $\mathrm{I}-40$ & mesa & 22.0 & 82.9 & NA \\
\hline 65 & 18 & G-34 & alluvial fan & 34.5 & 123.1 & NA \\
\hline 66 & 26 & L-17 & alluvial fan & 17.8 & 308.7 & NA \\
\hline 67 & 26 & L-17 & alluvial fan & 3.7 & 78.7 & NA \\
\hline 68 & 12 & G-39 & broken terrain & 22.3 & 322.4 & NA \\
\hline
\end{tabular}

3. Area and grid numbers come from long-established maps and are the primary location information used by Department of Energy employees in describing locations on NTS. 4. From analysis of the 10-m digital elevation model (see fig. 1). 5. NA, the plot is not in a closed drainage basin with a playa. The two major playas -- Frenchman and Yucca Lakes -- are identified. 
Table 2A. Climate stations in the vicinity of the Nevada Test Site.

\begin{tabular}{|c|c|c|c|c|c|c|}
\hline Site Name & $\begin{array}{l}\text { Station } \\
\text { ID }\end{array}$ & $\begin{array}{c}\text { Latitude } \\
\text { (North) }\end{array}$ & $\begin{array}{c}\text { Longitude } \\
\text { (West) }\end{array}$ & $\begin{array}{l}\text { Elevation } \\
\text { (m) }\end{array}$ & $\begin{array}{l}\text { Beginning } \\
\text { Date }\end{array}$ & Ending Date \\
\hline Buster Jangle $\mathrm{Y}$ & na & $37^{\circ} 03^{\prime} 46^{\prime \prime}$ & $116^{\circ} 03^{\circ} 09^{\prime \prime}$ & 1241 & $2 / 1960$ & current \\
\hline Well 5B & na & $36^{\circ} 48^{\prime} 07^{\prime \prime}$ & $115^{\circ} 57^{\prime} 55^{\prime \prime}$ & 939 & $10 / 1962$ & current \\
\hline Yucca Dry Lake & na & $36^{\circ} 57^{\prime} 23^{\prime \prime}$ & $116^{\circ} 02^{\prime} 51^{\prime \prime}$ & 1196 & $5 / 1958$ & current \\
\hline Rainier Mesa & na & $37^{\circ} 11^{\prime} 28^{\prime \prime}$ & $116^{\circ} 12^{\prime} 55^{\prime \prime}$ & 2283 & $3 / 1959$ & current \\
\hline Mid Valley & na & $36^{\circ} 58^{\prime} 21^{\prime \prime}$ & $116^{\circ} 110^{\prime} 19^{\prime \prime}$ & 1420 & $9 / 1964$ & current \\
\hline PHS Farm & na & $37^{\circ} 12^{\prime} 32^{\prime \prime}$ & $116^{\circ} 02^{\prime} 19^{\prime \prime}$ & 1392 & $10 / 1964$ & current \\
\hline Tippipah Springs 2 & na & $37^{\circ} 03^{\prime} 11^{\prime \prime}$ & $116^{\circ} 11^{\prime} 29^{\prime \prime}$ & 1518 & $5 / 1960$ & current \\
\hline Little Feller 2 & na & $37^{\circ} 07^{\prime} 05^{\prime \prime}$ & $116^{\circ} 18^{\prime} 14^{\prime \prime}$ & 1561 & $8 / 1976$ & current \\
\hline Pahute Mesa 1 & na & $37^{\circ} 14^{\prime} 56^{\prime \prime}$ & $116^{\circ} 26^{\prime} 15^{\prime \prime}$ & 1997 & $1 / 1964$ & current \\
\hline Mercury & na & $36^{\circ} 39^{\prime} 39^{\prime \prime}$ & $116^{\circ} 00^{\prime} 36^{\prime \prime}$ & 1149 & $9 / 1962$ & current \\
\hline Desert Rock & na & $36^{\circ} 37^{\prime} 16^{\prime \prime}$ & $116^{\circ} 01^{\prime} 33^{\prime \prime}$ & 991 & $10 / 1963$ & current \\
\hline Jackass Flats & na & $36^{\circ} 47^{\prime} 05^{\prime \prime}$ & $116^{\circ} 17^{\prime} 20^{\prime \prime}$ & 1043 & $12 / 1957$ & current \\
\hline Cane Springs & na & $36^{\circ} 48^{\prime} 44^{\prime \prime}$ & $116^{\circ} 05^{\prime} 29^{\prime \prime}$ & 1219 & $9 / 1964$ & current \\
\hline 40 Mile Canyon & na & $37^{\circ} 02^{\prime} 57^{\prime \prime}$ & $116^{\circ} 17^{\prime} 15^{\prime \prime}$ & 1469 & $2 / 1960$ & current \\
\hline Rock Valley & na & $36^{\circ} 41^{\prime} 07^{\prime \prime}$ & $116^{\circ} 11^{\prime} 32^{\prime \prime}$ & 1036 & $3 / 1963$ & current \\
\hline E Tunnel & na & $37^{\circ} 11^{\prime} 30^{\prime \prime}$ & $116^{\circ} 12^{\prime} 04^{\prime \prime}$ & 1905 & $12 / 1996$ & current \\
\hline Area 6 & na & $36^{\circ} 53^{\prime} 58^{\prime \prime}$ & $116^{\circ} 02^{\prime} 04^{\prime \prime}$ & 1131 & 2/1997 & current \\
\hline Amargosa Farms & 150 & $36^{\circ} 34^{\prime} 18^{\prime \prime}$ & $116^{\circ} 27^{\prime} 43^{\prime \prime}$ & 747 & $12 / 1 / 1965$ & $12 / 31 / 2000$ \\
\hline Beatty & 714 & $36^{\circ} 55^{\prime} 00^{\prime \prime}$ & $116^{\circ} 45^{\prime} 00^{\prime \prime}$ & 1007 & 7/1/1948 & $11 / 30 / 1972$ \\
\hline Beatty $8 \mathrm{~N}$ & 718 & $36^{\circ} 59^{\prime} 42^{\prime \prime}$ & $116^{\circ} 43^{\prime} 06^{\prime \prime}$ & 1082 & $12 / 1 / 1972$ & $12 / 31 / 2000$ \\
\hline Desert NWR & 2243 & $36^{\circ} 26^{\prime} 16^{\prime \prime}$ & $115^{\circ} 21^{\prime} 35^{\prime \prime}$ & 890 & 7/1/1948 & $12 / 31 / 2000$ \\
\hline Diablo & 2276 & $37^{\circ} 55^{\prime} 00^{\prime \prime}$ & $116^{\circ} 03^{\prime} 00^{\prime \prime}$ & 1556 & $9 / 1 / 1959$ & $3 / 22 / 1979$ \\
\hline Hiko & 3671 & $37^{\circ} 33^{\prime} 29^{\prime \prime}$ & $115^{\circ} 13^{\prime} 25^{\prime \prime}$ & 1189 & $9 / 1 / 1989$ & $12 / 31 / 2000$ \\
\hline Indian Springs & 3980 & $36^{\circ} 35^{\prime} 00^{\prime \prime}$ & $115^{\circ} 41^{\prime} 00^{\prime \prime}$ & 952 & $7 / 1 / 1948$ & $6 / 30 / 1964$ \\
\hline Key Pittman WMA & 4143 & $37^{\circ} 37^{\prime} 00^{\prime \prime}$ & $115^{\circ} 13^{\prime} 00^{\prime \prime}$ & 1204 & $3 / 1 / 1964$ & $5 / 31 / 1989$ \\
\hline Lathrop Wells & 4457 & $36^{\circ} 39^{\prime} 00^{\prime \prime}$ & $116^{\circ} 24^{\prime} 00^{\prime \prime}$ & 814 & 7/1/1948 & $1 / 31 / 1963$ \\
\hline Lathrop Wells 16 SSE & 4473 & $36^{\circ} 25^{\prime} 00^{\prime \prime}$ & $116^{\circ} 21^{\prime} 00^{\prime \prime}$ & 665 & $11 / 1 / 1970$ & $8 / 31 / 1977$ \\
\hline Pahrump & 5890 & $36^{\circ} 16^{\prime} 43^{\prime \prime}$ & $116^{\circ} 00^{\prime} 11^{\prime \prime}$ & 815 & $11 / 1 / 1948$ & $12 / 31 / 2000$ \\
\hline Penoyer Valley & 6130 & $37^{\circ} 38^{\prime} 55^{\prime \prime}$ & $115^{\circ} 48^{\prime} 03^{\prime \prime}$ & 1463 & $3 / 1 / 1967$ & $12 / 31 / 2000$ \\
\hline Tempiute 4 NW & 7983 & $37^{\circ} 41^{\prime} 00^{\prime \prime}$ & $115^{\circ} 43^{\prime} 00^{\prime \prime}$ & 1490 & $4 / 29 / 1972$ & $12 / 31 / 1985$ \\
\hline
\end{tabular}

Table 2B. Weather stations at Yucca Mountain, Nevada (Flint and Davies, 1996).

\begin{tabular}{cccccc}
\hline Station Name & $\begin{array}{c}\text { Northing } \\
\text { UTMz11 } \\
\text { NAD 27 }\end{array}$ & $\begin{array}{c}\text { Easting } \\
\text { UTM z11 } \\
\text { NAD 27 }\end{array}$ & $\begin{array}{c}\text { Elevation } \\
(\mathbf{m})\end{array}$ & $\begin{array}{c}\text { Beginning } \\
\text { Date }\end{array}$ & $\begin{array}{c}\text { Ending } \\
\text { Date }\end{array}$ \\
\hline Weather Station 1 (WX1) & 4076521 & 550424 & 1163 & $1 / 1 / 1988$ & $9 / 30 / 1994$ \\
Weather Station 2a (WX2a) & 4075931 & 564332 & 1154 & $4 / 18 / 1987$ & $3 / 7 / 1989$ \\
Weather Station 2b (WX2b) & 4071986 & 563430 & 1055 & $3 / 7 / 1989$ & $9 / 30 / 1994$ \\
Weather Station 3 (WX3) & 4080316 & 548038 & 1351 & $7 / 16 / 1987$ & $9 / 30 / 1994$ \\
Weather Station 4a (WX4a) & 4076856 & 547504 & 1498 & $7 / 1 / 1997$ & $6 / 8 / 1990$ \\
Weather Station 4b (WX4b) & 4075990 & 547481 & 1489 & $6 / 8 / 1990$ & $9 / 30 / 1994$ \\
Weather Station 5a (WX5a) & 4083775 & 546453 & 1789 & $6 / 23 / 1988$ & $10 / 4 / 1993$ \\
Weather Station 5b (WX5b) & 4082370 & 547984 & 1563 & $10 / 4 / 1993$ & $9 / 30 / 1994$ \\
\hline
\end{tabular}


APPENDIX 1 


\section{Explanation for Appendix Tables}

This appendix consists of tables and figures for each of the 68 permanent plots on the Nevada Test Site originally established by Dr. Janice C. Beatley in 1962. The figures are self explanatory photographic matches, and the photographic information appears in Table 69. The following explanations apply to the tables, which constitute the bulk of this appendix. Each plot is represented by at least one A table, which contains summary geospatial and attribute data, and at least one B table, which gives the summary data for perennial plants.

For the A tables, area and grid coordinates come from maps of NTS and are the primary locating coordinates for explaining where the plots are to Department of Energy personnel who control access to NTS. The GPS readings are from hand-held units that typically have an estimated position error (EPE) of \pm 3 and $\pm 5 \mathrm{~m}$. These readings were used to calculate the plot elevation from $10 \mathrm{~m}$ digital-elevation models (DEMs) of NTS. Similarly, plot slope and aspect were calculated from these DEMs. The exception is plot 32, which lies east of NTS on Nellis Air Force base and for which a 10-m DEM is unavailable. NA -- not recorded or not applicable. Annual precipitation was both measured and modeled as described in the text. The parent material and substrate on the alluvial fans was determined through inspection of the plot. The "Slate geologic unit" is determined from digital surficial geology maps published by Slate and others (1999) and available as Arc/Info files. The Beatley plant assemblage is derived from Beatley (1979) and does not necessarily reflect numerical dominance by cover; the same is true for the Ostler-Hanson plant-assemblage and land-unit classification, which is derived from digital vegetation-landform maps of NTS (Ostler and others, 2000). Other information on the A tables is self-explanatory.

The B tables represent the summarized plant data for each plot. As explained in the units and nomenclature section, the species list reflects mostly the species names used at the time Beatley originally measured her plots. We made the following exceptions for name changes that occurred since 1963 but that Beatley did not adopt: Erioneuron pulchellum = Tridens pulchellum; Ericameria cooperi and E. laricifolius $=$ Haplopappus cooperi and H. laricifolius, respectively; and Xylorhiza tortifolia = Machaeranthera tortifolia $=$ Aster abatus. The following name changes are included in Wethermax (2002) but are not adopted here: Achnatherum speciosum = Stipa speciosa, Achnatherum hymenoides $=$ Oryzopsis hymenoides, Elymus $=$ Sitanian, Escobaria vivipara $=$ Coryphantha vivipara, Pleuriphis jamesii $=$ Hilaria jamesii, and Krascheninnikovia lanata = Ceratoides lanata. See pages 3-4 for more discussion. Finally, although Yucca baccata v. vespertina is no longer recognized as a distinct subspecies, we follow Beatley's original designation. Although we question Beatley's identification of Artemisia nova on several plots (notably plot 61), and instead think the plants may be A. tridentata stunted owing to unfavorable edaphic conditions, we leave the former name intact. Number of plants $=$ the number of live plants and dead branches on live plants encountered in the line intercepts; average height $=$ the average of the maximum heights of all individuals of that species encountered on the line (not the maximum height along the intercept); cover $=$ total percent of intercept length occupied by that species irrespective of stature; and biomass index = coverage distance $(\mathrm{m})$ times maximum height $(\mathrm{m})$ and summed per species to yield an index of biomass. The cover contains some overlap among species owing to structure issues (e.g., Larrea shading of Grayia, but both are measured) and therefore is generally larger than absolute ground cover. 
Table A-1A. Site characteristics for Plot 1.

Location: Mercury Valley

NTS Area: 22

NTS Grid: S-7

USGS 7.5’ Quadrangle Name: Camp Desert Rock

\begin{tabular}{lllll} 
& & \multicolumn{2}{c}{ GPS Readings (Zone 11S) } & \\
& Location & Northing & Easting & Condition of Original Corners \\
Corner 1: & SW & 4055318 & 586082 & Standing \\
Corner 2: & NW & 4055347 & 586089 & Standing \\
Corner 3: & NE & 4055341 & 586118 & Standing \\
Corner 4: & SE & 4055310 & 586110 & Standing
\end{tabular}

DEM Plot Elevation: 1094 m

Plot Aspect: $308^{\circ}$

Elevation Above Playa (Playa Name): NA

Plot Slope: $17^{\circ}$

Annual Precipitation

Measured: $142 \mathrm{~mm}$

Modeled: $137 \mathrm{~mm}$

Parent Material Type: alluvial fan

Substrate: alluvial fan, limestone, desert pavement, shallow caliche layer

Slate Geologic Unit: Qai

Beatley Plant Assemblage: Larrea-Ambrosia

Ostler-Hanson Plant Association: Larrea-Yucca schidigera-Ambrosia

Ostler-Hanson Land Unit: 1137

Abundance of Biological Soil Crusts: occur throughout plot, with lichens, locally heavily

Type and Date of Disturbance: none

Plot Condition: good

Location of Transect 1: south side of plot

Direction Transects Are Read: west to east

Date(s) Plot Remeasured: 11 May 2000 
Figure A-1. Photographs showing Plot 1.

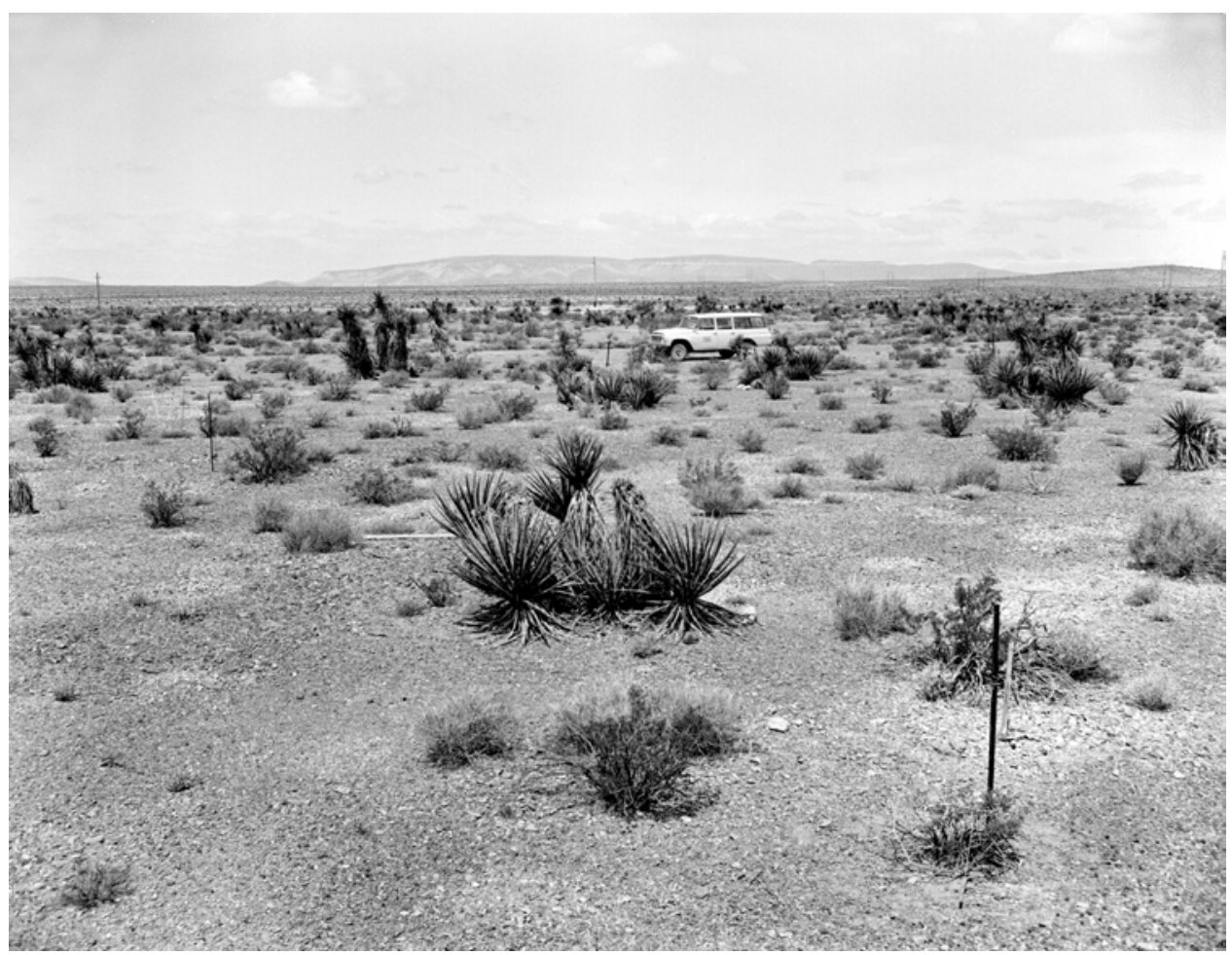

A. (April 19, 1964). View northwesterly across Plot 1, showing Mojave yucca (Yucca schidigera) in the foreground and Skull Mountain in the background. The vegetation assemblage is creosote bush (Larrea tridentata) - white bursage (Ambrosia dumosa) (Janice Beatley Collection, 13A).

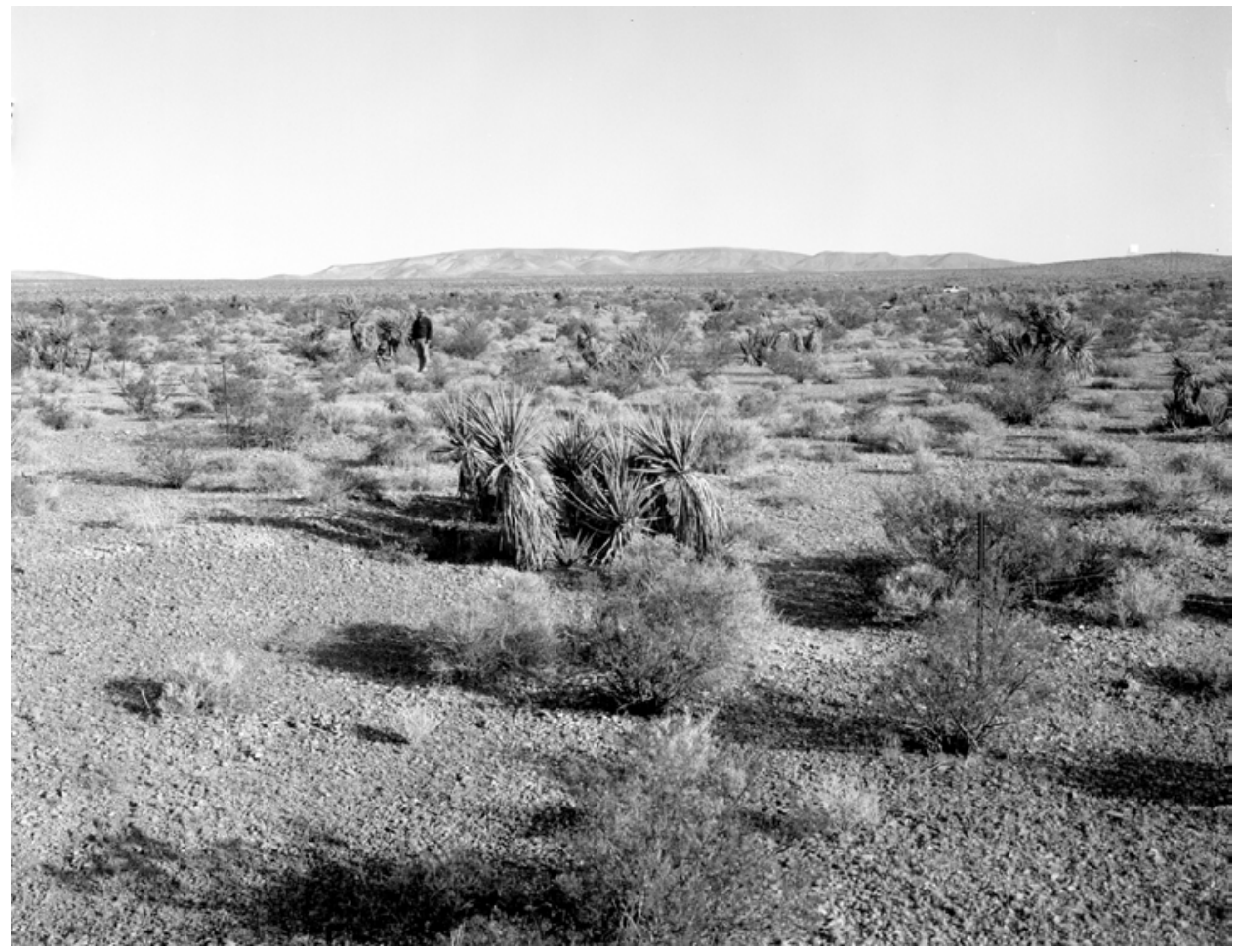

B. (May 12, 2000). Little change has occurred in the Mojave yuccas, and the creosote bushes in the foreground have grown considerably (R.H. Webb, Stake 4033A). 
Table A-1B. Summary plant data for Plot 1.

\begin{tabular}{|c|c|c|c|c|c|c|c|}
\hline \multicolumn{5}{|c|}{ Number of Plants } & \multicolumn{3}{|c|}{ Cover (\%) } \\
\hline SPECIES & 1963 & 1975 & 2000 & SPECIES & 1963 & 1975 & 2000 \\
\hline Acamptopappus shockleyi & 1 & 1 & 1 & Acamptopappus shockleyi & 0.09 & 0.09 & 0.09 \\
\hline Ambrosia dumosa & 13 & 26 & 46 & Ambrosia dumosa & 1.25 & 2.55 & 3.36 \\
\hline Echinocactus polycephalus & 1 & 1 & 1 & Echinocactus polycephalus & 0.13 & 0.18 & 0.17 \\
\hline Ephedra nevadensis & 11 & 11 & 9 & Ephedra nevadensis & 1.39 & 2.08 & 1.04 \\
\hline Erioneuron pulchellum & 0 & 0 & 115 & Erioneuron pulchellum & 0.00 & 0.00 & 1.16 \\
\hline Krameria parvifolia & 22 & 25 & 32 & Krameria parvifolia & 1.95 & 2.17 & 3.83 \\
\hline Larrea tridentata & 36 & 34 & 39 & Larrea tridentata & 6.98 & 6.24 & 9.10 \\
\hline Lycium andersonii & 11 & 10 & 10 & Lycium andersonii & 1.77 & 1.74 & 1.33 \\
\hline Menodora spinescens & 0 & 0 & 2 & Menodora spinescens & 0.00 & 0.00 & 0.13 \\
\hline Opuntia ramosissima & 2 & 1 & 1 & Opuntia ramosissima & 0.09 & 0.03 & 0.08 \\
\hline Oryzopsis hymenoides & 0 & 1 & 0 & Oryzopsis hymenoides & 0.00 & 0.09 & 0.00 \\
\hline Psorothamnus fremontii & 0 & 0 & 1 & Psorothamnus fremontii & 0.00 & 0.00 & 0.10 \\
\hline Sphaeralcea ambigua & 0 & 2 & 2 & Sphaeralcea ambigua & 0.00 & 0.05 & 0.03 \\
\hline Yucca schidigera & 21 & 16 & 12 & Yucca schidigera & 3.65 & 2.56 & 1.54 \\
\hline Total Live & 118 & 128 & 271 & Total Live & 17.32 & 17.77 & 21.95 \\
\hline Dead Shrub & 21 & 14 & 21 & Dead Shrub & 1.75 & 0.11 & 3.20 \\
\hline Total & 139 & 142 & 292 & Total & 19.07 & 17.89 & 25.15 \\
\hline \multicolumn{5}{|c|}{ Average Height (m) } & \multicolumn{3}{|c|}{ Biomass Index $\left(\mathbf{m}^{2}\right)$} \\
\hline SPECIES & 1963 & 1975 & 2000 & SPECIES & 1963 & 1975 & 2000 \\
\hline Acamptopappus shockleyi & 0.15 & 0.18 & 0.15 & Acamptopappus shockleyi & 0.05 & 0.05 & 0.05 \\
\hline Ambrosia dumosa & 0.23 & 0.29 & 0.33 & Ambrosia dumosa & 0.99 & 2.85 & 4.08 \\
\hline Echinocactus polycephalus & 0.25 & 0.36 & 0.36 & Echinocactus polycephalus & 0.11 & 0.22 & 0.21 \\
\hline Ephedra nevadensis & 0.48 & 0.55 & 0.50 & Ephedra nevadensis & 2.34 & 4.09 & 2.01 \\
\hline Erioneuron pulchellum & 0.00 & 0.00 & 0.03 & Erioneuron pulchellum & 0.00 & 0.00 & 0.12 \\
\hline Krameria parvifolia & 0.16 & 0.17 & 0.23 & Krameria parvifolia & 1.10 & 1.32 & 3.13 \\
\hline Larrea tridentata & 0.68 & 0.66 & 0.94 & Larrea tridentata & 17.12 & 14.76 & 29.64 \\
\hline Lycium andersonii & 0.41 & 0.42 & 0.40 & Lycium andersonii & 2.46 & 2.45 & 1.74 \\
\hline Menodora spinescens & 0.00 & 0.00 & 0.13 & Menodora spinescens & 0.00 & 0.00 & 0.05 \\
\hline Opuntia ramosissima & 0.10 & 0.36 & 0.36 & Opuntia ramosissima & 0.03 & 0.03 & 0.10 \\
\hline Oryzopsis hymenoides & 0.00 & 0.43 & 0.00 & Oryzopsis hymenoides & 0.00 & 0.13 & 0.00 \\
\hline Psorothamnus fremontii & 0.00 & 0.00 & 0.25 & Psorothamnus fremontii & 0.00 & 0.00 & 0.09 \\
\hline Sphaeralcea ambigua & 0.00 & 0.25 & 0.15 & Sphaeralcea ambigua & 0.00 & 0.06 & 0.01 \\
\hline \multirow[t]{2}{*}{ Yucca schidigera } & 1.05 & 1.16 & 1.06 & Yucca schidigera & 13.00 & 12.38 & 5.93 \\
\hline & & & & Total Live & 37.19 & 38.33 & 47.16 \\
\hline
\end{tabular}


Table A-2A. Site characteristics for Plot 2.

Location: Mercury Valley

NTS Area: 22

NTS Grid: N-8

USGS 7.5’ Quadrangle Name: Specter Range NW

\begin{tabular}{|c|c|c|c|c|}
\hline & & GPS Reac & Zone 11S) & \\
\hline & Location & Northing & Easting & Condition of Original Corners \\
\hline Corner 1: & SW & 4058539 & 577798 & On ground \\
\hline Corner 2: & NW & 4058569 & 577804 & On ground \\
\hline Corner 3: & $\mathrm{NE}$ & 4058562 & 577834 & Standing \\
\hline Corner 4: & SE & 4058533 & 577826 & On ground \\
\hline DEM Plo & vation: 10 & & & Plot Aspect: $162^{\circ}$ \\
\hline Elevation & e Playa ( & Name): & & Plot Slope: $2^{\circ}$ \\
\hline
\end{tabular}

Annual Precipitation

Measured: $179 \mathrm{~mm}$

Modeled: $145 \mathrm{~mm}$

Parent Material Type: alluvial fan

Substrate: alluvial fan, limestone, deep soil, lots of calcrete rubble on surface

Slate Geologic Unit: Tgy

Beatley Plant Assemblage: Larrea-Grayia-Lycium

Ostler-Hanson Plant Association: Grayia-Larrea-Lycium

Ostler-Hanson Land Unit: 3

Abundance of Biological Soil Crusts: occur throughout plot

Type and Date of Disturbance: none

Plot Condition: good

Location of Transect 1: west side of plot

Direction Transects Are Read: north to south

Date(s) Plot Remeasured: 12 May 2000 
Figure A-2. Photographs showing Plot 2.

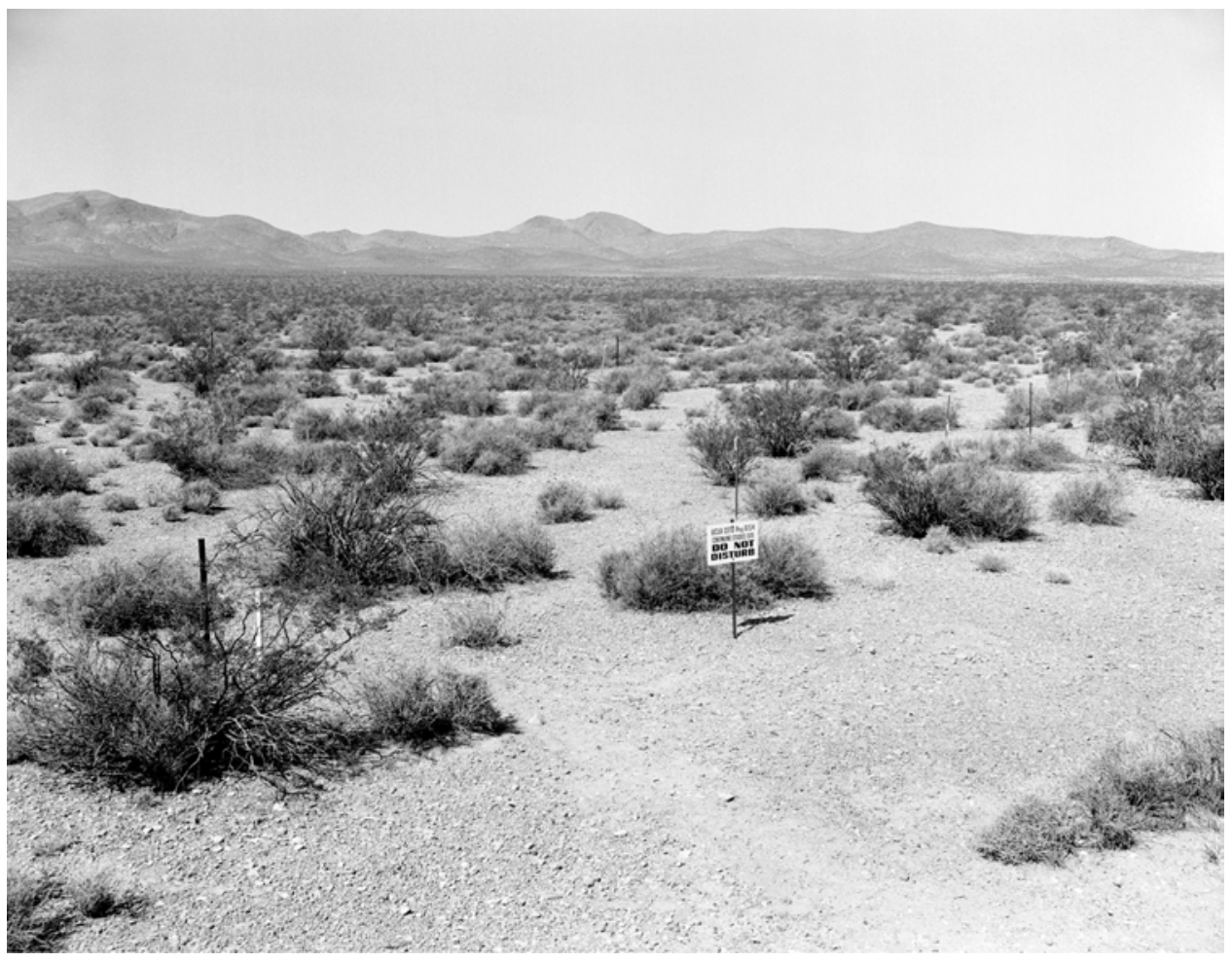

A. (May 14, 1964). View southwesterly across Plot 2 showing the original plot sign and a creosote bush - spiny hopsage (Grayia spinosa) wolfberry (Lycium andersoni) community. The Specter Range appears in the background (Janice Beatley Collection, 110-B).

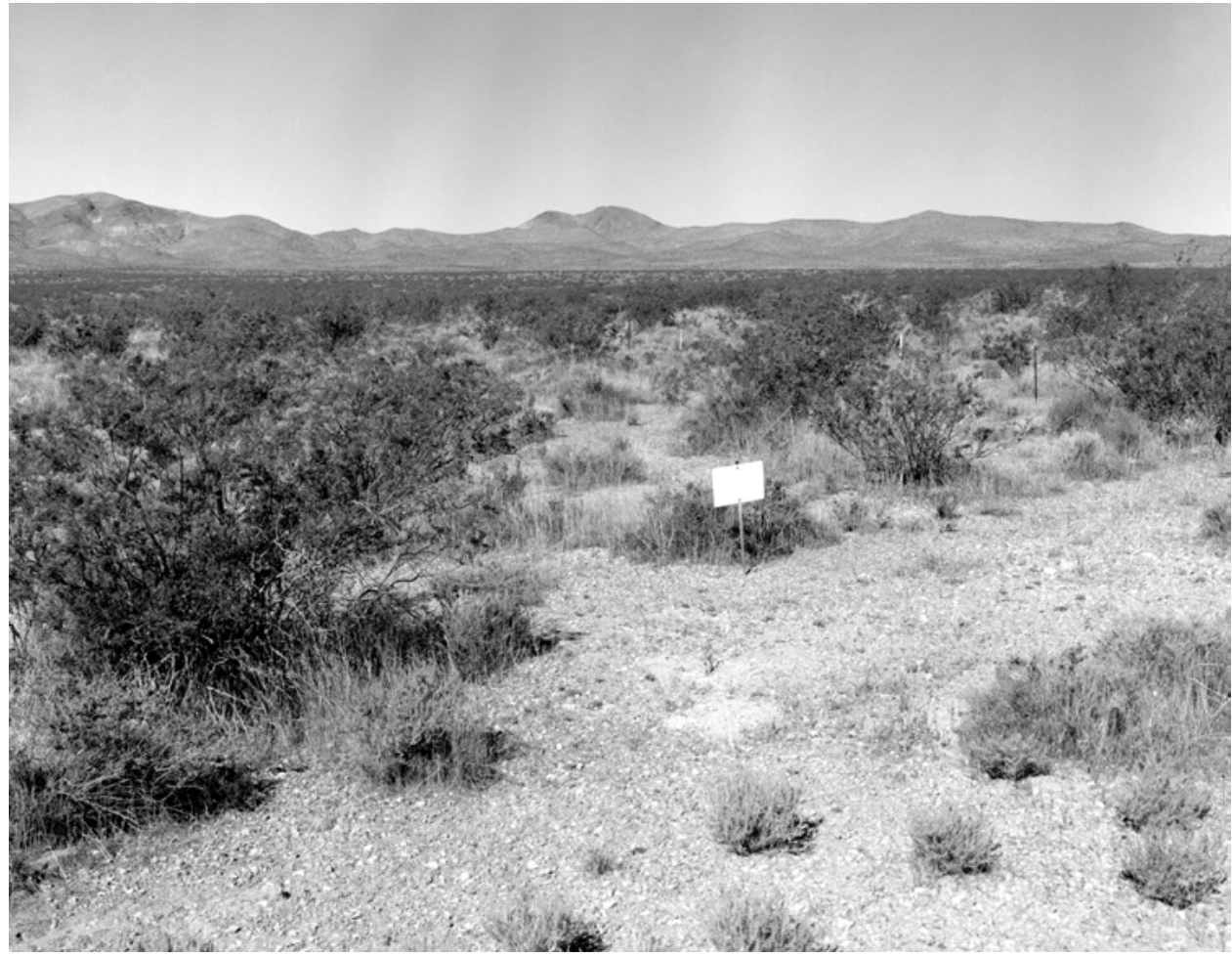

B. (May 12, 2000). The creosote bush have grown much larger, and more white bursage are apparent throughout the view. The species composition has shifted, owing to the extensive loss of spiny hopsage, to a creosote bush - white bursage assemblage (R.H. Webb, Stake 4034B). 
Table A-2B. Summary plant data for Plot 2.

\begin{tabular}{|c|c|c|c|c|c|c|c|}
\hline \multicolumn{5}{|c|}{ Number of Plants } & \multicolumn{3}{|c|}{ Cover (\%) } \\
\hline SPECIES & 1963 & 1975 & 2000 & SPECIES & 1963 & 1975 & 2000 \\
\hline Acamptopappus shockleyi & 19 & 21 & 9 & Acamptopappus shockleyi & 1.53 & 1.08 & 0.39 \\
\hline Ambrosia dumosa & 17 & 16 & 49 & Ambrosia dumosa & 1.65 & 1.56 & 3.86 \\
\hline Ceratoides lanata & 36 & 31 & 26 & Ceratoides lanata & 3.35 & 2.14 & 1.75 \\
\hline Dichelostemma pulchella & 0 & 3 & 0 & Dichelostemma pulchella & 0.00 & 0.04 & 0.00 \\
\hline Ephedra nevadensis & 1 & 6 & 10 & Ephedra nevadensis & 0.04 & 0.66 & 2.23 \\
\hline Grayia spinosa & 48 & 78 & 12 & Grayia spinosa & 9.55 & 11.66 & 1.57 \\
\hline Hymenoclea salsola & 0 & 1 & 5 & Hymenoclea salsola & 0.00 & 0.05 & 0.44 \\
\hline Larrea tridentata & 12 & 16 & 22 & Larrea tridentata & 3.40 & 5.39 & 9.49 \\
\hline Lycium andersonii & 14 & 20 & 13 & Lycium andersonii & 2.63 & 2.22 & 1.20 \\
\hline Mirabilis pudica & 0 & 0 & 3 & Mirabilis pudica & 0.00 & 0.00 & 0.33 \\
\hline Opuntia basilaris & 0 & 1 & 0 & Opuntia basilaris & 0.00 & 0.04 & 0.00 \\
\hline Oryzopsis hymenoides & 0 & 3 & 1 & Oryzopsis hymenoides & 0.00 & 0.21 & 0.09 \\
\hline Sitanion hystrix & 0 & 3 & 0 & Sitanion hystrix & 0.00 & 0.05 & 0.00 \\
\hline Sphaeralcea ambigua & 0 & 0 & 2 & Sphaeralcea ambigua & 0.00 & 0.00 & 0.09 \\
\hline Stanleya pinnata & 0 & 0 & 1 & Stanleya pinnata & 0.00 & 0.00 & 0.05 \\
\hline Stipa speciosa & 0 & 1 & 0 & Stipa speciosa & 0.00 & 0.03 & 0.00 \\
\hline Tetradymia axillaris & 1 & 1 & 1 & Tetradymia axillaris & 0.02 & 0.16 & 0.10 \\
\hline Total Live & 148 & 201 & 154 & Total Live & 22.15 & 25.29 & 21.58 \\
\hline Dead grass & 0 & 2 & 0 & Dead grass & 0.00 & 0.11 & 0.00 \\
\hline Dead shrub & 18 & 46 & 113 & Dead shrub & 5.28 & 4.74 & 10.39 \\
\hline \multirow[t]{2}{*}{ Total } & 166 & 249 & 267 & Total & 27.44 & 30.14 & 31.97 \\
\hline & \multicolumn{3}{|c|}{ Average Height (m) } & & \multicolumn{3}{|c|}{ Biomass Index $\left(\mathbf{m}^{2}\right)$} \\
\hline SPECIES & 1963 & 1975 & 2000 & SPECIES & 1963 & 1975 & 2000 \\
\hline Acamptopappus shockleyi & 0.24 & 0.21 & 0.31 & Acamptopappus shockleyi & 1.21 & 0.86 & 0.41 \\
\hline Ambrosia dumosa & 0.36 & 0.39 & 0.42 & Ambrosia dumosa & 2.22 & 2.28 & 5.81 \\
\hline Ceratoides lanata & 0.38 & 0.43 & 0.49 & Ceratoides lanata & 4.68 & 3.31 & 2.95 \\
\hline Dichelostemma pulchella & 0.00 & 0.19 & 0.00 & Dichelostemma pulchella & 0.00 & 0.02 & 0.00 \\
\hline Ephedra nevadensis & 0.41 & 0.42 & 0.68 & Ephedra nevadensis & 0.05 & 1.24 & 5.54 \\
\hline Grayia spinosa & 0.59 & 0.60 & 0.70 & Grayia spinosa & 20.25 & 24.83 & 4.47 \\
\hline Hymenoclea salsola & 0.00 & 0.56 & 0.47 & Hymenoclea salsola & 0.00 & 0.10 & 0.72 \\
\hline Larrea tridentata & 1.37 & 1.25 & 1.81 & Larrea tridentata & 17.99 & 28.12 & 62.54 \\
\hline Lycium andersonii & 0.50 & 0.40 & 0.46 & Lycium andersonii & 4.67 & 3.15 & 2.13 \\
\hline Mirabilis pudica & 0.00 & 0.00 & 0.34 & Mirabilis pudica & 0.00 & 0.00 & 0.33 \\
\hline Opuntia basilaris & 0.00 & 0.15 & 0.00 & Opuntia basilaris & 0.00 & 0.02 & 0.00 \\
\hline Oryzopsis hymenoides & 0.00 & 0.29 & 0.48 & Oryzopsis hymenoides & 0.00 & 0.21 & 0.15 \\
\hline Sitanion hystrix & 0.00 & 0.29 & 0.00 & Sitanion hystrix & 0.00 & 0.04 & 0.00 \\
\hline Sphaeralcea ambigua & 0.00 & 0.00 & 0.24 & Sphaeralcea ambigua & 0.00 & 0.00 & 0.11 \\
\hline Stanleya pinnata & 0.00 & 0.00 & 0.53 & Stanleya pinnata & 0.00 & 0.00 & 0.08 \\
\hline Stipa speciosa & 0.00 & 0.18 & 0.00 & Stipa speciosa & 0.00 & 0.02 & 0.00 \\
\hline \multirow[t]{2}{*}{ Tetradymia axillaris } & 0.41 & 0.53 & 0.91 & Tetradymia axillaris & 0.02 & 0.29 & 0.31 \\
\hline & & & & Total Live & 51.09 & 64.49 & 85.54 \\
\hline
\end{tabular}


Table A-3A. Site characteristics for Plot 3.

Location: Rock Valley

NTS Area: 25

NTS Grid: J-10

USGS 7.5’ Quadrangle Name: Specter Range NW

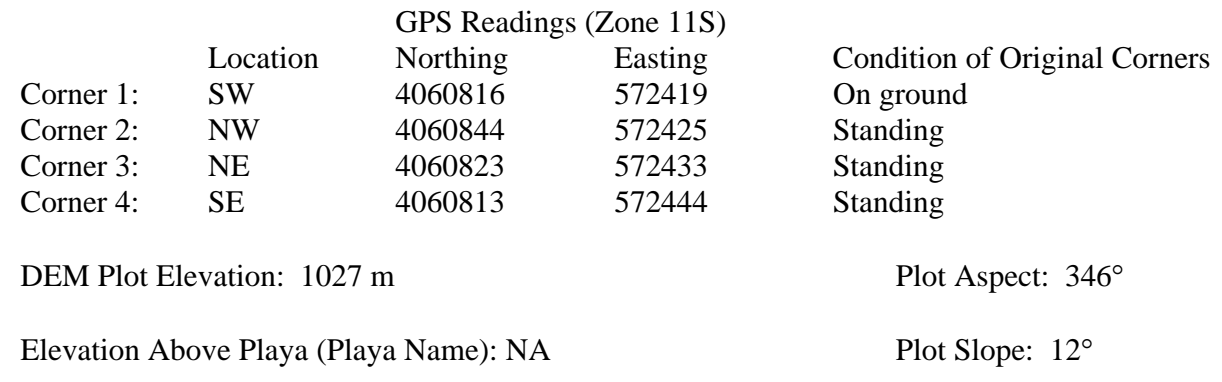

Annual Precipitation

Measured: $175 \mathrm{~mm}$

Modeled: $148 \mathrm{~mm}$

Parent Material Type: alluvial fan

Substrate: alluvial fan, volcanic, ryholite

Slate Geologic Unit: Qai

Beatley Plant Assemblage: Larrea-Ambrosia/Larrea-Lycium-Grayia

Ostler-Hanson Plant Association: Larrea-Psorothamnus-Lycium

Ostler-Hanson Land Unit: 333

Abundance of Biological Soil Crusts: moderate distribution of crusts

Type and Date of Disturbance: none

Plot Condition: good

Location of Transect 1: east side of plot

Direction Transects Are Read: south to north

Date(s) Plot Remeasured: 9 May 2000 
Figure A-3. Photographs showing Plot 3.

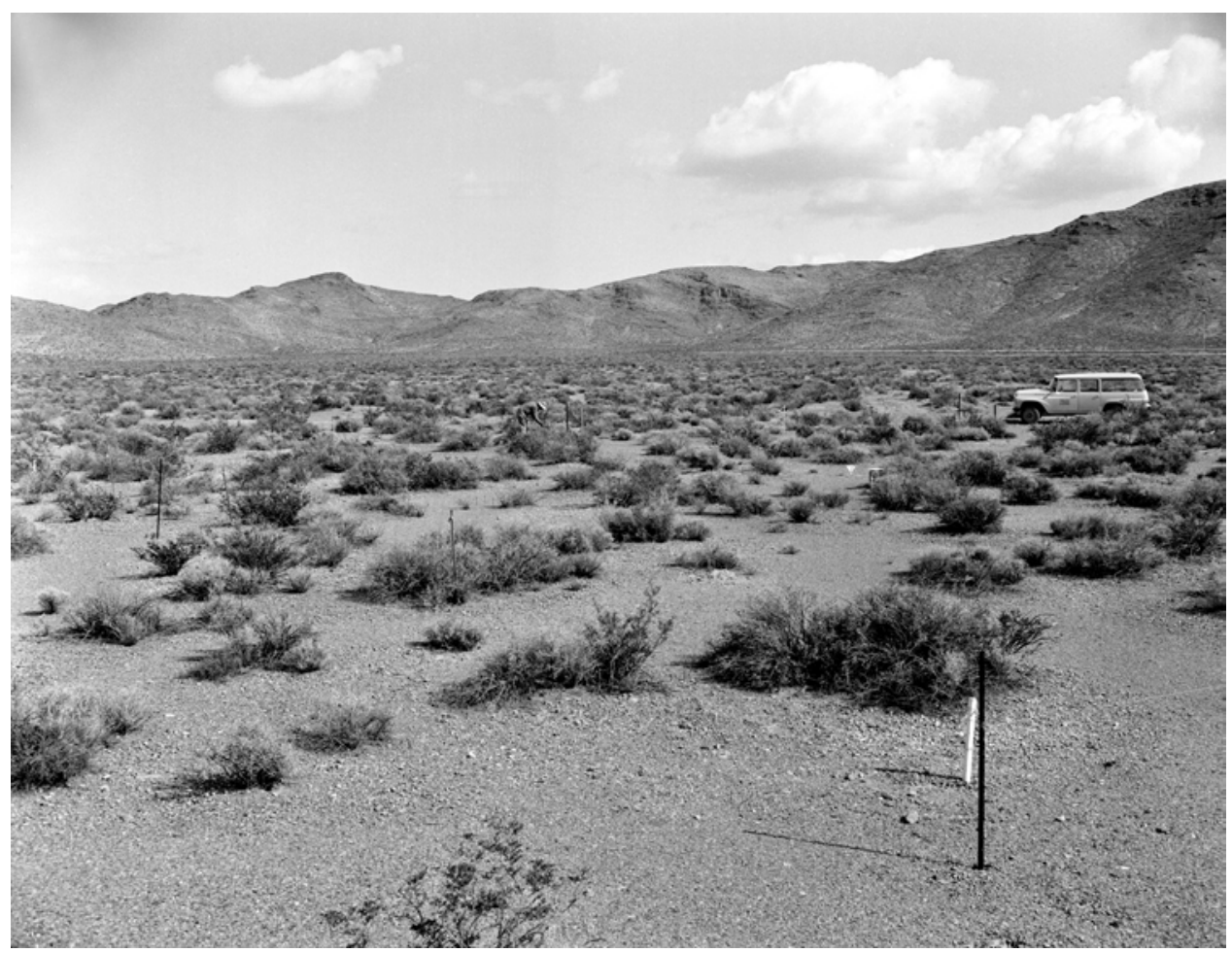

A. (April 19, 1964). View southeasterly across Plot 3 showing the typical mixed creosote bush community in that area and part of the Specter Range in the background (Janice Beatley Collection, 15-A).

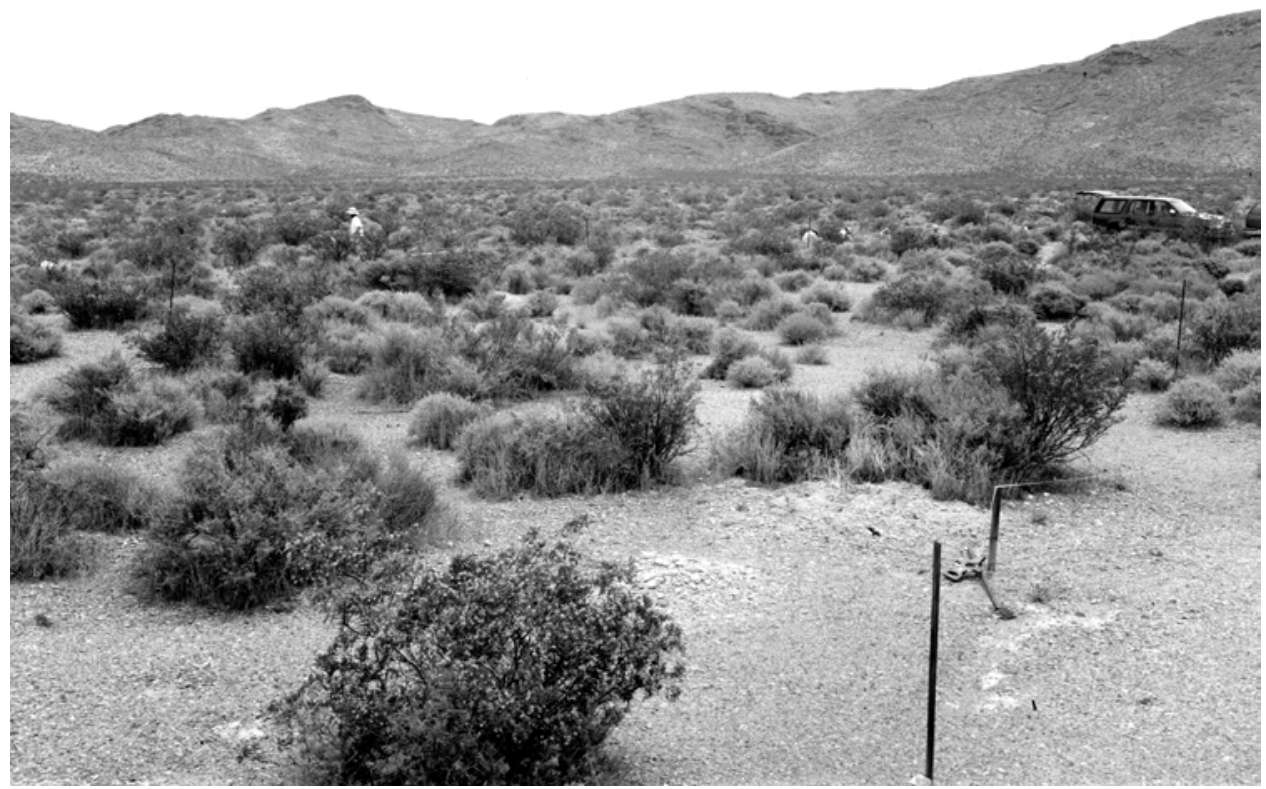

B. (May 9, 2000). The biomass throughout the plot has increased considerably since the original photograph was taken. The lighter colored material in the foreground is bioturbation from digging by an unknown animal species, possibly badger (R.H. Webb, Stake 4020B). 
Table A-3B. Summary plant data for Plot 3.

\begin{tabular}{|c|c|c|c|c|c|c|c|}
\hline \multicolumn{5}{|c|}{ Number of Plants } & \multicolumn{3}{|c|}{ Cover (\%) } \\
\hline SPECIES & 1963 & 1975 & 2000 & SPECIES & 1963 & 1975 & 2000 \\
\hline Acamptopappus shockleyi & 1 & 2 & 0 & Acamptopappus shockleyi & 0.34 & 0.32 & 0.00 \\
\hline Ambrosia dumosa & 38 & 40 & 102 & Ambrosia dumosa & 4.17 & 4.58 & 11.12 \\
\hline Ceratoides lanata & 3 & 4 & 1 & Ceratoides lanata & 0.28 & 0.40 & 0.15 \\
\hline Coleogyne ramosissima & 8 & 8 & 11 & Coleogyne ramosissima & 1.69 & 1.59 & 2.20 \\
\hline Cuscuta nevadensis & 0 & 0 & 0 & Cuscuta nevadensis & 0.00 & 0.00 & 0.00 \\
\hline Ephedra nevadensis & 27 & 23 & 23 & Ephedra nevadensis & 2.17 & 2.57 & 3.86 \\
\hline Grayia spinosa & 31 & 37 & 6 & Grayia spinosa & 3.78 & 4.07 & 0.76 \\
\hline Krameria parvifolia & 3 & 5 & 8 & Krameria parvifolia & 0.38 & 0.67 & 1.45 \\
\hline Larrea tridentata & 22 & 24 & 24 & Larrea tridentata & 2.45 & 5.29 & 6.56 \\
\hline Lycium andersonii & 27 & 20 & 16 & Lycium andersonii & 6.72 & 4.81 & 2.54 \\
\hline Lycium pallidum & 21 & 21 & 21 & Lycium pallidum & 2.64 & 2.90 & 4.16 \\
\hline Oryzopsis hymenoides & 0 & 0 & 1 & Oryzopsis hymenoides & 0.00 & 0.00 & 0.05 \\
\hline Psorothamnus fremontii & 2 & 3 & 4 & Psorothamnus fremontii & 0.22 & 0.45 & 0.80 \\
\hline Sphaeralcea ambigua & 0 & 0 & 6 & Sphaeralcea ambigua & 0.00 & 0.00 & 0.36 \\
\hline Stephanomeria species & 0 & 0 & 1 & Stephanomeria species & 0.00 & 0.00 & 0.04 \\
\hline Xylorhiza tortifolia & 1 & 4 & 4 & Xylorhiza tortifolia & 0.03 & 0.15 & 0.22 \\
\hline Total live & 184 & 191 & 228 & Total live & 24.86 & 27.81 & 34.26 \\
\hline Dead grass & 0 & 0 & 1 & Dead grass & 0.00 & 0.00 & 0.07 \\
\hline Dead shrub & 15 & 23 & 38 & Dead shrub & 5.55 & 3.07 & 5.18 \\
\hline Dead herbaceous & 0 & 1 & 0 & Dead herbaceous & 0.00 & 0.07 & 0.00 \\
\hline Total & 199 & 215 & 267 & Total & 30.41 & 30.95 & 39.52 \\
\hline \multicolumn{5}{|c|}{ Average Height (m) } & \multicolumn{3}{|c|}{ Biomass Index $\left(\mathbf{m}^{2}\right)$} \\
\hline SPECIES & 1963 & 1975 & 2000 & SPECIES & 1963 & 1975 & 2000 \\
\hline Acamptopappus shockleyi & 0.33 & 0.32 & 0.00 & Acamptopappus shockleyi & 0.37 & 0.39 & 0.00 \\
\hline Ambrosia dumosa & 0.36 & 0.40 & 0.42 & Ambrosia dumosa & 5.38 & 6.28 & 16.15 \\
\hline Ceratoides lanata & 0.40 & 0.48 & 0.69 & Ceratoides lanata & 0.37 & 0.66 & 0.33 \\
\hline Coleogyne ramosissima & 0.63 & 0.63 & 0.58 & Coleogyne ramosissima & 3.53 & 3.29 & 4.83 \\
\hline Cuscuta nevadensis & 0.00 & 0.00 & 0.00 & Cuscuta nevadensis & 0.00 & 0.00 & 0.00 \\
\hline Ephedra nevadensis & 0.40 & 0.49 & 0.57 & Ephedra nevadensis & 3.17 & 4.44 & 7.76 \\
\hline Grayia spinosa & 0.50 & 0.50 & 0.65 & Grayia spinosa & 7.04 & 7.50 & 1.80 \\
\hline Krameria parvifolia & 0.20 & 0.21 & 0.28 & Krameria parvifolia & 0.31 & 0.51 & 1.27 \\
\hline Larrea tridentata & 0.74 & 0.78 & 1.06 & Larrea tridentata & 6.42 & 14.53 & 26.12 \\
\hline Lycium andersonii & 0.71 & 0.58 & 0.54 & Lycium andersonii & 38.77 & 29.36 & 5.11 \\
\hline Lycium pallidum & 0.60 & 0.59 & 0.71 & Lycium pallidum & 5.02 & 5.78 & 10.44 \\
\hline Oryzopsis hymenoides & 0.00 & 0.00 & 0.48 & Oryzopsis hymenoides & 0.00 & 0.00 & 0.07 \\
\hline Psorothamnus fremontii & 0.23 & 0.30 & 0.41 & Psorothamnus fremontii & 0.19 & 0.51 & 1.25 \\
\hline Sphaeralcea ambigua & 0.00 & 0.00 & 0.34 & Sphaeralcea ambigua & 0.00 & 0.00 & 0.63 \\
\hline Stephanomeria species & 0.00 & 0.00 & 0.33 & Stephanomeria species & 0.00 & 0.00 & 0.04 \\
\hline \multirow[t]{2}{*}{ Xylorhiza tortifolia } & 0.13 & 0.37 & 0.37 & Xylorhiza tortifolia & 0.01 & 0.21 & 0.30 \\
\hline & & & & Total live & 70.56 & 73.46 & 76.11 \\
\hline
\end{tabular}


Table A-4A. Site characteristics for Plot 4.

Location: Rock Valley

NTS Area: 25

NTS Grid: J-11

USGS 7.5’ Quadrangle Name: Specter Range NW

\begin{tabular}{|c|c|c|c|c|}
\hline & & GPS Reac & Zone 11S) & \\
\hline & Location & Northing & Easting & Condition of Original Corners \\
\hline Corner 1: & SW & 4061662 & 573575 & Missing \\
\hline Corner 2: & NW & 4061692 & 573582 & Missing \\
\hline Corner 3: & $\mathrm{NE}$ & 4061681 & 573610 & Standing \\
\hline Corner 4: & SE & 4061656 & 573606 & Standing \\
\hline DEM Plo & vation: 10 & & & Plot Aspect: $63^{\circ}$ \\
\hline Elevation & e Playa ( & Name): & & Plot Slope: $6^{\circ}$ \\
\hline
\end{tabular}

Annual Precipitation

Measured: $151 \mathrm{~mm}$

Modeled: $147 \mathrm{~mm}$

Parent Material Type: alluvial fan

Substrate: alluvial fan, basalt, old geomorphic surface, well-varnished gravels

Slate Geologic Unit: Qai

Beatley Plant Assemblage: Larrea-Ambrosia/Larrea-Lycium-Grayia

Ostler-Hanson Plant Association: Ceratoides-Grayia-Ambrosia

Ostler-Hanson Land Unit: 301

Abundance of Biological Soil Crusts: none

Type and Date of Disturbance: none

Plot Condition: good

Location of Transect 1: north side of plot

Direction Transects Are Read: east to west

Date(s) Plot Remeasured: 11 May 2000 
Figure A-4. Photographs showing Plot 4.

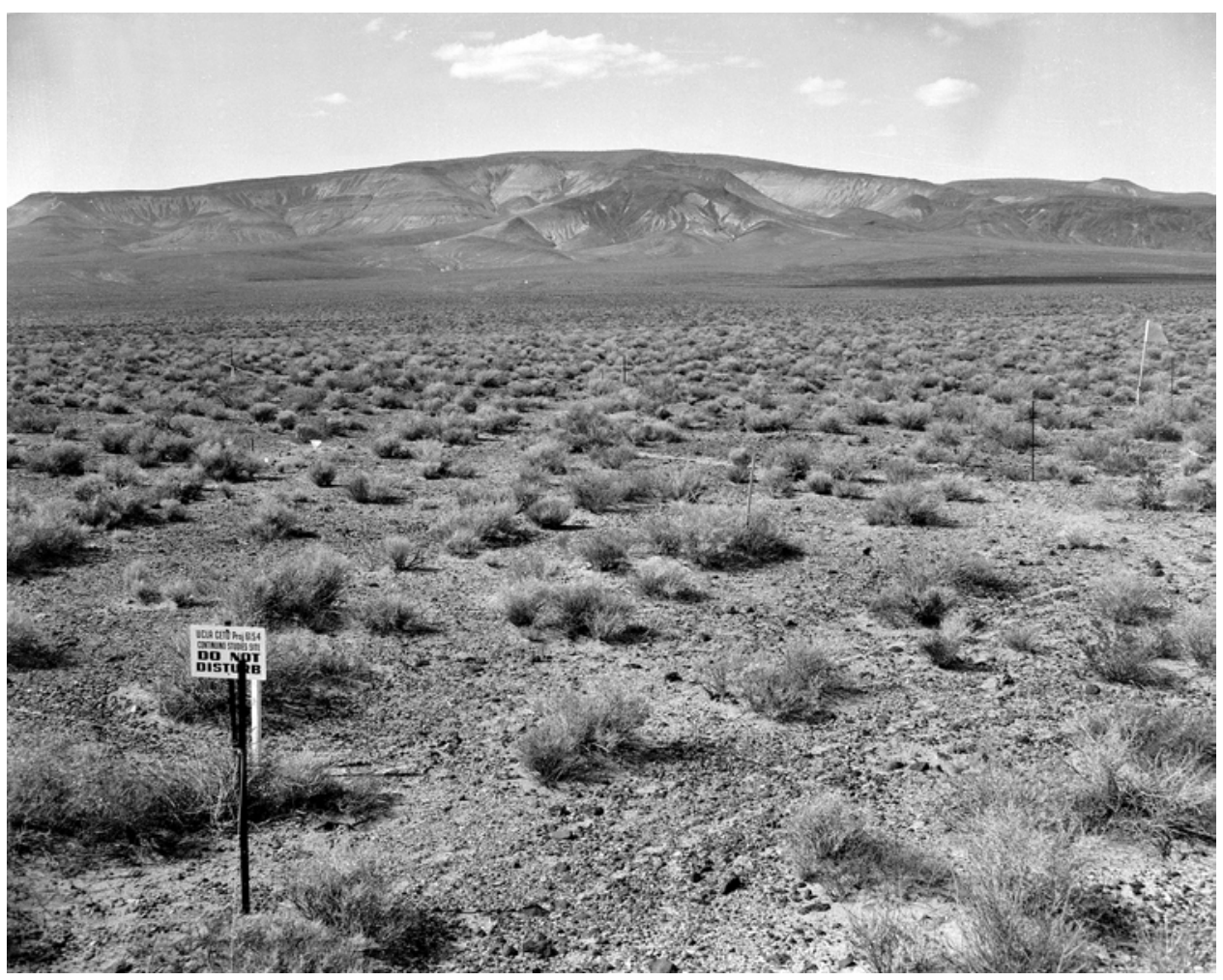

A. (April 22, 1964). View northerly across Plot 4 showing Skull Mountain in the background. The vegetation is a typical mixed-shrub community with scattered creosote bushes (Janice Beatley Collection, 22-B).

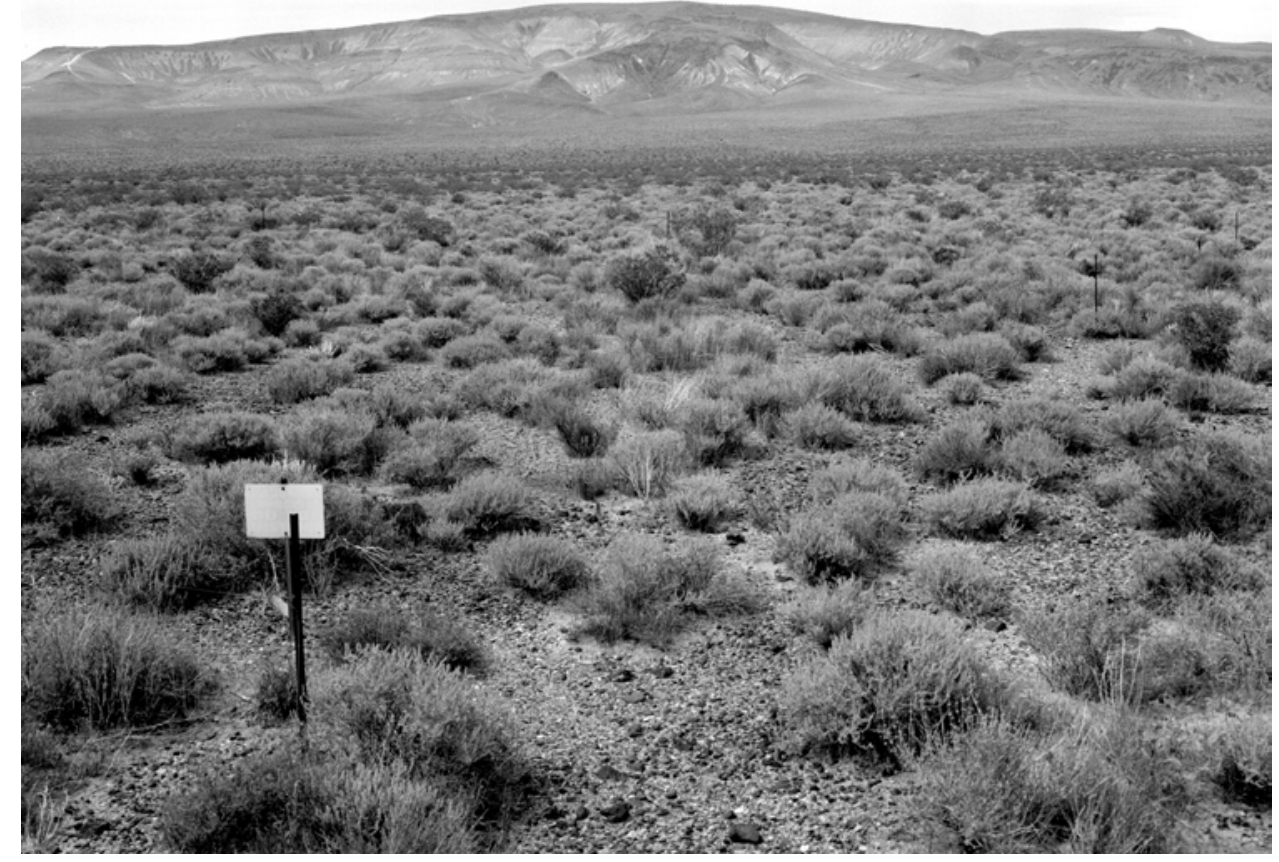

B. (May 9, 2000). Cover and biomass of the undisturbed vegetation has increased considerably (R.H. Webb, Stake 4021A). 
Table A-4B. Summary plant data for Plot 4.

\begin{tabular}{|c|c|c|c|c|c|c|c|}
\hline \multicolumn{5}{|c|}{ Number of Plants } & \multicolumn{3}{|c|}{ Cover (\%) } \\
\hline SPECIES & 1963 & 1975 & 2000 & SPECIES & 1963 & 1975 & 2000 \\
\hline Acamptopappus shockleyi & 1 & 1 & 1 & Acamptopappus shockleyi & 0.13 & 0.15 & 0.07 \\
\hline Ambrosia dumosa & 30 & 40 & 95 & Ambrosia dumosa & 3.03 & 3.68 & 11.12 \\
\hline Ceratoides lanata & 37 & 73 & 9 & Ceratoides lanata & 3.18 & 5.41 & 0.31 \\
\hline Ephedra nevadensis & 8 & 11 & 10 & Ephedra nevadensis & 1.34 & 1.61 & 1.49 \\
\hline Grayia spinosa & 30 & 47 & 2 & Grayia spinosa & 4.50 & 4.90 & 0.16 \\
\hline Krameria parvifolia & 32 & 20 & 28 & Krameria parvifolia & 3.00 & 1.99 & 2.89 \\
\hline Larrea tridentata & 12 & 12 & 13 & Larrea tridentata & 2.07 & 1.58 & 3.48 \\
\hline Lycium andersonii & 5 & 6 & 7 & Lycium andersonii & 0.70 & 0.63 & 0.68 \\
\hline Lycium pallidum & 21 & 21 & 14 & Lycium pallidum & 2.55 & 2.05 & 2.11 \\
\hline Oryzopsis hymenoides & 0 & 15 & 5 & Oryzopsis hymenoides & 0.10 & 1.22 & 0.39 \\
\hline Total live & 176 & 246 & 184 & Total live & 20.49 & 23.23 & 22.71 \\
\hline Dead shrubs & 15 & 32 & 112 & Dead shrubs & 2.05 & 3.32 & 10.18 \\
\hline Total & 191 & 278 & 296 & Total & 22.54 & 26.55 & 32.89 \\
\hline \multicolumn{5}{|c|}{ Average Height (m) } & \multicolumn{3}{|c|}{ Biomass Index $\left(\mathbf{m}^{2}\right)$} \\
\hline SPECIES & 1963 & 1975 & 2000 & SPECIES & 1963 & 1975 & 2000 \\
\hline Acamptopappus shockleyi & 0.41 & 0.41 & 0.33 & Acamptopappus shockleyi & 0.17 & 0.21 & 0.08 \\
\hline Ambrosia dumosa & 0.29 & 0.31 & 0.40 & Ambrosia dumosa & 3.08 & 4.37 & 15.62 \\
\hline Ceratoides lanata & 0.34 & 0.37 & 0.40 & Ceratoides lanata & 3.85 & 7.22 & 0.49 \\
\hline Ephedra nevadensis & 0.45 & 0.55 & 0.58 & Ephedra nevadensis & 2.15 & 3.11 & 3.03 \\
\hline Grayia spinosa & 0.50 & 0.45 & 0.52 & Grayia spinosa & 7.73 & 7.94 & 0.28 \\
\hline Krameria parvifolia & 0.13 & 0.16 & 0.27 & Krameria parvifolia & 1.34 & 1.19 & 2.65 \\
\hline Larrea tridentata & 0.62 & 0.62 & 0.90 & Larrea tridentata & 4.23 & 3.67 & 11.24 \\
\hline Lycium andersonii & 0.49 & 0.43 & 0.43 & Lycium andersonii & 1.17 & 1.05 & 1.02 \\
\hline Lycium pallidum & 0.48 & 0.47 & 0.53 & Lycium pallidum & 4.48 & 3.26 & 3.85 \\
\hline \multirow[t]{2}{*}{ Oryzopsis hymenoides } & 0.00 & 0.34 & 0.48 & Oryzopsis hymenoides & 0.10 & 1.48 & 0.66 \\
\hline & & & & Total live & 28.22 & 33.50 & 38.92 \\
\hline
\end{tabular}


Table A-5A. Site characteristics for Plot 5.

Location: Jackass Flats

NTS Area: 25

NTS Grid: G-13

USGS 7.5’ Quadrangle Name: Specter Range NW

\begin{tabular}{|c|c|c|c|c|}
\hline & & GPS Reac & Zone 11S) & \\
\hline & Location & Northing & Easting & Condition of Original Corners \\
\hline Corner 1: & SW & 4067288 & 567150 & Standing \\
\hline Corner 2: & NW & 4067308 & 567172 & Standing \\
\hline Corner 3: & $\mathrm{NE}$ & 4067287 & 567193 & Standing \\
\hline Corner 4: & SE & 4067267 & 567173 & Standing \\
\hline DEM Plo & vation: 11 & & & Plot Aspect: $145^{\circ}$ \\
\hline Elevation & e Playa ( & Name): $\mathrm{N}$ & & Plot Slope: $6^{\circ}$ \\
\hline
\end{tabular}

Annual Precipitation

Measured: $142 \mathrm{~mm}$

Modeled: $144 \mathrm{~mm}$

Parent Material Type: alluvial fan

Substrate: alluvial fan, basaltic alluvium, rocks old \& varnished

Slate Geologic Unit: Qay

Beatley Plant Assemblage: Larrea-Lycium-Grayia

Ostler-Hanson Plant Association: Larrea-Lycium pallidum-Krameria

Ostler-Hanson Land Unit: 215

Abundance of Biological Soil Crusts: none

Type and Date of Disturbance: none

Plot Condition: good

Location of Transect 1: southwest side of plot

Direction Transects Are Read: southeast to northwest

Date(s) Plot Remeasured: 12 May 2000 
Figure A-5. Photographs showing Plot 5.

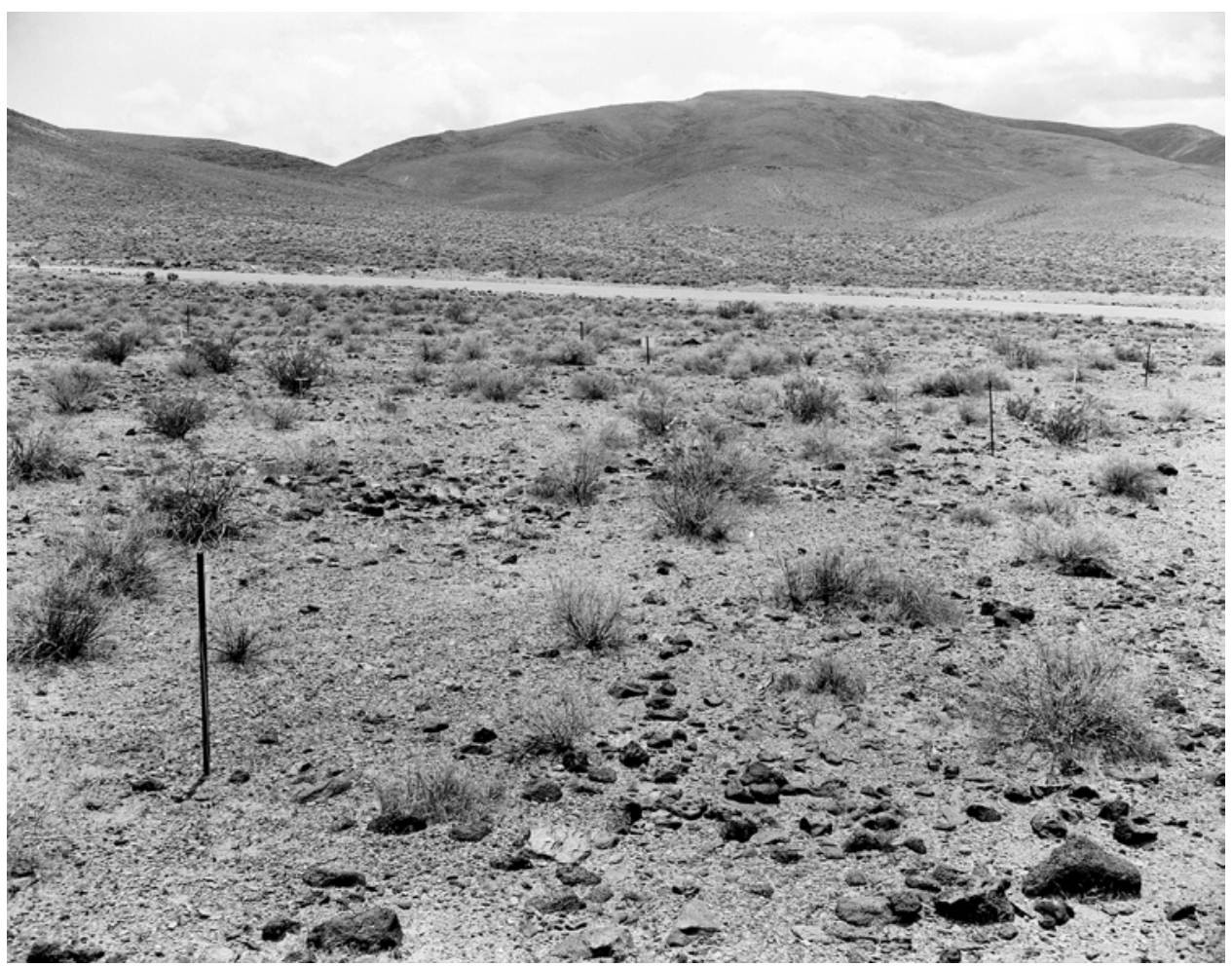

A. (May 7, 1964). This southerly view across Plot 5 shows the flanks of Little Skull Mountain in the background. The vegetation is dominated by creosote bush, and the rocky alluvium has a considerable coarse-particle content dominated by basalt (Janice Beatley Collection, 61-B).

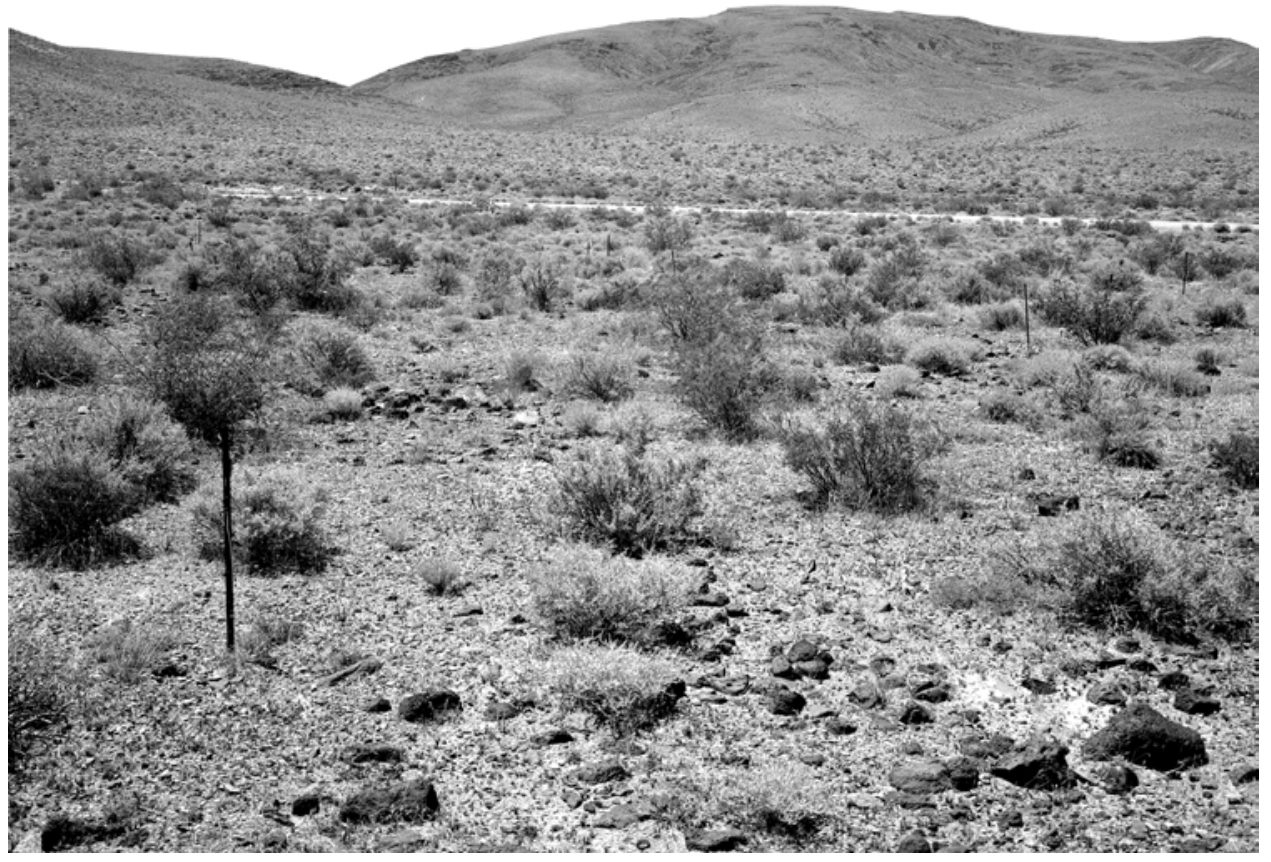

B. (May 12, 2000). Although most of the rocks depicted in the first view are in the same places, the vegetation biomass has increased considerably. The species composition has changed, with spiny hopsage diminishing in importance and range ratany (Krameria parviflora) increasing on the plot (R.H. Webb, Stake 4037A). 
Table A-5B. Summary plant data for Plot 5.

\begin{tabular}{|c|c|c|c|c|c|c|c|}
\hline \multicolumn{5}{|c|}{ Number of Plants } & \multicolumn{3}{|c|}{ Cover (\%) } \\
\hline SPECIES & 1963 & 1975 & 2000 & SPECIES & 1963 & 1975 & 2000 \\
\hline Acamptopappus shockleyi & 0 & 3 & 1 & Acamptopappus shockleyi & 0.00 & 0.03 & 0.12 \\
\hline Ambrosia dumosa & 3 & 4 & 15 & Ambrosia dumosa & 0.25 & 0.38 & 1.44 \\
\hline Ceratoides lanata & 0 & 1 & 0 & Ceratoides lanata & 0.00 & 0.07 & 0.00 \\
\hline Encelia frutescens & 0 & 0 & 2 & Encelia frutescens & 0.00 & 0.00 & 0.15 \\
\hline Ephedra nevadensis & 1 & 0 & 2 & Ephedra nevadensis & 0.11 & 0.00 & 0.15 \\
\hline Eriogonum inflatum & 3 & 6 & 10 & Eriogonum inflatum & 0.13 & 0.12 & 0.47 \\
\hline Grayia spinosa & 6 & 1 & 0 & Grayia spinosa & 0.91 & 0.20 & 0.00 \\
\hline Hymenoclea salsola & 0 & 1 & 0 & Hymenoclea salsola & 0.00 & 0.10 & 0.00 \\
\hline Krameria parvifolia & 12 & 10 & 21 & Krameria parvifolia & 1.64 & 1.45 & 2.66 \\
\hline Larrea tridentata & 9 & 11 & 22 & Larrea tridentata & 1.91 & 2.90 & 5.33 \\
\hline Lycium andersonii & 4 & 5 & 6 & Lycium andersonii & 0.88 & 1.16 & 1.02 \\
\hline Lycium pallidum & 16 & 12 & 6 & Lycium pallidum & 2.75 & 2.44 & 0.76 \\
\hline Opuntia basilaris & 1 & 0 & 0 & Opuntia basilaris & 0.05 & 0.00 & 0.00 \\
\hline Opuntia echinocarpa & 0 & 0 & 1 & Opuntia echinocarpa & 0.00 & 0.00 & 0.02 \\
\hline Oryzopsis hymenoides & 0 & 2 & 0 & Oryzopsis hymenoides & 0.00 & 0.07 & 0.00 \\
\hline Psorothamnus fremontii & 0 & 0 & 1 & Psorothamnus fremontii & 0.00 & 0.00 & 0.06 \\
\hline Sphaeralcea ambigua & 0 & 11 & 44 & Sphaeralcea ambigua & 0.00 & 0.42 & 1.23 \\
\hline Total live & 55 & 67 & 131 & Total live & 8.62 & 9.35 & 13.41 \\
\hline Dead shrubs & 10 & 12 & 24 & Dead shrubs & 1.15 & 1.50 & 1.46 \\
\hline Total & 65 & 79 & 155 & Total & 9.77 & 10.85 & 14.87 \\
\hline \multicolumn{5}{|c|}{ Average Height (m) } & \multicolumn{3}{|c|}{ Biomass Index $\left(\mathrm{m}^{2}\right)$} \\
\hline SPECIES & 1963 & 1975 & 2000 & SPECIES & 1963 & 1975 & 2000 \\
\hline Acamptopappus shockleyi & 0.00 & 0.06 & 0.36 & Acamptopappus shockleyi & 0.00 & 0.01 & 0.14 \\
\hline Ambrosia dumosa & 0.23 & 0.27 & 0.32 & Ambrosia dumosa & 0.21 & 0.36 & 1.79 \\
\hline Ceratoides lanata & 0.00 & 0.36 & 0.00 & Ceratoides lanata & 0.00 & 0.09 & 0.00 \\
\hline Encelia frutescens & 0.00 & 0.00 & 0.18 & Encelia frutescens & 0.00 & 0.00 & 0.13 \\
\hline Ephedra nevadensis & 0.43 & 0.00 & 0.44 & Ephedra nevadensis & 0.16 & 0.00 & 0.23 \\
\hline Eriogonum inflatum & 0.18 & 0.08 & 0.45 & Eriogonum inflatum & 0.08 & 0.05 & 0.74 \\
\hline Grayia spinosa & 0.46 & 0.46 & 0.00 & Grayia spinosa & 1.51 & 0.31 & 0.00 \\
\hline Hymenoclea salsola & 0.00 & 0.51 & 0.00 & Hymenoclea salsola & 0.00 & 0.17 & 0.00 \\
\hline Krameria parvifolia & 0.19 & 0.20 & 0.23 & Krameria parvifolia & 1.12 & 1.00 & 2.30 \\
\hline Larrea tridentata & 0.94 & 0.80 & 0.88 & Larrea tridentata & 6.91 & 9.06 & 16.99 \\
\hline Lycium andersonii & 0.69 & 0.64 & 0.65 & Lycium andersonii & 2.05 & 2.57 & 2.28 \\
\hline Lycium pallidum & 0.59 & 0.60 & 0.70 & Lycium pallidum & 5.94 & 5.14 & 1.93 \\
\hline Opuntia basilaris & 0.13 & 0.00 & 0.00 & Opuntia basilaris & 0.02 & 0.00 & 0.00 \\
\hline Opuntia echinocarpa & 0.00 & 0.00 & 0.30 & Opuntia echinocarpa & 0.00 & 0.00 & 0.02 \\
\hline Oryzopsis hymenoides & 0.00 & 0.17 & 0.00 & Oryzopsis hymenoides & 0.00 & 0.06 & 0.00 \\
\hline Psorothamnus fremontii & 0.00 & 0.00 & 0.23 & Psorothamnus fremontii & 0.00 & 0.00 & 0.05 \\
\hline \multirow[t]{2}{*}{ Sphaeralcea ambigua } & 0.00 & 0.12 & 0.18 & Sphaeralcea ambigua & 0.00 & 0.23 & 0.91 \\
\hline & & & & Total live & 17.98 & 19.03 & 27.52 \\
\hline
\end{tabular}


Table A-6A. Site characteristics for Plot 6 .

Location: Jackass Flats

NTS Area: 25

NTS Grid: E-15

USGS 7.5’ Quadrangle Name: Jackass Flats

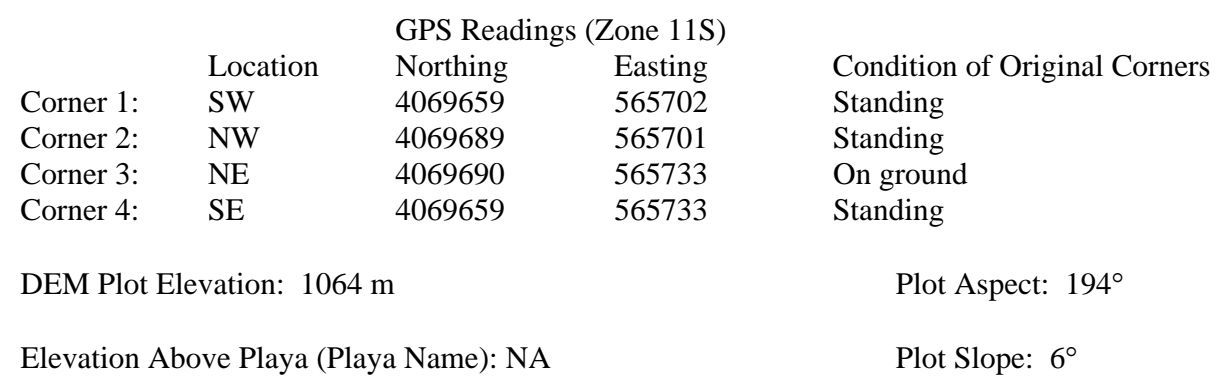

Annual Precipitation

Measured: $139 \mathrm{~mm}$

Modeled: $139 \mathrm{~mm}$

Parent Material Type: alluvial fan

Substrate: alluvial fan, volcanic, young geomorphic surface

Slate Geologic Unit: Qai

Beatley Plant Assemblage: Larrea-Ambrosia/Larrea-Lycium-Grayia

Ostler-Hanson Plant Association: Ambrosia-Larrea-Grayia

Ostler-Hanson Land Unit: 230

Abundance of Biological Soil Crusts: none

Type and Date of Disturbance: none

Plot Condition: good

Location of Transect 1: south side of plot

Direction Transects Are Read: west to east

Date(s) Plot Remeasured: 2 April 2001 
Figure A-6. Photographs showing Plot 6.

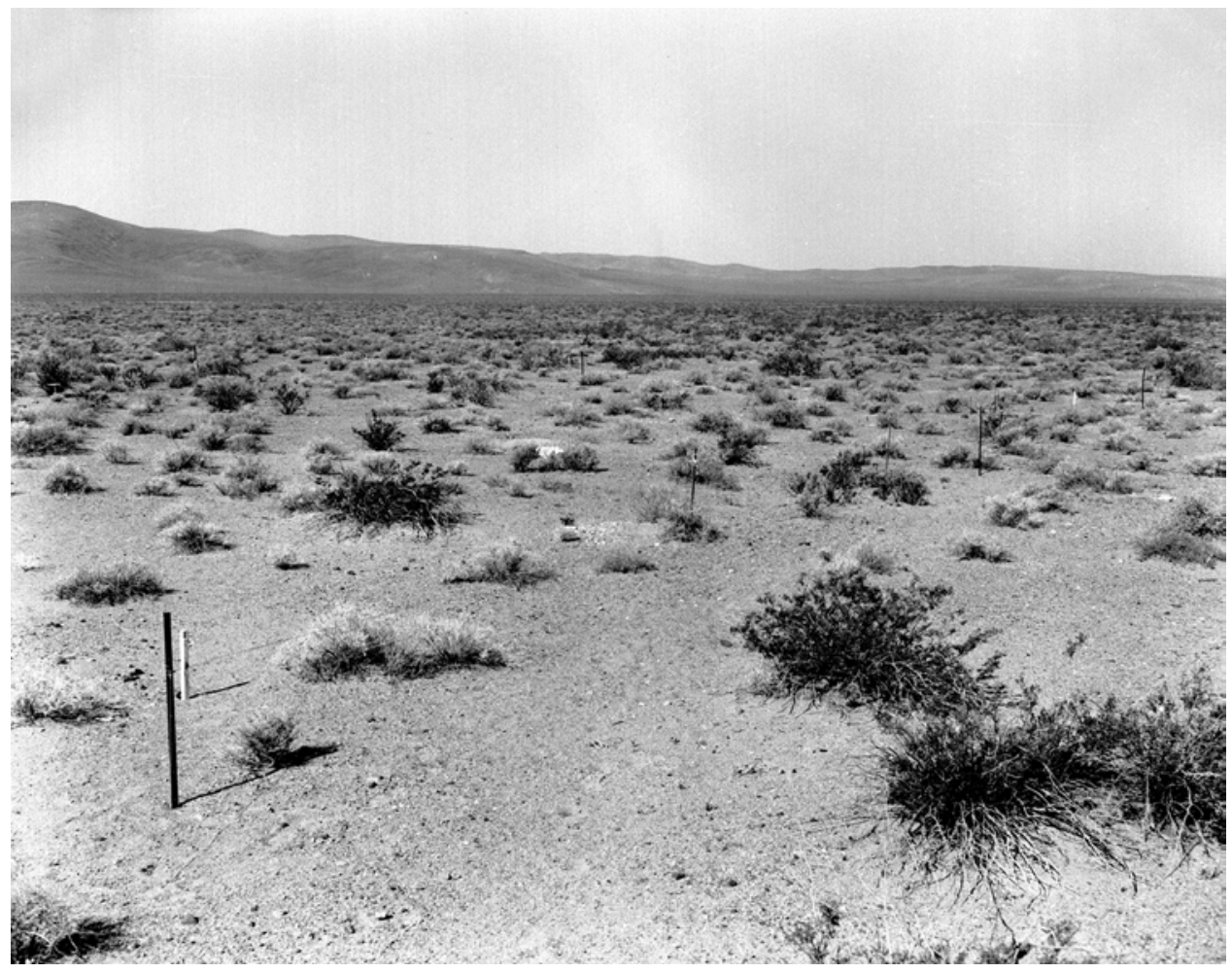

A. (May 14, 1964). In this southwesterly view across Plot 6, a creosote bush - white bursage community appears in the foreground and the westerly flanks of Little Skull Mountain appear in the background (Janice Beatley Collection, 112-B).

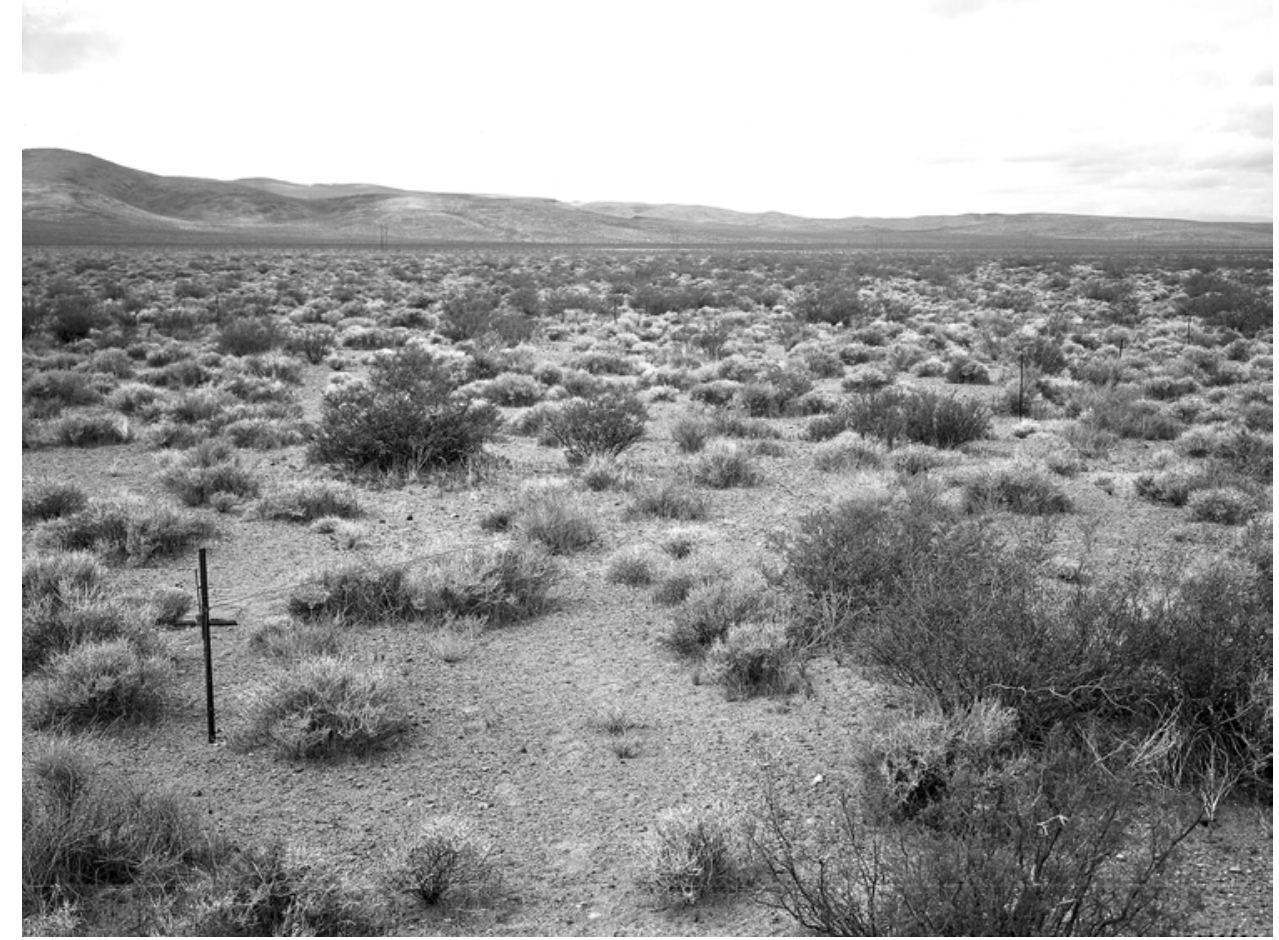

B. (April 5, 2001). Most of the individual plants depicted in the original view are much larger in 2001, and in general the increase in biomass is striking (R.H. Webb, Stake 4103B). 
Table A-6B. Summary plant data for Plot 6.

\author{
SPECIES \\ Ambrosia dumosa \\ Ephedra nevadensis \\ Grayia spinosa \\ Krameria parvifolia \\ Larrea tridentata \\ Lycium andersonii \\ Psorothamnus fremontii \\ Total live \\ Dead shrubs \\ Total
}

\section{SPECIES}

Ambrosia dumosa

Ephedra nevadensis

Grayia spinosa

Krameria parvifolia

Larrea tridentata

Lycium andersonii

Psorothamnus fremontii

\section{SPECIES}

Ambrosia dumosa

Ephedra nevadensis

Grayia spinosa

Krameria parvifolia

Larrea tridentata

Lycium andersonii

Psorothamnus fremontii

Total live

Dead shrubs

Total

\section{SPECIES}

Ambrosia dumosa

Ephedra nevadensis

Grayia spinosa

Krameria parvifolia

Larrea tridentata

Lycium andersonii

Psorothamnus fremontii

Total live

\section{Number of Plants}

1963

45

5

13

22

27

5

0

117

10

127

1963

0.33

0.38

0.46

0.18

0.78

0.55

0.00

1963

4.68

0.51

1.39

2.24

3.26

1.05

0.00

13.14

5.16

18.30

1963

5.62

0.68

2.16

1.29

9.30

1.87

0.00

20.91
1975

65

11

16

19

27

11

1

150

10

160

Average Height (m)

1975

0.36

0.40

0.58

0.18

0.71

0.47

0.18

Cover (\%)

1975

7.33

1.18

2.69

2.06

5.90

1.76

0.12

21.05

1.01

22.05

2001

0.37

0.47

0.68

0.24

1.07

0.52

0.48
2001

3

17

24

6

1

183

19
2001

10.72

2.89

0.34

2.14

5.65

0.67

0.02

22.42

2.46

24.88

Biomass Index $\left(\mathrm{m}^{2}\right)$

19752001

$9.39 \quad 13.53$

$2.02 \quad 4.91$

$5.31 \quad 0.77$

$1.32 \quad 1.89$

$15.03 \quad 20.71$

$2.85 \quad 1.01$

$0.07 \quad 0.03$

$35.99 \quad 42.85$ 
Table A-7A. Site characteristics for Plot 7.

Location: Jackass Flats

NTS Area: 25

NTS Grid: ZZ-15

USGS 7.5’ Quadrangle Name: Jackass Flats

\begin{tabular}{lllc} 
& & \multicolumn{2}{c}{ GPS Readings (Zone 11S) } \\
& Location & Northing & Easting \\
Corner 1: & SW & 4069717 & 557352 \\
Corner 2: & NW & 4069748 & 557353 \\
Corner 3: & NE & 4069749 & 557382 \\
Corner 4: & SE & 4069717 & 557383
\end{tabular}

DEM Plot Elevation: 913 m

Condition of Original Corners
Standing
Standing
Standing
Standing

Plot Aspect: $98^{\circ}$

Elevation Above Playa (Playa Name): NA

Plot Slope: $5^{\circ}$

Annual Precipitation

Measured: $122 \mathrm{~mm}$

Modeled: $130 \mathrm{~mm}$

Parent Material Type: alluvial fan

Substrate: alluvial fan, sandy mixed, mostly volcanic

Slate Geologic Unit: Qay

Beatley Plant Assemblage: Larrea-Ambrosia

Ostler-Hanson Plant Association: Menodora-Ambrosia-Larrea

Ostler-Hanson Land Unit: 351

Abundance of Biological Soil Crusts: none

Type and Date of Disturbance: none

Plot Condition: good

Location of Transect 1: south side of plot

Direction Transects Are Read: west to east

Date(s) Plot Remeasured: 4 April 2001 
Figure A-7. Photographs showing Plot 7.

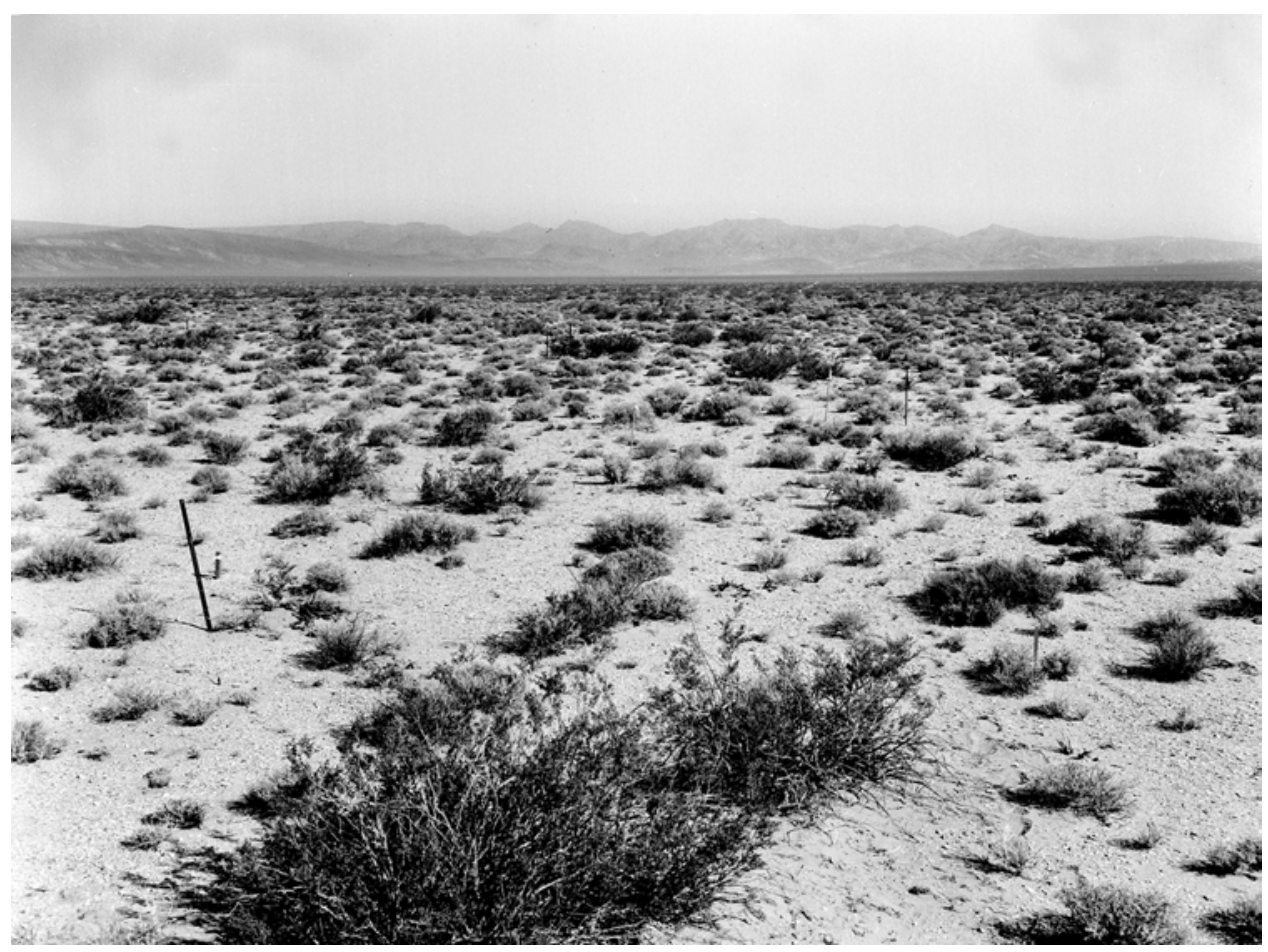

A. (May 14, 1964). This view is north across Plot 7 towards the Calico Hills and Shoshone Mountain in the background. The vegetation is creosote bush - white bursage with several other species present in the sandy substrate (Janice Beatley Collection, 107-B).

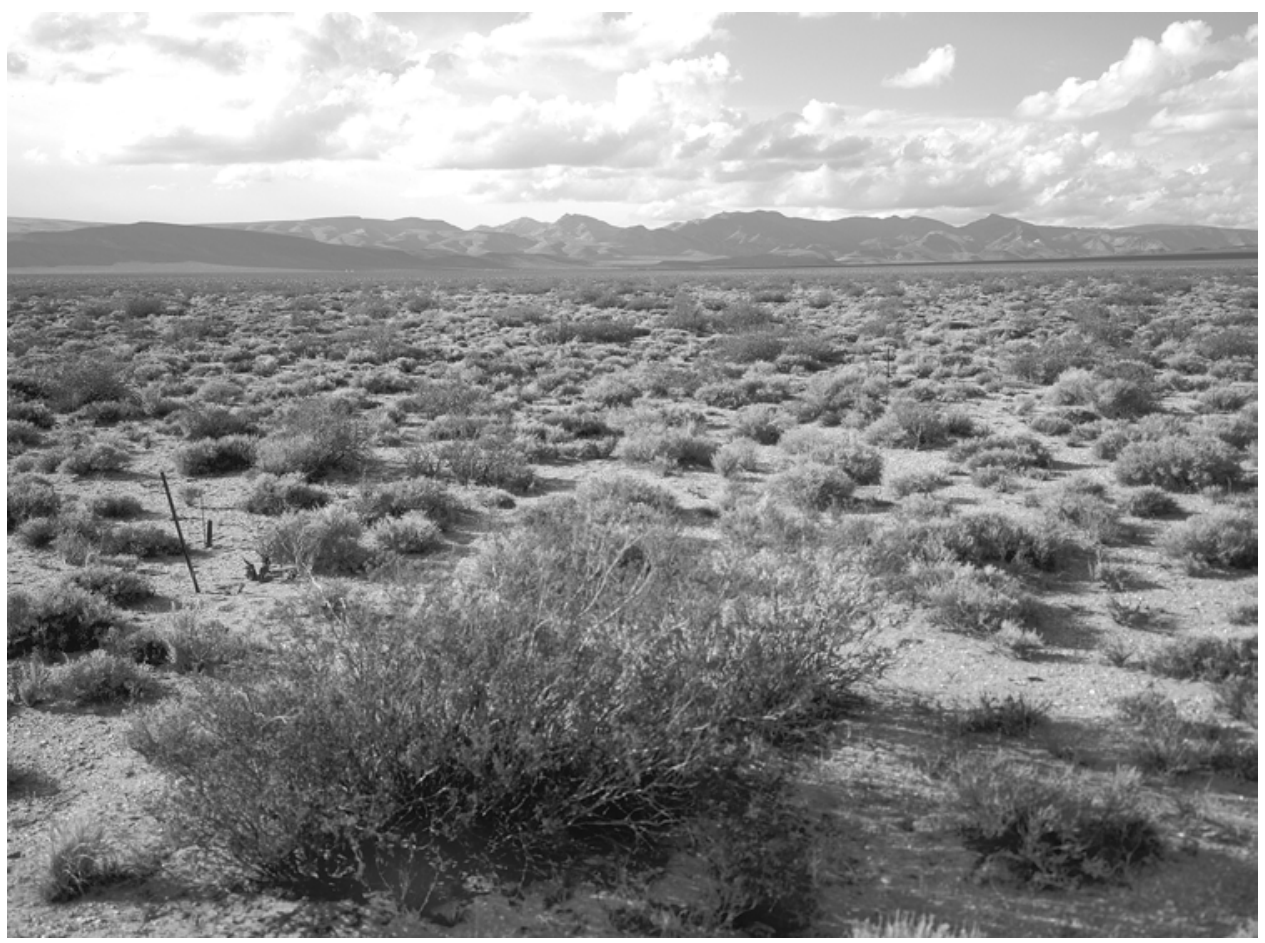

B. (April 5, 2001). The density, cover, and biomass of perennial vegetation has greatly increased, and numerous shrubs that were present in 1964 are still present. In 2001, spiny menodora (Menodora spinescens) has increased to become the most abundant species by cover (R.H. Webb, Stake 4109B). 
Table A-7B. Summary plant data for Plot 7.

\begin{tabular}{|c|c|c|c|c|c|c|c|}
\hline \multicolumn{5}{|c|}{ Number of Plants } & \multicolumn{3}{|c|}{ Cover (\%) } \\
\hline SPECIES & 1963 & 1975 & 2001 & SPECIES & 1963 & 1975 & 2001 \\
\hline Acamptopappus shockleyi & 55 & 51 & 22 & Acamptopappus shockleyi & 4.37 & 3.25 & 0.61 \\
\hline Ambrosia dumosa & 33 & 42 & 56 & Ambrosia dumosa & 4.16 & 4.67 & 3.78 \\
\hline Atriplex canescens & 2 & 2 & 3 & Atriplex canescens & 0.37 & 0.52 & 0.00 \\
\hline Ceratoides lanata & 5 & 8 & 4 & Ceratoides lanata & 0.38 & 0.55 & 0.12 \\
\hline Larrea tridentata & 18 & 24 & 24 & Larrea tridentata & 3.84 & 4.65 & 3.78 \\
\hline Menodora spinescens & 44 & 48 & 105 & Menodora spinescens & 7.18 & 6.84 & 7.88 \\
\hline Oryzopsis hymenoides & 9 & 30 & 27 & Oryzopsis hymenoides & 0.42 & 2.59 & 0.96 \\
\hline Sphaeralcea emoryi & 0 & 1 & 1 & Sphaeralcea emoryi & 0.00 & 0.10 & 0.02 \\
\hline Total Live & 166 & 206 & 242 & Total Live & 20.73 & 23.15 & 17.15 \\
\hline Dead Grass & 0 & 0 & 7 & Dead Grass & 0.00 & 0.00 & 0.15 \\
\hline Dead Shrub & 10 & 22 & 103 & Dead Shrub & 1.81 & 2.22 & 11.79 \\
\hline Total & 176 & 228 & 352 & Total & 22.54 & 25.37 & 29.09 \\
\hline \multicolumn{5}{|c|}{ Average Height (m) } & \multicolumn{3}{|c|}{ Biomass Index $\left(\mathrm{m}^{2}\right)$} \\
\hline SPECIES & 1963 & 1975 & 2001 & SPECIES & 1963 & 1975 & 2001 \\
\hline Acamptopappus shockleyi & 0.18 & 0.18 & 0.21 & Acamptopappus shockleyi & 2.81 & 2.04 & 0.44 \\
\hline Ambrosia dumosa & 0.33 & 0.30 & 0.29 & Ambrosia dumosa & 4.66 & 5.04 & 4.02 \\
\hline Atriplex canescens & 0.47 & 0.83 & 0.56 & Atriplex canescens & 0.58 & 1.44 & 0.00 \\
\hline Ceratoides lanata & 0.38 & 0.33 & 0.38 & Ceratoides lanata & 0.48 & 0.68 & 0.16 \\
\hline Larrea tridentata & 0.67 & 0.77 & 0.84 & Larrea tridentata & 9.43 & 12.79 & 11.35 \\
\hline Menodora spinescens & 0.15 & 0.17 & 0.21 & Menodora spinescens & 3.83 & 3.96 & 6.38 \\
\hline Oryzopsis hymenoides & 0.28 & 0.30 & 0.33 & Oryzopsis hymenoides & 0.40 & 2.86 & 1.09 \\
\hline \multirow[t]{2}{*}{ Sphaeralcea emoryi } & 0.00 & 0.33 & 0.06 & Sphaeralcea emoryi & 0.00 & 0.11 & 0.00 \\
\hline & & & & Total Live & 22.20 & 28.90 & 23.45 \\
\hline
\end{tabular}


Table A-8A. Site characteristics for Plot 8.

Location: Jackass Flats

NTS Area: 25

NTS Grid: XX-14

USGS 7.5’ Quadrangle Name: Busted Butte

\begin{tabular}{|c|c|c|c|c|}
\hline & & GPS Reac & Zone 11S) & \\
\hline & Location & Northing & Easting & Condition of Original Corners \\
\hline Corner 1: & SW & 4067758 & 555685 & Standing \\
\hline Corner 2: & NW & 4067788 & 555685 & Standing \\
\hline Corner 3: & $\mathrm{NE}$ & 4067789 & 555715 & Standing \\
\hline Corner 4: & SE & 4067759 & 555715 & Standing \\
\hline DEM Plo & vation: 93 & & & Plot Aspect: $0^{\circ}$ \\
\hline Elevation & e Playa & Name): I & & Plot Slope: $1^{\circ}$ \\
\hline
\end{tabular}

Annual Precipitation

Measured: $117 \mathrm{~mm}$

Modeled: $127 \mathrm{~mm}$

Parent Material Type: alluvial fan

Substrate: alluvial fan, mixed sand mostly volcanic aeolin sand

Slate Geologic Unit: Qai

Beatley Plant Assemblage: Larrea-Ambrosia

Ostler-Hanson Plant Association: Ambrosia-Oryzopsis-Acamptopappus

Ostler-Hanson Land Unit: 352

Abundance of Biological Soil Crusts: none

Type and Date of Disturbance: none

Plot Condition: good

Location of Transect 1: south side of plot

Direction Transects Are Read: east to west

Date(s) Plot Remeasured: 4 April 2001 
Figure A-8. Photographs showing Plot 8.

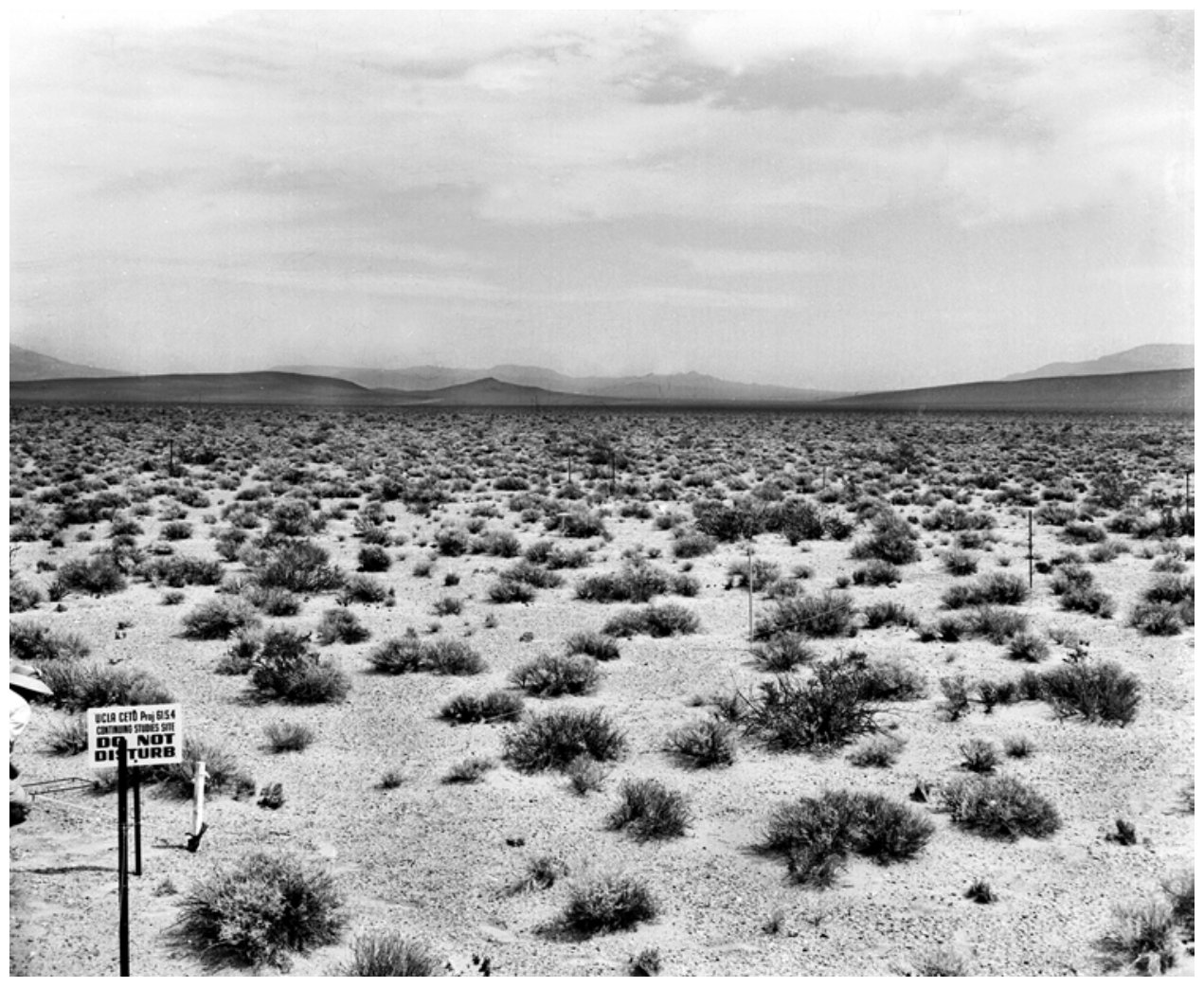

A. (April 17, 1964). This view is northeasterly across Plot 8 and Jackass Flats towards the Calico Hills, Shoshone Mountain, and Lookout Peak. The vegetation is creosote bush dominated with several other species present. Janice Beatley appears at the extreme left side measuring annuals (Janice Beatley Collection, 8-B).

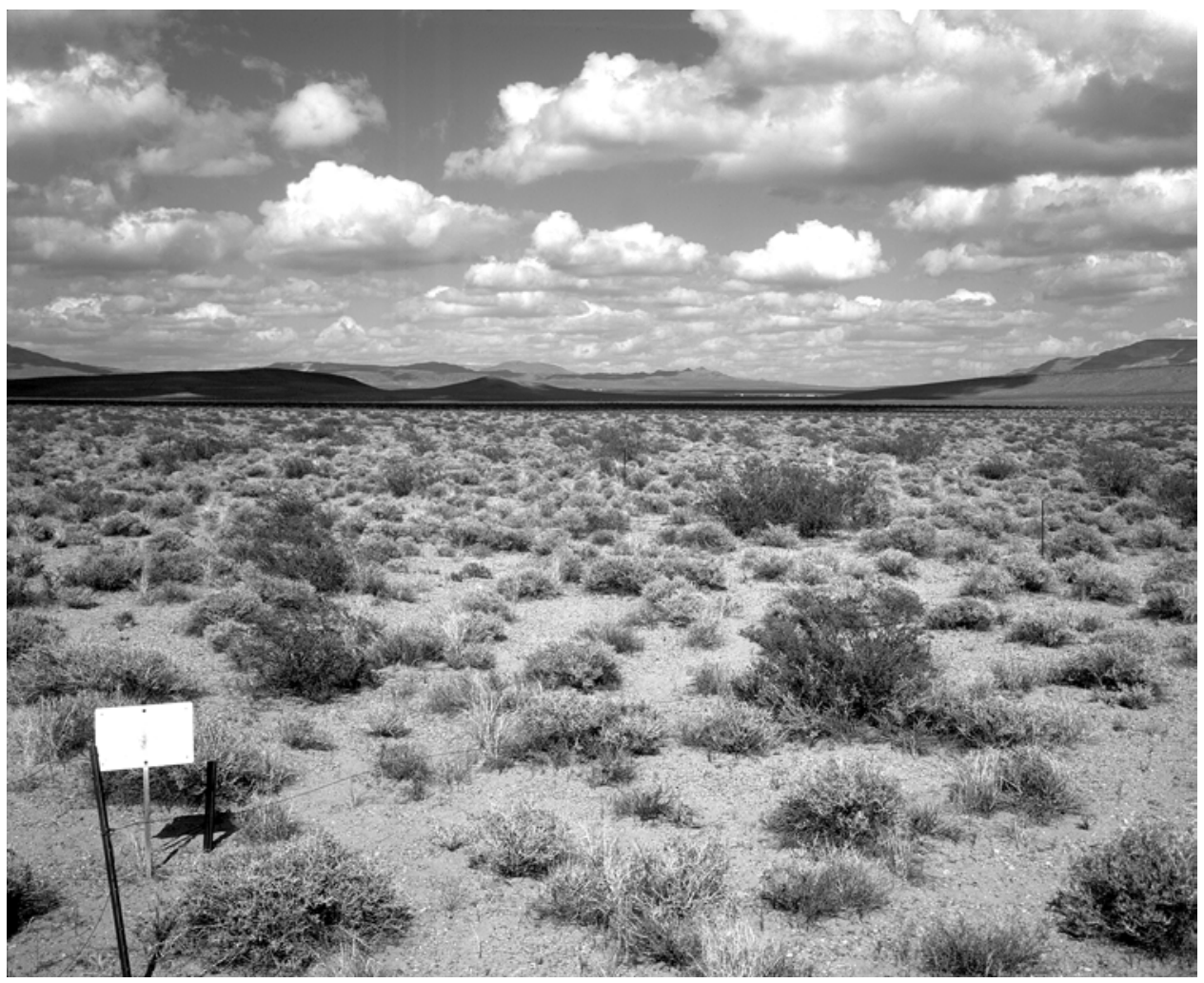

B. (April 5, 2001). The increase in cover, density, and biomass of the perennial species is striking in the 37 years between the original view and its match (R.H. Webb, Stake 4107A). 
Table A-8B. Summary plant data for Plot 8.

\author{
SPECIES \\ Acamptopappus shockleyi \\ Ambrosia dumosa \\ Ceratoides lanata \\ Larrea tridentata \\ Menodora spinescens \\ Oryzopsis hymenoides \\ Sphaeralcea emoryi \\ Total Live \\ Dead Grass \\ Dead Shrub \\ Total
}

\section{SPECIES}

Acamptopappus shockleyi

Ambrosia dumosa

Ceratoides lanata

Larrea tridentata

Menodora spinescens

Oryzopsis hymenoides

Sphaeralcea emoryi

\section{SPECIES}

Acamptopappus shockleyi

Ambrosia dumosa

Ceratoides lanata

Larrea tridentata

Menodora spinescens

Oryzopsis hymenoides

Sphaeralcea emoryi

Total Live

Dead Grass

Dead Shrub

Total

\section{SPECIES}

Acamptopappus shockleyi

Ambrosia dumosa

Ceratoides lanata

Larrea tridentata

Menodora spinescens

Oryzopsis hymenoides

Sphaeralcea emoryi

Total Live
1963

59

62

3

14

1

17

0

156

0

9

165

1963

0.25

0.31

0.41

0.55

0.23

0.25

0.00

1963

5.85

8.94

0.22

1.57

0.25

0.91

0.00

17.74

0.00

0.89

18.63

\section{3}

5.96

10.13

0.32

2.82

0.20

0.89

0.00

20.31

\section{Number of Plants}

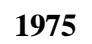

2001

68

47

107

14

24

3

60

0

255

16

40

311

\section{Average Height (m)}

19752001

$0.22 \quad 0.26$

$0.29 \quad 0.35$

$0.41 \quad 0.43$

$0.67 \quad 0.74$

$0.11 \quad 0.18$

$0.37 \quad 0.36$

$0.48 \quad 0.00$

Cover (\%)

19752001

$5.31 \quad 2.25$

$8.17 \quad 12.55$

$0.85 \quad 0.86$

$3.53 \quad 3.34$

$\begin{array}{ll}0.07 & 0.54\end{array}$

$5.74 \quad 2.86$

$0.06 \quad 0.00$

$23.74 \quad 22.40$

$0.13 \quad 0.56$

$1.43 \quad 5.75$

$25.29 \quad 28.72$

Biomass Index $\left(\mathrm{m}^{2}\right)$

19752001

$4.08 \quad 2.07$

$8.93 \quad 15.65$

$1.29 \quad 1.22$

$8.09 \quad 8.80$

$0.03 \quad 0.34$

$\begin{array}{ll}7.58 & 3.95\end{array}$

$0.10 \quad 0.00$

$30.11 \quad 32.03$ 
Table A-9A. Site characteristics for Plot 9 .

Location: Jackass Flats

NTS Area: 25

NTS Grid: ZZ-16

USGS 7.5’ Quadrangle Name: Jackass Flats

\begin{tabular}{lllc} 
& & \multicolumn{2}{c}{ GPS Readings (Zone 11S) } \\
& Location & Northing & Easting \\
Corner 1: & SW & 4072154 & 556655 \\
Corner 2: & NW & 4072190 & 556660 \\
Corner 3: & NE & 4072185 & 556690 \\
Corner 4: & SE & 4072154 & 556685
\end{tabular}

DEM Plot Elevation: 992 m

Condition of Original Corners
Standing
On ground, replaced in original position
Standing
Standing

Plot Aspect: $135^{\circ}$

Plot Slope: $3^{\circ}$

Elevation Above Playa (Playa Name): NA

Annual Precipitation

Measured: $130 \mathrm{~mm}$

Modeled: $132 \mathrm{~mm}$

Parent Material Type: alluvial fan

Substrate: NA

Slate Geologic Unit: Qay

Beatley Plant Assemblage: Menodora-Ephedra

Ostler-Hanson Plant Association: Menodora-Ephedra-Krameria

Ostler-Hanson Land Unit: 313

Abundance of Biological Soil Crusts: NA

Type and Date of Disturbance: NA

Plot Condition: good

Location of Transect 1: south side of plot

Direction Transects Are Read: west to east

Date(s) Plot Remeasured: 7 April 2001 
Figure A-9. Photographs showing Plot 9.

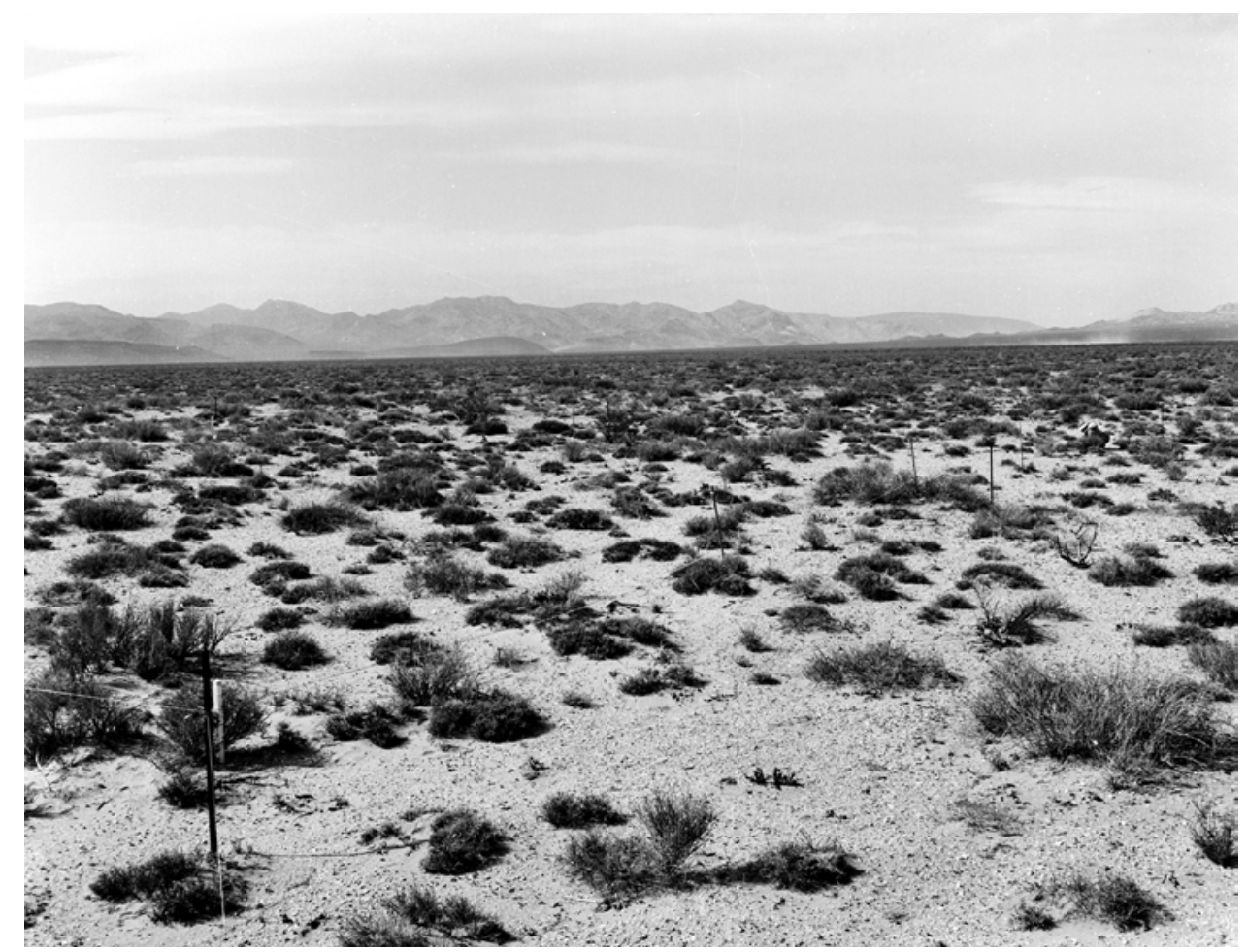

A. (April 17, 1964). This northerly view across Plot 9 and Jackass Flats shows a vegetation assemblage dominated by spiny menodora and Mormon tea (Ephedra nevadensis) with the Calico Hills in the background. An individual is measuring annual plants at right midground (Janice Beatley Collection, 9-B).

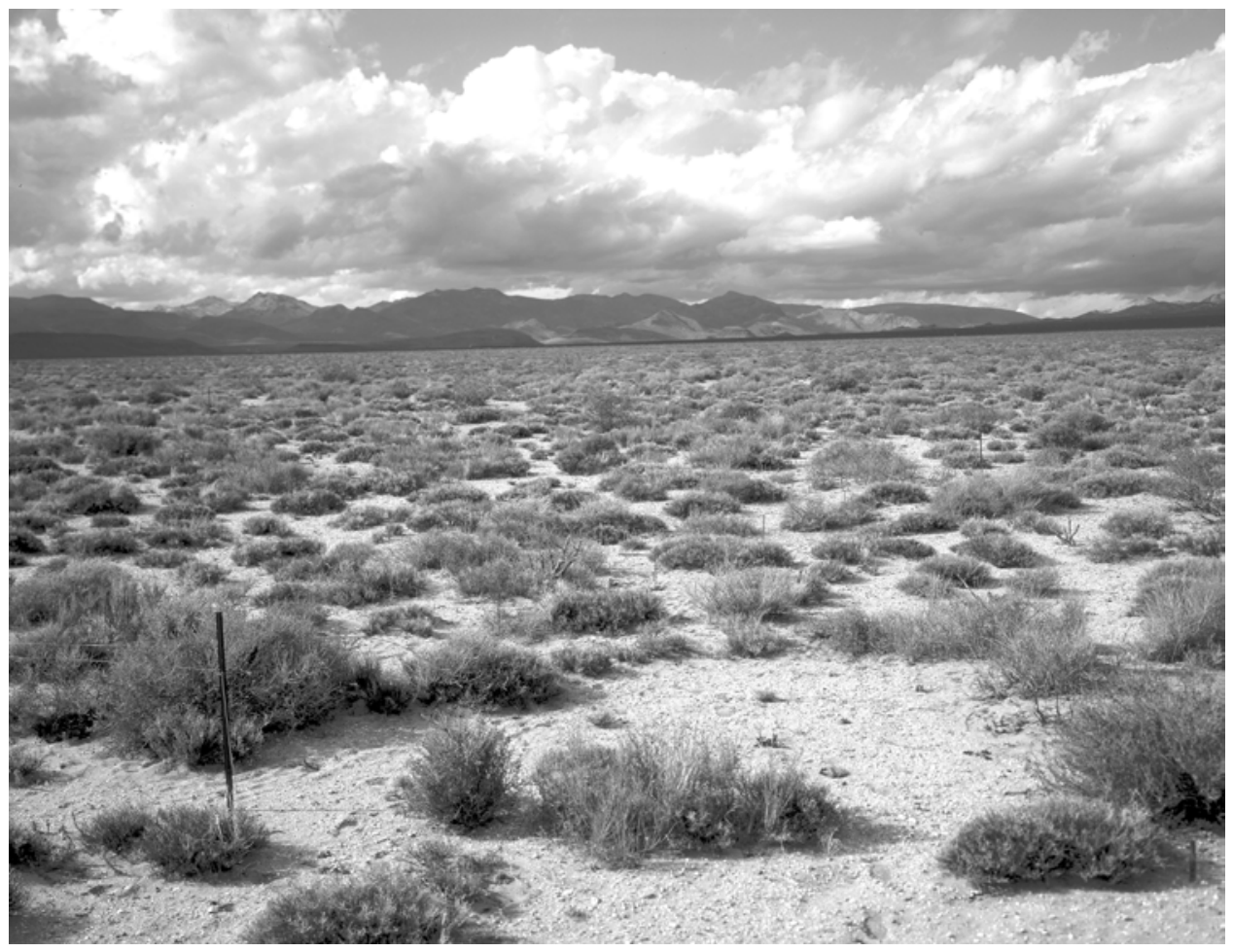

B. (April 7, 2001). Despite the fact that the spiny menodora has died back owing either to recent drought or freezes in 1989-1990, the biomass of perennial vegetation is still much higher than it was in 1964 (R.H. Webb, Stake 4126B). 
Table A-9B. Summary plant data for Plot 9.

\begin{tabular}{|c|c|c|c|c|c|c|c|}
\hline \multicolumn{5}{|c|}{ Number of Plants } & \multicolumn{3}{|c|}{ Cover (\%) } \\
\hline SPECIES & 1963 & 1975 & 2001 & SPECIES & 1963 & 1975 & 2001 \\
\hline Ambrosia dumosa & 1 & 0 & 2 & Ambrosia dumosa & 0.01 & 0.00 & 0.35 \\
\hline Ceratoides lanata & 1 & 1 & 0 & Ceratoides lanata & 0.13 & 0.08 & 0.00 \\
\hline Coleogyne ramosissima & 1 & 1 & 5 & Coleogyne ramosissima & 0.08 & 0.11 & 0.65 \\
\hline Ephedra nevadensis & 31 & 42 & 53 & Ephedra nevadensis & 5.79 & 6.85 & 6.49 \\
\hline Krameria parvifolia & 22 & 25 & 25 & Krameria parvifolia & 3.12 & 2.99 & 2.88 \\
\hline Larrea tridentata & 2 & 3 & 2 & Larrea tridentata & 0.01 & 0.29 & 0.16 \\
\hline Menodora spinescens & 61 & 75 & 97 & Menodora spinescens & 9.45 & 10.03 & 6.51 \\
\hline Opuntia echinocarpa & 1 & 0 & 0 & Opuntia echinocarpa & 0.25 & 0.00 & 0.00 \\
\hline Oryzopsis hymenoides & 9 & 23 & 35 & Oryzopsis hymenoides & 0.53 & 1.39 & 1.41 \\
\hline Sphaeralcea emoryi & 0 & 1 & 0 & Sphaeralcea emoryi & 0.00 & 0.01 & 0.00 \\
\hline Total Live & 129 & 171 & 219 & Total Live & 19.36 & 21.75 & 18.46 \\
\hline Dead Grass & 0 & 0 & 7 & Dead Grass & 0.00 & 0.00 & 0.17 \\
\hline Dead Shrub & 8 & 15 & 66 & Dead Shrub & 2.28 & 1.94 & 12.89 \\
\hline Total & 137 & 186 & 292 & Total & 21.65 & 23.69 & 31.53 \\
\hline \multicolumn{5}{|c|}{ Average Height (m) } & \multicolumn{3}{|c|}{ Biomass Index (m²) } \\
\hline SPECIES & 1963 & 1975 & 2001 & SPECIES & 1963 & 1975 & 2001 \\
\hline Ambrosia dumosa & 0.20 & 0.00 & 0.42 & Ambrosia dumosa & 0.01 & 0.00 & 0.55 \\
\hline Ceratoides lanata & 0.36 & 0.41 & 0.00 & Ceratoides lanata & 0.15 & 0.11 & 0.00 \\
\hline Coleogyne ramosissima & 0.51 & 0.66 & 0.61 & Coleogyne ramosissima & 0.14 & 0.24 & 1.59 \\
\hline Ephedra nevadensis & 0.41 & 0.50 & 0.48 & Ephedra nevadensis & 9.71 & 12.54 & 11.79 \\
\hline Krameria parvifolia & 0.21 & 0.21 & 0.25 & Krameria parvifolia & 2.35 & 2.21 & 2.51 \\
\hline Larrea tridentata & 0.67 & 0.54 & 0.55 & Larrea tridentata & 0.01 & 0.52 & 0.34 \\
\hline Menodora spinescens & 0.17 & 0.15 & 0.14 & Menodora spinescens & 5.65 & 5.60 & 3.60 \\
\hline Opuntia echinocarpa & 0.30 & 0.00 & 0.00 & Opuntia echinocarpa & 0.25 & 0.00 & 0.00 \\
\hline Oryzopsis hymenoides & 0.34 & 0.27 & 0.34 & Oryzopsis hymenoides & 0.58 & 1.30 & 1.74 \\
\hline \multirow[t]{2}{*}{ Sphaeralcea emoryi } & 0.00 & 0.33 & 0.00 & Sphaeralcea emoryi & 0.00 & 0.01 & 0.00 \\
\hline & & & & Total Live & 18.86 & 22.54 & 22.12 \\
\hline
\end{tabular}


Table A-10A. Site characteristics for Plot 10.

Location: Jackass Flats

NTS Area: 25

NTS Grid: B-16

USGS 7.5’ Quadrangle Name: Jackass Flats

\begin{tabular}{lllll} 
& & \multicolumn{2}{l}{ GPS Readings (Zone 11S) } & \\
& Location & Northing & Easting & Condition of Original Corners \\
Corner 1: & SW & 4072305 & 560773 & Standing \\
Corner 2: & NW & 4072331 & 560790 & Missing \\
Corner 3: & NE & 4072316 & 560814 & Standing \\
Corner 4: & SE & 4072289 & 560799 & Standing
\end{tabular}

DEM Plot Elevation: 1038 m

Plot Aspect: $270^{\circ}$

Elevation Above Playa (Playa Name): NA

Plot Slope: $1^{\circ}$

Annual Precipitation

Measured: $125 \mathrm{~mm}$

Modeled: $136 \mathrm{~mm}$

Parent Material Type: alluvial fan

Substrate: alluvial fan, mixed mostly volcanic eolian sand

Slate Geologic Unit: Qay

Beatley Plant Assemblage: Larrea-Ambrosia/Larrea-Lycium-Grayia

Ostler-Hanson Plant Association: Ambrosia-Larrea-Oryzopsis

Ostler-Hanson Land Unit: 322

Abundance of Biological Soil Crusts: none

Type and Date of Disturbance: none

Plot Condition: good

Location of Transect 1: northeast side of plot

Direction Transects Are Read: southeast to northwest

Date(s) Plot Remeasured: 4 April 2001 
Figure A-10. Photographs showing Plot 10.

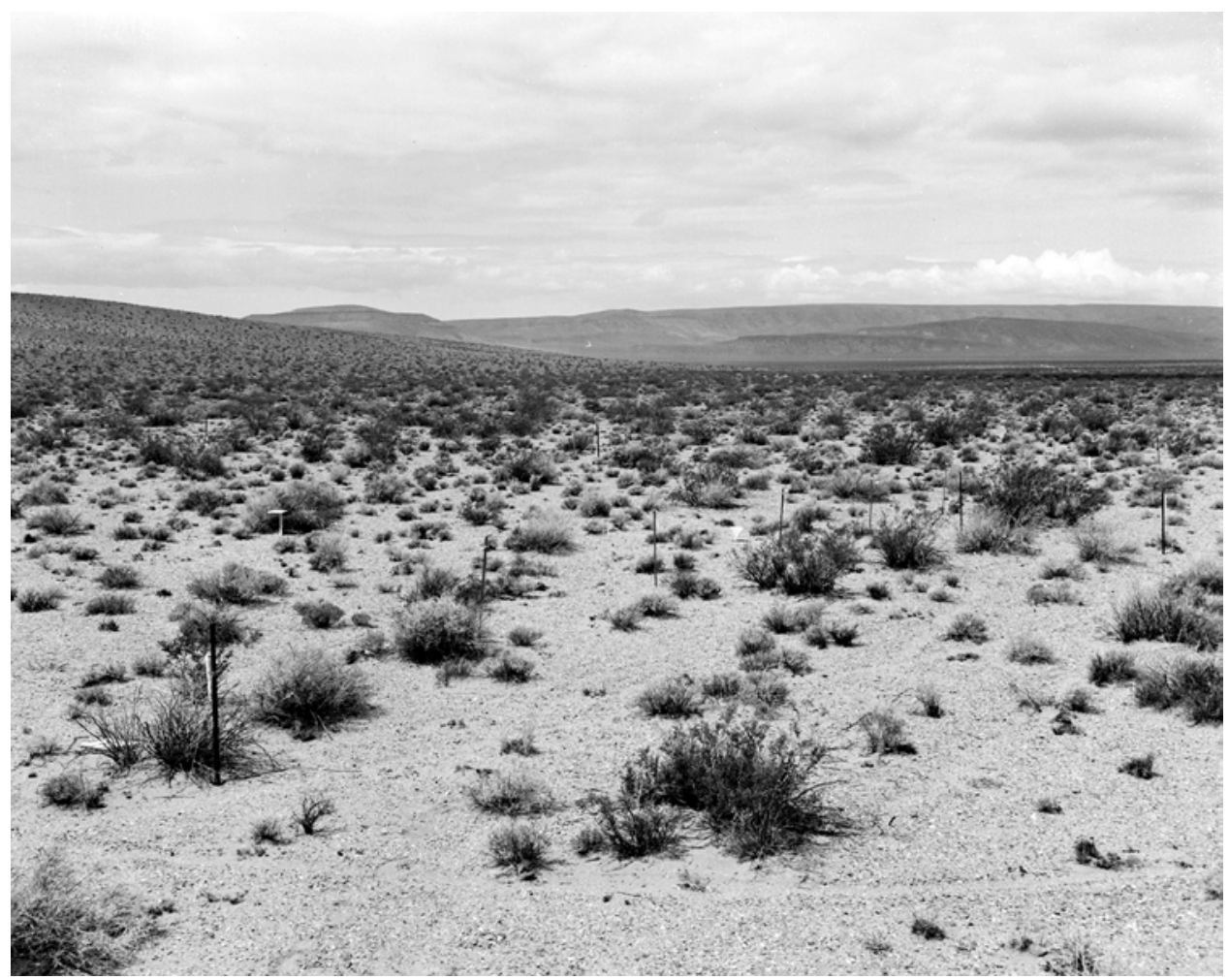

A. (April 18, 1964). This westerly view across Plot 10 shows a mixed creosote-bush assemblage in the plot and Yucca Mountain in the background (Janice Beatley Collection, 12-B).

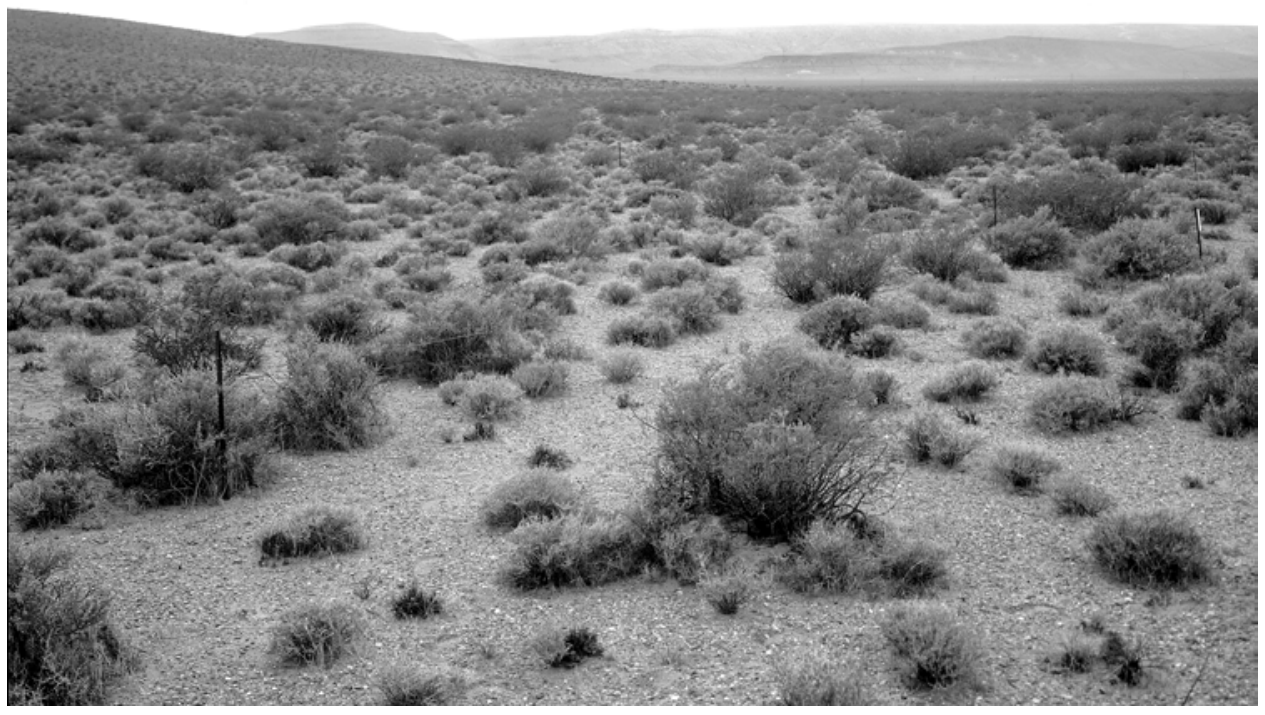

B. (April 6, 2001). The increase in density, cover, and biomass of perennial vegetation is striking, and white bursage, in the foreground, has particularly increased (R.H. Webb, Stake 4123A). 
Table A-10B. Summary plant data for Plot 10.

\begin{tabular}{|c|c|c|c|c|c|c|c|}
\hline \multicolumn{5}{|c|}{ Number of Plants } & \multicolumn{3}{|c|}{ Cover (\%) } \\
\hline SPECIES & 1963 & 1975 & 2001 & SPECIES & 1963 & 1975 & 2001 \\
\hline Acamptopappus shockleyi & 12 & 18 & 45 & Acamptopappus shockleyi & 1.08 & 1.61 & 3.93 \\
\hline Ambrosia dumosa & 61 & 75 & 98 & Ambrosia dumosa & 4.95 & 5.88 & 5.95 \\
\hline Ceratoides lanata & 23 & 22 & 3 & Ceratoides lanata & 1.65 & 1.64 & 0.16 \\
\hline Ericameria cooperi & 2 & 2 & 3 & Ericameria cooperi & 0.20 & 0.16 & 0.31 \\
\hline Grayia spinosa & 12 & 17 & 7 & Grayia spinosa & 2.64 & 2.18 & 0.60 \\
\hline Larrea tridentata & 20 & 25 & 22 & Larrea tridentata & 4.84 & 5.85 & 5.26 \\
\hline Lycium pallidum & 16 & 19 & 17 & Lycium pallidum & 3.43 & 3.54 & 3.97 \\
\hline Menodora spinescens & 2 & 1 & 19 & Menodora spinescens & 0.21 & 0.08 & 1.65 \\
\hline Oryzopsis hymenoides & 9 & 45 & 12 & Oryzopsis hymenoides & 0.26 & 3.63 & 0.35 \\
\hline Sphaeralcea emoryi & 0 & 3 & 0 & Sphaeralcea emoryi & 0.00 & 0.06 & 0.00 \\
\hline Total Live & 157 & 227 & 226 & Total Live & 19.25 & 24.63 & 22.18 \\
\hline Dead Grass & 0 & 2 & 15 & Dead Grass & 0.00 & 0.07 & 0.47 \\
\hline Dead Shrub & 1 & 8 & 44 & Dead Shrub & 0.15 & 1.34 & 6.97 \\
\hline Total & 158 & 237 & 285 & Total & 19.41 & 26.04 & 29.63 \\
\hline \multicolumn{5}{|c|}{ Average Height (m) } & \multicolumn{3}{|c|}{ Biomass Index $\left(\mathrm{m}^{2}\right)$} \\
\hline SPECIES & 1963 & 1975 & 2001 & SPECIES & 1963 & 1975 & 2001 \\
\hline Acamptopappus shockleyi & 0.23 & 0.27 & 0.32 & Acamptopappus shockleyi & 0.88 & 1.57 & 4.31 \\
\hline Ambrosia dumosa & 0.23 & 0.28 & 0.29 & Ambrosia dumosa & 3.95 & 5.72 & 5.88 \\
\hline Ceratoides lanata & 0.37 & 0.38 & 0.41 & Ceratoides lanata & 2.17 & 2.32 & 0.23 \\
\hline Ericameria cooperi & 0.22 & 0.36 & 0.35 & Ericameria cooperi & 0.15 & 0.17 & 0.39 \\
\hline Grayia spinosa & 0.63 & 0.66 & 0.62 & Grayia spinosa & 5.68 & 5.03 & 1.34 \\
\hline Larrea tridentata & 0.78 & 0.89 & 1.06 & Larrea tridentata & 13.63 & 18.88 & 19.45 \\
\hline Lycium pallidum & 0.67 & 0.71 & 0.71 & Lycium pallidum & 7.86 & 8.49 & 9.56 \\
\hline Menodora spinescens & 0.18 & 0.28 & 0.23 & Menodora spinescens & 0.12 & 0.08 & 1.34 \\
\hline Oryzopsis hymenoides & 0.22 & 0.37 & 0.29 & Oryzopsis hymenoides & 0.21 & 4.97 & 0.35 \\
\hline \multirow[t]{2}{*}{ Sphaeralcea emoryi } & 0.00 & 0.28 & 0.00 & Sphaeralcea emoryi & 0.00 & 0.07 & 0.00 \\
\hline & & & & Total Live & 34.67 & 47.31 & 42.86 \\
\hline
\end{tabular}


Table A-11A. Site characteristics for Plot 11.

Location: Jackass Flats

NTS Area: 25

NTS Grid: F-19

USGS 7.5’ Quadrangle Name: Jackass Flats

\begin{tabular}{lllc} 
& & \multicolumn{2}{c}{ GPS Readings (Zone 11S) } \\
& Location & Northing & Easting \\
Corner 1: & SW & 4077098 & 565850 \\
Corner 2: & NW & 4077127 & 565858 \\
Corner 3: & NE & 4077119 & 565888 \\
Corner 4: & SE & 4077091 & 565881
\end{tabular}

DEM Plot Elevation: 1193 m

Condition of Original Corners
Standing
Standing
Standing
Standing

Elevation Above Playa (Playa Name): NA

Plot Aspect: $104^{\circ}$

Plot Slope: $12^{\circ}$

Annual Precipitation

Measured: $142 \mathrm{~mm}$

Modeled: $152 \mathrm{~mm}$

Parent Material Type: alluvial fan

Substrate: alluvial fan, mixed volcanic alluvium

Slate Geologic Unit: Qay

Beatley Plant Assemblage: Larrea-Lycium-Grayia

Ostler-Hanson Plant Association: Larrea-Krameria-Ephedra

Ostler-Hanson Land Unit: 227

Abundance of Biological Soil Crusts: none

Type and Date of Disturbance: none

Plot Condition: good

Location of Transect 1: north side of plot

Direction Transects Are Read: west to east

Date(s) Plot Remeasured: 3 April 2001 
Figure A-11. Photographs showing Plot 11.

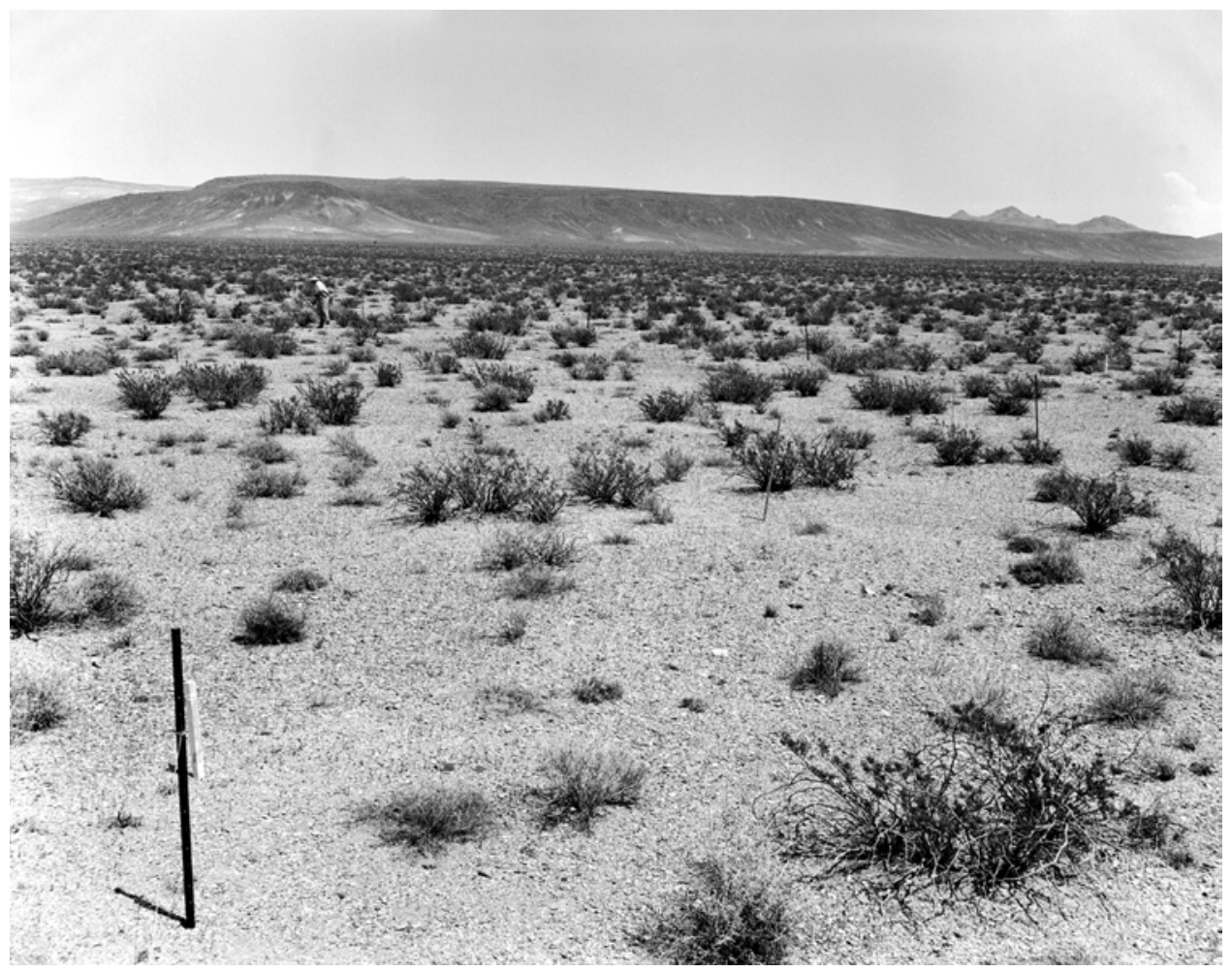

A. (April 30, 1964). This easterly view across Plot 11 shows a mixed creosote bush scrub assemblage with Kiwi Mesa in the background. Janice Beatley is standing in the photograph at left midground (Janice Beatley Collection, 46-B).

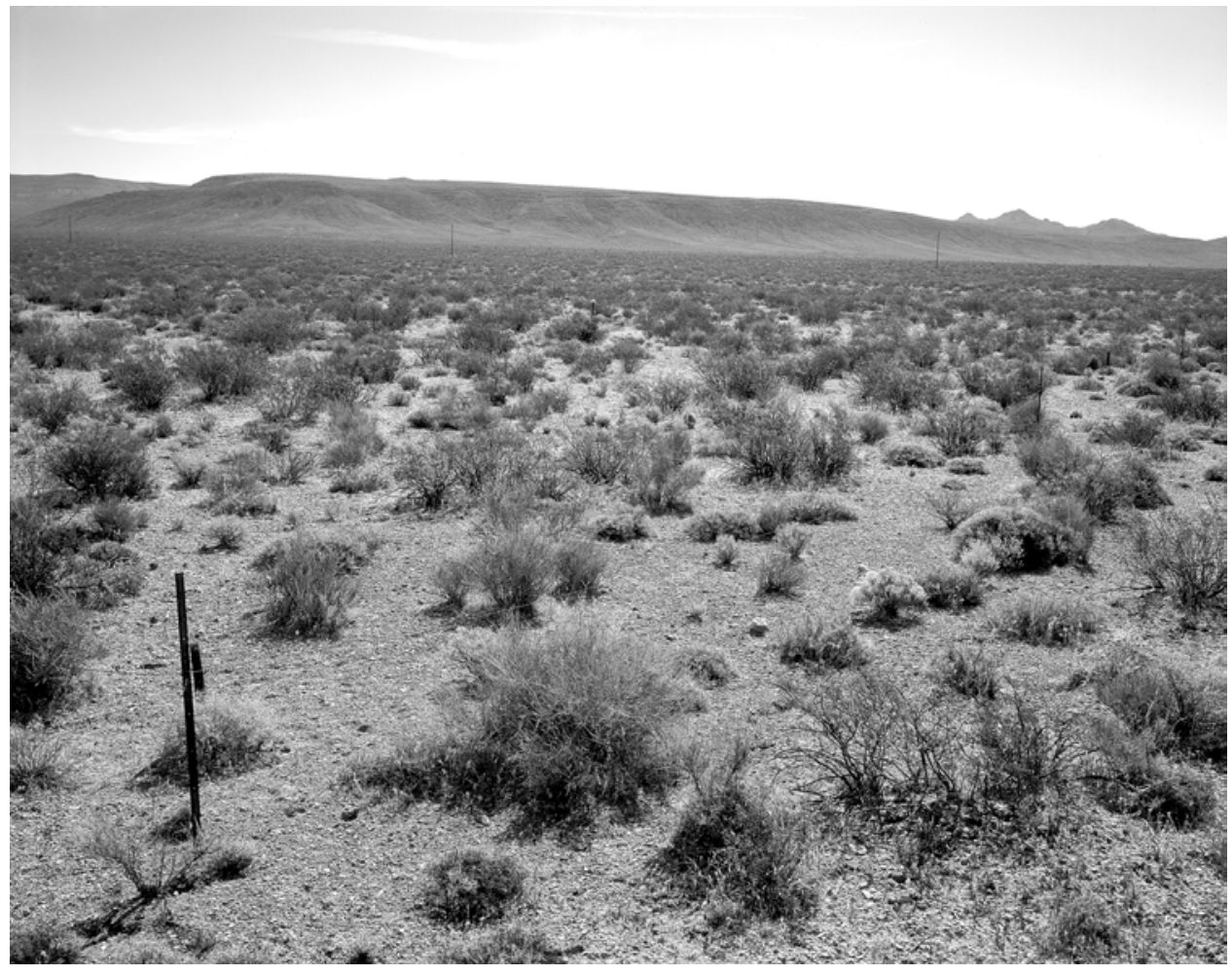

B. (June 8, 2001). The density, cover, and biomass of the perennial vegetation has increased dramatically in the 37 years that have elapsed between the original and matched photograph. Wolfberry and spiny hopsage decrease significantly on this plot, indicating that most of the increases are in creosote bush, range ratany, and Mormon tea (Dominic Oldershaw, Stake 4051B). 
Table A-11B. Summary plant data for Plot 11.

\begin{tabular}{|c|c|c|c|c|c|c|c|}
\hline \multicolumn{5}{|c|}{ Number of Plants } & \multicolumn{3}{|c|}{ Cover (\%) } \\
\hline SPECIES & 1963 & 1975 & 2001 & SPECIES & 1963 & 1975 & 2001 \\
\hline Acamptopappus shockleyi & 16 & 18 & 17 & Acamptopappus shockleyi & 0.92 & 0.90 & 1.11 \\
\hline Ambrosia dumosa & 0 & 1 & 6 & Ambrosia dumosa & 0.00 & 0.02 & 0.34 \\
\hline Ceratoides lanata & 0 & 2 & 1 & Ceratoides lanata & 0.00 & 0.13 & 0.09 \\
\hline Coleogyne ramosissima & 1 & 1 & 1 & Coleogyne ramosissima & 0.15 & 0.19 & 0.32 \\
\hline Ephedra nevadensis & 19 & 25 & 30 & Ephedra nevadensis & 1.91 & 3.24 & 4.72 \\
\hline Eriogonum inflatum & 0 & 2 & 3 & Eriogonum inflatum & 0.00 & 0.02 & 0.13 \\
\hline Grayia spinosa & 0 & 2 & 2 & Grayia spinosa & 0.00 & 0.05 & 0.11 \\
\hline Krameria parvifolia & 33 & 37 & 38 & Krameria parvifolia & 3.56 & 4.40 & 4.30 \\
\hline Larrea tridentata & 39 & 43 & 42 & Larrea tridentata & 5.28 & 6.67 & 6.10 \\
\hline Lycium andersonii & 12 & 15 & 15 & Lycium andersonii & 1.79 & 2.67 & 1.44 \\
\hline Menodora spinescens & 6 & 11 & 27 & Menodora spinescens & 0.25 & 0.95 & 2.73 \\
\hline Oryzopsis hymenoides & 7 & 20 & 11 & Oryzopsis hymenoides & 0.15 & 1.94 & 0.50 \\
\hline Xylorhiza tortifolia & 0 & 1 & 2 & Xylorhiza tortifolia & 0.00 & 0.02 & 0.06 \\
\hline Total Live & 133 & 178 & 195 & Total Live & 14.03 & 21.19 & 21.94 \\
\hline Dead Grass & 0 & 0 & 3 & Dead Grass & 0.00 & 0.00 & 0.15 \\
\hline Dead Shrub & 9 & 6 & 21 & Dead Shrub & 1.45 & 2.90 & 2.95 \\
\hline Total & 142 & 184 & 219 & Total & 15.48 & 24.09 & 25.03 \\
\hline \multicolumn{5}{|c|}{ Average Height (m) } & \multicolumn{3}{|c|}{ Biomass Index $\left(\mathrm{m}^{2}\right)$} \\
\hline SPECIES & 1963 & 1975 & 2001 & SPECIES & 1963 & 1975 & 2001 \\
\hline Acamptopappus shockleyi & 0.14 & 0.18 & 0.25 & Acamptopappus shockleyi & 0.46 & 0.57 & 0.95 \\
\hline Ambrosia dumosa & 0.00 & 0.30 & 0.26 & Ambrosia dumosa & 0.00 & 0.02 & 0.27 \\
\hline Ceratoides lanata & 0.00 & 0.38 & 0.78 & Ceratoides lanata & 0.00 & 0.16 & 0.14 \\
\hline Coleogyne ramosissima & 0.53 & 0.48 & 0.87 & Coleogyne ramosissima & 0.28 & 0.31 & 0.88 \\
\hline Ephedra nevadensis & 0.32 & 0.46 & 0.51 & Ephedra nevadensis & 2.09 & 5.42 & 8.79 \\
\hline Eriogonum inflatum & 0.00 & 0.08 & 0.36 & Eriogonum inflatum & 0.00 & 0.00 & 0.15 \\
\hline Grayia spinosa & 0.00 & 0.43 & 0.85 & Grayia spinosa & 0.00 & 0.08 & 0.32 \\
\hline Krameria parvifolia & 0.17 & 0.20 & 0.26 & Krameria parvifolia & 2.09 & 3.10 & 3.77 \\
\hline Larrea tridentata & 0.62 & 0.63 & 0.74 & Larrea tridentata & 11.31 & 14.53 & 16.40 \\
\hline Lycium andersonii & 0.45 & 0.47 & 0.41 & Lycium andersonii & 2.79 & 4.29 & 2.16 \\
\hline Menodora spinescens & 0.09 & 0.14 & 0.20 & Menodora spinescens & 0.09 & 0.45 & 1.87 \\
\hline Oryzopsis hymenoides & 0.16 & 0.31 & 0.31 & Oryzopsis hymenoides & 0.09 & 2.14 & 0.57 \\
\hline \multirow[t]{2}{*}{ Xylorhiza tortifolia } & 0.00 & 0.10 & 0.34 & Xylorhiza tortifolia & 0.00 & 0.01 & 0.07 \\
\hline & & & & Total Live & 19.19 & 31.08 & 36.34 \\
\hline
\end{tabular}


Table A-12A. Site characteristics for Plot 12.

Location: Jackass Flats

NTS Area: 25

NTS Grid: F-19

USGS 7.5’ Quadrangle Name: Jackass Flats

\begin{tabular}{lllll} 
& & \multicolumn{2}{c}{ GPS Readings (Zone 11S) } & \\
& Location & Northing & Easting & Condition of Original Corners \\
Corner 1: & SW & 4077726 & 565982 & Standing \\
Corner 2: & NW & 4077753 & 565996 & Standing \\
Corner 3: & NE & 4077738 & 566024 & Standing \\
Corner 4: & SE & 4077713 & 566010 & Standing
\end{tabular}

DEM Plot Elevation: 1209 m

Plot Aspect: $230^{\circ}$

Elevation Above Playa (Playa Name): NA

Plot Slope: $12^{\circ}$

Annual Precipitation

Measured: $149 \mathrm{~mm}$

Modeled: $154 \mathrm{~mm}$

Parent Material Type: alluvial fan

Substrate: alluvial fan, mixed volcanic alluvium

Slate Geologic Unit: Qay

Beatley Plant Assemblage: Larrea-Lycium-Grayia

Ostler-Hanson Plant Association: Larrea-Krameria-Lycium

Ostler-Hanson Land Unit: 227

Abundance of Biological Soil Crusts: none

Type and Date of Disturbance: none

Plot Condition: good

Location of Transect 1: northeast side of plot

Direction Transects Are Read: northwest to southeast

Date(s) Plot Remeasured: 3 April 2001 
Figure A-12. Photographs showing Plot 12.

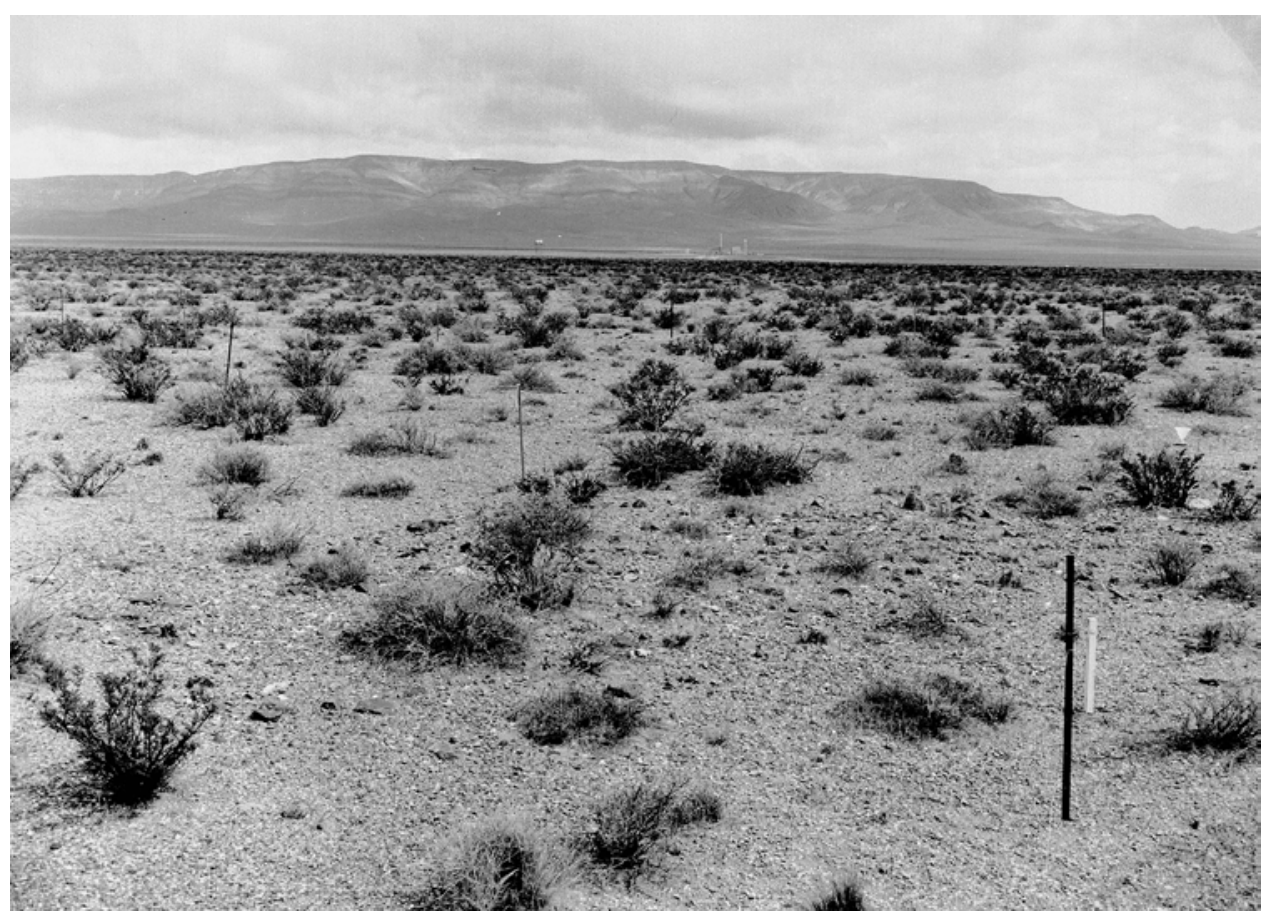

A. (May 6, 1964). This southeasterly view across Plot 12 and Jackass Flats shows the north side of Skull Mountain in the distance. The vegetation assemblage is creosote bush with other species present (Janice Beatley Collection, 51-A).

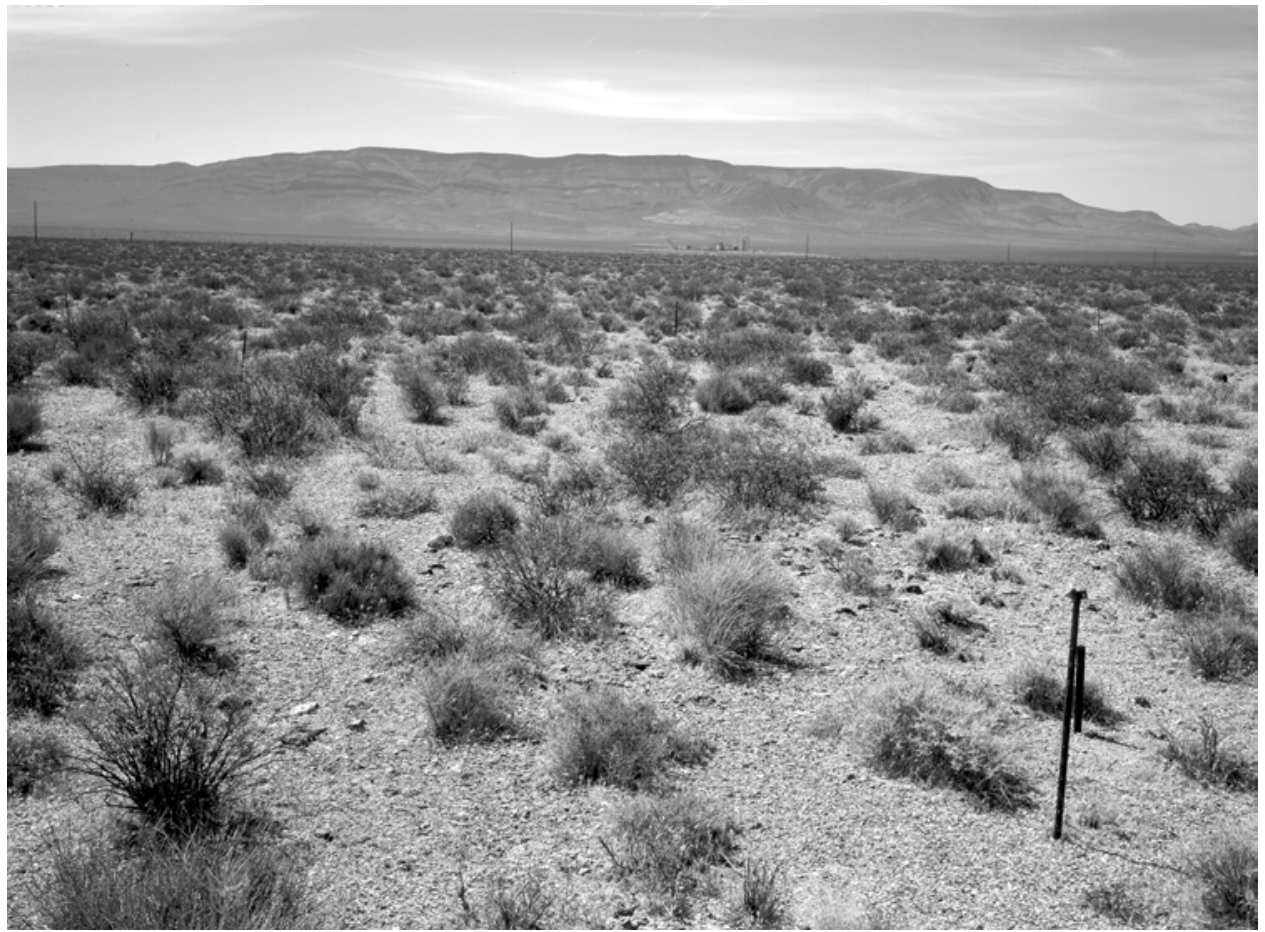

B. (June 8, 2001). The density, cover, and biomass of the perennial vegetation has increased dramatically in the intervening 37 years between the original and the match photograph. Again, wolfberry and spiny hopsage have decreased while other species have greatly increased (Dominic Oldershaw, Stake 4052A). 
Table A-12B. Summary plant data for Plot 12.

\begin{tabular}{|c|c|c|c|c|c|c|c|}
\hline \multicolumn{5}{|c|}{ Number of Plants } & \multicolumn{3}{|c|}{ Cover (\%) } \\
\hline SPECIES & 1963 & 1975 & 2001 & SPECIES & 1963 & 1975 & 2001 \\
\hline Acamptopappus shockleyi & 7 & 7 & 7 & Acamptopappus shockleyi & 0.25 & 0.35 & 0.33 \\
\hline Ambrosia dumosa & 1 & 0 & 18 & Ambrosia dumosa & 0.08 & 0.00 & 1.42 \\
\hline Ceratoides lanata & 8 & 10 & 1 & Ceratoides lanata & 0.35 & 0.71 & 0.05 \\
\hline Coleogyne ramosissima & 0 & 0 & 3 & Coleogyne ramosissima & 0.00 & 0.00 & 0.41 \\
\hline Ephedra nevadensis & 10 & 16 & 31 & Ephedra nevadensis & 0.63 & 1.01 & 3.85 \\
\hline Eriogonum inflatum & 1 & 3 & 0 & Eriogonum inflatum & 0.06 & 0.07 & 0.00 \\
\hline Grayia spinosa & 6 & 10 & 3 & Grayia spinosa & 0.75 & 0.95 & 0.22 \\
\hline Krameria parvifolia & 34 & 38 & 33 & Krameria parvifolia & 3.26 & 4.35 & 3.64 \\
\hline Larrea tridentata & 58 & 60 & 59 & Larrea tridentata & 3.49 & 10.48 & 8.30 \\
\hline Lycium andersonii & 19 & 22 & 14 & Lycium andersonii & 3.37 & 3.91 & 0.90 \\
\hline Oryzopsis hymenoides & 18 & 40 & 8 & Oryzopsis hymenoides & 0.74 & 2.93 & 0.25 \\
\hline Xylorhiza tortifolia & 0 & 1 & 0 & Xylorhiza tortifolia & 0.00 & 0.04 & 0.00 \\
\hline Total Live & 162 & 207 & 177 & Total Live & 12.98 & 24.78 & 19.35 \\
\hline Dead Grass & 0 & 0 & 10 & Dead Grass & 0.00 & 0.00 & 0.26 \\
\hline Dead Shrub & 5 & 5 & 30 & Dead Shrub & 6.76 & 0.46 & 4.98 \\
\hline Total & 167 & 212 & 217 & Total & 19.75 & 25.25 & 24.60 \\
\hline \multicolumn{5}{|c|}{ Average Height (m) } & \multicolumn{3}{|c|}{ Biomass Index $\left(\mathrm{m}^{2}\right)$} \\
\hline SPECIES & 1963 & 1975 & 2001 & SPECIES & 1963 & 1975 & 2001 \\
\hline Acamptopappus shockleyi & 0.12 & 0.21 & 0.23 & Acamptopappus shockleyi & 0.11 & 0.23 & 0.29 \\
\hline Ambrosia dumosa & 0.18 & 0.00 & 0.32 & Ambrosia dumosa & 0.05 & 0.00 & 1.64 \\
\hline Ceratoides lanata & 0.29 & 0.36 & 0.40 & Ceratoides lanata & 0.35 & 0.85 & 0.06 \\
\hline Coleogyne ramosissima & 0.00 & 0.00 & 0.71 & Coleogyne ramosissima & 0.00 & 0.00 & 0.92 \\
\hline Ephedra nevadensis & 0.34 & 0.37 & 0.51 & Ephedra nevadensis & 0.72 & 1.36 & 7.57 \\
\hline Eriogonum inflatum & 0.33 & 0.05 & 0.00 & Eriogonum inflatum & 0.07 & 0.01 & 0.00 \\
\hline Grayia spinosa & 0.48 & 0.45 & 0.62 & Grayia spinosa & 1.26 & 1.50 & 0.51 \\
\hline Krameria parvifolia & 0.17 & 0.19 & 0.25 & Krameria parvifolia & 1.89 & 2.80 & 3.11 \\
\hline Larrea tridentata & 0.60 & 0.63 & 0.71 & Larrea tridentata & 6.44 & 22.71 & 20.12 \\
\hline Lycium andersonii & 0.42 & 0.46 & 0.31 & Lycium andersonii & 5.04 & 6.13 & 0.90 \\
\hline Oryzopsis hymenoides & 0.22 & 0.29 & 0.24 & Oryzopsis hymenoides & 0.60 & 2.94 & 0.23 \\
\hline \multirow[t]{2}{*}{ Xylorhiza tortifolia } & 0.00 & 0.28 & 0.00 & Xylorhiza tortifolia & 0.00 & 0.03 & 0.00 \\
\hline & & & & Total Live & 16.52 & 38.56 & 35.34 \\
\hline
\end{tabular}


Table A-13A. Site characteristics for Plot 13.

Location: Jackass Flats

NTS Area: 25

NTS Grid: G-19

USGS 7.5’ Quadrangle Name: Skull Mountain

\begin{tabular}{lllc} 
& & \multicolumn{2}{c}{ GPS Readings (Zone 11S) } \\
& Location & Northing & Easting \\
Corner 1: & SW & 4078985 & 567967 \\
Corner 2: & NW & 4079005 & 567990 \\
Corner 3: & NE & 4078978 & 568006 \\
Corner 4: & SE & 4078959 & 567982
\end{tabular}

DEM Plot Elevation: 1288 m

Elevation Above Playa (Playa Name): NA

Condition of Original Corners
On ground or missing
On ground or missing
On ground or missing
Standing

Plot Aspect: $104^{\circ}$

Plot Slope: $6^{\circ}$

Annual Precipitation

Measured: $167 \mathrm{~mm}$

Modeled: $164 \mathrm{~mm}$

Parent Material Type: alluvial fan

Substrate: NA

Slate Geologic Unit: Qai

Beatley Plant Assemblage: Coleogyne/Larrea-Grayia-Lycium

Ostler-Hanson Plant Association: Coleogyne-Lycium-Ephedra

Ostler-Hanson Land Unit: 233

Abundance of Biological Soil Crusts: NA

Type and Date of Disturbance: none

Plot Condition: good

Location of Transect 1: south side of plot

Direction Transects Are Read: east to west

Date(s) Plot Remeasured: 8 May 2001 
Figure A-13. Photographs showing Plot 13.

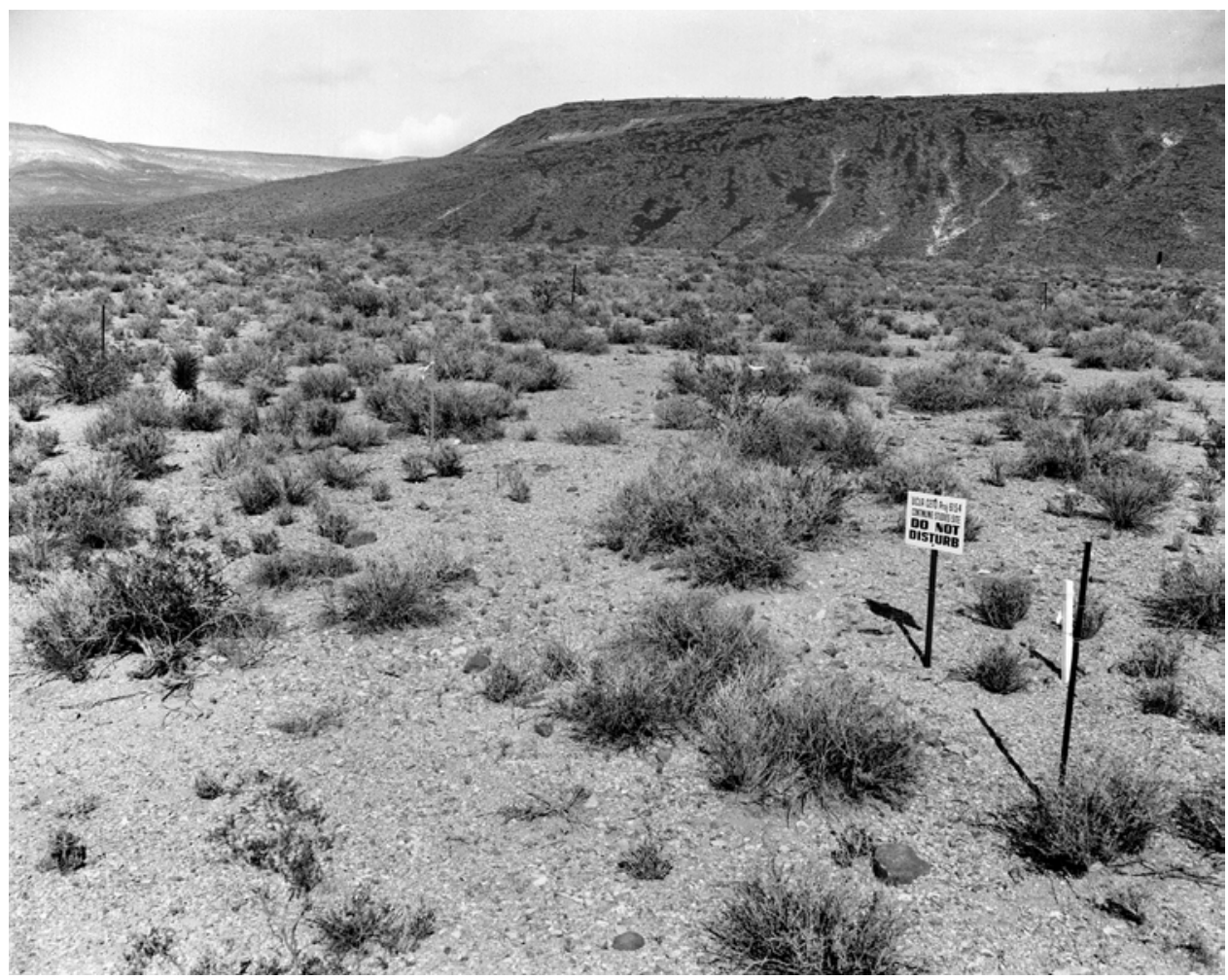

A. (May 6, 1964). This easterly view across Plot 13 shows a narrow valley with a mixed shrub community dominated by blackbrush (Coleogyne ramosissima) and creosote bush. Kiwi Mesa appears in the distance. Joshua trees (Yucca brevifolia) and creosote bush are two of the most obvious species present (Janice Beatley Collection, 53-A).

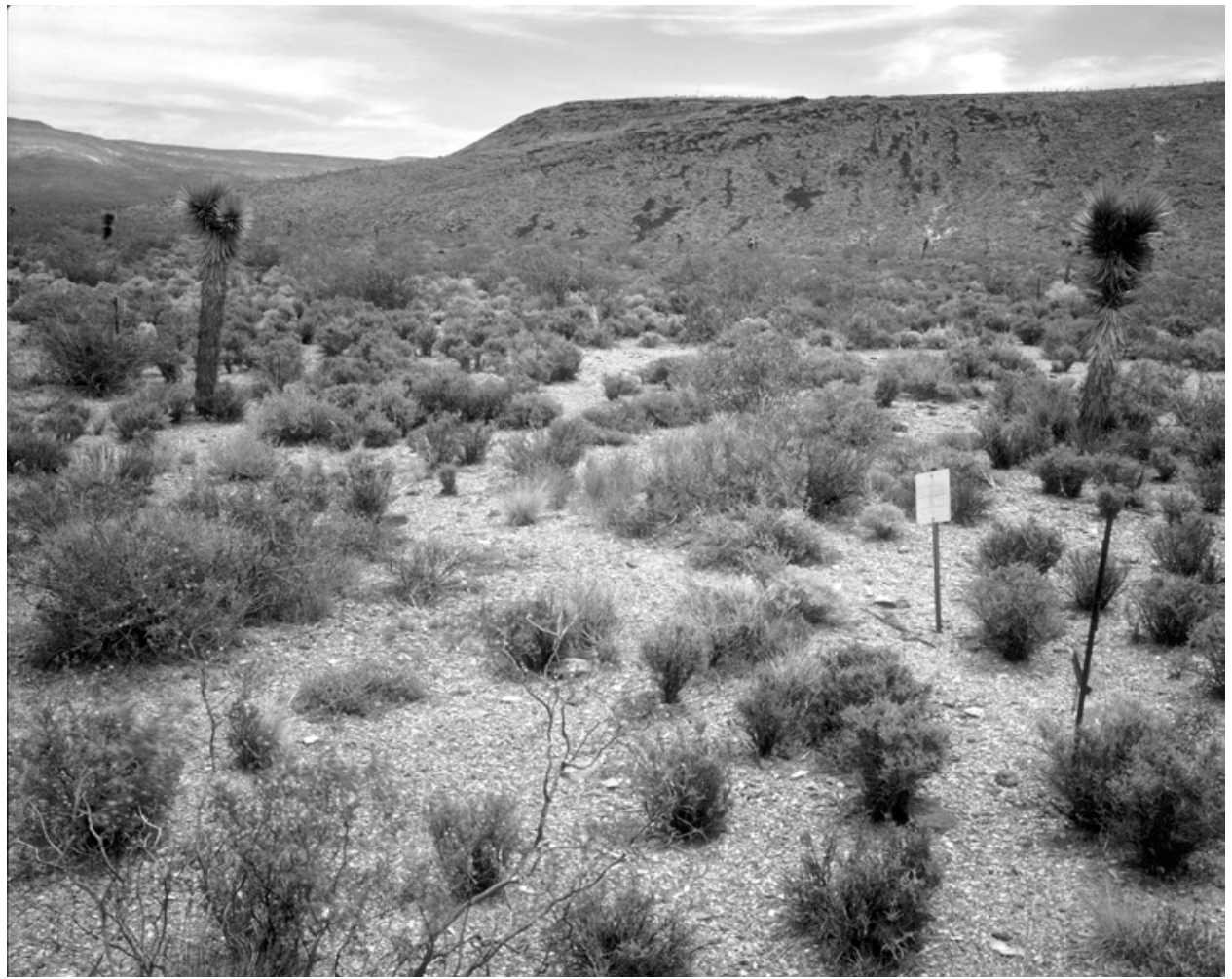

B. (June 8, 2001). The Joshua trees have grown considerably, and several new ones can be seen in the distance. The density, cover, and biomass of perennial vegetation has increased considerably, and many changes have occurred in the relative dominance of species on the plot (Dominic Oldershaw, Stake 4055A). 
Table A-13B. Summary plant data for Plot 13.

\begin{tabular}{|c|c|c|c|}
\hline SPECIES & 1963 & 1975 & 2001 \\
\hline Ambrosia dumosa & 5 & 6 & 22 \\
\hline Ceratoides lanata & 1 & 1 & 0 \\
\hline Coleogyne ramosissima & 81 & 87 & 92 \\
\hline Ephedra nevadensis & 18 & 19 & 22 \\
\hline Ericameria cooperi & 4 & 20 & 18 \\
\hline Eriogonum fasciculatum & 0 & 0 & 2 \\
\hline Grayia spinosa & 17 & 25 & 5 \\
\hline Krameria parvifolia & 0 & 0 & 2 \\
\hline Larrea tridentata & 5 & 7 & 10 \\
\hline Lycium andersonii & 34 & 35 & 32 \\
\hline Oryzopsis hymenoides & 0 & 1 & 2 \\
\hline Stipa speciosa & 4 & 4 & 16 \\
\hline Xylorhiza tortifolia & 1 & 9 & 14 \\
\hline Total Live & 170 & 214 & 237 \\
\hline Dead Grass & 0 & 0 & 0 \\
\hline Dead Shrub & 17 & 27 & 34 \\
\hline Total & 187 & 241 & 271 \\
\hline & \multicolumn{3}{|c|}{ Average Height (m) } \\
\hline SPECIES & 1963 & 1975 & 2001 \\
\hline Ambrosia dumosa & 0.28 & 0.29 & 0.24 \\
\hline Ceratoides lanata & 0.25 & 0.41 & 0.00 \\
\hline Coleogyne ramosissima & 0.41 & 0.43 & 0.50 \\
\hline Ephedra nevadensis & 0.43 & 0.54 & 0.58 \\
\hline Ericameria cooperi & 0.32 & 0.19 & 0.32 \\
\hline Eriogonum fasciculatum & 0.00 & 0.00 & 0.48 \\
\hline Grayia spinosa & 0.50 & 0.45 & 0.58 \\
\hline Krameria parvifolia & 0.00 & 0.00 & 0.20 \\
\hline Larrea tridentata & 1.12 & 0.95 & 1.34 \\
\hline Lycium andersonii & 0.42 & 0.47 & 0.36 \\
\hline Oryzopsis hymenoides & 0.00 & 0.28 & 0.45 \\
\hline Stipa speciosa & 0.27 & 0.29 & 0.47 \\
\hline Xylorhiza tortifolia & 0.23 & 0.25 & 0.28 \\
\hline
\end{tabular}

\section{SPECIES}

Ambrosia dumosa

Ceratoides lanata

Coleogyne ramosissima

Ephedra nevadensis

Ericameria cooperi

Eriogonum fasciculatum

Grayia spinosa

Krameria parvifolia

Larrea tridentata

Lycium andersonii

Oryzopsis hymenoides

Stipa speciosa

Xylorhiza tortifolia

Total Live

Dead Grass

Dead Shrub

Total

\section{SPECIES}

Ambrosia dumosa

Ceratoides lanata

Coleogyne ramosissima

Ephedra nevadensis

Ericameria cooperi

Eriogonum fasciculatum

Grayia spinosa

Krameria parvifolia

Larrea tridentata

Lycium andersonii

Oryzopsis hymenoides

Stipa speciosa

Xylorhiza tortifolia

Total Live
Cover (\%)

1975

0.54

0.02

10.36

2.96

1.07

0.00

2.62

0.00

1.62

7.05

0.05

0.40

0.35

27.05

0.00

2.82

29.87

2001

0.97

0.00

8.90

3.14

1.52

0.06

0.46

0.07

3.11

3.47

0.07

1.44

0.63

23.85

0.00

8.64

32.48

Biomass Index $\left(\mathrm{m}^{2}\right)$

$\begin{array}{ccc}\mathbf{1 9 6 3} & \mathbf{1 9 7 5} & \mathbf{2 0 0 1} \\ 0.60 & 0.56 & 0.84 \\ 0.05 & 0.02 & 0.00 \\ 15.14 & 16.99 & 15.44 \\ 3.25 & 5.78 & 6.49 \\ 0.20 & 0.89 & 1.70 \\ 0.00 & 0.00 & 0.10 \\ 3.75 & 3.91 & 0.94 \\ 0.00 & 0.00 & 0.05 \\ 5.77 & 5.29 & 16.13 \\ 10.48 & 11.49 & 4.33 \\ 0.00 & 0.05 & 0.11 \\ 0.23 & 0.42 & 2.41 \\ 0.05 & 0.36 & 0.62 \\ 39.51 & 45.76 & 49.16\end{array}$


Table A-14A. Site characteristics for Plot 14.

Location: Mid Valley

NTS Area: 25

NTS Grid: I-21

USGS 7.5’ Quadrangle Name: Mine Mountain

\begin{tabular}{llll} 
& & \multicolumn{2}{l}{ GPS Readings (Zone 11S) } \\
& Location & Northing & Easting \\
Corner 1: & SW & 4082123 & 570335 \\
Corner 2: & NW & NA & NA \\
Corner 3: & NE & 4082145 & 570374 \\
Corner 4: & SE & 4082116 & 570367
\end{tabular}

DEM Plot Elevation: 1457 m

Condition of Original Corners
Standing
Standing
On ground
On ground

Plot Aspect: $18^{\circ}$

Elevation Above Playa (Playa Name): NA

Plot Slope: $11^{\circ}$

Annual Precipitation

Measured: $189 \mathrm{~mm}$

Modeled: $186 \mathrm{~mm}$

Parent Material Type: broken terrain

Substrate: NA

Slate Geologic Unit: Qay

Beatley Plant Assemblage: Coleogyne/Grayia-Lycium

Ostler-Hanson Plant Association: Coleogyne-Lycium-Ephedra

Ostler-Hanson Land Unit: 240

Abundance of Biological Soil Crusts: NA

Type and Date of Disturbance: NA

Plot Condition: good

Location of Transect 1: NA

Direction Transects Are Read: southwest to northeast

Date(s) Plot Remeasured: 8 May 2001 
Figure A-14. Photographs showing Plot 14.

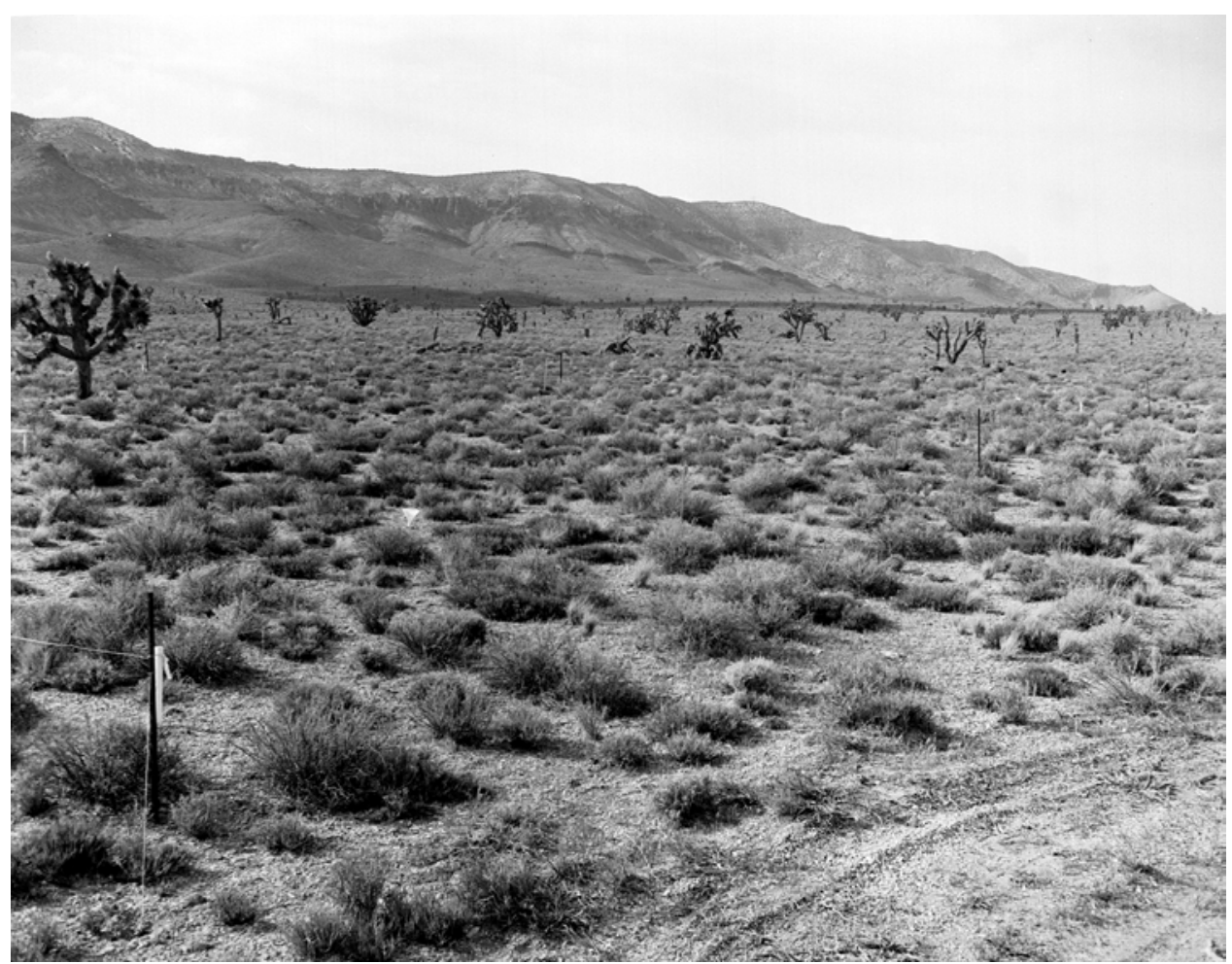

A. (May 6, 1964). This northwesterly view across Plot 14 shows scattered Joshua trees and a mixed shrub community dominated by blackbrush, spiny hopsage, wolfberry, and spiny menodora. This view is above the upper elevation limit for creosote bush in this area. Part of the access road to the plot appears at right foreground, and Shoshone Mountain appears in the background (Janice Beatley Collection, 56-B).

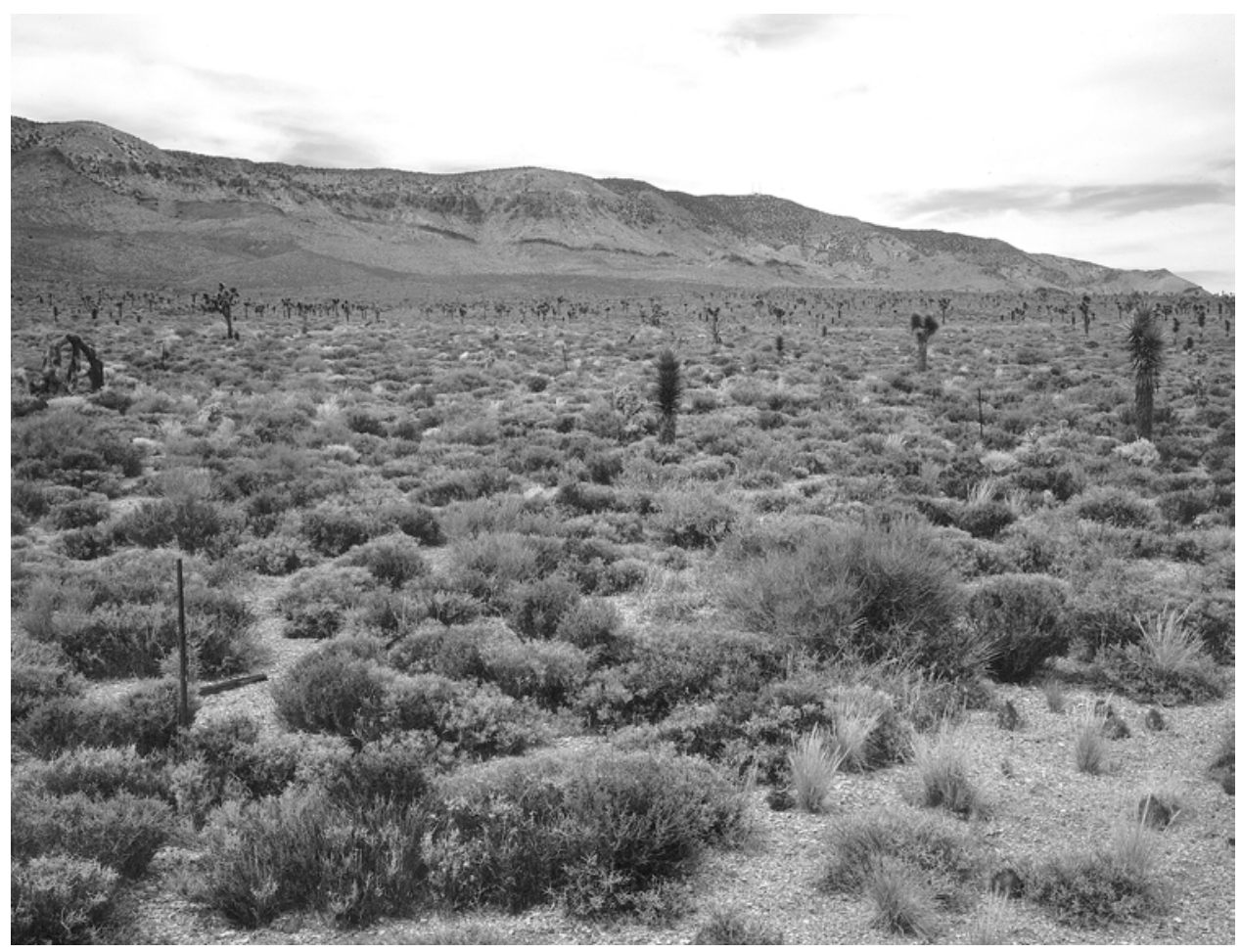

B. (June 8, 2001). Several changes are apparent in this match. The large Joshua tree at left midground has died, and its remains are still present. In general, Joshua trees have increased considerably in the background. Spiny menodora has become the dominant species in terms of cover. The biomass and cover of perennial shrubs has increased throughout the plot, despite the fact that spiny hopsage has decreased considerably here. The access road has partially revegetated, mostly with perennial grasses and short-lived shrubs (Dominic Oldershaw, Stake 4057B). 
Table A-14B. Summary plant data for Plot 14.

\begin{tabular}{|c|c|c|c|c|c|c|c|}
\hline \multicolumn{5}{|c|}{ Number of Plants } & \multicolumn{3}{|c|}{ Cover (\%) } \\
\hline SPECIES & 1963 & 1975 & 2001 & SPECIES & 1963 & 1975 & 2001 \\
\hline Arabis pulchra munciensis & 0 & 1 & 0 & Arabis pulchra munciensis & 0.00 & 0.01 & 0.00 \\
\hline Astragalus lentiginosus & 0 & 4 & 0 & Astragalus lentiginosus & 0.00 & 0.10 & 0.00 \\
\hline Ceratoides lanata & 7 & 13 & 10 & Ceratoides lanata & 0.45 & 0.92 & 0.73 \\
\hline Coleogyne ramosissima & 56 & 57 & 68 & Coleogyne ramosissima & 8.45 & 7.48 & 7.21 \\
\hline Ephedra nevadensis & 10 & 11 & 18 & Ephedra nevadensis & 1.25 & 1.28 & 2.14 \\
\hline Ericameria cooperi & 0 & 0 & 1 & Ericameria cooperi & 0.00 & 0.00 & 0.02 \\
\hline Grayia spinosa & 54 & 67 & 12 & Grayia spinosa & 8.31 & 9.92 & 1.30 \\
\hline Lycium andersonii & 24 & 29 & 32 & Lycium andersonii & 2.76 & 2.82 & 2.45 \\
\hline Menodora spinescens & 139 & 198 & 232 & Menodora spinescens & 17.99 & 25.42 & 29.95 \\
\hline Oryzopsis hymenoides & 0 & 1 & 1 & Oryzopsis hymenoides & 0.00 & 0.03 & 0.07 \\
\hline Phlox stansburyi & 0 & 32 & 23 & Phlox stansburyi & 0.00 & 0.49 & 0.37 \\
\hline Sitanion hystrix & 5 & 6 & 9 & Sitanion hystrix & 0.24 & 0.41 & 0.40 \\
\hline Sphaeralcea ambigua & 0 & 0 & 1 & Sphaeralcea ambigua & 0.00 & 0.00 & 0.01 \\
\hline Stipa speciosa & 34 & 55 & 12 & Stipa speciosa & 1.54 & 2.52 & 0.68 \\
\hline Yucca brevifolia & 3 & 2 & 4 & Yucca brevifolia & 0.54 & 0.72 & 0.42 \\
\hline Total Live & 332 & 476 & 423 & Total Live & 41.51 & 52.11 & 45.75 \\
\hline Dead Grass & 0 & 0 & 6 & Dead Grass & 0.00 & 0.00 & 0.15 \\
\hline Dead Shrub & 16 & 9 & 48 & Dead Shrub & 2.93 & 1.45 & 9.69 \\
\hline Total & 348 & 485 & 477 & Total & 44.44 & 53.56 & 55.59 \\
\hline \multicolumn{5}{|c|}{ Average Height (m) } & \multicolumn{3}{|c|}{ Biomass Index $\left(\mathbf{m}^{2}\right)$} \\
\hline SPECIES & 1963 & 1975 & 2001 & SPECIES & 1963 & 1975 & 2001 \\
\hline Arabis pulchra munciensis & 0.00 & 0.25 & 0.00 & Arabis pulchra munciensis & 0.00 & 0.01 & 0.00 \\
\hline Astragalus lentiginosus & 0.00 & 0.10 & 0.00 & Astragalus lentiginosus & 0.00 & 0.03 & 0.00 \\
\hline Ceratoides lanata & 0.35 & 0.40 & 0.46 & Ceratoides lanata & 0.54 & 1.38 & 1.26 \\
\hline Coleogyne ramosissima & 0.41 & 0.37 & 0.47 & Coleogyne ramosissima & 12.34 & 9.86 & 11.68 \\
\hline Ephedra nevadensis & 0.37 & 0.45 & 0.54 & Ephedra nevadensis & 1.80 & 1.99 & 4.40 \\
\hline Ericameria cooperi & 0.00 & 0.00 & 0.30 & Ericameria cooperi & 0.00 & 0.00 & 0.02 \\
\hline Grayia spinosa & 0.43 & 0.56 & 0.57 & Grayia spinosa & 12.25 & 18.94 & 2.49 \\
\hline Lycium andersonii & 0.36 & 0.35 & 0.36 & Lycium andersonii & 3.43 & 3.56 & 3.04 \\
\hline Menodora spinescens & 0.18 & 0.21 & 0.28 & Menodora spinescens & 11.78 & 18.80 & 29.44 \\
\hline Oryzopsis hymenoides & 0.00 & 0.20 & 0.54 & Oryzopsis hymenoides & 0.00 & 0.02 & 0.13 \\
\hline Phlox stansburyi & 0.00 & 0.09 & 0.17 & Phlox stansburyi & 0.00 & 0.13 & 0.24 \\
\hline Sitanion hystrix & 0.27 & 0.25 & 0.39 & Sitanion hystrix & 0.23 & 0.36 & 0.51 \\
\hline Sphaeralcea ambigua & 0.00 & 0.00 & 0.05 & Sphaeralcea ambigua & 0.00 & 0.00 & 0.00 \\
\hline Stipa speciosa & 0.27 & 0.29 & 0.39 & Stipa speciosa & 1.48 & 2.52 & 0.91 \\
\hline \multirow[t]{2}{*}{ Yucca brevifolia } & 0.35 & 2.11 & 1.01 & Yucca brevifolia & 0.56 & 7.67 & 2.17 \\
\hline & & & & Total Live & 44.40 & 65.28 & 56.28 \\
\hline
\end{tabular}


Table A-15A. Site characteristics for Plot 15.

Location: Jackass Flats

NTS Area: 25

NTS Grid: F-15

USGS 7.5’ Quadrangle Name: Jackass Flats

\begin{tabular}{|c|c|c|c|c|}
\hline & & GPS Reac & Zone 11S) & \\
\hline & Location & Northing & Easting & Condition of Original Corners \\
\hline Corner 1: & SW & 4070942 & 566051 & Standing \\
\hline Corner 2: & NW & 4070970 & 566062 & Standing \\
\hline Corner 3: & $\mathrm{NE}$ & 4070967 & 566089 & Standing \\
\hline Corner 4: & SE & 4070934 & 566082 & Standing \\
\hline DEM Plo & evation: 10 & & & Plot Aspect: $135^{\circ}$ \\
\hline Elevation & e Playa & Name): I & & Plot Slope: $5^{\circ}$ \\
\hline
\end{tabular}

Annual Precipitation

Measured: $132 \mathrm{~mm}$

Modeled: $140 \mathrm{~mm}$

Parent Material Type: alluvial fan

Substrate: alluvial fan, volcanic alluvium

Slate Geologic Unit: Qay

Beatley Plant Assemblage: Larrea-Ambrosia/Larrea-Lycium-Grayia

Ostler-Hanson Plant Association: Larrea-Lycium-Ambrosia

Ostler-Hanson Land Unit: 229

Abundance of Biological Soil Crusts: none

Type and Date of Disturbance: none

Plot Condition: good

Location of Transect 1: west side of plot

Direction Transects Are Read: south to north

Date(s) Plot Remeasured: 2 April 2001 
Figure A-15. Photographs showing Plot 15.

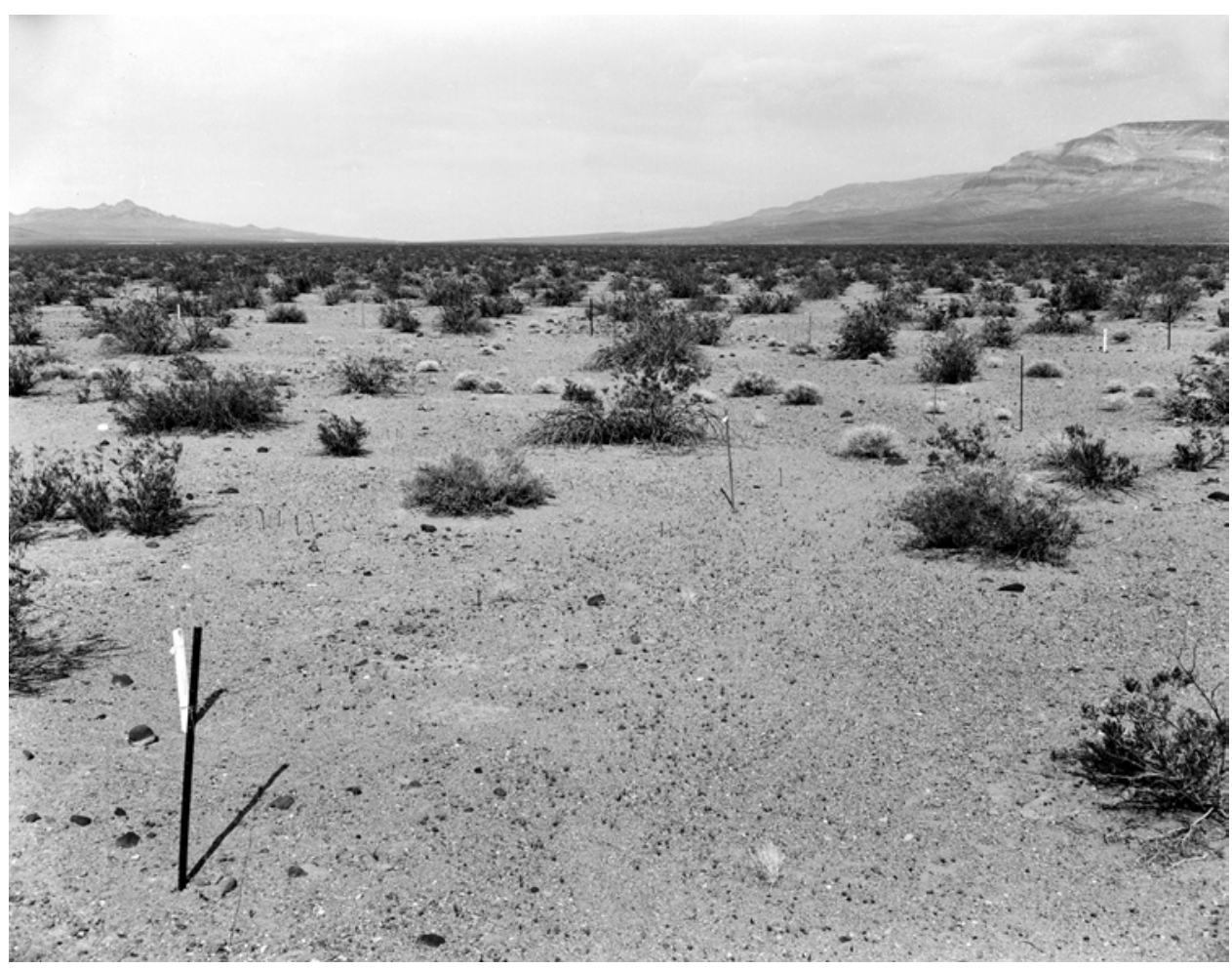

A. (April 28, 1964). This easterly view across Plot 15 shows Skull Mountain in the right background and Lookout Peak in the left background. The vegetation is creosote bush - white bursage with several other species present (Janice Beatley Collection, 38-B).

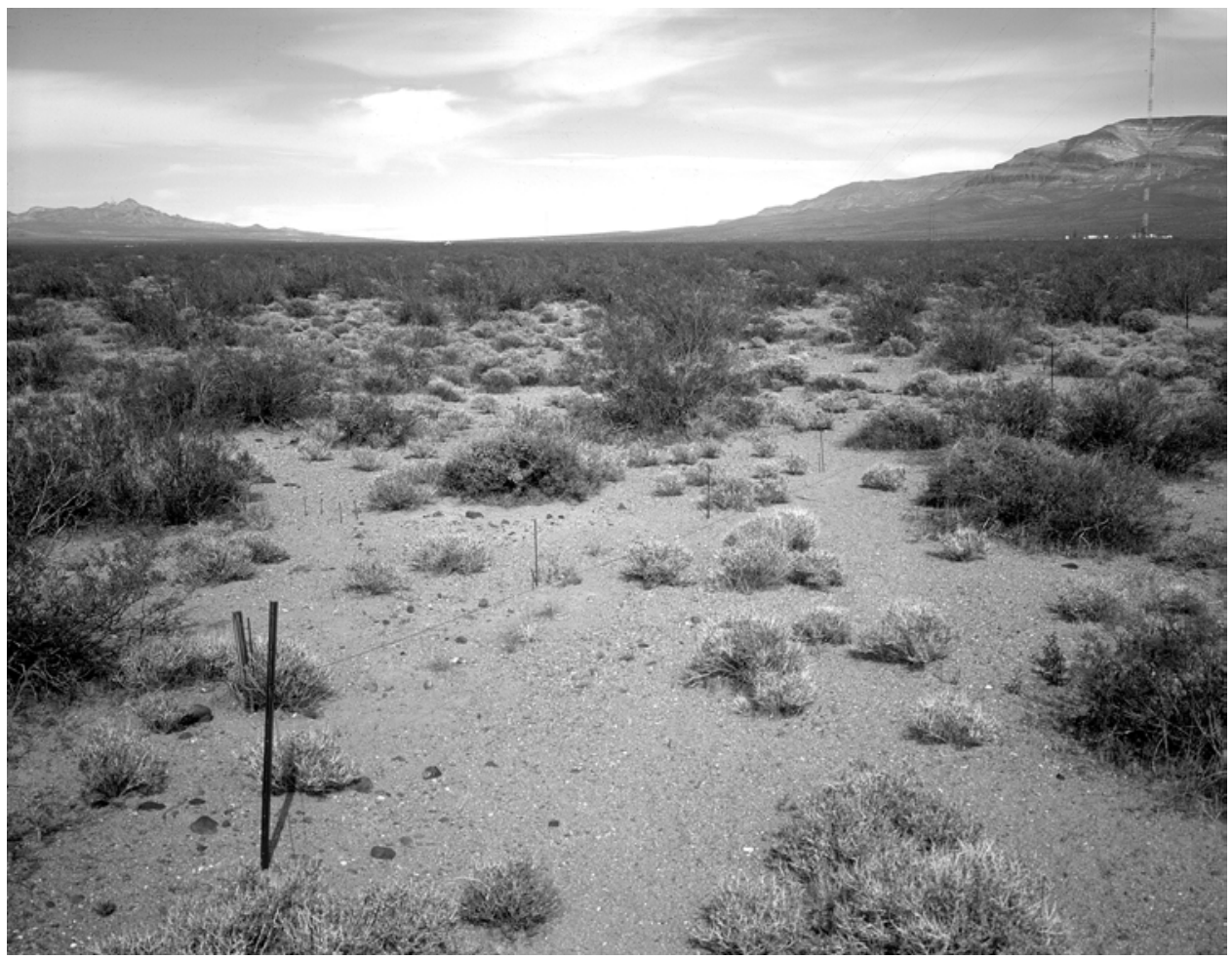

B. (April 6, 2001). The density, cover, and biomass of perennial vegetation has increased considerably in the 37 years that span the original photograph and its match. Spiny hopsage decreased here while wolfberry has increased to become a subdominant (R.H. Webb, Stake 4121B). 
Table A-15B. Summary plant data for Plot 15.

\author{
SPECIES \\ Acamptopappus shockleyi \\ Ambrosia dumosa \\ Grayia spinosa \\ Larrea tridentata \\ Lycium andersonii \\ Opuntia echinocarpa \\ Oryzopsis hymenoides \\ Total Live \\ Dead Grass \\ Dead Shrub \\ Total
}

\section{SPECIES}

Acamptopappus shockleyi

Ambrosia dumosa

Grayia spinosa

Larrea tridentata

Lycium andersonii

Opuntia echinocarpa

Oryzopsis hymenoides

\section{SPECIES}

Acamptopappus shockleyi

Ambrosia dumosa

Grayia spinosa

Larrea tridentata

Lycium andersonii

Opuntia echinocarpa

Oryzopsis hymenoides

Total Live

Dead Grass

Dead Shrub

Total

\section{SPECIES}

Acamptopappus shockleyi

Ambrosia dumosa

Grayia spinosa

Larrea tridentata

Lycium andersonii

Opuntia echinocarpa

Oryzopsis hymenoides

Total Live

Number of Plants

1963

1

6

0

32

9

0

6

54

0

1

55

1963

0.18

0.31

0.00

1.05

0.51

0.00

0.26

1963

0.04

0.77

0.00

9.62

1.62

0.00

0.20

12.25

0.00

1.46

13.71

1963

0.02

1.00

0.00

37.90

2.75

0.00

0.18

41.85
1975

4

$9 \quad 105$

15

$40 \quad 47$

$7 \quad 10$

$1 \quad 0$

$9 \quad 16$

$71 \quad 199$

$0 \quad 1$

$1 \quad 10$

$72 \quad 210$

Average Height (m)

19752001

$0.18 \quad 0.27$

$0.36 \quad 0.29$

$0.48 \quad 0.76$

$0.93 \quad 1.38$

$0.61 \quad 0.61$

$0.23 \quad 0.00$

$0.30 \quad 0.32$

\section{Cover (\%)}

19752001

$0.18 \quad 0.90$

$1.13 \quad 8.92$

$0.09 \quad 0.65$

$11.71 \quad 12.11$

$1.40 \quad 1.96$

$0.03 \quad 0.00$

$0.63 \quad 0.86$

$15.16 \quad 25.40$

$0.00 \quad 0.05$

$0.06 \quad 3.68$

$15.23 \quad 29.13$

Biomass Index $\left(\mathrm{m}^{2}\right)$

19752001

$0.14 \quad 0.77$

$1.44 \quad 9.43$

$0.15 \quad 1.70$

$39.55 \quad 56.79$

$2.89 \quad 4.41$

$0.02 \quad 0.00$

$0.70 \quad 0.95$

$44.90 \quad 74.05$ 
Table A-16A. Site characteristics for Plot 16.

Location: Jackass Flats

NTS Area: 25

NTS Grid: H-17

USGS 7.5’ Quadrangle Name: Skull Mountain

\begin{tabular}{lllc} 
& & \multicolumn{2}{c}{ GPS Readings (Zone 11S) } \\
& Location & Northing & Easting \\
Corner 1: & SW & 4072553 & 568887 \\
Corner 2: & NW & 4072581 & 568895 \\
Corner 3: & NE & 4072576 & 568925 \\
Corner 4: & SE & 4072547 & 568918
\end{tabular}

DEM Plot Elevation: 1162 m

Elevation Above Playa (Playa Name): NA

Condition of Original Corners
Standing
Standing
Standing
Standing

Plot Aspect: $135^{\circ}$

Plot Slope: $3^{\circ}$

Annual Precipitation

Measured: $147 \mathrm{~mm}$

Modeled: $149 \mathrm{~mm}$

Parent Material Type: alluvial fan

Substrate: alluvial fan, mixed volcanic alluvium

Slate Geologic Unit: Qay

Beatley Plant Assemblage: Larrea-Lycium-Grayia

Ostler-Hanson Plant Association: Larrea-Oryzopsis

Ostler-Hanson Land Unit: 181

Abundance of Biological Soil Crusts: none

Type and Date of Disturbance: none

Plot Condition: good

Location of Transect 1: west side of plot

Direction Transects Are Read: south to north

Date(s) Plot Remeasured: 5 April 2001 
Figure A-16. Photographs showing Plot 16.

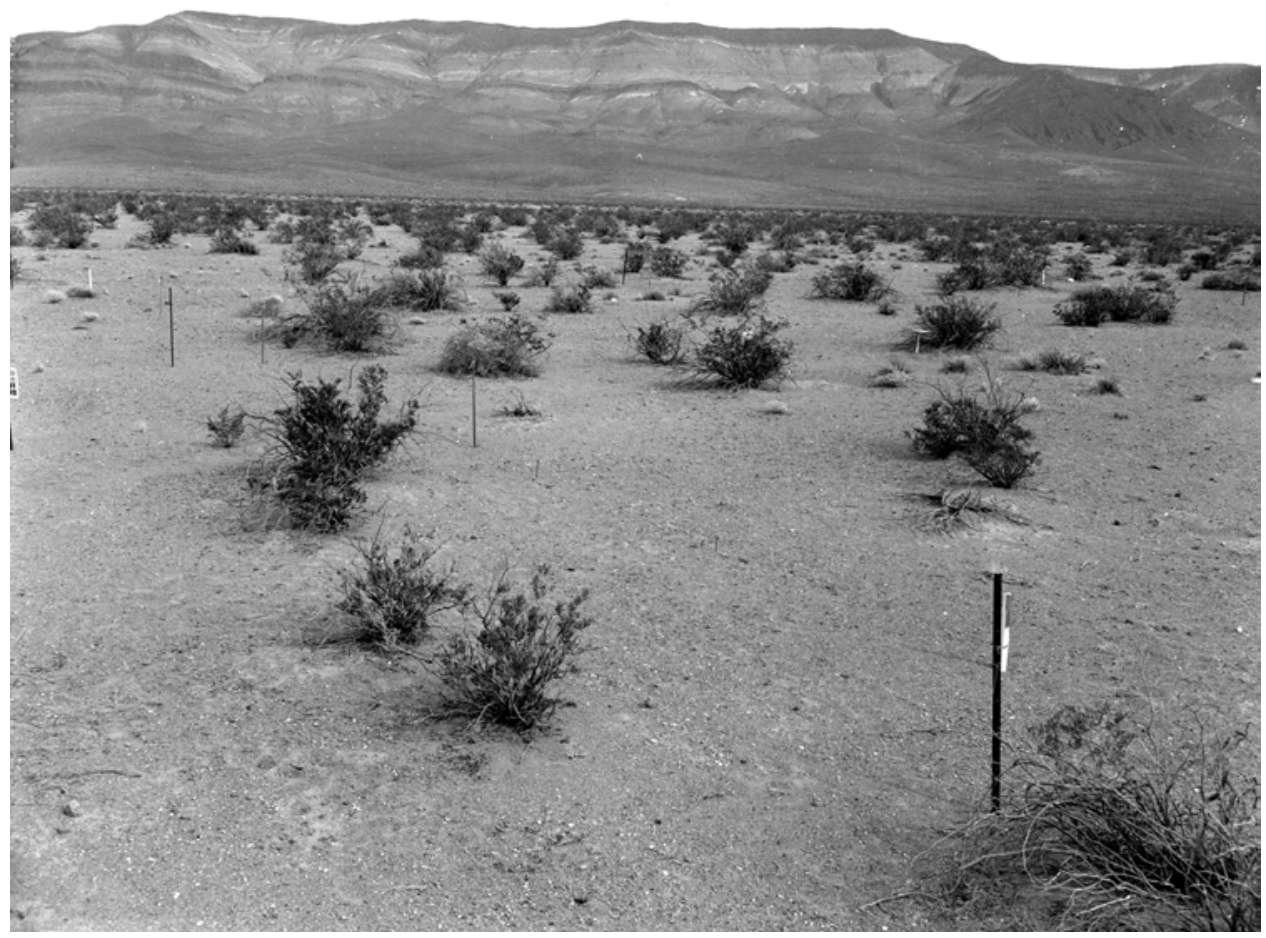

A. (April 28, 1964). This southeasterly view across Plot 16 shows creosote bush - white bursage vegetation with Skull Mountain in the background. Spiny hopsage is a significant subdominant species here (Janice Beatley Collection, 39-A).

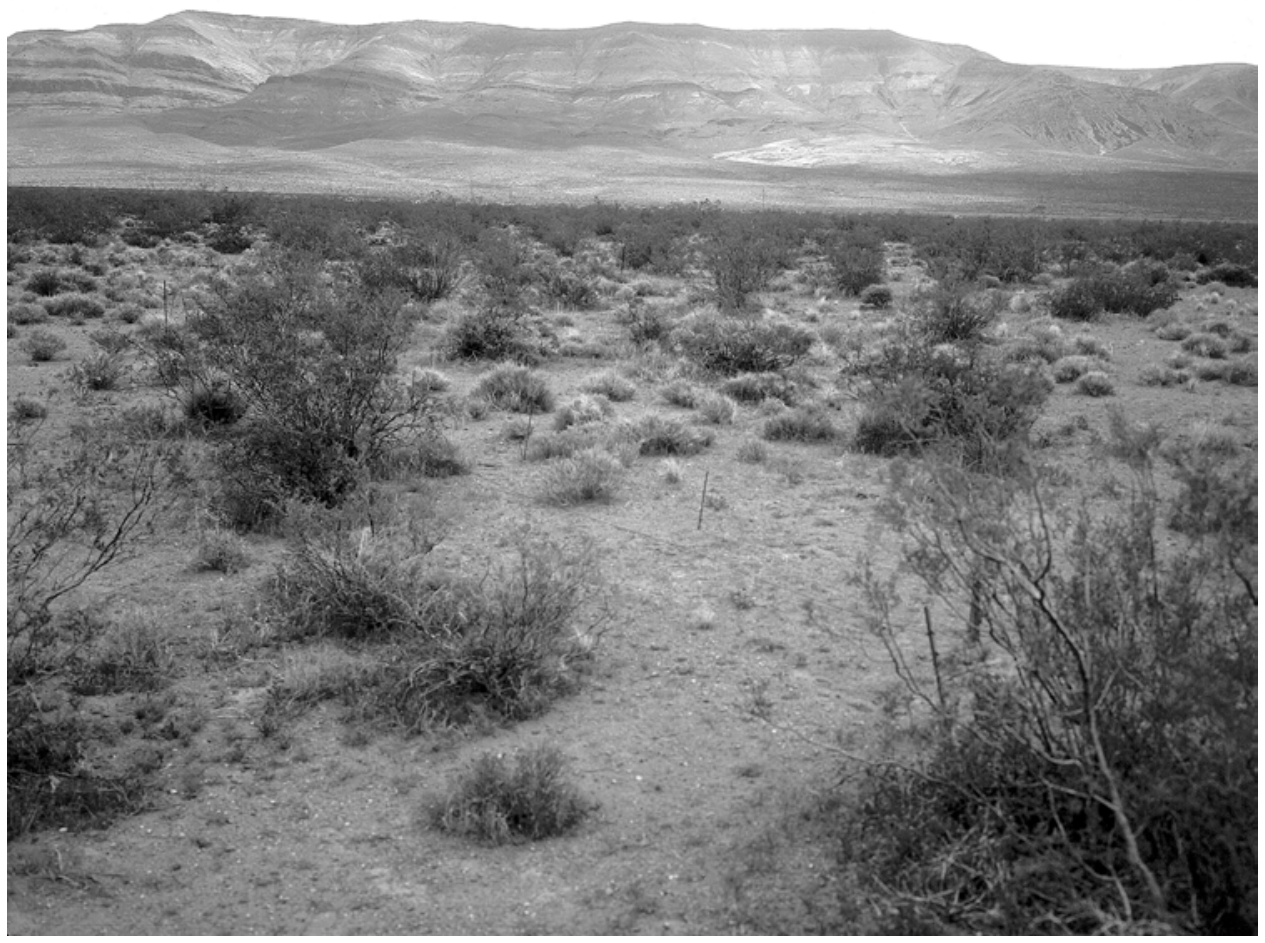

B. (April 5, 2001). The density, cover, and biomass of the perennial vegetation has increased considerably. The increase is particularly noteworthy in creosote bush, which now partially blocks the fence posts in the view. Spiny hopsage has decreased here while wolfberry has increased (R.H. Webb, Stake 4101B). 
Table A-16B. Summary plant data for Plot 16.

\begin{tabular}{|c|c|c|c|c|c|c|c|}
\hline \multicolumn{5}{|c|}{ Number of Plants } & \multicolumn{3}{|c|}{ Cover (\%) } \\
\hline SPECIES & 1963 & 1975 & 2001 & SPECIES & 1963 & 1975 & 2001 \\
\hline Acamptopappus shockleyi & 0 & 5 & 9 & Acamptopappus shockleyi & 0.00 & 0.06 & 0.66 \\
\hline Ambrosia dumosa & 1 & 0 & 55 & Ambrosia dumosa & 0.02 & 0.00 & 4.74 \\
\hline Grayia spinosa & 3 & 1 & 0 & Grayia spinosa & 0.45 & 0.13 & 0.00 \\
\hline Hymenoclea salsola & 0 & 0 & 3 & Hymenoclea salsola & 0.00 & 0.00 & 0.26 \\
\hline Larrea tridentata & 25 & 31 & 40 & Larrea tridentata & 6.02 & 8.13 & 10.92 \\
\hline Lycium andersonii & 6 & 7 & 7 & Lycium andersonii & 0.85 & 0.79 & 1.03 \\
\hline Oryzopsis hymenoides & 5 & 18 & 19 & Oryzopsis hymenoides & 0.12 & 1.77 & 0.94 \\
\hline Total Live & 40 & 62 & 133 & Total Live & 7.45 & 10.88 & 18.55 \\
\hline Dead Grass & 0 & 1 & 0 & Dead Grass & 0.00 & 0.05 & 0.00 \\
\hline Dead Shrub & 3 & 3 & 11 & Dead Shrub & 2.00 & 0.38 & 3.41 \\
\hline Total & 43 & 66 & 144 & Total & 9.45 & 11.31 & 21.95 \\
\hline \multicolumn{5}{|c|}{ Average Height (m) } & \multicolumn{3}{|c|}{ Biomass Index (m²) } \\
\hline SPECIES & 1963 & 1975 & 2001 & SPECIES & 1963 & 1975 & 2001 \\
\hline Acamptopappus shockleyi & 0.00 & 0.09 & 0.31 & Acamptopappus shockleyi & 0.00 & 0.03 & 0.75 \\
\hline Ambrosia dumosa & 0.25 & 0.00 & 0.32 & Ambrosia dumosa & 0.02 & 0.00 & 5.52 \\
\hline Grayia spinosa & 0.62 & 0.46 & 0.00 & Grayia spinosa & 0.98 & 0.20 & 0.00 \\
\hline Hymenoclea salsola & 0.00 & 0.00 & 0.36 & Hymenoclea salsola & 0.00 & 0.00 & 0.35 \\
\hline Larrea tridentata & 1.11 & 1.13 & 1.45 & Larrea tridentata & 22.80 & 32.71 & 56.86 \\
\hline Lycium andersonii & 0.52 & 0.54 & 0.49 & Lycium andersonii & 1.45 & 1.36 & 1.84 \\
\hline \multirow[t]{2}{*}{ Oryzopsis hymenoides } & 0.26 & 0.31 & 0.28 & Oryzopsis hymenoides & 0.10 & 1.90 & 0.90 \\
\hline & & & & Total Live & 25.34 & 36.19 & 66.22 \\
\hline
\end{tabular}


Table A-17A. Site characteristics for Plot 17.

Location: Jackass Flats

NTS Area: 25

NTS Grid: I-17

USGS 7.5’ Quadrangle Name: Skull Mountain

\begin{tabular}{lllc} 
& & \multicolumn{2}{c}{ GPS Readings (Zone 11S) } \\
& Location & Northing & Easting \\
Corner 1: & SW & 4073825 & 571148 \\
Corner 2: & NW & 4073855 & 571147 \\
Corner 3: & NE & 4073856 & 571177 \\
Corner 4: & SE & 4073825 & 571179
\end{tabular}

DEM Plot Elevation: 1244 m

Elevation Above Playa (Playa Name): NA

Condition of Original Corners
Standing
Standing
Standing
Standing

Plot Aspect: $315^{\circ}$

Plot Slope: $10^{\circ}$

Annual Precipitation

Measured: $141 \mathrm{~mm}$

Modeled: $159 \mathrm{~mm}$

Parent Material Type: alluvial fan

Substrate: NA

Slate Geologic Unit: Qay

Beatley Plant Assemblage: Larrea-Lycium-Grayia

Ostler-Hanson Plant Association: Larrea-Lycium-Grayia

Ostler-Hanson Land Unit: 179

Abundance of Biological Soil Crusts: none

Type and Date of Disturbance: none

Plot Condition: good

Location of Transect 1: south side of plot

Direction Transects Are Read: east to west

Date(s) Plot Remeasured: 5 April 2001 
Figure A-17. Photographs showing Plot 17.

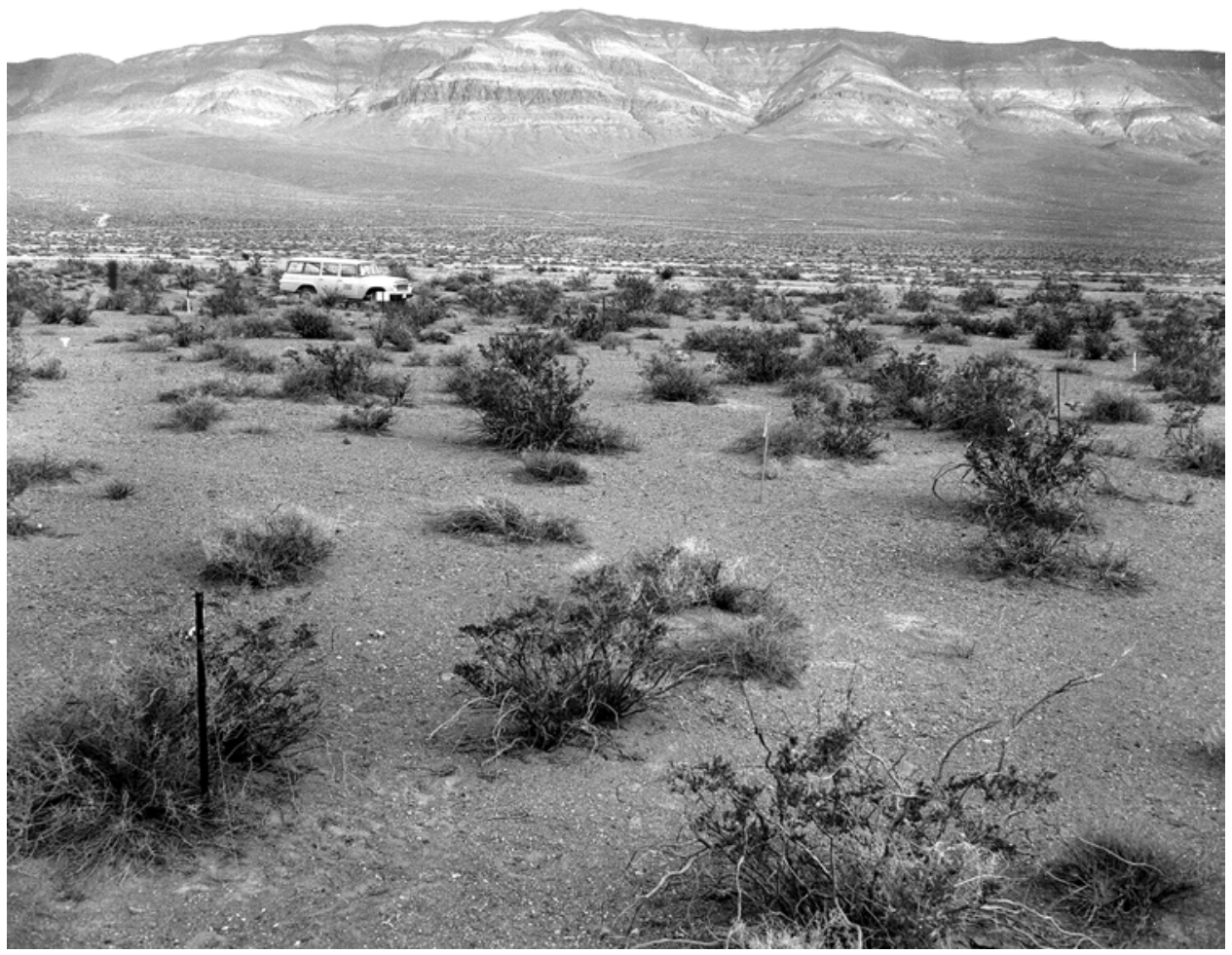

A. (April 28, 1964). This southeasterly view across Plot 17 shows the north flank of Skull Mountain in the background. The vegetation is a mixed creosote bush scrub association and is dominated by relatively few, large individuals. The Cane Springs road crosses the midground (Janice Beatley Collection, 41-B).

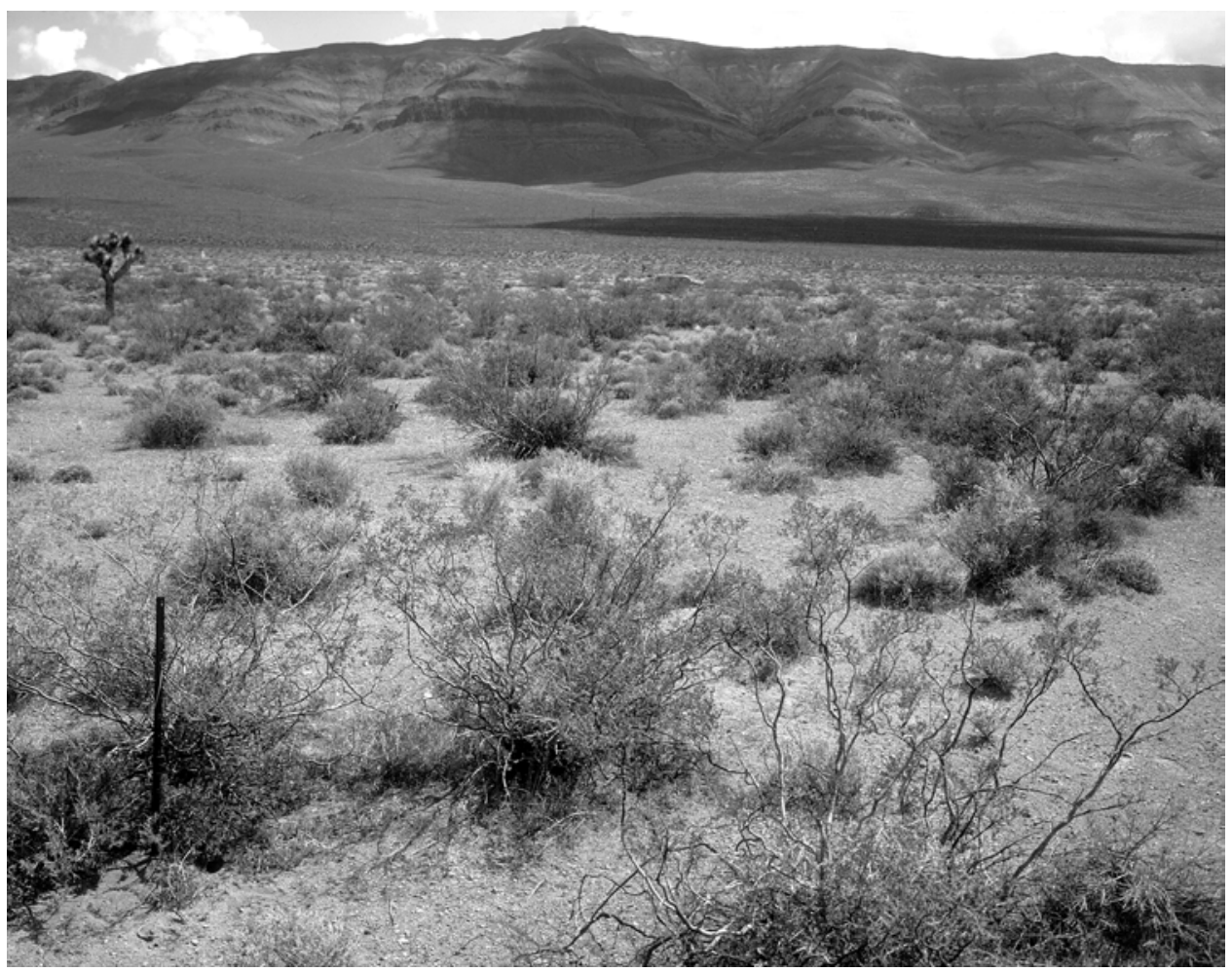

B. (April 5, 2001). The cover, density, and biomass of perennial vegetation is large enough to obscure the Cane Springs road. The dominants now are creosote bush, wolfberry, and Mormon tea, and spiny hopsage, which once was a subdominant here, has greatly decreased. Much of the increase is attributable to an increase in size of persisting creosote bush (R.H. Webb, Stake 4105A). 
Table A-17B. Summary plant data for Plot 17.

\begin{tabular}{|c|c|c|c|c|c|c|c|}
\hline \multicolumn{5}{|c|}{ Number of Plants } & \multicolumn{3}{|c|}{ Cover (\%) } \\
\hline SPECIES & 1963 & 1975 & 2001 & SPECIES & 1963 & 1975 & 2001 \\
\hline Acamptopappus shockleyi & 2 & 3 & 9 & Acamptopappus shockleyi & 0.09 & 0.18 & 0.53 \\
\hline Ambrosia dumosa & 0 & 1 & 13 & Ambrosia dumosa & 0.00 & 0.11 & 1.07 \\
\hline Atriplex canescens & 0 & 1 & 7 & Atriplex canescens & 0.00 & 0.16 & 0.35 \\
\hline Ceratoides lanata & 2 & 7 & 6 & Ceratoides lanata & 0.05 & 0.39 & 0.26 \\
\hline Ephedra nevadensis & 5 & 6 & 12 & Ephedra nevadensis & 0.53 & 0.55 & 1.85 \\
\hline Grayia spinosa & 24 & 13 & 14 & Grayia spinosa & 3.09 & 1.69 & 1.46 \\
\hline Krameria parvifolia & 3 & 6 & 10 & Krameria parvifolia & 0.39 & 0.62 & 0.95 \\
\hline Larrea tridentata & 31 & 35 & 42 & Larrea tridentata & 8.38 & 9.59 & 12.00 \\
\hline Lepidium fremontii & & 2 & 3 & Lepidium fremontii & 0.00 & 0.41 & 0.40 \\
\hline Lycium andersonii & 32 & 32 & 39 & Lycium andersonii & 8.73 & 5.41 & 5.65 \\
\hline Lycium pallidum & 0 & 0 & 1 & Lycium pallidum & 0.00 & 0.00 & 0.16 \\
\hline Oryzopsis hymenoides & 0 & 4 & 2 & Oryzopsis hymenoides & 0.01 & 0.59 & 0.05 \\
\hline Thamnosma montana & 1 & 2 & 2 & Thamnosma montana & 0.11 & 0.25 & 0.38 \\
\hline Xylorhiza tortifolia & 1 & 0 & 1 & Xylorhiza tortifolia & 0.05 & 0.00 & 0.04 \\
\hline Total Live & 101 & 112 & 161 & Total Live & 21.42 & 19.95 & 25.15 \\
\hline Dead Grass & 0 & 0 & 0 & Dead Grass & 0.00 & 0.00 & 0.00 \\
\hline Dead Shrub & 6 & 19 & 27 & Dead Shrub & 1.72 & 4.22 & 5.48 \\
\hline Total & 107 & 131 & 188 & Total & 23.14 & 24.16 & 30.63 \\
\hline \multicolumn{5}{|c|}{ Average Height (m) } & \multicolumn{3}{|c|}{ Biomass Index $\left(\mathrm{m}^{2}\right)$} \\
\hline SPECIES & 1963 & 1975 & 2001 & SPECIES & 1963 & 1975 & 2001 \\
\hline Acamptopappus shockleyi & 0.28 & 0.29 & 0.32 & Acamptopappus shockleyi & 0.09 & 0.22 & 0.62 \\
\hline Ambrosia dumosa & 0.00 & 0.51 & 0.32 & Ambrosia dumosa & 0.00 & 0.19 & 1.28 \\
\hline Atriplex canescens & 0.00 & 0.91 & 0.56 & Atriplex canescens & 0.00 & 0.50 & 0.63 \\
\hline Ceratoides lanata & 0.42 & 0.38 & 0.41 & Ceratoides lanata & 0.08 & 0.54 & 0.36 \\
\hline Ephedra nevadensis & 0.38 & 0.46 & 0.53 & Ephedra nevadensis & 0.74 & 1.03 & 3.59 \\
\hline Grayia spinosa & 0.51 & 0.57 & 0.59 & Grayia spinosa & 5.69 & 3.44 & 3.21 \\
\hline Krameria parvifolia & 0.19 & 0.19 & 0.23 & Krameria parvifolia & 0.26 & 0.39 & 0.79 \\
\hline Larrea tridentata & 1.01 & 0.96 & 1.30 & Larrea tridentata & 29.87 & 33.38 & 55.28 \\
\hline Lepidium fremontii & 0.00 & 0.69 & 0.52 & Lepidium fremontii & 0.00 & 0.91 & 0.70 \\
\hline Lycium andersonii & 0.52 & 0.45 & 0.48 & Lycium andersonii & 15.49 & 9.10 & 9.14 \\
\hline Lycium pallidum & 0.00 & 0.00 & 0.30 & Lycium pallidum & 0.00 & 0.00 & 0.16 \\
\hline Oryzopsis hymenoides & 0.00 & 0.36 & 0.26 & Oryzopsis hymenoides & 0.01 & 0.71 & 0.03 \\
\hline Thamnosma montana & 0.25 & 0.25 & 0.34 & Thamnosma montana & 0.09 & 0.21 & 0.41 \\
\hline \multirow[t]{2}{*}{ Xylorhiza tortifolia } & 0.28 & 0.00 & 0.30 & Xylorhiza tortifolia & 0.04 & 0.00 & 0.04 \\
\hline & & & & Total Live & 52.35 & 50.62 & 76.25 \\
\hline
\end{tabular}


Table A-18A. Site characteristics for Plot 18.

Location: Topopah Valley

NTS Area: 25

NTS Grid: F-23

USGS 7.5’ Quadrangle Name: Topopah Spring

\begin{tabular}{lllll} 
& & \multicolumn{2}{l}{ GPS Readings (Zone 11S) } \\
Cocation & Northing & Easting & Condition of Original Corners \\
Corner 1: & SW & 4084205 & 564458 & NA \\
Corner 2: & NW & NA & NA & NA \\
Corner 3: & NE & NA & NA & NA \\
Corner 4: & SE & NA & NA & NA
\end{tabular}

DEM Plot Elevation: 1459 m

Plot Aspect: $276^{\circ}$

Elevation Above Playa (Playa Name): NA

Plot Slope: $20^{\circ}$

Annual Precipitation

Measured: $228 \mathrm{~mm}$

Modeled: $189 \mathrm{~mm}$

Parent Material Type: alluvial fan

Substrate: NA

Slate Geologic Unit: Qay

Beatley Plant Assemblage: Coleogyne

Ostler-Hanson Plant Association: Coleogyne-Ephedra

Ostler-Hanson Land Unit: 437

Abundance of Biological Soil Crusts: light scattered crust cover

Type and Date of Disturbance: none (control for Plot 19)

Plot Condition: good

Location of Transect 1: NA

Direction Transects Are Read: NA

Date(s) Plot Remeasured: 20 May 00 
Figure A-18. Photographs showing Plot 18.

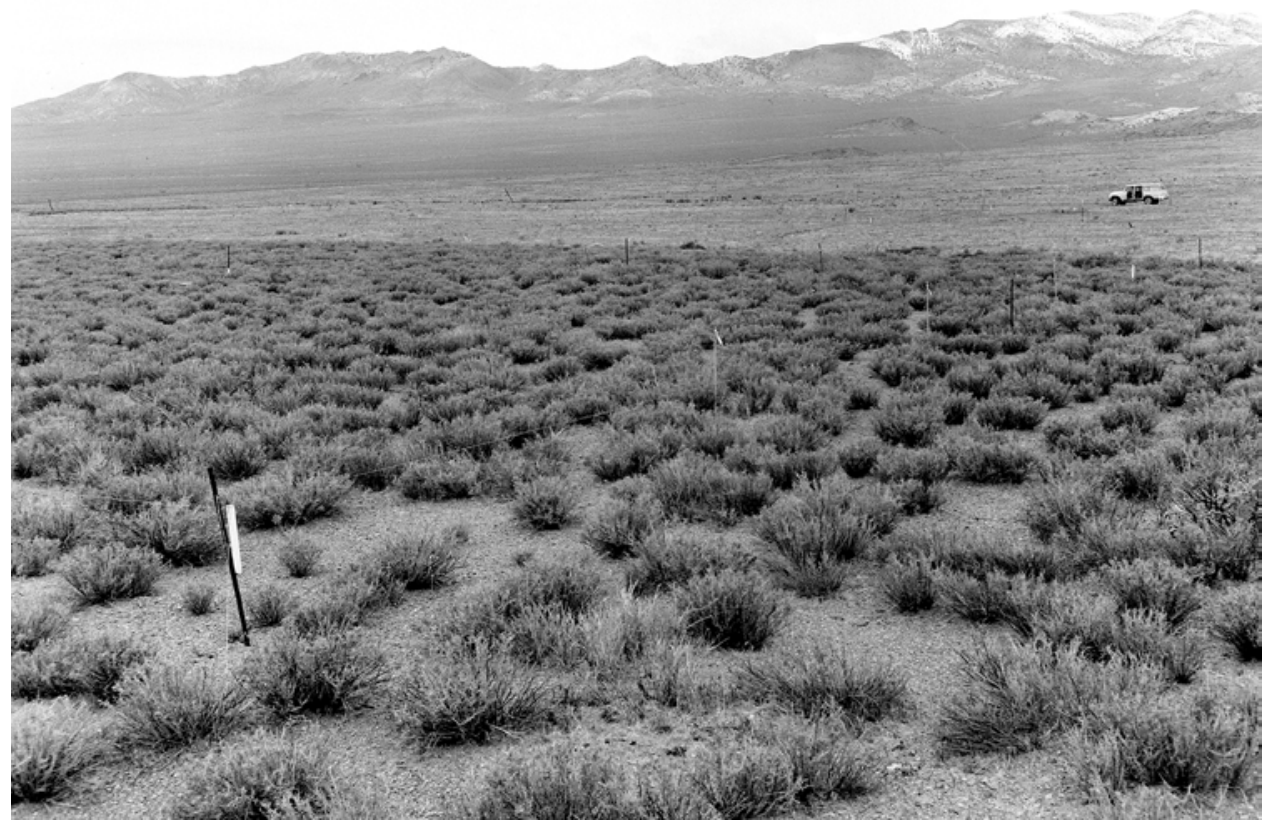

A. (May 7, 1964). This westerly view across Plot 18 and Topopah Valley shows the Calico Hills in the distance. This view shows snow on the hills, indicating that a late spring storm occurred just before the photograph was taken. The vegetation is a nearly monospecific blackbrush stand. The bare areas in the background are the result of a fire in the mid-1950s, and this plot combined with Plot 19 represent a fire-recovery study (Janice Beatley Collection, 60-B).

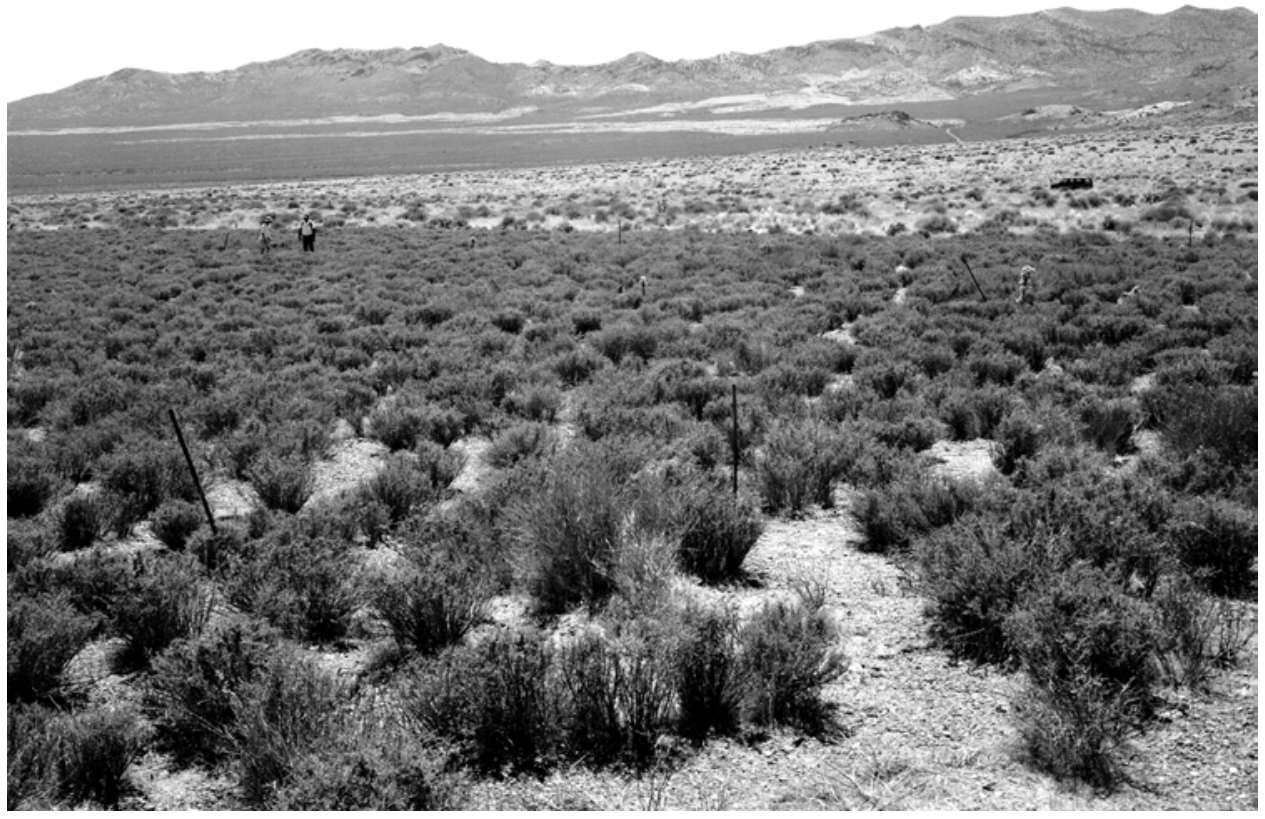

B. (June 20, 2000). Other fires that occurred after 1964 are apparent in the background, although the plot has remained undisturbed. Individual blackbrush appear to be larger in 2000, but overall little change is apparent in the undisturbed vegetation. Mormon tea has increased in importance on the plot. Several chollas (Opuntia echinocarpa) now protrude above the blackbrush canopy in the midground (R.H. Webb, Stake 4043B). 
Table A-18B. Summary plant data for Plot 18.

\author{
SPECIES \\ Calochortus species \\ Coleogyne ramosissima \\ Coryphantha vivipara rosea \\ Ephedra nevadensis \\ Opuntia echinocarpa \\ Stipa speciosa \\ Total Live \\ Dead \\ Total
}

\section{SPECIES}

Calochortus species

Coleogyne ramosissima

Coryphantha vivipara rosea

Ephedra nevadensis

Opuntia echinocarpa

Stipa speciosa

\section{SPECIES}

Calochortus species

Coleogyne ramosissima

Coryphantha vivipara rosea

Ephedra nevadensis

Opuntia echinocarpa

Stipa speciosa

Total Live

Dead

Total

\section{SPECIES}

Calochortus species

Coleogyne ramosissima

Coryphantha vivipara rosea

Ephedra nevadensis

Opuntia echinocarpa

Stipa speciosa

Total Live

Number of Plants

1963

0

278

0

16

0

0

294

1

295

1963

0.00

0.38

0.00

0.42

0.00

1963

0.00

42.95

0.00

2.07

0.00

0.00

45.03

0.10

45.13

1963

0.00

56.54

0.00

3.18

0.00

0.00

59.72
1975

0

393

1

16

0

5

415

7

422

Average Height (m)

\section{5}

0.00

0.37

0.13

0.49

0.00

0.47

Cover (\%)

1975

0.00

43.88

0.01

1.90

0.00

0.27

46.06

0.85

46.91

Biomass Index $\left(\mathrm{m}^{2}\right)$

1975

0.00

56.75

0.00

3.24

0.00

0.43

60.42
2000

6

294

0

19

2

1

322

41

322

\section{0}

0.34

0.43

0.00

0.53

0.46

0.47

2000

0.07

32.80

0.00

2.06

0.07

0.05

35.06

2.23

37.29

2000

0.08

49.20

0.00

3.78

0.12

0.09

53.27 
Table A-19A. Site characteristics for Plot 19.

Location: Topopah Valley

NTS Area: 25

NTS Grid: F-23

USGS 7.5’ Quadrangle Name: Topopah Spring

\begin{tabular}{lllll} 
& & \multicolumn{2}{l}{ GPS Readings (Zone 11S) } & \\
& Location & Northing & Easting & Condition of Original Corners \\
Corner 1: & SW & 4084216 & 564392 & NA \\
Corner 2: & NW & NA & NA & NA \\
Corner 3: & NE & NA & NA & NA \\
Corner 4: & SE & NA & NA & NA \\
\multicolumn{2}{ll}{ DEM Plot Elevation: 1466 m } & & \multicolumn{2}{c}{ Plot Aspect: $135^{\circ}$} \\
\multicolumn{2}{l}{ Elevation Above Playa (Playa Name): NA } & & \multicolumn{2}{c}{ Plot Slope: $9^{\circ}$}
\end{tabular}

Annual Precipitation

Measured: $219 \mathrm{~mm}$

Modeled: $189 \mathrm{~mm}$

Parent Material Type: alluvial fan

Substrate: NA

Slate Geologic Unit: Qay

Beatley Plant Assemblage: Ephedra

Ostler-Hanson Plant Association: NA

Ostler-Hanson Land Unit: 437

Abundance of Biological Soil Crusts: none

Type and Date of Disturbance: fire in 1959

Plot Condition: good

Location of Transect 1: south edge of plot

Direction Transects Are Read: east to west

Date(s) Plot Remeasured: 20-Jun-00 
Figure A-19. Photographs showing Plot 19.

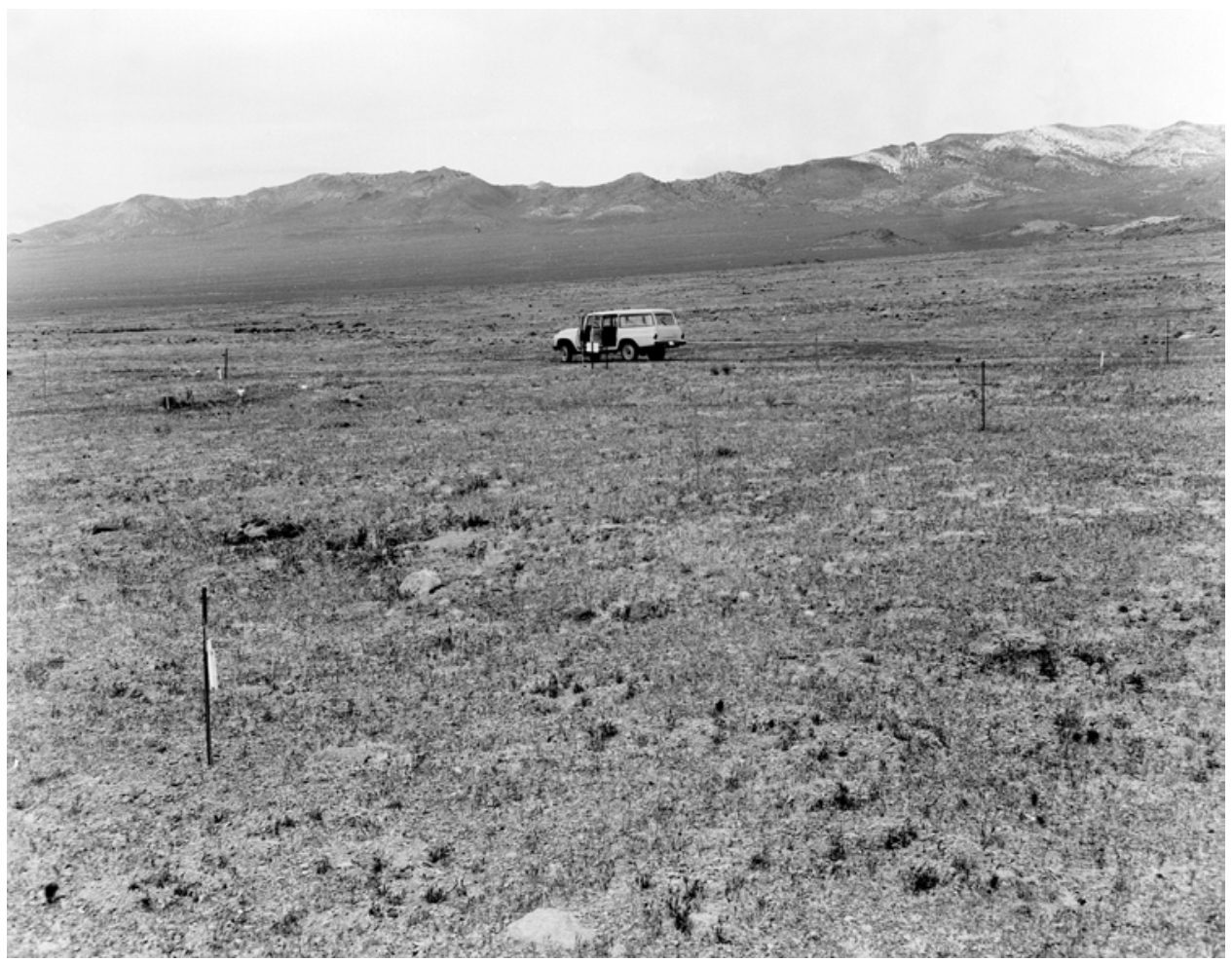

A. (May 7, 1964). This westerly view across Plot 19 shows a nearly denuded area following a fire in the mid-1950s. The Calico Hills appear in the background (Janice Beatley Collection, 58-B).

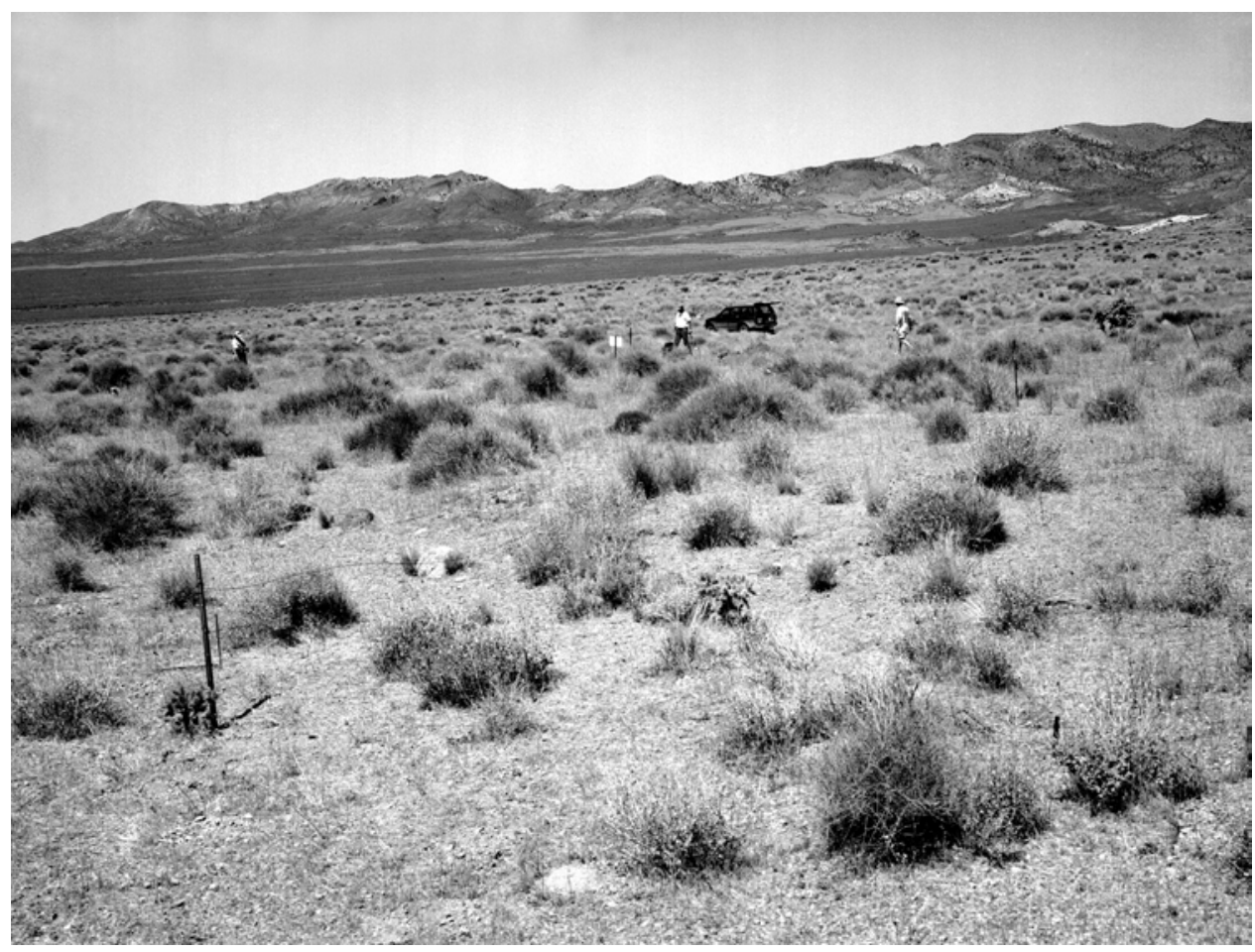

B. (June 20, 2000). Some recovery of perennial vegetation has occurred in the intervening 36 years between the original and matched photograph. Most of the shrubs now prominent are Mormon tea, although several other species are also present (R.H. Webb, Stake 4038A). 
Table A-19B. Summary plant data for Plot 19.

\begin{tabular}{|c|c|c|c|c|c|c|c|c|}
\hline SPECIES & 1963 & 1964 & 1965 & 1966 & 1967 & 1970 & 1975 & 2000 \\
\hline Acamptopappus shockleyi & 0 & 0 & 0 & 0 & 0 & 0 & 0 & 1 \\
\hline Oryzopsis hymenoides & 0 & 0 & 0 & 0 & 0 & 0 & 0 & 1 \\
\hline Astragalus lentiginosus fremontii & 0 & 0 & 0 & 0 & 1 & 0 & 0 & 1 \\
\hline Calochortus species & 0 & 0 & 0 & 0 & 0 & 0 & 0 & 1 \\
\hline Coleogyne ramosissima & 0 & 0 & 1 & 1 & 1 & 1 & 1 & 2 \\
\hline Dichelostemma pulchella & 0 & 0 & 9 & 0 & 0 & 0 & 0 & 0 \\
\hline Encelia virginensis & 0 & 0 & 0 & 0 & 0 & 0 & 0 & 65 \\
\hline Ephedra nevadensis & 23 & 22 & 15 & 21 & 23 & 22 & 17 & 35 \\
\hline Ericameria cooperi & 0 & 0 & 0 & 0 & 0 & 0 & 0 & 1 \\
\hline Hymenoclea salsola & 0 & 0 & 0 & 0 & 0 & 1 & 1 & 2 \\
\hline Opuntia echinocarpa & 0 & 0 & 0 & 0 & 0 & 0 & 0 & 1 \\
\hline Salazaria mexicana & 0 & 0 & 0 & 0 & 0 & 0 & 0 & 1 \\
\hline Sitanion hystrix & 0 & 0 & 0 & 0 & 0 & 0 & 0 & 1 \\
\hline Sphaeralcea ambigua & 3 & 1 & 11 & 18 & 16 & 0 & 22 & 111 \\
\hline Stephanomeria parryi & 0 & 0 & 0 & 1 & 0 & 0 & 0 & 0 \\
\hline Stipa speciosa & 0 & 0 & 6 & 11 & 9 & 6 & 7 & 13 \\
\hline Total Live & 26 & 23 & 42 & 52 & 50 & 30 & 48 & 237 \\
\hline Dead & 1 & 0 & 0 & 0 & 2 & 0 & 1 & 11 \\
\hline Total & 27 & 23 & 42 & 52 & 52 & 30 & 49 & 248 \\
\hline & \multicolumn{8}{|c|}{ Average Height (m) } \\
\hline SPECIES & 1963 & 1964 & 1965 & 1966 & 1967 & 1970 & 1975 & 2000 \\
\hline Acamptopappus shockleyi & 0.00 & 0.00 & 0.00 & 0.00 & 0.00 & 0.00 & 0.00 & 0.30 \\
\hline Oryzopsis hymenoides & 0.00 & 0.00 & 0.00 & 0.00 & 0.00 & 0.00 & 0.00 & 0.24 \\
\hline Astragalus lentiginosus fremontii & 0.00 & 0.00 & 0.00 & 0.00 & 0.15 & 0.00 & 0.00 & 0.31 \\
\hline Calochortus species & 0.00 & 0.00 & 0.00 & 0.00 & 0.00 & 0.00 & 0.00 & 0.31 \\
\hline Coleogyne ramosissima & 0.00 & 0.00 & 0.25 & 0.30 & 0.28 & 0.38 & 0.46 & 0.65 \\
\hline Dichelostemma pulchella & 0.00 & 0.00 & 0.11 & 0.00 & 0.00 & 0.00 & 0.00 & 0.00 \\
\hline Encelia virginensis & 0.00 & 0.00 & 0.00 & 0.00 & 0.00 & 0.00 & 0.00 & 0.32 \\
\hline Ephedra nevadensis & 0.29 & 0.30 & 0.30 & 0.28 & 0.40 & 0.46 & 0.50 & 0.61 \\
\hline Ericameria cooperi & 0.00 & 0.00 & 0.00 & 0.00 & 0.00 & 0.00 & 0.00 & 0.33 \\
\hline Hymenoclea salsola & 0.00 & 0.00 & 0.00 & 0.00 & 0.00 & 0.43 & 0.13 & 0.63 \\
\hline Opuntia echinocarpa & 0.08 & 0.00 & 0.00 & 0.00 & 0.00 & 0.00 & 0.00 & 0.26 \\
\hline Salazaria mexicana & 0.00 & 0.00 & 0.00 & 0.00 & 0.00 & 0.00 & 0.00 & 1.10 \\
\hline Sitanion hystrix & 0.00 & 0.00 & 0.00 & 0.00 & 0.00 & 0.00 & 0.00 & 0.55 \\
\hline Sphaeralcea ambigua & 0.27 & 0.28 & 0.16 & 0.36 & 0.32 & 0.00 & 0.05 & 0.34 \\
\hline Stephanomeria parryi & 0.00 & 0.00 & 0.00 & 0.13 & 0.00 & 0.00 & 0.00 & 0.00 \\
\hline Stipa speciosa & 0.00 & 0.00 & 0.15 & 0.30 & 0.27 & 0.33 & 0.44 & 0.46 \\
\hline
\end{tabular}


Table A-19B (continued). Summary plant data for Plot 19.

\begin{tabular}{|c|c|c|c|c|c|c|c|c|}
\hline \multirow[b]{2}{*}{ SPECIES } & \\
\hline & 1963 & 1964 & 1965 & 1966 & 1967 & 1970 & 1975 & 2000 \\
\hline Acamptopappus shockleyi & 0.00 & 0.00 & 0.00 & 0.00 & 0.00 & 0.00 & 0.00 & 0.14 \\
\hline Oryzopsis hymenoides & 0.00 & 0.00 & 0.00 & 0.00 & 0.00 & 0.00 & 0.00 & 0.01 \\
\hline Astragalus lentiginosus fremontii & 0.00 & 0.00 & 0.00 & 0.00 & 0.01 & 0.00 & 0.00 & 0.09 \\
\hline Calochortus species & 0.00 & 0.00 & 0.00 & 0.00 & 0.00 & 0.00 & 0.00 & 0.01 \\
\hline Coleogyne ramosissima & 0.00 & 0.00 & 0.04 & 0.01 & 0.02 & 0.02 & 0.10 & 0.53 \\
\hline Dichelostemma pulchella & 0.00 & 0.00 & 0.12 & 0.00 & 0.00 & 0.00 & 0.00 & 0.00 \\
\hline Encelia virginensis & 0.00 & 0.00 & 0.00 & 0.00 & 0.00 & 0.00 & 0.00 & 5.45 \\
\hline Ephedra nevadensis & 1.87 & 1.60 & 1.14 & 1.75 & 2.09 & 2.75 & 2.21 & 7.18 \\
\hline Ericameria cooperi & 0.00 & 0.00 & 0.00 & 0.00 & 0.00 & 0.00 & 0.00 & 0.22 \\
\hline Hymenoclea salsola & 0.00 & 0.00 & 0.00 & 0.00 & 0.00 & 0.07 & 0.02 & 0.39 \\
\hline Opuntia echinocarpa & 0.00 & 0.00 & 0.00 & 0.00 & 0.00 & 0.00 & 0.00 & 0.01 \\
\hline Salazaria mexicana & 0.00 & 0.00 & 0.00 & 0.00 & 0.00 & 0.00 & 0.00 & 0.23 \\
\hline Sitanion hystrix & 0.00 & 0.00 & 0.00 & 0.00 & 0.00 & 0.00 & 0.00 & 0.12 \\
\hline Sphaeralcea ambigua & 0.15 & 0.10 & 0.26 & 0.88 & 1.01 & 0.00 & 0.43 & 3.39 \\
\hline Stephanomeria parryi & 0.00 & 0.00 & 0.00 & 0.04 & 0.00 & 0.00 & 0.00 & 0.00 \\
\hline Stipa speciosa & 0.00 & 0.00 & 0.22 & 0.63 & 0.54 & 0.67 & 0.78 & 1.05 \\
\hline Total Live & 2.03 & 1.70 & 1.77 & 3.31 & 3.66 & 3.52 & 3.54 & 18.82 \\
\hline Unknown Dead & 0.01 & 0.00 & 0.00 & 0.00 & 0.00 & 0.00 & 0.29 & 1.85 \\
\hline \multirow[t]{2}{*}{ Total } & 2.04 & 1.70 & 1.77 & 3.31 & 3.66 & 3.52 & 3.83 & 20.67 \\
\hline & \multicolumn{8}{|c|}{ Biomass Index $\left(\mathrm{m}^{2}\right)$} \\
\hline SPECIES & 1963 & 1964 & 1965 & 1966 & 1967 & 1970 & 1975 & 2000 \\
\hline Acamptopappus shockleyi & 0.00 & 0.00 & 0.00 & 0.00 & 0.00 & 0.00 & 0.00 & 0.14 \\
\hline Oryzopsis hymenoides & 0.00 & 0.00 & 0.00 & 0.00 & 0.00 & 0.00 & 0.00 & 0.01 \\
\hline Astragalus lentiginosus fremontii & 0.00 & 0.00 & 0.00 & 0.00 & 0.00 & 0.00 & 0.00 & 0.09 \\
\hline Calochortus species & 0.00 & 0.00 & 0.00 & 0.00 & 0.00 & 0.00 & 0.00 & 0.01 \\
\hline Coleogyne ramosissima & 0.00 & 0.00 & 0.03 & 0.01 & 0.02 & 0.02 & 0.15 & 1.15 \\
\hline Dichelostemma pulchella & 0.00 & 0.00 & 0.04 & 0.00 & 0.00 & 0.00 & 0.00 & 0.00 \\
\hline Encelia virginensis & 0.00 & 0.00 & 0.00 & 0.00 & 0.00 & 0.00 & 0.00 & 7.85 \\
\hline Ephedra nevadensis & 1.97 & 1.77 & 1.25 & 1.93 & 3.22 & 4.75 & 17.00 & 18.32 \\
\hline Ericameria cooperi & 0.00 & 0.00 & 0.00 & 0.00 & 0.00 & 0.00 & 0.00 & 0.18 \\
\hline Hymenoclea salsola & 0.00 & 0.00 & 0.00 & 0.00 & 0.00 & 0.11 & 0.01 & 0.82 \\
\hline Opuntia echinocarpa & 0.00 & 0.00 & 0.00 & 0.00 & 0.00 & 0.00 & 0.00 & 0.01 \\
\hline Salazaria mexicana & 0.00 & 0.00 & 0.00 & 0.00 & 0.00 & 0.00 & 0.00 & 0.84 \\
\hline Sitanion hystrix & 0.00 & 0.00 & 0.00 & 0.00 & 0.00 & 0.00 & 0.00 & 0.22 \\
\hline Sphaeralcea ambigua & 0.15 & 0.09 & 0.17 & 1.15 & 1.20 & 0.00 & 0.09 & 4.34 \\
\hline Stephanomeria parryi & 0.00 & 0.00 & 0.00 & 0.02 & 0.00 & 0.00 & 0.00 & 0.00 \\
\hline Stipa speciosa & 0.00 & 0.00 & 0.11 & 0.70 & 0.54 & 0.69 & 1.20 & 1.72 \\
\hline Total Live & 2.12 & 1.87 & 1.60 & 3.81 & 4.99 & 5.57 & 18.45 & 35.69 \\
\hline
\end{tabular}


Table A-20A. Site characteristics for Plot 20.

Location: Frenchman Flat

NTS Area: 5

NTS Grid: T-14

USGS 7.5’ Quadrangle Name: Cane Spring

\begin{tabular}{|c|c|c|c|c|}
\hline & & GPS Reac & Zone 11S) & \\
\hline & Location & Northing & Easting & Condition of Original Corners \\
\hline Corner 1: & SW & 4067866 & 587326 & Standing \\
\hline Corner 2: & NW & 4067856 & 587332 & Wood on ground; angle iron standing \\
\hline Corner 3: & $\mathrm{NE}$ & 4067886 & 587360 & Standing \\
\hline Corner 4: & SE & 4067857 & 587355 & Standing \\
\hline DEM Plot & vation: 99 & & & Plot Aspect: $315^{\circ}$ \\
\hline Elevation & e Playa ( & Name): 5 & Frenchman) & Plot Slope: $6^{\circ}$ \\
\hline
\end{tabular}

Annual Precipitation

Measured: $139 \mathrm{~mm}$

Modeled: $133 \mathrm{~mm}$

Parent Material Type: alluvial fan

Substrate: lower alluvial fan, very sandy w/pebbles, volcanic

Slate Geologic Unit: Qay

Beatley Plant Assemblage: Larrea-Ambrosia/Larrea-Lycium-Grayia

Ostler-Hanson Plant Association: Larrea-Grayia-Ceratoides

Ostler-Hanson Land Unit: 6

Abundance of Biological Soil Crusts: none

Type and Date of Disturbance: none

Plot Condition: good

Location of Transect 1: east side of plot

Direction Transects Are Read: north to south

Date(s) Plot Remeasured: 16 April 2002 
Figure A-20. Photographs showing Plot 20.

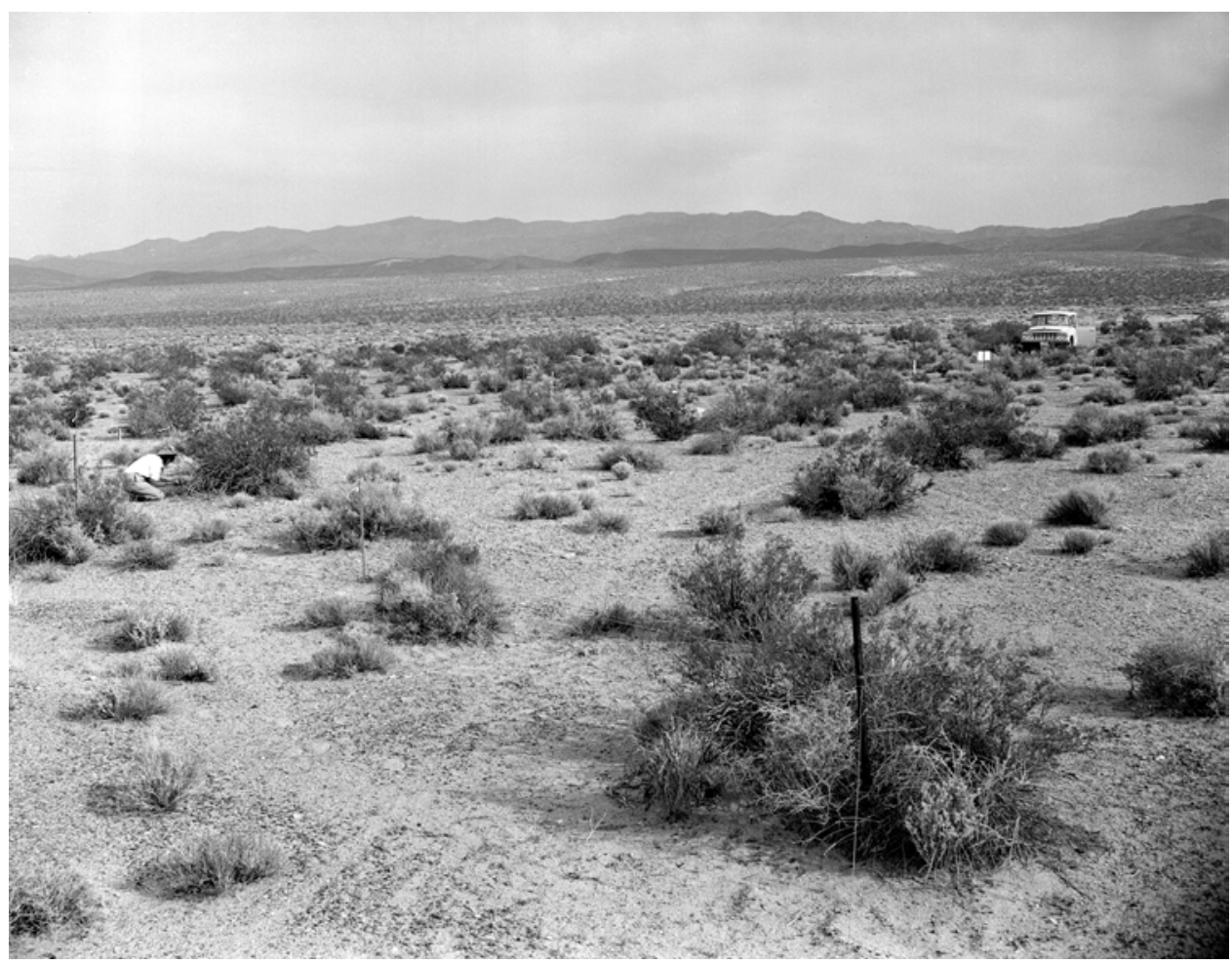

A. (April 16, 1964). This southeasterly view across Plot 20 and the southwesterly cover of Frenchman Flat shows a mixed creosote bush scrub with white bursage as the subdominant. The Spotted Range appears in the background. Janice Beatley is measuring annuals at left (Janice Beatley Collection, 4-A).

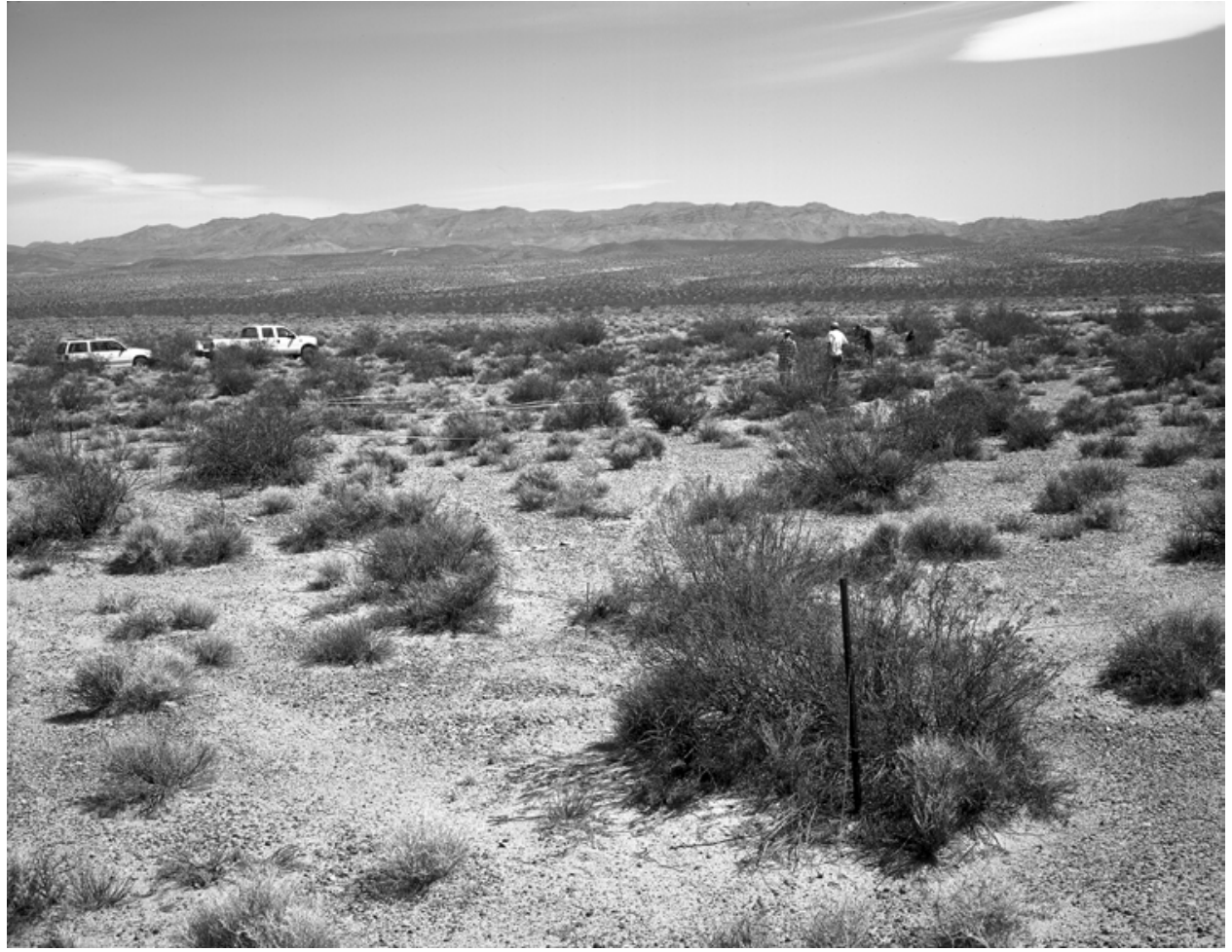

B. (April 16, 2002). The change here is less visible than at other plots in this type of vegetation, although comparison of the corner posts with the heights of shrubs shows that creosote bush, in particularly, has increased in size. The vegetation is now dominated by creosote bush and white bursage, with winterfat (Ceratoides lanata) as an important subdominant (Dominic Oldershaw, Stake 4100A). 
Table A-20B. Summary plant data for Plot 20.

\begin{tabular}{|c|c|c|c|c|c|c|c|}
\hline \multicolumn{5}{|c|}{ Number of Plants } & \multicolumn{3}{|c|}{ Cover (\%) } \\
\hline SPECIES & 1963 & 1975 & 2002 & SPECIES & 1963 & 1975 & 2002 \\
\hline Acamptopappus shockleyi & 18 & 30 & 14 & Acamptopappus shockleyi & 1.51 & 2.45 & 0.89 \\
\hline Ambrosia dumosa & 21 & 28 & 72 & Ambrosia dumosa & 1.62 & 2.46 & 5.02 \\
\hline Atriplex canescens & 0 & 2 & 0 & Atriplex canescens & 0.00 & 0.16 & 0.00 \\
\hline Ceratoides lanata & 29 & 40 & 25 & Ceratoides lanata & 2.06 & 3.84 & 1.51 \\
\hline Grayia spinosa & 26 & 28 & 6 & Grayia spinosa & 3.57 & 4.15 & 0.60 \\
\hline Larrea tridentata & 27 & 42 & 44 & Larrea tridentata & 8.33 & 10.27 & 6.92 \\
\hline Lycium andersonii & 10 & 12 & 10 & Lycium andersonii & 1.20 & 1.62 & 0.65 \\
\hline Mirabilis pudica & 1 & 2 & 2 & Mirabilis pudica & 0.03 & 0.12 & 0.09 \\
\hline Oryzopsis hymenoides & 8 & 40 & 11 & Oryzopsis hymenoides & 0.29 & 2.39 & 0.50 \\
\hline Sitanion hystrix & 0 & 0 & 2 & Sitanion hystrix & 0.00 & 0.00 & 0.06 \\
\hline Tetradymia axillaris & 1 & 0 & 1 & Tetradymia axillaris & 0.04 & 0.00 & 0.13 \\
\hline Total Live & 141 & 224 & 187 & Total Live & 18.65 & 27.46 & 16.36 \\
\hline Dead Grass & 0 & 19 & 5 & Dead Grass & 0.00 & 2.09 & 0.10 \\
\hline Dead Shrub & 10 & 1 & 44 & Dead Shrub & 2.24 & 0.04 & 3.25 \\
\hline Total & 151 & 256 & 236 & Total & 20.88 & 29.59 & 19.71 \\
\hline \multicolumn{5}{|c|}{ Average Height (m) } & \multicolumn{3}{|c|}{ Biomass Index $\left(\mathbf{m}^{2}\right)$} \\
\hline SPECIES & 1963 & 1975 & 2002 & SPECIES & 1963 & 1975 & 2002 \\
\hline Acamptopappus shockleyi & 0.28 & 0.28 & 0.26 & Acamptopappus shockleyi & 1.63 & 2.47 & 0.93 \\
\hline Ambrosia dumosa & 0.25 & 0.30 & 0.32 & Ambrosia dumosa & 1.73 & 2.69 & 5.82 \\
\hline Atriplex canescens & n.d. & 0.87 & n.d. & Atriplex canescens & 0.00 & 0.46 & 0.00 \\
\hline Ceratoides lanata & 0.42 & 0.50 & 0.44 & Ceratoides lanata & 3.11 & 6.84 & 2.27 \\
\hline Grayia spinosa & 0.64 & 0.68 & 0.48 & Grayia spinosa & 8.35 & 9.61 & 1.11 \\
\hline Larrea tridentata & 1.04 & 1.01 & 1.07 & Larrea tridentata & 29.53 & 35.78 & 25.40 \\
\hline Lycium andersonii & 0.56 & 0.53 & 0.44 & Lycium andersonii & 2.31 & 2.84 & 1.02 \\
\hline Mirabilis pudica & 0.20 & 0.09 & 0.25 & Mirabilis pudica & 0.02 & 0.03 & 0.07 \\
\hline Oryzopsis hymenoides & 0.20 & 0.26 & 0.21 & Oryzopsis hymenoides & 0.24 & 2.18 & 0.37 \\
\hline Sitanion hystrix & n.d. & n.d. & 0.36 & Sitanion hystrix & 0.00 & 0.00 & 0.08 \\
\hline \multirow[t]{2}{*}{ Tetradymia axillaris } & 0.36 & n.d. & 0.59 & Tetradymia axillaris & 0.04 & 0.00 & 0.25 \\
\hline & & & & Total Live & 46.95 & 62.90 & 37.34 \\
\hline
\end{tabular}


Table A-21A. Site characteristics for Plot 21.

Location: Frenchman Flat

NTS Area: 5

NTS Grid: U-15

USGS 7.5’ Quadrangle Name: Cane Spring

\begin{tabular}{lllr} 
& & \multicolumn{2}{c}{ GPS Readings (Zone 11S) } \\
& Location & Northing & Easting \\
Corner 1: & SW & 4070661 & 588169 \\
Corner 2: & NW & 4070691 & 588176 \\
Corner 3: & NE & 4070686 & 588205 \\
Corner 4: & SE & 4070656 & 588198
\end{tabular}

DEM Plot Elevation: $981 \mathrm{~m}$

Condition of Original Corners

Angle iron standing; 3 wood corners standing Angle iron standing; 3 wood corners standing Angle iron standing; 3 wood corners standing Angle iron standing; 3 wood corners standing

Plot Aspect: $0^{\circ}$

Elevation Above Playa (Playa Name): 40 m (Frenchman)

Plot Slope: $3^{\circ}$

Annual Precipitation

Measured: $133 \mathrm{~mm}$

Modeled: $127 \mathrm{~mm}$

Parent Material Type: alluvial fan

Substrate: not much rock varnish, pavement not well developed, very sandy alluvial, w/volcanic pebbles veneer

Slate Geologic Unit: Qay

Beatley Plant Assemblage: Larrea-Lycium-Grayia

Ostler-Hanson Plant Association: Larrea-Oryzopsis-Grayia

Ostler-Hanson Land Unit: 101

Abundance of Biological Soil Crusts: none

Type and Date of Disturbance: none

Plot Condition: good

Location of Transect 1: east side of plot

Direction Transects Are Read: south to north

Date(s) Plot Remeasured: 16 April 2002 
Figure A-21. Photographs showing Plot 21.

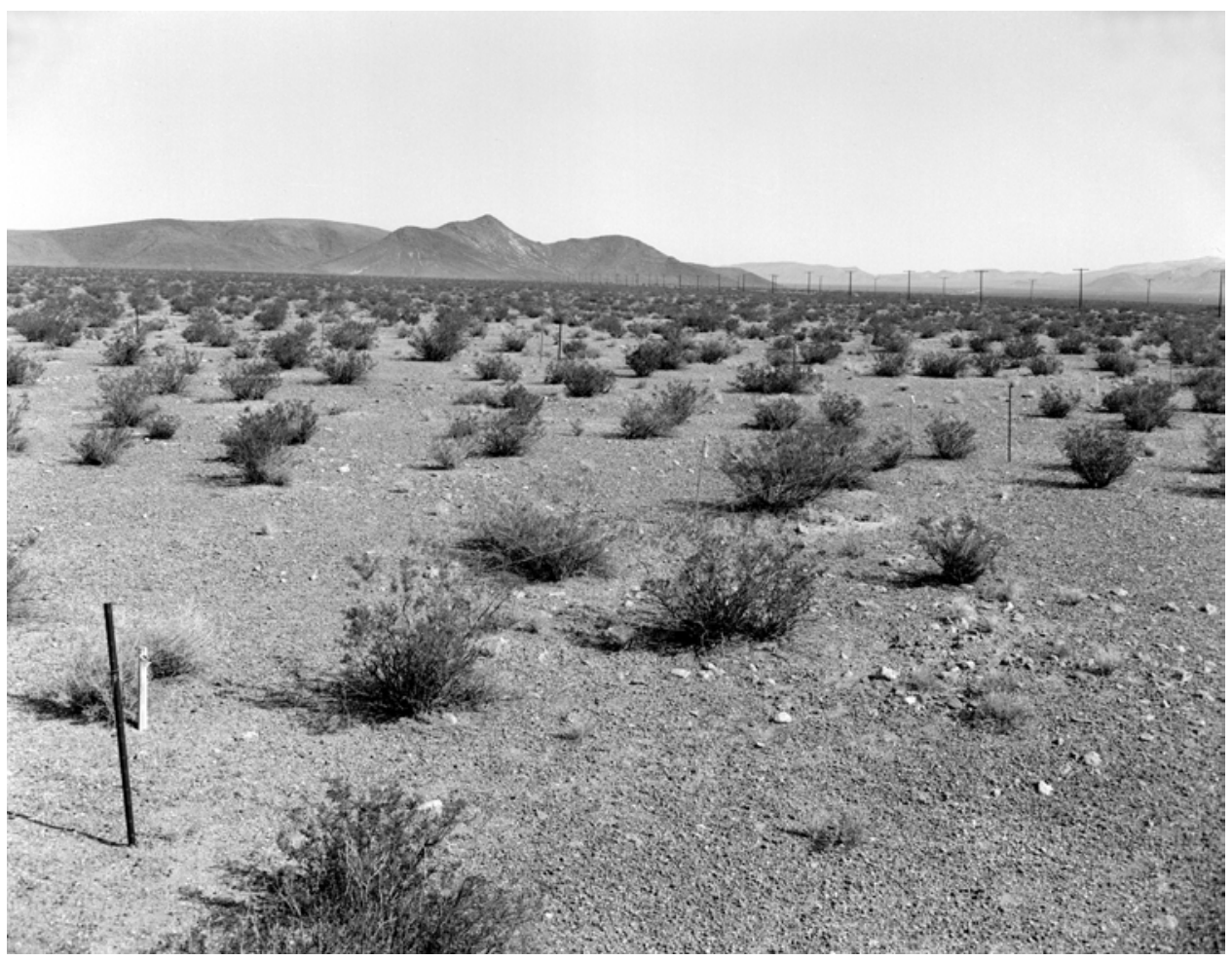

A. (April 29, 1964). This westerly view across Plot 21 shows a mixed shrub assemblage in western Frenchman Flat with some low hills that are east of Mount Salyer. The vegetation is dominated by creosote bush, wolfberry, and spiny hopsage (Janice Beatley Collection, 44-B).

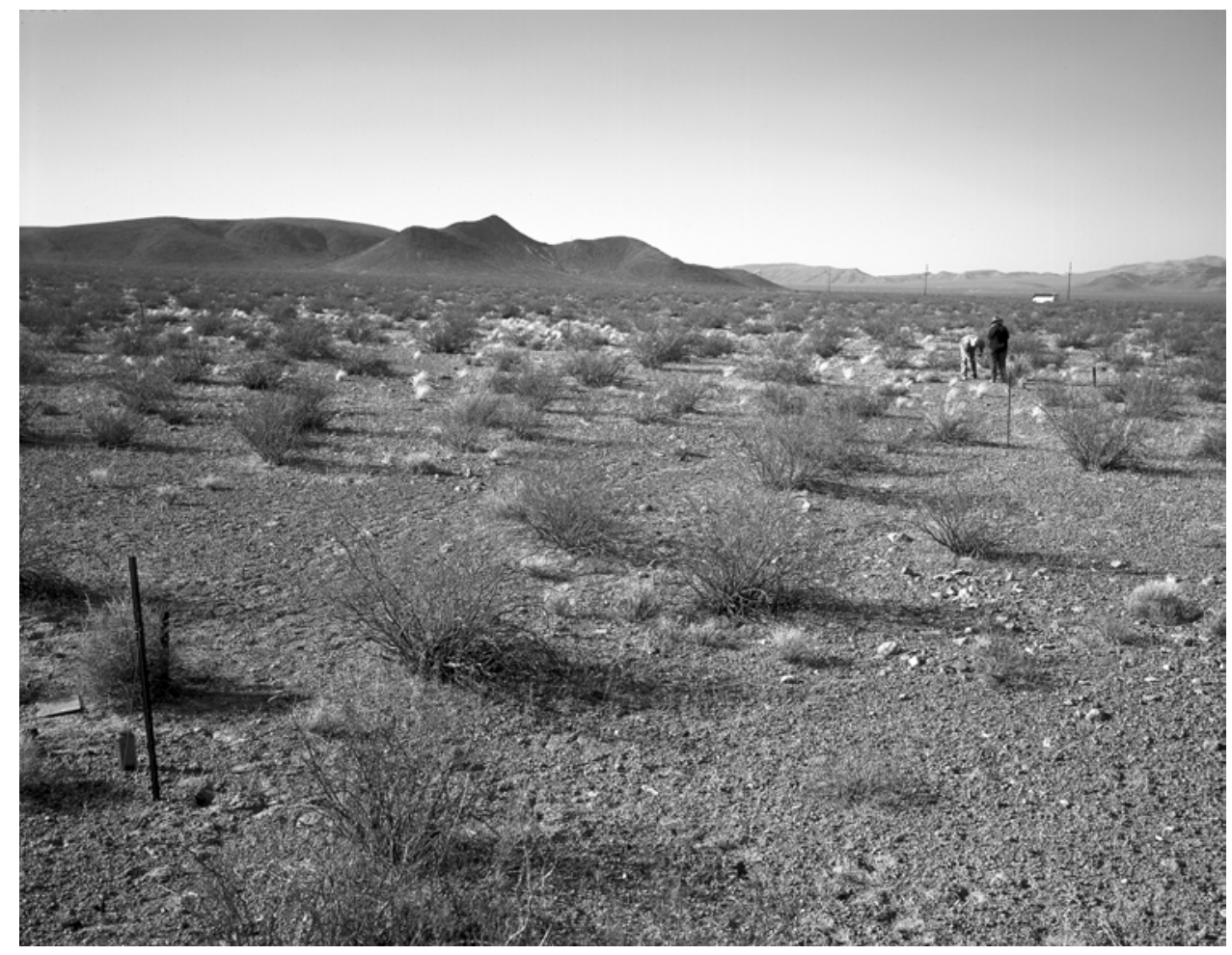

B. (April 16, 2002). Most of the individuals of creosote bush are in the same place and are only slightly larger. Wolfberry and spiny hopsage have greatly decreased here, and Indian rice grass (Stipa hymenoides) has significant cover. White bursage has increased in density, but not as much as other plots in this vegetation association (Dominic Oldershaw, Stake 4213A). 
Table A-21B. Summary plant data for Plot 21.

\begin{tabular}{|c|c|c|c|c|c|c|c|}
\hline \multicolumn{5}{|c|}{ Number of Plants } & \multicolumn{3}{|c|}{ Cover (\%) } \\
\hline SPECIES & 1963 & 1975 & 2002 & SPECIES & 1963 & 1975 & 2002 \\
\hline Acamptopappus shockleyi & 1 & 2 & 4 & Acamptopappus shockleyi & 0.08 & 0.18 & 0.30 \\
\hline Ambrosia dumosa & 0 & 1 & 8 & Ambrosia dumosa & 0.00 & 0.02 & 0.43 \\
\hline Grayia spinosa & 10 & 16 & 3 & Grayia spinosa & 1.08 & 1.86 & 0.35 \\
\hline Larrea tridentata & 35 & 43 & 46 & Larrea tridentata & 7.49 & 9.72 & 8.63 \\
\hline Lycium andersonii & 3 & 4 & 5 & Lycium andersonii & 0.61 & 0.83 & 0.75 \\
\hline Mirabilis pudica & 0 & 1 & 0 & Mirabilis pudica & 0.00 & 0.07 & 0.00 \\
\hline Oryzopsis hymenoides & 11 & 36 & 19 & Oryzopsis hymenoides & 0.54 & 2.93 & 1.00 \\
\hline Total Live & 60 & 103 & 85 & Total Live & 9.80 & 15.61 & 11.45 \\
\hline Dead Grass & 0 & 3 & 1 & Dead Grass & 0.00 & 0.23 & 0.02 \\
\hline Dead Shrub & 3 & 2 & 1 & Dead Shrub & 0.74 & 0.30 & 0.30 \\
\hline Total & 63 & 108 & 87 & Total & 10.54 & 16.14 & 11.77 \\
\hline \multicolumn{5}{|c|}{ Average Height (m) } & \multicolumn{3}{|c|}{ Biomass Index $\left(\mathrm{m}^{2}\right)$} \\
\hline SPECIES & 1963 & 1975 & 2002 & SPECIES & 1963 & 1975 & 2002 \\
\hline Acamptopappus shockleyi & 0.25 & 0.24 & 0.29 & Acamptopappus shockleyi & 0.07 & 0.16 & 0.33 \\
\hline Ambrosia dumosa & 0.00 & 0.20 & 0.27 & Ambrosia dumosa & 0.00 & 0.01 & 0.32 \\
\hline Grayia spinosa & 0.44 & 0.56 & 0.64 & Grayia spinosa & 1.66 & 3.63 & 0.81 \\
\hline Larrea tridentata & 0.79 & 0.77 & 0.95 & Larrea tridentata & 21.48 & 26.53 & 29.81 \\
\hline Lycium andersonii & 0.40 & 0.51 & 0.55 & Mirabilis pudica & 0.82 & 1.46 & 0.00 \\
\hline Mirabilis pudica & 0.00 & 0.10 & 0.00 & Lycium andersonii & 0.00 & 0.02 & 1.61 \\
\hline \multirow[t]{2}{*}{ Oryzopsis hymenoides } & 0.26 & 0.27 & 0.25 & Oryzopsis hymenoides & 0.48 & 2.95 & 0.84 \\
\hline & & & & Total Live & 24.51 & 34.77 & 33.72 \\
\hline
\end{tabular}


Table A-22A. Site characteristics for Plot 22.

Location: Frenchman Flat

NTS Area: 5

NTS Grid: T-16

USGS 7.5’ Quadrangle Name: Cane Spring

\begin{tabular}{lllll} 
& & \multicolumn{2}{l}{ GPS Readings (Zone 11S) } & \\
& Location & Northing & Easting & Condition of Original Corners \\
Corner 1: & SW & 4072526 & 587612 & Standing \\
Corner 2: & NW & 4072555 & 587621 & Standing \\
Corner 3: & NE & 4072520 & 587643 & Standing \\
Corner 4: & SE & 4072549 & 587650 & Standing
\end{tabular}

DEM Plot Elevation: 999 m

Plot Aspect: $278^{\circ}$

Elevation Above Playa (Playa Name): 40 m (Frenchman)

Plot Slope: $5^{\circ}$

Annual Precipitation

Measured: $131 \mathrm{~mm}$

Modeled: $134 \mathrm{~mm}$

Parent Material Type: alluvial fan

Substrate: sandy alluvial, w/pebbles and rocks, some varnished, volcanic, older than plot 20 and 21

Slate Geologic Unit: Qai

Beatley Plant Assemblage: Larrea-Ambrosia

Ostler-Hanson Plant Association: Larrea-Ambrosia-Grayia

Ostler-Hanson Land Unit: 476

Abundance of Biological Soil Crusts: none

Type and Date of Disturbance: none

Plot Condition: good

Location of Transect 1: east side of plot

Direction Transects Are Read: south to north

Date(s) Plot Remeasured: 18 April 2002 
Figure A-22. Photographs showing Plot 22.

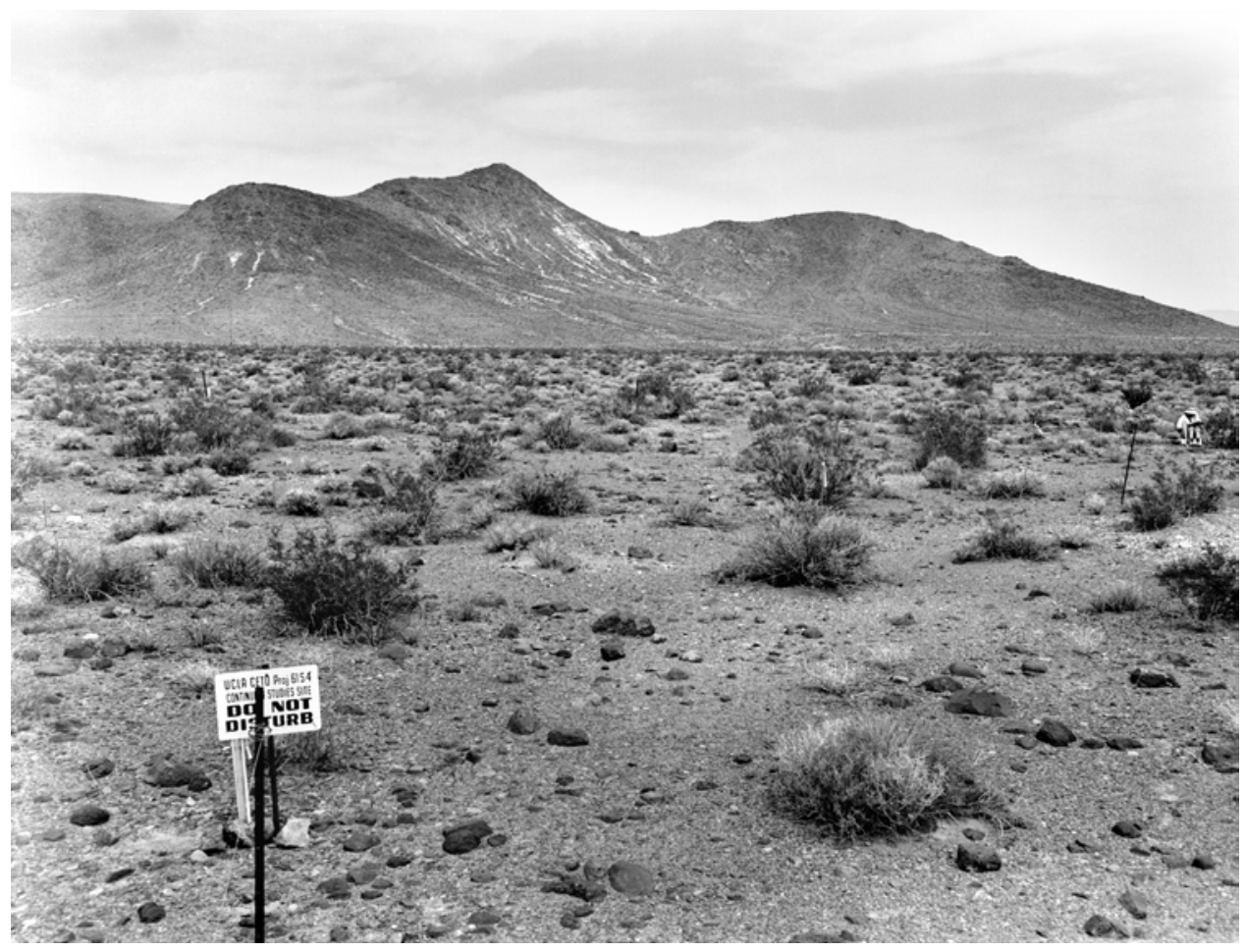

A. (April 16, 1964). This northwesterly view across Plot 22 shows a creosote bush - white bursage vegetation assemblage in western Frenchman Flat. The same low hills shown in Figure 24 are now closer in this view. Volcanic clasts litter the soil surface in the foreground, and Janice Beatley is measuring annual vegetation in the right midground (Janice Beatley Collection, 6-B).

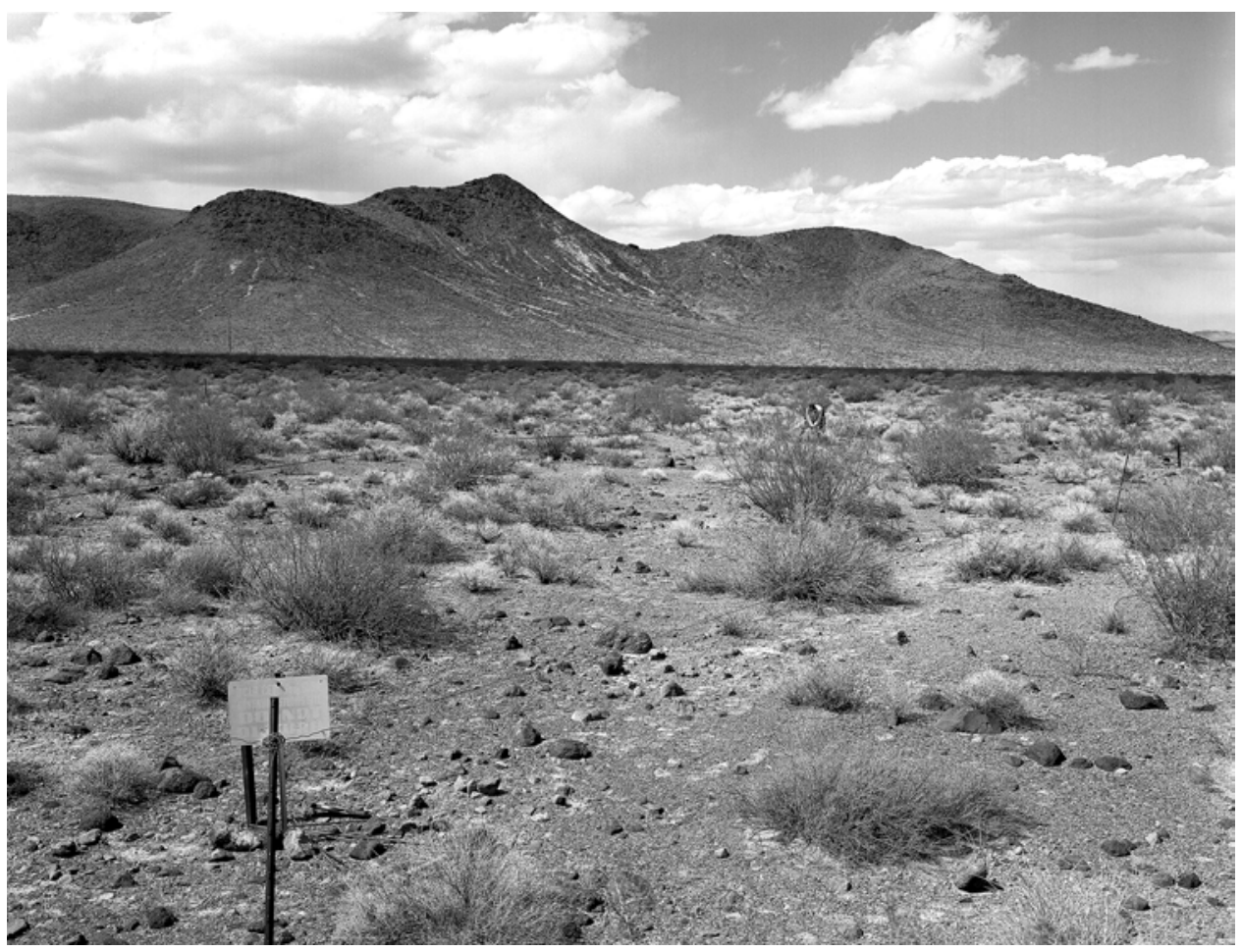

B. (April 18, 2002). The individual creosote bushes are much larger now, and white bursage has increased in density and size. Fremont's dalea (Psorothamnus fremontii) has become a subdominant here (Dominic Oldershaw, Stake 4158B). 
Table A-22B. Summary plant data for Plot 22.

\begin{tabular}{|c|c|c|c|c|c|c|c|}
\hline \multicolumn{5}{|c|}{ Number of Plants } & \multicolumn{3}{|c|}{ Cover (\%) } \\
\hline SPECIES & 1963 & 1975 & 2002 & SPECIES & 1963 & 1975 & 2002 \\
\hline Ambrosia dumosa & 40 & 64 & 60 & Ambrosia dumosa & 4.64 & 4.90 & 4.63 \\
\hline Atriplex confertifolia & 1 & 6 & 5 & Atriplex confertifolia & 0.09 & 0.63 & 0.38 \\
\hline Eriogonum inflatum & 0 & 2 & 2 & Eriogonum inflatum & 0.00 & 0.07 & 0.31 \\
\hline Ephedra nevadensis & 1 & 0 & 0 & Ephedra nevadensis & 0.13 & 0.00 & 0.00 \\
\hline Grayia spinosa & 9 & 19 & 4 & Grayia spinosa & 0.89 & 2.14 & 0.59 \\
\hline Krameria parvifolia & 1 & 2 & 4 & Krameria parvifolia & 0.23 & 0.26 & 0.57 \\
\hline Larrea tridentata & 24 & 25 & 30 & Larrea tridentata & 6.96 & 6.50 & 7.20 \\
\hline Lycium andersonii & 4 & 6 & 6 & Lycium andersonii & 0.81 & 1.12 & 0.66 \\
\hline Lycium pallidum oligospermum & 1 & 1 & 2 & Lycium pallidum oligospermum & 0.37 & 0.43 & 0.69 \\
\hline Mirabilis bigelovii & 0 & 1 & 0 & Mirabilis bigelovii & 0.00 & 0.09 & 0.00 \\
\hline Psorothamnus fremontii & 8 & 13 & 9 & Psorothamnus fremontii & 0.61 & 1.46 & 1.25 \\
\hline Stipa speciosa & 0 & 1 & 0 & Stipa speciosa & 0.00 & 0.09 & 0.00 \\
\hline Total Live & 89 & 140 & 122 & Total Live & 14.73 & 17.69 & 16.28 \\
\hline Dead Grass & 0 & 0 & 0 & Dead Grass & 0.00 & 0.00 & 0.00 \\
\hline Dead Shrub & 16 & 21 & 56 & Dead Shrub & 3.29 & 2.15 & 7.68 \\
\hline Total & 105 & 161 & 178 & Total & 18.02 & 19.84 & 23.96 \\
\hline \multicolumn{5}{|c|}{ Average Height (m) } & \multicolumn{3}{|c|}{ Biomass Index $\left(\mathrm{m}^{2}\right)$} \\
\hline SPECIES & 1963 & 1975 & 2002 & SPECIES & 1963 & 1975 & 2002 \\
\hline Ambrosia dumosa & 0.33 & 0.32 & 0.33 & Ambrosia dumosa & 5.43 & 5.68 & 5.41 \\
\hline Atriplex confertifolia & 0.46 & 0.51 & 0.32 & Atriplex confertifolia & 0.14 & 1.06 & 0.40 \\
\hline Eriogonum inflatum & 0.00 & 0.20 & 0.49 & Eriogonum inflatum & 0.00 & 0.05 & 0.54 \\
\hline Ephedra nevadensis & 0.41 & 0.00 & 0.00 & Ephedra nevadensis & 0.17 & 0.00 & 0.00 \\
\hline Grayia spinosa & 0.45 & 0.46 & 0.60 & Grayia spinosa & 1.40 & 3.57 & 1.22 \\
\hline Krameria parvifolia & 0.38 & 0.34 & 0.37 & Krameria parvifolia & 0.29 & 0.36 & 0.74 \\
\hline Larrea tridentata & 0.83 & 0.85 & 1.11 & Larrea tridentata & 21.48 & 18.95 & 27.09 \\
\hline Lycium andersonii & 0.54 & 0.55 & 0.44 & Lycium andersonii & 1.48 & 2.10 & 1.03 \\
\hline Lycium pallidum oligospermum & 0.84 & 0.97 & 0.78 & Lycium pallidum oligospermum & 1.05 & 1.38 & 1.94 \\
\hline Mirabilis bigelovii & 0.00 & 0.38 & 0.00 & Mirabilis bigelovii & 0.00 & 0.12 & 0.00 \\
\hline Psorothamnus fremontii & 0.23 & 0.34 & 0.44 & Psorothamnus fremontii & 0.54 & 1.82 & 2.11 \\
\hline \multirow[t]{2}{*}{ Stipa speciosa } & 0.00 & 0.36 & 0.00 & Stipa speciosa & 0.00 & 0.11 & 0.00 \\
\hline & & & & Total Live & 31.98 & 35.20 & 40.48 \\
\hline
\end{tabular}


Table A-23A. Site characteristics for Plot 23.

Location: Frenchman Flat

NTS Area: 5

NTS Grid: V-17

USGS 7.5’ Quadrangle Name: Frenchman Lake

\begin{tabular}{lllll} 
& & \multicolumn{2}{c}{ GPS Readings (Zone 11S) } & \\
& Location & Northing & Easting & Condition of Original Corners \\
Corner 1: & SW & 4074412 & 590158 & Standing \\
Corner 2: & NW & 4074442 & 590165 & Standing \\
Corner 3: & NE & 4074433 & 590196 & Standing \\
Corner 4: & SE & 4074404 & 590188 & Standing
\end{tabular}

DEM Plot Elevation: 968 m

Plot Aspect: $90^{\circ}$

Elevation Above Playa (Playa Name): 24 m (Frenchman)

Plot Slope: $10^{\circ}$

Annual Precipitation

Measured: $123 \mathrm{~mm}$

Modeled: $125 \mathrm{~mm}$

Parent Material Type: alluvial fan

Substrate: sandy alluvium w/volcanic pebble veneer

Slate Geologic Unit: Qay

Beatley Plant Assemblage: Larrea-Ambrosia/Larrea-Lycium-Grayia

Ostler-Hanson Plant Association: Larrea-Ambrosia-Acamptopappus

Ostler-Hanson Land Unit: 103

Abundance of Biological Soil Crusts: none

Type and Date of Disturbance: none

Plot Condition: good

Location of Transect 1: east side of plot

Direction Transects Are Read: south to north

Date(s) Plot Remeasured: 17 April 2002 
Figure A-23. Photographs showing Plot 23.

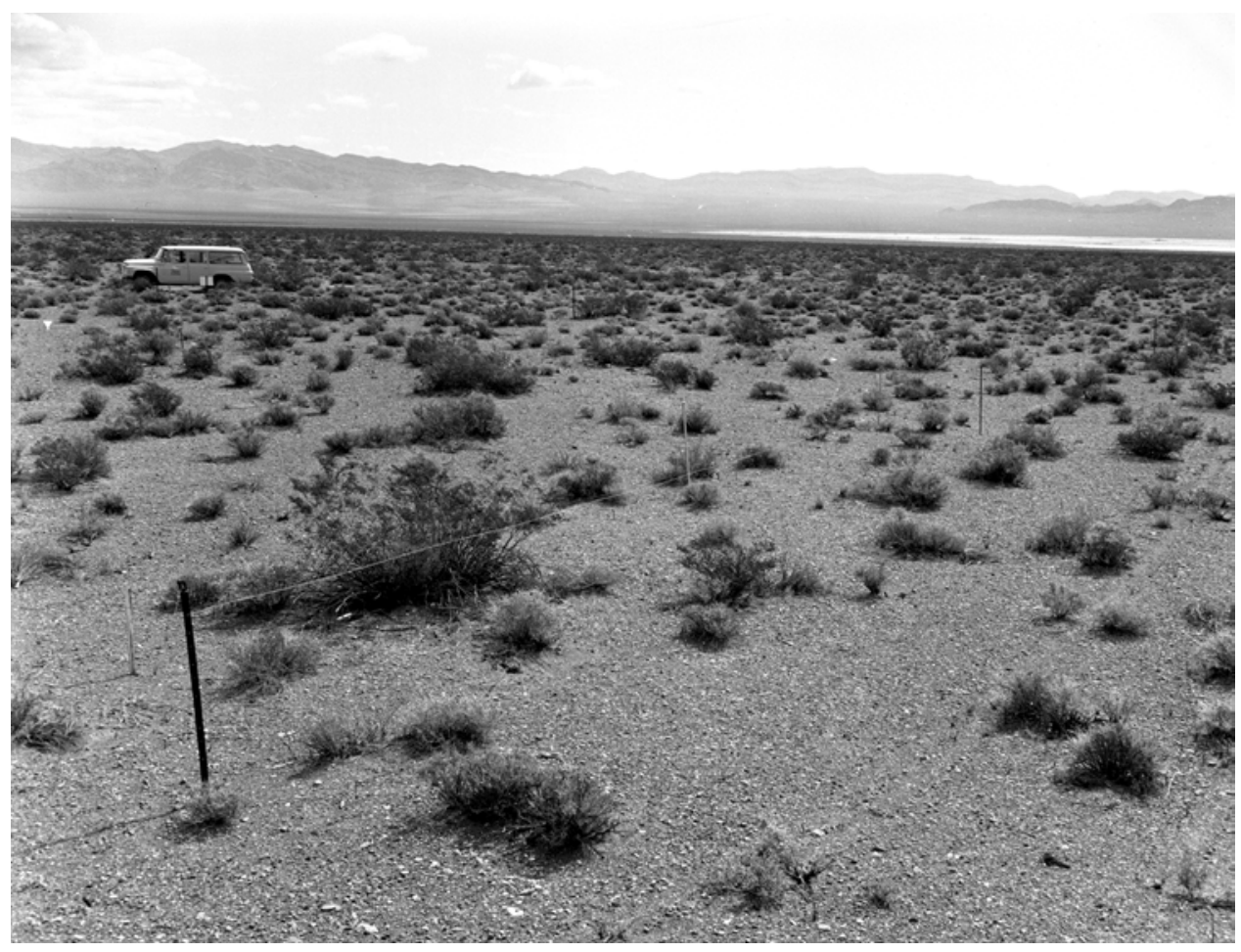

A. (April 25, 1964). This east-northeast view across Plot 23 shows a mixed creosote bush scrub assemblage with Frenchman Lake in the background. The Ranger Mountains appear in the extreme right background, and the Buried Hills appear in the center-left background (Janice Beatley Collection, 30-B).

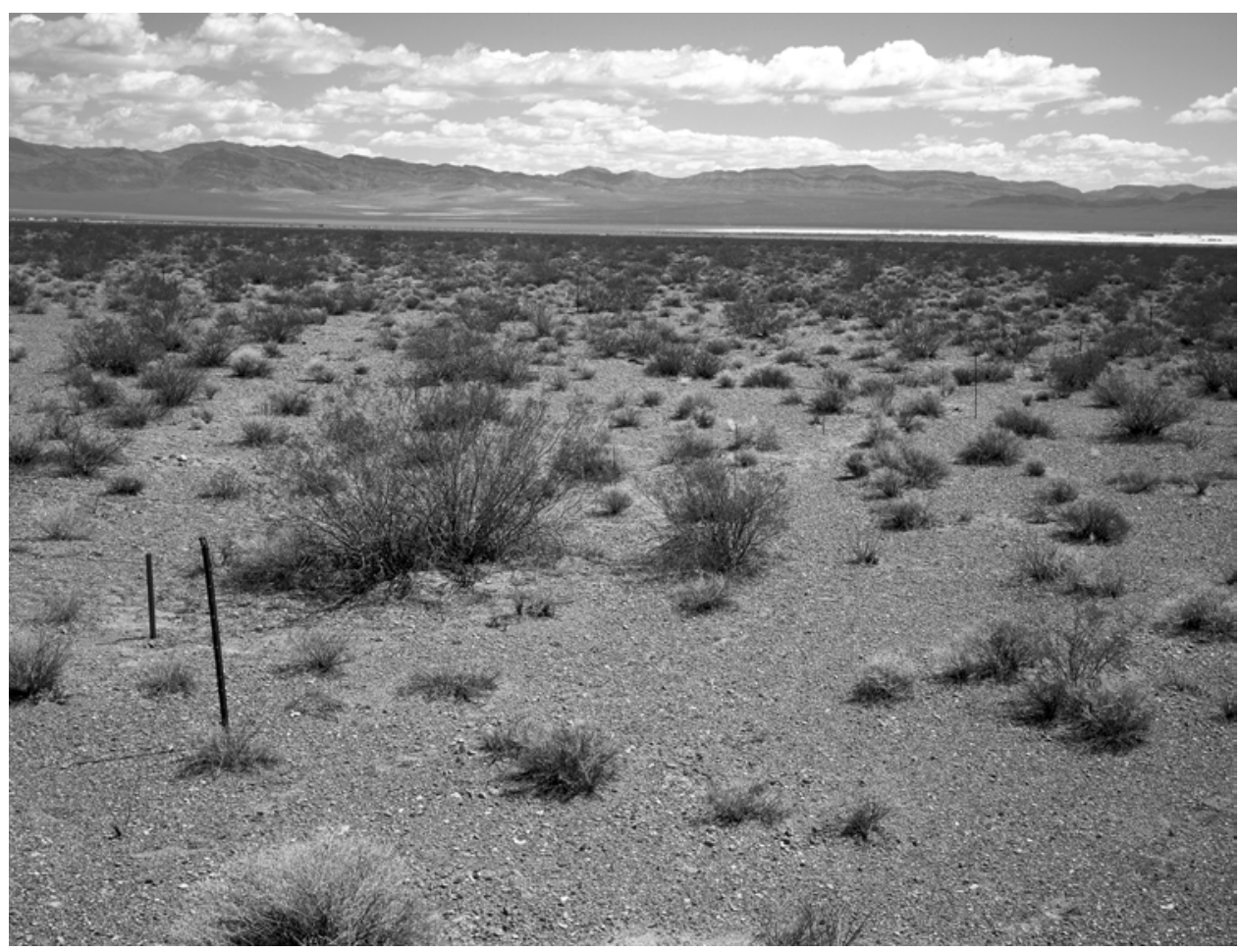

B. (April 20, 2002). Individual creosote bushes have increased considerably in size, and white bursage has increased in density in this matched view. Wolfberry and spiny hopsage have greatly decreased on this plot (Dominic Oldershaw, Stake 4176B). 
Table A-23B. Summary plant data for Plot 23.

\begin{tabular}{|c|c|c|c|c|c|c|c|}
\hline \multicolumn{5}{|c|}{ Number of Plants } & \multicolumn{3}{|c|}{ Cover (\%) } \\
\hline SPECIES & 1963 & 1975 & 2002 & SPECIES & 1963 & 1975 & 2002 \\
\hline Acamptopappus shockleyi & 22 & 28 & 9 & Acamptopappus shockleyi & 1.88 & 2.52 & 0.53 \\
\hline Ambrosia dumosa & 27 & 38 & 43 & Ambrosia dumosa & 2.45 & 3.25 & 3.31 \\
\hline Ceratoides lanata & 3 & 5 & 7 & Ceratoides lanata & 0.18 & 0.43 & 0.41 \\
\hline Ephedra nevadensis & 2 & 3 & 3 & Ephedra nevadensis & 0.13 & 0.39 & 0.40 \\
\hline Grayia spinosa & 9 & 14 & 4 & Grayia spinosa & 0.90 & 1.62 & 0.15 \\
\hline Hymenoclea salsola & 7 & 15 & 3 & Hymenoclea salsola & 0.49 & 1.05 & 0.31 \\
\hline Larrea tridentata & 22 & 20 & 21 & Larrea tridentata & 2.65 & 3.73 & 5.63 \\
\hline Lycium andersonii & 5 & 7 & 4 & Lycium andersonii & 0.78 & 0.96 & 0.40 \\
\hline Oryzopsis hymenoides & 2 & 9 & 3 & Oryzopsis hymenoides & 0.10 & 0.45 & 0.07 \\
\hline Psorothamnus fremontii & 1 & 1 & 0 & Psorothamnus fremontii & 0.11 & 0.11 & 0.00 \\
\hline Total Live & 100 & 140 & 97 & Total Live & 9.67 & 14.50 & 11.21 \\
\hline Dead Grass & 0 & 0 & 1 & Dead Grass & 0.00 & 0.00 & 0.02 \\
\hline Dead Shrub & 27 & 10 & 45 & Dead Shrub & 2.41 & 0.77 & 4.87 \\
\hline Total & 127 & 150 & 143 & Total & 12.08 & 15.27 & 16.10 \\
\hline \multicolumn{5}{|c|}{ Average Height (m) } & \multicolumn{3}{|c|}{ Biomass Index $\left(\mathrm{m}^{2}\right)$} \\
\hline SPECIES & 1963 & 1975 & 2002 & SPECIES & 1963 & 1975 & 2002 \\
\hline Acamptopappus shockleyi & 0.25 & 0.26 & 0.24 & Acamptopappus shockleyi & 1.72 & 2.35 & 0.45 \\
\hline Ambrosia dumosa & 0.30 & 0.34 & 0.34 & Ambrosia dumosa & 2.56 & 3.84 & 3.91 \\
\hline Ceratoides lanata & 0.32 & 0.48 & 0.44 & Ceratoides lanata & 0.23 & 0.80 & 0.59 \\
\hline Ephedra nevadensis & 0.43 & 0.47 & 0.42 & Ephedra nevadensis & 0.19 & 0.78 & 0.54 \\
\hline Grayia spinosa & 0.41 & 0.50 & 0.50 & Grayia spinosa & 1.29 & 3.06 & 0.23 \\
\hline Hymenoclea salsola & 0.33 & 0.36 & 0.37 & Hymenoclea salsola & 0.53 & 1.46 & 0.44 \\
\hline Larrea tridentata & 0.71 & 0.73 & 0.93 & Larrea tridentata & 6.62 & 9.25 & 17.86 \\
\hline Lycium andersonii & 0.43 & 0.36 & 0.43 & Lycium andersonii & 1.33 & 1.35 & 0.56 \\
\hline Oryzopsis hymenoides & 0.30 & 0.23 & 0.24 & Oryzopsis hymenoides & 0.10 & 0.37 & 0.06 \\
\hline \multirow[t]{2}{*}{ Psorothamnus fremontii } & 0.25 & 0.36 & 0.00 & Psorothamnus fremontii & 0.09 & 0.13 & 0.00 \\
\hline & & & & Total Live & 14.67 & 23.39 & 24.64 \\
\hline
\end{tabular}


Table A-24A. Site characteristics for Plot 24.

Location: Frenchman Flat

NTS Area: 5

NTS Grid: U-20

USGS 7.5’ Quadrangle Name: Cane Spring

\begin{tabular}{|c|c|c|c|c|}
\hline & & GPS Read & Zone 11S) & \\
\hline & Location & Northing & Easting & Condition of Original Corners \\
\hline Corner 1: & SW & 4079061 & 588105 & Standing \\
\hline Corner 2: & NW & 4079092 & 588114 & Standing \\
\hline Corner 3: & $\mathrm{NE}$ & 4079090 & 588143 & Standing \\
\hline Corner 4: & SE & 4079060 & 588137 & Standing \\
\hline DEM Plot & vation: 10 & & & Plot Aspect: $326^{\circ}$ \\
\hline Elevation & re Playa ( & Name): 8 & enchman) & Plot Slope: $10^{\circ}$ \\
\hline
\end{tabular}

Annual Precipitation

Measured: $141 \mathrm{~mm}$

Modeled: $139 \mathrm{~mm}$

Parent Material Type: alluvial fan

Substrate: silty alluvium, weak desert pavement

Slate Geologic Unit: Qai

Beatley Plant Assemblage: Larrea-Ambrosia/Larrea-Lycium-Grayia

Ostler-Hanson Plant Association: Larrea-Lycium-Ambrosia

Ostler-Hanson Land Unit: 139

Abundance of Biological Soil Crusts: none

Type and Date of Disturbance: none

Plot Condition: minor damage from road construction on south side of plot, 1964

Location of Transect 1: east side of plot

Direction Transects Are Read: south to north

Date(s) Plot Remeasured: 18 April 2002 
Figure A-24. Photographs showing Plot 24.

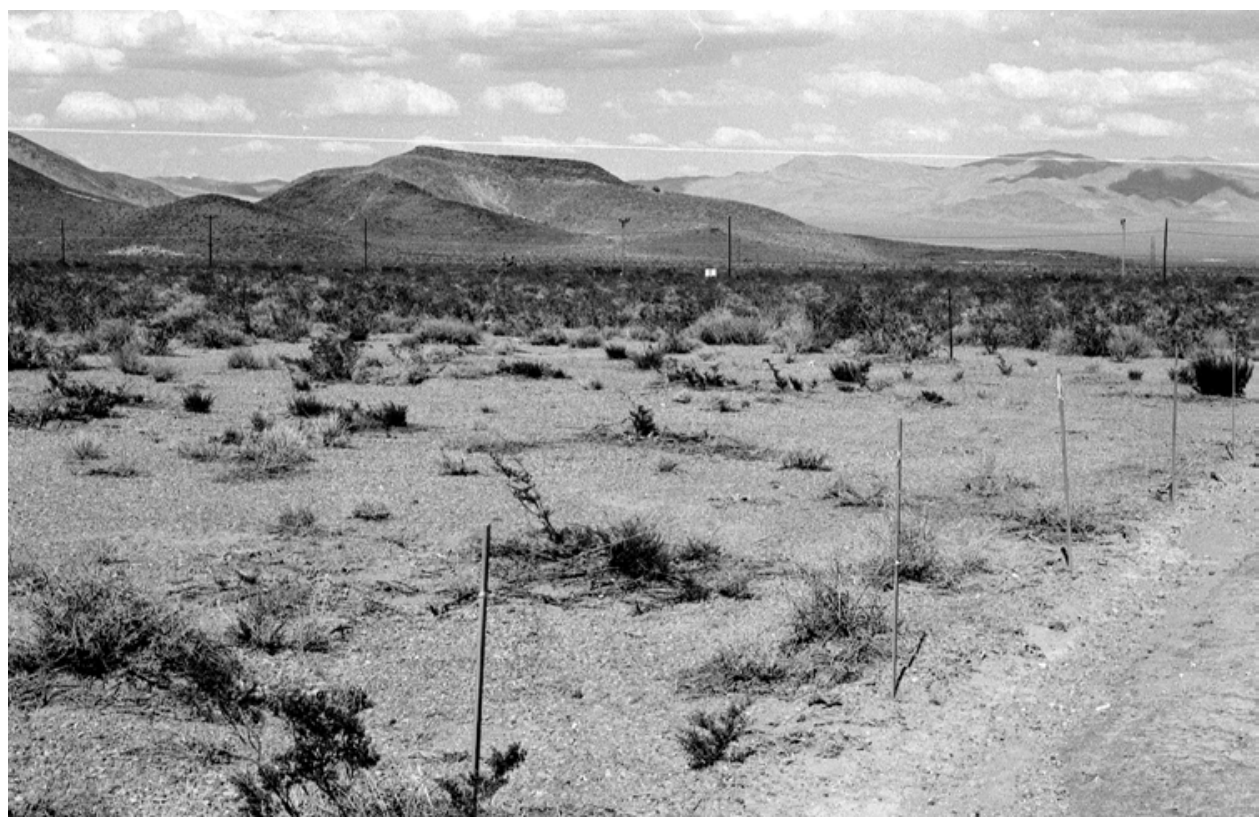

A. (1974). This northeasterly view across Plot 24 shows damage caused by construction after the plot was established. The plot is in the undisturbed vegetation in the midground. The vegetation is another mixed creosote bush scrub association. The Mercury Highway crosses the view at the line of power poles in the midground. Massachusetts Mountain appears in the left background, and the Buried Hills appear in the right background (Janice Beatley Collection, 16A).

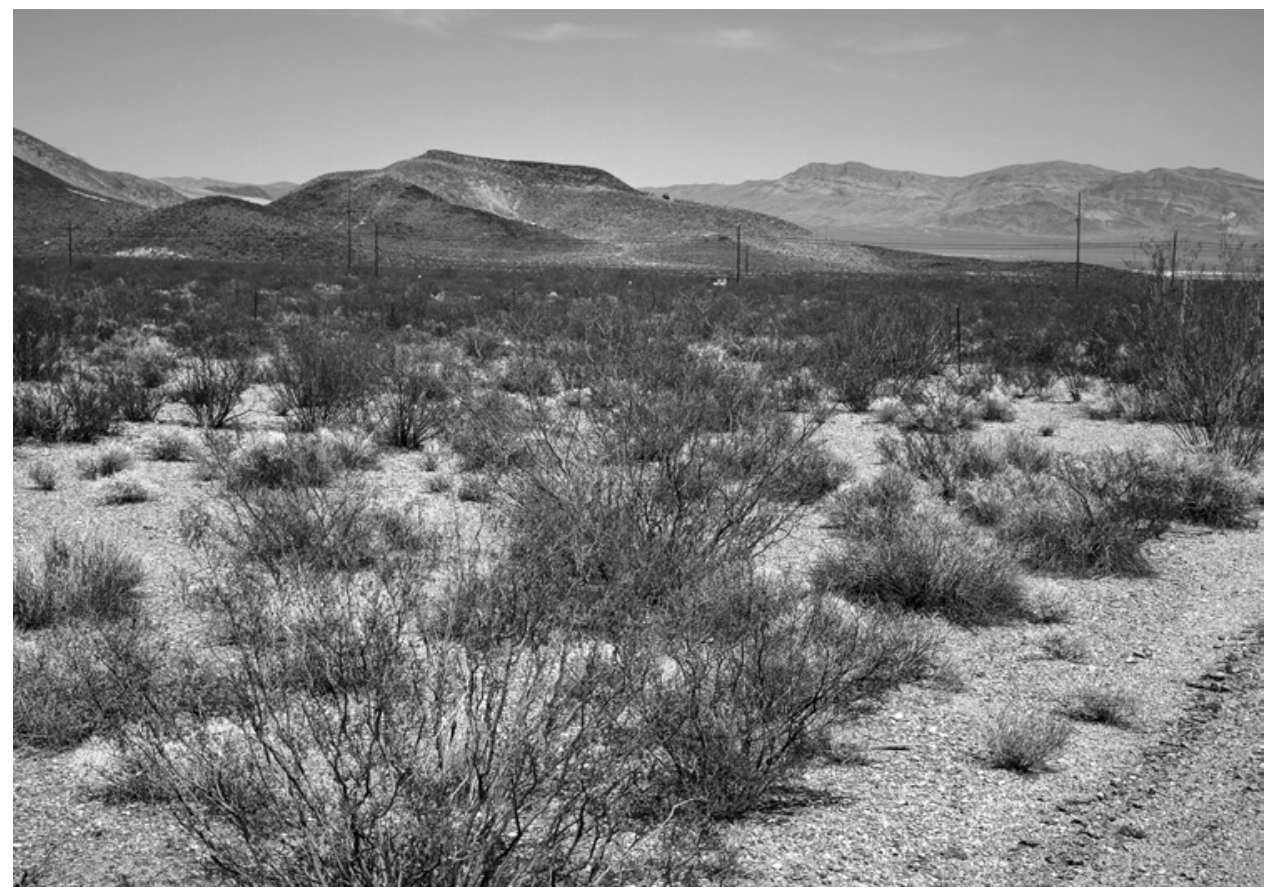

B. (April 21, 2002). The formerly disturbed vegetation has increased in biomass, in part because runoff from the newly paved road enhanced the adjacent vegetation owing to water harvesting. The road that just appears in the lower right was lightly paved, allowing runoff to move into the adjacent vegetation. Again, wolfberry and spiny hopsage decrease, and Mormon tea has increased on this plot (Dominic Oldershaw, Stake 4191B). 
Table A-24B. Summary plant data for Plot 24.

\begin{tabular}{|c|c|c|c|c|c|c|c|}
\hline \multicolumn{5}{|c|}{ Number of Plants } & \multicolumn{3}{|c|}{ Cover (\%) } \\
\hline SPECIES & 1963 & 1975 & 2002 & SPECIES & 1963 & 1975 & 2002 \\
\hline Acamptopappus shockleyi & 3 & 4 & 0 & Acamptopappus shockleyi & 0.20 & 0.29 & 0.00 \\
\hline Ambrosia dumosa & 22 & 24 & 35 & Ambrosia dumosa & 1.62 & 2.18 & 3.34 \\
\hline Ceratoides lanata & 0 & 0 & 1 & Ceratoides lanata & 0.00 & 0.00 & 0.01 \\
\hline Ephedra nevadensis & 10 & 21 & 12 & Ephedra nevadensis & 1.20 & 1.40 & 1.43 \\
\hline Eriogonum inflatum & 0 & 1 & 0 & Eriogonum inflatum & 0.00 & 0.05 & 0.00 \\
\hline Grayia spinosa & 5 & 18 & 2 & Grayia spinosa & 0.51 & 1.72 & 0.15 \\
\hline Larrea tridentata & 49 & 54 & 64 & Larrea tridentata & 9.63 & 10.11 & 12.09 \\
\hline Lycium andersonii & 11 & 17 & 11 & Lycium andersonii & 2.06 & 2.95 & 0.97 \\
\hline Total Live & 100 & 139 & 125 & Total Live & 15.22 & 18.71 & 17.98 \\
\hline Dead Grass & 0 & 0 & 0 & Dead Grass & 0.00 & 0.00 & 0.00 \\
\hline Dead Shrub & 26 & 18 & 41 & Dead Shrub & 3.83 & 1.57 & 6.14 \\
\hline Total & 126 & 157 & 166 & Total & 19.05 & 20.28 & 24.12 \\
\hline \multicolumn{5}{|c|}{ Average Height (m) } & \multicolumn{3}{|c|}{ Biomass Index $\left(\mathrm{m}^{2}\right)$} \\
\hline SPECIES & 1963 & 1975 & 2002 & SPECIES & 1963 & 1975 & 2002 \\
\hline Acamptopappus shockleyi & 0.22 & 0.27 & 0.00 & Acamptopappus shockleyi & 0.15 & 0.26 & 0.00 \\
\hline Ambrosia dumosa & 0.27 & 0.31 & 0.33 & Ambrosia dumosa & 1.71 & 2.50 & 3.98 \\
\hline Ceratoides lanata & 0.00 & 0.00 & 0.48 & Ceratoides lanata & 0.00 & 0.00 & 0.01 \\
\hline Ephedra nevadensis & 0.36 & 0.33 & 0.48 & Ephedra nevadensis & 1.52 & 1.84 & 2.32 \\
\hline Eriogonum inflatum & 0.00 & 0.05 & 0.00 & Eriogonum inflatum & 0.00 & 0.01 & 0.00 \\
\hline Grayia spinosa & 0.44 & 0.49 & 0.64 & Grayia spinosa & 0.74 & 2.99 & 0.32 \\
\hline Larrea tridentata & 0.78 & 0.77 & 0.93 & Larrea tridentata & 25.47 & 27.10 & 39.18 \\
\hline \multirow[t]{2}{*}{ Lycium andersonii } & 0.47 & 0.50 & 0.43 & Lycium andersonii & 3.37 & 5.10 & 1.48 \\
\hline & & & & Total Live & 32.97 & 39.80 & 47.30 \\
\hline
\end{tabular}


Table A-25A. Site characteristics for Plot 25.

Location: Frenchman Flat

NTS Area: 5

NTS Grid: R-19

USGS 7.5’ Quadrangle Name: Cane Spring

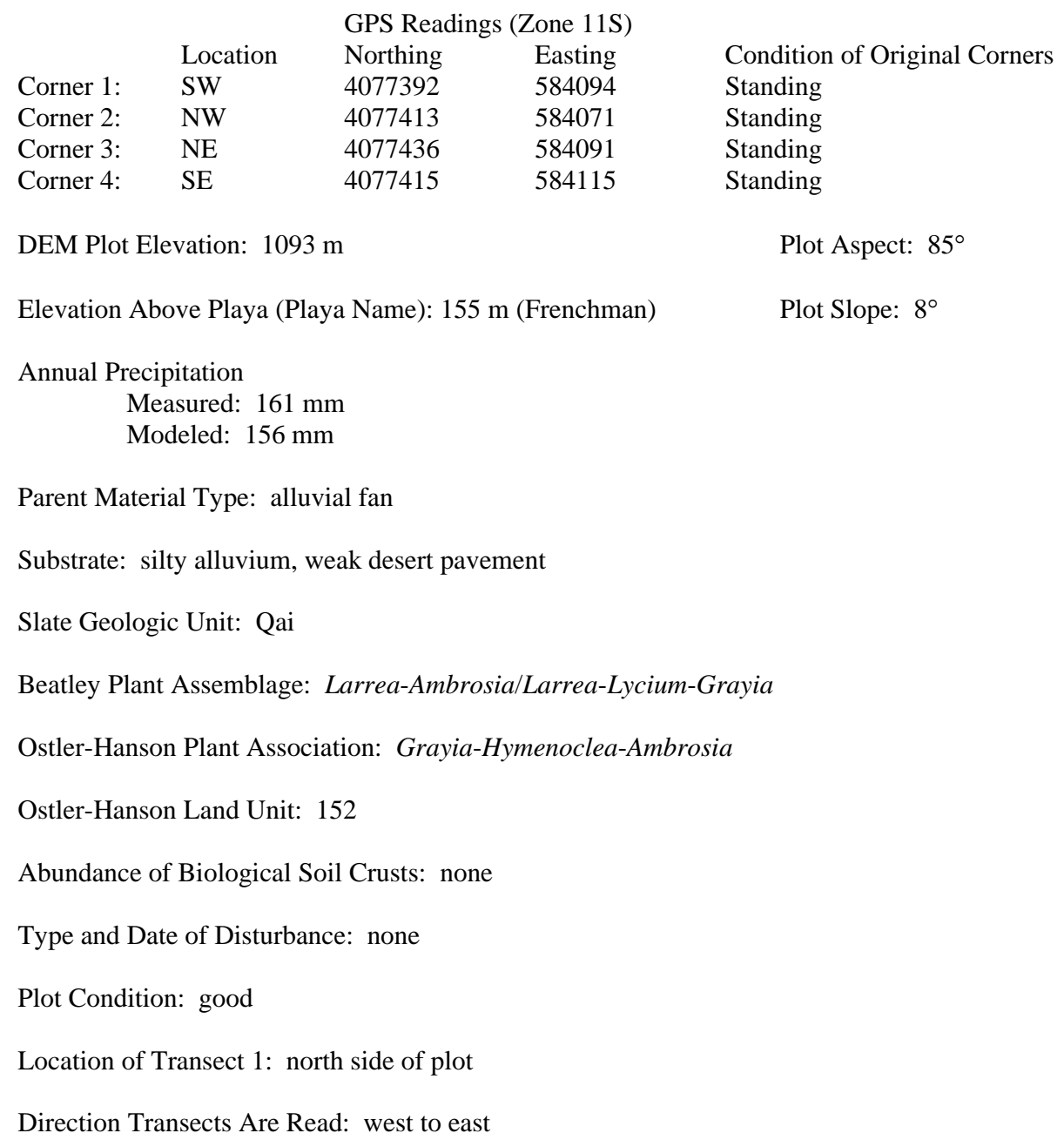

Date(s) Plot Remeasured: 20 April 2002 
Figure A-25. Photographs showing Plot 25.

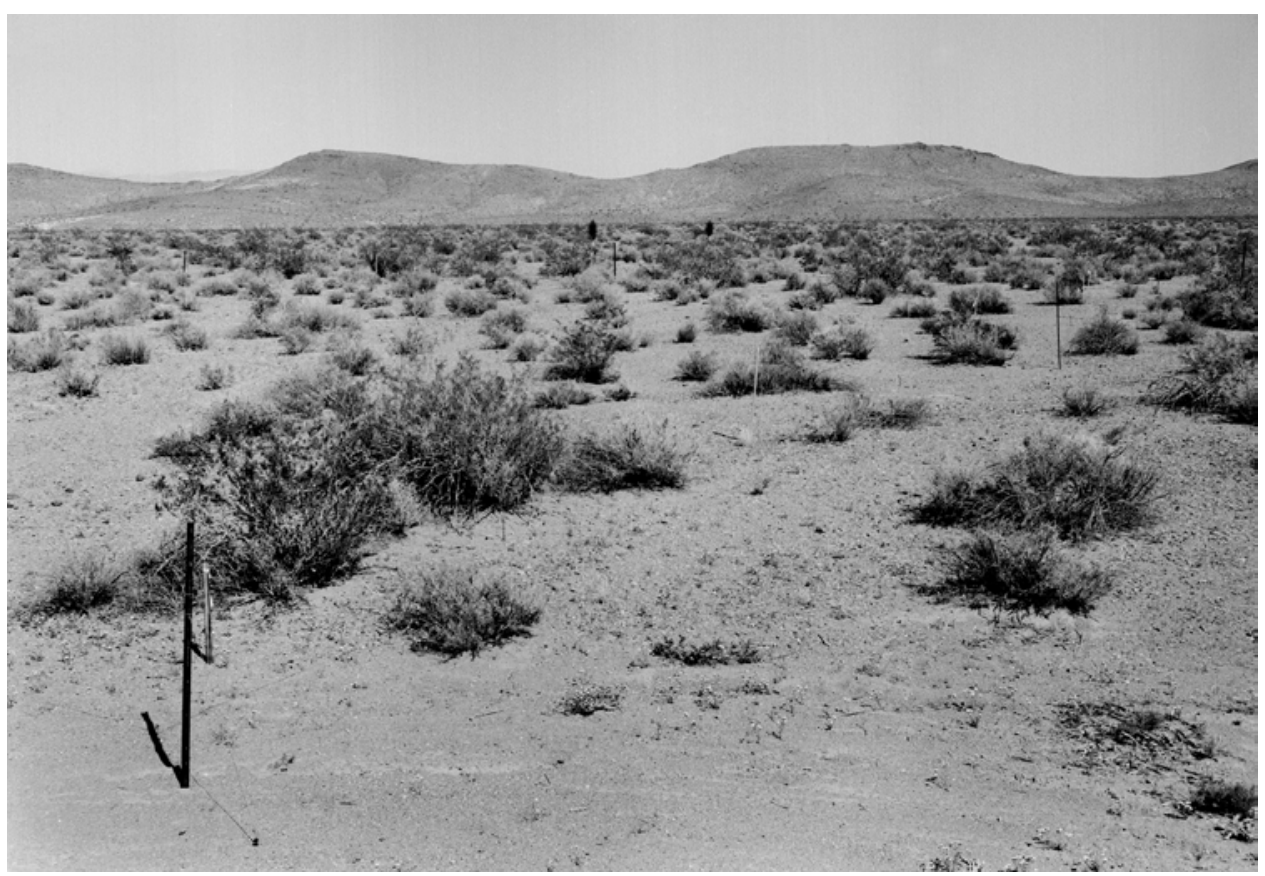

A. (May 10, 1964). This southeasterly view across Plot 25 shows scattered Joshua trees in the distance and a mixed-shrub assemblage dominated by creosote bush in the plot. The hills in the distance are part of an unnamed mountain range that has Mount Salyer as its summit (Janice Beatley Collection, no number).

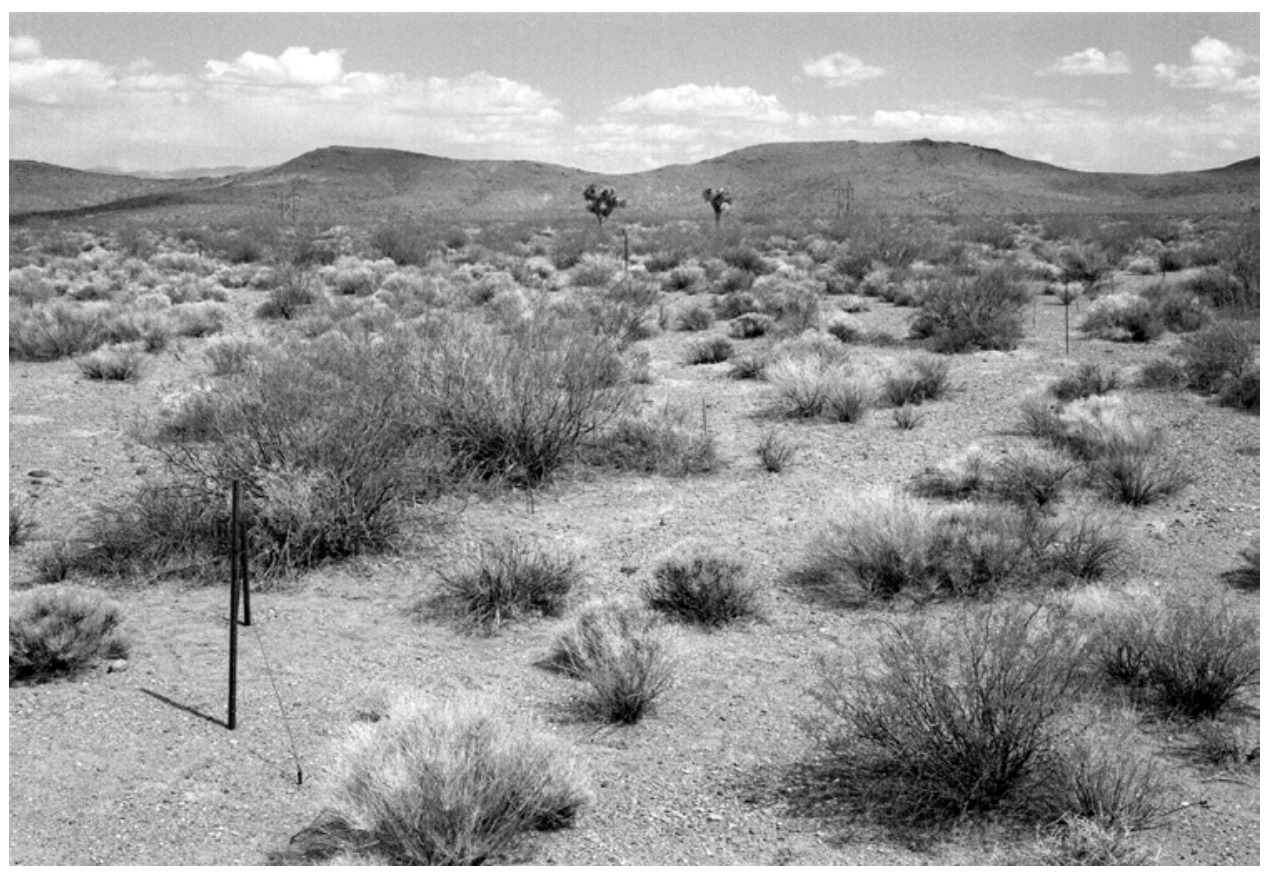

B. (April 20, 2002). There has been a considerable increase in density, cover, and biomass of perennial vegetation in the plot. White bursage has greatly increased here and is now the numerical dominant in terms of cover. The Joshua trees in the midground have increased in stature. A new powerline crosses the view in the distance (Dominic Oldershaw, Stake 4180B). 
Table A-25B. Summary plant data for Plot 25.

\begin{tabular}{|c|c|c|c|c|c|c|c|}
\hline \multicolumn{5}{|c|}{ Number of Plants } & \multicolumn{3}{|c|}{ Cover (\%) } \\
\hline SPECIES & 1963 & 1975 & 2002 & SPECIES & 1963 & 1975 & 2002 \\
\hline Acamptopappus shockleyi & 0 & 0 & 1 & Acamptopappus shockleyi & 0.00 & 0.00 & 0.06 \\
\hline Ambrosia dumosa & 22 & 41 & 62 & Ambrosia dumosa & 2.05 & 3.15 & 5.33 \\
\hline Dyssodia cooperi & 0 & 1 & 0 & Dyssodia cooperi & 0.00 & 0.04 & 0.00 \\
\hline Ephedra nevadensis & 7 & 9 & 18 & Ephedra nevadensis & 0.92 & 1.76 & 2.77 \\
\hline Ericameria cooperi & 2 & 6 & 11 & Ericameria cooperi & 0.23 & 0.63 & 0.86 \\
\hline Grayia spinosa & 27 & 39 & 16 & Grayia spinosa & 3.83 & 4.15 & 1.44 \\
\hline Hymenoclea salsola & 12 & 28 & 20 & Hymenoclea salsola & 1.45 & 3.65 & 2.34 \\
\hline Larrea tridentata & 7 & 14 & 11 & Larrea tridentata & 0.87 & 3.32 & 4.12 \\
\hline Lycium andersonii & 8 & 19 & 18 & Lycium andersonii & 1.29 & 2.27 & 1.15 \\
\hline Mirabilis pudica & 0 & 0 & 1 & Mirabilis pudica & 0.00 & 0.00 & 0.02 \\
\hline Oryzopsis hymenoides & 2 & 7 & 1 & Oryzopsis hymenoides & 0.07 & 0.43 & 0.05 \\
\hline Psorothamnus fremontii & 1 & 3 & 4 & Psorothamnus fremontii & 0.04 & 0.35 & 0.56 \\
\hline Stephanomeria parryi & 0 & 6 & 0 & Stephanomeria parryi & 0.00 & 0.06 & 0.00 \\
\hline Stipa speciosa & 0 & 0 & 4 & Stipa speciosa & 0.00 & 0.00 & 0.18 \\
\hline Yucca brevifolia & 0 & 0 & 1 & Yucca brevifolia & 0.00 & 0.00 & 0.00 \\
\hline Total Live & 88 & 173 & 168 & Total Live & 10.75 & 19.81 & 18.87 \\
\hline Dead Grass & 0 & 0 & 1 & Dead Grass & 0.00 & 0.00 & 0.02 \\
\hline Dead Shrub & 12 & 18 & 41 & Dead Shrub & 3.15 & 3.10 & 8.59 \\
\hline Total & 100 & 191 & 210 & Total & 13.89 & 22.91 & 27.48 \\
\hline \multicolumn{5}{|c|}{ Average Height (m) } & \multicolumn{3}{|c|}{ Biomass Index $\left(\mathrm{m}^{2}\right)$} \\
\hline SPECIES & 1963 & 1975 & 2002 & SPECIES & 1963 & 1975 & 2002 \\
\hline Acamptopappus shockleyi & 0.00 & 0.00 & 0.37 & Acamptopappus shockleyi & 0.00 & 0.00 & 0.08 \\
\hline Ambrosia dumosa & 0.34 & 0.34 & 0.42 & Ambrosia dumosa & 2.39 & 4.00 & 7.59 \\
\hline Dyssodia cooperi & 0.00 & 0.08 & 0.00 & Dyssodia cooperi & 0.00 & 0.01 & 0.00 \\
\hline Ephedra nevadensis & 0.53 & 0.64 & 0.59 & Ephedra nevadensis & 1.58 & 4.08 & 6.18 \\
\hline Ericameria cooperi & 0.34 & 0.41 & 0.36 & Ericameria cooperi & 0.26 & 0.93 & 1.08 \\
\hline Grayia spinosa & 0.53 & 0.58 & 0.70 & Grayia spinosa & 7.12 & 8.38 & 3.51 \\
\hline Hymenoclea salsola & 0.41 & 0.53 & 0.60 & Hymenoclea salsola & 2.11 & 6.80 & 5.07 \\
\hline Larrea tridentata & 1.18 & 1.23 & 1.57 & Larrea tridentata & 3.11 & 12.93 & 17.96 \\
\hline Lycium andersonii & 0.47 & 0.50 & 0.46 & Lycium andersonii & 2.20 & 3.95 & 1.76 \\
\hline Mirabilis pudica & 0.00 & 0.00 & 0.15 & Mirabilis pudica & 0.00 & 0.00 & 0.01 \\
\hline Oryzopsis hymenoides & 0.20 & 0.31 & 0.19 & Oryzopsis hymenoides & 0.05 & 0.49 & 0.03 \\
\hline Psorothamnus fremontii & 0.15 & 0.30 & 0.41 & Psorothamnus fremontii & 0.02 & 0.43 & 0.88 \\
\hline Stephanomeria parryi & 0.00 & 0.03 & 0.00 & Stephanomeria parryi & 0.00 & 0.01 & 0.00 \\
\hline Stipa speciosa & 0.00 & 0.00 & 0.43 & Stipa speciosa & 0.00 & 0.00 & 0.27 \\
\hline Yucca brevifolia & 0.00 & 0.00 & 0.98 & Yucca brevifolia & 0.00 & 0.00 & 0.00 \\
\hline & & & & Total Live & 18.84 & 42.00 & 44.42 \\
\hline
\end{tabular}


Table A-26A. Site characteristics for Plot 26.

Location:

NTS Area: 5

NTS Grid: O-17

USGS 7.5’ Quadrangle Name: Cane Spring

\begin{tabular}{lllll} 
& & \multicolumn{2}{l}{ GPS Readings (Zone 11S) } & \\
& Location & Northing & Easting & Condition of Original Corners \\
Corner 1: & SW & 4074175 & 579802 & Leaning \\
Corner 2: & NW & 4074208 & 579810 & Standing \\
Corner 3: & NE & 4074196 & 579842 & Missing \\
Corner 4: & SE & 4074169 & 579832 & Standing
\end{tabular}

DEM Plot Elevation: 1212 m

Plot Aspect: $219^{\circ}$

Elevation Above Playa (Playa Name): 271 m (Frenchman)

Plot Slope: $10^{\circ}$

Annual Precipitation

Measured: $185 \mathrm{~mm}$

Modeled: $180 \mathrm{~mm}$

Parent Material Type: alluvial fan

Substrate: sandy, silty alluvium

Slate Geologic Unit: Qai

Beatley Plant Assemblage: Grayia-Lycium

Ostler-Hanson Plant Association: Hymenoclea-Ephedra-Stipa

Ostler-Hanson Land Unit: 157

Abundance of Biological Soil Crusts: none

Type and Date of Disturbance: none

Plot Condition: good

Location of Transect 1: west side of plot

Direction Transects Are Read: south to north

Date(s) Plot Remeasured: 19 April 2002 
Figure A-26. Photographs showing Plot 26.

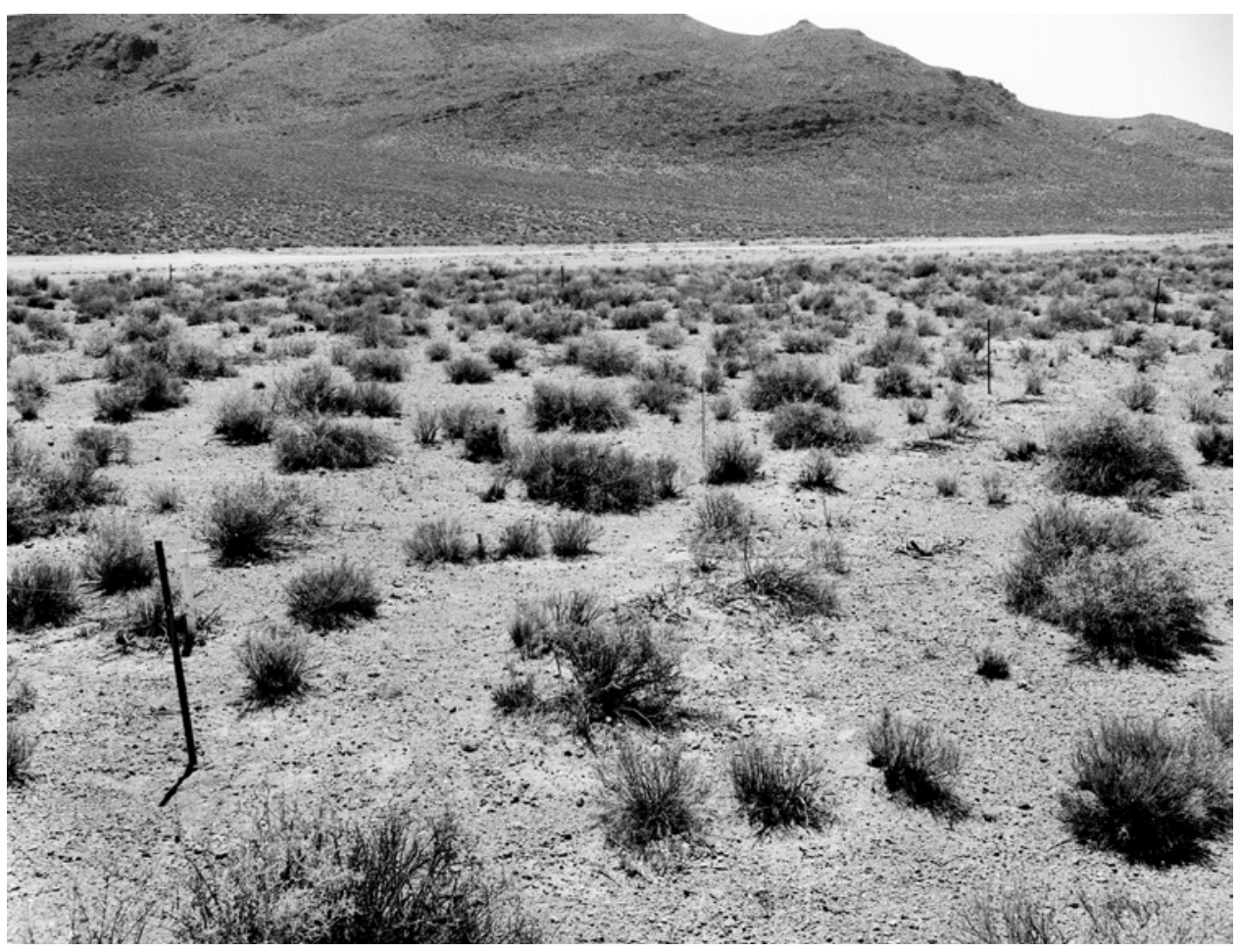

A. (May 10, 1964). The view is west-southwest across Plot 26 across Wahmonie Flat towards the eastern edge of Skull Mountain. The powerlines mark the Cane Springs Road, which crosses the view from east to west. The vegetation assemblage is spiny hopsage and wolfberry (Janice Beatley Collection, 88-B).

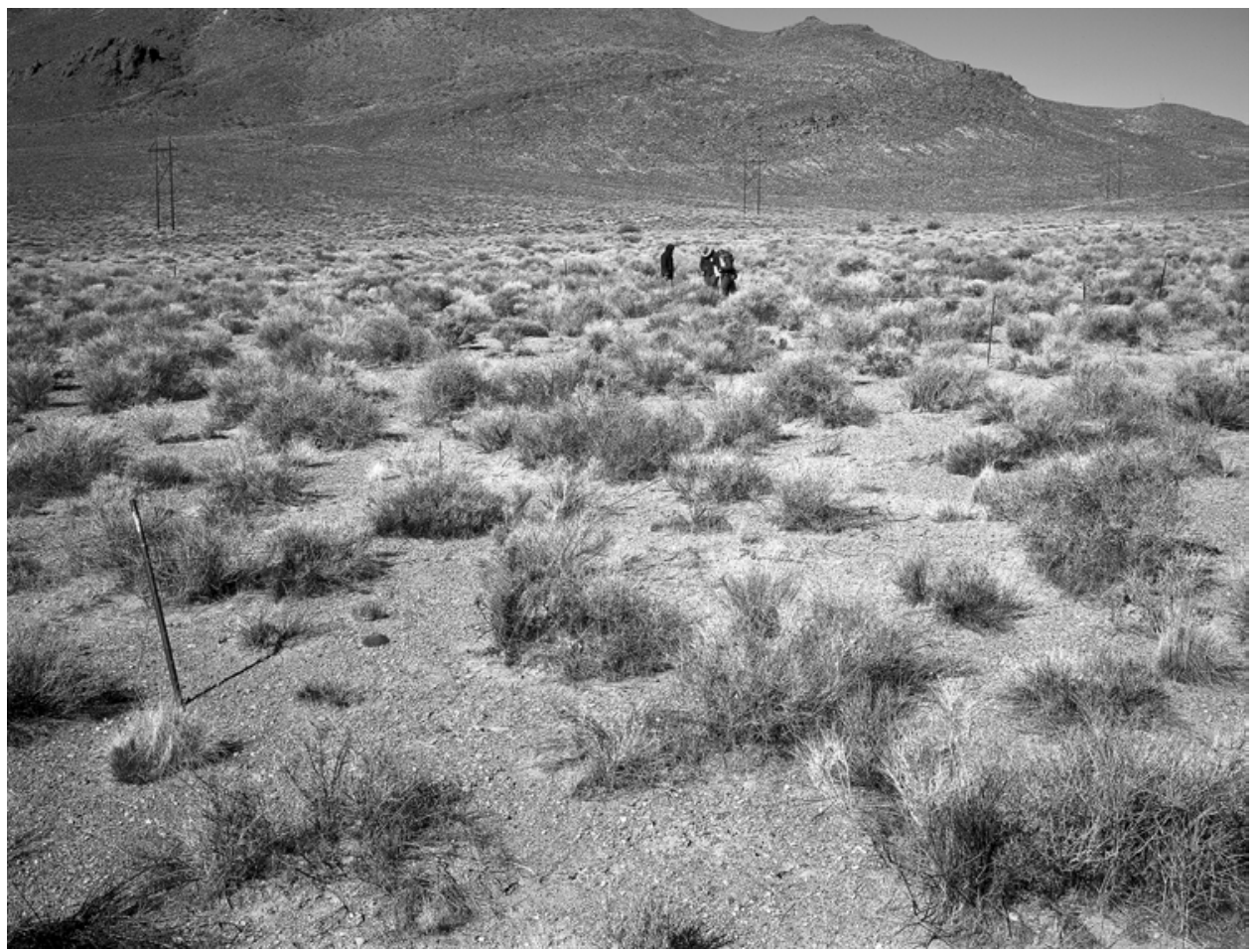

B. (April 19, 2002). Many individuals persist on this plot and are now larger. The dominants on this plot have shifted to Mormon tea, cheesebush (Hymenoclea salsola), and wolfberry (Dominic Oldershaw, Stake 4161B). 
Table A-26B. Summary plant data for Plot 26.

\begin{tabular}{|c|c|c|c|}
\hline SPECIES & 1963 & 1975 & 2002 \\
\hline Arabis glaucovalvula & 0 & 1 & 1 \\
\hline Atriplex canescens & 0 & 1 & 4 \\
\hline Coleogyne ramosissima & 0 & 1 & 3 \\
\hline Dyssodia cooperi & 0 & 0 & 3 \\
\hline Ephedra nevadensis & 32 & 56 & 84 \\
\hline Grayia spinosa & 44 & 32 & 7 \\
\hline Hymenoclea salsola & 40 & 82 & 52 \\
\hline Krameria parvifolia & 0 & 0 & 1 \\
\hline Lycium andersonii & 9 & 16 & 15 \\
\hline Mirabilis pudica & 3 & 1 & 0 \\
\hline Oryzopsis hymenoides & 9 & 21 & 5 \\
\hline Psorothamnus fremontii & 0 & 6 & 7 \\
\hline Sitanion hystrix & 1 & 2 & 2 \\
\hline Stephanomeria parryi & 0 & 23 & 0 \\
\hline Stipa speciosa & 3 & 75 & 12 \\
\hline Tetradymia axillaris & 0 & 0 & 2 \\
\hline Thamnosma montana & 17 & 32 & 39 \\
\hline Xylorhiza tortifolia & 0 & 0 & 2 \\
\hline Total Live & 158 & 349 & 239 \\
\hline Dead Grass & 0 & 0 & 11 \\
\hline Dead Shrub & 22 & 20 & 43 \\
\hline Total & 180 & 369 & 293 \\
\hline & \multicolumn{3}{|c|}{ Average Height (m) } \\
\hline SPECIES & 1963 & 1975 & 2002 \\
\hline Arabis glaucovalvula & 0.00 & 0.28 & 0.32 \\
\hline Atriplex canescens & 0.00 & 0.97 & 0.80 \\
\hline Coleogyne ramosissima & 0.00 & 0.51 & 0.55 \\
\hline Dyssodia cooperi & 0.00 & 0.00 & 0.26 \\
\hline Ephedra nevadensis & 0.45 & 0.56 & 0.62 \\
\hline Grayia spinosa & 0.59 & 0.56 & 0.61 \\
\hline Hymenoclea salsola & 0.46 & 0.49 & 0.57 \\
\hline Krameria parvifolia & 0.00 & 0.00 & 0.32 \\
\hline Lycium andersonii & 0.56 & 0.51 & 0.48 \\
\hline Mirabilis pudica & 0.23 & 0.05 & 0.00 \\
\hline Oryzopsis hymenoides & 0.24 & 0.33 & 0.30 \\
\hline Psorothamnus fremontii & 0.00 & 0.28 & 0.50 \\
\hline Sitanion hystrix & 0.28 & 0.38 & 0.29 \\
\hline Stephanomeria parryi & 0.00 & 0.04 & 0.00 \\
\hline Stipa speciosa & 0.27 & 0.43 & 0.45 \\
\hline Tetradymia axillaris & 0.00 & 0.00 & 0.72 \\
\hline Thamnosma montana & 0.41 & 0.44 & 0.41 \\
\hline Xylorhiza tortifolia & 0.00 & 0.00 & 0.41 \\
\hline
\end{tabular}

SPECIES

Arabis glaucovalvula

Atriplex canescens

Coleogyne ramosissima

Dyssodia cooperi

Ephedra nevadensis

Grayia spinosa

Hymenoclea salsola

Krameria parvifolia

Lycium andersonii

Mirabilis pudica

Oryzopsis hymenoides

Psorothamnus fremontii

Sitanion hystrix

Stephanomeria parryi

Stipa speciosa

Tetradymia axillaris

Thamnosma montana

Xylorhiza tortifolia

Total Live

Dead Grass

Dead Shrub

Total

\section{SPECIES}

Arabis glaucovalvula

Atriplex canescens

Coleogyne ramosissima

Dyssodia cooperi

Ephedra nevadensis

Grayia spinosa

Hymenoclea salsola

Krameria parvifolia

Lycium andersonii

Mirabilis pudica

Oryzopsis hymenoides

Psorothamnus fremontii

Sitanion hystrix

Stephanomeria parryi

Stipa speciosa

Tetradymia axillaris

Thamnosma montana

Xylorhiza tortifolia

Total Live
Cover (\%)

1975

2002

1963

0.03

0.01

0.00

0.00

0.00

3.05

8.10

3.99

0.00

1.58

0.19

0.49

0.00

0.08

0.00

0.20

0.00

2.31

0.00

19.99

0.00

2.74

22.73

0.20

0.05

0.00

9.61

4.44

10.84

0.00

1.83

0.02

2.04

0.41

0.15

0.34

7.02

0.00

4.75

0.29

0.44

0.24

12.95

0.50

6.20

0.23

1.36

0.00

0.17

0.86

0.05

0.00

0.73

0.00

4.38

0.00

0.18

41.70

28.60

0.00

0.30

3.03

6.65

44.73

35.55

\begin{tabular}{ccc}
\multicolumn{3}{c}{ Biomass Index $\left(\mathbf{m}^{2}\right)$} \\
$\mathbf{1 9 6 3}$ & $\mathbf{1 9 7 5}$ & $\mathbf{2 0 0 2}$ \\
0.00 & 0.03 & 0.01 \\
0.00 & 0.65 & 0.70 \\
0.00 & 0.08 & 0.89 \\
0.00 & 0.00 & 0.21 \\
4.95 & 19.66 & 30.04 \\
16.78 & 8.71 & 1.03 \\
6.20 & 19.53 & 12.22 \\
0.00 & 0.00 & 0.24 \\
3.04 & 3.15 & 2.07 \\
0.17 & 0.00 & 0.00 \\
0.43 & 2.31 & 0.15 \\
0.00 & 0.61 & 1.57 \\
0.08 & 0.20 & 0.06 \\
0.00 & 0.04 & 0.00 \\
0.24 & 10.59 & 1.15 \\
0.00 & 0.00 & 0.00 \\
3.30 & 7.21 & 6.28 \\
0.00 & 0.00 & 0.26 \\
35.18 & 72.75 & 56.88
\end{tabular}


Table A-27A. Site characteristics for Plot 27.

Location: Frenchman Flat

NTS Area: 6

NTS Grid: R-22

USGS 7.5’ Quadrangle Name: Yucca Lake

\begin{tabular}{|c|c|c|c|c|}
\hline & & GPS Reac & Zone 11S) & \\
\hline & Location & Northing & Easting & Condition of Original Corners \\
\hline Corner 1: & SW & 4083280 & 584745 & Standing \\
\hline Corner 2: & NW & 4083310 & 584749 & Standing \\
\hline Corner 3: & $\mathrm{NE}$ & 4083308 & 584780 & Standing \\
\hline Corner 4: & SE & 4083276 & 584775 & Standing \\
\hline DEM Plo & vation: 11 & & & Plot Aspect: $0^{\circ}$ \\
\hline Elevation & e Playa ( & Name): 1 & (Frenchman) & Plot Slope: $3^{\circ}$ \\
\hline
\end{tabular}

Annual Precipitation

Measured: $154 \mathrm{~mm}$

Modeled: $152 \mathrm{~mm}$

Parent Material Type: alluvial fan

Substrate: mixed mostly volcanic alluvium w/some limestone

Slate Geologic Unit: Qay

Beatley Plant Assemblage: Larrea-Lycium-Grayia

Ostler-Hanson Plant Association: Larrea-Krameria-Grayia

Ostler-Hanson Land Unit: 126

Abundance of Biological Soil Crusts: none

Type and Date of Disturbance: none

Plot Condition: vehicle drove through center of plot, second set of tracks noticed 11 Dec 01

Location of Transect 1: east side of plot

Direction Transects Are Read: north to south

Date(s) Plot Remeasured: 20 April 2002 
Figure A-27. Photographs showing Plot 27.



A. (May 10, 1964). This northwesterly view across Plot 27 shows a creosote bush - wolfberry - spiny hopsage assemblage in the northern part of Frenchman Flat. Scattered Joshua trees appear in the midground and distance. A low range of unnamed mountains appears in the distance (Janice Beatley Collection, no number).

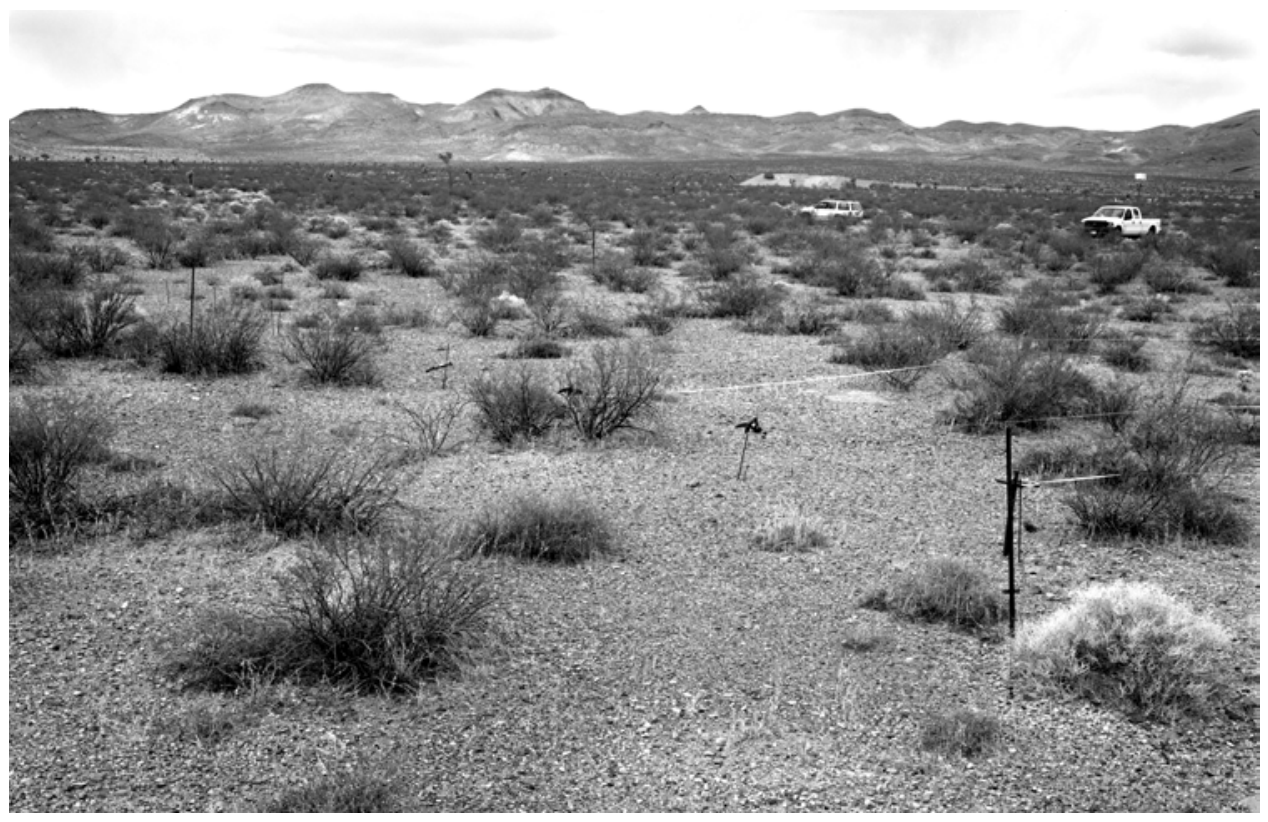

B. (April 20, 2002). Many individual creosote bushes are much larger in 2002. Both wolfberry and spiny hopsage have decreased here, and now range ratany and white bursage are the subdominants. The Joshua trees in the distance are no longer as apparent as they once were (Dominic Oldershaw, Stake 4177A). 
Table A-27B. Summary plant data for Plot 27.

\begin{tabular}{lccc} 
& \multicolumn{3}{c}{ Number of Plants } \\
SPECIES & $\mathbf{1 9 6 3}$ & $\mathbf{1 9 7 5}$ & $\mathbf{2 0 0 2}$ \\
Ambrosia dumosa & 2 & 7 & 9 \\
Erioneuron pulchellum & 0 & 1 & 1 \\
Grayia spinosa & 3 & 21 & 0 \\
Krameria parvifolia & 16 & 17 & 28 \\
Larrea tridentata & 44 & 46 & 43 \\
Lycium andersonii & 19 & 11 & 8 \\
Opuntia echinocarpa & 0 & 0 & 1 \\
Oryzopsis hymenoides & 0 & 0 & 1 \\
Total Live & 84 & 103 & 91 \\
Dead Grass & 0 & 0 & 0 \\
Dead Shrub & 10 & 11 & 30 \\
Total & 94 & 114 & 121 \\
& & & \\
SPECIES & & Average Height (m) \\
Ambrosia dumosa & $\mathbf{1 9 6 3}$ & $\mathbf{1 9 7 5}$ & $\mathbf{2 0 0 2}$ \\
Erioneuron pulchellum & 0.25 & 0.30 & 0.38 \\
Grayia spinosa & 0.00 & 0.03 & 0.00 \\
Krameria parvifolia & 0.47 & 0.55 & 0.00 \\
Larrea tridentata & 0.22 & 0.24 & 0.25 \\
Lycium andersonii & 0.44 & 0.80 & 0.90 \\
Opuntia echinocarpa & 0.00 & 0.44 & 0.35 \\
Oryzopsis hymenoides & 0.00 & 0.00 & 0.51 \\
& & 0.00 & 0.31 \\
& & &
\end{tabular}

SPECIES
Ambrosia dumosa
Erioneuron pulchellum
Grayia spinosa
Krameria parvifolia
Larrea tridentata
Lycium andersonii
Opuntia echinocarpa
Oryzopsis hymenoides
Total Live
Dead Grass
Dead Shrub
Total

$\begin{array}{ccc} & \text { Cover (\%) } & \\ \mathbf{1 9 6 3} & \mathbf{1 9 7 5} & \mathbf{2 0 0 2} \\ 0.14 & 0.65 & 0.92 \\ 0.00 & 0.01 & 0.01 \\ 0.27 & 2.28 & 0.00 \\ 2.18 & 3.05 & 4.30 \\ 7.96 & 8.94 & 7.55 \\ 3.67 & 2.11 & 0.38 \\ 0.00 & 0.00 & 0.06 \\ 0.00 & 0.00 & 0.05 \\ 14.23 & 17.03 & 13.28 \\ 0.00 & 0.00 & 0.00 \\ 2.55 & 1.92 & 5.55 \\ 16.78 & 18.95 & 18.84\end{array}$

$\begin{array}{lccc}\text { SPECIES } & \mathbf{1 9 6 3} & \mathbf{1 9 7 5} & \mathbf{2 0 0 2} \\ \text { Ambrosia dumosa } & 0.15 & 0.68 & 1.16 \\ \text { Erioneuron pulchellum } & 0.00 & 0.00 & 0.00 \\ \text { Grayia spinosa } & 0.44 & 4.38 & 0.00 \\ \text { Krameria parvifolia } & 1.59 & 2.55 & 4.22 \\ \text { Larrea tridentata } & 24.89 & 25.66 & 21.84 \\ \text { Lycium andersonii } & 5.60 & 3.20 & 0.41 \\ \text { Opuntia echinocarpa } & 0.00 & 0.00 & 0.11 \\ \text { Oryzopsis hymenoides } & 0.00 & 0.00 & 0.06 \\ \text { Total Live } & 32.67 & 36.47 & 27.80\end{array}$


Table A-28A. Site characteristics for Plot 28.

Location: Frenchman Flat

NTS Area: 5

NTS Grid: Z-19

USGS 7.5’ Quadrangle Name: Frenchman Lake

\begin{tabular}{|c|c|c|c|c|}
\hline & & GPS Reac & Zone 11S) & \\
\hline & Location & Northing & Easting & Condition of Original Corners \\
\hline Corner 1: & SW & 4079198 & 596258 & Standing \\
\hline Corner 2: & NW & 4079228 & 596267 & Standing \\
\hline Corner 3: & $\mathrm{NE}$ & 4079223 & 596296 & Standing \\
\hline Corner 4: & SE & 4079194 & 596289 & Standing \\
\hline DEM Plo & evation: 98 & & & Plot Aspect: $345^{\circ}$ \\
\hline Elevation & e Playa & Name): & Frenchman) & Plot Slope: $8^{\circ}$ \\
\hline
\end{tabular}

Annual Precipitation

Measured: $121 \mathrm{~mm}$

Modeled: $131 \mathrm{~mm}$

Parent Material Type: alluvial fan

Substrate: sandy alluvium w/pebbles, volcanics

Slate Geologic Unit: Qay

Beatley Plant Assemblage: Larrea-Ambrosia

Ostler-Hanson Plant Association: Larrea-Acamptopappus-Ambrosia

Ostler-Hanson Land Unit: NA

Abundance of Biological Soil Crusts: none

Type and Date of Disturbance: none

Plot Condition: good

Location of Transect 1: south side of plot

Direction Transects Are Read: east to west

Date(s) Plot Remeasured: 18 April 2002 
Figure A-28. Photographs showing Plot 28.

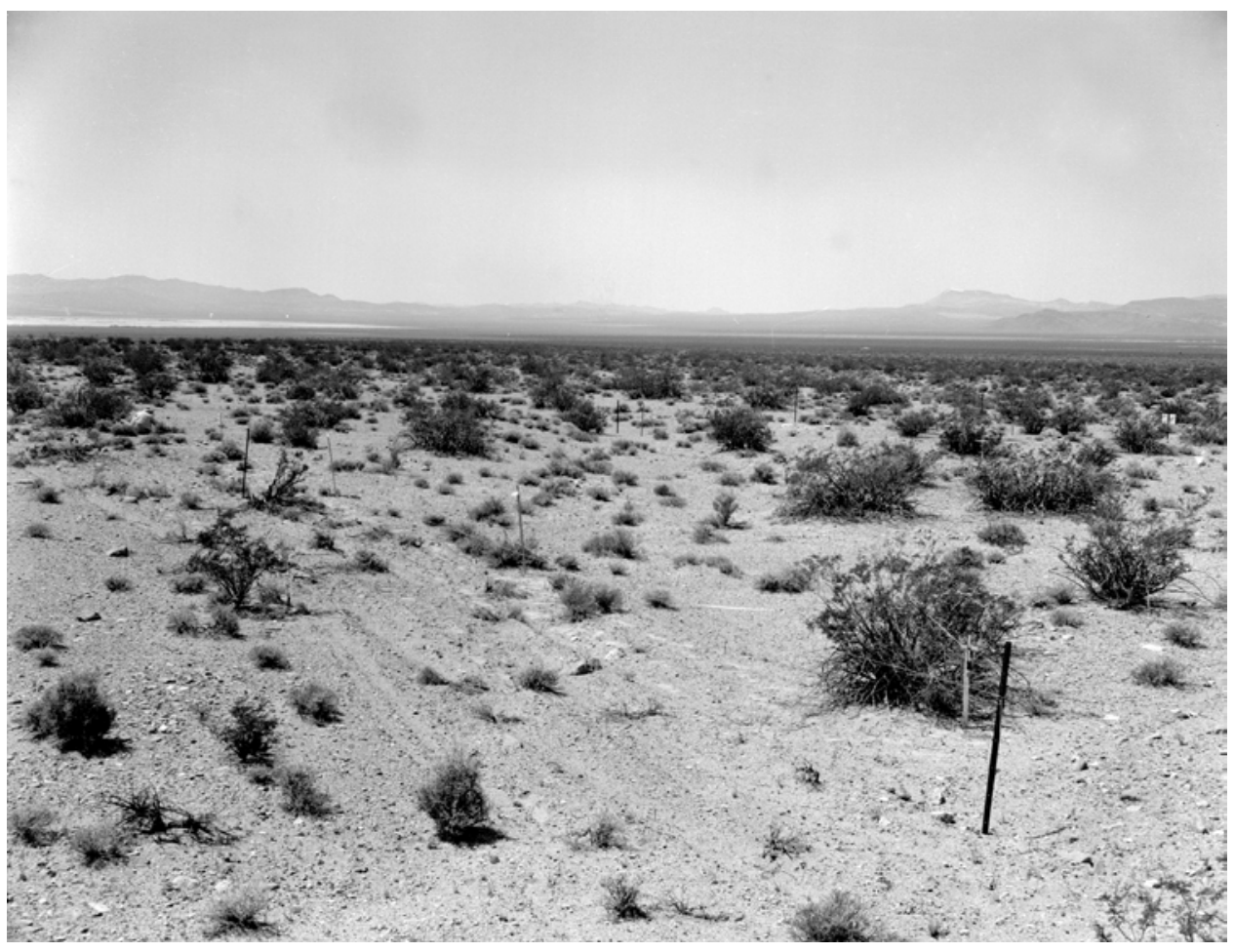

A. (April 22, 1964). In this south-southwesterly view across Plot 28 and the northern part of Frenchman Flat, a cresote bush - white bursage assemblage is shown. Frenchman Lake appears in the distance, with (from right to left) the Specter Range, Red Mountain, and the Ranger Mountains in the distance (Janice Beatley Collection, 32-A).

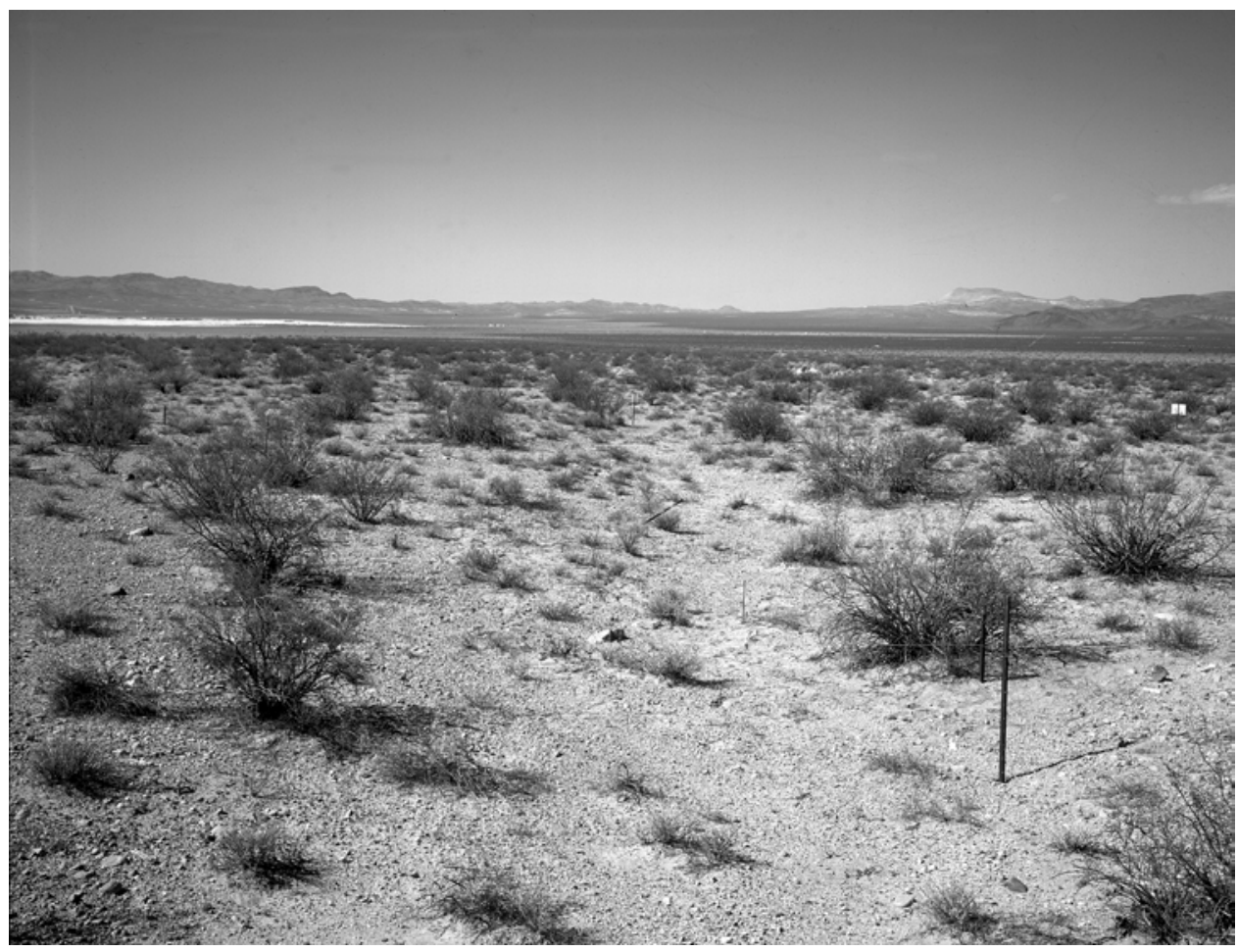

B. (April 18, 2002). This is one of the few plots on the Nevada Test Site within the range of creosote bush that has not changed dramatically from a visual perspective, although a careful comparison reveals that the creosote bush are taller and the total cover on the plot is larger (Dominic Oldershaw, Stake 4153A). 
Table A-28B. Summary plant data for Plot 28.

\begin{tabular}{|c|c|c|c|c|c|c|c|}
\hline \multicolumn{5}{|c|}{ Number of Plants } & \multicolumn{3}{|c|}{ Cover (\%) } \\
\hline SPECIES & 1963 & 1975 & 2002 & SPECIES & 1963 & 1975 & 2002 \\
\hline Acamptopappus shockleyi & 37 & 76 & 1 & Acamptopappus shockleyi & 2.08 & 4.62 & 0.03 \\
\hline Ambrosia dumosa & 43 & 35 & 26 & Ambrosia dumosa & 4.08 & 2.76 & 1.28 \\
\hline Atriplex canescens & 4 & 6 & 9 & Atriplex canescens & 0.29 & 0.74 & 0.93 \\
\hline Atriplex confertifolia & 3 & 1 & 3 & Atriplex confertifolia & 0.43 & 0.11 & 0.19 \\
\hline Ceratoides lanata & 1 & 1 & 1 & Ceratoides lanata & 0.04 & 0.03 & 0.00 \\
\hline Ephedra nevadensis & 1 & 1 & 0 & Ephedra nevadensis & 0.17 & 0.06 & 0.00 \\
\hline Grayia spinosa & 9 & 16 & 1 & Grayia spinosa & 1.04 & 1.75 & 0.12 \\
\hline Krameria parvifolia & 2 & 3 & 6 & Krameria parvifolia & 0.12 & 0.21 & 0.40 \\
\hline Larrea tridentata & 23 & 24 & 23 & Larrea tridentata & 5.92 & 7.15 & 5.69 \\
\hline Mirabilis pudica & 5 & 6 & 0 & Mirabilis pudica & 0.32 & 0.48 & 0.00 \\
\hline Oryzopsis hymenoides & 6 & 2 & 0 & Oryzopsis hymenoides & 0.20 & 0.04 & 0.00 \\
\hline Total Live & 134 & 171 & 70 & Total Live & 14.68 & 17.95 & 8.64 \\
\hline Dead Grass & 0 & 0 & 0 & Dead Grass & 0.00 & 0.00 & 0.00 \\
\hline Dead Shrub & 19 & 20 & 133 & Dead Shrub & 2.74 & 2.04 & 11.54 \\
\hline Total & 153 & 191 & 203 & Total & 17.42 & 19.98 & 20.17 \\
\hline \multicolumn{5}{|c|}{ Average Height (m) } & \multicolumn{3}{|c|}{ Biomass Index $\left(\mathrm{m}^{2}\right)$} \\
\hline SPECIES & 1963 & 1975 & 2002 & SPECIES & 1963 & 1975 & 2002 \\
\hline Acamptopappus shockleyi & 0.17 & 0.18 & 0.23 & Acamptopappus shockleyi & 1.62 & 3.02 & 0.02 \\
\hline Ambrosia dumosa & 0.25 & 0.25 & 0.26 & Ambrosia dumosa & 3.57 & 2.52 & 1.22 \\
\hline Atriplex canescens & 0.41 & 0.50 & 0.53 & Atriplex canescens & 0.47 & 1.32 & 1.81 \\
\hline Atriplex confertifolia & 0.35 & 0.43 & 0.32 & Atriplex confertifolia & 0.49 & 0.16 & 0.20 \\
\hline Ceratoides lanata & 0.23 & 0.30 & 0.44 & Ceratoides lanata & 0.03 & 0.03 & 0.00 \\
\hline Ephedra nevadensis & 0.25 & 0.18 & 0.00 & Ephedra nevadensis & 0.15 & 0.04 & 0.00 \\
\hline Grayia spinosa & 0.50 & 0.54 & 0.42 & Grayia spinosa & 1.81 & 3.24 & 0.17 \\
\hline Krameria parvifolia & 0.19 & 0.25 & 0.21 & Krameria parvifolia & 0.08 & 0.17 & 0.39 \\
\hline Larrea tridentata & 1.06 & 1.11 & 1.12 & Larrea tridentata & 20.95 & 26.72 & 22.11 \\
\hline Mirabilis pudica & 0.27 & 0.08 & 0.00 & Mirabilis pudica & 0.33 & 0.14 & 0.00 \\
\hline \multirow[t]{2}{*}{ Oryzopsis hymenoides } & 0.18 & 0.11 & 0.00 & Oryzopsis hymenoides & 0.13 & 0.01 & 0.00 \\
\hline & & & & Total Live & 29.61 & 37.40 & 25.93 \\
\hline
\end{tabular}


Table A-29A. Site characteristics for Plot 29.

Location: Frenchman Flat

NTS Area: 5

NTS Grid: Z-19

USGS 7.5’ Quadrangle Name: Frenchman Lake

\begin{tabular}{|c|c|c|c|c|}
\hline & & GPS Reac & Zone 11S) & \\
\hline & Location & Northing & Easting & Condition of Original Corners \\
\hline Corner 1: & SW & 4079369 & 595913 & NA \\
\hline Corner 2: & NW & 4079399 & 595922 & NA \\
\hline Corner 3: & $\mathrm{NE}$ & 4079392 & 595950 & NA \\
\hline Corner 4: & SE & 4079361 & 595941 & NA \\
\hline DEM Plot & vation: 99 & & & Plot Aspect: $135^{\circ}$ \\
\hline Elevation & e Playa ( & Name): 4 & renchman) & Plot Slope: $13^{\circ}$ \\
\hline
\end{tabular}

Annual Precipitation

Measured: $126 \mathrm{~mm}$

Modeled: $131 \mathrm{~mm}$

Parent Material Type: alluvial fan

Substrate: silty sandy w/jagged pebble/cobble surface

Slate Geologic Unit: Qai

Beatley Plant Assemblage: Larrea-Atriplex

Ostler-Hanson Plant Association: Ephedra-Krameria-Atriplex confertifolia

Ostler-Hanson Land Unit: NA

Abundance of Biological Soil Crusts: NA

Type and Date of Disturbance: NA

Plot Condition: good

Location of Transect 1: north side of plot

Direction Transects Are Read: east to west

Date(s) Plot Remeasured: 18 April 2002 
Figure A-29. Photographs showing Plot 29.

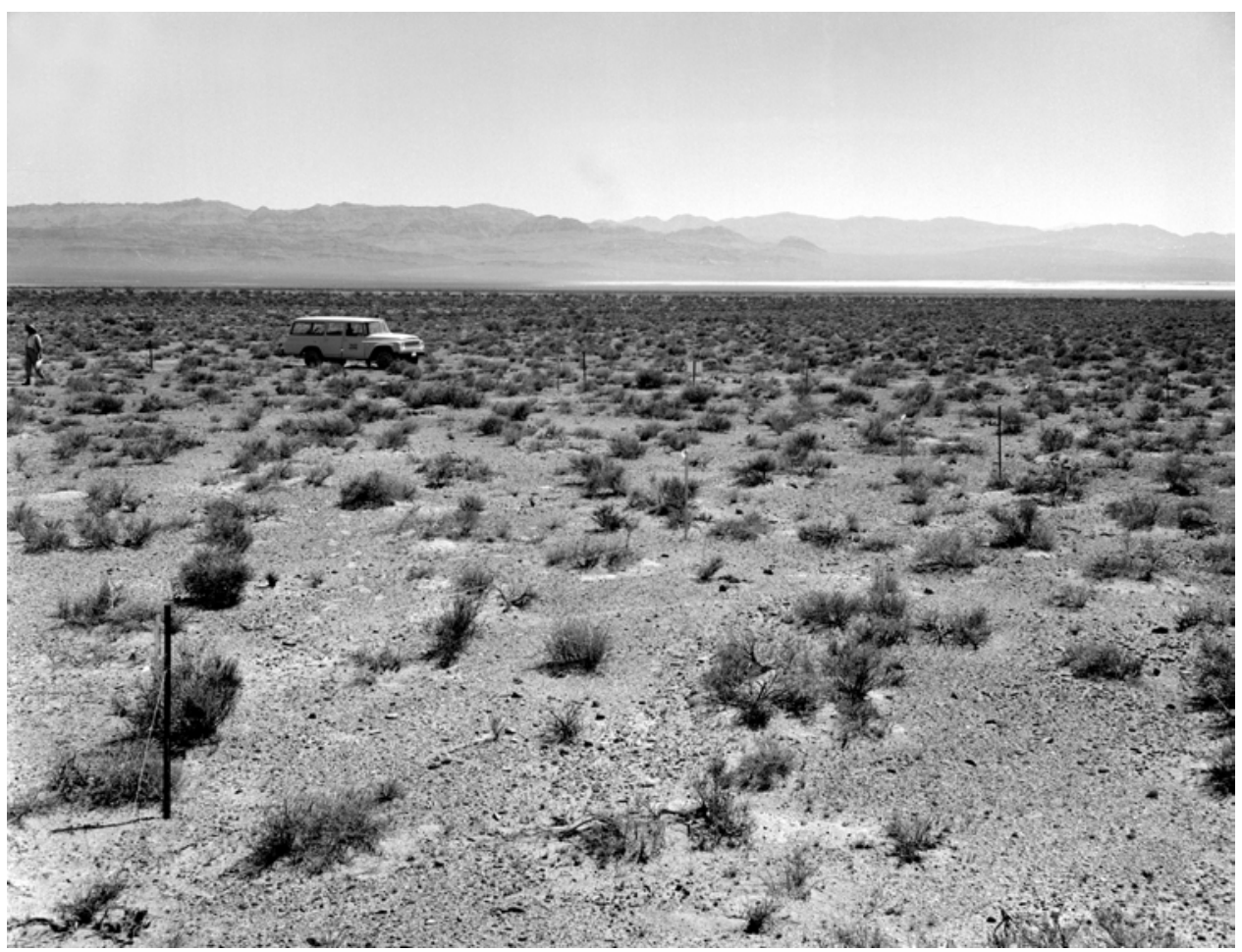

A. (April 22, 1964). In this southerly view across Plot 29, a creosote bush - shadscale (Atriplex confertifolia) community appears north of Frenchman Lake. In 1964, the plot was dominated by Mormon tea, range ratany, and shadscale. The Ranger Mountains appear in the distance (Janice Beatley Collection, 34-A).

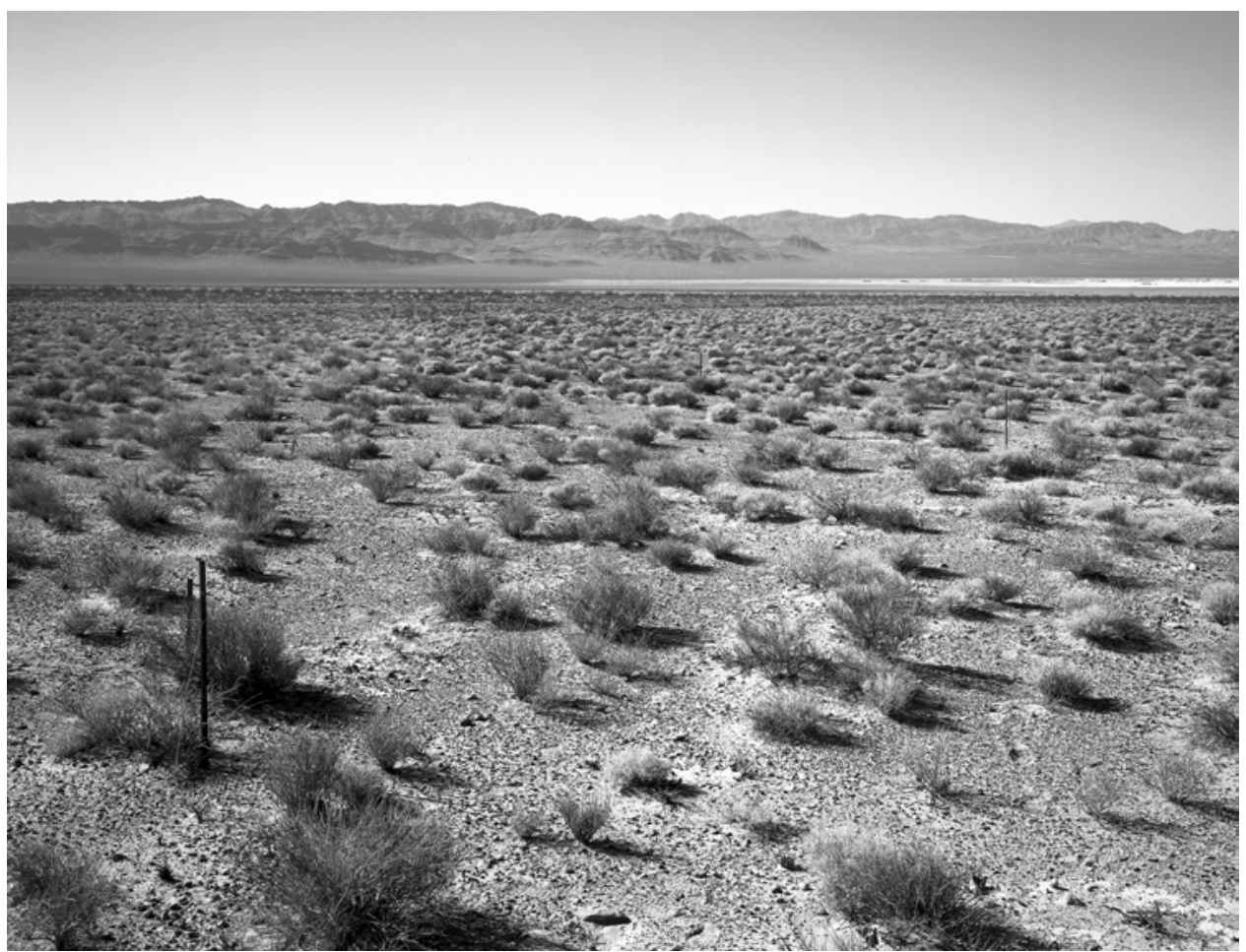

B. (April 18, 2002). The three dominant species are still present and still dominate. Visually, the biomass appears to have increased at least slightly (Dominic Oldershaw, Stake 4151B). 
Table A-29B. Summary plant data for Plot 29.

\author{
SPECIES \\ Ambrosia dumosa \\ Atriplex confertifolia \\ Ephedra nevadensis \\ Krameria parvifolia \\ Larrea tridentata \\ Total Live \\ Dead Grass \\ Dead Shrub \\ Total
}

\section{SPECIES}

Ambrosia dumosa

Atriplex confertifolia

Ephedra nevadensis

Krameria parvifolia

Larrea tridentata

\section{SPECIES}

Ambrosia dumosa

Atriplex confertifolia

Ephedra nevadensis

Krameria parvifolia

Larrea tridentata

Total Live

Dead Grass

Dead Shrub

Total

\section{SPECIES}

Ambrosia dumosa

Atriplex confertifolia

Ephedra nevadensis

Krameria parvifolia

Larrea tridentata

Total Live

\section{Number of Plants}

1963

3

19

50

59

4

135

0

71

206

1963

0.14

0.33

0.40

0.19

0.36

1963

0.15

1.95

6.73

5.93

0.51

15.26

0.00

6.05

21.32

1963

0.07

2.28

9.44

3.99

0.61

16.39

1975

3

18

75

45

1

142

0

45

187

1975

0.16

0.31

0.41

0.19

0.20

\section{Cover (\%)}

1975

0.16

1975

0.09

2.70

15.01

3.10

0.01

20.90
Average Height (m)

2002

0.34

0.30

0.39

0.17

0.56

2002

0.15

4.28

4.80

1.24

0.57

11.04

0.00

7.72

18.75

Biomass Index (m²)

2002

0.16

4.54

6.75

0.76

1.14

13.35 
Table A-30A. Site characteristics for Plot 30.

Location: Frenchman Flat

NTS Area: 5

NTS Grid: Y-18

USGS 7.5’ Quadrangle Name: Frenchman Lake

\begin{tabular}{lllc} 
& & \multicolumn{2}{c}{ GPS Readings (Zone 11S) } \\
& Location & Northing & Easting \\
Corner 1: & SW & 4076734 & 594561 \\
Corner 2: & NW & 4076764 & 594567 \\
Corner 3: & NE & 4076762 & 594597 \\
Corner 4: & SE & 4076731 & 594592
\end{tabular}

DEM Plot Elevation: 9471 m

Condition of Original Corners
Standing
Standing
Standing
Standing

Plot Aspect: $45^{\circ}$

Elevation Above Playa (Playa Name): 6 m (Frenchman)

Plot Slope: $4^{\circ}$

Annual Precipitation

Measured: $126 \mathrm{~mm}$

Modeled: $125 \mathrm{~mm}$

Parent Material Type: alluvial fan

Substrate: silty alluvium w/rocks \& pebbles, volcanic

Slate Geologic Unit: Qay

Beatley Plant Assemblage: Atriplex canescens

Ostler-Hanson Plant Association: Atriplex canescens

Ostler-Hanson Land Unit: 75

Abundance of Biological Soil Crusts: none

Type and Date of Disturbance: none

Plot Condition: good

Location of Transect 1: north side of plot

Direction Transects Are Read: east to west

Date(s) Plot Remeasured: 18 April 02 
Figure A-30. Photographs showing Plot 30.

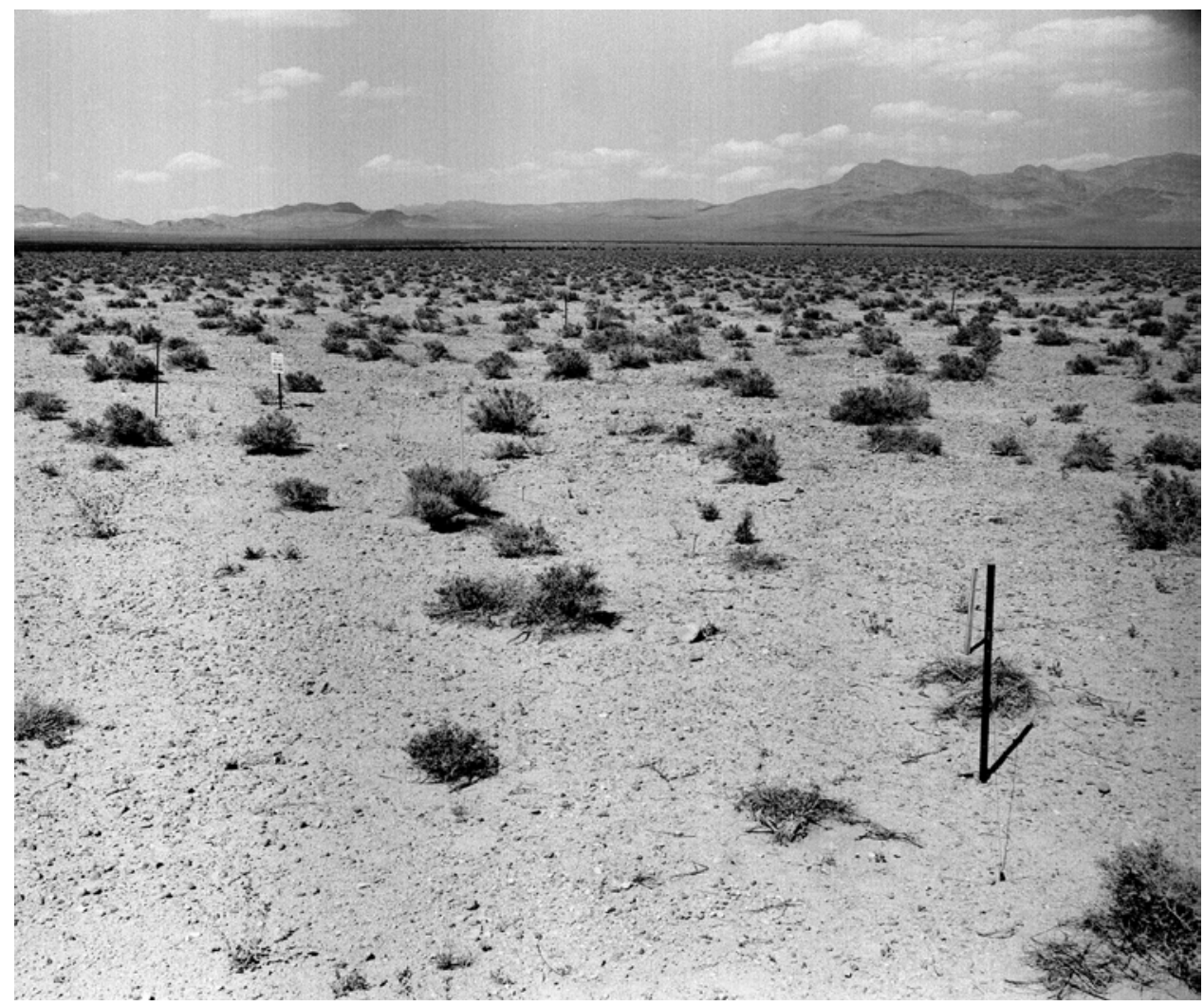

A. (May 11, 1964). In this northeasterly view across Plot 30, a four-wing saltbush (Atriplex canescens) assemblage appears north of Frenchman Flat. The Buried Hills appear in the distance (Janice Beatley Collection, 63-A).

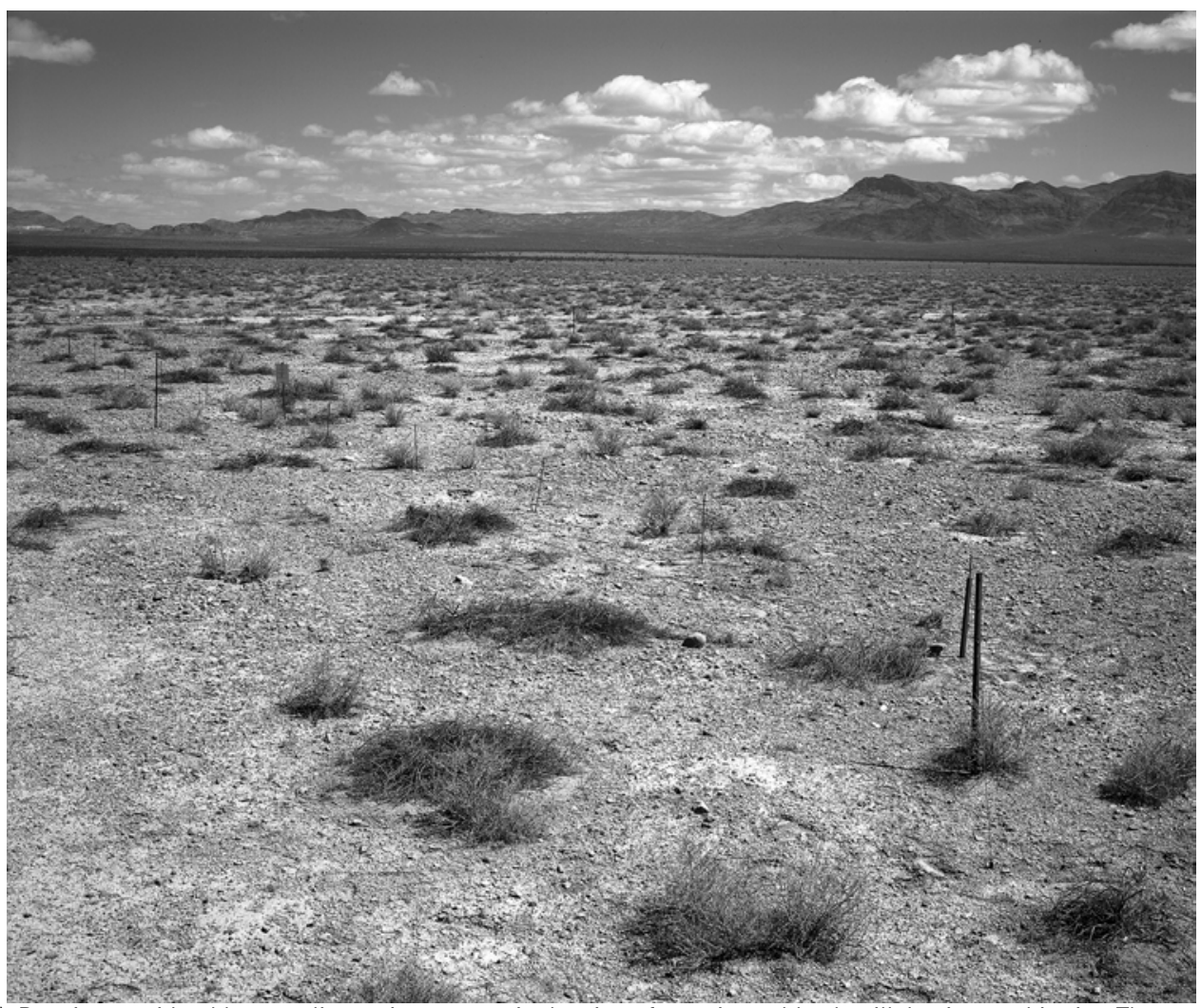

B. (April 18, 2002). Despite considerable mortality, as is apparent in the view, four-wing saltbush still dominates this site. The apparent increase in gravel lag cover is the result of periodic windstorms that add or remove the type of sand cover that was present in 1964 (Dominic Oldershaw, Stake 4155A). 
Table A-30B. Summary plant data for Plot 30.

\begin{tabular}{|c|c|c|c|c|c|c|c|}
\hline \multicolumn{5}{|c|}{ Number of Plants } & \multicolumn{3}{|c|}{ Cover (\%) } \\
\hline SPECIES & 1963 & 1975 & 2002 & SPECIES & 1963 & 1975 & 2002 \\
\hline Ambrosia dumosa & 1 & 1 & 0 & Ambrosia dumosa & 0.14 & 0.12 & 0.00 \\
\hline Astragalus lentiginosus fremontii & 0 & 1 & 0 & Astragalus lentiginosus fremontii & 0.00 & 0.02 & 0.00 \\
\hline Atriplex canescens & 38 & 52 & 34 & Atriplex canescens & 4.45 & 6.13 & 0.96 \\
\hline Atriplex confertifolia & 2 & 3 & 6 & Atriplex confertifolia & 0.26 & 0.36 & 0.17 \\
\hline Mirabilis pudica & 2 & 7 & 0 & Mirabilis pudica & 0.07 & 0.70 & 0.00 \\
\hline Oryzopsis hymenoides & 0 & 3 & 1 & Oryzopsis hymenoides & 0.00 & 0.14 & 0.03 \\
\hline Polygala subspinosa heterorhyncha & 0 & 5 & 2 & Polygala subspinosa heterorhyncha & 0.00 & 0.17 & 0.00 \\
\hline Sphaeralcea parvifolia & 0 & 4 & 2 & Sphaeralcea parvifolia & 0.00 & 0.10 & 0.02 \\
\hline Total Live & 43 & 76 & 43 & Total Live & 4.92 & 7.74 & 1.18 \\
\hline Dead & 4 & 5 & 63 & Dead & 0.52 & 0.25 & 2.10 \\
\hline Total & 47 & 81 & 106 & Total & 5.44 & 7.98 & 3.28 \\
\hline \multicolumn{5}{|c|}{ Average Height (m) } & \multicolumn{3}{|c|}{ Biomass Index (m²) } \\
\hline SPECIES & 1963 & 1975 & 2002 & SPECIES & 1963 & 1975 & 2002 \\
\hline Ambrosia dumosa & 0.23 & 0.23 & 0.00 & Ambrosia dumosa & 0.10 & 0.40 & 0.00 \\
\hline Astragalus lentiginosus fremontii & 0.00 & 0.05 & 0.00 & Astragalus lentiginosus fremontii & 0.00 & 0.06 & 0.00 \\
\hline Atriplex canescens & 0.36 & 0.37 & 0.40 & Atriplex canescens & 5.86 & 20.54 & 4.35 \\
\hline Atriplex confertifolia & 0.37 & 0.29 & 0.27 & Atriplex confertifolia & 0.35 & 1.22 & 0.49 \\
\hline Mirabilis pudica & 0.24 & 0.11 & 0.00 & Mirabilis pudica & 0.06 & 2.35 & 0.00 \\
\hline Oryzopsis hymenoides & 0.00 & 0.13 & 0.10 & Oryzopsis hymenoides & 0.00 & 0.46 & 0.01 \\
\hline Polygala subspinosa heterorhyncha & 0.00 & 0.04 & 0.00 & Polygala subspinosa heterorhyncha & 0.00 & 0.58 & 0.00 \\
\hline \multirow[t]{2}{*}{ Sphaeralcea parvifolia } & 0.00 & 0.06 & 0.07 & Sphaeralcea parvifolia & 0.00 & 0.34 & 0.00 \\
\hline & & & & Total Live & 6.37 & 25.94 & 4.85 \\
\hline
\end{tabular}


Table A-31A. Site characteristics for Plot 31.

Location: Frenchman Flat

NTS Area: 5

NTS Grid: Y-18

USGS 7.5’ Quadrangle Name: Frenchman Lake

\begin{tabular}{lllll} 
& & \multicolumn{2}{l}{ GPS Readings (Zone 11S) } & \\
& Location & Northing & Easting & Condition of Original Corners \\
Corner 1: & SW & 4075956 & 595086 & Standing \\
Corner 2: & NW & 4075983 & 595095 & NA \\
Corner 3: & NE & 4075982 & 595126 & NA \\
Corner 4: & SE & 4075955 & 595117 & Standing
\end{tabular}

DEM Plot Elevation: 942 m

Plot Aspect: $129^{\circ}$

Elevation Above Playa (Playa Name): 2 m (Frenchman)

Plot Slope: $10^{\circ}$

Annual Precipitation

Measured: $130 \mathrm{~mm}$

Modeled: $124 \mathrm{~mm}$

Parent Material Type: playa

Substrate: silty alluvium w/pebbles; rocks, mostly volcanic

Slate Geologic Unit: Qay

Beatley Plant Assemblage: Atriplex confertifolia

Ostler-Hanson Plant Association: Atriplex confertifolia

Ostler-Hanson Land Unit: 75

Abundance of Biological Soil Crusts: none

Type and Date of Disturbance: none

Plot Condition: good

Location of Transect 1: south side of plot

Direction Transects Are Read: west to east

Date(s) Plot Remeasured: 18 April 2002 
Figure A-31. Photographs showing Plot 31.

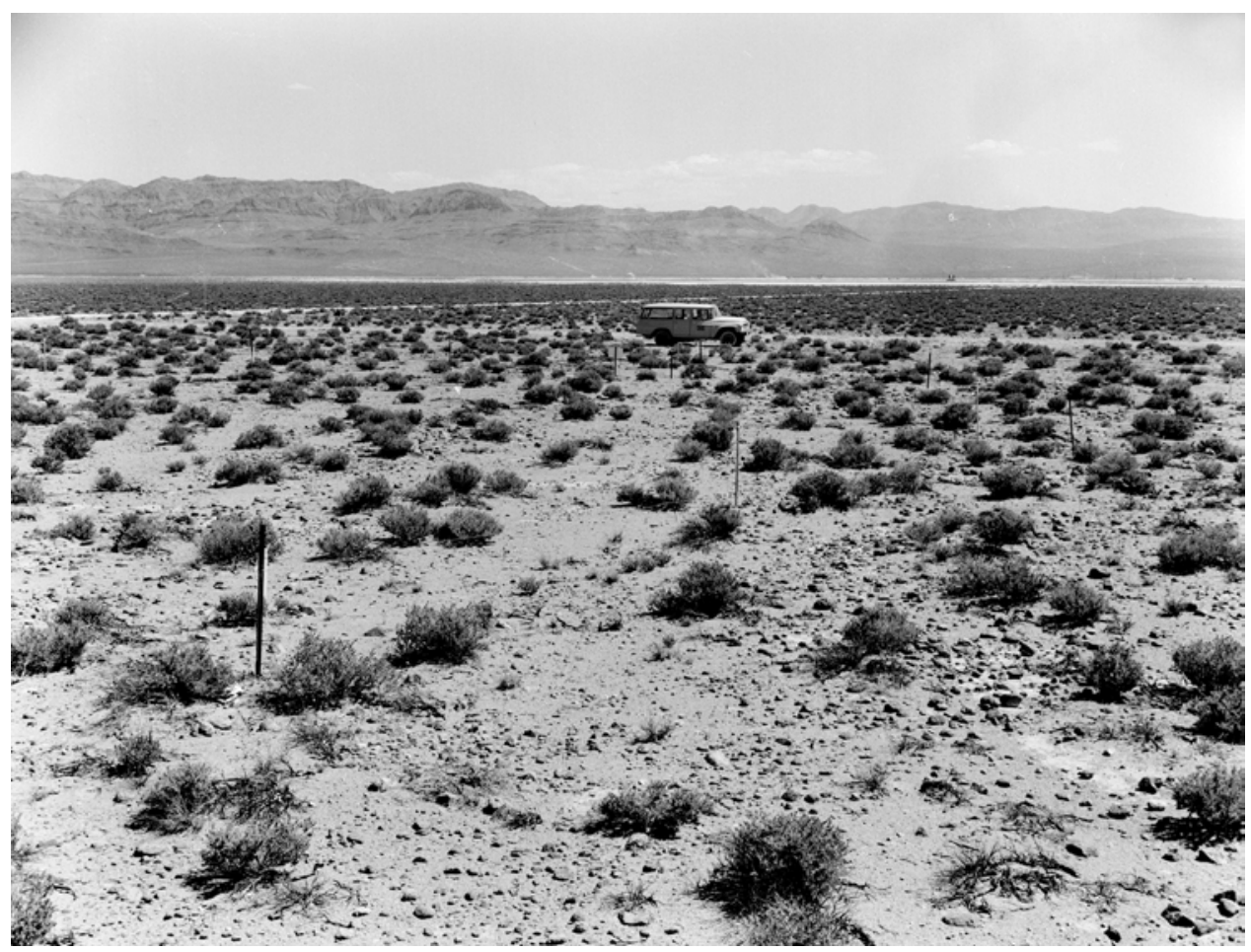

A. (May 11, 1964). In this southerly view across Plot 31, Frenchman Lake appears in the low point of the valley with the Ranger Mountains in the distance. The vegetation here is shadscale (Janice Beatley Collection, 72-B).

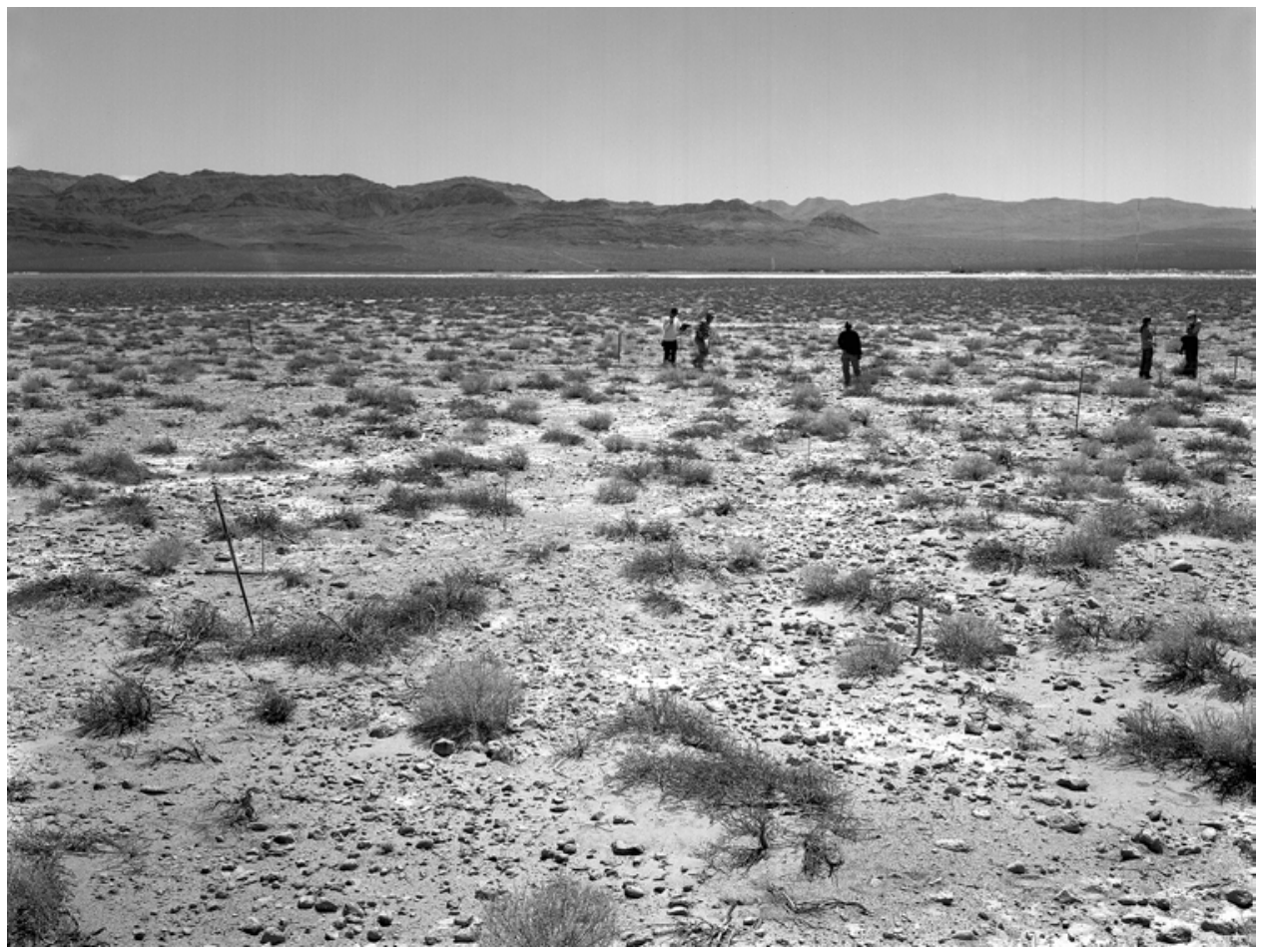

B. (April 18, 2002). Shadscale continues to dominate this plot despite considerable mortality in this species in the intervening 38 years (Dominic Oldershaw, Stake 4156B). 
Table A-31B. Summary plant data for Plot 31.

\author{
SPECIES \\ Astragalus lentiginosus fremontii \\ Atriplex confertifolia \\ Oryzopsis hymenoides \\ Sphaeralcea parvifolia \\ Total Live \\ Dead Grass \\ Dead Shrubs \\ Total
}

\section{SPECIES}

Astragalus lentiginosus fremontii Atriplex confertifolia

Oryzopsis hymenoides

Sphaeralcea parvifolia

\section{SPECIES}

Astragalus lentiginosus fremontii Atriplex confertifolia

Oryzopsis hymenoides

Sphaeralcea parvifolia

Total Live

Dead Grass

Dead Shrubs

Total

SPECIES

Astragalus lentiginosus fremontii Atriplex confertifolia Oryzopsis hymenoides Sphaeralcea parvifolia Total Live

\section{Number of Plants}

1975

2002

0

12

65

2

5

84

0

10

94

0

34

3

0

37

0

70

107

Average Height (m)

1963

0.00

0.33

0.21

0.00

1975

2002

0.09

0.00

0.33

0.33

0.09

0.18

0.09

0.00

\section{Cover (\%)}

1975

2002

1963

0.00

6.16

0.07

0.00

6.24

0.00

2.48

8.72

0.37

7.45

0.07

0.09

7.98

0.00

0.98

8.96

0.00

4.16

0.11

0.00

4.27

0.00

7.26

11.54

\section{Biomass Index $\left(\mathrm{m}^{2}\right)$}

1963

0.00

1975

2002

0.13

0.00

9.93

4.80

7.50

0.03

0.08

0.00

0.03

0.00

7.55

10.11

4.88 
Table A-32A. Site characteristics for Plot 32.

Location: Frenchman Flat

NTS Area: Nellis

NTS Grid: W64C

USGS 7.5’ Quadrangle Name: Frenchman Lake SE

\begin{tabular}{|c|c|c|c|c|}
\hline & & GPS Reac & Zone 11S) & \\
\hline & Location & Northing & Easting & Condition of Original Corners \\
\hline Corner 1: & SW & 4077331 & 601322 & Standing \\
\hline Corner 2: & NW & 4077361 & 601328 & Standing \\
\hline Corner 3: & $\mathrm{NE}$ & 4077357 & 601358 & Standing \\
\hline Corner 4: & SE & 4077327 & 601350 & Standing \\
\hline GPS Plot & yation: 103 & & & Plot Aspect: $\mathrm{NA}^{\circ}$ \\
\hline Elevation & e Playa & Name): & enchman) & Plot Slope: $\mathrm{NA}^{\circ}$ \\
\hline
\end{tabular}

Annual Precipitation

Measured: $122 \mathrm{~mm}$

Modeled: NA mm

Parent Material Type: alluvial fan

Substrate: sandy, no soil crusts

Slate Geologic Unit: NA

Beatley Plant Assemblage: Larrea-Ambrosia

Ostler-Hanson Plant Association: Ambrosia-Psorothamnus-Menodora

Ostler-Hanson Land Unit: NA

Abundance of Biological Soil Crusts: NA

Type and Date of Disturbance: NA

Plot Condition: good

Location of Transect 1: west side of plot

Direction Transects Are Read: south to north

Date(s) Plot Remeasured: 19 April 2002 
Figure A-32. Photographs showing Plot 32.

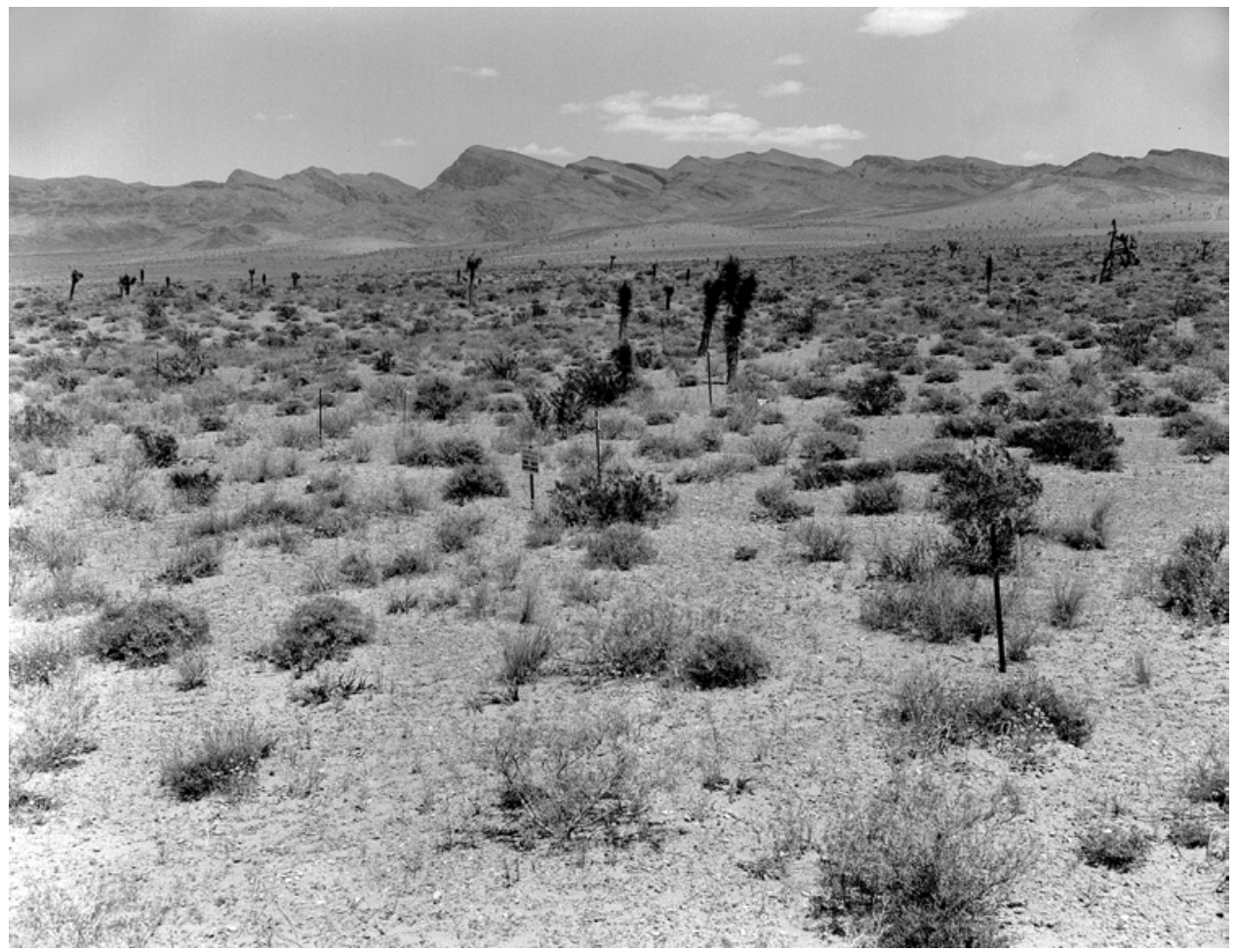

A. (May 11, 1964). In this north-northeasterly view across Plot 32, a creosote bush - white bursage assemblage appears south of the Buried Hills. Joshua trees are scattered across the landscape (Janice Beatley Collection, 70-A).

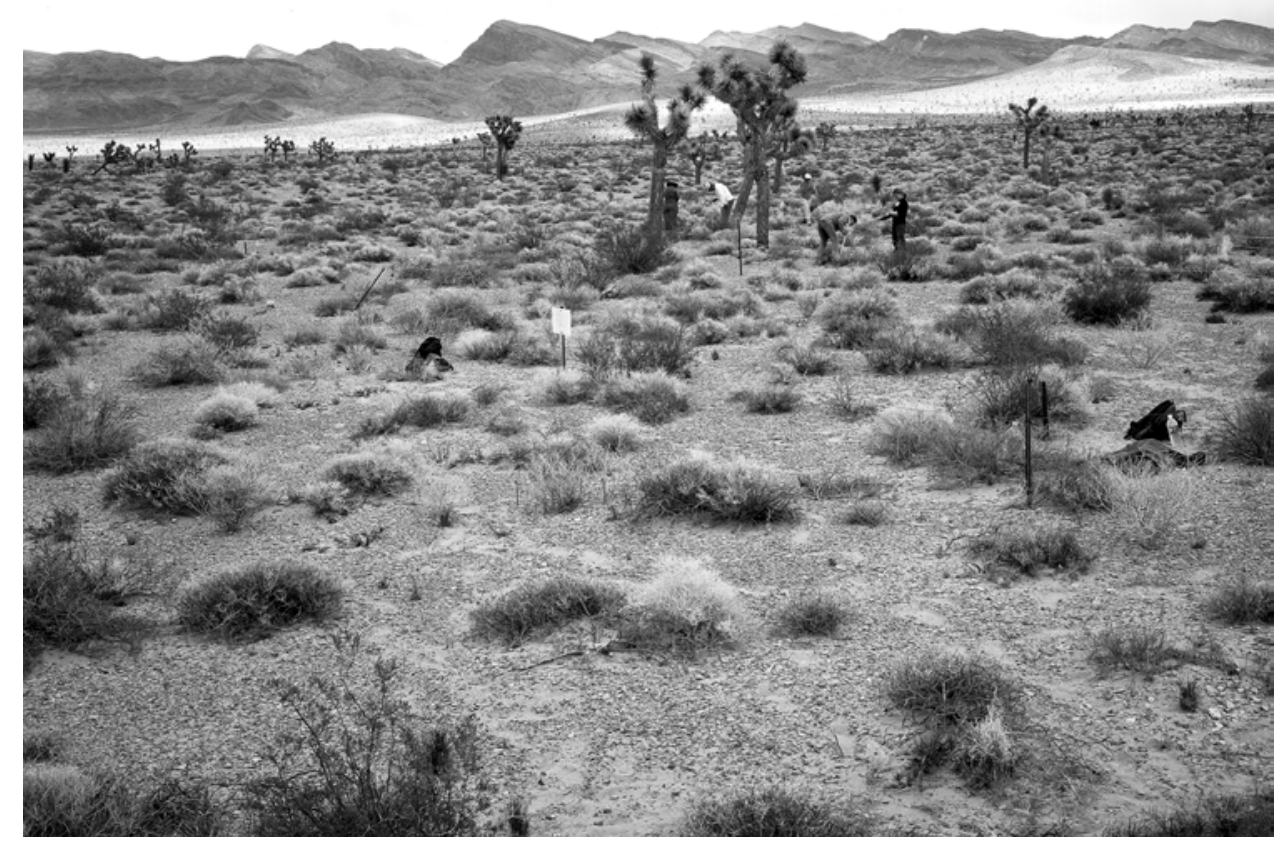

B. (April 19, 2002). White bursage, range ratany, and spiny menodora have increased here. The Joshua trees have increased in density and size in the intervening 38 years. This is one of the few sites at which creosote bush has decreased significantly in cover. The light color on the background hills is sunlight piercing through a generally overcast sky (Dominic Oldershaw, Stake 4164A). 
Table A-32B. Summary plant data for Plot 32.

\begin{tabular}{|c|c|c|c|}
\hline SPECIES & 1963 & 1975 & 2002 \\
\hline Ambrosia dumosa & 46 & 61 & 69 \\
\hline Ceratoides lanata & 0 & 0 & 1 \\
\hline Ephedra nevadensis & 2 & 4 & 5 \\
\hline Grayia spinosa & 0 & 0 & 1 \\
\hline Krameria parvifolia & 16 & 15 & 25 \\
\hline Larrea tridentata & 9 & 10 & 10 \\
\hline Menodora spinescens & 27 & 23 & 32 \\
\hline Oryzopsis hymenoides & 10 & 37 & 8 \\
\hline Psorothamnus fremontii & 18 & 20 & 20 \\
\hline Sphaeralcea ambigua & 0 & 0 & 0 \\
\hline Sporobolus airoides & 0 & 0 & 12 \\
\hline Stipa speciosa & 0 & 0 & 1 \\
\hline Yucca brevifolia & 2 & 2 & 5 \\
\hline Total Live & 130 & 172 & 189 \\
\hline Dead Grass & 0 & 6 & 8 \\
\hline Dead Shrub & 20 & 15 & 45 \\
\hline Total & 150 & 193 & 242 \\
\hline & \multicolumn{3}{|c|}{ Average Height (m) } \\
\hline SPECIES & 1963 & 1975 & 2002 \\
\hline Ambrosia dumosa & 0.31 & 0.30 & 0.34 \\
\hline Ceratoides lanata & 0.00 & 0.00 & 0.36 \\
\hline Ephedra nevadensis & 0.74 & 0.50 & 0.49 \\
\hline Grayia spinosa & 0.00 & 0.00 & 0.46 \\
\hline Krameria parvifolia & 0.32 & 0.27 & 0.32 \\
\hline Larrea tridentata & 0.63 & 0.64 & 0.74 \\
\hline Menodora spinescens & 0.20 & 0.18 & 0.22 \\
\hline Oryzopsis hymenoides & 0.30 & 0.29 & 0.20 \\
\hline Psorothamnus fremontii & 0.45 & 0.44 & 0.54 \\
\hline Sphaeralcea ambigua & 0.00 & 0.00 & 0.00 \\
\hline Sporobolus airoides & 0.00 & 0.00 & 0.32 \\
\hline Stipa speciosa & 0.00 & 0.00 & 0.48 \\
\hline Yucca brevifolia & 2.51 & 3.68 & 3.67 \\
\hline
\end{tabular}

\section{SPECIES}

Ambrosia dumosa

Ceratoides lanata

Ephedra nevadensis

Grayia spinosa

Krameria parvifolia

Larrea tridentata

Menodora spinescens

Oryzopsis hymenoides

Psorothamnus fremontii

Sphaeralcea ambigua

Sporobolus airoides

Stipa speciosa

Yucca brevifolia

Total Live

Dead Grass

Dead Shrub

Total
Cover (\%)

1975

7.07

0.00

0.46

0.00

2.24

1.98

2.75

2.05

3.11

0.00

0.00

0.00

0.53

20.18

0.24

1.36

21.78

2002

6.93

0.05

0.54

0.04

2.85

0.79

3.13

0.22

2.30

0.00

0.40

0.01

1.86

19.10

0.27

5.40

24.77
Biomass Index $\left(\mathrm{m}^{2}\right)$

$\begin{array}{ccc}\mathbf{1 9 6 3} & \mathbf{1 9 7 5} & \mathbf{2 0 0 2} \\ 6.36 & 7.75 & 8.19 \\ 0.00 & 0.00 & 0.05 \\ 1.05 & 0.90 & 0.90 \\ 0.00 & 0.00 & 0.06 \\ 2.59 & 2.18 & 3.14 \\ 3.21 & 4.79 & 1.93 \\ 2.10 & 1.80 & 2.47 \\ 0.52 & 2.19 & 0.13 \\ 4.12 & 5.44 & 4.28 \\ 0.00 & 0.00 & 0.00 \\ 0.00 & 0.00 & 0.44 \\ 0.00 & 0.00 & 0.01 \\ 2.65 & 6.60 & 24.99 \\ 22.60 & 31.67 & 46.61\end{array}$


Table A-33A. Site characteristics for Plot 33.

Location: Frenchman Flat

NTS Area: Nellis

NTS Grid: W64B

USGS 7.5’ Quadrangle Name: Frenchman Lake

\begin{tabular}{|c|c|c|c|c|}
\hline & & GPS Reac & Zone 11S) & \\
\hline & Location & Northing & Easting & Condition of Original Corners \\
\hline Corner 1: & SW & 4075464 & 599351 & Standing \\
\hline Corner 2: & NW & 4075492 & 599356 & Standing \\
\hline Corner 3: & $\mathrm{NE}$ & 4075488 & 599387 & Standing \\
\hline Corner 4: & SE & 4075457 & 599381 & Standing \\
\hline DEM Plo & evation: 98 & & & Plot Aspect: $104^{\circ}$ \\
\hline Elevation & e Playa & Name): & enchman) & Plot Slope: $6^{\circ}$ \\
\hline
\end{tabular}

Annual Precipitation

Measured: $119 \mathrm{~mm}$

Modeled: $130 \mathrm{~mm}$

Parent Material Type: alluvial fan

Substrate: desert pavement, limestone

Slate Geologic Unit: Qai

Beatley Plant Assemblage: Larrea-Psorothamnus

Ostler-Hanson Plant Association: Larrea-Psorothamnus-Krameria

Ostler-Hanson Land Unit: NA

Abundance of Biological Soil Crusts: good cover

Type and Date of Disturbance: none

Plot Condition: good

Location of Transect 1: east side of plot

Direction Transects Are Read: north to south

Date(s) Plot Remeasured: 19 April 2002 
Figure A-33. Photographs showing Plot 33.

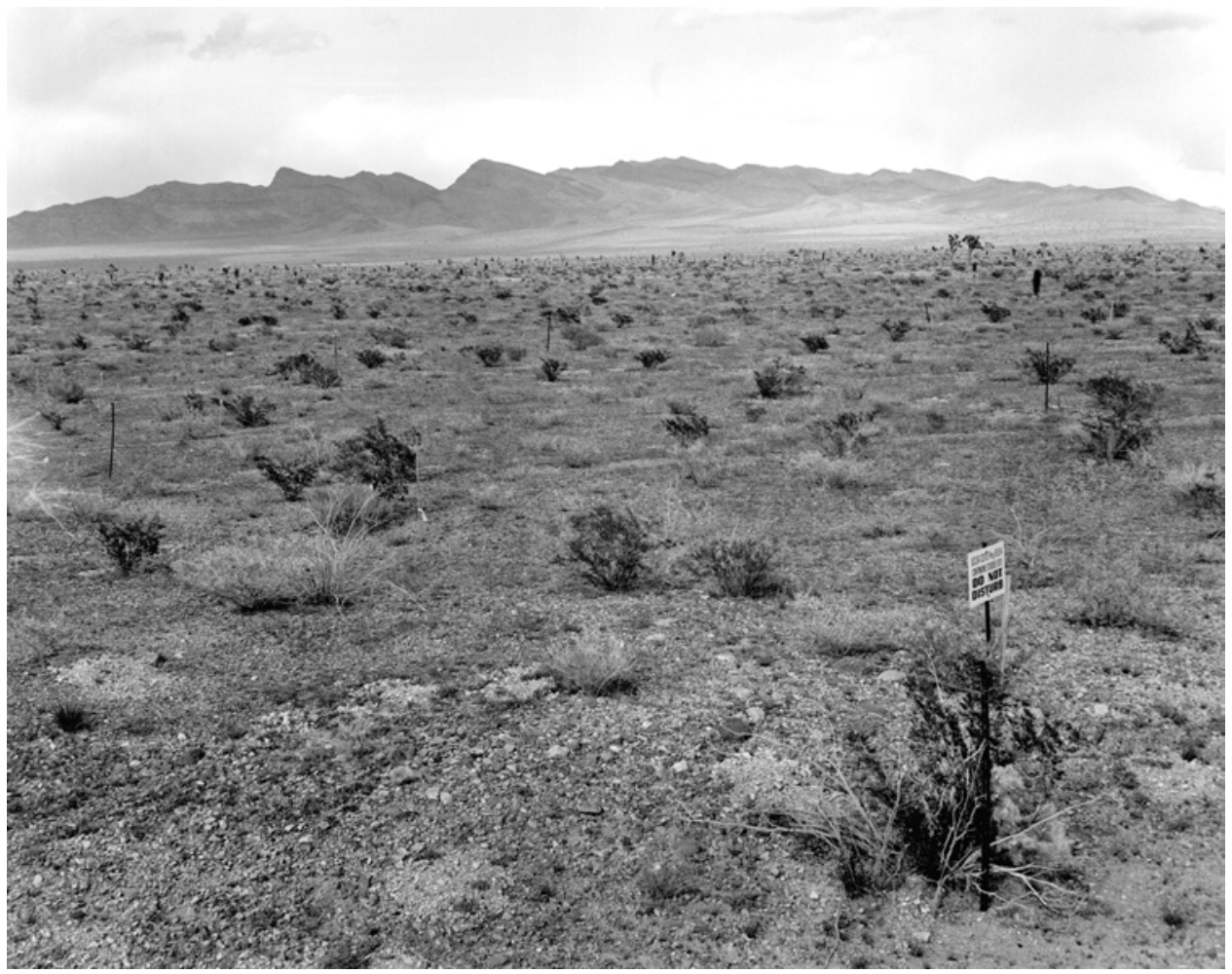

A. (April 24, 1964). In this northeasterly view across Plot 33, a sparse creosote bush assemblage appears in eastern Frenchman Flat. Fremont's dalea (Psorothamnus fremontii) is a subdominant here, and Joshua trees appear in the distance (Janice Beatley Collection, 26-A).

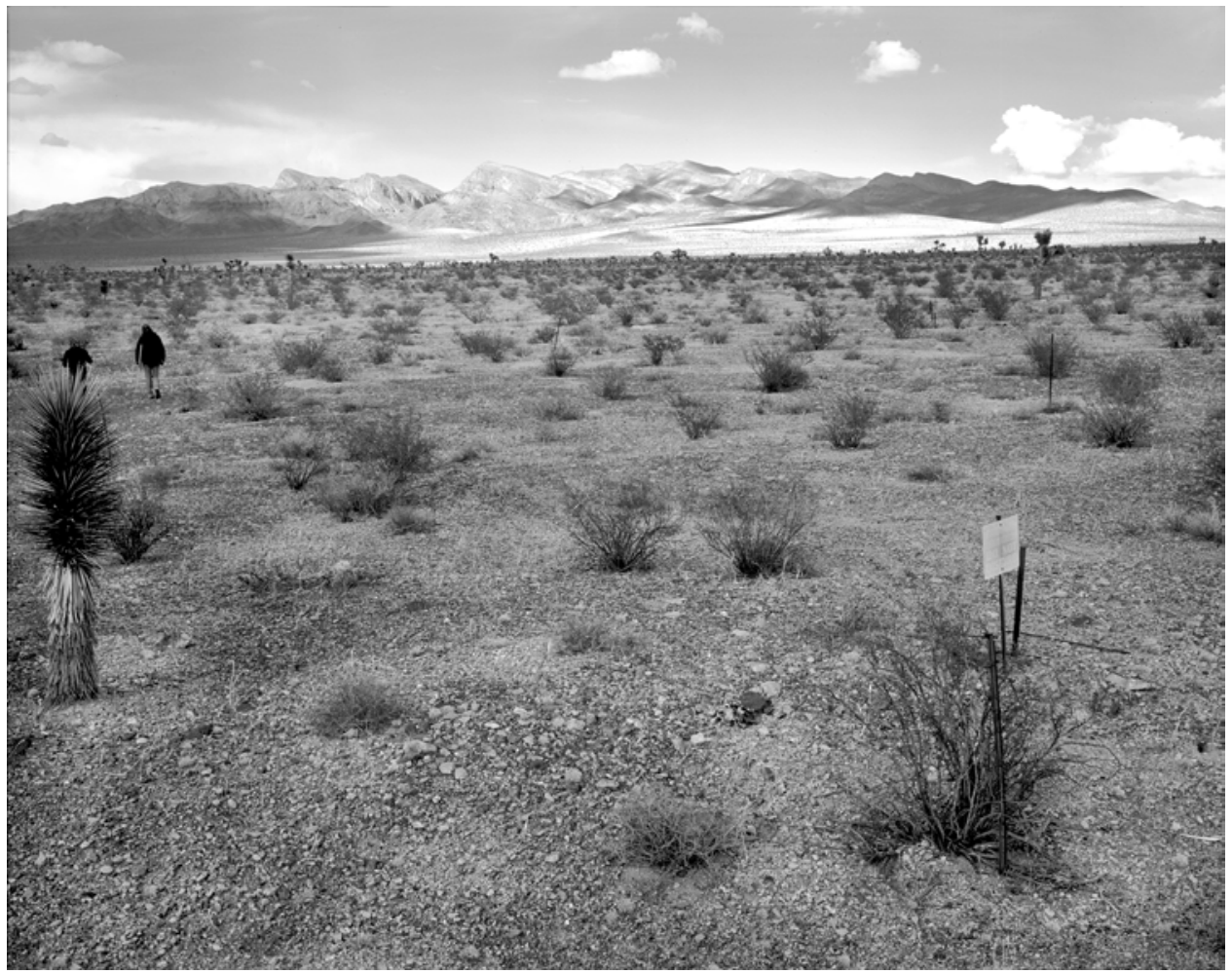

B. (April 19, 2002). The size of individual creosote bushes has increased, and range ratany has become the subdominant. Joshua trees have increased in size and density in the distance. Note the Joshua tree in the left foreground that was a nondescript seedling in 1964 (Dominic Oldershaw, Stake 4167A). 
Table A-33B. Summary plant data for Plot 33.

\begin{tabular}{|c|c|c|c|c|c|c|c|}
\hline \multicolumn{5}{|c|}{ Number of Plants } & \multicolumn{3}{|c|}{ Cover (\%) } \\
\hline SPECIES & 1963 & 1975 & 2002 & SPECIES & 1963 & 1975 & 2002 \\
\hline Ambrosia dumosa & 0 & 2 & 2 & Ambrosia dumosa & 0.00 & 0.07 & 0.21 \\
\hline Ceratoides lanata & 0 & 0 & 1 & Ceratoides lanata & 0.00 & 0.00 & 0.11 \\
\hline Ephedra nevadensis & 1 & 2 & 3 & Ephedra nevadensis & 0.18 & 0.59 & 0.15 \\
\hline Eriogonum inflatum & 0 & 5 & 2 & Eriogonum inflatum & 0.00 & 0.11 & 0.03 \\
\hline Erioneuron pulchellum & 0 & 0 & 64 & Erioneuron pulchellum & 0.00 & 0.00 & 1.54 \\
\hline Grayia spinosa & 0 & 0 & 1 & Grayia spinosa & 0.00 & 0.00 & 0.01 \\
\hline Krameria parvifolia & 12 & 13 & 16 & Krameria parvifolia & 1.15 & 1.66 & 1.70 \\
\hline Larrea tridentata & 11 & 14 & 13 & Larrea tridentata & 2.05 & 3.38 & 2.50 \\
\hline Opuntia echinocarpa & 0 & 0 & 1 & Opuntia echinocarpa & 0.00 & 0.00 & 0.05 \\
\hline Opuntia ramosissima & 1 & 2 & 0 & Opuntia ramosissima & 0.07 & 0.16 & 0.00 \\
\hline Oryzopsis hymenoides & 2 & 1 & 0 & Oryzopsis hymenoides & 0.09 & 0.07 & 0.00 \\
\hline Psorothamnus fremontii & 24 & 26 & 0 & Psorothamnus fremontii & 2.49 & 3.14 & 0.00 \\
\hline Total Live & 51 & 65 & 101 & Total Live & 6.04 & 9.19 & 6.05 \\
\hline Dead Grass & 0 & 1 & 1 & Dead Grass & 0.00 & 0.05 & 0.02 \\
\hline Dead Shrubs & 8 & 9 & 23 & Dead Shrubs & 1.25 & 0.95 & 2.57 \\
\hline Total & 59 & 75 & 125 & Total & 7.28 & 10.19 & 8.64 \\
\hline \multicolumn{5}{|c|}{ Average Height (m) } & \multicolumn{3}{|c|}{ Biomass Index $\left(\mathbf{m}^{2}\right)$} \\
\hline SPECIES & 1963 & 1975 & 2002 & SPECIES & 1963 & 1975 & 2002 \\
\hline Ambrosia dumosa & 0.00 & 0.15 & 0.22 & Ambrosia dumosa & 0.00 & 0.04 & 0.16 \\
\hline Ceratoides lanata & 0.00 & 0.00 & 0.32 & Ceratoides lanata & 0.00 & 0.00 & 0.12 \\
\hline Ephedra nevadensis & 0.46 & 0.64 & 0.43 & Ephedra nevadensis & 0.28 & 1.26 & 0.17 \\
\hline Eriogonum inflatum & 0.00 & 0.11 & 0.00 & Eriogonum inflatum & 0.00 & 0.04 & 0.00 \\
\hline Erioneuron pulchellum & 0.00 & 0.00 & 0.00 & Erioneuron pulchellum & 0.00 & 0.00 & 0.00 \\
\hline Grayia spinosa & 0.00 & 0.00 & 0.00 & Grayia spinosa & 0.00 & 0.00 & 0.00 \\
\hline Krameria parvifolia & 0.19 & 0.26 & 0.27 & Krameria parvifolia & 0.78 & 1.44 & 1.55 \\
\hline Larrea tridentata & 0.69 & 0.69 & 0.91 & Larrea tridentata & 5.09 & 8.30 & 7.10 \\
\hline Opuntia echinocarpa & 0.00 & 0.00 & 0.12 & Opuntia echinocarpa & 0.00 & 0.00 & 0.02 \\
\hline Opuntia ramosissima & 0.36 & 0.22 & 0.00 & Opuntia ramosissima & 0.09 & 0.19 & 0.00 \\
\hline Oryzopsis hymenoides & 0.37 & 0.33 & 0.00 & Oryzopsis hymenoides & 0.11 & 0.08 & 0.00 \\
\hline \multirow[t]{2}{*}{ Psorothamnus fremontii } & 0.36 & 0.42 & 0.00 & Psorothamnus fremontii & 3.33 & 4.61 & 0.00 \\
\hline & & & & Total Live & 9.68 & 15.96 & 8.95 \\
\hline
\end{tabular}


Table A-34A. Site characteristics for Plot 34.

Location: Frenchman Flat

NTS Area: 5

NTS Grid: Z-16

USGS 7.5’ Quadrangle Name: Frenchman Lake

\begin{tabular}{|c|c|c|c|c|}
\hline & & GPS Reac & Zone 11S) & \\
\hline & Location & Northing & Easting & Condition of Original Corners \\
\hline Corner 1: & SW & 4073028 & 597687 & NA \\
\hline Corner 2: & NW & 4073056 & 597694 & NA \\
\hline Corner 3: & $\mathrm{NE}$ & 4073049 & 597722 & NA \\
\hline Corner 4: & SE & 4073021 & 597716 & NA \\
\hline DEM Plot & vation: 94 & & & Plot Aspect: $115^{\circ}$ \\
\hline Elevation & re Playa ( & Name): 2 & nchman) & Plot Slope: $12^{\circ}$ \\
\hline
\end{tabular}

Annual Precipitation

Measured: $124 \mathrm{~mm}$

Modeled: $124 \mathrm{~mm}$

Parent Material Type: playa

Substrate: pebbles, well developed crust

Slate Geologic Unit: Qay

Beatley Plant Assemblage: Larrea-Lycium shockleyi-Atriplex

Ostler-Hanson Plant Association: Lycium shockleyi-Larrea

Ostler-Hanson Land Unit: NA

Abundance of Biological Soil Crusts: well developed

Type and Date of Disturbance: NA

Plot Condition: good

Location of Transect 1: east side of plot

Direction Transects Are Read: south to north

Date(s) Plot Remeasured: 17 April 2002 
Figure A-34. Photographs showing Plot 34.

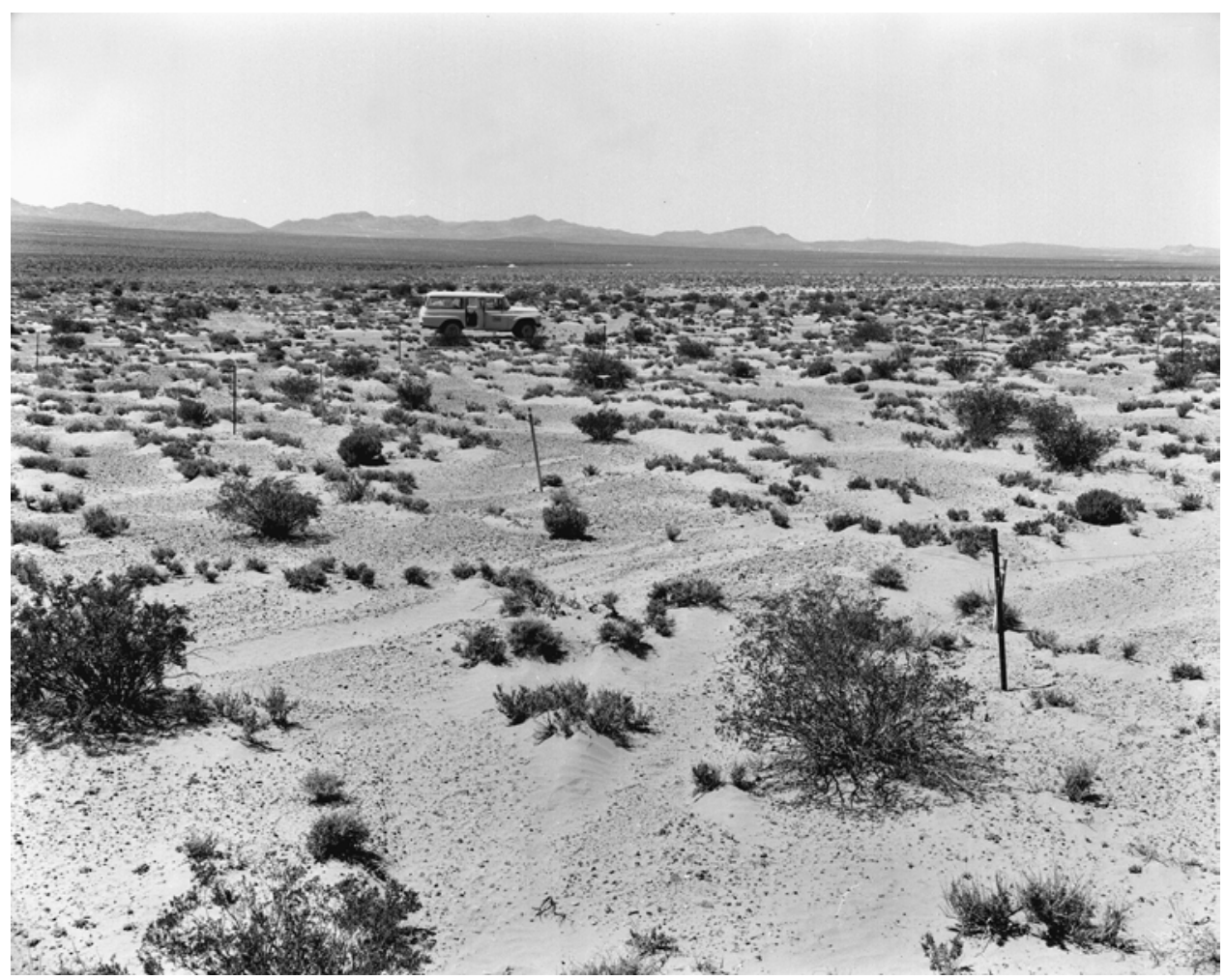

A. (May 11, 1964). The view shown is southwesterly across Plot 34 in southern Frenchman Flat. This plot is on the southeast corner of Frenchman Lake. The mountains in the distance are part of the Mercury Ridge north of Mercury, Nevada. The vegetation assemblage is a mixture of creosote bush, Shockley's desert thorn (Lycium shockleyi), and shadscale (Janice Beatley Collection, 73-A).

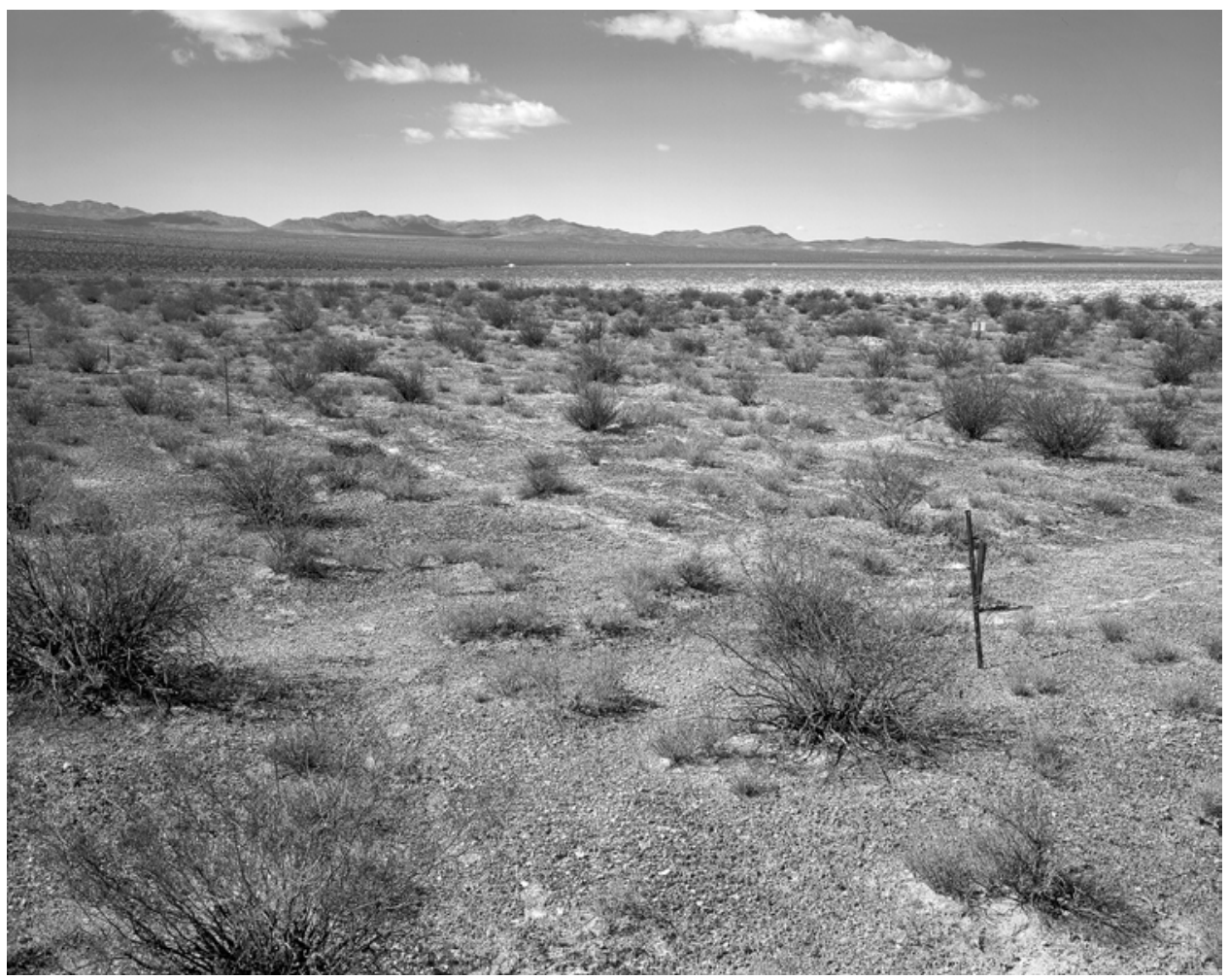

B. (April 20, 2002). The creosote bush have grown considerably, and Shockley's desert thorn is now the dominant species. The darkened ground surface is the result of the increase in prominence of biological soil crusts in this view. This increase may be largely the result of a difference in the type of photographic film, but the change appears to be real (Dominic Oldershaw, Stake 4175A). 
Table A-34B. Summary plant data for Plot 34.

\author{
SPECIES \\ Ambrosia dumosa \\ Atriplex canescens \\ Atriplex confertifolia \\ Ceratoides lanata \\ Krameria parvifolia \\ Larrea tridentata \\ Lycium shockleyi \\ Total Live \\ Dead Grass \\ Dead Shrub \\ Total
}

\section{SPECIES}

Ambrosia dumosa

Atriplex canescens

Atriplex confertifolia

Ceratoides lanata

Krameria parvifolia

Larrea tridentata

Lycium shockleyi

\section{SPECIES}

Ambrosia dumosa

Atriplex canescens

Atriplex confertifolia

Ceratoides lanata

Krameria parvifolia

Larrea tridentata

Lycium shockleyi

Total Live

Dead Grass

Dead Shrub

Total

\section{SPECIES}

Ambrosia dumosa

Atriplex canescens

Atriplex confertifolia

Ceratoides lanata

Krameria parvifolia

Larrea tridentata

Lycium shockleyi

Total Live
1963

5

0

6

1

0

10

90

112

0

0

112

1963

0.18

0.00

0.22

0.28

0.00

0.74

0.16

1963

0.47

0.00

0.43

0.04

0.00

1.96

7.75

10.65

0.00

0.00

10.65

1963

0.28

0.00

0.37

0.03

0.00

5.21

4.46

10.35

\section{Number of Plants}

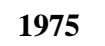

2002

$9 \quad 6$

20

$5 \quad 1$

4 1

11

$11 \quad 22$

$101 \quad 106$

$133 \quad 137$

$0 \quad 0$

$1 \quad 57$

$134 \quad 194$

\section{Average Height (m)}

1975

2002

$0.23 \quad 0.21$

$0.33 \quad 0.00$

$0.36 \quad 0.12$

$0.27 \quad 0.28$

$0.41 \quad 0.35$

$0.91 \quad 0.96$

$0.23 \quad 0.26$

\section{Cover (\%)}

19752002

$0.72 \quad 0.28$

$0.15 \quad 0.00$

$0.55 \quad 0.04$

$0.20 \quad 0.08$

$0.11 \quad 0.08$

$2.57 \quad 3.73$

$12.76 \quad 7.66$

$17.06 \quad 11.87$

$0.00 \quad 0.00$

$0.09 \quad 6.19$

$17.15 \quad 18.06$

Biomass Index (m²)

19752002

$0.57 \quad 0.15$

$0.20 \quad 0.00$

$0.80 \quad 0.01$

$0.23 \quad 0.08$

$0.15 \quad 0.10$

$8.43 \quad 12.99$

$10.24 \quad 7.03$

$20.60 \quad 20.36$ 
Table A-35A. Site characteristics for Plot 35.

Location: Frenchman Flat

NTS Area: 5

NTS Grid: Z-15

USGS 7.5’ Quadrangle Name: Frenchman Lake

\begin{tabular}{|c|c|c|c|c|}
\hline & & GPS Reac & Zone 11S) & \\
\hline & Location & Northing & Easting & Condition of Original Corners \\
\hline Corner 1: & SW & 4070888 & 597631 & NA \\
\hline Corner 2: & NW & 4070916 & 597639 & Standing \\
\hline Corner 3: & NE & 4070905 & 597669 & Standing \\
\hline Corner 4: & SE & 4070876 & 597663 & Standing \\
\hline DEM Plot & evation: 98 & & & Plot Aspect: $190^{\circ}$ \\
\hline Elevation & bve Playa ( & a Name): 6 & renchman) & Plot Slope: $31^{\circ}$ \\
\hline Annual Pre & $\begin{array}{l}\text { itation } \\
\text { sured: } 13 \\
\text { eled: } 130\end{array}$ & & & \\
\hline
\end{tabular}

Parent Material Type: broken terrain

Substrate: desert pavement, limestone

Slate Geologic Unit: QTa

Beatley Plant Assemblage: Larrea-Lycium shockleyi-Atriplex

Ostler-Hanson Plant Association: Menodora-Lycium-Atriplex confertifolia

Ostler-Hanson Land Unit: NA

Abundance of Biological Soil Crusts: patchy

Type and Date of Disturbance: none

Plot Condition: good

Location of Transect 1: northwest side of plot

Direction Transects Are Read: southwest to northeast

Date(s) Plot Remeasured: 17 April 2002 
Figure A-35. Photographs showing Plot 35.

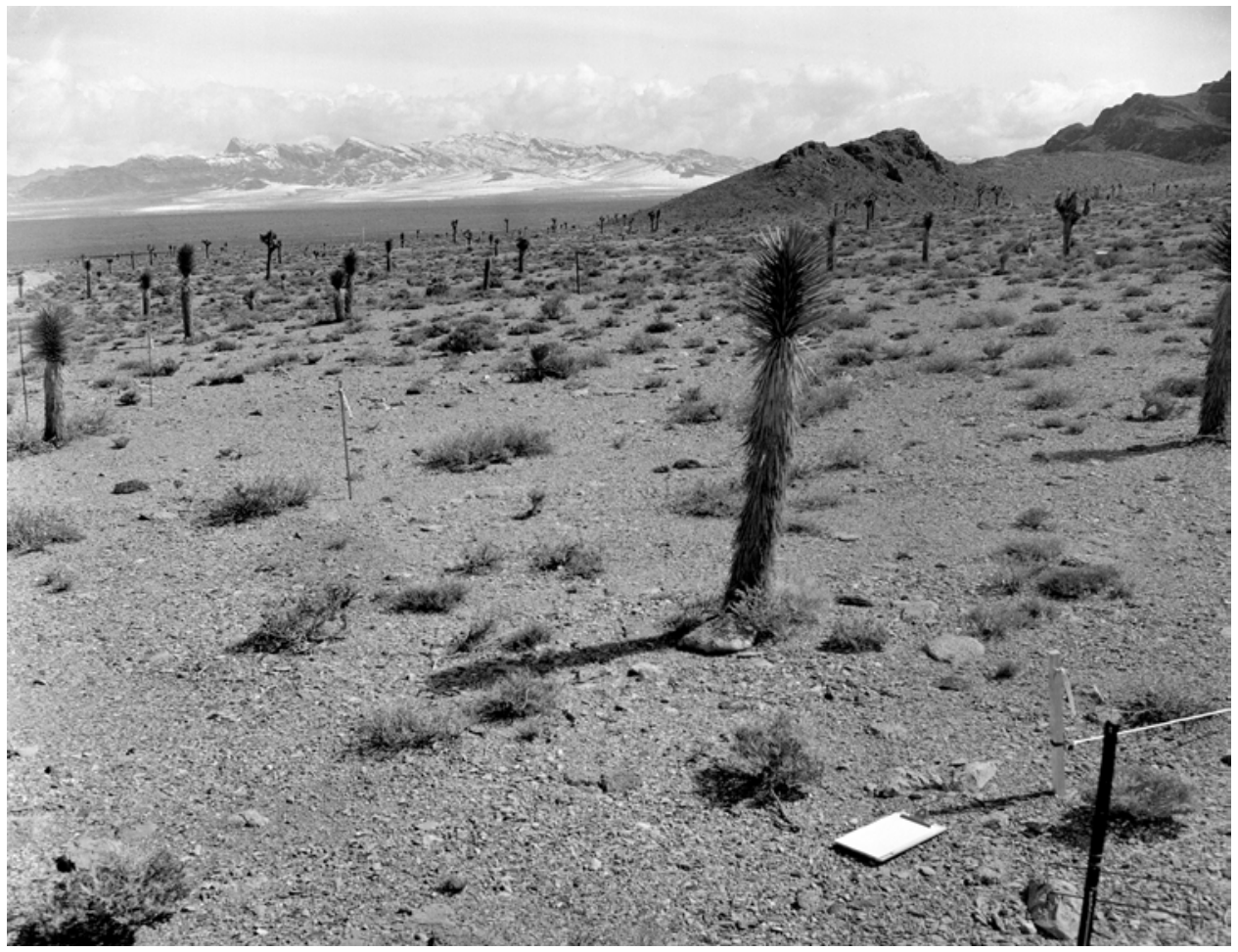

A. (April 24, 1964). In this northeasterly view across Plot 35, a mixture of creosote bush, Shockley's desert thorn, and shadscale occurs between scattered Joshua trees. This plot is near the foothills of the Ranger Mountains, which are shown in the background (Janice Beatley Collection, 23-A).

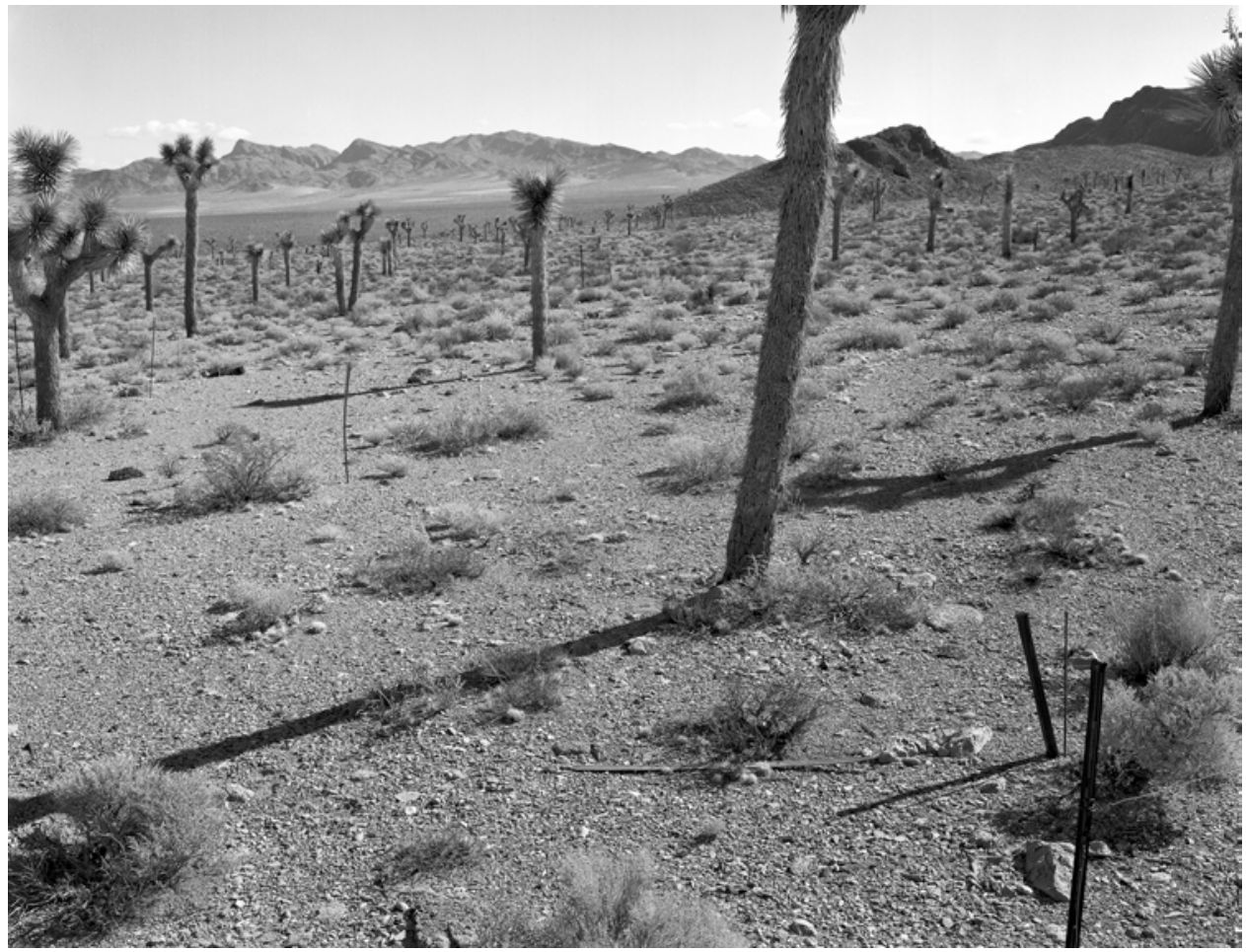

B. (April 20, 2002). Shadscale now dominates the vegetation on this plot. The Joshua trees have greatly increased in height and density (Dominic Oldershaw, Stake 4172A). 
Table A-35B. Summary plant data for Plot 35.

SPECIES
Astragalus tidestromii
Atriplex confertifolia
Cryptantha virginensis
Ephedra torreyana
Eriogonum inflatum
Erioneuron pulchellum
Krameria parvifolia
Larrea tridentata
Lepidium fremontii
Lycium shockleyi
Menodora spinescens
Opuntia basilaris
Oryzopsis hymenoides
Psorothamnus fremontii
Sphaeralcea parvifolia
Stipa speciosa
Yucca brevifolia
Yucca schidigera
Total Live
Dead Grass
Dead Shrub
Total

$\begin{array}{ccc}\mathbf{1 9 6 3} & \text { Number of Plants } & \\ 1 & \mathbf{1 9 7 5} & \mathbf{2 0 0 2} \\ 23 & 6 & 0 \\ 0 & 32 & 58 \\ 3 & 4 & 0 \\ 0 & 5 & 1 \\ 0 & 14 & 1 \\ 15 & 0 & 122 \\ 3 & 14 & 7 \\ 0 & 5 & 3 \\ 40 & 1 & 1 \\ 33 & 42 & 14 \\ 1 & 44 & 8 \\ 1 & 0 & 2 \\ 2 & 0 & 0 \\ 0 & 0 & 0 \\ 0 & 7 & 3 \\ 0 & 1 & 0 \\ 0 & 1 & 5 \\ 122 & 0 & 1 \\ 0 & 176 & 226 \\ 10 & 0 & 1 \\ 132 & 12 & 129 \\ & 188 & 356\end{array}$

SPECIES
Astragalus tidestromii
Atriplex confertifolia
Cryptantha virginensis
Ephedra torreyana
Eriogonum inflatum
Erioneuron pulchellum
Krameria parvifolia
Larrea tridentata
Lepidium fremontii
Lycium shockleyi
Menodora spinescens
Opuntia basilaris
Oryzopsis hymenoides
Psorothamnus fremontii
Sphaeralcea parvifolia
Stipa speciosa
Yucca brevifolia
Yucca schidigera
Total Live
Dead Grass
Dead Shrub
Total

$\begin{array}{ccc} & \text { Cover (\%) } & \\ \mathbf{1 9 6 3} & \mathbf{1 9 7 5} & \mathbf{2 0 0 2} \\ 0.02 & 0.13 & 0.00 \\ 1.52 & 2.84 & 2.62 \\ 0.00 & 0.09 & 0.00 \\ 0.29 & 0.33 & 0.21 \\ 0.00 & 0.32 & 0.01 \\ 0.00 & 0.00 & 1.56 \\ 1.35 & 1.05 & 0.35 \\ 0.32 & 0.39 & 0.35 \\ 0.00 & 0.05 & 0.00 \\ 4.24 & 3.38 & 0.53 \\ 2.05 & 3.73 & 0.53 \\ 0.02 & 0.00 & 0.06 \\ 0.05 & 0.00 & 0.00 \\ 0.14 & 0.00 & 0.00 \\ 0.00 & 0.25 & 0.02 \\ 0.00 & 0.08 & 0.00 \\ 0.00 & 0.10 & 0.61 \\ 0.00 & 0.00 & 0.01 \\ 9.98 & 12.74 & 6.85 \\ 0.00 & 0.00 & 0.03 \\ 0.77 & 0.75 & 8.95 \\ 10.75 & 13.48 & 15.82\end{array}$

$\begin{array}{llll}\text { SPECIES } & \mathbf{1 9 6 3} & \mathbf{1 9 7 5} & \mathbf{2 0 0 2} \\ \text { Astragalus tidestromii } & 0.08 & 0.09 & 0.00 \\ \text { Atriplex confertifolia } & 0.19 & 0.26 & 0.19 \\ \text { Cryptantha virginensis } & 0.00 & 0.10 & 0.00 \\ \text { Ephedra torreyana } & 0.40 & 0.35 & 0.43 \\ \text { Eriogonum inflatum } & 0.00 & 0.16 & 0.09 \\ \text { Erioneuron pulchellum } & 0.00 & 0.00 & 0.03 \\ \text { Krameria parvifolia } & 0.16 & 0.17 & 0.25 \\ \text { Larrea tridentata } & 0.42 & 0.33 & 0.59 \\ \text { Lepidium fremontii } & 0.00 & 0.30 & 0.19 \\ \text { Lycium shockleyi } & 0.21 & 0.20 & 0.26 \\ \text { Menodora spinescens } & 0.13 & 0.13 & 0.14 \\ \text { Opuntia basilaris } & 0.05 & 0.00 & 0.12 \\ \text { Oryzopsis hymenoides } & 0.23 & 0.00 & 0.00 \\ \text { Psorothamnus fremontii } & 0.18 & 0.00 & 0.00 \\ \text { Sphaeralcea parvifolia } & 0.00 & 0.20 & 0.10 \\ \text { Stipa speciosa } & 0.00 & 0.41 & 0.00 \\ \text { Yucca brevifolia } & 0.00 & 2.46 & 2.61 \\ \text { Yucca schidigera } & 0.00 & 0.00 & 0.16\end{array}$

$\begin{array}{llll}\text { SPECIES } & \mathbf{1 9 6 3} & \mathbf{1 9 7 5} & \mathbf{2 0 0 2} \\ \text { Astragalus tidestromii } & 0.00 & 0.06 & 0.00 \\ \text { Atriplex confertifolia } & 1.05 & 2.55 & 1.89 \\ \text { Cryptantha virginensis } & 0.00 & 0.03 & 0.00 \\ \text { Ephedra torreyana } & 0.37 & 0.43 & 0.30 \\ \text { Eriogonum inflatum } & 0.00 & 0.17 & 0.00 \\ \text { Erioneuron pulchellum } & 0.00 & 0.00 & 0.03 \\ \text { Krameria parvifolia } & 0.73 & 0.66 & 0.25 \\ \text { Larrea tridentata } & 0.49 & 0.48 & 0.72 \\ \text { Lepidium fremontii } & 0.00 & 0.06 & 0.00 \\ \text { Lycium shockleyi } & 3.06 & 2.33 & 0.47 \\ \text { Menodora spinescens } & 1.02 & 1.83 & 0.26 \\ \text { Opuntia basilaris } & 0.00 & 0.00 & 0.03 \\ \text { Oryzopsis hymenoides } & 0.04 & 0.00 & 0.00 \\ \text { Psorothamnus fremontii } & 0.10 & 0.00 & 0.00 \\ \text { Sphaeralcea parvifolia } & 0.00 & 0.19 & 0.00 \\ \text { Stipa speciosa } & 0.00 & 0.11 & 0.00 \\ \text { Yucca brevifolia } & 0.00 & 0.83 & 5.86 \\ \text { Yucca schidigera } & 0.00 & 0.00 & 0.00 \\ \text { Total Live } & 6.87 & 9.73 & 9.83\end{array}$


Table A-36A. Site characteristics for Plot 36.

Location: Frenchman Flat

NTS Area: 5

NTS Grid: Z-15

USGS 7.5’ Quadrangle Name: Frenchman Lake

\begin{tabular}{|c|c|c|c|c|}
\hline & & GPS Reac & Zone 11S) & \\
\hline & Location & Northing & Easting & Condition of Original Corners \\
\hline Corner 1: & SW & 4071195 & 596037 & NA \\
\hline Corner 2: & NW & 4071226 & 596046 & NA \\
\hline Corner 3: & $\mathrm{NE}$ & 4071221 & 596075 & NA \\
\hline Corner 4: & SE & 4071193 & 596067 & NA \\
\hline DEM Plot & vation: 93 & & & Plot Aspect: $108^{\circ}$ \\
\hline Elevation & e Playa ( & Name): 3 & nchman) & Plot Slope: $2^{\circ}$ \\
\hline
\end{tabular}

Annual Precipitation

Measured: $126 \mathrm{~mm}$

Modeled: $123 \mathrm{~mm}$

Parent Material Type: playa

Substrate: cobbles, well developed crust

Slate Geologic Unit: Qay

Beatley Plant Assemblage: Larrea-Lycium shockleyi-Atriplex

Ostler-Hanson Plant Association: Lycium shockleyi-Larrea-Atriplex confertifolia

Ostler-Hanson Land Unit: NA

Abundance of Biological Soil Crusts: well developed

Type and Date of Disturbance: none

Plot Condition: good

Location of Transect 1: north side of plot

Direction Transects Are Read: east to west

Date(s) Plot Remeasured: 17 April 2002 
Figure A-36. Photographs showing Plot 36.

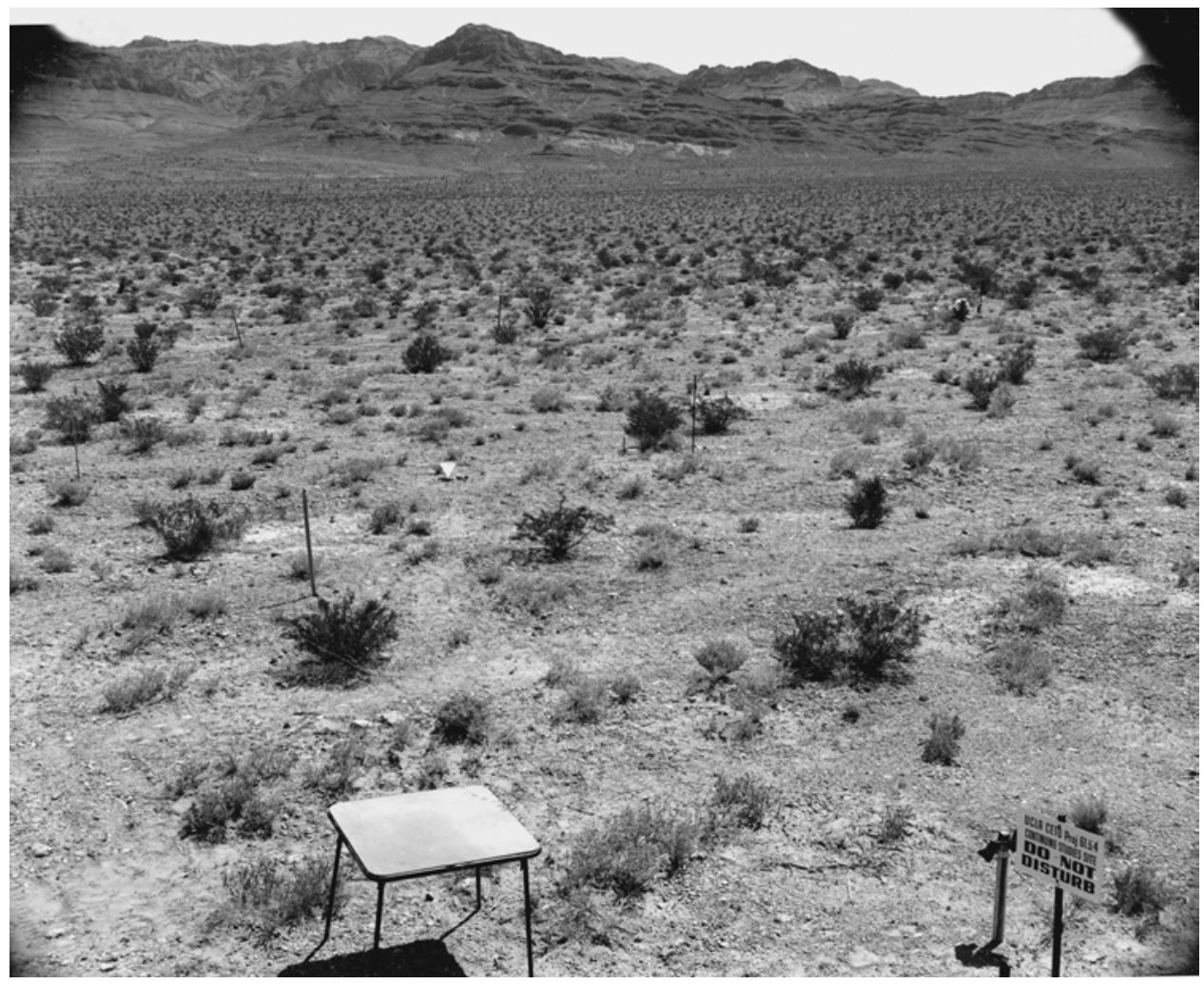

A. (April 15, 1964). This southeasterly view across Plot 36 shows an alluvial fan with the Ranger Mountains in the background. The vegetation is dominated by creosote bush, Shockley's desert thorn, and shadscale. (Janice Beatley Collection, 1-A).

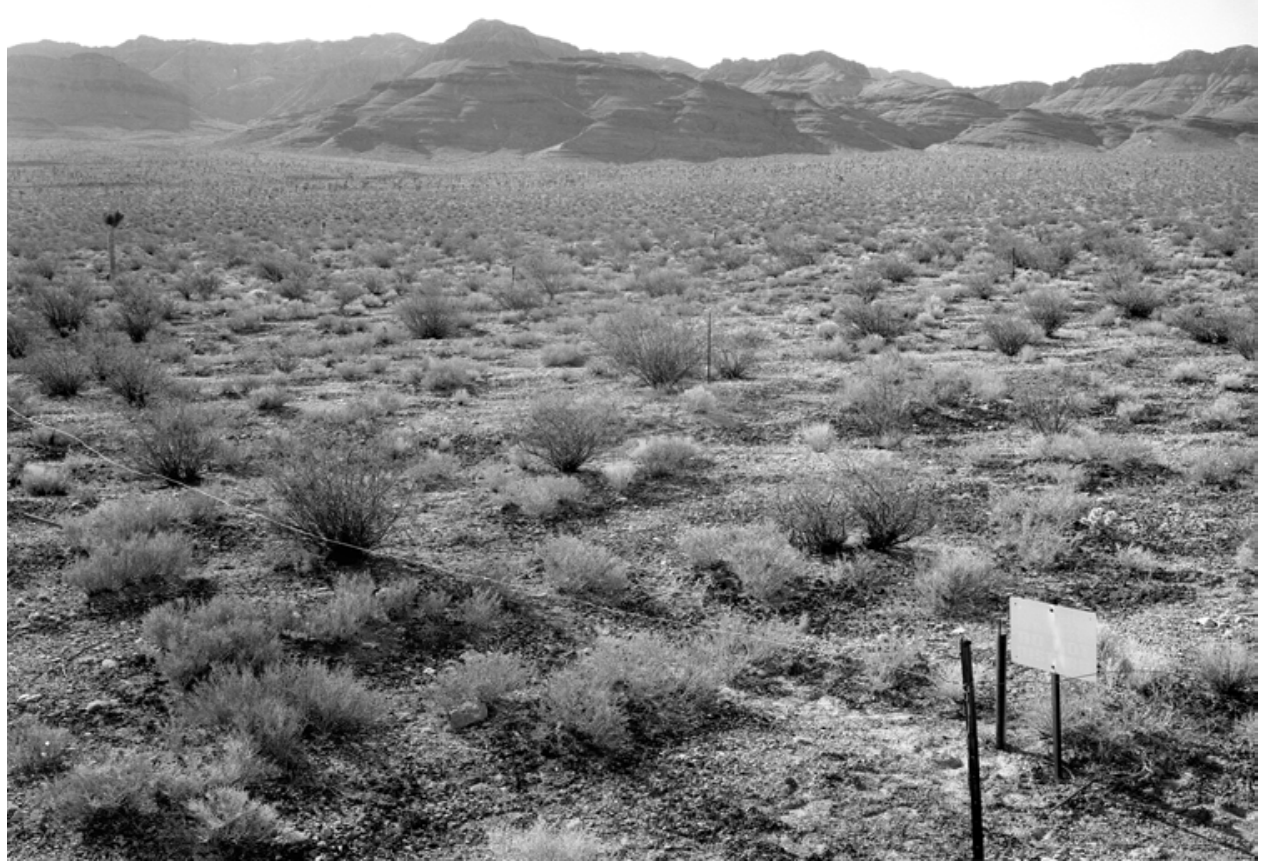

B. (April 20, 2002). The camera station is behind the original camera station, leading to a difference in perspective on the signs in the lower right foreground. Creosote bush have increased in size on the plot, and now white bursage is more numerically dominant than shadscale. Biological soil crusts are prominent in the foreground of this view and appear to have increased following the foot-traffic disturbance that affected the plot during its original measurements (Dominic Oldershaw, Stake 4169B). 
Table A-36B. Summary plant data for Plot 36.

\begin{tabular}{|c|c|c|c|c|c|c|c|}
\hline \multicolumn{5}{|c|}{ Number of Plants } & \multicolumn{3}{|c|}{ Cover (\%) } \\
\hline SPECIES & 1963 & 1975 & 2002 & SPECIES & 1963 & 1975 & 2002 \\
\hline Acamptopappus shockleyi & 27 & 38 & 4 & Acamptopappus shockleyi & 1.80 & 1.99 & 0.09 \\
\hline Ambrosia dumosa & 8 & 14 & 37 & Ambrosia dumosa & 0.73 & 0.79 & 2.49 \\
\hline Atriplex confertifolia & 15 & 12 & 12 & Atriplex confertifolia & 1.05 & 1.16 & 0.56 \\
\hline Baileya multiradiata & 0 & 1 & 0 & Baileya multiradiata & 0.00 & 0.01 & 0.00 \\
\hline Eriogonum inflatum & 0 & 6 & 1 & Eriogonum inflatum & 0.00 & 0.15 & 0.01 \\
\hline Erioneuron pulchellum & 0 & 9 & 28 & Erioneuron pulchellum & 0.00 & 0.18 & 0.36 \\
\hline Hymenoclea salsola & 1 & 1 & 0 & Hymenoclea salsola & 0.05 & 0.10 & 0.00 \\
\hline Krameria parvifolia & 0 & 0 & 1 & Krameria parvifolia & 0.00 & 0.00 & 0.00 \\
\hline Larrea tridentata & 14 & 20 & 22 & Larrea tridentata & 2.65 & 4.10 & 5.39 \\
\hline Lycium shockleyi & 50 & 58 & 48 & Lycium shockleyi & 4.45 & 5.43 & 4.06 \\
\hline Mirabilis pudica & 0 & 0 & 1 & Mirabilis pudica & 0.00 & 0.00 & 0.00 \\
\hline Opuntia echinocarpa & 1 & 1 & 0 & Opuntia echinocarpa & 0.04 & 0.01 & 0.00 \\
\hline Opuntia ramosissima & 1 & 1 & 1 & Opuntia ramosissima & 0.04 & 0.05 & 0.02 \\
\hline Oryzopsis hymenoides & 1 & 2 & 0 & Oryzopsis hymenoides & 0.05 & 0.07 & 0.00 \\
\hline Psorothamnus fremontii & 2 & 3 & 0 & Psorothamnus fremontii & 0.29 & 0.43 & 0.00 \\
\hline Stephanomeria pauciflora & 0 & 0 & 0 & Stephanomeria pauciflora & 0.00 & 0.00 & 0.00 \\
\hline Thamnosma montana & 4 & 7 & 0 & Thamnosma montana & 0.35 & 0.63 & 0.00 \\
\hline Total Live & 124 & 173 & 155 & Total Live & 11.49 & 15.09 & 12.99 \\
\hline Dead Grass & 0 & 0 & 0 & Dead Grass & 0.00 & 0.00 & 0.00 \\
\hline Dead Shrub & 2 & 8 & 78 & Dead Shrub & 0.30 & 0.59 & 6.21 \\
\hline Total & 126 & 181 & 233 & Total & 11.79 & 15.68 & 19.20 \\
\hline \multicolumn{5}{|c|}{ Average Height (m) } & \multicolumn{3}{|c|}{ Biomass Index $\left(\mathrm{m}^{2}\right)$} \\
\hline SPECIES & 1963 & 1975 & 2002 & SPECIES & 1963 & 1975 & 2002 \\
\hline Acamptopappus shockleyi & 0.15 & 0.16 & 0.14 & Acamptopappus shockleyi & 0.95 & 1.17 & 0.05 \\
\hline Ambrosia dumosa & 0.21 & 0.20 & 0.26 & Ambrosia dumosa & 0.50 & 0.57 & 2.37 \\
\hline Atriplex confertifolia & 0.26 & 0.29 & 0.26 & Atriplex confertifolia & 0.93 & 1.28 & 0.59 \\
\hline Baileya multiradiata & 0.00 & 0.08 & 0.00 & Baileya multiradiata & 0.00 & 0.00 & 0.00 \\
\hline Eriogonum inflatum & 0.00 & 0.05 & 0.00 & Eriogonum inflatum & 0.00 & 0.03 & 0.00 \\
\hline Erioneuron pulchellum & 0.00 & 0.03 & 0.00 & Erioneuron pulchellum & 0.00 & 0.02 & 0.00 \\
\hline Hymenoclea salsola & 0.28 & 0.30 & 0.00 & Hymenoclea salsola & 0.05 & 0.10 & 0.00 \\
\hline Krameria parvifolia & 0.00 & 0.00 & 0.33 & Krameria parvifolia & 0.00 & 0.00 & 0.00 \\
\hline Larrea tridentata & 0.58 & 0.81 & 1.21 & Larrea tridentata & 5.54 & 11.47 & 22.59 \\
\hline Lycium shockleyi & 0.19 & 0.23 & 0.26 & Lycium shockleyi & 3.01 & 4.33 & 3.68 \\
\hline Mirabilis pudica & 0.00 & 0.00 & 0.21 & Mirabilis pudica & 0.00 & 0.00 & 0.00 \\
\hline Opuntia echinocarpa & 0.13 & 0.13 & 0.00 & Opuntia echinocarpa & 0.02 & 0.00 & 0.00 \\
\hline Opuntia ramosissima & 0.13 & 0.15 & 0.05 & Opuntia ramosissima & 0.02 & 0.02 & 0.00 \\
\hline Oryzopsis hymenoides & 0.23 & 0.20 & 0.00 & Oryzopsis hymenoides & 0.03 & 0.05 & 0.00 \\
\hline Psorothamnus fremontii & 0.33 & 0.36 & 0.00 & Psorothamnus fremontii & 0.31 & 0.53 & 0.00 \\
\hline Stephanomeria pauciflora & 0.00 & 0.00 & 0.00 & Stephanomeria pauciflora & 0.00 & 0.00 & 0.00 \\
\hline Thamnosma montana & 0.23 & 0.29 & 0.00 & Thamnosma montana & 0.29 & 0.64 & 0.00 \\
\hline & & & & Total Live & 11.65 & 20.22 & 29.28 \\
\hline
\end{tabular}


Table A-37A. Site characteristics for Plot 37.

Location: Frenchman Flat

NTS Area: 5

NTS Grid: Z-15

USGS 7.5’ Quadrangle Name: Frenchman Lake

\begin{tabular}{|c|c|c|c|c|}
\hline & & GPS Reac & Zone 11S) & \\
\hline & Location & Northing & Easting & Condition of Original Corners \\
\hline Corner 1: & SW & 4071377 & 596085 & Standing \\
\hline Corner 2: & NW & 4071405 & 596094 & Standing \\
\hline Corner 3: & $\mathrm{NE}$ & 4071396 & 596126 & Standing \\
\hline Corner 4: & SE & 4071368 & 596115 & Standing \\
\hline DEM Plo & vation: 93 & & & Plot Aspect: $315^{\circ}$ \\
\hline Elevation & e Playa ( & Name): 3 & nchman) & Plot Slope: $6^{\circ}$ \\
\hline
\end{tabular}

Annual Precipitation

Measured: $129 \mathrm{~mm}$

Modeled: $123 \mathrm{~mm}$

Parent Material Type: playa

Substrate: relatively old laggard surface, silty alluvial w/solid gravel veneer and limestone quartzite

Slate Geologic Unit: Qay

Beatley Plant Assemblage: Lycium shockleyi-Atriplex

Ostler-Hanson Plant Association: Lycium shockleyi-Atriplex confertifolia

Ostler-Hanson Land Unit: NA

Abundance of Biological Soil Crusts: lots

Type and Date of Disturbance: none

Plot Condition: good

Location of Transect 1: south side of plot

Direction Transects Are Read: west to east

Date(s) Plot Remeasured: 17 April 2002 
Figure A-37. Photographs showing Plot 37.

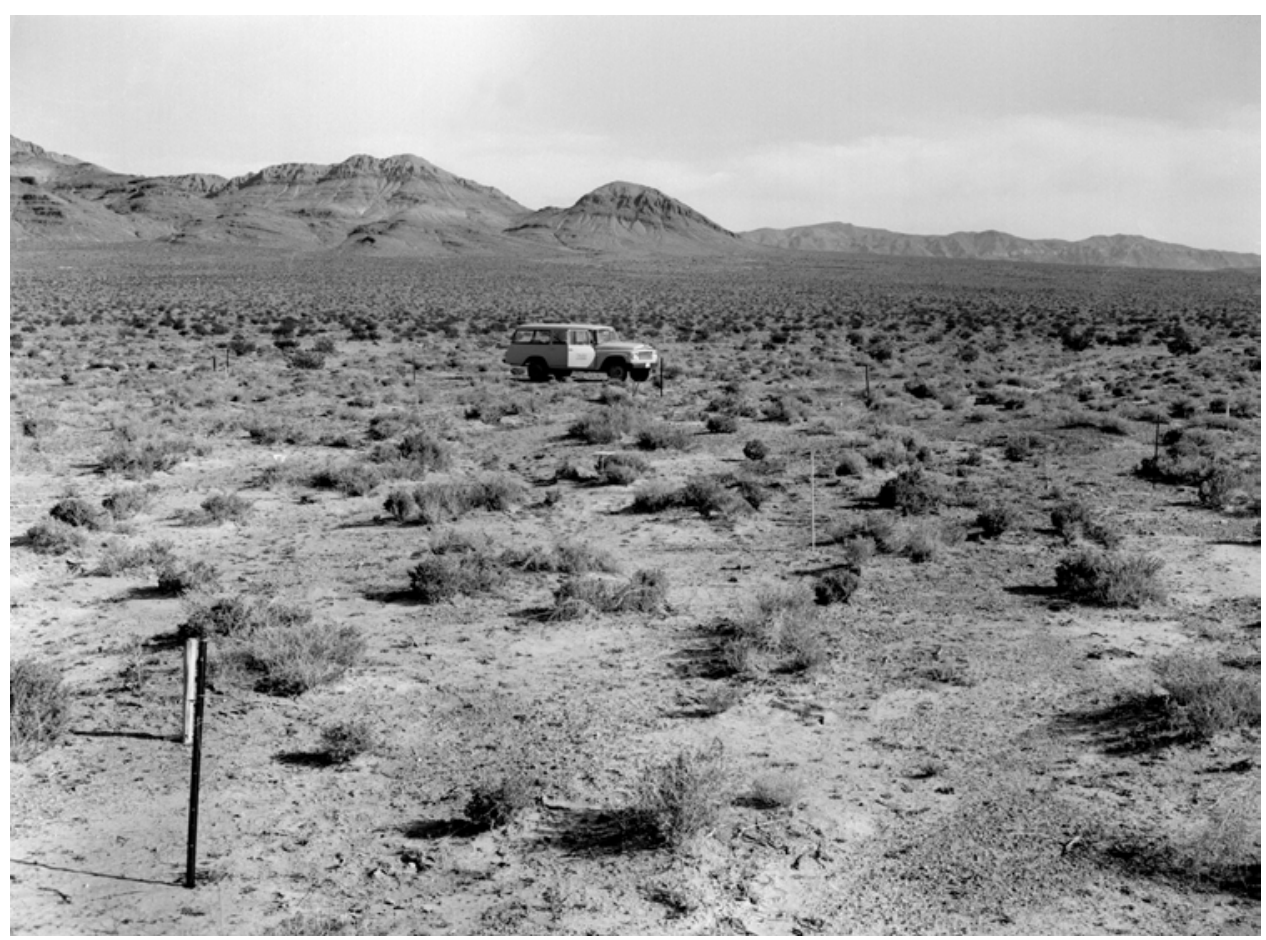

A. (April 22, 1964). This southeasterly view across Plot 37 shows an assemblage dominated by Shockley's desert thorn and shadscale. No Joshua trees are apparent in this view (Janice Beatley Collection, 35-B).

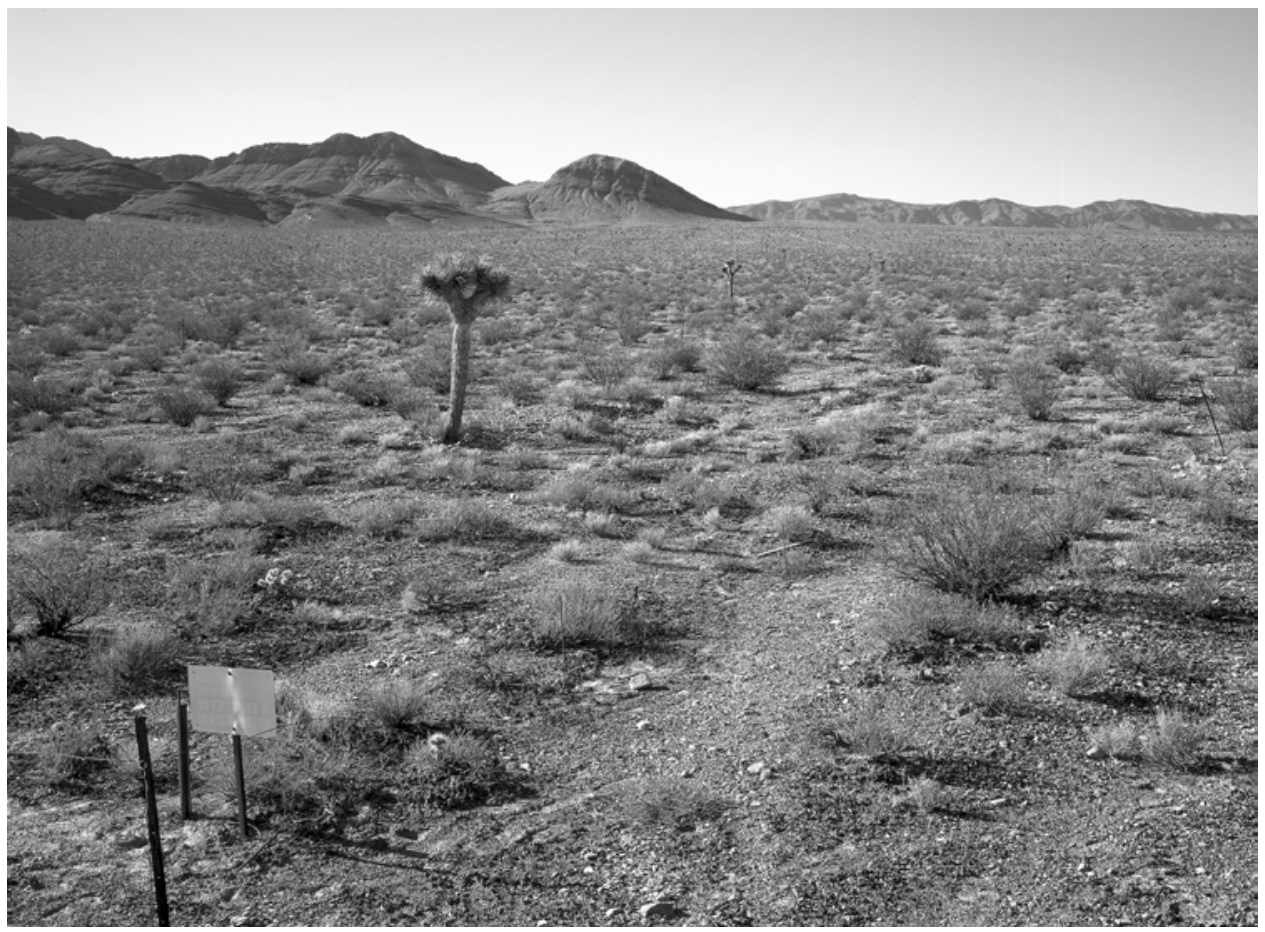

B. (April 20, 2002). Biological soil crusts now prominently appear throughout the plot. Shadscale has greatly decreased here, and several new Joshua trees appear (Dominic Oldershaw, Stake 4170B). 
Table A-37B. Summary plant data for Plot 37.

\begin{tabular}{|c|c|c|c|c|c|c|c|}
\hline \multicolumn{5}{|c|}{ Number of Plants } & \multicolumn{3}{|c|}{ Cover (\%) } \\
\hline SPECIES & 1963 & 1975 & 2002 & SPECIES & 1963 & 1975 & 2002 \\
\hline Acamptopappus shockleyi & 0 & 2 & 0 & Acamptopappus shockleyi & 0.00 & 0.04 & 0.00 \\
\hline Ambrosia dumosa & 0 & 0 & 2 & Ambrosia dumosa & 0.00 & 0.00 & 0.08 \\
\hline Atriplex confertifolia & 20 & 30 & 0 & Atriplex confertifolia & 1.55 & 2.52 & 0.00 \\
\hline Ceratoides lanata & 3 & 3 & 1 & Ceratoides lanata & 0.26 & 0.25 & 0.09 \\
\hline Larrea tridentata & 0 & 2 & 1 & Larrea tridentata & 0.00 & 0.05 & 0.22 \\
\hline Lycium shockleyi & 79 & 97 & 136 & Lycium shockleyi & 10.10 & 14.08 & 9.25 \\
\hline Oryzopsis hymenoides & 6 & 8 & 0 & Oryzopsis hymenoides & 0.17 & 0.35 & 0.00 \\
\hline Sphaeralcea parvifolia & 0 & 3 & 0 & Sphaeralcea parvifolia & 0.00 & 0.06 & 0.00 \\
\hline Stanleya pinnata & 0 & 5 & 0 & Stanleya pinnata & 0.00 & 0.34 & 0.00 \\
\hline Total Live & 108 & 150 & 140 & Total Live & 12.09 & 17.68 & 9.65 \\
\hline Dead Grass & 0 & 0 & 1 & Dead Grass & 0.00 & 0.00 & 0.01 \\
\hline Dead Shrub & 0 & 6 & 71 & Dead Shrub & 0.00 & 0.47 & 8.20 \\
\hline Total & 108 & 156 & 212 & Total & 12.09 & 18.15 & 17.85 \\
\hline \multicolumn{5}{|c|}{ Average Height (m) } & \multicolumn{3}{|c|}{ Biomass Index $\left(\mathrm{m}^{2}\right)$} \\
\hline SPECIES & 1963 & 1975 & 2002 & SPECIES & 1963 & 1975 & 2002 \\
\hline Acamptopappus shockleyi & 0.00 & 0.11 & 0.00 & Acamptopappus shockleyi & 0.00 & 0.02 & 0.00 \\
\hline Ambrosia dumosa & 0.00 & 0.00 & 0.21 & Ambrosia dumosa & 0.00 & 0.00 & 0.06 \\
\hline Atriplex confertifolia & 0.28 & 0.30 & 0.00 & Atriplex confertifolia & 1.62 & 3.29 & 0.00 \\
\hline Ceratoides lanata & 0.33 & 0.52 & 0.66 & Ceratoides lanata & 0.30 & 0.44 & 0.20 \\
\hline Larrea tridentata & 0.00 & 0.04 & 0.82 & Larrea tridentata & 0.00 & 0.01 & 0.60 \\
\hline Lycium shockleyi & 0.24 & 0.31 & 0.30 & Lycium shockleyi & 9.07 & 15.93 & 9.80 \\
\hline Oryzopsis hymenoides & 0.19 & 0.26 & 0.00 & Oryzopsis hymenoides & 0.12 & 0.32 & 0.00 \\
\hline Sphaeralcea parvifolia & 0.00 & 0.16 & 0.00 & Sphaeralcea parvifolia & 0.00 & 0.03 & 0.00 \\
\hline \multirow[t]{2}{*}{ Stanleya pinnata } & 0.00 & 0.43 & 0.00 & Stanleya pinnata & 0.00 & 0.48 & 0.00 \\
\hline & & & & Total Live & 11.10 & 20.52 & 10.66 \\
\hline
\end{tabular}


Table A-38A. Site characteristics for Plot 38.

Location: Frenchman Flat

NTS Area: 5

NTS Grid: W-16

USGS 7.5’ Quadrangle Name: Frenchman Lake

\begin{tabular}{|c|c|c|c|c|}
\hline & & GPS Reac & Zone 11S) & \\
\hline & Location & Northing & Easting & Condition of Original Corners \\
\hline Corner 1: & SW & 4072424 & 590614 & Standing \\
\hline Corner 2: & NW & 4072454 & 570619 & Standing \\
\hline Corner 3: & $\mathrm{NE}$ & 4072431 & 590648 & Standing \\
\hline Corner 4: & SE & 4072420 & 590644 & Standing \\
\hline DEM Plot & vation: 95 & & & Plot Aspect: $153^{\circ}$ \\
\hline Elevation & e Playa ( & Name): 6 & nchman) & Plot Slope: $3^{\circ}$ \\
\hline
\end{tabular}

Annual Precipitation

Measured: $121 \mathrm{~mm}$

Modeled: $123 \mathrm{~mm}$

Parent Material Type: alluvial fan

Substrate: mixed alluvium, sandy w/pebbles

Slate Geologic Unit: Qay

Beatley Plant Assemblage: Lycium pallidum-Grayia

Ostler-Hanson Plant Association: Lycium pallidum-Psorothamnus-Grayia

Ostler-Hanson Land Unit: 80

Abundance of Biological Soil Crusts: none

Type and Date of Disturbance: one motorcycle track through plot, some rodent activity

Plot Condition: good

Location of Transect 1: south side of plot

Direction Transects Are Read: east to west

Date(s) Plot Remeasured: 17 April 2002 
Figure A-38. Photographs showing Plot 38.

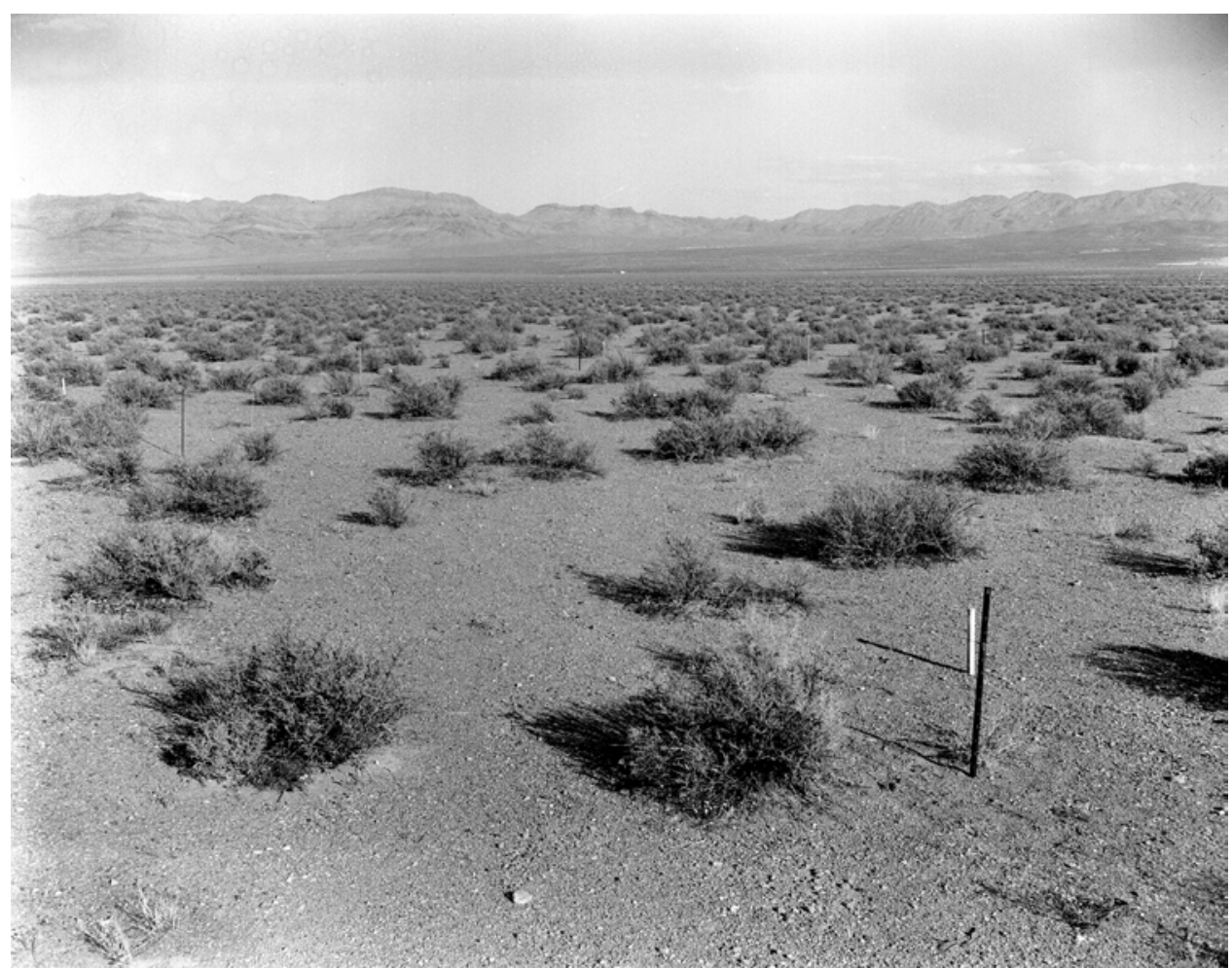

A. (May 11, 1964). In this south-southeasterly view across Plot 38, Frenchman Lake and the Ranger Mountains appear in the distance. Rabbit thorn (Lycium pallidum v. oligospermum) and spiny hopsage are the dominants on this plot (Janice Beatley Collection, 68-A).

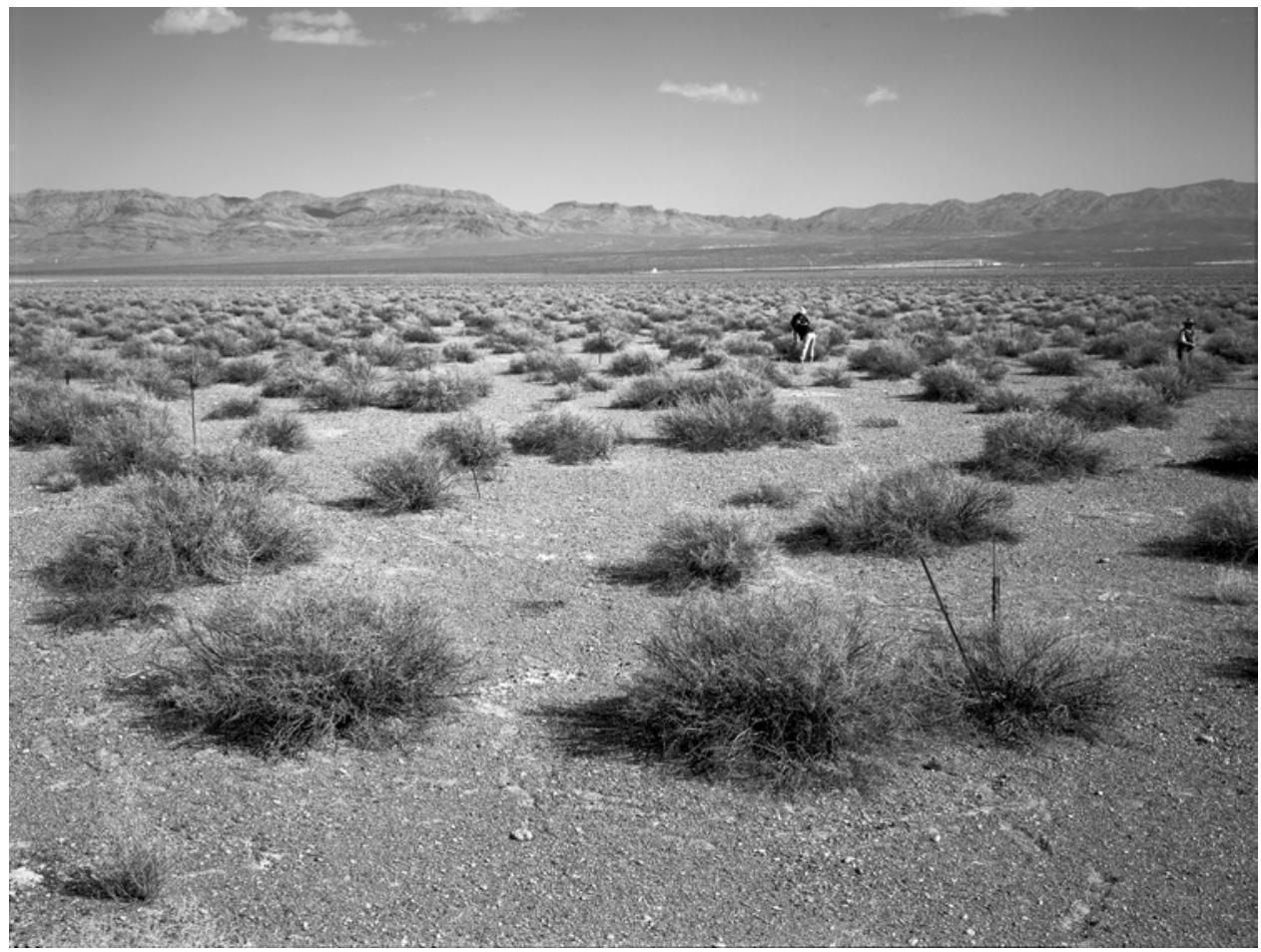

B. (April 17, 2002). The plot is still dominated by rabbit thorn and spiny hopsage, although many individuals of the latter species have died (Dominic Oldershaw, Stake 4147A). 
Table A-38B. Summary plant data for Plot 38.

\begin{tabular}{lccc} 
& \multicolumn{3}{c}{ Number of Plants } \\
SPECIES & $\mathbf{1 9 6 3}$ & $\mathbf{1 9 7 5}$ & $\mathbf{2 0 0 2}$ \\
Ambrosia dumosa & 0 & 0 & 1 \\
Atriplex canescens & 4 & 14 & 1 \\
Ceratoides lanata & 1 & 0 & 1 \\
Grayia spinosa & 20 & 22 & 12 \\
Lycium andersonii & 2 & 0 & 0 \\
Lycium pallidum oligospermum & 48 & 48 & 58 \\
Mirabilis pudica & 4 & 9 & 1 \\
Oryzopsis hymenoides & 0 & 1 & 0 \\
Psorothamnus polydenius & 3 & 2 & 1 \\
Total Live & 82 & 96 & 75 \\
Dead Grass & 0 & 0 & 0 \\
Dead Shrub & 4 & 9 & 27 \\
Total & 86 & 105 & 102 \\
& & & \\
SPECIES & Average Height & $(\mathbf{m})$ \\
Ambrosia dumosa & $\mathbf{1 9 6 3}$ & $\mathbf{1 9 7 5}$ & $\mathbf{2 0 0 2}$ \\
Atriplex canescens & 0.00 & 0.00 & 0.25 \\
Ceratoides lanata & 0.60 & 0.59 & 0.51 \\
Grayia spinosa & 0.36 & 0.00 & 0.22 \\
Lycium andersonii & 0.54 & 0.61 & 0.58 \\
Lycium pallidum oligospermum & 0.67 & 0.73 & 0.77 \\
Mirabilis pudica & 0.27 & 0.09 & 0.00 \\
Oryzopsis hymenoides & 0.00 & 0.15 & 0.00 \\
Psorothamnus polydenius & 0.27 & 0.39 & 0.45 \\
& & &
\end{tabular}

SPECIES
Ambrosia dumosa
Atriplex canescens
Ceratoides lanata
Grayia spinosa
Lycium andersonii
Lycium pallidum oligospermum
Mirabilis pudica
Oryzopsis hymenoides
Psorothamnus polydenius
Total Live
Dead Grass
Dead Shrub
Total

\section{SPECIES}

Ambrosia dumosa

Atriplex canescens

Ceratoides lanata

Grayia spinosa

Lycium andersonii

Lycium pallidum oligospermum

Mirabilis pudica

Oryzopsis hymenoides

Psorothamnus polydenius

Total Live
Cover (\%)

1975

2002

$\begin{array}{lll}1963 & 0.00 & 0.00\end{array}$

$\begin{array}{lll}0.45 & 1.38 & 0.09\end{array}$

$\begin{array}{lll}0.05 & 0.00 \quad 0.00\end{array}$

$2.41 \quad 2.52 \quad 1.21$

$\begin{array}{lll}0.30 & 0.00 \quad 0.00\end{array}$

$10.45 \quad 13.13 \quad 15.67$

$\begin{array}{lll}0.25 & 0.72 & 0.07\end{array}$

$\begin{array}{lll}0.00 & 0.07 & 0.00\end{array}$

$\begin{array}{lll}0.29 & 0.35 & 0.01\end{array}$

$14.20 \quad 18.16 \quad 17.12$

$\begin{array}{lll}0.00 & 0.00 & 0.00\end{array}$

$\begin{array}{lll}0.34 & 1.13 & 5.75\end{array}$

$14.54 \quad 19.29 \quad 22.86$

\section{Biomass Index $\left(\mathrm{m}^{2}\right)$}

$\begin{array}{ccc}\mathbf{1 9 6 3} & \mathbf{1 9 7 5} & \mathbf{2 0 0 2} \\ 0.00 & 0.00 & 0.05 \\ 0.94 & 2.83 & 0.16 \\ 0.07 & 0.00 & 0.00 \\ 4.51 & 5.72 & 2.49 \\ 0.59 & 0.00 & 0.00 \\ 24.25 & 33.97 & 41.80 \\ 0.24 & 0.22 & 0.00 \\ 0.00 & 0.04 & 0.00 \\ 0.25 & 0.45 & 0.01 \\ 30.85 & 43.22 & 44.50\end{array}$


Table A-39A. Site characteristics for Plot 39.

Location: Mid Valley

NTS Area: 14

NTS Grid: L-24

USGS 7.5’ Quadrangle Name: Mine Mountain

\begin{tabular}{lllll} 
& & \multicolumn{2}{l}{ GPS Readings (Zone 11S) } & \\
& Location & Northing & Easting & Condition of Original Corners \\
Corner 1: & SW & 4086870 & 574057 & Standing \\
Corner 2: & NW & 4086900 & 574064 & Standing \\
Corner 3: & NE & 4086890 & 574093 & Standing \\
Corner 4: & SE & 4086862 & 574086 & Standing
\end{tabular}

DEM Plot Elevation: $1381 \mathrm{~m}$

Plot Aspect: $329^{\circ}$

Elevation Above Playa (Playa Name): NA

Plot Slope: $8^{\circ}$

Annual Precipitation

Measured: $204 \mathrm{~mm}$

Modeled: $189 \mathrm{~mm}$

Parent Material Type: alluvial fan

Substrate: alluvial fan, rhyolite

Slate Geologic Unit: Qai

Beatley Plant Assemblage: Coleogyne/Grayia-Lycium

Ostler-Hanson Plant Association: Coleogyne-Grayia-Ephedra

Ostler-Hanson Land Unit: 455

Abundance of Biological Soil Crusts: patchy but locally heavy

Type and Date of Disturbance: none (control for Plot 40)

Plot Condition: good

Location of Transect 1: west side of plot

Direction Transects Are Read: south to north

Date(s) Plot Remeasured: 13 May 2000 
Figure A-39. Photograph showing Plot 39.

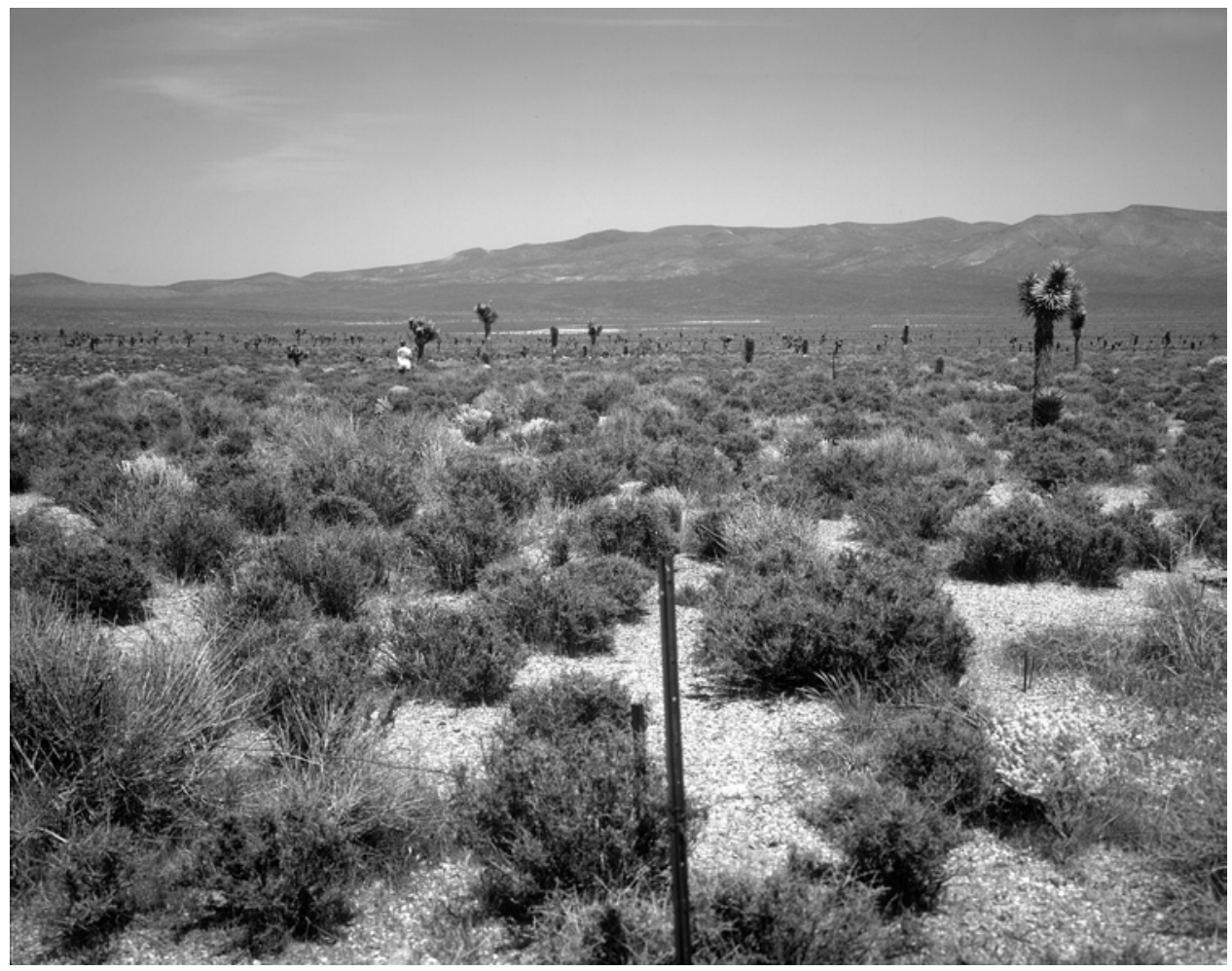

(May 10, 2000). This northeasterly view across Plot 39 shows a blackbrush assemblage in Mid Valley. Mine Mountain appears in the distance. Mormon tea and wolfberry are the subdominants on this plot. This plot either was not photographed in 1964 or the negatives have been lost (R.H. Webb, Stake 4029). 
Table A-39B. Summary plant data for Plot 39.

\begin{tabular}{|c|c|c|c|}
\hline SPECIES & 1963 & 1975 & 2000 \\
\hline Arabis pulchra munciensis & 0 & 1 & 0 \\
\hline Artemisia spinescens & 10 & 7 & 2 \\
\hline Ceratoides lanata & 11 & 21 & 7 \\
\hline Coleogyne ramosissima & 144 & 154 & 165 \\
\hline Ephedra nevadensis & 25 & 28 & 27 \\
\hline Ericameria cooperi & 10 & 9 & 10 \\
\hline Grayia spinosa & 47 & 51 & 6 \\
\hline Lycium andersonii & 23 & 23 & 19 \\
\hline Opuntia echinocarpa & 0 & 0 & 1 \\
\hline Oryzopsis hymenoides & 0 & 0 & 1 \\
\hline Sitanion hystrix & 2 & 12 & 0 \\
\hline Stephanomeria parryi & 0 & 1 & 0 \\
\hline Stipa speciosa & 14 & 37 & 2 \\
\hline Stipa x Oryzopsis hybrid & 1 & 4 & 0 \\
\hline Tetradymia axillaris & 2 & 1 & 0 \\
\hline Tetradymia glabrata & 1 & 2 & 0 \\
\hline Total Live & 290 & 351 & 240 \\
\hline Dead Grass & 0 & 0 & 13 \\
\hline Dead Shrubs & 33 & 10 & 62 \\
\hline Total & 323 & 361 & 315 \\
\hline & \multicolumn{3}{|c|}{ Average Height (m) } \\
\hline SPECIES & 1963 & 1975 & 2000 \\
\hline Arabis pulchra munciensis & 0.00 & 0.46 & 0.00 \\
\hline Artemisia spinescens & 0.17 & 0.16 & 0.15 \\
\hline Ceratoides lanata & 0.34 & 0.36 & 0.49 \\
\hline Coleogyne ramosissima & 0.38 & 0.36 & 0.47 \\
\hline Ephedra nevadensis & 0.43 & 0.44 & 0.60 \\
\hline Ericameria cooperi & 0.23 & 0.28 & 0.36 \\
\hline Grayia spinosa & 0.55 & 0.59 & 0.55 \\
\hline Lycium andersonii & 0.33 & 0.35 & 0.36 \\
\hline Opuntia echinocarpa & 0.00 & 0.00 & 0.69 \\
\hline Oryzopsis hymenoides & 0.00 & 0.00 & 0.46 \\
\hline Sitanion hystrix & 0.32 & 0.34 & 0.00 \\
\hline Stephanomeria parryi & 0.00 & 0.03 & 0.00 \\
\hline Stipa speciosa & 0.34 & 0.36 & 0.32 \\
\hline Stipa x Oryzopsis hybrid & 0.53 & 0.60 & 0.00 \\
\hline Tetradymia axillaris & 0.33 & 0.64 & 0.00 \\
\hline Tetradymia glabrata & 0.53 & 0.56 & 0.00 \\
\hline
\end{tabular}

SPECIES

Arabis pulchra munciensis

Artemisia spinescens

Ceratoides lanata

Coleogyne ramosissima

Ephedra nevadensis

Ericameria cooperi

Grayia spinosa

Lycium andersonii

Opuntia echinocarpa

Oryzopsis hymenoides

Sitanion hystrix

Stephanomeria parryi

Stipa speciosa

Stipa x Oryzopsis hybrid

Tetradymia axillaris

Tetradymia glabrata

Total Live

Dead Grass

Dead Shrubs

Total

SPECIES
Arabis pulchra munciensis
Artemisia spinescens
Ceratoides lanata
Coleogyne ramosissima
Ephedra nevadensis
Ericameria cooperi
Grayia spinosa
Lycium andersonii
Opuntia echinocarpa
Oryzopsis hymenoides
Sitanion hystrix
Stephanomeria parryi
Stipa speciosa
Stipa x Oryzopsis hybrid
Tetradymia axillaris
Tetradymia glabrata
Total Live

Cover (\%)

1963

0.00

0.62

0.55

16.89

3.72

0.68

6.75

2.46

0.00

0.00

0.13

0.00

0.75

0.06

0.07

0.11

32.79

0.00

3.76

36.55
1975

0.02

0.44

1.62

18.41

4.59

1.25

8.47

2.96

0.00

0.00

0.96

0.01

2.64

0.73

0.02

0.25

42.36

0.00

1.31

43.67
2000

0.00

0.05

0.71

20.67

4.45

1.09

0.50

1.45

0.02

0.02

0.00

0.00

0.06

0.00

0.00

0.00

29.03

0.51

10.07

39.61

\section{Biomass Index $\left(\mathrm{m}^{2}\right)$}

$\begin{array}{ccc}\mathbf{1 9 6 3} & \mathbf{1 9 7 5} & \mathbf{2 0 0 0} \\ 0.00 & 0.03 & 0.00 \\ 0.36 & 0.26 & 0.03 \\ 0.62 & 2.19 & 1.13 \\ 24.57 & 24.16 & 34.40 \\ 6.71 & 8.00 & 9.47 \\ 0.57 & 1.23 & 1.34 \\ 12.92 & 17.23 & 1.06 \\ 2.94 & 3.60 & 1.84 \\ 0.00 & 0.00 & 0.04 \\ 0.00 & 0.00 & 0.03 \\ 0.14 & 1.09 & 0.00 \\ 0.00 & 0.00 & 0.00 \\ 1.03 & 3.37 & 0.07 \\ 0.11 & 1.58 & 0.00 \\ 0.08 & 0.04 & 0.00 \\ 0.20 & 0.49 & 0.00 \\ 50.24 & 63.27 & 49.41\end{array}$


Table A-40A. Site characteristics for Plot 40.

Location: Mid Valley

NTS Area: 14

NTS Grid: L-24

USGS 7.5’ Quadrangle Name: Mine Mountain

\begin{tabular}{lllc} 
& & \multicolumn{2}{c}{ GPS Readings (Zone 11S) } \\
& Location & Northing & Easting \\
Corner 1: & SW & 4086764 & 574031 \\
Corner 2: & NW & 4086794 & 574040 \\
Corner 3: & NE & 4086789 & 574096 \\
Corner 4: & SE & 4086757 & 574061
\end{tabular}

DEM Plot Elevation: 1383 m

Condition of Original Corners
Standing
Standing
Standing
Standing

Elevation Above Playa (Playa Name): NA

Plot Aspect: $180^{\circ}$

Plot Slope: $14^{\circ}$

Annual Precipitation

Measured: $198 \mathrm{~mm}$

Modeled: $188 \mathrm{~mm}$

Parent Material Type: alluvial fan

Substrate: alluvial fan, rhyolite

Slate Geologic Unit: Qai

Beatley Plant Assemblage: Ephedra-Lycium

Ostler-Hanson Plant Association: NA

Ostler-Hanson Land Unit: 455

Abundance of Biological Soil Crusts: scattered, locally heavy

Type and Date of Disturbance: fire occurred in 1959

Plot Condition: good

Location of Transect 1: west side of plot

Direction Transects Are Read: south to north

Date(s) Plot Remeasured: 13 May 2000 
Figure A-40. Photographs showing Plot 40.

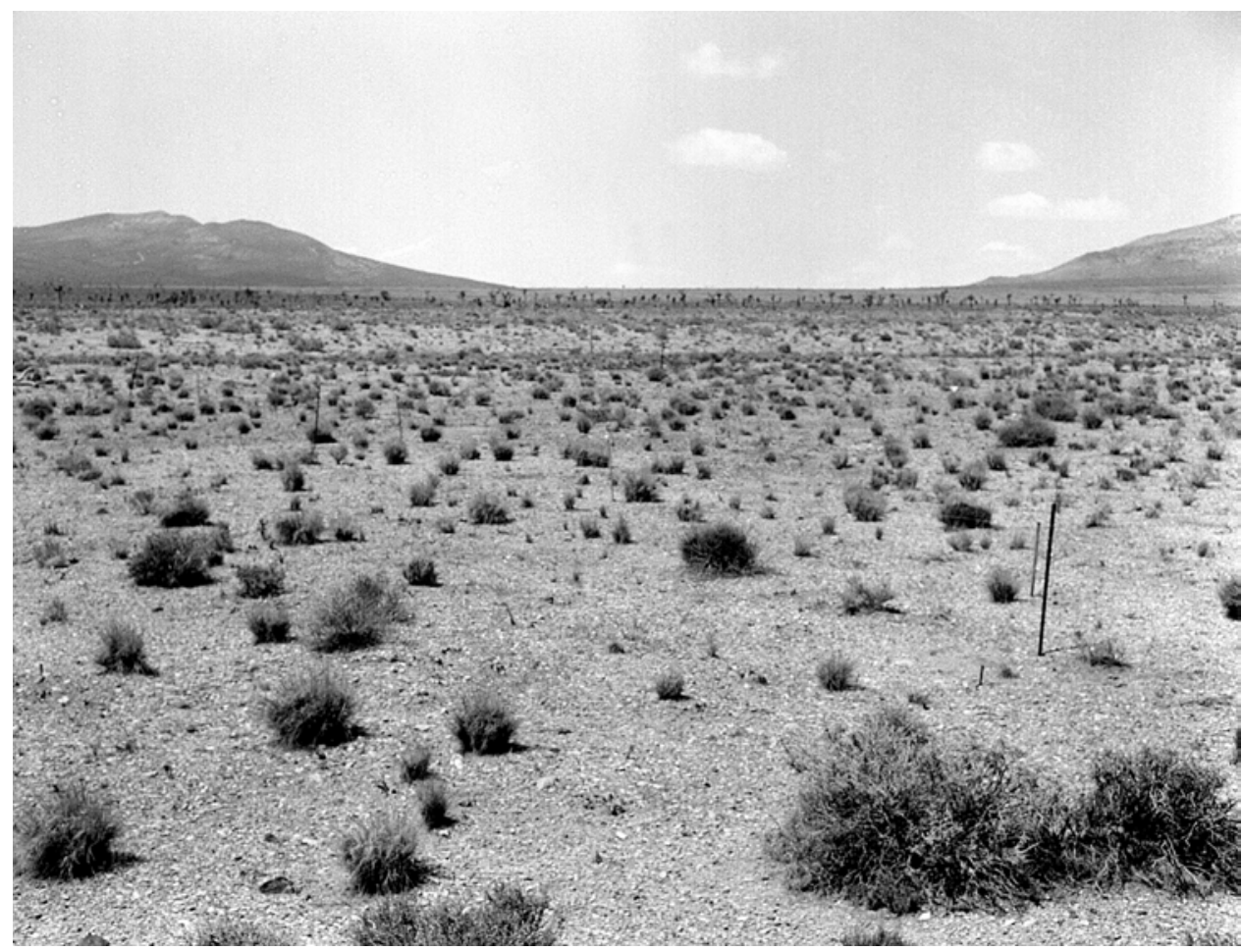

A. (1964). This southwesterly view across Plot 40 shows an area recovering from a fire that occurred in 1959. The southern flanks of Shoshone Mountain appear in the distance at right, and an unnamed mountain range that has Lookout Peak as its summit appears in the distance at left. Scattered Mormon tea and perennial grasses (mostly needlegrass, Stipa speciosa) appear in the foreground (Janice Beatley Collection, 40-2).

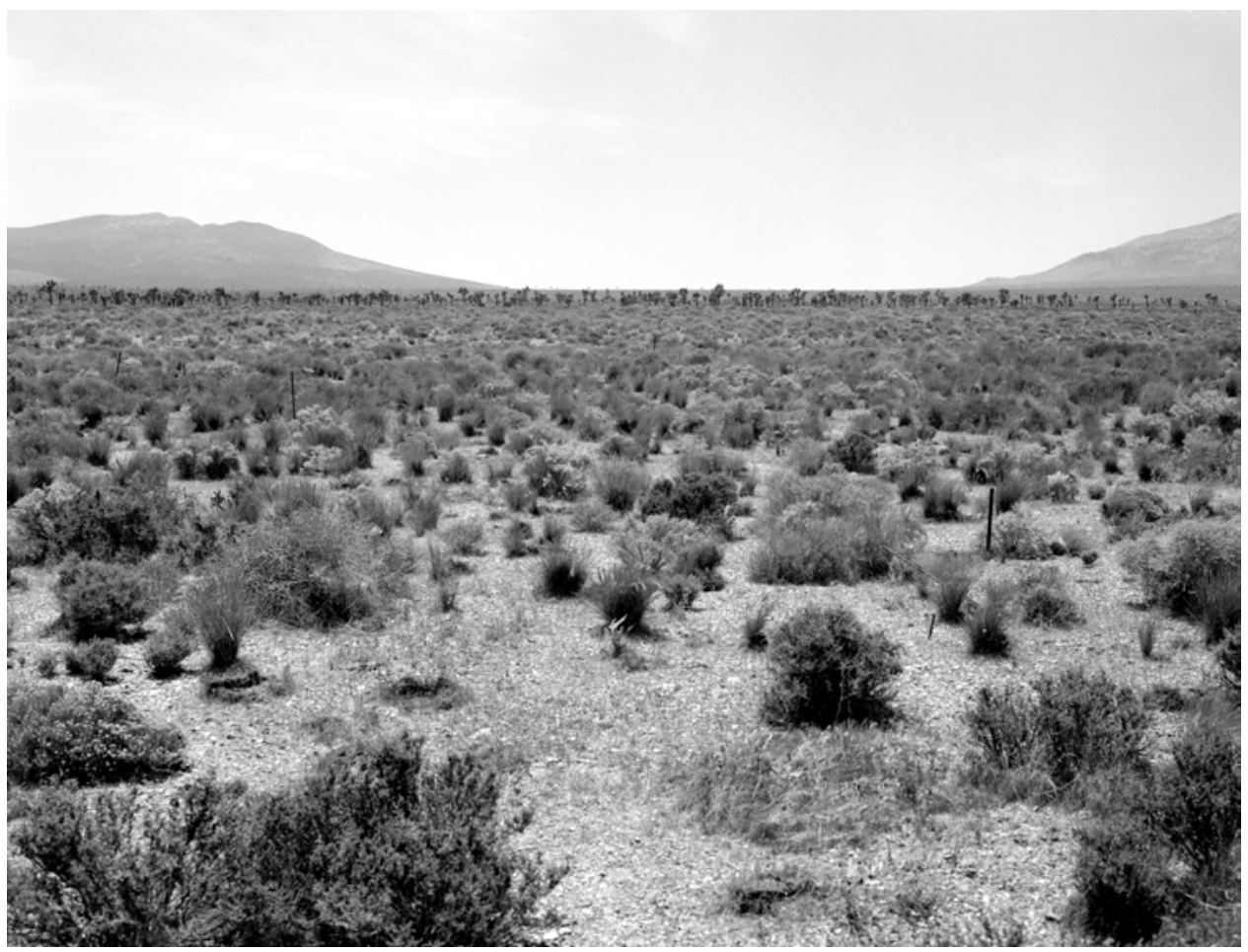

B. (May 10, 2000). Considerable change has occurred here as the disturbed assemblage has recovered some of its cover of perennial vegetation. Needlegrass, Mormon tea, cheesebush, and turpintinebroom (Thamnosma montana) dominate the vegetation. In the background, Joshua trees have increased considerably in stature and number (R.H. Webb, Stake 4027A). 
Table A-40B. Summary plant data for Plot 40.

\begin{tabular}{|c|c|c|c|c|c|c|c|c|}
\hline SPECIES & 1963 & 1964 & 1965 & 1966 & 1967 & 1970 & 1975 & 2000 \\
\hline Arabis pulchra munciensis & 0 & 0 & 0 & 0 & 0 & 0 & 1 & 0 \\
\hline Artemisia spinescens & 0 & 0 & 0 & 0 & 0 & 0 & 1 & 2 \\
\hline Atriplex canescens & 0 & 0 & 0 & 0 & 0 & 0 & 0 & 2 \\
\hline Calochortus flexuosus & 0 & 0 & 0 & 1 & 0 & 0 & 2 & 0 \\
\hline Chrysothamnus viscidiflorus & 0 & 0 & 0 & 0 & 0 & 0 & 0 & 26 \\
\hline Coleogyne ramosissima & 1 & 1 & 1 & 1 & 1 & 1 & 1 & 4 \\
\hline Ephedra nevadensis & 12 & 10 & 10 & 17 & 14 & 17 & 20 & 28 \\
\hline Ericameria cooperi & 0 & 0 & 0 & 0 & 0 & 0 & 0 & 3 \\
\hline Eriogonum inflatum & 0 & 0 & 0 & 0 & 0 & 0 & 0 & 4 \\
\hline Haplopappus sp. & 0 & 0 & 0 & 0 & 0 & 0 & 0 & 4 \\
\hline Hymenoclea salsola & 6 & 4 & 6 & 6 & 7 & 11 & 13 & 20 \\
\hline Linum lewisii & 0 & 0 & 0 & 0 & 0 & 0 & 0 & 1 \\
\hline Lycium andersonii & 8 & 8 & 8 & 8 & 9 & 8 & 10 & 8 \\
\hline Oryzopsis hymenoides & 0 & 2 & 6 & 7 & 8 & 5 & 8 & 13 \\
\hline Sitanion hystrix & 14 & 10 & 19 & 27 & 23 & 14 & 29 & 0 \\
\hline Sphaeralcea ambigua & 1 & 20 & 26 & 30 & 34 & 0 & 9 & 21 \\
\hline Stephanomeria parryi & 0 & 0 & 0 & 4 & 1 & 0 & 7 & 0 \\
\hline Stipa speciosa & 68 & 179 & 98 & 110 & 114 & 103 & 176 & 122 \\
\hline Stipa x Oryzopsis & 4 & 0 & 1 & 1 & 1 & 1 & 0 & 0 \\
\hline Tetradymia glabrata & 0 & 0 & 0 & 0 & 0 & 1 & 1 & 0 \\
\hline Thamnosma montana & 1 & 1 & 1 & 1 & 1 & 1 & 0 & 28 \\
\hline Yucca brevifolia & 0 & 0 & 0 & 0 & 0 & 0 & 0 & 3 \\
\hline Total Live & 115 & 235 & 176 & 213 & 213 & 162 & 278 & 289 \\
\hline Dead Grass & 0 & 2 & 1 & 0 & 0 & 0 & 1 & 19 \\
\hline Dead Shrub & 3 & 1 & 0 & 0 & 0 & 1 & 0 & 16 \\
\hline Total & 118 & 238 & 177 & 213 & 213 & 163 & 279 & 324 \\
\hline & \multicolumn{8}{|c|}{ Average Height (m) } \\
\hline SPECIES & 1963 & 1964 & 1965 & 1966 & 1967 & 1970 & 1975 & 2000 \\
\hline Arabis pulchra munciensis & 0.00 & 0.00 & 0.00 & 0.00 & 0.00 & 0.00 & 0.18 & 0.00 \\
\hline Artemisia spinescens & 0.00 & 0.00 & 0.00 & 0.00 & 0.00 & 0.00 & 0.15 & 0.17 \\
\hline Atriplex canescens & 0.00 & 0.00 & 0.00 & 0.00 & 0.00 & 0.00 & 0.00 & 0.53 \\
\hline Calochortus flexuosus & 0.00 & 0.00 & 0.00 & 0.10 & 0.00 & 0.00 & 0.03 & 0.00 \\
\hline Chrysothamnus viscidiflorus & 0.00 & 0.00 & 0.00 & 0.00 & 0.00 & 0.00 & 0.00 & 0.28 \\
\hline Coleogyne ramosissima & 0.41 & 0.36 & 0.38 & 0.43 & 0.41 & 0.43 & 0.48 & 0.45 \\
\hline Ephedra nevadensis & 0.33 & 0.31 & 0.39 & 0.40 & 0.47 & 0.53 & 0.53 & 0.51 \\
\hline Ericameria cooperi & 0.00 & 0.00 & 0.00 & 0.00 & 0.00 & 0.00 & 0.00 & 0.27 \\
\hline Eriogonum inflatum & 0.00 & 0.00 & 0.00 & 0.00 & 0.00 & 0.00 & 0.00 & 0.00 \\
\hline Haplopappus sp. & 0.00 & 0.00 & 0.00 & 0.00 & 0.00 & 0.00 & 0.00 & 0.36 \\
\hline Hymenoclea salsola & 0.37 & 0.36 & 0.45 & 0.40 & 0.41 & 0.47 & 0.45 & 0.51 \\
\hline Linum lewisii & 0.00 & 0.00 & 0.00 & 0.00 & 0.00 & 0.00 & 0.00 & 0.28 \\
\hline Lycium andersonii & 0.30 & 0.29 & 0.35 & 0.36 & 0.35 & 0.41 & 0.44 & 0.38 \\
\hline Oryzopsis hymenoides & 0.00 & 0.15 & 0.40 & 0.45 & 0.41 & 0.45 & 0.37 & 0.46 \\
\hline Sitanion hystrix & 0.22 & 0.21 & 0.28 & 0.30 & 0.28 & 0.29 & 0.24 & 0.00 \\
\hline Sphaeralcea ambigua & 0.15 & 0.05 & 0.17 & 0.20 & 0.23 & 0.00 & 0.07 & 0.19 \\
\hline Stephanomeria parryi & 0.00 & 0.00 & 0.00 & 0.07 & 0.10 & 0.00 & 0.03 & 0.00 \\
\hline Stipa speciosa & 0.48 & 0.19 & 0.40 & 0.44 & 0.43 & 0.42 & 0.43 & 0.51 \\
\hline
\end{tabular}


Table A-40B (continued). Summary plant data for Plot 40.

\begin{tabular}{|c|c|c|c|c|c|c|c|c|}
\hline \multirow{2}{*}{\multicolumn{9}{|c|}{ SPFCIFS }} \\
\hline & 1963 & 1964 & 1965 & 1966 & 1967 & 1970 & 1975 & 2000 \\
\hline Stipa x Oryzopsis & 0.39 & 0.00 & 0.51 & 0.76 & 0.69 & 0.74 & 0.00 & 0.00 \\
\hline Tetradymia glabrata & 0.00 & 0.00 & 0.00 & 0.00 & 0.00 & 0.25 & 0.38 & 0.00 \\
\hline Thamnosma montana & 0.25 & 0.33 & 0.33 & 0.30 & 0.33 & 0.33 & 0.00 & 0.32 \\
\hline \multirow[t]{2}{*}{ Yucca brevifolia } & 0.00 & 0.10 & 0.00 & 0.00 & 0.00 & 0.00 & 0.00 & 0.42 \\
\hline & \multicolumn{8}{|c|}{ Cover (\%) } \\
\hline SPECIES & 1963 & 1964 & 1965 & 1966 & 1967 & 1970 & 1975 & 2000 \\
\hline Arabis pulchra munciensis & 0.00 & 0.00 & 0.00 & 0.00 & 0.00 & 0.00 & 0.01 & 0.00 \\
\hline Artemisia spinescens & 0.00 & 0.00 & 0.00 & 0.00 & 0.00 & 0.00 & 0.04 & 0.16 \\
\hline Atriplex canescens & 0.00 & 0.00 & 0.00 & 0.00 & 0.00 & 0.00 & 0.00 & 0.14 \\
\hline Calochortus flexuosus & 0.00 & 0.00 & 0.00 & 0.01 & 0.00 & 0.00 & 0.02 & 0.00 \\
\hline Chrysothamnus viscidiflorus & 0.00 & 0.00 & 0.00 & 0.00 & 0.00 & 0.00 & 0.00 & 1.62 \\
\hline Coleogyne ramosissima & 0.03 & 0.06 & 0.12 & 0.05 & 0.08 & 0.09 & 0.14 & 0.61 \\
\hline Ephedra nevadensis & 1.28 & 1.14 & 1.90 & 1.90 & 2.12 & 2.61 & 3.97 & 4.63 \\
\hline Ericameria cooperi & 0.00 & 0.00 & 0.00 & 0.00 & 0.00 & 0.00 & 0.00 & 0.25 \\
\hline Eriogonum inflatum & 0.00 & 0.00 & 0.00 & 0.00 & 0.00 & 0.00 & 0.00 & 0.05 \\
\hline Haplopappus sp. & 0.00 & 0.00 & 0.00 & 0.00 & 0.00 & 0.00 & 0.00 & 0.12 \\
\hline Hymenoclea salsola & 0.65 & 0.52 & 0.66 & 0.75 & 0.79 & 1.39 & 2.19 & 2.52 \\
\hline Linum lewisii & 0.00 & 0.00 & 0.00 & 0.00 & 0.00 & 0.00 & 0.00 & 0.01 \\
\hline Lycium andersonii & 0.76 & 0.85 & 0.95 & 0.93 & 1.05 & 1.03 & 1.58 & 0.57 \\
\hline Oryzopsis hymenoides & 0.00 & 0.05 & 0.45 & 0.47 & 0.65 & 0.48 & 0.91 & 0.66 \\
\hline Sitanion hystrix & 0.75 & 0.69 & 1.21 & 2.09 & 1.90 & 0.98 & 1.94 & 0.00 \\
\hline Sphaeralcea ambigua & 0.03 & 0.35 & 0.70 & 0.86 & 1.19 & 0.00 & 0.15 & 0.35 \\
\hline Stephanomeria parryi & 0.00 & 0.00 & 0.00 & 0.05 & 0.02 & 0.00 & 0.07 & 0.00 \\
\hline Stipa speciosa & 6.94 & 7.60 & 8.93 & 10.58 & 10.63 & 9.71 & 19.03 & 6.78 \\
\hline Stipa x Oryzopsis & 0.28 & 0.00 & 0.11 & 0.22 & 0.16 & 0.19 & 0.00 & 0.00 \\
\hline Tetradymia glabrata & 0.00 & 0.00 & 0.00 & 0.00 & 0.00 & 0.01 & 0.13 & 0.00 \\
\hline Thamnosma montana & 0.05 & 0.07 & 0.07 & 0.09 & 0.08 & 0.11 & 0.00 & 2.22 \\
\hline Yucca brevifolia & 0.00 & 0.00 & 0.00 & 0.00 & 0.00 & 0.00 & 0.00 & 0.14 \\
\hline Total Live & 10.76 & 11.33 & 15.11 & 17.99 & 18.67 & 16.60 & 30.17 & 20.81 \\
\hline Dead Grass & 0.00 & 0.04 & 0.04 & 0.00 & 0.00 & 0.00 & 0.07 & 2.97 \\
\hline Dead Shrub & 0.13 & 0.05 & 0.00 & 0.00 & 0.00 & 0.02 & 0.00 & 2.55 \\
\hline Total & 10.89 & 11.41 & 15.15 & 17.99 & 18.67 & 16.62 & 30.25 & 26.33 \\
\hline
\end{tabular}


Table A-40B (continued). Summary plant data for Plot 40.

\begin{tabular}{|c|c|c|c|c|c|c|c|c|}
\hline SPECIES & 1963 & 1964 & 1965 & 1966 & 1967 & 1970 & 1975 & 2000 \\
\hline Arabis pulchra munciensis & 0.00 & 0.00 & 0.00 & 0.00 & 0.00 & 0.00 & 0.01 & 0.00 \\
\hline Artemisia spinescens & 0.00 & 0.00 & 0.00 & 0.00 & 0.00 & 0.00 & 0.02 & 0.11 \\
\hline Atriplex canescens & 0.00 & 0.00 & 0.00 & 0.00 & 0.00 & 0.00 & 0.00 & 0.24 \\
\hline Calochortus flexuosus & 0.00 & 0.00 & 0.00 & 0.00 & 0.00 & 0.00 & 0.00 & 0.00 \\
\hline Chrysothamnus viscidiflorus & 0.00 & 0.00 & 0.00 & 0.00 & 0.00 & 0.00 & 0.00 & 1.88 \\
\hline Coleogyne ramosissima & 0.04 & 0.08 & 0.15 & 0.07 & 0.11 & 0.13 & 0.22 & 1.17 \\
\hline Ephedra nevadensis & 1.64 & 1.44 & 2.49 & 2.86 & 3.30 & 4.84 & 7.34 & 9.13 \\
\hline Ericameria cooperi & 0.00 & 0.00 & 0.00 & 0.00 & 0.00 & 0.00 & 0.00 & 0.26 \\
\hline Eriogonum inflatum & 0.00 & 0.00 & 0.00 & 0.00 & 0.00 & 0.00 & 0.00 & 0.00 \\
\hline Haplopappus sp. & 0.00 & 0.00 & 0.00 & 0.00 & 0.00 & 0.00 & 0.00 & 0.14 \\
\hline Hymenoclea salsola & 0.90 & 0.69 & 1.13 & 1.09 & 1.25 & 2.40 & 3.75 & 4.85 \\
\hline Linum lewisii & 0.00 & 0.00 & 0.00 & 0.00 & 0.00 & 0.00 & 0.00 & 0.01 \\
\hline Lycium andersonii & 0.83 & 0.90 & 1.21 & 1.25 & 1.37 & 1.47 & 2.43 & 0.82 \\
\hline Oryzopsis hymenoides & 0.00 & 0.03 & 0.68 & 0.80 & 0.92 & 0.77 & 1.12 & 1.20 \\
\hline Sitanion hystrix & 0.58 & 0.58 & 1.23 & 2.29 & 1.96 & 0.95 & 1.84 & 0.00 \\
\hline Stephanomeria parryi & 0.00 & 0.00 & 0.00 & 0.70 & 1.15 & 0.00 & 0.05 & 0.00 \\
\hline Sphaeralcea ambigua & 0.00 & 0.05 & 0.45 & 0.01 & 0.01 & 0.00 & 0.01 & 0.25 \\
\hline Stipa speciosa & 12.00 & 9.44 & 14.56 & 18.64 & 18.17 & 15.51 & 29.75 & 12.07 \\
\hline Stipa x Oryzopsis & 0.41 & 0.00 & 0.19 & 0.56 & 0.38 & 0.47 & 0.00 & 0.00 \\
\hline Tetradymia glabrata & 0.00 & 0.00 & 0.00 & 0.00 & 0.00 & 0.01 & 0.16 & 0.00 \\
\hline Thamnosma montana & 0.05 & 0.08 & 0.08 & 0.09 & 0.09 & 0.12 & 0.00 & 2.49 \\
\hline Yucca brevifolia & 0.00 & 0.00 & 0.00 & 0.00 & 0.00 & 0.00 & 0.00 & 0.22 \\
\hline Total Live & 16.44 & 13.29 & 22.17 & 28.35 & 28.71 & 26.68 & 46.70 & 34.84 \\
\hline
\end{tabular}


Table A-41A. Site characteristics for Plot 41.

Location: Mid Valley

NTS Area: 14

NTS Grid: K-26

USGS 7.5’ Quadrangle Name: Mine Mountain

\begin{tabular}{|c|c|c|c|c|}
\hline & & GPS Rea & Zone 11S) & \\
\hline & Location & Northing & Easting & Condition of Original Corners \\
\hline Corner 1: & SW & 4090825 & 572644 & Standing \\
\hline Corner 2: & NW & 4090854 & 572651 & Standing \\
\hline Corner 3: & $\mathrm{NE}$ & 4090850 & 572680 & Standing \\
\hline Corner 4: & SE & 4090818 & 572674 & Standing \\
\hline DEM Plo & vation: 14 & & & Plot Aspect: $27^{\circ}$ \\
\hline Elevation & re Playa ( & Name): I & & Plot Slope: $16^{\circ}$ \\
\hline
\end{tabular}

Annual Precipitation

Measured: $241 \mathrm{~mm}$

Modeled: $212 \mathrm{~mm}$

Parent Material Type: alluvial fan

Substrate: alluvial fan, mixed volcanic w/granite? Mostly rhyolite no basalt

Slate Geologic Unit: Qai

Beatley Plant Assemblage: Coleogyne

Ostler-Hanson Plant Association: Coleogyne-Ephedra

Ostler-Hanson Land Unit: 844

Abundance of Biological Soil Crusts: NA

Type and Date of Disturbance: none (control for Plot 42)

Plot Condition: good

Location of Transect 1: west side of plot

Direction Transects Are Read: north to south

Date(s) Plot Remeasured: 13 May 2000 
Figure A-41. Photograph showing Plot 41.

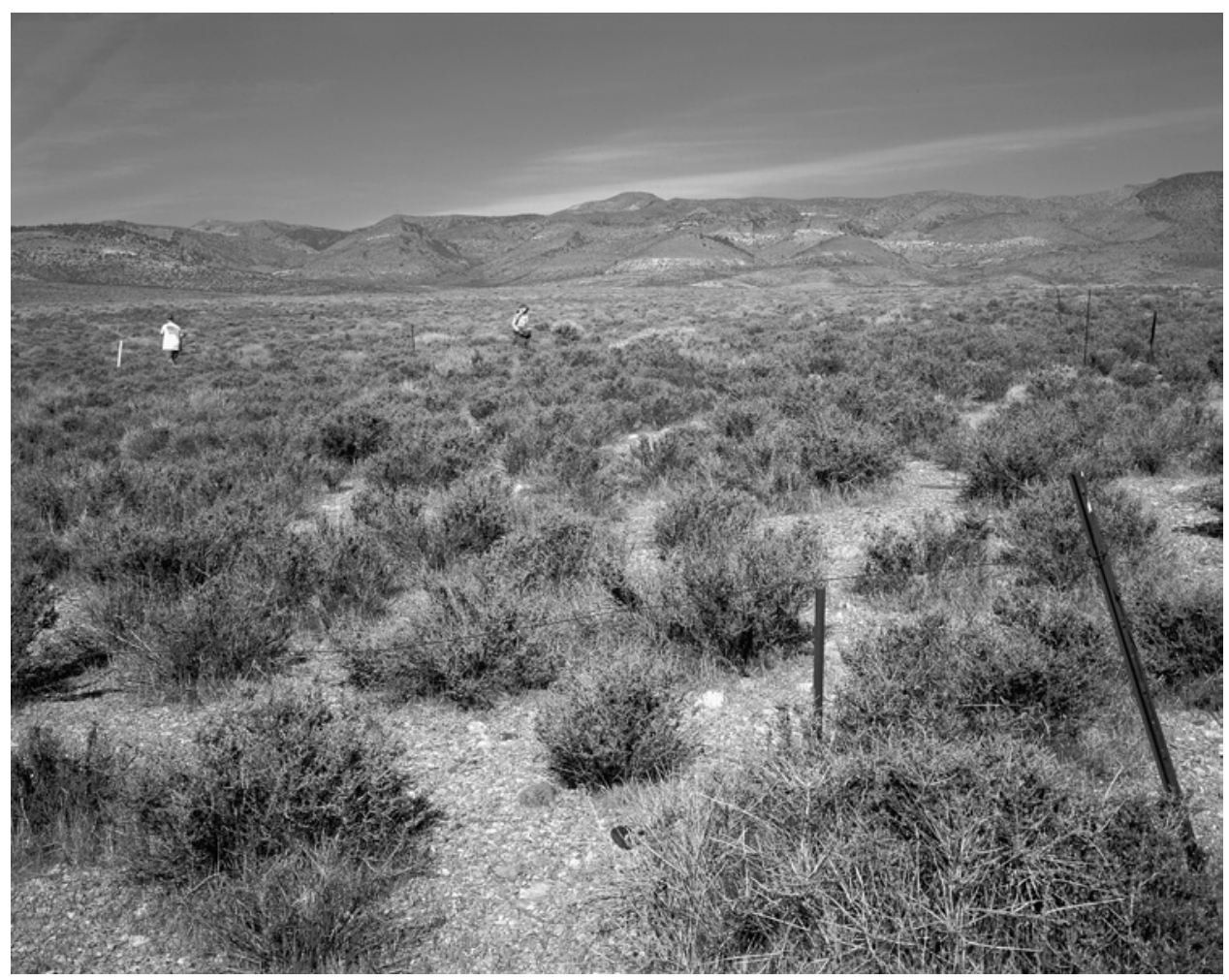

(May 10, 2000). This westerly view across Plot 41 and the northern part of Mid Valley shows a blackbrush assemblage with Mormon tea. Shoshone Mountain appears in the background. Apparently no photographs were taken of this plot in 1964 (R.H. Webb, Stake 4023A). 
Table A-41B. Summary plant data for Plot 41.

\begin{tabular}{|c|c|c|c|c|c|c|c|}
\hline \multicolumn{5}{|c|}{ Number of Plants } & \multicolumn{3}{|c|}{ Cover (\%) } \\
\hline SPECIES & 1963 & 1975 & 2000 & SPECIES & 1963 & 1975 & 2000 \\
\hline Artemisia tridentata & 0 & 0 & 2 & Artemisia tridentata & 0.00 & 0.00 & 0.15 \\
\hline Chrysothamnus viscidiflorus & 1 & 1 & 1 & Chrysothamnus viscidiflorus & 0.11 & 0.12 & 0.04 \\
\hline Coleogyne ramosissima & 356 & 368 & 321 & Coleogyne ramosissima & 44.88 & 44.15 & 31.64 \\
\hline Coryphantha sp. & 0 & 0 & 2 & Coryphantha sp. & 0.00 & 0.00 & 0.04 \\
\hline Ephedra nevadensis & 26 & 43 & 52 & Ephedra nevadensis & 2.62 & 4.59 & 4.66 \\
\hline Opuntia echinocarpa & 0 & 1 & 2 & Opuntia echinocarpa & 0.00 & 0.04 & 0.08 \\
\hline Sitanion hystrix & 1 & 0 & 0 & Sitanion hystrix & 0.04 & 0.00 & 0.00 \\
\hline Stipa speciosa & 0 & 1 & 0 & Stipa speciosa & 0.00 & 0.04 & 0.00 \\
\hline Total Live & 383 & 414 & 380 & Total Live & 47.61 & 48.94 & 36.60 \\
\hline Dead Grass & 0 & 0 & 0 & Dead Grass & 0.00 & 0.00 & 0.00 \\
\hline Dead Shrub & 26 & 9 & 36 & Dead Shrub & 2.50 & 0.84 & 5.00 \\
\hline Total & 409 & 423 & 416 & Total & 50.11 & 49.77 & 41.60 \\
\hline \multicolumn{5}{|c|}{ Average Height (m) } & \multicolumn{3}{|c|}{ Biomass Index $\left(\mathbf{m}^{2}\right)$} \\
\hline SPECIES & 1963 & 1975 & 2000 & SPECIES & 1963 & 1975 & 2000 \\
\hline Artemisia tridentata & 0.00 & 0.00 & 0.66 & Artemisia tridentata & 0.00 & 0.00 & 0.20 \\
\hline Chrysothamnus viscidiflorus & 0.38 & 0.41 & 0.36 & Chrysothamnus viscidiflorus & 0.14 & 0.16 & 0.04 \\
\hline Coleogyne ramosissima & 0.42 & 0.37 & 0.43 & Coleogyne ramosissima & 69.05 & 60.54 & 49.82 \\
\hline Coryphantha sp. & 0.00 & 0.00 & 0.06 & Coryphantha sp. & 0.00 & 0.00 & 0.01 \\
\hline Ephedra nevadensis & 0.36 & 0.43 & 0.52 & Ephedra nevadensis & 3.82 & 7.49 & 9.02 \\
\hline Opuntia echinocarpa & 0.00 & 0.18 & 0.30 & Opuntia echinocarpa & 0.00 & 0.02 & 0.08 \\
\hline Sitanion hystrix & 0.25 & 0.00 & 0.00 & Sitanion hystrix & 0.03 & 0.00 & 0.00 \\
\hline \multirow[t]{2}{*}{ Stipa speciosa } & 0.00 & 0.30 & 0.00 & Stipa speciosa & 0.00 & 0.04 & 0.00 \\
\hline & & & & Total Live & 73.01 & 68.25 & 59.17 \\
\hline
\end{tabular}


Table A-42A. Site characteristics for Plot 42.

Location: Mid Valley

NTS Area: 14

NTS Grid: K-26

USGS 7.5’ Quadrangle Name: Mine Mountain

\begin{tabular}{|c|c|c|c|c|}
\hline & & GPS Reac & Zone 11S & \\
\hline & Location & Northing & Easting & Condition of Original Corners \\
\hline Corner 1: & SW & 4090789 & 572634 & Standing \\
\hline Corner 2: & NW & 4090794 & 572635 & Standing \\
\hline Corner 3: & $\mathrm{NE}$ & 4090785 & 572638 & Standing \\
\hline Corner 4: & SE & 4090761 & 572661 & Standing \\
\hline DEM Plo & vation: 14 & & & Plot Aspect: $297^{\circ}$ \\
\hline Elevation & e Playa ( & Name): I & & Plot Slope: $6^{\circ}$ \\
\hline
\end{tabular}

Annual Precipitation

Measured: $233 \mathrm{~mm}$

Modeled: $211 \mathrm{~mm}$

Parent Material Type: alluvial fan

Substrate: alluvial fan, mixed volcanic w/granite? Mostly rhyolite no basalt

Slate Geologic Unit: Qai

Beatley Plant Assemblage: Ephedra

Ostler-Hanson Plant Association: NA

Ostler-Hanson Land Unit: 844

Abundance of Biological Soil Crusts: none

Type and Date of Disturbance: fire occurred in 1959

Plot Condition: good

Location of Transect 1: west side of plot

Direction Transects Are Read: north to south

Date(s) Plot Remeasured: 13 May 2000 
Figure A-42. Photograph showing Plot 42.

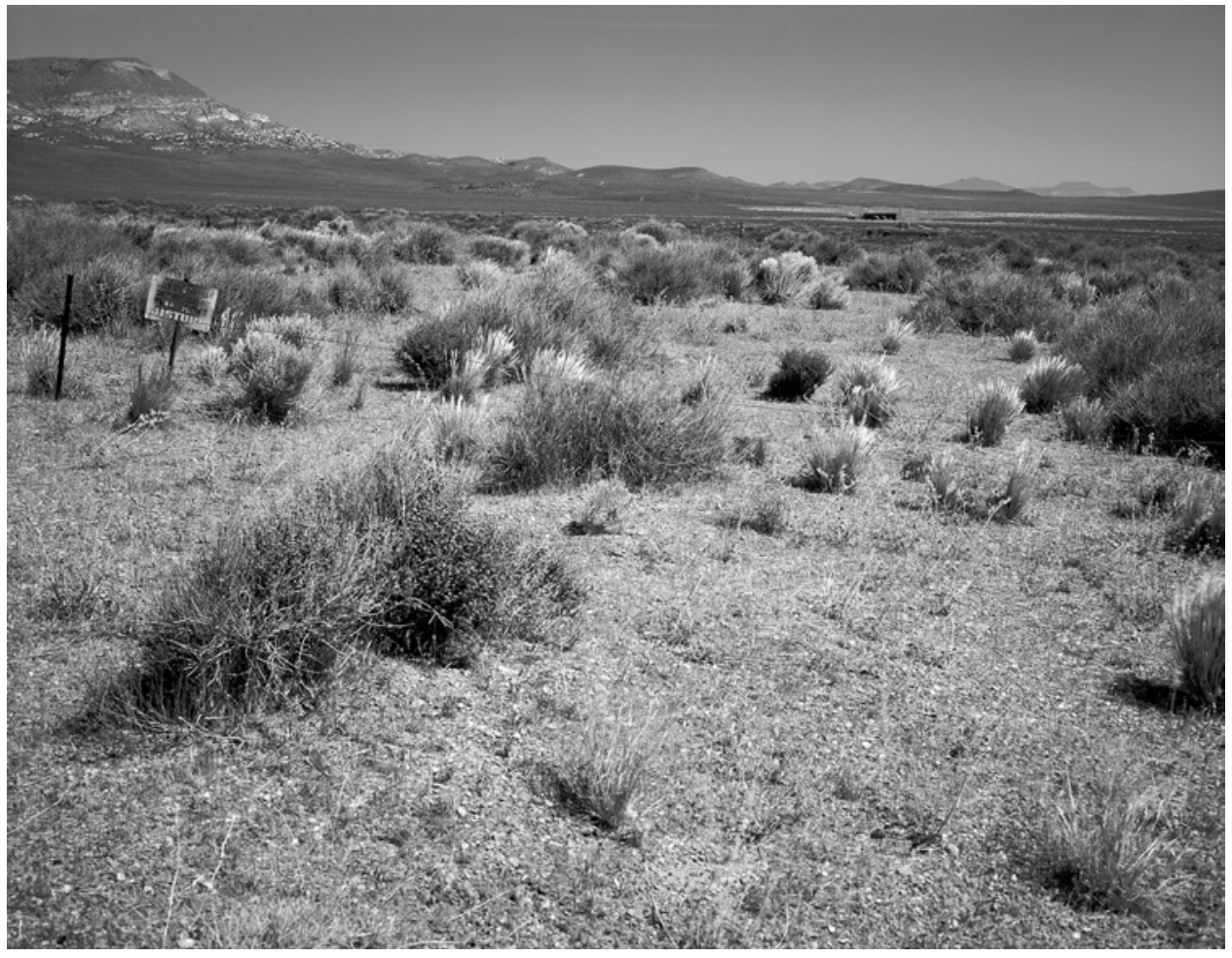

(May 9, 2000). This northwesterly view across Plot 42 shows an area burned in the late 1950s in Mid Valley. The assemblage is dominated by Mormon tea and needlegrass. Apparently no photographs were taken of this plot in 1964 (R.H. Webb, Stake 4025). 
Table A-42B. Summary plant data for Plot 42.

\author{
SPECIES \\ Astragalus lentiginosus fremontii \\ Calochortus flexuosus \\ Chrysothamnus nauseosus \\ Coleogyne ramosissima \\ Ephedra nevadensis \\ Ephedra viridis \\ Ericameria laricifolia \\ Erioneuron pulchellum \\ Oryzopsis hymenoides \\ Sitanion hystrix \\ Sphaeralcea ambigua monticola \\ Stephanomeria parryi \\ Stipa speciosa \\ Total Live \\ Dead Grass \\ Dead Shrub \\ Total
}

\section{SPECIES}

Astragalus lentiginosus fremontii

Calochortus flexuosus

Chrysothamnus nauseosus

Coleogyne ramosissima

Ephedra nevadensis

Ephedra viridis

Ericameria laricifolia

Erioneuron pulchellum

Oryzopsis hymenoides

Sitanion hystrix

Sphaeralcea ambigua monticola

Stephanomeria parryi

Stipa speciosa

\section{Number of Plants}

$\begin{array}{cccccccc}\mathbf{1 9 6 3} & \mathbf{1 9 6 4} & \mathbf{1 9 6 5} & \mathbf{1 9 6 6} & \mathbf{1 9 6 7} & \mathbf{1 9 7 0} & \mathbf{1 9 7 5} & \mathbf{2 0 0 0} \\ 0 & 1 & 3 & 123 & 130 & 0 & 45 & 2 \\ 0 & 0 & 0 & 3 & 1 & 0 & 0 & 0 \\ 0 & 0 & 0 & 0 & 0 & 0 & 2 & 6 \\ 0 & 0 & 0 & 0 & 0 & 0 & 0 & 5 \\ 26 & 16 & 26 & 39 & 38 & 31 & 39 & 61 \\ 0 & 0 & 0 & 0 & 0 & 0 & 0 & 2 \\ 0 & 0 & 0 & 0 & 0 & 0 & 0 & 1 \\ 0 & 0 & 0 & 0 & 0 & 0 & 0 & 3 \\ 0 & 0 & 0 & 0 & 0 & 0 & 0 & 1 \\ 0 & 0 & 0 & 0 & 0 & 0 & 1 & 0 \\ 3 & 6 & 26 & 58 & 135 & 0 & 29 & 113 \\ 0 & 0 & 2 & 8 & 2 & 0 & 0 & 0 \\ 0 & 0 & 0 & 1 & 4 & 4 & 8 & 19 \\ 29 & 23 & 57 & 232 & 310 & 35 & 124 & 213 \\ 0 & 0 & 0 & 0 & 0 & 0 & 0 & 8 \\ 7 & 15 & 1 & 0 & 7 & 1 & 0 & 5 \\ 36 & 38 & 58 & 232 & 317 & 36 & 124 & 226\end{array}$

\section{Average Height (m)}

1963

0.00

0.00

0.00

0.00

0.26

0.00

0.00

0.00

0.00

0.00

0.14

0.00

0.00
1964

0.08

0.00

0.00

0.00

0.26

0.00

0.00

0.00

0.00

0.00

0.15

0.00

0.00
1965

0.07

0.00

0.00

0.00

0.28

0.00

0.00

0.00

0.00

0.00

0.17

0.08

0.00

\begin{abstract}
1966
\end{abstract}
0.16

1967

0.09

0.00

0.00

0.37

0.00

0.00

0.00

0.00

0.00

0.27

0.09

0.18

0.20

0.15

0.00

0.00

0.41

0.00

0.00

0.00

0.00

0.00

0.40

0.08

0.34
1970

1975

2000

$\begin{array}{lll}0.00 & 0.08 & 0.19\end{array}$

$\begin{array}{lll}0.00 & 0.00 & 0.00\end{array}$

$\begin{array}{lll}0.00 & 0.32 & 0.70\end{array}$

$\begin{array}{lll}0.00 & 0.68 & 0.38\end{array}$

$\begin{array}{lll}0.59 & 0.00 & 0.75\end{array}$

$\begin{array}{lll}0.00 & 0.00 & 0.89\end{array}$

$\begin{array}{lll}0.00 & 0.00 & 0.84\end{array}$

$\begin{array}{lll}0.00 & 0.00 & 0.03\end{array}$

$\begin{array}{lll}0.00 & 0.00 & 0.51\end{array}$

$\begin{array}{lll}0.00 & 0.18 & 0.00\end{array}$

$\begin{array}{lll}0.00 & 0.05 & 0.32\end{array}$

$\begin{array}{lll}0.00 & 0.00 & 0.00\end{array}$

$\begin{array}{lll}0.45 & 0.37 & 0.56\end{array}$ 
Table A-42B (continued). Summary plant data for Plot 42.

SPECIES
Astragalus lentiginosus fremontii
Calochortus flexuosus
Chrysothamnus nauseosus
Coleogyne ramosissima
Ephedra nevadensis
Ephedra viridis
Ericameria laricifolia
Erioneuron pulchellum
Oryzopsis hymenoides
Sitanion hystrix
Sphaeralcea ambigua monticola
Stephanomeria parryi
Stipa speciosa
Total Live
Dead Grass
Dead Shrub
Total

\section{SPECIES}

Astragalus lentiginosus fremontii

Calochortus flexuosus

Chrysothamnus nauseosus

Coleogyne ramosissima

Ephedra nevadensis

Ephedra viridis

Ericameria laricifolia

Erioneuron pulchellum

Oryzopsis hymenoides

Sitanion hystrix

Sphaeralcea ambigua monticola

Stephanomeria parryi

Stipa speciosa

Total Live

$\begin{array}{cccccccc}\mathbf{1 9 6 3} & \mathbf{1 9 6 4} & \mathbf{1 9 6 5} & \mathbf{1 9 6 6} & \mathbf{1 9 6 7} & \mathbf{1 9 7 0} & \mathbf{1 9 7 5} & \mathbf{2 0 0 0} \\ 0.00 & 0.01 & 0.06 & 5.34 & 10.24 & 0.00 & 0.99 & 0.12 \\ 0.00 & 0.00 & 0.00 & 0.07 & 0.05 & 0.00 & 0.00 & 0.00 \\ 0.00 & 0.00 & 0.00 & 0.00 & 0.00 & 0.00 & 0.09 & 1.12 \\ 0.00 & 0.00 & 0.00 & 0.00 & 0.00 & 0.00 & 9.84 & 0.62 \\ 1.72 & 1.27 & 1.56 & 3.24 & 3.89 & 5.84 & 0.00 & 17.72 \\ 0.00 & 0.00 & 0.00 & 0.00 & 0.00 & 0.00 & 0.00 & 0.73 \\ 0.00 & 0.00 & 0.00 & 0.00 & 0.00 & 0.00 & 0.00 & 0.34 \\ 0.00 & 0.00 & 0.00 & 0.00 & 0.00 & 0.00 & 0.00 & 0.04 \\ 0.00 & 0.00 & 0.00 & 0.00 & 0.00 & 0.00 & 0.00 & 0.10 \\ 0.00 & 0.00 & 0.00 & 0.00 & 0.00 & 0.00 & 0.02 & 0.00 \\ 0.00 & 0.16 & 0.54 & 2.52 & 11.70 & 0.00 & 0.88 & 3.21 \\ 0.00 & 0.00 & 0.02 & 0.15 & 0.04 & 0.00 & 0.00 & 0.00 \\ 0.00 & 0.00 & 0.00 & 0.05 & 0.21 & 0.25 & 0.74 & 1.78 \\ 1.72 & 1.45 & 2.18 & 11.36 & 26.13 & 6.09 & 12.55 & 25.76 \\ 0.00 & 0.00 & 0.00 & 0.00 & 0.00 & 0.00 & 0.00 & 0.46 \\ 0.58 & 0.73 & 0.03 & 0.00 & 0.50 & 0.07 & 0.00 & 1.12 \\ 2.30 & 2.17 & 2.21 & 11.36 & 26.63 & 6.16 & 12.55 & 27.34\end{array}$

$\begin{array}{cccccccc}\mathbf{1 9 6 3} & \mathbf{1 9 6 4} & \mathbf{1 9 6 5} & \mathbf{1 9 6 6} & \mathbf{1 9 6 7} & \mathbf{1 9 7 0} & \mathbf{1 9 7 5} & \mathbf{2 0 0 0} \\ 0.00 & 0.00 & 0.02 & 3.08 & 7.30 & 0.00 & 0.35 & 0.08 \\ 0.00 & 0.00 & 0.00 & 0.02 & 0.03 & 0.00 & 0.00 & 0.00 \\ 0.00 & 0.00 & 0.00 & 0.00 & 0.00 & 0.00 & 0.14 & 2.89 \\ 0.00 & 0.00 & 0.00 & 0.00 & 0.00 & 0.00 & 23.03 & 1.28 \\ 1.72 & 1.25 & 1.63 & 4.34 & 5.87 & 12.12 & 0.00 & 47.91 \\ 0.00 & 0.00 & 0.00 & 0.00 & 0.00 & 0.00 & 0.00 & 2.31 \\ 0.00 & 0.00 & 0.00 & 0.00 & 0.00 & 0.00 & 0.00 & 0.95 \\ 0.00 & 0.00 & 0.00 & 0.00 & 0.00 & 0.00 & 0.00 & 0.00 \\ 0.00 & 0.00 & 0.00 & 0.00 & 0.00 & 0.00 & 0.00 & 0.17 \\ 0.00 & 0.00 & 0.00 & 0.00 & 0.00 & 0.00 & 0.01 & 0.00 \\ 0.00 & 0.07 & 0.44 & 2.73 & 16.96 & 0.00 & 0.17 & 3.98 \\ 0.00 & 0.00 & 0.00 & 0.04 & 0.01 & 0.00 & 0.00 & 0.00 \\ 0.00 & 0.00 & 0.00 & 0.03 & 0.24 & 0.39 & 1.00 & 3.56 \\ 1.72 & 1.32 & 2.10 & 10.25 & 30.41 & 12.51 & 24.71 & 63.13\end{array}$


Table A-43A. Site characteristics for Plot 43.

Location: Yucca Flat

NTS Area: 6

NTS Grid: Q-26

USGS 7.5’ Quadrangle Name: Yucca Lake

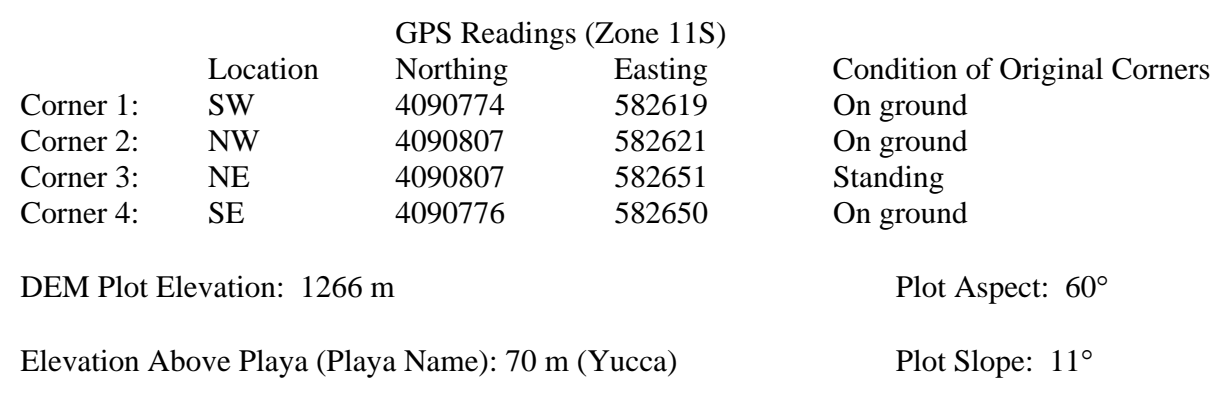

Annual Precipitation

Measured: $180 \mathrm{~mm}$

Modeled: $174 \mathrm{~mm}$

Parent Material Type: alluvial fan

Substrate: alluvial fan

Slate Geologic Unit: QTa

Beatley Plant Assemblage: Larrea-Atriplex/Coleogyne

Ostler-Hanson Plant Association: Atriplex confertifolia-Ephedra-Larrea

Ostler-Hanson Land Unit: 552

Abundance of Biological Soil Crusts: moderate to heavy cover

Type and Date of Disturbance: none

Plot Condition: good

Location of Transect 1: east side of plot

Direction Transects Are Read: south to north

Date(s) Plot Remeasured: 7 May 2001 
Figure A-43. Photographs showing Plot 43.

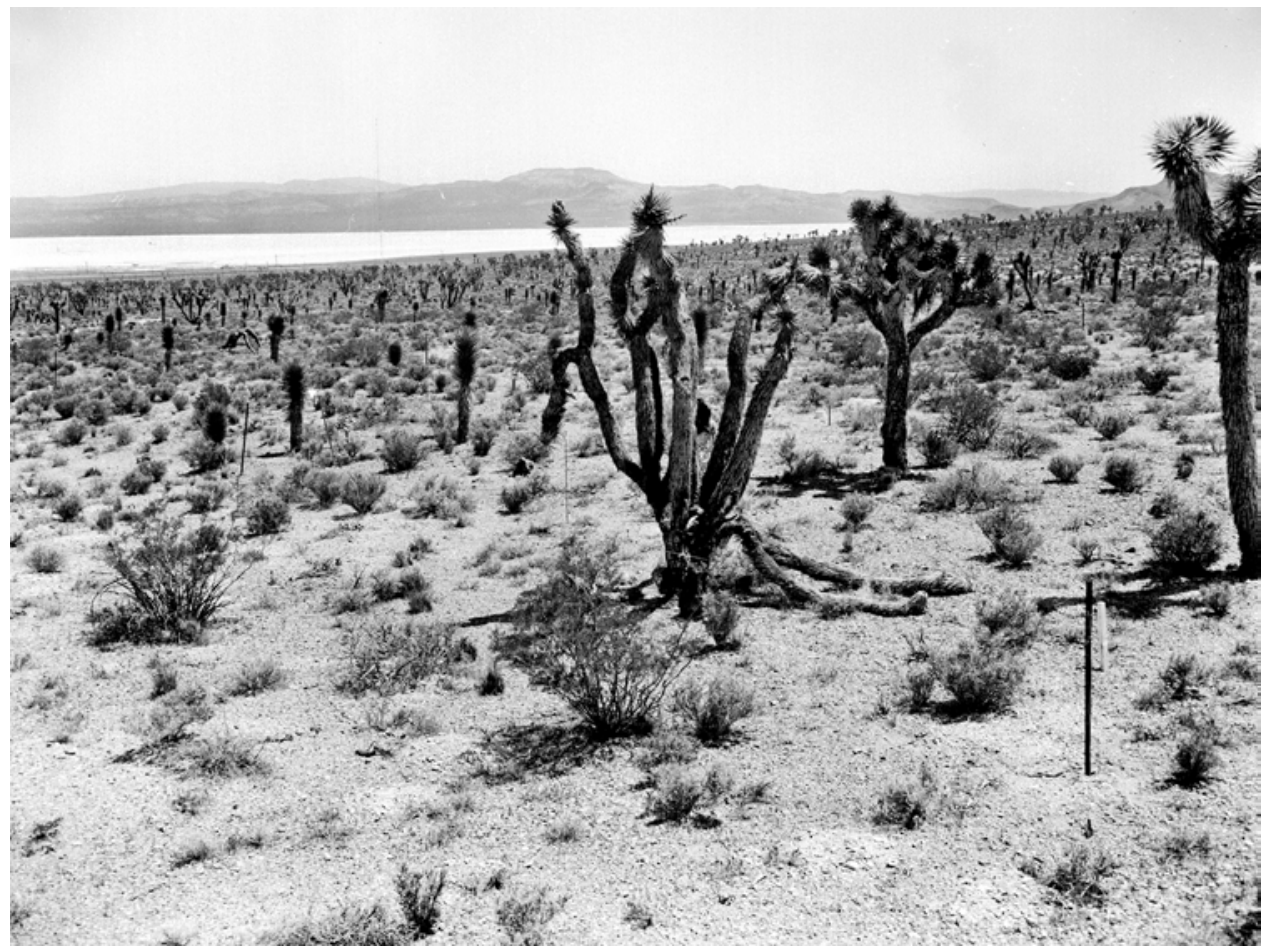

A. (May 12, 1964). This southeasterly view across Plot 43 shows Yucca Lake in the bottom of the valley with a collection of different mountains in the distance. These include the Half Pint Range in the far distance and the southerly extension of Paiute Ridge at left. The vegetation is a creosote bush mixed assemblage with shadscale, blackbrush, and a large number of Joshua trees on the plot and in the distance (Janice Beatley Collection, 99-A).



B. (June 7, 2001). The assemblage composition has changed, with shadscale reduced in abundance while Mormon tea has increased. Although many of the Joshua trees on the plot appear larger now, the number of Joshua trees has not noticeably increased (Dominic Oldershaw, Stake 4044A). 
Table A-43B. Summary plant data for Plot 43.

\begin{tabular}{|c|c|c|c|c|c|c|c|}
\hline \multicolumn{5}{|c|}{ Number of Plants } & \multicolumn{3}{|c|}{ Cover (\%) } \\
\hline SPECIES & 1963 & 1975 & 2001 & SPECIES & 1963 & 1975 & 2001 \\
\hline Acamptopappus shockleyi & 42 & 26 & 10 & Acamptopappus shockleyi & 2.61 & 1.20 & 0.82 \\
\hline Artemisia spinescens & 7 & 6 & 0 & Artemisia spinescens & 0.42 & 0.36 & 0.00 \\
\hline Atriplex confertifolia & 64 & 74 & 34 & Atriplex confertifolia & 7.29 & 12.95 & 2.16 \\
\hline Coleogyne ramosissima & 24 & 26 & 34 & Coleogyne ramosissima & 2.23 & 2.71 & 3.90 \\
\hline Coryphantha vivipara rosea & 0 & 1 & 0 & Coryphantha vivipara rosea & 0.00 & 0.02 & 0.00 \\
\hline Ephedra nevadensis & 17 & 33 & 28 & Ephedra nevadensis & 2.05 & 3.73 & 6.48 \\
\hline Eriogonum inflatum & 2 & 9 & 10 & Eriogonum inflatum & 0.11 & 0.35 & 0.39 \\
\hline Erioneuron pulchellum & 0 & 11 & 2 & Erioneuron pulchellum & 0.00 & 0.25 & 0.03 \\
\hline Grayia spinosa & 1 & 0 & 1 & Grayia spinosa & 0.13 & 0.00 & 0.09 \\
\hline Krameria parvifolia & 0 & 0 & 1 & Krameria parvifolia & 0.00 & 0.00 & 0.05 \\
\hline Larrea tridentata & 13 & 17 & 30 & Larrea tridentata & 2.15 & 3.71 & 9.65 \\
\hline Lycium andersonii & 1 & 3 & 5 & Lycium andersonii & 0.12 & 0.25 & 0.27 \\
\hline Menodora spinescens & 4 & 5 & 10 & Menodora spinescens & 0.16 & 0.23 & 1.42 \\
\hline Oryzopsis hymenoides & 6 & 10 & 2 & Oryzopsis hymenoides & 0.19 & 0.83 & 0.17 \\
\hline Psorothamnus fremontii & 0 & 0 & 1 & Psorothamnus fremontii & 0.00 & 0.00 & 0.16 \\
\hline Sphaeralcea ambigua & 5 & 4 & 3 & Sphaeralcea ambigua & 0.11 & 0.11 & 0.05 \\
\hline Stipa speciosa & 14 & 39 & 4 & Stipa speciosa & 0.83 & 3.84 & 0.41 \\
\hline Viguiera deltoidea & 0 & 0 & 1 & Viguiera deltoidea & 0.00 & 0.00 & 0.05 \\
\hline Yucca brevifolia & 4 & 4 & 5 & Yucca brevifolia & 0.39 & 0.95 & 1.16 \\
\hline Total Live & 204 & 268 & 181 & Total Live & 18.78 & 31.47 & 27.27 \\
\hline Dead Grass & 0 & 1 & 1 & Dead Grass & 0.00 & 0.03 & 0.03 \\
\hline Dead Shrub & 38 & 18 & 82 & Dead Shrub & 2.77 & 2.17 & 9.06 \\
\hline Total & 242 & 287 & 264 & Total & 21.55 & 33.67 & 36.36 \\
\hline \multicolumn{5}{|c|}{ Average Height (m) } & \multicolumn{3}{|c|}{ Biomass Index $\left(\mathbf{m}^{2}\right)$} \\
\hline SPECIES & 1963 & 1975 & 2001 & SPECIES & 1963 & 1975 & 2001 \\
\hline Acamptopappus shockleyi & 0.15 & 0.20 & 0.27 & Acamptopappus shockleyi & 1.45 & 0.83 & 0.80 \\
\hline Artemisia spinescens & 0.21 & 0.23 & 0.00 & Artemisia spinescens & 0.30 & 0.31 & 0.00 \\
\hline Atriplex confertifolia & 0.35 & 0.44 & 0.39 & Atriplex confertifolia & 9.16 & 20.32 & 3.22 \\
\hline Coleogyne ramosissima & 0.41 & 0.44 & 0.55 & Coleogyne ramosissima & 3.36 & 4.22 & 7.55 \\
\hline Coryphantha vivipara rosea & 0.00 & 0.05 & 0.00 & Coryphantha vivipara rosea & 0.00 & 0.00 & 0.00 \\
\hline Ephedra nevadensis & 0.33 & 0.46 & 0.63 & Ephedra nevadensis & 2.55 & 5.95 & 13.74 \\
\hline Eriogonum inflatum & 0.23 & 0.08 & 0.33 & Eriogonum inflatum & 0.08 & 0.12 & 0.48 \\
\hline Erioneuron pulchellum & 0.00 & 0.04 & 0.05 & Erioneuron pulchellum & 0.00 & 0.04 & 0.00 \\
\hline Grayia spinosa & 0.53 & 0.00 & 0.20 & Grayia spinosa & 0.23 & 0.00 & 0.06 \\
\hline Krameria parvifolia & 0.00 & 0.00 & 0.51 & Krameria parvifolia & 0.00 & 0.00 & 0.09 \\
\hline Larrea tridentata & 0.90 & 1.22 & 1.52 & Larrea tridentata & 6.51 & 14.98 & 50.85 \\
\hline Lycium andersonii & 0.30 & 0.31 & 0.13 & Lycium andersonii & 0.12 & 0.28 & 0.30 \\
\hline Menodora spinescens & 0.10 & 0.16 & 0.24 & Menodora spinescens & 0.06 & 0.14 & 1.10 \\
\hline Oryzopsis hymenoides & 0.19 & 0.36 & 0.46 & Oryzopsis hymenoides & 0.13 & 1.06 & 0.41 \\
\hline Psorothamnus fremontii & 0.00 & 0.00 & 0.43 & Psorothamnus fremontii & 0.00 & 0.00 & 0.02 \\
\hline Sphaeralcea ambigua & 0.14 & 0.08 & 0.11 & Sphaeralcea ambigua & 0.05 & 0.03 & 0.03 \\
\hline Stipa speciosa & 0.27 & 0.44 & 0.44 & Stipa speciosa & 0.84 & 6.07 & 0.68 \\
\hline Viguiera deltoidea & 0.00 & 0.00 & 0.22 & Viguiera deltoidea & 0.00 & 0.00 & 3.51 \\
\hline \multirow[t]{2}{*}{ Yucca brevifolia } & 2.29 & 2.71 & 2.87 & Yucca brevifolia & 3.30 & 9.64 & 9.87 \\
\hline & & & & Total Live & 28.13 & 63.96 & 92.71 \\
\hline
\end{tabular}


Table A-44A. Site characteristics for Plot 44.

Location: Yucca Flat

NTS Area: 6

NTS Grid: P-27

USGS 7.5’ Quadrangle Name: Yucca Lake

\begin{tabular}{lllll} 
& & \multicolumn{2}{c}{ GPS Readings (Zone 11S) } & \\
& Location & Northing & Easting & Condition of Original Corners \\
Corner 1: & SW & 4092999 & 580985 & Standing \\
Corner 2: & NW & 4093018 & 580990 & Standing \\
Corner 3: & NE & 4093011 & 581018 & Standing \\
Corner 4: & SE & 4092982 & 581011 & Standing
\end{tabular}

DEM Plot Elevation: 1257 m

Plot Aspect: $252^{\circ}$

Elevation Above Playa (Playa Name): 61 m (Yucca)

Plot Slope: $5^{\circ}$

Annual Precipitation

Measured: $182 \mathrm{~mm}$

Modeled: $171 \mathrm{~mm}$

Parent Material Type: alluvial fan

Substrate: alluvial fan, strong desert pavement, heavy AV horizon

Slate Geologic Unit: QTa

Beatley Plant Assemblage: Coleogyne/Larrea-Grayia-Lycium

Ostler-Hanson Plant Association: Coleogyne-Lycium-Grayia

Ostler-Hanson Land Unit: 554

Abundance of Biological Soil Crusts: none

Type and Date of Disturbance: none

Plot Condition: good

Location of Transect 1: northeast side of plot

Direction Transects Are Read: southeast to northwest

Date(s) Plot Remeasured: 9 May 2001 
Figure A-44. Photographs showing Plot 44.

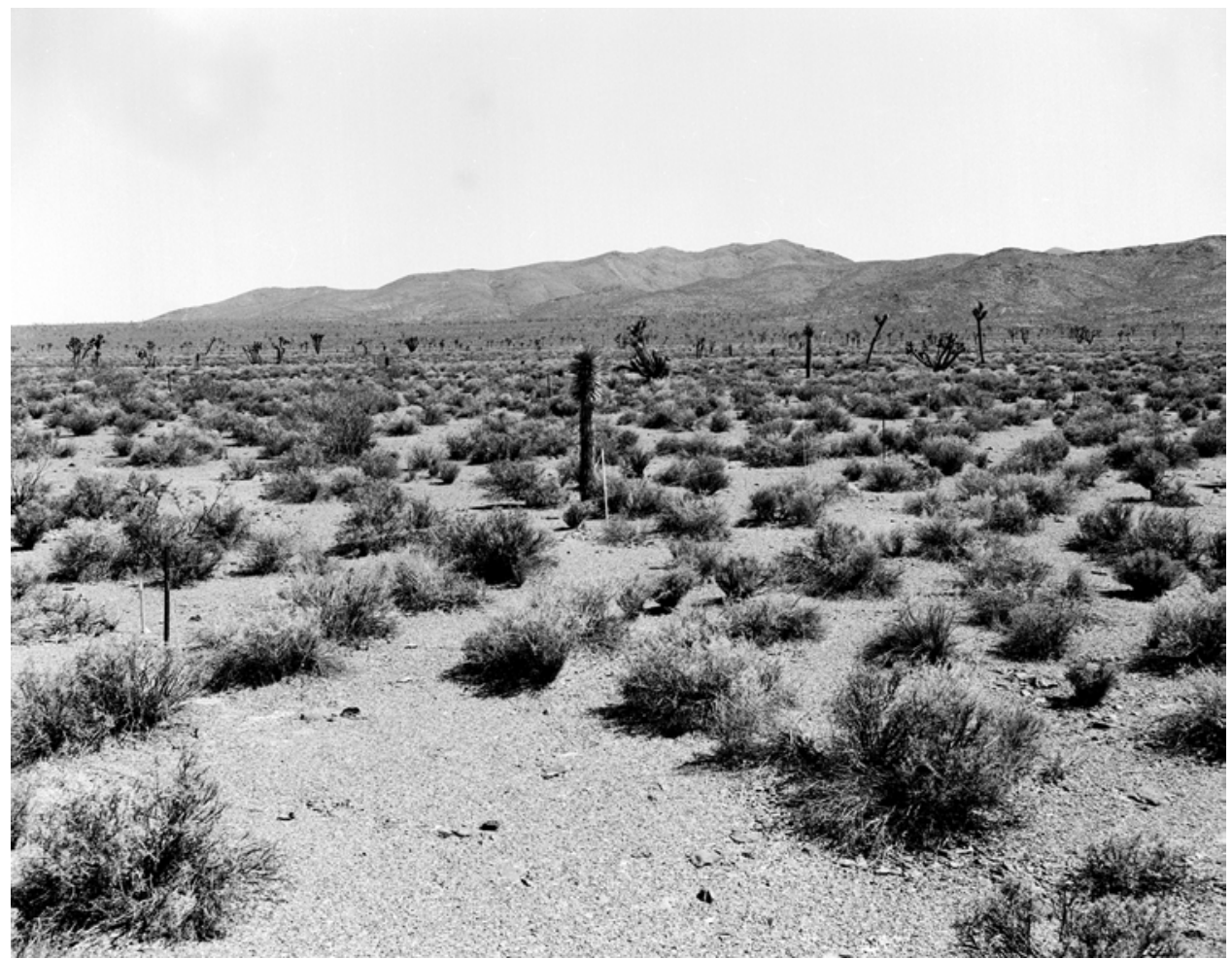

A. (May 12, 1964). The view is southerly across Plot 44 and towards the CP Hills and unnamed mountains on the southwestern side of Yucca Flat. The Tippipah Highway crosses the view in the midground. The vegetation association is blackbrush with creosote bush, spiny hopsage, and wolfberry. Scattered Joshua trees appear on the plot and in the midground (Janice Beatley Collection, 90-B).

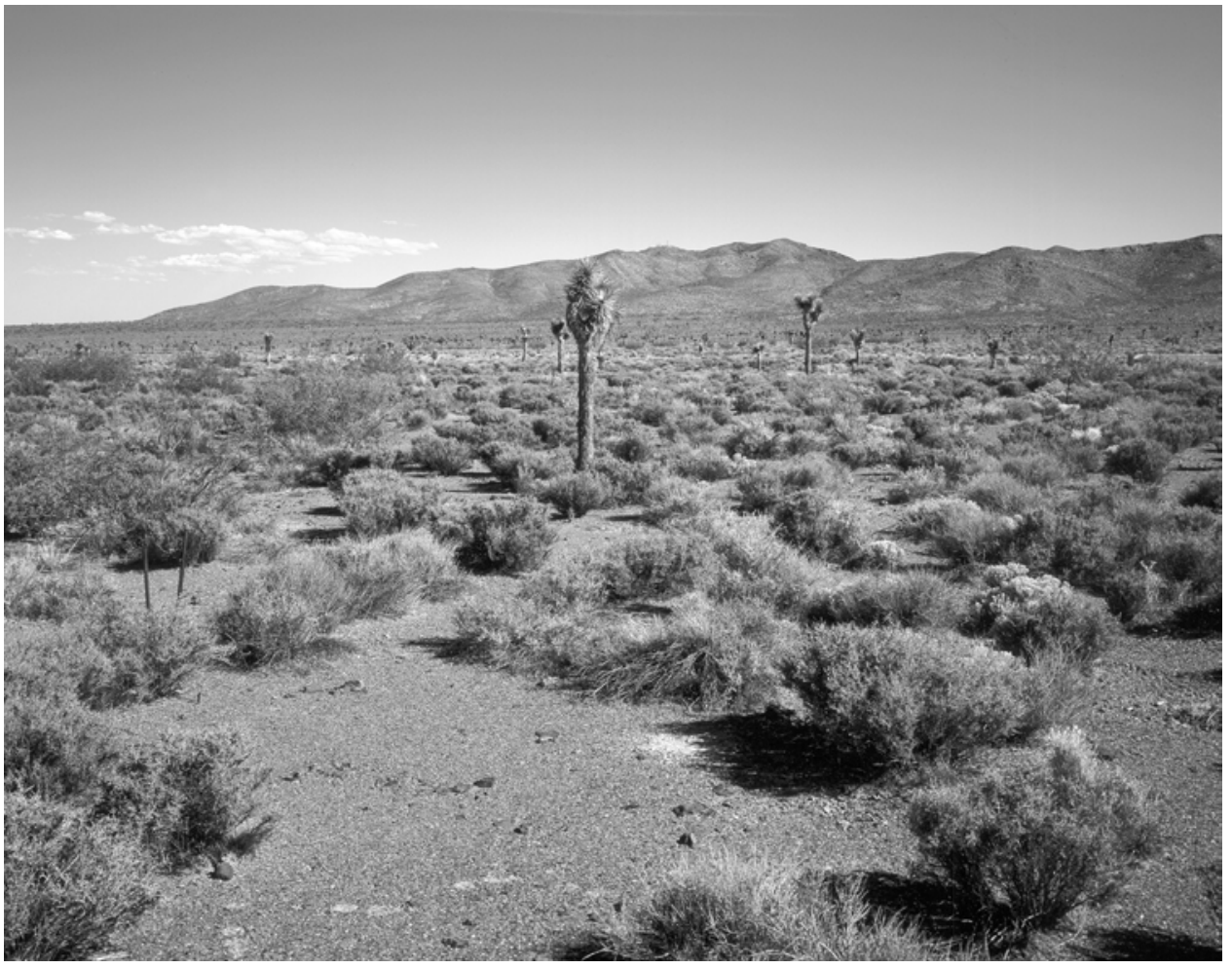

B. (June 7, 2001). Overall, the sizes of individuals appear to have increased through most of the plot. Of the species on this plot, Mormon tea has increased while spiny hopsage has decreased. The Joshua trees may have decreased slightly in density while most persisting individuals have increased in height (Dominic Oldershaw, Stake 4047B). 
Table A-44B. Summary plant data for Plot 44.

\begin{tabular}{|c|c|c|c|c|c|c|c|}
\hline \multicolumn{5}{|c|}{ Number of Plants } & \multicolumn{3}{|c|}{ Cover (\%) } \\
\hline SPECIES & 1963 & 1975 & 2001 & SPECIES & 1963 & 1975 & 2001 \\
\hline Acamptopappus shockleyi & 0 & 1 & 0 & Acamptopappus shockleyi & 0.00 & 0.05 & 0.00 \\
\hline Arabis pulchra munciensis & 0 & 8 & 0 & Arabis pulchra munciensis & 0.00 & 0.15 & 0.00 \\
\hline Artemisia spinescens & 10 & 6 & 3 & Artemisia spinescens & 0.86 & 0.36 & 0.22 \\
\hline Coleogyne ramosissima & 61 & 68 & 85 & Coleogyne ramosissima & 9.37 & 10.76 & 11.45 \\
\hline Delphinium parishii & 0 & 2 & 0 & Delphinium parishii & 0.00 & 0.04 & 0.00 \\
\hline Echinocereus engelmanii & 0 & 1 & 0 & Echinocereus engelmanii & 0.00 & 0.10 & 0.00 \\
\hline Ephedra nevadensis & 16 & 20 & 40 & Ephedra nevadensis & 2.20 & 2.54 & 5.01 \\
\hline Ericameria cooperi & 6 & 4 & 4 & Ericameria cooperi & 0.51 & 0.36 & 0.30 \\
\hline Grayia spinosa & 32 & 39 & 12 & Grayia spinosa & 4.92 & 4.64 & 0.95 \\
\hline Hymenoclea salsola & 0 & 1 & 1 & Hymenoclea salsola & 0.00 & 0.02 & 0.02 \\
\hline Larrea tridentata & 4 & 6 & 9 & Larrea tridentata & 1.25 & 1.85 & 5.31 \\
\hline Lycium andersonii & 22 & 35 & 32 & Lycium andersonii & 2.68 & 6.02 & 3.04 \\
\hline Menodora spinescens & 2 & 2 & 4 & Menodora spinescens & 0.09 & 0.13 & 0.29 \\
\hline Opuntia echinocarpa & 1 & 0 & 0 & Opuntia echinocarpa & 0.05 & 0.00 & 0.00 \\
\hline Oryzopsis hymenoides & 0 & 1 & 0 & Oryzopsis hymenoides & 0.00 & 0.10 & 0.00 \\
\hline Sitanion hystrix & 1 & 1 & 3 & Sitanion hystrix & 0.05 & 0.14 & 0.19 \\
\hline Stephanomeria parryi & 0 & 2 & 0 & Stephanomeria parryi & 0.00 & 0.03 & 0.00 \\
\hline Stipa speciosa & 7 & 14 & 12 & Stipa speciosa & 0.26 & 1.26 & 0.83 \\
\hline Tetradymia axillaris & 1 & 1 & 1 & Tetradymia axillaris & 0.11 & 0.30 & 0.00 \\
\hline Xylorhiza tortifolia & 1 & 4 & 3 & Xylorhiza tortifolia & 0.05 & 0.09 & 0.15 \\
\hline Total Live & 164 & 216 & 209 & Total Live & 22.42 & 28.94 & 27.75 \\
\hline Dead Grass & 0 & 0 & 0 & Dead Grass & 0.00 & 0.00 & 0.00 \\
\hline Dead Shrub & 39 & 7 & 34 & Dead Shrub & 4.28 & 1.11 & 6.34 \\
\hline Total & 203 & 223 & 243 & Total & 26.70 & 30.05 & 34.08 \\
\hline \multicolumn{5}{|c|}{ Average Height (m) } & \multicolumn{3}{|c|}{ Biomass Index $\left(\mathbf{m}^{2}\right)$} \\
\hline SPECIES & 1963 & 1975 & 2001 & SPECIES & 1963 & 1975 & 2001 \\
\hline Acamptopappus shockleyi & 0.00 & 0.28 & 0.00 & Acamptopappus shockleyi & 0.00 & 0.04 & 0.00 \\
\hline Arabis pulchra munciensis & 0.00 & 0.38 & 0.00 & Arabis pulchra munciensis & 0.00 & 0.18 & 0.00 \\
\hline Artemisia spinescens & 0.27 & 0.22 & 0.28 & Artemisia spinescens & 0.82 & 0.31 & 0.20 \\
\hline Coleogyne ramosissima & 0.52 & 0.47 & 0.60 & Coleogyne ramosissima & 17.35 & 18.18 & 24.16 \\
\hline Delphinium parishii & 0.00 & 0.27 & 0.00 & Delphinium parishii & 0.00 & 0.03 & 0.00 \\
\hline Echinocereus engelmanii & 0.00 & 0.25 & 0.00 & Echinocereus engelmanii & 0.00 & 0.09 & 0.00 \\
\hline Ephedra nevadensis & 0.47 & 0.51 & 0.57 & Ephedra nevadensis & 3.77 & 4.74 & 10.29 \\
\hline Ericameria cooperi & 0.30 & 0.27 & 0.38 & Ericameria cooperi & 0.53 & 0.35 & 0.39 \\
\hline Grayia spinosa & 0.52 & 0.53 & 0.56 & Grayia spinosa & 8.84 & 8.63 & 1.71 \\
\hline Hymenoclea salsola & 0.00 & 0.66 & 0.68 & Hymenoclea salsola & 0.00 & 0.04 & 0.04 \\
\hline Larrea tridentata & 1.35 & 1.56 & 2.00 & Larrea tridentata & 5.05 & 9.45 & 31.70 \\
\hline Lycium andersonii & 0.40 & 0.50 & 0.37 & Lycium andersonii & 3.70 & 10.38 & 3.66 \\
\hline Menodora spinescens & 0.17 & 0.14 & 0.26 & Menodora spinescens & 0.05 & 0.05 & 0.25 \\
\hline Opuntia echinocarpa & 0.13 & 0.00 & 0.00 & Opuntia echinocarpa & 0.02 & 0.00 & 0.00 \\
\hline Oryzopsis hymenoides & 0.00 & 0.33 & 0.00 & Oryzopsis hymenoides & 0.00 & 0.11 & 0.00 \\
\hline Sitanion hystrix & 0.38 & 0.38 & 0.55 & Sitanion hystrix & 0.07 & 0.17 & 0.34 \\
\hline Stephanomeria parryi & 0.00 & 0.06 & 0.00 & Stephanomeria parryi & 0.00 & 0.00 & 0.00 \\
\hline Stipa speciosa & 0.26 & 0.34 & 0.54 & Stipa speciosa & 0.23 & 1.53 & 1.49 \\
\hline Tetradymia axillaris & 0.66 & 0.94 & 0.70 & Tetradymia axillaris & 0.24 & 0.95 & 0.00 \\
\hline Xylorhiza tortifolia & 0.15 & 0.23 & 0.26 & Xylorhiza tortifolia & 0.03 & 0.07 & 0.12 \\
\hline & & & & Total Live & 40.71 & 55.31 & 74.36 \\
\hline
\end{tabular}


Table A-45A. Site characteristics for Plot 45.

Location: Yucca Flat

NTS Area: 6

NTS Grid: P-27

USGS 7.5’ Quadrangle Name: Yucca Lake

\begin{tabular}{lllc} 
& & \multicolumn{2}{c}{ GPS Readings (Zone 11S) } \\
& Location & Northing & Easting \\
Corner 1: & SW & 4093297 & 580112 \\
Corner 2: & NW & 4093327 & 580118 \\
Corner 3: & NE & 4093321 & 580147 \\
Corner 4: & SE & 4093290 & 580142
\end{tabular}

DEM Plot Elevation: 1277 m

Condition of Original Corners
Standing
Standing
Standing
Standing

Plot Aspect: $169^{\circ}$

Elevation Above Playa (Playa Name): 82 m (Yucca)

Plot Slope: $4^{\circ}$

Annual Precipitation

Measured: $187 \mathrm{~mm}$

Modeled: $174 \mathrm{~mm}$

Parent Material Type: alluvial fan

Substrate: alluvial fan, mixed volcanic alluvium

Slate Geologic Unit: Qai

Beatley Plant Assemblage: Coleogyne/Grayia-Lycium

Ostler-Hanson Plant Association: Coleogyne-Lycium-Ephedra

Ostler-Hanson Land Unit: 558

Abundance of Biological Soil Crusts: light

Type and Date of Disturbance: none

Plot Condition: good

Location of Transect 1: west side of plot

Direction Transects Are Read: south to north

Date(s) Plot Remeasured: 10 May 2001 
Figure A-45. Photographs showing Plot 45.

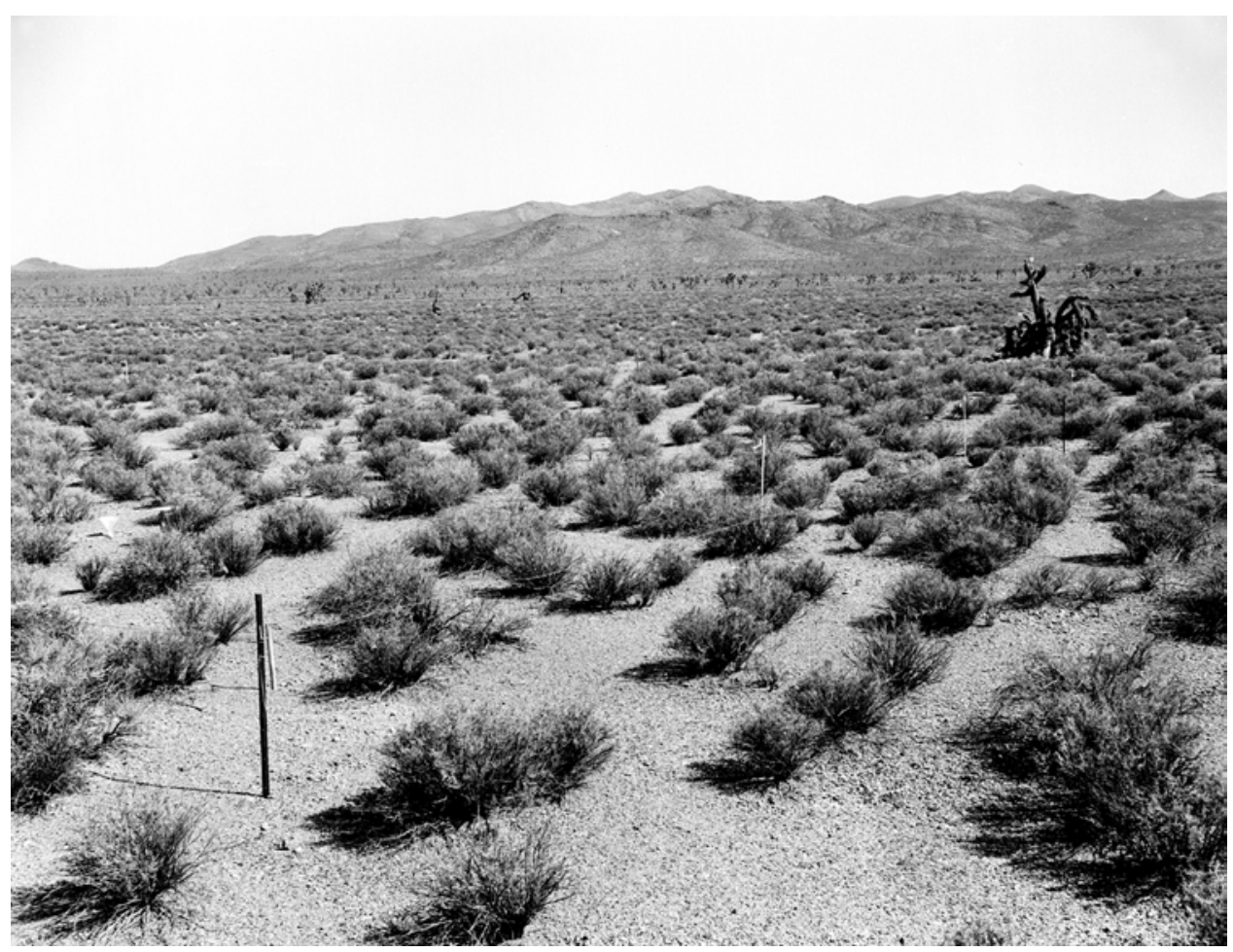

A. (May 12, 1964). This southerly view across Plot 45 shows a blackbrush assemblage with spiny hopsage and wolfberry as subdominants. The mountains in the distance are the same as those depicted in Figure 47 (Janice Beatley Collection, 91-B).

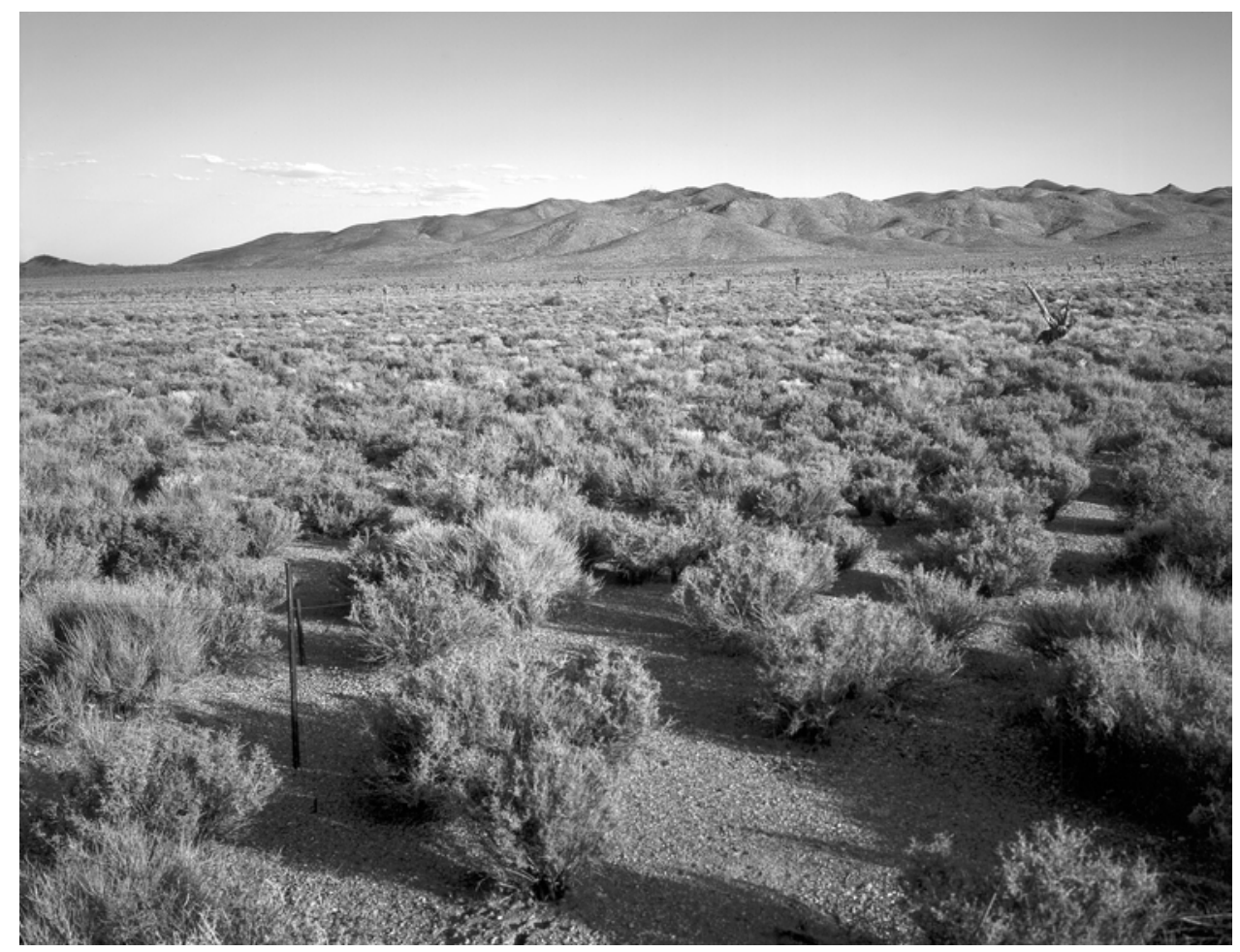

B. (June 7, 2001). Although the blackbrush has not changed much here, spiny hopsage has decreased and Mormon tea has increased on this plot. The Joshua trees in the distance appear little changed (Dominic Oldershaw, Stake 4049B). 
Table A-45B. Summary plant data for Plot 45.

SPECIES
Arabis pulchra munciensis
Ceratoides lanata
Coleogyne ramosissima
Ephedra nevadensis
Eriogonum inflatum
Grayia spinosa
Hymenoclea salsola
Lycium andersonii
Oryzopsis hymenoides
Sitanion hystrix
Stephanomeria parryi
Stipa speciosa
Tetradymia axillaris
Yucca brevifolia
Total Live
Dead Grass
Dead Shrub
Total

\begin{tabular}{ccc}
\multicolumn{3}{c}{ Number of Plants } \\
$\mathbf{1 9 6 3}$ & $\mathbf{1 9 7 5}$ & $\mathbf{2 0 0 1}$ \\
0 & 1 & 0 \\
14 & 29 & 11 \\
111 & 122 & 137 \\
16 & 24 & 32 \\
0 & 0 & 1 \\
18 & 24 & 16 \\
0 & 0 & 6 \\
56 & 63 & 45 \\
1 & 5 & 2 \\
0 & 0 & 6 \\
0 & 3 & 0 \\
0 & 2 & 0 \\
3 & 2 & 1 \\
0 & 0 & 1 \\
219 & 275 & 258 \\
0 & 0 & 0 \\
33 & 16 & 35 \\
252 & 291 & 293 \\
& &
\end{tabular}

SPECIES
Arabis pulchra munciensis
Ceratoides lanata
Coleogyne ramosissima
Ephedra nevadensis
Eriogonum inflatum
Grayia spinosa
Hymenoclea salsola
Lycium andersonii
Oryzopsis hymenoides
Sitanion hystrix
Stephanomeria parryi
Stipa speciosa
Tetradymia axillaris
Yucca brevifolia

\section{Average Height (m)}

$\begin{array}{lll}\mathbf{1 9 6 3} & \mathbf{1 9 7 5} & \mathbf{2 0 0 1} \\ 0.00 & 0.51 & 0.00 \\ 0.46 & 0.48 & 0.60 \\ 0.52 & 0.50 & 0.60 \\ 0.49 & 0.58 & 0.70 \\ 0.00 & 0.00 & 0.31 \\ 0.50 & 0.58 & 0.63 \\ 0.00 & 0.00 & 0.51 \\ 0.43 & 0.52 & 0.41 \\ 0.28 & 0.42 & 0.46 \\ 0.00 & 0.00 & 0.48 \\ 0.00 & 0.08 & 0.00 \\ 0.00 & 0.30 & 0.00 \\ 0.56 & 0.71 & 0.42 \\ 0.00 & 0.00 & 0.68\end{array}$

SPECIES
Arabis pulchra munciensis
Ceratoides lanata
Coleogyne ramosissima
Ephedra nevadensis
Eriogonum inflatum
Grayia spinosa
Hymenoclea salsola
Lycium andersonii
Oryzopsis hymenoides
Sitanion hystrix
Stephanomeria parryi
Stipa speciosa
Tetradymia axillaris
Yucca brevifolia
Total Live
Dead Grass
Dead Shrub
Total

SPECIES
Arabis pulchra munciensis
Ceratoides lanata
Coleogyne ramosissima
Ephedra nevadensis
Eriogonum inflatum
Grayia spinosa
Hymenoclea salsola
Lycium andersonii
Oryzopsis hymenoides
Sitanion hystrix
Stephanomeria parryi
Stipa speciosa
Tetradymia axillaris
Yucca brevifolia
Total Live

$\begin{array}{ccc} & \text { Cover (\%) } \\ \mathbf{1 9 6 3} & \mathbf{1 9 7 5} & \mathbf{2 0 0 1} \\ 0.00 & 0.02 & 0.00 \\ 1.20 & 1.98 & 0.72 \\ 18.14 & 18.24 & 20.66 \\ 2.73 & 3.45 & 5.45 \\ 0.00 & 0.00 & 0.03 \\ 2.08 & 2.69 & 1.77 \\ 0.00 & 0.00 & 0.11 \\ 9.39 & 12.45 & 3.90 \\ 0.05 & 0.53 & 0.09 \\ 0.00 & 0.00 & 0.17 \\ 0.00 & 0.05 & 0.00 \\ 0.00 & 0.05 & 0.00 \\ 0.46 & 0.35 & 0.00 \\ 0.00 & 0.00 & 0.08 \\ 34.05 & 39.80 & 32.98 \\ 0.00 & 0.00 & 0.00 \\ 3.51 & 1.63 & 9.94 \\ 37.56 & 41.43 & 42.92\end{array}$

\section{Biomass Index $\left(\mathrm{m}^{2}\right)$}

$\begin{array}{ccc}\mathbf{1 9 6 3} & \mathbf{1 9 7 5} & \mathbf{2 0 0 1} \\ 0.00 & 0.03 & 0.00 \\ 1.84 & 3.62 & 1.42 \\ 32.52 & 31.23 & 41.21 \\ 5.08 & 7.20 & 13.01 \\ 0.00 & 0.00 & 0.03 \\ 3.69 & 5.42 & 3.87 \\ 0.00 & 0.00 & 0.18 \\ 14.85 & 23.07 & 5.47 \\ 0.05 & 0.79 & 0.15 \\ 0.00 & 0.00 & 0.28 \\ 0.00 & 0.01 & 0.00 \\ 0.00 & 0.06 & 0.00 \\ 0.90 & 0.82 & 0.00 \\ 0.00 & 0.00 & 0.19 \\ 58.93 & 72.24 & 65.80\end{array}$


Table A-46A. Site characteristics for Plot 46.

Location: Yucca Flat

NTS Area: 1

NTS Grid: P-29

USGS 7.5’ Quadrangle Name: Yucca Flat

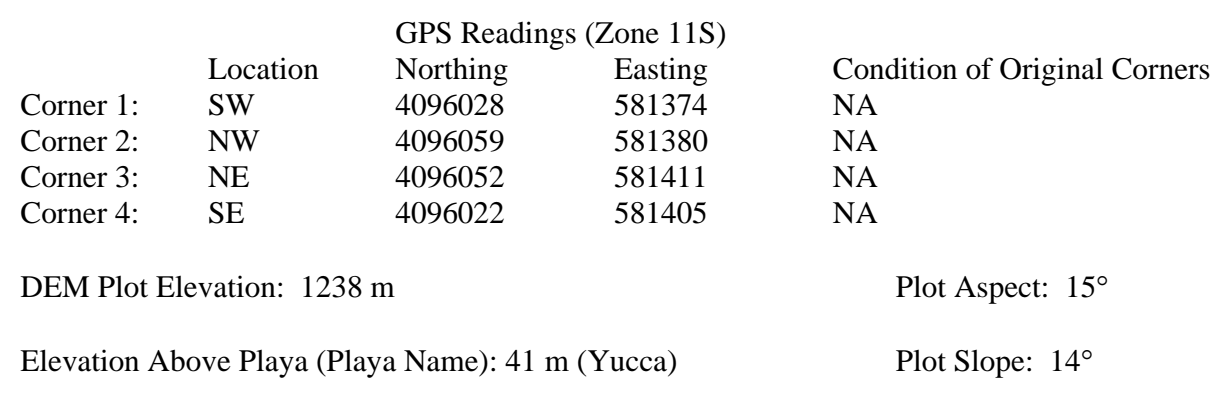

Annual Precipitation

Measured: $179 \mathrm{~mm}$

Modeled: $166 \mathrm{~mm}$

Parent Material Type: alluvial fan

Substrate: alluvial fan, desert pavement

Slate Geologic Unit: Qai

Beatley Plant Assemblage: Grayia-Lycium

Ostler-Hanson Plant Association: Grayia-Lycium-Ceratoides

Ostler-Hanson Land Unit: 581

Abundance of Biological Soil Crusts: moderate

Type and Date of Disturbance: none

Plot Condition: good

Location of Transect 1: west side of plot

Direction Transects Are Read: north to south

Date(s) Plot Remeasured: 9 April 2001 
Figure A-46. Photographs showing Plot 46.

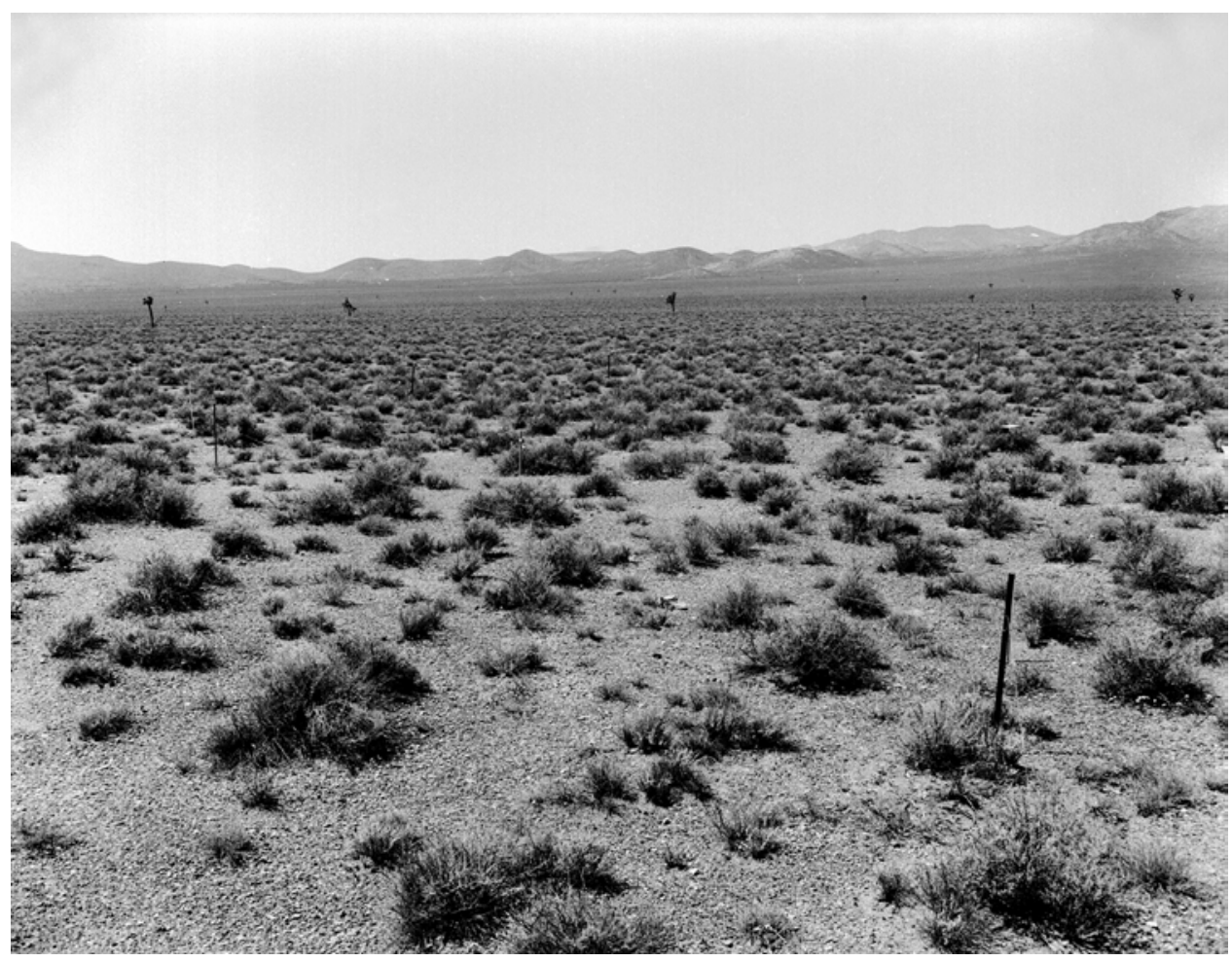

A. (May 12, 1964). In this southwesterly view across Plot 46, the southern part of Yucca Flat is dominated by spiny hopsage and wolfberry. Scattered Joshua trees appear in the distance. Mine Mountain appears in the background (Janice Beatley Collection, 98-A).

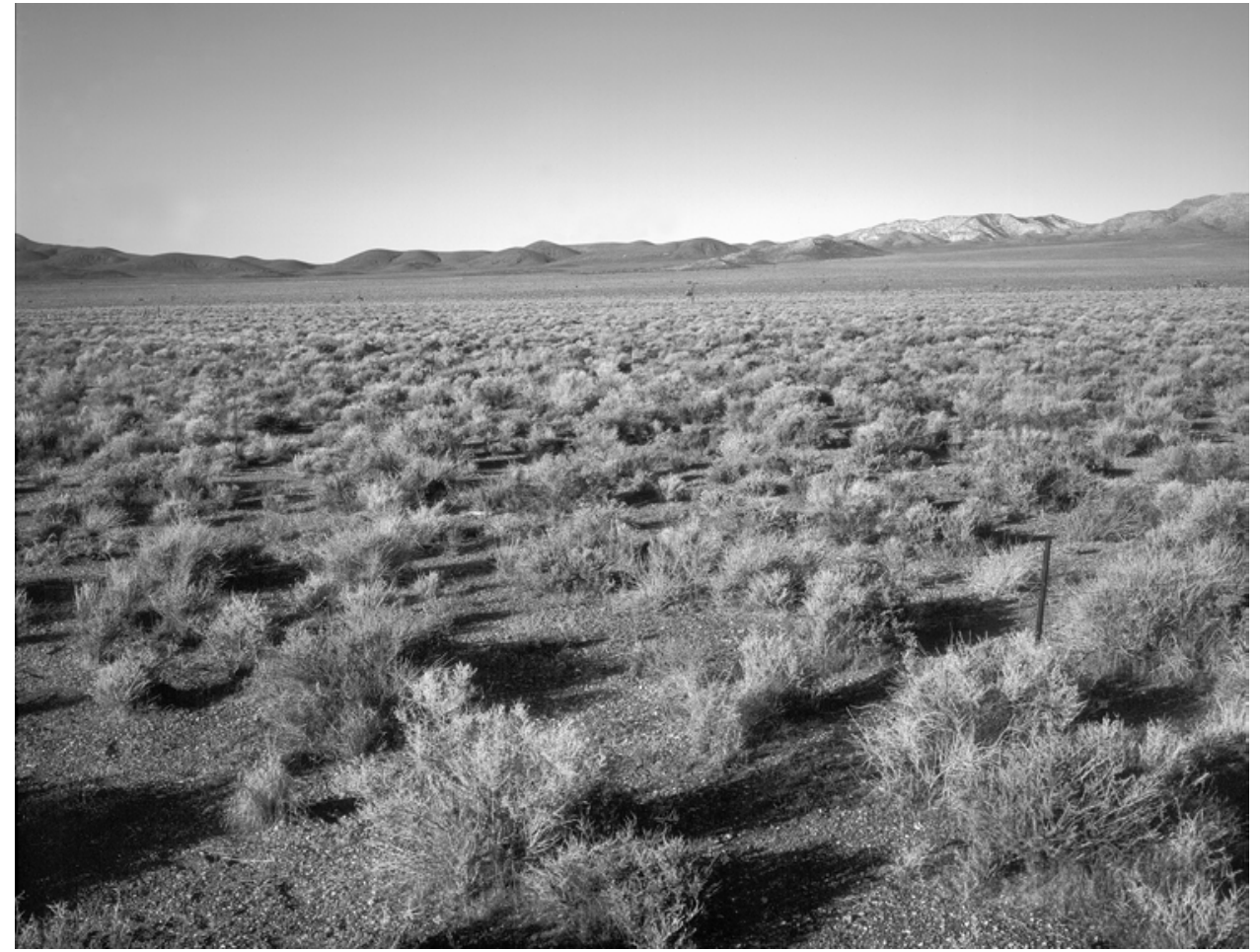

B. (April 8, 2001). Mormon tea has assumed dominance, as both spiny hopsage and wolfberry have declined. The few Joshua trees that once appeared in the distance are now mostly gone, and only one is readily apparent now (R.H. Webb, Stake 4127A). 
Table A-46B. Summary plant data for Plot 46.

$\begin{array}{lcccl}\text { SPECIES } & \mathbf{1 9 6 3} & \mathbf{1 9 7 5} & \mathbf{2 0 0 1} & \text { SPECIES } \\ \text { Acamptopappus shockleyi } & 65 & 66 & 32 & \text { Acamptopappus shockleyi } \\ \text { Artemisia spinescens } & 11 & 20 & 6 & \text { Artemisia spinescens } \\ \text { Atriplex canescens } & 0 & 3 & 15 & \text { Atriplex canescens } \\ \text { Ceratoides lanata } & 84 & 96 & 39 & \text { Ceratoides lanata } \\ \text { Chrysothamnus viscidiflorus } & 0 & 1 & 0 & \text { Chrysothamnus viscidiflorus } \\ \text { Ephedra nevadensis } & 13 & 21 & 48 & \text { Ephedra nevadensis } \\ \text { Ericameria cooperi } & 1 & 0 & 0 & \text { Ericameria cooperi } \\ \text { Grayia spinosa } & 75 & 106 & 64 & \text { Grayia spinosa } \\ \text { Hymenoclea salsola } & 0 & 2 & 4 & \text { Hymenoclea salsola } \\ \text { Lycium andersonii } & 36 & 53 & 46 & \text { Lycium andersonii } \\ \text { Opuntia echinocarpa } & 0 & 0 & 1 & \text { Opuntia echinocarpa } \\ \text { Oryzopsis hymenoides } & 0 & 1 & 16 & \text { Oryzopsis hymenoides } \\ \text { Sitanion hystrix } & 2 & 6 & 0 & \text { Sitanion hystrix } \\ \text { Sphaeralcea ambigua } & 0 & 2 & 3 & \text { Sphaeralcea ambigua } \\ \text { Stipa comata } & 0 & 0 & 12 & \text { Stipa comata } \\ \text { Stipa speciosa } & 0 & 3 & 4 & \text { Stipa speciosa } \\ \text { Tetradymia axillaris } & 3 & 5 & 3 & \text { Tetradymia axillaris } \\ \text { Tetradymia glabrata } & 1 & 1 & 0 & \text { Tetradymia glabrata } \\ \text { Unknown herbaceous perennial } & 0 & 0 & 1 & \text { Unknown herbaceous perennial } \\ \text { Total Live } & 291 & 386 & 294 & \text { Total Live } \\ \text { Dead Grass } & 0 & 0 & 6 & \text { Dead Grass } \\ \text { Dead Shrub } & 46 & 18 & 129 & \text { Dead Shrub } \\ \text { Total } & 337 & 404 & 429 & \text { Total }\end{array}$

$\begin{array}{ccc} & \text { Cover (\%) } & \\ \mathbf{1 9 6 3} & \mathbf{1 9 7 5} & \mathbf{2 0 0 1} \\ 4.24 & 3.82 & 1.89 \\ 0.67 & 0.85 & 0.35 \\ 0.00 & 0.45 & 1.55 \\ 6.04 & 6.22 & 2.14 \\ 0.00 & 0.01 & 0.00 \\ 1.61 & 3.45 & 5.51 \\ 0.03 & 0.00 & 0.00 \\ 11.00 & 12.72 & 4.95 \\ 0.00 & 0.09 & 0.58 \\ 3.63 & 7.34 & 5.32 \\ 0.00 & 0.00 & 0.03 \\ 0.01 & 0.06 & 0.50 \\ 0.05 & 0.21 & 0.00 \\ 0.00 & 0.02 & 0.06 \\ 0.00 & 0.00 & 0.62 \\ 0.00 & 0.24 & 0.29 \\ 0.60 & 0.47 & 0.09 \\ 0.07 & 0.18 & 0.00 \\ 0.00 & 0.00 & 0.02 \\ 27.93 & 36.12 & 23.89 \\ 0.00 & 0.00 & 0.15 \\ 3.46 & 1.40 & 13.26 \\ 31.39 & 37.52 & 37.31\end{array}$

\section{Average Height (m)}

\section{SPECIES}

Acamptopappus shockleyi

Artemisia spinescens

Atriplex canescens

Ceratoides lanata

Chrysothamnus viscidiflorus

Ephedra nevadensis

Ericameria cooperi

Grayia spinosa

Hymenoclea salsola

Lycium andersonii

Opuntia echinocarpa

Oryzopsis hymenoides

Sitanion hystrix

Sphaeralcea ambigua

Stipa comata

Stipa speciosa

Tetradymia axillaris

Tetradymia glabrata

Unknown herbaceous perennial

$\begin{array}{lll}\mathbf{1 9 6 3} & \mathbf{1 9 7 5} & \mathbf{2 0 0 1} \\ 0.18 & 0.20 & 0.24 \\ 0.21 & 0.16 & 0.29 \\ 0.00 & 0.71 & 0.59 \\ 0.29 & 0.34 & 0.40 \\ 0.00 & 0.43 & 0.00 \\ 0.38 & 0.51 & 0.54 \\ 0.13 & 0.00 & 0.00 \\ 0.45 & 0.50 & 0.50 \\ 0.00 & 0.44 & 0.49 \\ 0.37 & 0.46 & 0.39 \\ 0.00 & 0.00 & 0.49 \\ 0.00 & 0.36 & 0.25 \\ 0.29 & 0.25 & 0.00 \\ 0.00 & 0.03 & 0.05 \\ 0.00 & 0.00 & 0.34 \\ 0.00 & 0.30 & 0.30 \\ 0.67 & 0.57 & 0.57 \\ 0.38 & 0.56 & 0.00 \\ 0.00 & 0.00 & 0.35\end{array}$

$\begin{array}{lccc}\text { SPECIES } & \mathbf{1 9 6 3} & \mathbf{1 9 7 5} & \mathbf{2 0 0 1} \\ \text { Acamptopappus shockleyi } & 2.72 & 2.63 & 1.58 \\ \text { Artemisia spinescens } & 0.49 & 0.54 & 0.36 \\ \text { Atriplex canescens } & 0.00 & 1.08 & 3.56 \\ \text { Ceratoides lanata } & 6.06 & 7.61 & 3.03 \\ \text { Chrysothamnus viscidiflorus } & 0.00 & 0.01 & 0.00 \\ \text { Ephedra nevadensis } & 2.27 & 6.15 & 11.25 \\ \text { Ericameria cooperi } & 0.01 & 0.00 & 0.00 \\ \text { Grayia spinosa } & 17.60 & 22.83 & 8.65 \\ \text { Hymenoclea salsola } & 0.00 & 0.13 & 1.01 \\ \text { Lycium andersonii } & 4.80 & 11.70 & 7.14 \\ \text { Opuntia echinocarpa } & 0.00 & 0.00 & 0.04 \\ \text { Oryzopsis hymenoides } & 0.01 & 0.08 & 0.46 \\ \text { Sitanion hystrix } & 0.04 & 0.17 & 0.00 \\ \text { Sphaeralcea ambigua } & 0.00 & 0.00 & 0.01 \\ \text { Stipa comata } & 0.00 & 0.00 & 0.69 \\ \text { Stipa speciosa } & 0.00 & 0.26 & 0.30 \\ \text { Tetradymia axillaris } & 1.33 & 0.91 & 0.19 \\ \text { Tetradymia glabrata } & 0.09 & 0.34 & 0.00 \\ \text { Unknown herbaceous perennial } & 0.00 & 0.00 & 0.02 \\ \text { Total Live } & 35.42 & 54.44 & 38.33\end{array}$


Table A-47A. Site characteristics for Plot 47.

Location: Yucca Flat

NTS Area: 16

NTS Grid: K-30

USGS 7.5’ Quadrangle Name: Tippipah Spring

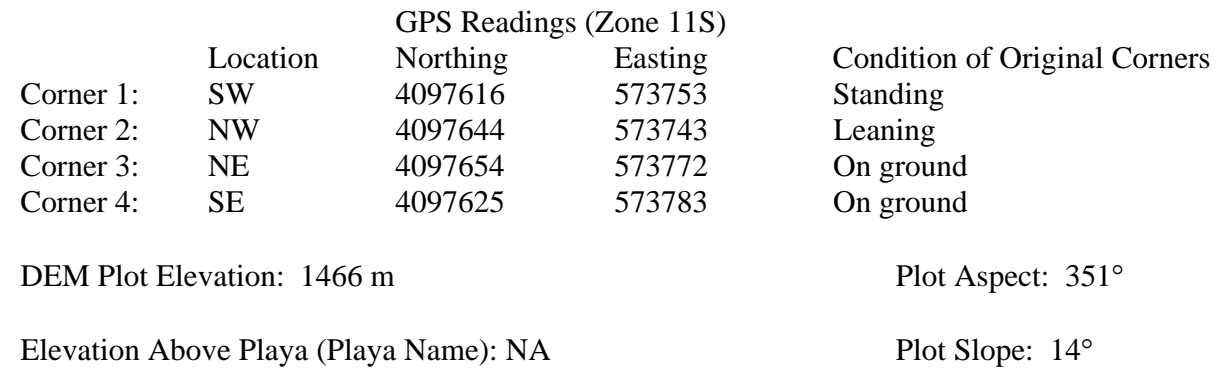

Annual Precipitation

Measured: $232 \mathrm{~mm}$

Modeled: $199 \mathrm{~mm}$

Parent Material Type: alluvial fan

Substrate: NA

Slate Geologic Unit: Qai

Beatley Plant Assemblage: Grayia-Stipa-Coleogyne

Ostler-Hanson Plant Association: Grayia-Stipa-Coleogyne

Ostler-Hanson Land Unit: 782

Abundance of Biological Soil Crusts: no crusts, some near road

Type and Date of Disturbance: NA

Plot Condition: good

Location of Transect 1: east side of plot

Direction Transects Are Read: south to north

Date(s) Plot Remeasured: 10 May 2001 
Figure A-47. Photographs showing Plot 47.

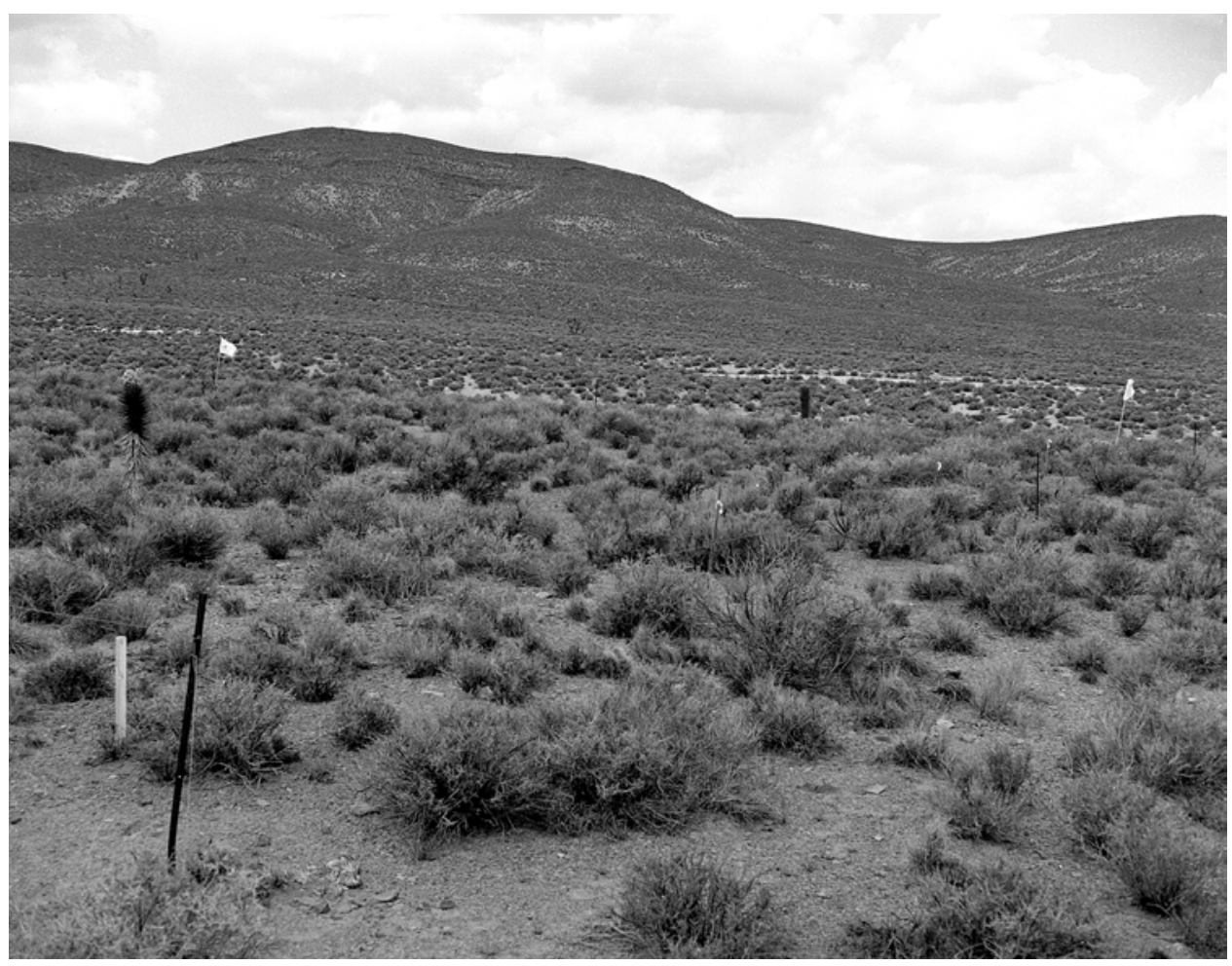

A. (April 21, 1964). This northwesterly view across Plot 47 shows a mixture of shrubs with low hills in front of Syncline Ridge. This plot is at the extreme western edge of Yucca Flat. The vegetation is dominated by spiny hopsage, needlegrass, and blackbrush. A few Joshua trees are present (Janice Beatley Collection, 17-B).

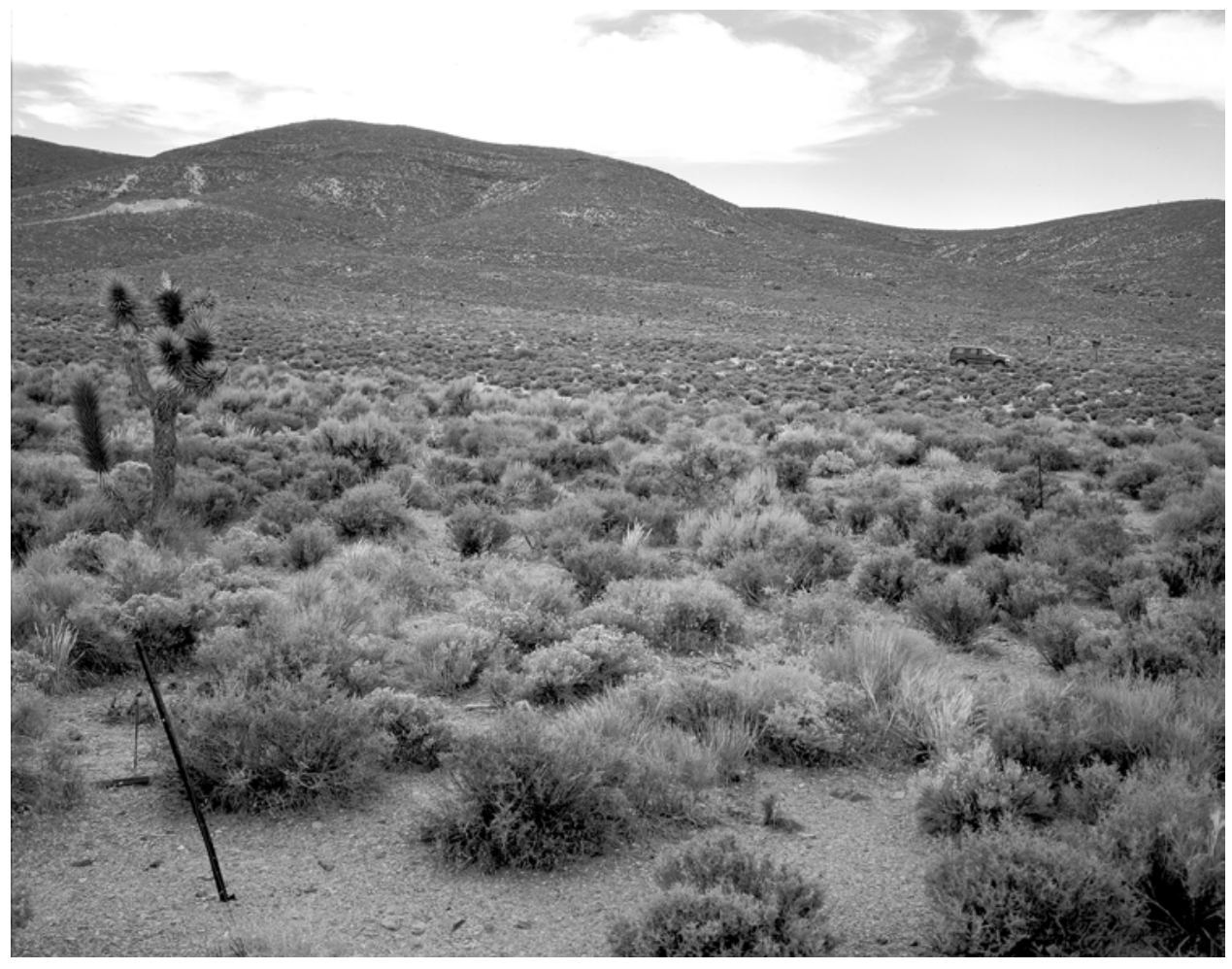

B. (June 8, 2001). Blackbrush now dominates the plot, along with Cooper's goldenbush (Ericameria cooperi) and Great Basin sagebrush (Artemisia tridentata). The one persisting Joshua tree in the view has grown and branched (Dominic Oldershaw, Stake 4059B). 
Table A-47B. Summary plant data for Plot 47.

SPECIES
Artemisia spinescens
Artemisia tridentata
Calochortus flexuosus
Chrysothamnus viscidiflorus
Coleogyne ramosissima
Delphinium parishii
Ephedra nevadensis
Ericameria cooperi
Grayia spinosa
Hymenoclea salsola
Lycium andersonii
Oryzopsis hymenoides
Phlox stansburyi
Sitanion hystrix
Stipa speciosa
Tetradymia glabrata
Yucca brevifolia
Total Live
Dead Grass
Dead Shrub
Total

\begin{tabular}{ccc}
\multicolumn{3}{c}{ Number of Plants } \\
$\mathbf{1 9 6 3}$ & $\mathbf{1 9 7 5}$ & $\mathbf{2 0 0 1}$ \\
0 & 0 & 4 \\
16 & 19 & 17 \\
0 & 0 & 1 \\
0 & 0 & 1 \\
64 & 63 & 94 \\
0 & 1 & 0 \\
25 & 32 & 29 \\
45 & 44 & 35 \\
49 & 56 & 15 \\
5 & 7 & 9 \\
5 & 5 & 3 \\
0 & 0 & 1 \\
0 & 0 & 6 \\
8 & 10 & 15 \\
75 & 131 & 18 \\
4 & 6 & 3 \\
1 & 1 & 1 \\
297 & 375 & 252 \\
0 & 0 & 24 \\
55 & 22 & 83 \\
352 & 397 & 359
\end{tabular}

SPECIES
Artemisia spinescens
Artemisia tridentata
Calochortus flexuosus
Chrysothamnus viscidiflorus
Coleogyne ramosissima
Delphinium parishii
Ephedra nevadensis
Ericameria cooperi
Grayia spinosa
Hymenoclea salsola
Lycium andersonii
Oryzopsis hymenoides
Phlox stansburyi
Sitanion hystrix
Stipa speciosa
Tetradymia glabrata
Yucca brevifolia
Total Live
Dead Grass
Dead Shrub
Total

$\begin{array}{ccc} & \text { Cover (\%) } & \\ \mathbf{1 9 6 3} & \mathbf{1 9 7 5} & \mathbf{2 0 0 1} \\ 0.00 & 0.00 & 1.02 \\ 3.40 & 3.85 & 4.08 \\ 0.00 & 0.00 & 0.01 \\ 0.00 & 0.00 & 0.11 \\ 9.85 & 9.82 & 13.44 \\ 0.00 & 0.02 & 0.00 \\ 2.77 & 3.31 & 3.81 \\ 4.10 & 4.75 & 4.62 \\ 8.42 & 11.78 & 1.30 \\ 0.35 & 0.82 & 1.04 \\ 1.20 & 1.06 & 0.09 \\ 0.00 & 0.00 & 0.12 \\ 0.00 & 0.00 & 0.45 \\ 0.53 & 0.68 & 1.30 \\ 3.76 & 11.48 & 1.48 \\ 1.05 & 1.34 & 0.38 \\ 0.14 & 0.18 & 0.20 \\ 35.56 & 49.10 & 33.45 \\ 0.00 & 0.00 & 1.15 \\ 6.45 & 3.57 & 12.62 \\ 42.02 & 52.67 & 47.21\end{array}$

\section{SPECIES}

Artemisia spinescens

Artemisia tridentata

Calochortus flexuosus

Chrysothamnus viscidiflorus

Coleogyne ramosissima

Delphinium parishii

Ephedra nevadensis

Ericameria cooperi

Grayia spinosa

Hymenoclea salsola

Lycium andersonii

Oryzopsis hymenoides

Phlox stansburyi

Sitanion hystrix

Stipa speciosa

Tetradymia glabrata

Yucca brevifolia

\section{Average Height (m)}

$\begin{array}{lll}\mathbf{1 9 6 3} & \mathbf{1 9 7 5} & \mathbf{2 0 0 1} \\ 0.00 & 0.00 & 0.78 \\ 0.71 & 0.75 & 0.93 \\ 0.00 & 0.00 & 0.32 \\ 0.00 & 0.00 & 0.27 \\ 0.52 & 0.50 & 0.61 \\ 0.00 & 0.10 & 0.00 \\ 0.38 & 0.48 & 0.63 \\ 0.31 & 0.34 & 0.40 \\ 0.62 & 0.65 & 0.54 \\ 0.40 & 0.48 & 0.59 \\ 0.56 & 0.56 & 0.37 \\ 0.00 & 0.00 & 0.53 \\ 0.00 & 0.00 & 0.23 \\ 0.35 & 0.30 & 0.46 \\ 0.35 & 0.38 & 0.49 \\ 0.67 & 0.68 & 0.59 \\ 1.55 & 2.39 & 3.33\end{array}$

SPECIES
Artemisia spinescens
Artemisia tridentata
Calochortus flexuosus
Chrysothamnus viscidiflorus
Coleogyne ramosissima
Delphinium parishii
Ephedra nevadensis
Ericameria cooperi
Grayia spinosa
Hymenoclea salsola
Lycium andersonii
Oryzopsis hymenoides
Phlox stansburyi
Sitanion hystrix
Stipa speciosa
Tetradymia glabrata
Yucca brevifolia
Total Live

\begin{tabular}{lcc}
\multicolumn{3}{c}{ Biomass Index $\left(\mathbf{m}^{2}\right)$} \\
$\mathbf{1 9 6 3}$ & $\mathbf{1 9 7 5}$ & $\mathbf{2 0 0 1}$ \\
0.00 & 0.00 & 2.82 \\
8.79 & 10.98 & 12.84 \\
0.00 & 0.00 & 0.01 \\
0.00 & 0.00 & 0.10 \\
18.47 & 17.36 & 28.23 \\
0.00 & 0.01 & 0.00 \\
4.27 & 6.12 & 8.75 \\
4.65 & 5.78 & 6.67 \\
17.87 & 27.15 & 2.72 \\
0.50 & 1.35 & 1.95 \\
2.69 & 2.10 & 0.11 \\
0.00 & 0.00 & 0.21 \\
0.00 & 0.00 & 0.37 \\
0.63 & 0.76 & 2.14 \\
4.76 & 15.17 & 2.56 \\
2.39 & 3.13 & 0.71 \\
0.71 & 1.46 & 2.23 \\
65.71 & 91.36 & 72.42
\end{tabular}


Table A-48A. Site characteristics for Plot 48.

Location: Yucca Flat

NTS Area: 1

NTS Grid: P-32

USGS 7.5’ Quadrangle Name: Yucca Flat

\begin{tabular}{lllc} 
& & \multicolumn{2}{c}{ GPS Readings (Zone 11S) } \\
& Location & Northing & Easting \\
Corner 1: & SW & 4101300 & 580831 \\
Corner 2: & NW & 4101330 & 580825 \\
Corner 3: & NE & 4101321 & 580856 \\
Corner 4: & SE & 4101291 & 580859
\end{tabular}

DEM Plot Elevation: 1277 m

Elevation Above Playa (Playa Name): 82 m (Yucca)

Condition of Original Corners
Standing
Standing
Standing, but in wrong place (3.8 feet off)
Standing

Plot Aspect: $112^{\circ}$

Plot Slope: $8^{\circ}$

Annual Precipitation

Measured: $164 \mathrm{~mm}$

Modeled: $165 \mathrm{~mm}$

Parent Material Type: alluvial fan

Substrate: alluvial fan

Slate Geologic Unit: Qai

Beatley Plant Assemblage: Grayia-Larrea

Ostler-Hanson Plant Association: NA

Ostler-Hanson Land Unit: 588

Abundance of Biological Soil Crusts: NA

Type and Date of Disturbance: above-ground testing effects from T1 site

Plot Condition: good

Location of Transect 1: south side of plot

Direction Transects Are Read: east to west

Date(s) Plot Remeasured: 6 April 2001 
Figure A-48. Photographs showing Plot 48.

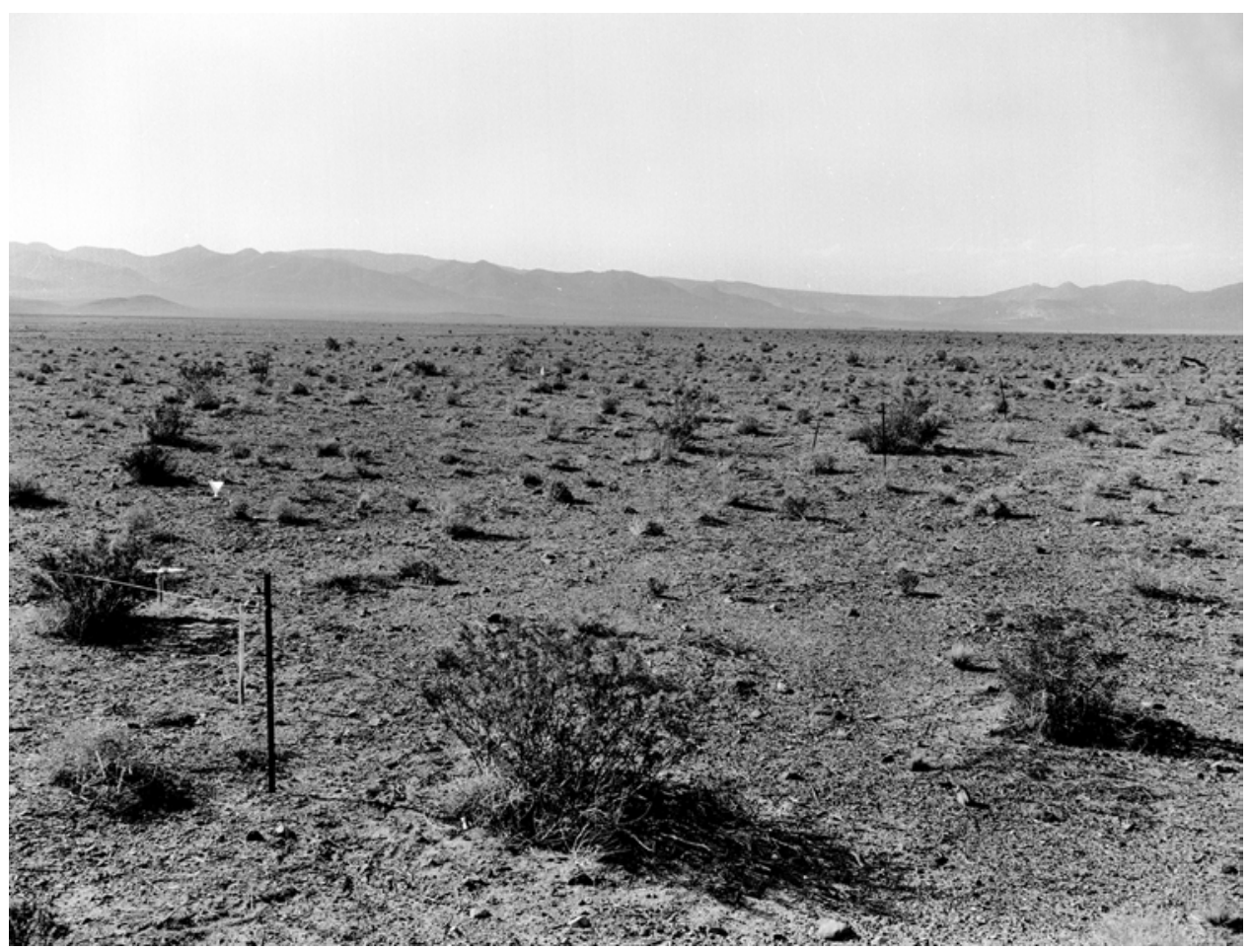

A. (May 12, 1964). In this northwesterly view across Plot 48, disturbance associated with above-ground nuclear testing, 1952-1957, is apparent on Yucca Flat. Approximately 1 mile west of this site, four tests were conducted at T-1 Ground Zero under the names of Easy, Simon, Apple II, and Galileo. The Eleana Range (right background) and Syncline Ridge (left background) appear in the distance. The funnel shown in the view is a

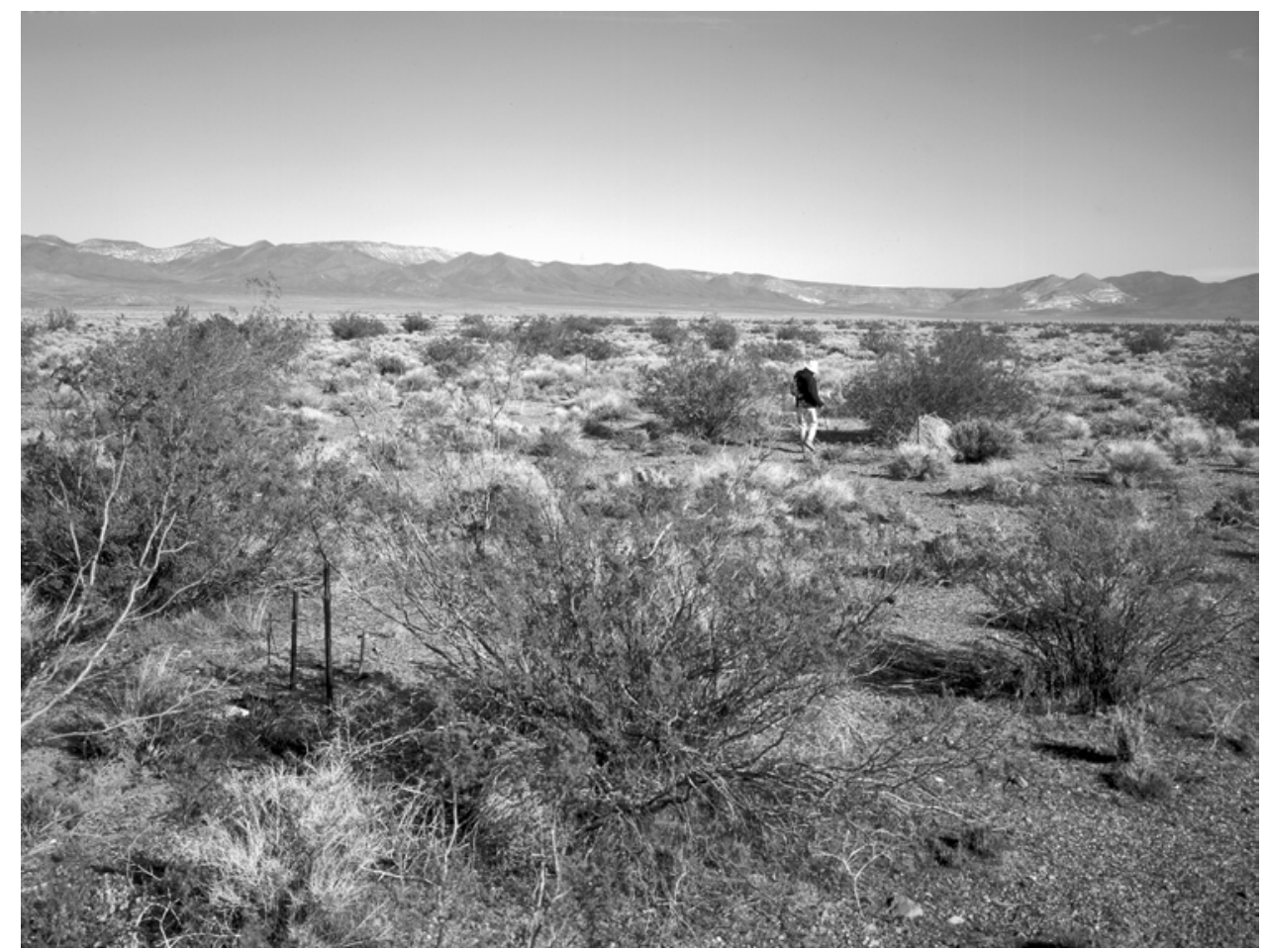

storage rainfall gage. The disturbed vegetation assemblage is dominated by spiny hopsage and creosote bush (Janice Beatley Collection, 93-B). B. (April 6, 2001). The increase in cover and biomass is striking here and reflects disturbance recovery. Creosote bush and cheesebush dominate this plot (R.H. Webb, Stake 4111B). 
Table A-48B. Summary plant data for Plot 48.

\begin{tabular}{|c|c|c|c|c|c|c|c|c|}
\hline SPECIES & 1963 & 1964 & 1965 & 1966 & 1967 & 1970 & 1975 & 2001 \\
\hline Astragalus lentiginosus & 0 & 0 & 0 & 3 & 0 & 0 & 0 & 0 \\
\hline Ceratoides lanata & 0 & 0 & 0 & 1 & 1 & 0 & 0 & 1 \\
\hline Chrysothamnus viscidiflorus & 1 & 2 & 2 & 2 & 2 & 2 & 2 & 16 \\
\hline Coleogyne ramosissima & 1 & 1 & 1 & 3 & 1 & 2 & 2 & 3 \\
\hline Dichelostemma pulchella & 0 & 0 & 0 & 0 & 0 & 0 & 0 & 4 \\
\hline Ephedra nevadensis & 0 & 0 & 0 & 0 & 0 & 0 & 0 & 1 \\
\hline Ericameria cooperi & 3 & 2 & 3 & 2 & 3 & 2 & 2 & 0 \\
\hline Eriogonum inflatum & 0 & 0 & 0 & 0 & 0 & 0 & 0 & 1 \\
\hline Grayia spinosa & 16 & 17 & 20 & 23 & 24 & 27 & 27 & 2 \\
\hline Hymenoclea salsola & 8 & 8 & 7 & 9 & 10 & 11 & 11 & 52 \\
\hline Larrea tridentata & 4 & 3 & 5 & 6 & 6 & 9 & 9 & 14 \\
\hline Lycium andersonii & 1 & 1 & 2 & 3 & 2 & 3 & 3 & 6 \\
\hline Opuntia basilaris & 0 & 0 & 0 & 0 & 0 & 0 & 0 & 1 \\
\hline Oryzopsis hymenoides & 0 & 0 & 0 & 1 & 1 & 0 & 0 & 5 \\
\hline Sphaeralcea ambigua & 0 & 0 & 2 & 3 & 2 & 0 & 0 & 1 \\
\hline Stephanomeria parryi & 0 & 0 & 0 & 0 & 0 & 0 & 0 & 0 \\
\hline Stipa speciosa & 3 & 4 & 3 & 5 & 3 & 5 & 5 & 5 \\
\hline Total Live & 37 & 38 & 45 & 61 & 55 & 61 & 61 & 112 \\
\hline Dead Grass & 0 & 0 & 0 & 0 & 0 & 0 & 0 & 4 \\
\hline Dead Shrub & 0 & 3 & 1 & 0 & 0 & 0 & 0 & 26 \\
\hline Total & 37 & 41 & 46 & 66 & 55 & 61 & 61 & 142 \\
\hline & \multicolumn{8}{|c|}{ Average Height (m) } \\
\hline SPECIES & 1963 & 1964 & 1965 & 1966 & 1967 & 1970 & 1975 & 2001 \\
\hline Astragalus lentiginosus & 0.00 & 0.00 & 0.00 & 0.14 & 0.00 & 0.00 & 0.00 & 0.00 \\
\hline Ceratoides lanata & 0.00 & 0.00 & 0.00 & 0.20 & 0.23 & 0.00 & 0.30 & 0.17 \\
\hline Chrysothamnus viscidiflorus & 0.30 & 0.30 & 0.39 & 0.43 & 0.46 & 0.47 & 0.42 & 0.31 \\
\hline Coleogyne ramosissima & 0.25 & 0.28 & 0.36 & 0.30 & 0.38 & 0.38 & 0.44 & 0.81 \\
\hline Dichelostemma pulchella & 0.00 & 0.00 & 0.00 & 0.00 & 0.00 & 0.00 & 0.00 & 0.20 \\
\hline Ephedra nevadensis & 0.00 & 0.00 & 0.00 & 0.00 & 0.00 & 0.00 & 0.00 & 0.65 \\
\hline Ericameria cooperi & 0.21 & 0.15 & 0.21 & 0.17 & 0.20 & 0.20 & 0.26 & 0.00 \\
\hline Eriogonum inflatum & 0.00 & 0.00 & 0.00 & 0.00 & 0.00 & 0.00 & 0.00 & 0.42 \\
\hline Grayia spinosa & 0.43 & 0.42 & 0.50 & 0.57 & 0.58 & 0.60 & 0.64 & 0.47 \\
\hline Hymenoclea salsola & 0.37 & 0.37 & 0.39 & 0.45 & 0.44 & 0.50 & 0.48 & 0.51 \\
\hline Larrea tridentata & 1.06 & 0.91 & 1.19 & 1.05 & 1.43 & 1.67 & 2.09 & 2.77 \\
\hline Lycium andersonii & 0.15 & 0.13 & 0.23 & 0.32 & 0.28 & 0.35 & 0.46 & 0.42 \\
\hline Opuntia basilaris & 0.00 & 0.00 & 0.00 & 0.00 & 0.00 & 0.00 & 0.00 & 0.15 \\
\hline Oryzopsis hymenoides & 0.00 & 0.00 & 0.00 & 0.33 & 0.41 & 0.00 & 0.33 & 0.31 \\
\hline Sphaeralcea ambigua & 0.00 & 0.00 & 0.20 & 0.21 & 0.27 & 0.00 & 0.00 & 0.45 \\
\hline Stephanomeria parryi & 0.00 & 0.00 & 0.00 & 0.00 & 0.00 & 0.00 & 0.05 & 0.00 \\
\hline Stipa speciosa & 0.20 & 0.14 & 0.30 & 0.35 & 0.33 & 0.28 & 0.38 & 0.39 \\
\hline
\end{tabular}


Table A-48B (continued). Summary plant data for Plot 48.

\begin{tabular}{|c|c|c|c|c|c|c|c|c|}
\hline SPECIES & 1963 & 1964 & 1965 & 1966 & 1967 & 1970 & 1975 & 2001 \\
\hline Astragalus lentiginosus & 0.00 & 0.00 & 0.00 & 0.09 & 0.00 & 0.00 & 0.00 & 0.00 \\
\hline Ceratoides lanata & 0.00 & 0.00 & 0.00 & 0.01 & 0.02 & 0.00 & 0.02 & 1.61 \\
\hline Chrysothamnus viscidiflorus & 0.07 & 0.15 & 0.17 & 0.20 & 0.22 & 0.32 & 0.34 & 0.00 \\
\hline Coleogyne ramosissima & 0.06 & 0.05 & 0.07 & 0.17 & 0.08 & 0.11 & 0.17 & 0.83 \\
\hline Dichelostemma pulchella & 0.00 & 0.00 & 0.00 & 0.00 & 0.00 & 0.00 & 0.00 & 0.04 \\
\hline Ephedra nevadensis & 0.00 & 0.00 & 0.00 & 0.00 & 0.00 & 0.00 & 0.00 & 0.31 \\
\hline Ericameria cooperi & 0.16 & 0.10 & 0.14 & 0.11 & 0.12 & 0.14 & 0.25 & 0.00 \\
\hline Eriogonum inflatum & 0.00 & 0.00 & 0.00 & 0.00 & 0.00 & 0.00 & 0.00 & 0.07 \\
\hline Grayia spinosa & 2.25 & 2.35 & 3.13 & 3.96 & 4.11 & 5.11 & 7.80 & 0.05 \\
\hline Hymenoclea salsola & 0.85 & 0.90 & 0.95 & 1.05 & 1.23 & 1.55 & 2.15 & 5.56 \\
\hline Larrea tridentata & 0.55 & 0.45 & 0.94 & 0.93 & 1.74 & 2.62 & 4.65 & 9.35 \\
\hline Lycium andersonii & 0.11 & 0.10 & 0.17 & 0.24 & 0.18 & 0.31 & 0.47 & 0.75 \\
\hline Opuntia basilaris & 0.00 & 0.00 & 0.00 & 0.00 & 0.00 & 0.14 & 0.00 & 0.03 \\
\hline Oryzopsis hymenoides & 0.00 & 0.00 & 0.00 & 0.05 & 0.07 & 1.55 & 0.24 & 0.27 \\
\hline Sphaeralcea ambigua & 0.00 & 0.00 & 0.09 & 0.08 & 0.03 & 2.62 & 0.00 & 0.14 \\
\hline Stephanomeria parryi & 0.00 & 0.00 & 0.00 & 0.00 & 0.00 & 0.31 & 0.01 & 0.00 \\
\hline Stipa speciosa & 0.10 & 0.12 & 0.19 & 0.27 & 0.10 & 0.22 & 1.37 & 0.36 \\
\hline Total Live & 4.15 & 4.22 & 5.85 & 7.15 & 7.89 & 10.37 & 17.46 & 19.37 \\
\hline Dead Grass & 0.00 & 0.00 & 0.00 & 0.00 & 0.00 & 0.00 & 0.00 & 0.24 \\
\hline Dead Shrub & 0.00 & 0.39 & 0.05 & 0.00 & 0.00 & 0.00 & 0.00 & 8.05 \\
\hline Total & 4.15 & 4.61 & 5.90 & 7.15 & 7.89 & 10.37 & 17.46 & 27.66 \\
\hline & \multicolumn{8}{|c|}{ Biomass Index $\left(\mathrm{m}^{2}\right)$} \\
\hline SPECIES & 1963 & 1964 & 1965 & 1966 & 1967 & 1970 & 1975 & 2001 \\
\hline Astragalus lentiginosus & 0.00 & 0.00 & 0.00 & 0.04 & 0.00 & 0.00 & 0.00 & 0.00 \\
\hline Ceratoides lanata & 0.00 & 0.00 & 0.00 & 0.01 & 0.01 & 0.00 & 0.02 & 0.00 \\
\hline Chrysothamnus viscidiflorus & 0.07 & 0.15 & 0.23 & 0.29 & 0.34 & 0.51 & 0.57 & 2.03 \\
\hline Coleogyne ramosissima & 0.05 & 0.05 & 0.09 & 0.17 & 0.10 & 0.15 & 0.27 & 2.25 \\
\hline Dichelostemma pulchella & 0.00 & 0.00 & 0.00 & 0.00 & 0.00 & 0.00 & 0.00 & 0.02 \\
\hline Ephedra nevadensis & 0.00 & 0.00 & 0.00 & 0.00 & 0.00 & 0.00 & 0.00 & 0.67 \\
\hline Ericameria cooperi & 0.13 & 0.05 & 0.10 & 0.06 & 0.08 & 0.09 & 0.21 & 0.00 \\
\hline Eriogonum inflatum & 0.00 & 0.00 & 0.00 & 0.00 & 0.00 & 0.00 & 0.00 & 0.10 \\
\hline Grayia spinosa & 3.43 & 3.48 & 5.61 & 8.19 & 8.54 & 10.96 & 18.05 & 0.08 \\
\hline Hymenoclea salsola & 1.15 & 1.20 & 1.33 & 1.70 & 1.92 & 2.73 & 3.63 & 10.16 \\
\hline Larrea tridentata & 2.16 & 1.09 & 3.43 & 4.32 & 8.76 & 16.13 & 33.89 & 77.81 \\
\hline Lycium andersonii & 0.06 & 0.04 & 0.13 & 0.25 & 0.17 & 0.36 & 0.74 & 1.09 \\
\hline Opuntia basilaris & 0.00 & 0.00 & 0.00 & 0.00 & 0.00 & 0.00 & 0.00 & 0.01 \\
\hline Oryzopsis hymenoides & 0.00 & 0.00 & 0.00 & 0.05 & 0.10 & 0.00 & 0.30 & 0.27 \\
\hline Sphaeralcea ambigua & 0.00 & 0.00 & 0.09 & 0.07 & 0.02 & 0.00 & 0.00 & 0.21 \\
\hline Stephanomeria parryi & 0.00 & 0.00 & 0.00 & 0.00 & 0.00 & 0.00 & 0.00 & 0.00 \\
\hline Stipa speciosa & 0.07 & 0.06 & 0.19 & 0.37 & 0.10 & 0.21 & 1.76 & 0.46 \\
\hline Total Live & 7.13 & 6.12 & 11.21 & 15.52 & 20.14 & 31.16 & 59.46 & 95.17 \\
\hline
\end{tabular}


Table A-49A. Site characteristics for Plot 49.

Location: Yucca Flat

NTS Area: 1

NTS Grid: P-32

USGS 7.5’ Quadrangle Name: Yucca Flat

\begin{tabular}{lllll} 
& & \multicolumn{2}{l}{ GPS Readings (Zone 11S) } \\
& Location & Northing & Easting & Condition of Original Corners \\
Corner 1: & SW & NA & NA & NA \\
Corner 2: & NW & NA & NA & NA \\
Corner 3: & NE & 4101986 & 581981 & NA \\
Corner 4: & SE & NA & NA & NA
\end{tabular}

DEM Plot Elevation: 1265 m

Plot Aspect: $135^{\circ}$

Elevation Above Playa (Playa Name): 67 m (Yucca)

Plot Slope: $3^{\circ}$

Annual Precipitation

Measured: $166 \mathrm{~mm}$

Modeled: $162 \mathrm{~mm}$

Parent Material Type: alluvial fan

Substrate: alluvial fan

Slate Geologic Unit: QTa

Beatley Plant Assemblage: Larrea-Grayia-Lycium

Ostler-Hanson Plant Association: Grayia-Lycium-Larrea

Ostler-Hanson Land Unit: 589

Abundance of Biological Soil Crusts: NA

Type and Date of Disturbance: none (control for Plot 48)

Plot Condition: good

Location of Transect 1: south side of plot

Direction Transects Are Read: east to west

Date(s) Plot Remeasured: 6 April 2001 
Figure A-49. Photographs showing Plot 49.

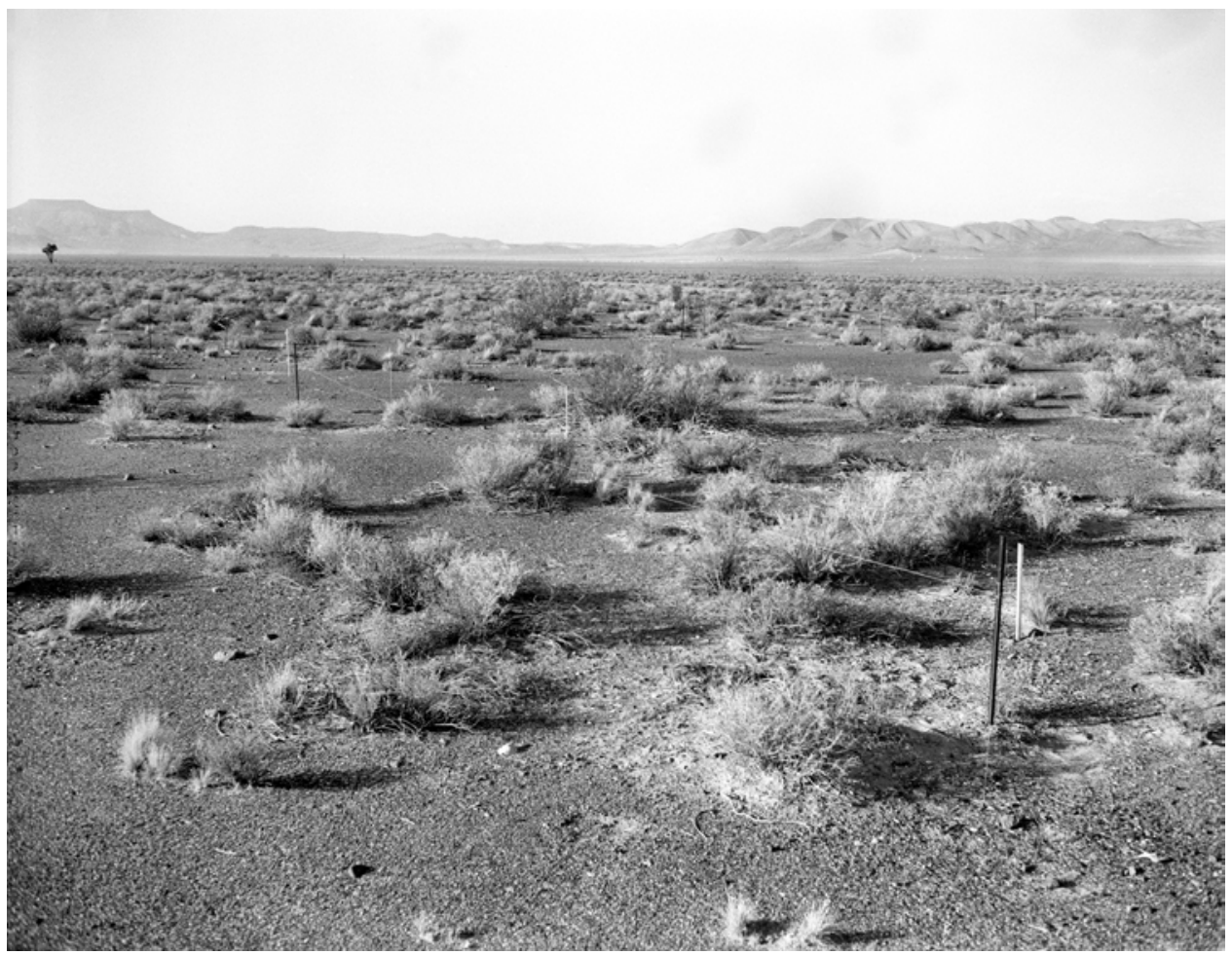

A. (May 12, 1964). This northeasterly view across Plot 49 shows the undisturbed control area to which Plot 48 is compared. The vegetation here is dominated by creosote bush, spiny hopsage, and wolfberry. Syncline Ridge and the Eleana Range appear in the distance in this view across Yucca Flat. Only two Joshua trees are readily apparent in this view (Janice Beatley Collection, 96-A).

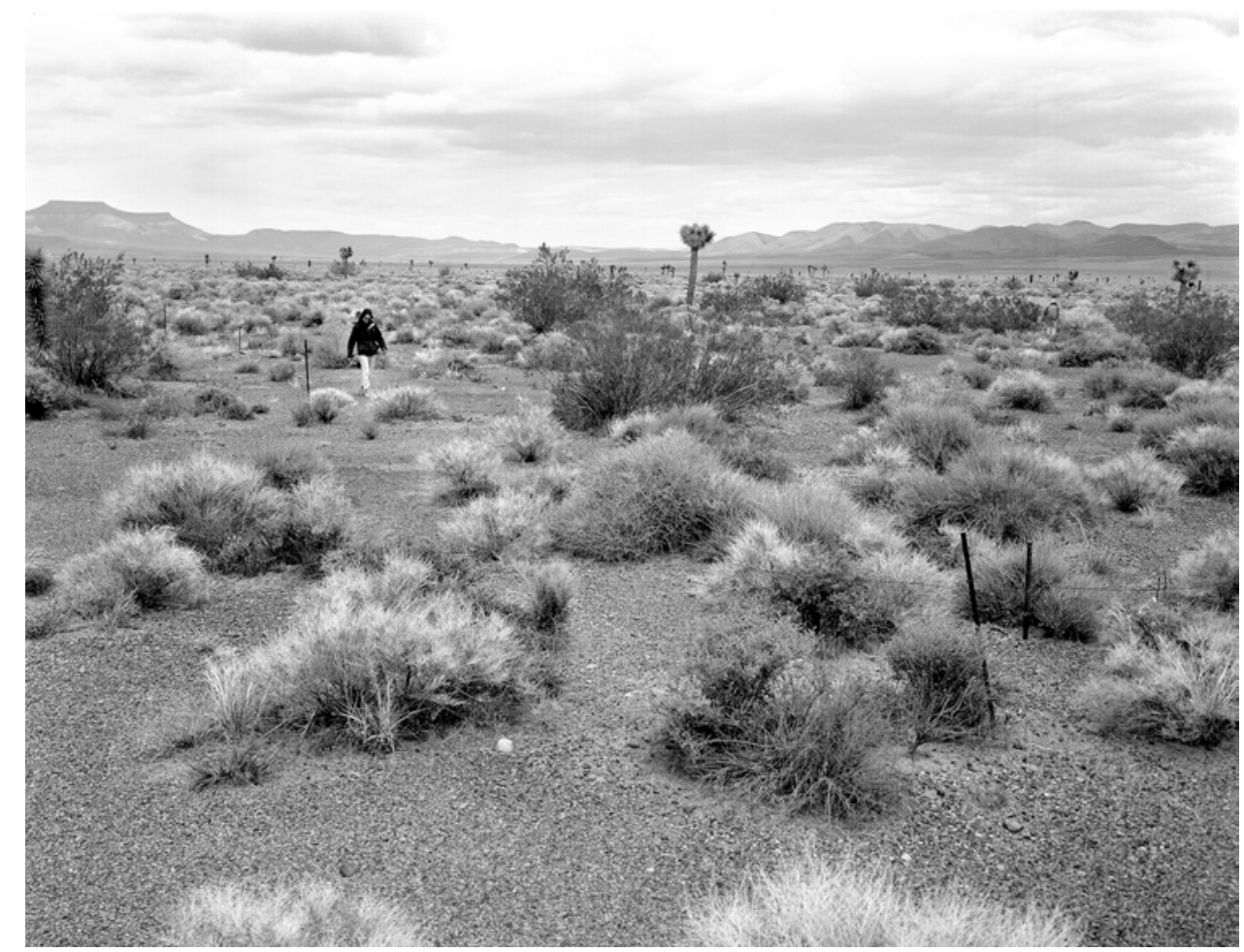

B. (April 6, 2001). Creosote bush has increased significantly in size in the intervening 37 years. Spiny hopsage and wolfberry have declined, while Mormon tea has greatly increased. The Joshua trees have increased significantly in height and density in the view (R.H. Webb, Stake $4116 b)$. 
Table A-49B. Summary plant data for Plot 49.

SPECIES
Atriplex canescens
Ceratoides lanata
Chrysothamnus viscidiflorus
Ephedra nevadensis
Grayia spinosa
Hymenoclea salsola
Larrea tridentata
Lycium andersonii
Mirabilis pudica
Oryzopsis hymenoides
Sphaeralcea ambigua
Stipa speciosa
Tetradymia axillaris
Xylorhiza tortifolia
Total Live
Dead Grass
Dead Shrub
Total

\begin{tabular}{ccc}
\multicolumn{3}{c}{ Number of Plants } \\
$\mathbf{1 9 6 3}$ & $\mathbf{1 9 7 5}$ & $\mathbf{2 0 0 1}$ \\
0 & 2 & 8 \\
1 & 2 & 1 \\
0 & 1 & 0 \\
15 & 16 & 24 \\
35 & 52 & 11 \\
19 & 20 & 19 \\
7 & 9 & 16 \\
23 & 22 & 23 \\
0 & 1 & 0 \\
2 & 2 & 20 \\
0 & 1 & 1 \\
6 & 12 & 3 \\
2 & 3 & 0 \\
1 & 2 & 5 \\
111 & 145 & 131 \\
0 & 0 & 1 \\
15 & 11 & 54 \\
126 & 156 & 186
\end{tabular}

SPECIES
Atriplex canescens
Ceratoides lanata
Chrysothamnus viscidiflorus
Ephedra nevadensis
Grayia spinosa
Hymenoclea salsola
Larrea tridentata
Lycium andersonii
Mirabilis pudica
Oryzopsis hymenoides
Sphaeralcea ambigua
Stipa speciosa
Tetradymia axillaris
Xylorhiza tortifolia
Total Live
Dead Grass
Dead Shrub
Total

\begin{tabular}{ccc}
\multicolumn{3}{c}{ Cover (\%) } \\
$\mathbf{1 9 6 3}$ & $\mathbf{1 9 7 5}$ & $\mathbf{2 0 0 1}$ \\
0.00 & 0.42 & 0.83 \\
0.03 & 0.23 & 0.08 \\
0.00 & 0.05 & 0.00 \\
1.86 & 2.49 & 6.25 \\
4.76 & 7.54 & 1.44 \\
1.85 & 2.54 & 1.95 \\
1.97 & 2.84 & 6.47 \\
4.46 & 5.50 & 2.96 \\
0.00 & 0.01 & 0.00 \\
0.08 & 0.40 & 1.43 \\
0.00 & 0.05 & 0.13 \\
0.29 & 1.00 & 0.29 \\
0.14 & 0.47 & 0.00 \\
0.09 & 0.11 & 0.46 \\
15.54 & 23.64 & 22.29 \\
0.00 & 0.00 & 0.05 \\
2.32 & 1.55 & 8.13 \\
17.85 & 25.19 & 30.47
\end{tabular}

SPECIES
Atriplex canescens
Ceratoides lanata
Chrysothamnus viscidiflorus
Ephedra nevadensis
Grayia spinosa
Hymenoclea salsola
Larrea tridentata
Lycium andersonii
Mirabilis pudica
Oryzopsis hymenoides
Sphaeralcea ambigua
Stipa speciosa
Tetradymia axillaris
Xylorhiza tortifolia

\section{Average Height (m)}

$\begin{array}{lll}\mathbf{1 9 6 3} & \mathbf{1 9 7 5} & \mathbf{2 0 0 1} \\ 0.00 & 0.64 & 0.58 \\ 0.38 & 0.43 & 0.65 \\ 0.00 & 0.10 & 0.00 \\ 0.44 & 0.49 & 0.68 \\ 0.56 & 0.54 & 0.63 \\ 0.39 & 0.49 & 0.62 \\ 1.32 & 1.51 & 2.02 \\ 0.49 & 0.52 & 0.46 \\ 0.00 & 0.05 & 0.00 \\ 0.19 & 0.43 & 0.34 \\ 0.00 & 0.20 & 0.26 \\ 0.32 & 0.40 & 0.37 \\ 0.51 & 0.75 & 0.00 \\ 0.20 & 0.17 & 0.33\end{array}$

SPECIES
Atriplex canescens
Ceratoides lanata
Chrysothamnus viscidiflorus
Ephedra nevadensis
Grayia spinosa
Hymenoclea salsola
Larrea tridentata
Lycium andersonii
Mirabilis pudica
Oryzopsis hymenoides
Sphaeralcea ambigua
Stipa speciosa
Tetradymia axillaris
Xylorhiza tortifolia
Total Live

\section{Biomass Index $\left(\mathrm{m}^{2}\right)$}

$\begin{array}{ccc}\mathbf{1 9 6 3} & \mathbf{1 9 7 5} & \mathbf{2 0 0 1} \\ 0.00 & 0.87 & 1.69 \\ 0.03 & 0.33 & 0.18 \\ 0.00 & 0.02 & 0.00 \\ 3.18 & 4.54 & 14.93 \\ 9.32 & 14.52 & 3.31 \\ 2.59 & 4.28 & 4.41 \\ 9.86 & 16.10 & 46.49 \\ 7.71 & 9.52 & 4.97 \\ 0.00 & 0.00 & 0.00 \\ 0.06 & 0.61 & 1.69 \\ 0.00 & 0.04 & 0.11 \\ 0.31 & 1.44 & 0.37 \\ 0.22 & 1.21 & 0.00 \\ 0.06 & 0.07 & 0.51 \\ 33.34 & 53.55 & 78.66\end{array}$


Table A-50A. Site characteristics for Plot 50.

Location: Yucca Flat

NTS Area: 2

NTS Grid: P-37

USGS 7.5’ Quadrangle Name: Yucca Flat

\begin{tabular}{|c|c|c|c|c|}
\hline & & GPS Reac & Zone 11S) & \\
\hline & Location & Northing & Easting & Condition of Original Corners \\
\hline Corner 1: & SW & 4103919 & 583394 & On ground \\
\hline Corner 2: & NW & 4103948 & 583401 & Standing \\
\hline Corner 3: & $\mathrm{NE}$ & 4103937 & 583431 & Standing \\
\hline Corner 4: & SE & 4103910 & 583424 & Standing \\
\hline DEM Plo & vation: 12 & & & Plot Aspect: $135^{\circ}$ \\
\hline Elevation & e Playa & Name): & ıсса) & Plot Slope: $1^{\circ}$ \\
\hline
\end{tabular}

Annual Precipitation

Measured: $165 \mathrm{~mm}$

Modeled: $161 \mathrm{~mm}$

Parent Material Type: alluvial fan

Substrate: alluvial fan, mixed alluvium volcanic paleozoic

Slate Geologic Unit: Qai

Beatley Plant Assemblage: Grayia-Lycium

Ostler-Hanson Plant Association: Grayia-Atriplex canescens-Ceratoides

Ostler-Hanson Land Unit: 759

Abundance of Biological Soil Crusts: NA

Type and Date of Disturbance: none

Plot Condition: road just touches outside plot at SW corner, evidence of gophers throughout plot

Location of Transect 1: east side of plot

Direction Transects Are Read: north to south

Date(s) Plot Remeasured: 6-8 April 2001 
Figure A-50. Photographs showing Plot 50.

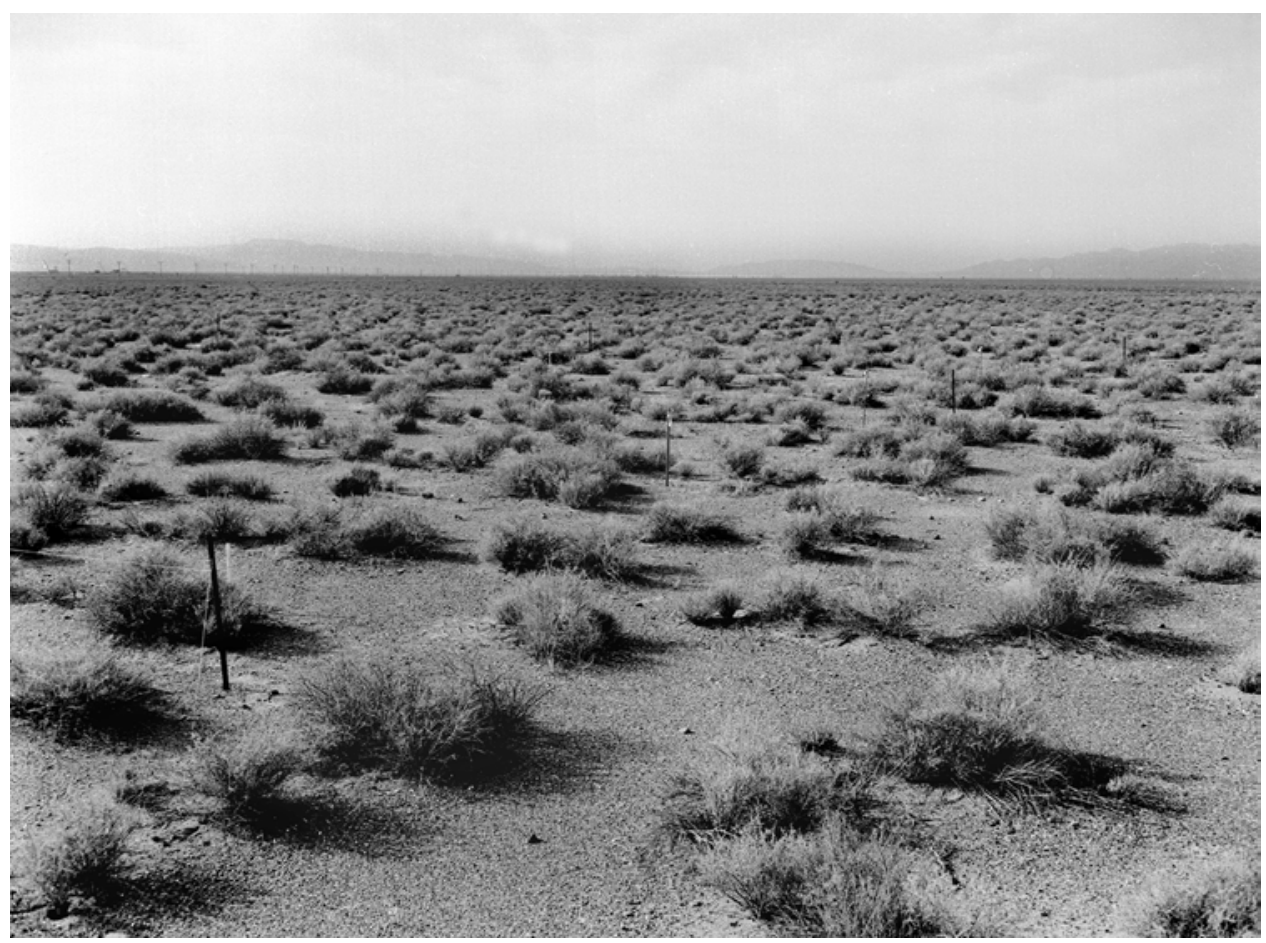

A. (May 13, 1964). In this southeasterly view across Plot 50 and Yucca Flat, a spiny hopsage - wolfberry assemblage appears in the foreground. The background mountains are Massachusetts Mountain (left) and the CP Hills (right); the CP Hogback is at right center (Janice Beatley Collection, 103-B).

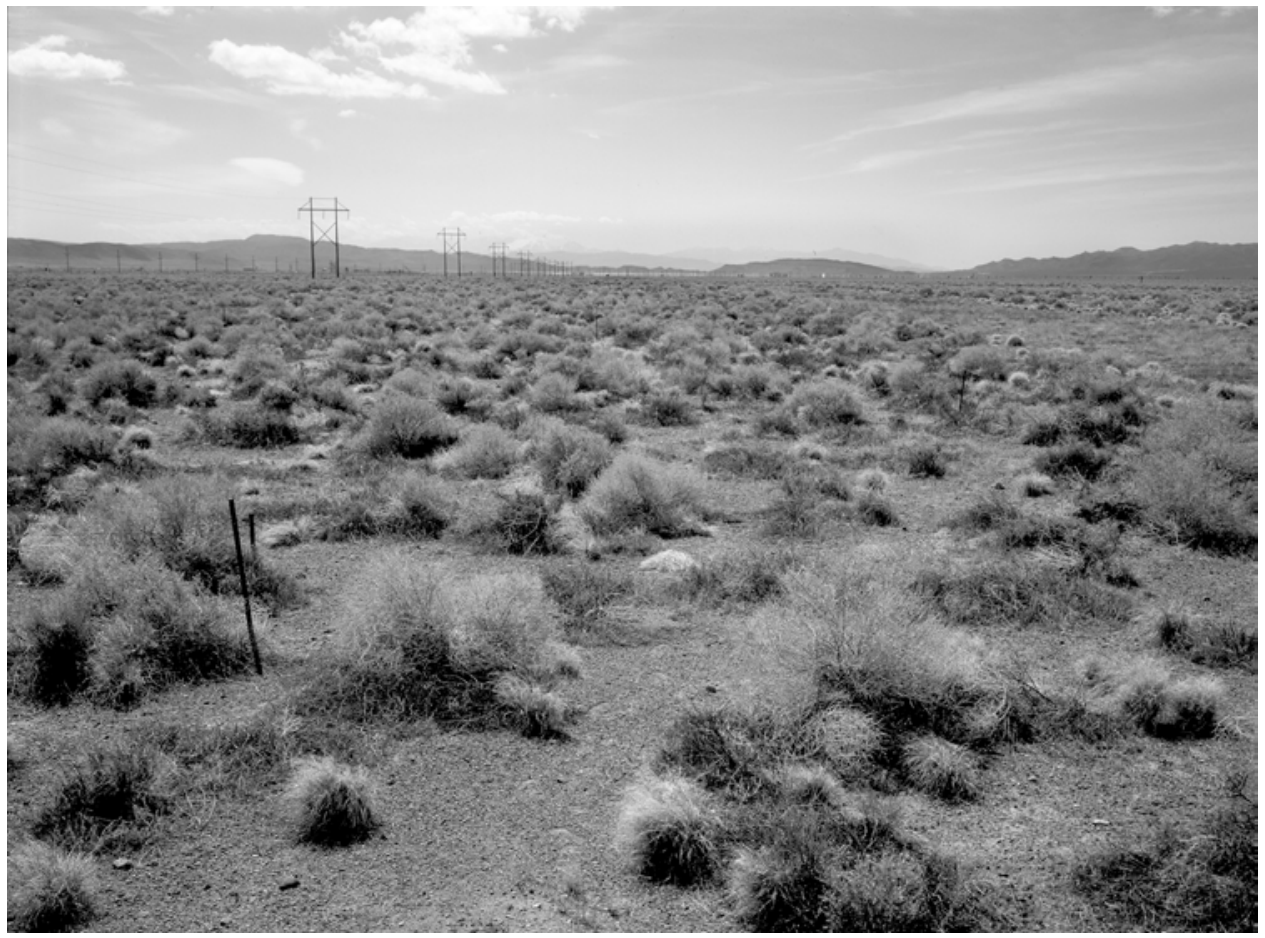

B. (April 6, 2001). Owing to the decline of spiny hopsage, Indian rice grass is the dominant species on this plot, followed by four-wing saltbush (R.H. Webb, Stake 4115b). 
Table A-50B. Summary plant data for Plot 50.

\begin{tabular}{|c|c|c|c|c|c|c|c|}
\hline \multicolumn{5}{|c|}{ Number of Plants } & \multicolumn{3}{|c|}{ Cover (\%) } \\
\hline SPECIES & 1963 & 1975 & 2001 & SPECIES & 1963 & 1975 & 2001 \\
\hline Artemisia spinescens & 17 & 20 & 0 & Artemisia spinescens & 1.17 & 1.50 & 0.00 \\
\hline Atriplex canescens & 20 & 39 & 29 & Atriplex canescens & 1.86 & 5.85 & 3.95 \\
\hline Ceratoides lanata & 48 & 65 & 18 & Ceratoides lanata & 4.33 & 5.04 & 1.75 \\
\hline Grayia spinosa & 85 & 120 & 9 & Grayia spinosa & 12.35 & 16.85 & 0.88 \\
\hline Lycium andersonii & 31 & 42 & 40 & Lycium andersonii & 3.84 & 4.55 & 3.81 \\
\hline Mirabilis pudica & 4 & 13 & 0 & Mirabilis pudica & 0.14 & 0.77 & 0.00 \\
\hline Oryzopsis hymenoides & 1 & 0 & 72 & Oryzopsis hymenoides & 0.09 & 0.00 & 6.46 \\
\hline Stephanomeria parryi & 0 & 4 & 0 & Stephanomeria parryi & 0.00 & 0.04 & 0.00 \\
\hline Sphaeralcea ambigua & 0 & 0 & 0 & Sphaeralcea ambigua & 0.00 & 0.00 & 0.00 \\
\hline Stipa speciosa & 0 & 2 & 1 & Stipa speciosa & 0.00 & 0.09 & 0.10 \\
\hline Tetradymia axillaris & 1 & 1 & 0 & Tetradymia axillaris & 0.06 & 0.13 & 0.00 \\
\hline Sitanion hystrix & 0 & 0 & 15 & Sitanion hystrix & 0.00 & 0.00 & 1.09 \\
\hline Total Live & 206 & 305 & 168 & Total Live & 23.78 & 34.60 & 16.85 \\
\hline Dead Grass & 0 & 0 & 10 & Dead Grass & 0.00 & 0.00 & 0.80 \\
\hline Dead Shrub & 15 & 9 & 125 & Dead Shrub & 1.15 & 0.99 & 17.79 \\
\hline Total & 221 & 314 & 303 & Total & 24.93 & 35.59 & 35.44 \\
\hline \multicolumn{5}{|c|}{ Average Height (m) } & \multicolumn{3}{|c|}{ Biomass Index $\left(\mathrm{m}^{2}\right)$} \\
\hline SPECIES & 1963 & 1975 & 2001 & SPECIES & 1963 & 1975 & 2001 \\
\hline Artemisia spinescens & 0.25 & 0.26 & 0.00 & Artemisia spinescens & 1.01 & 1.34 & 0.00 \\
\hline Atriplex canescens & 0.40 & 0.62 & 0.73 & Atriplex canescens & 2.61 & 12.35 & 10.03 \\
\hline Ceratoides lanata & 0.35 & 0.41 & 0.48 & Ceratoides lanata & 5.47 & 7.26 & 2.91 \\
\hline Grayia spinosa & 0.50 & 0.55 & 0.57 & Grayia spinosa & 21.67 & 32.68 & 1.64 \\
\hline Lycium andersonii & 0.46 & 0.48 & 0.45 & Lycium andersonii & 6.18 & 7.35 & 5.66 \\
\hline Mirabilis pudica & 0.19 & 0.10 & 0.00 & Mirabilis pudica & 0.09 & 0.28 & 0.00 \\
\hline Oryzopsis hymenoides & 0.20 & 0.00 & 0.36 & Oryzopsis hymenoides & 0.06 & 0.00 & 8.34 \\
\hline Stephanomeria parryi & 0.00 & 0.03 & 0.00 & Stephanomeria parryi & 0.00 & 0.00 & 0.00 \\
\hline Sphaeralcea ambigua & 0.00 & 0.00 & 0.00 & Sphaeralcea ambigua & 0.00 & 0.00 & 0.00 \\
\hline Stipa speciosa & 0.00 & 0.32 & 0.35 & Stipa speciosa & 0.00 & 0.10 & 0.12 \\
\hline Tetradymia axillaris & 0.43 & 0.53 & 0.00 & Tetradymia axillaris & 0.09 & 0.23 & 0.00 \\
\hline \multirow[t]{2}{*}{ Sitanion hystrix } & & & 0.36 & Sitanion hystrix & 0.00 & 0.00 & 1.43 \\
\hline & & & & Total Live & 37.08 & 61.27 & 28.58 \\
\hline
\end{tabular}


Table A-51A. Site characteristics for Plot 51.

Location: Yucca Flat

NTS Area: 12

NTS Grid: M-39

USGS 7.5’ Quadrangle Name: Rainier Mesa

\begin{tabular}{lllc} 
& & \multicolumn{2}{c}{ GPS Readings (Zone 11S) } \\
& Location & Northing & Easting \\
Corner 1: & SW & 4115181 & 576238 \\
Corner 2: & NW & 4115212 & 576245 \\
Corner 3: & NE & 4115208 & 576275 \\
Corner 4: & SE & 4115177 & 576269
\end{tabular}

DEM Plot Elevation: 1520 m

Elevation Above Playa (Playa Name): 317 m (Yucca)

Condition of Original Corners
Missing
Missing
Standing, but in wrong place
Standing

Plot Aspect: $162^{\circ}$

Plot Slope: $2^{\circ}$

Annual Precipitation

Measured: $225 \mathrm{~mm}$

Modeled: $194 \mathrm{~mm}$

Parent Material Type: alluvial fan

Substrate: alluvial fan, volcanic

Slate Geologic Unit: QTa

Beatley Plant Assemblage: Coleogyne

Ostler-Hanson Plant Association: Coleogyne-Ephedra

Ostler-Hanson Land Unit: 640

Abundance of Biological Soil Crusts: NA

Type and Date of Disturbance: none

Plot Condition: good

Location of Transect 1: east side of plot

Direction Transects Are Read: north to south

Date(s) Plot Remeasured: 19 April 2002 
Figure A-51. Photographs showing Plot 51.

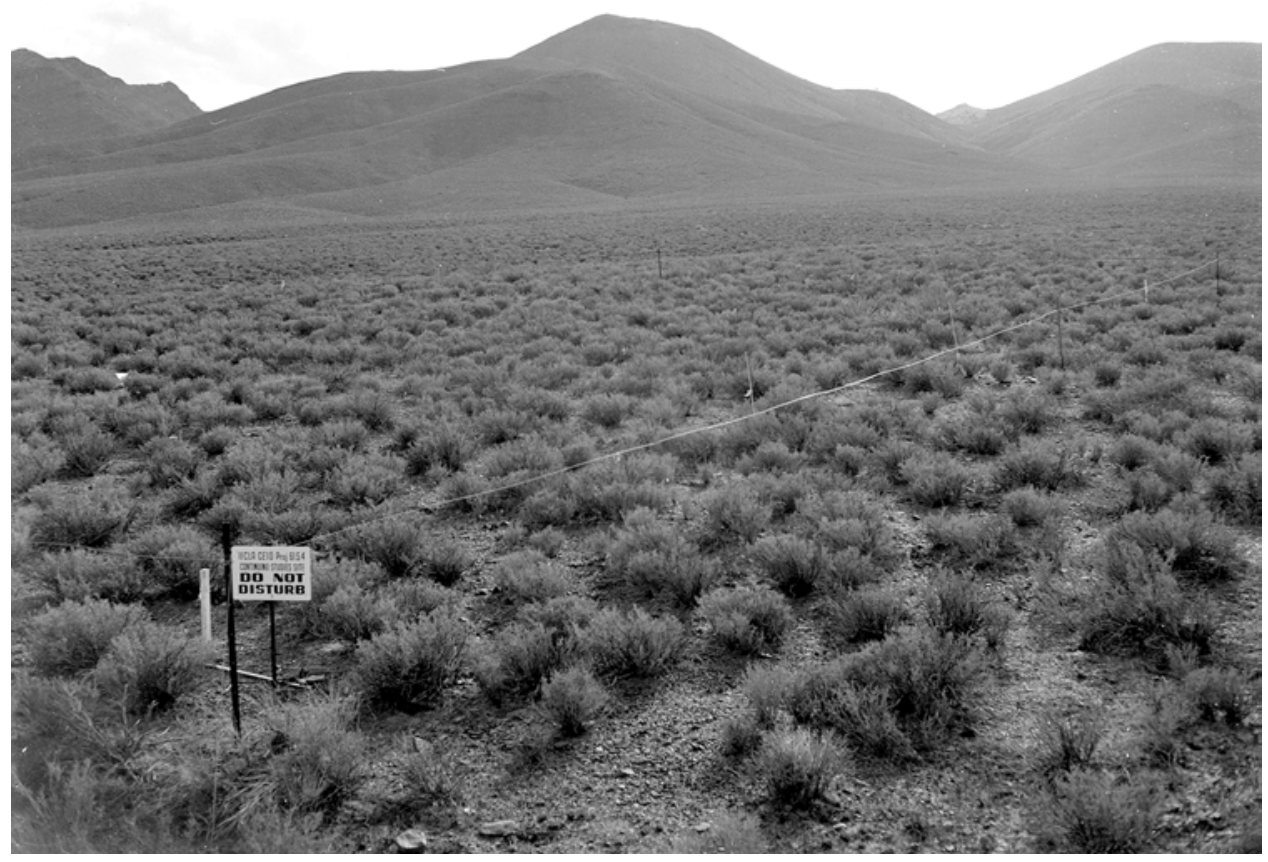

A. (April 21, 1964). Plot 51 is in the northwestern corner of Yucca Flat. This westerly view shows a blackbrush assemblage in the foreground, the roadcut for the Tippipah Highway behind, and the Eleana Range in the background. Rainier Mesa appears behind the notch saddle on the right side (Janice Beatley Collection, 20-B).

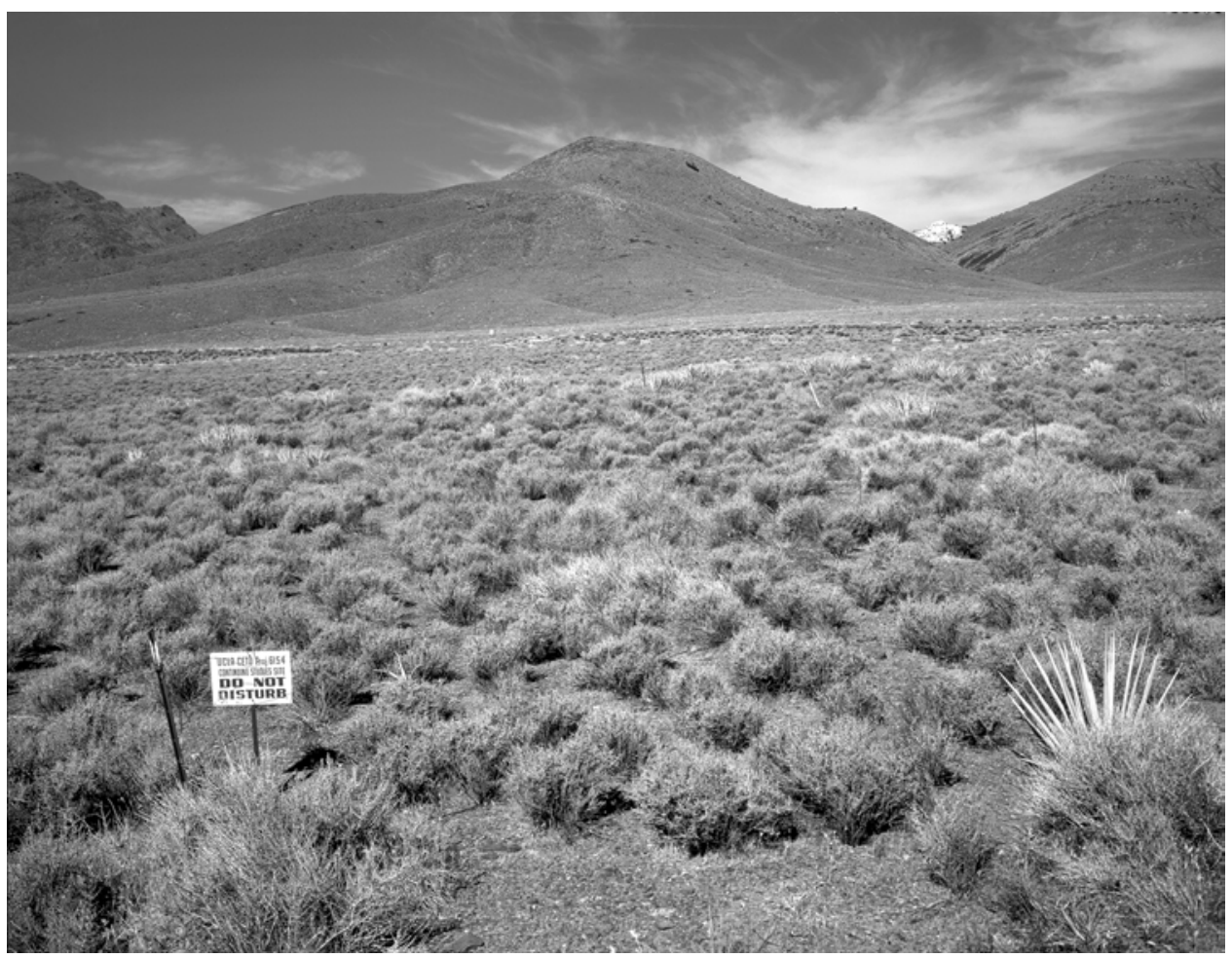

B. (June 10, 2001). Blackbrush has not changed significantly in the intervening 37 years, but Mormon tea has increased. Banana yucca (Yucca baccata) now appears in the foreground and is more prominent in the view (Dominic Oldershaw, Stake 4074B). 
Table A-51B. Summary plant data for Plot 51.

\begin{tabular}{|c|c|c|c|c|c|c|c|}
\hline \multicolumn{5}{|c|}{ Number of Plants } & \multicolumn{3}{|c|}{ Cover (\%) } \\
\hline SPECIES & 1963 & 1975 & 2002 & SPECIES & 1963 & 1975 & 2002 \\
\hline Coleogyne ramosissima & 299 & 396 & 318 & Coleogyne ramosissima & 40.05 & 42.86 & 30.28 \\
\hline Echinocereus engelmanii & 0 & 1 & 1 & Echinocereus engelmanii & 0.00 & 0.04 & 0.16 \\
\hline Ephedra nevadensis & 15 & 15 & 58 & Ephedra nevadensis & 1.44 & 2.42 & 7.15 \\
\hline Grayia spinosa & 1 & 0 & 0 & Grayia spinosa & 0.19 & 0.00 & 0.00 \\
\hline Sphaeralcea ambigua monticola & 0 & 1 & 0 & Sphaeralcea ambigua monticola & 0.00 & 0.01 & 0.00 \\
\hline Stephanomeria parryi & 0 & 1 & 0 & Stephanomeria parryi & 0.00 & 0.01 & 0.00 \\
\hline Stipa speciosa & 5 & 4 & 0 & Stipa speciosa & 0.29 & 0.45 & 0.00 \\
\hline Yucca baccata vespertina & 3 & 3 & 10 & Yucca baccata vespertina & 0.58 & 0.65 & 0.55 \\
\hline Total Live & 323 & 421 & 387 & Total Live & 42.55 & 46.43 & 38.14 \\
\hline Dead Grass & 1 & 0 & 0 & Dead Grass & 0.04 & 0.00 & 0.00 \\
\hline Dead Shrub & 27 & 14 & 0 & Dead Shrub & 2.81 & 1.35 & 14.61 \\
\hline Total & 351 & 435 & 387 & Total & 45.40 & 47.78 & 52.75 \\
\hline \multicolumn{5}{|c|}{ Average Height (m) } & \multicolumn{3}{|c|}{ Biomass Index $\left(\mathrm{m}^{2}\right)$} \\
\hline SPECIES & 1963 & 1975 & 2002 & SPECIES & 1963 & 1975 & 2002 \\
\hline Coleogyne ramosissima & 0.37 & 0.38 & 0.46 & Coleogyne ramosissima & 54.37 & 57.33 & 48.38 \\
\hline Echinocereus engelmanii & 0.00 & 0.10 & 0.14 & Echinocereus engelmanii & 0.00 & 0.01 & 0.08 \\
\hline Ephedra nevadensis & 0.26 & 0.45 & 0.48 & Ephedra nevadensis & 1.39 & 3.81 & 13.67 \\
\hline Grayia spinosa & 0.46 & 0.00 & 0.00 & Grayia spinosa & 0.29 & 0.00 & 0.00 \\
\hline Sphaeralcea ambigua monticola & 0.00 & 0.05 & 0.00 & Sphaeralcea ambigua monticola & 0.00 & 0.00 & 0.00 \\
\hline Stephanomeria parryi & 0.00 & 0.03 & 0.00 & Stephanomeria parryi & 0.00 & 0.00 & 0.00 \\
\hline Stipa speciosa & 0.37 & 0.43 & 0.00 & Stipa speciosa & 0.33 & 0.66 & 0.00 \\
\hline \multirow[t]{2}{*}{ Yucca baccata vespertina } & 0.56 & 0.75 & 0.68 & Yucca baccata vespertina & 1.15 & 1.77 & 1.39 \\
\hline & & & & Total Live & 57.53 & 63.59 & 63.51 \\
\hline
\end{tabular}


Table A-52A. Site characteristics for Plot 52.

Location: Yucca Flat

NTS Area: 10

NTS Grid: T-39

USGS 7.5’ Quadrangle Name: Oak Spring

\begin{tabular}{|c|c|c|c|c|}
\hline & & GPS Read & Zone 11S & \\
\hline & Location & Northing & Easting & Condition of Original Corners \\
\hline Corner 1: & SW & 4114990 & 586207 & Standing \\
\hline Corner 2: & NW & 4115020 & 586215 & Standing \\
\hline Corner 3: & $\mathrm{NE}$ & 4115014 & 586245 & Standing \\
\hline Corner 4: & SE & 4114985 & 586237 & Standing \\
\hline DEM Plo & vation: 13 & & & Plot Aspect: $121^{\circ}$ \\
\hline Elevation & e Playa ( & Name): 1 & (ucca) & Plot Slope: $8^{\circ}$ \\
\hline
\end{tabular}

Annual Precipitation

Measured: $167 \mathrm{~mm}$

Modeled: $170 \mathrm{~mm}$

Parent Material Type: alluvial fan

Substrate: alluvial fan, volcanic, predominately rhyolite with some sedimentary rocks, sandy surface

Slate Geologic Unit: Qay

Beatley Plant Assemblage: Hymenoclea-Lycium

Ostler-Hanson Plant Association: NA

Ostler-Hanson Land Unit: 665

Abundance of Biological Soil Crusts: none

Type and Date of Disturbance: blast disturbance (Sedan 1962)

Plot Condition: good

Location of Transect 1: south side of plot

Direction Transects Are Read: east to west

Date(s) Plot Remeasured: 8 April 2001 
Figure A-52. Photographs showing Plot 52.

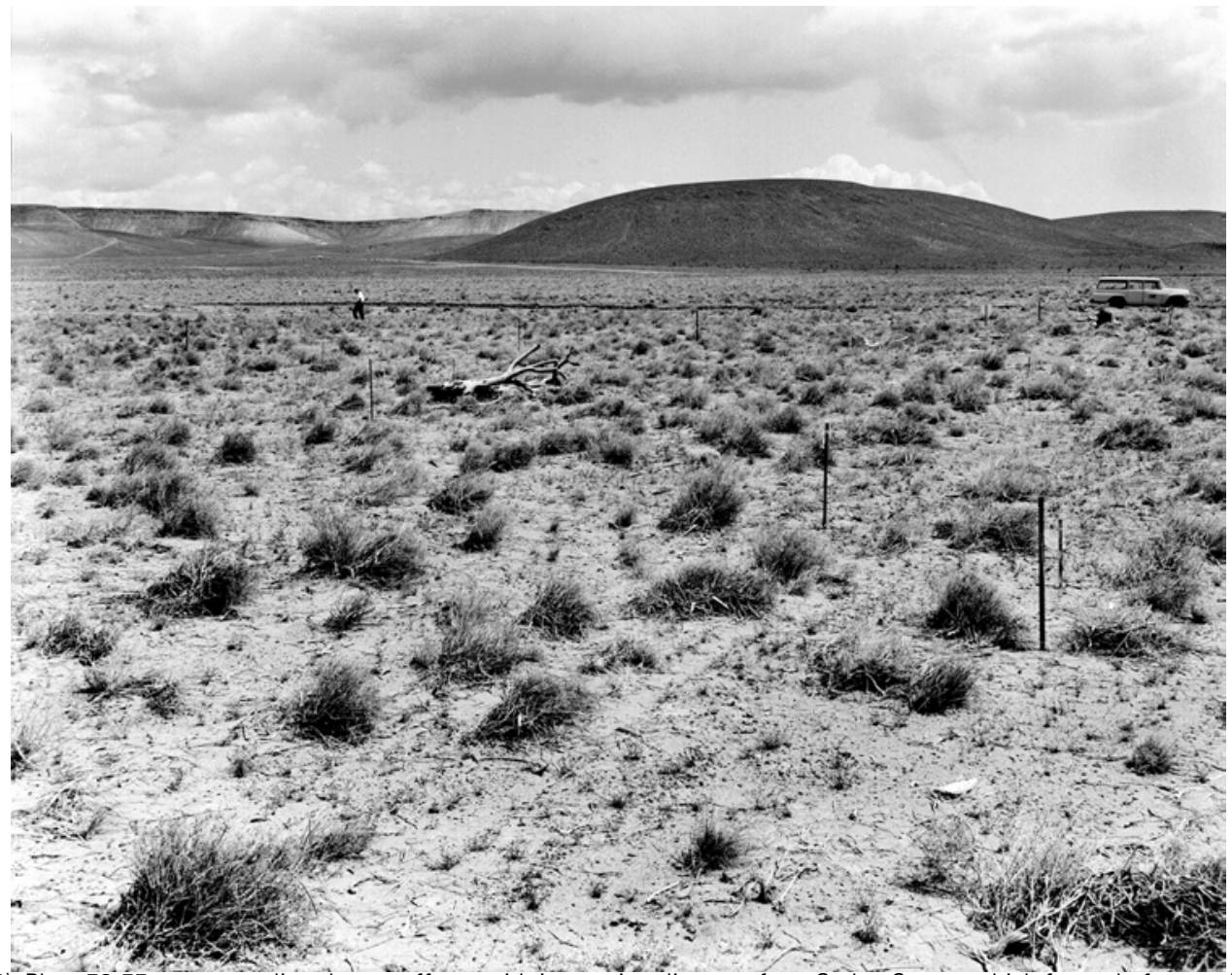

A. (May 8, 1964). Plots $52-55$ represent disturbance effects with increasing distance from Sedan Crater, which formed after testing on July 6 , 1962. The Sedan test, a part of the Plowshare Program, was an underground detonation designed to break the surface and create a crater to demonstrate the feasibility of peacetime use of atomic weapons to create infrastructure, in this case a depression for a lake. This northeasterly view across Plot 52 show the Rhyolite Hills on the northern side of Yucca Flat. Plot 52 represents the combined disturbance effects of shockwave damage, sand blasting, and significant overburden deposition. The vegetation is dominated by cheesebush and wolfberry. The carcass at left center is a Joshua tree flattened by the shock wave and dust cloud emanating from the detonation (Janice Beatley Collection, 78-A).

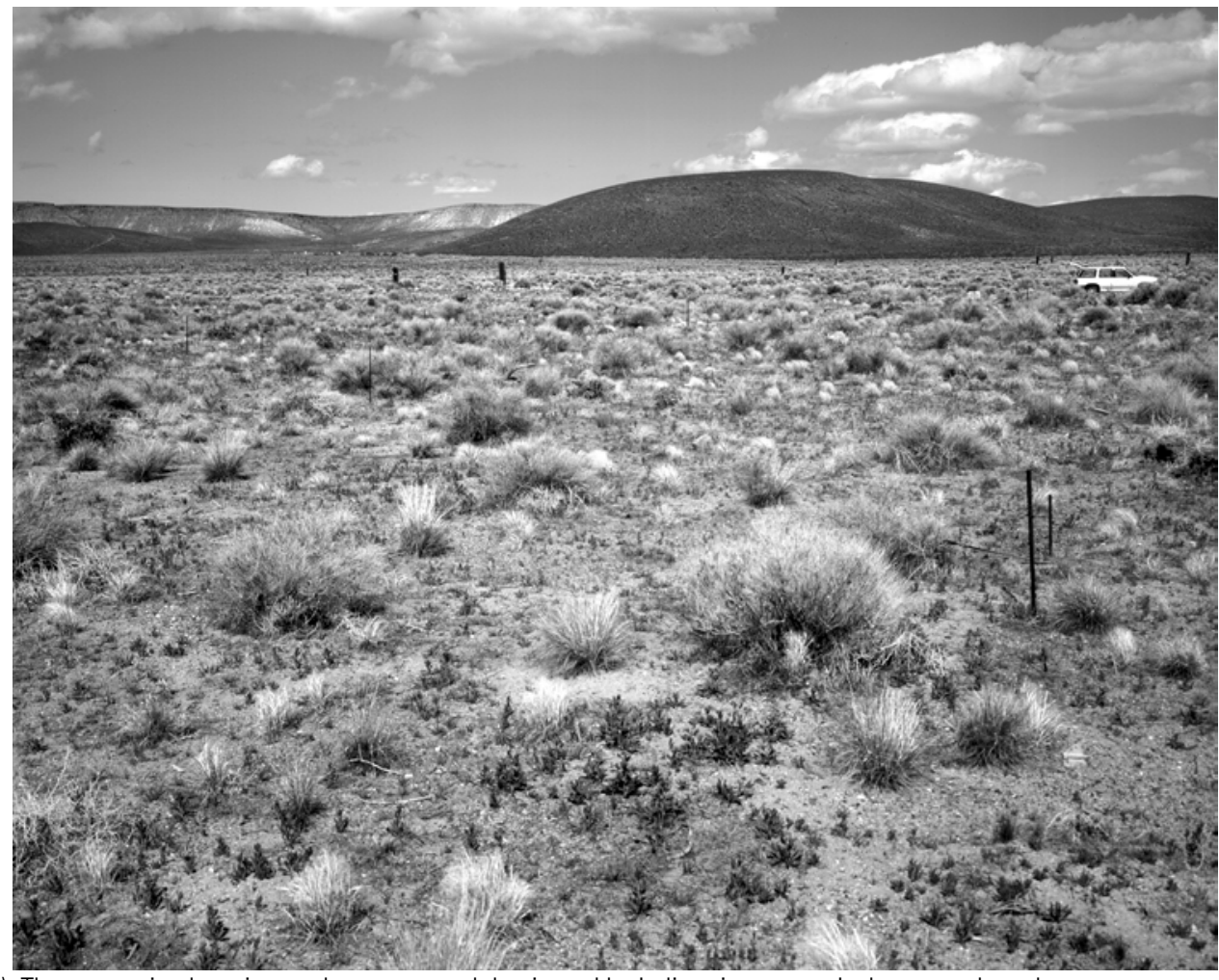

B. (April 8, 2001). The vegetation here is mostly grasses and dominated by Indian rice grass. Joshua trees have begun to resprout in this area (R.H. Webb, Stake 4139A). 
Table A-52B. Summary plant data for Plot 52.

\begin{tabular}{|c|c|c|c|c|c|c|c|c|}
\hline SPECIES & 1963 & 1964 & 1965 & 1966 & 1967 & 1970 & 1975 & 2001 \\
\hline Acamptopappus shockleyi & 0 & 0 & 0 & 0 & 0 & 0 & 0 & 1 \\
\hline Artemisia spinescens & 0 & 0 & 0 & 0 & 0 & 0 & 0 & 0 \\
\hline Astragalus lentiginosus fremontii & 0 & 0 & 0 & 2 & 0 & 0 & 0 & 0 \\
\hline Calochortus flexuosus & 0 & 0 & 1 & 0 & 0 & 0 & 1 & 0 \\
\hline Ceratoides lanata & 2 & 2 & 2 & 2 & 2 & 2 & 2 & 0 \\
\hline Coleogyne ramosissima & 0 & 0 & 0 & 0 & 0 & 0 & 0 & 0 \\
\hline Ephedra nevadensis & 0 & 0 & 0 & 0 & 0 & 0 & 0 & 0 \\
\hline Ericameria cooperi & 0 & 0 & 0 & 0 & 0 & 0 & 0 & 0 \\
\hline Grayia spinosa & 0 & 0 & 0 & 0 & 0 & 0 & 0 & 0 \\
\hline Hymenoclea salsola & 0 & 1 & 1 & 2 & 2 & 3 & 3 & 24 \\
\hline Lepidium fremontii & 0 & 0 & 0 & 0 & 0 & 1 & 0 & 0 \\
\hline Lycium andersonii & 0 & 2 & 1 & 4 & 1 & 2 & 5 & 3 \\
\hline Oryzopsis hymenoides & 33 & 59 & 103 & 108 & 114 & 94 & 102 & 99 \\
\hline Sitanion hystrix & 3 & 0 & 0 & 0 & 0 & 0 & 0 & 0 \\
\hline Stephanomeria parryi & 0 & 0 & 0 & 0 & 0 & 0 & 1 & 0 \\
\hline Stipa speciosa & 1 & 1 & 1 & 1 & 1 & 0 & 3 & 7 \\
\hline Tetradymia axillaris & 0 & 0 & 0 & 0 & 0 & 0 & 0 & 0 \\
\hline Tetradymia glabrata & 0 & 0 & 0 & 0 & 0 & 0 & 0 & 0 \\
\hline Yucca brevifolia & 0 & 0 & 0 & 0 & 0 & 0 & 0 & 0 \\
\hline Total Live & 39 & 65 & 109 & 119 & 120 & 102 & 117 & 134 \\
\hline Dead Grass & 0 & 2 & 0 & 1 & 0 & 0 & 7 & 15 \\
\hline Dead Shrubs & 144 & 149 & 93 & 0 & 0 & 0 & 0 & 9 \\
\hline Total & 183 & 216 & 202 & 120 & 120 & 102 & 124 & 158 \\
\hline & \multicolumn{8}{|c|}{ Average Height (m) } \\
\hline SPECIES & 1963 & 1964 & 1965 & 1966 & 1967 & 1970 & 1975 & 2001 \\
\hline Acamptopappus shockleyi & 0.00 & 0.00 & 0.00 & 0.00 & 0.00 & 0.00 & 0.00 & 0.08 \\
\hline Artemisia spinescens & 0.00 & 0.00 & 0.00 & 0.00 & 0.00 & 0.00 & 0.00 & 0.00 \\
\hline Astragalus lentiginosus fremontii & 0.00 & 0.00 & 0.00 & 0.15 & 0.00 & 0.00 & 0.00 & 0.00 \\
\hline Calochortus flexuosus & 0.00 & 0.00 & 0.05 & 0.00 & 0.00 & 0.00 & 0.05 & 0.00 \\
\hline Ceratoides lanata & 0.34 & 0.14 & 0.23 & 0.17 & 0.22 & 0.29 & 0.38 & 0.00 \\
\hline Coleogyne ramosissima & 0.00 & 0.00 & 0.00 & 0.00 & 0.00 & 0.00 & 0.00 & 0.00 \\
\hline Ephedra nevadensis & 0.00 & 0.00 & 0.00 & 0.00 & 0.00 & 0.00 & 0.00 & 0.00 \\
\hline Ericameria cooperi & 0.00 & 0.00 & 0.00 & 0.00 & 0.00 & 0.00 & 0.00 & 0.00 \\
\hline Grayia spinosa & 0.00 & 0.00 & 0.00 & 0.00 & 0.00 & 0.00 & 0.00 & 0.00 \\
\hline Hymenoclea salsola & 0.00 & 0.15 & 0.20 & 0.28 & 0.33 & 0.54 & 0.58 & 0.65 \\
\hline Lepidium fremontii & 0.00 & 0.00 & 0.00 & 0.00 & 0.00 & 0.46 & 0.00 & 0.00 \\
\hline Lycium andersonii & 0.00 & 0.33 & 0.15 & 0.24 & 0.25 & 0.32 & 0.35 & 0.54 \\
\hline Oryzopsis hymenoides & 0.23 & 0.25 & 0.30 & 0.35 & 0.34 & 0.38 & 0.28 & 0.27 \\
\hline Sitanion hystrix & 0.18 & 0.00 & 0.00 & 0.00 & 0.00 & 0.00 & 0.00 & 0.00 \\
\hline Stephanomeria parryi & 0.00 & 0.00 & 0.00 & 0.00 & 0.00 & 0.00 & 0.05 & 0.00 \\
\hline Stipa speciosa & 0.28 & 0.25 & 0.30 & 0.36 & 0.28 & 0.00 & 0.47 & 0.47 \\
\hline Tetradymia axillaris & 0.00 & 0.00 & 0.00 & 0.00 & 0.00 & 0.00 & 0.00 & 0.00 \\
\hline Tetradymia glabrata & 0.00 & 0.00 & 0.00 & 0.00 & 0.00 & 0.00 & 0.00 & 0.00 \\
\hline Yucca brevifolia & 0.00 & 0.00 & 0.00 & 0.00 & 0.00 & 0.00 & 0.00 & 0.00 \\
\hline
\end{tabular}


Table A-52B (continued). Summary plant data for Plot 52.

\begin{tabular}{|c|c|c|c|c|c|c|c|c|}
\hline & & & & & & & & \\
\hline SPECIES & 1963 & 1964 & 1965 & 1966 & 1967 & 1970 & 1975 & 2001 \\
\hline Acamptopappus shockleyi & 0.00 & 0.00 & 0.00 & 0.00 & 0.00 & 0.00 & 0.00 & 0.02 \\
\hline Artemisia spinescens & 0.00 & 0.00 & 0.00 & 0.00 & 0.00 & 0.00 & 0.00 & 0.00 \\
\hline Astragalus lentiginosus fremontii & 0.00 & 0.00 & 0.00 & 0.08 & 0.00 & 0.00 & 0.00 & 0.00 \\
\hline Calochortus flexuosus & 0.00 & 0.00 & 0.01 & 0.00 & 0.00 & 0.00 & 0.01 & 0.00 \\
\hline Ceratoides lanata & 0.12 & 0.03 & 0.12 & 0.05 & 0.08 & 0.15 & 0.20 & 0.00 \\
\hline Coleogyne ramosissima & 0.00 & 0.00 & 0.00 & 0.00 & 0.00 & 0.00 & 0.00 & 0.00 \\
\hline Ephedra nevadensis & 0.00 & 0.00 & 0.00 & 0.00 & 0.00 & 0.00 & 0.00 & 0.00 \\
\hline Ericameria cooperi & 0.00 & 0.00 & 0.00 & 0.00 & 0.00 & 0.00 & 0.00 & 0.00 \\
\hline Grayia spinosa & 0.00 & 0.00 & 0.00 & 0.00 & 0.00 & 0.00 & 0.00 & 0.00 \\
\hline Hymenoclea salsola & 0.00 & 0.02 & 0.04 & 0.08 & 0.23 & 0.49 & 0.66 & 3.05 \\
\hline Lepidium fremontii & 0.00 & 0.00 & 0.00 & 0.00 & 0.00 & 0.07 & 0.00 & 0.00 \\
\hline Lycium andersonii & 0.00 & 0.19 & 0.15 & 0.17 & 0.04 & 0.02 & 0.38 & 0.61 \\
\hline Oryzopsis hymenoides & 1.39 & 3.75 & 9.05 & 10.52 & 11.00 & 7.42 & 7.39 & 6.83 \\
\hline Sitanion hystrix & 0.08 & 0.00 & 0.00 & 0.00 & 0.00 & 0.00 & 0.00 & 0.00 \\
\hline Stephanomeria parryi & 0.00 & 0.00 & 0.00 & 0.00 & 0.00 & 0.00 & 0.01 & 0.00 \\
\hline Stipa speciosa & 0.12 & 0.12 & 0.11 & 0.11 & 0.11 & 0.00 & 0.25 & 0.79 \\
\hline Tetradymia axillaris & 0.00 & 0.00 & 0.00 & 0.00 & 0.00 & 0.00 & 0.00 & 0.00 \\
\hline Tetradymia glabrata & 0.00 & 0.00 & 0.00 & 0.00 & 0.00 & 0.00 & 0.00 & 0.00 \\
\hline Yucca brevifolia & 0.00 & 0.00 & 0.00 & 0.00 & 0.00 & 0.00 & 0.00 & 0.00 \\
\hline Total Live & 1.71 & 4.11 & 9.46 & 11.02 & 11.45 & 8.15 & 8.91 & 11.29 \\
\hline Dead Grass & 0.00 & 0.05 & 0.00 & 0.05 & 0.00 & 0.00 & 0.40 & 0.56 \\
\hline Dead Shrubs & 13.79 & 13.86 & 9.85 & 0.00 & 0.00 & 0.00 & 0.00 & 2.40 \\
\hline Total & 15.50 & 18.02 & 19.32 & 11.07 & 11.45 & 8.15 & 9.31 & 14.25 \\
\hline & & & & Biomas & $\operatorname{dex}\left(\mathrm{m}^{2}\right)$ & & & \\
\hline SPECIES & 1963 & 1964 & 1965 & 1966 & 1967 & 1970 & 1975 & 2001 \\
\hline Acamptopappus shockleyi & 0.00 & 0.00 & 0.00 & 0.00 & 0.00 & 0.00 & 0.00 & 0.00 \\
\hline Artemisia spinescens & 0.00 & 0.00 & 0.00 & 0.00 & 0.00 & 0.00 & 0.00 & 0.00 \\
\hline Astragalus lentiginosus fremontii & 0.00 & 0.00 & 0.00 & 0.05 & 0.00 & 0.00 & 0.00 & 0.00 \\
\hline Calochortus flexuosus & 0.00 & 0.00 & 0.00 & 0.00 & 0.00 & 0.00 & 0.00 & 0.00 \\
\hline Ceratoides lanata & 0.13 & 0.01 & 0.09 & 0.03 & 0.06 & 0.15 & 0.26 & 0.00 \\
\hline Coleogyne ramosissima & 0.00 & 0.00 & 0.00 & 0.00 & 0.00 & 0.00 & 0.00 & 0.00 \\
\hline Ephedra nevadensis & 0.00 & 0.00 & 0.00 & 0.00 & 0.00 & 0.00 & 0.00 & 0.00 \\
\hline Ericameria cooperi & 0.00 & 0.00 & 0.00 & 0.00 & 0.00 & 0.00 & 0.00 & 0.00 \\
\hline Grayia spinosa & 0.00 & 0.00 & 0.00 & 0.00 & 0.00 & 0.00 & 0.00 & 0.00 \\
\hline Hymenoclea salsola & 0.00 & 0.01 & 0.02 & 0.08 & 0.25 & 1.00 & 1.42 & 7.68 \\
\hline Lepidium fremontii & 0.00 & 0.00 & 0.00 & 0.00 & 0.00 & 0.11 & 0.00 & 0.00 \\
\hline Lycium andersonii & 0.00 & 0.21 & 0.00 & 0.12 & 0.03 & 0.02 & 0.50 & 1.15 \\
\hline Oryzopsis hymenoides & 1.11 & 3.37 & 9.68 & 12.63 & 13.00 & 9.65 & 7.66 & 6.48 \\
\hline Sitanion hystrix & 0.05 & 0.00 & 0.00 & 0.00 & 0.00 & 0.00 & 0.00 & 0.00 \\
\hline Stephanomeria parryi & 0.00 & 0.00 & 0.00 & 0.00 & 0.00 & 0.00 & 0.00 & 0.00 \\
\hline Stipa speciosa & 0.11 & 0.10 & 0.11 & 0.13 & 0.10 & 0.00 & 0.40 & 1.33 \\
\hline Tetradymia axillaris & 0.00 & 0.00 & 0.00 & 0.00 & 0.00 & 0.00 & 0.00 & 0.00 \\
\hline Tetradymia glabrata & 0.00 & 0.00 & 0.00 & 0.00 & 0.00 & 0.00 & 0.00 & 0.00 \\
\hline Yucca brevifolia & 0.00 & 0.00 & 0.00 & 0.00 & 0.00 & 0.00 & 0.00 & 0.00 \\
\hline Total Live & 1.41 & 3.71 & 9.91 & 13.04 & 13.44 & 10.92 & 10.25 & 16.64 \\
\hline
\end{tabular}


Table A-53A. Site characteristics for Plot 53.

Location: Yucca Flat

NTS Area: 10

NTS Grid: T-38

USGS 7.5’ Quadrangle Name: Oak Spring

\begin{tabular}{|c|c|c|c|c|}
\hline & & GPS Read & Zone 11S & \\
\hline & Location & Northing & Easting & Condition of Original Corners \\
\hline Corner 1: & SW & 4113778 & 586167 & Standing \\
\hline Corner 2: & NW & 4113810 & 586170 & Standing \\
\hline Corner 3: & $\mathrm{NE}$ & 4113805 & 586201 & Standing \\
\hline Corner 4: & SE & 4113774 & 586196 & Standing \\
\hline DEM Plot & vation: 13 & & & Plot Aspect: $333^{\circ}$ \\
\hline Elevation & e Playa ( & Name): 1 & Yucca) & Plot Slope: $6^{\circ}$ \\
\hline
\end{tabular}

Annual Precipitation

Measured: $164 \mathrm{~mm}$

Modeled: $169 \mathrm{~mm}$

Parent Material Type: alluvial fan

Substrate: alluvial fan, volcanic, predominately rhyolite with some sedimentary rocks

Slate Geologic Unit: Qai

Beatley Plant Assemblage: Ceratoides-Hymenoclea

Ostler-Hanson Plant Association: NA

Ostler-Hanson Land Unit: 670

Abundance of Biological Soil Crusts: none

Type and Date of Disturbance: blast disturbance (Sedan 1962)

Plot Condition: good

Location of Transect 1: west side of plot

Direction Transects Are Read: south to north

Date(s) Plot Remeasured: 18 April 2001 
Figure A-53. Photographs showing Plot 53.

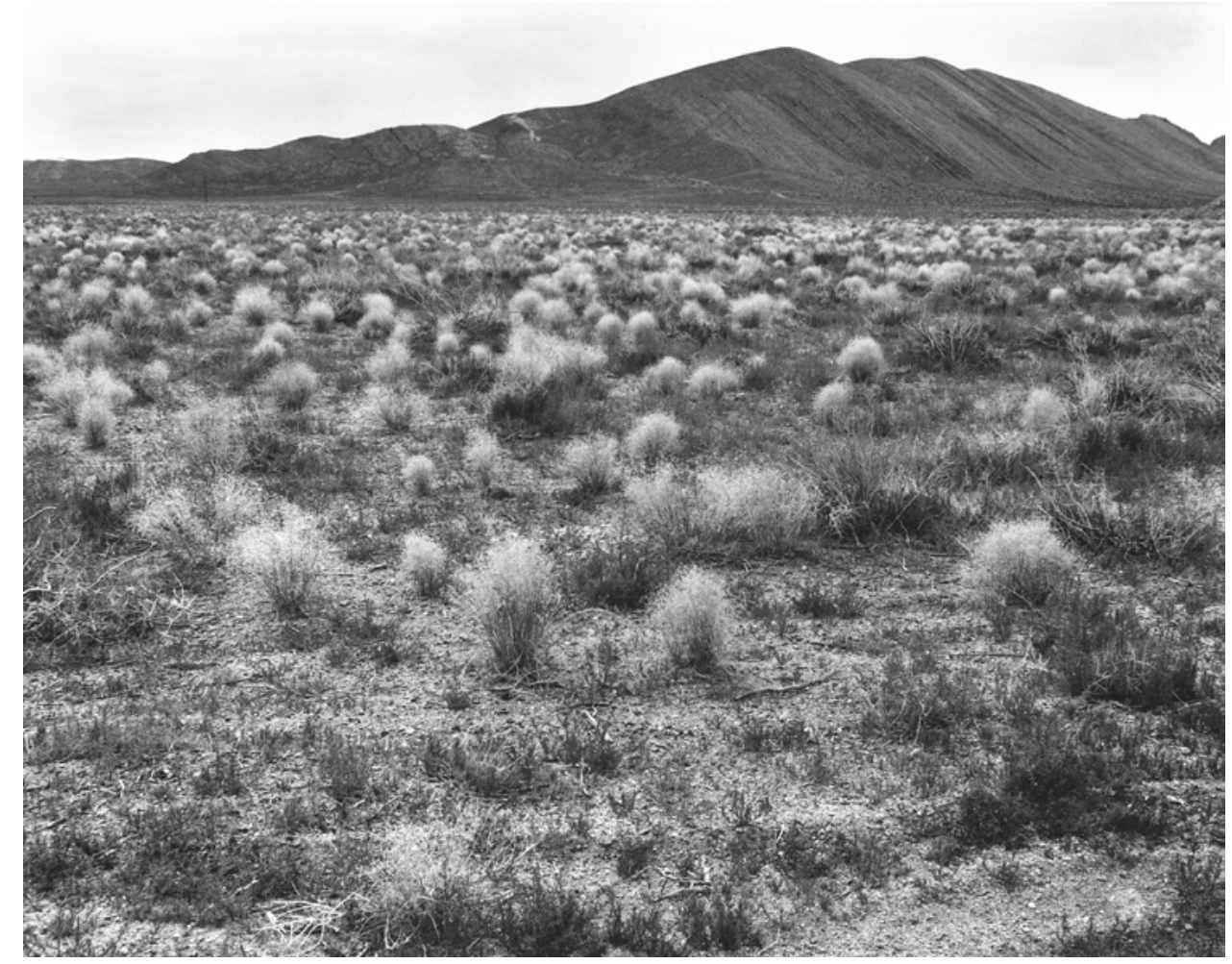

A. (July 16, 1965). This southeasterly view across Plot 53 shows a site that was damaged by sand blasting and the shock wave from the Sedan Test in July 1962. Banded Mountain appears in the distance. The vegetation is dominated by Indian rice grass; the most significant perennial shrubs are winterfat and cheesebush (Janice Beatley Collection, 3074-6).

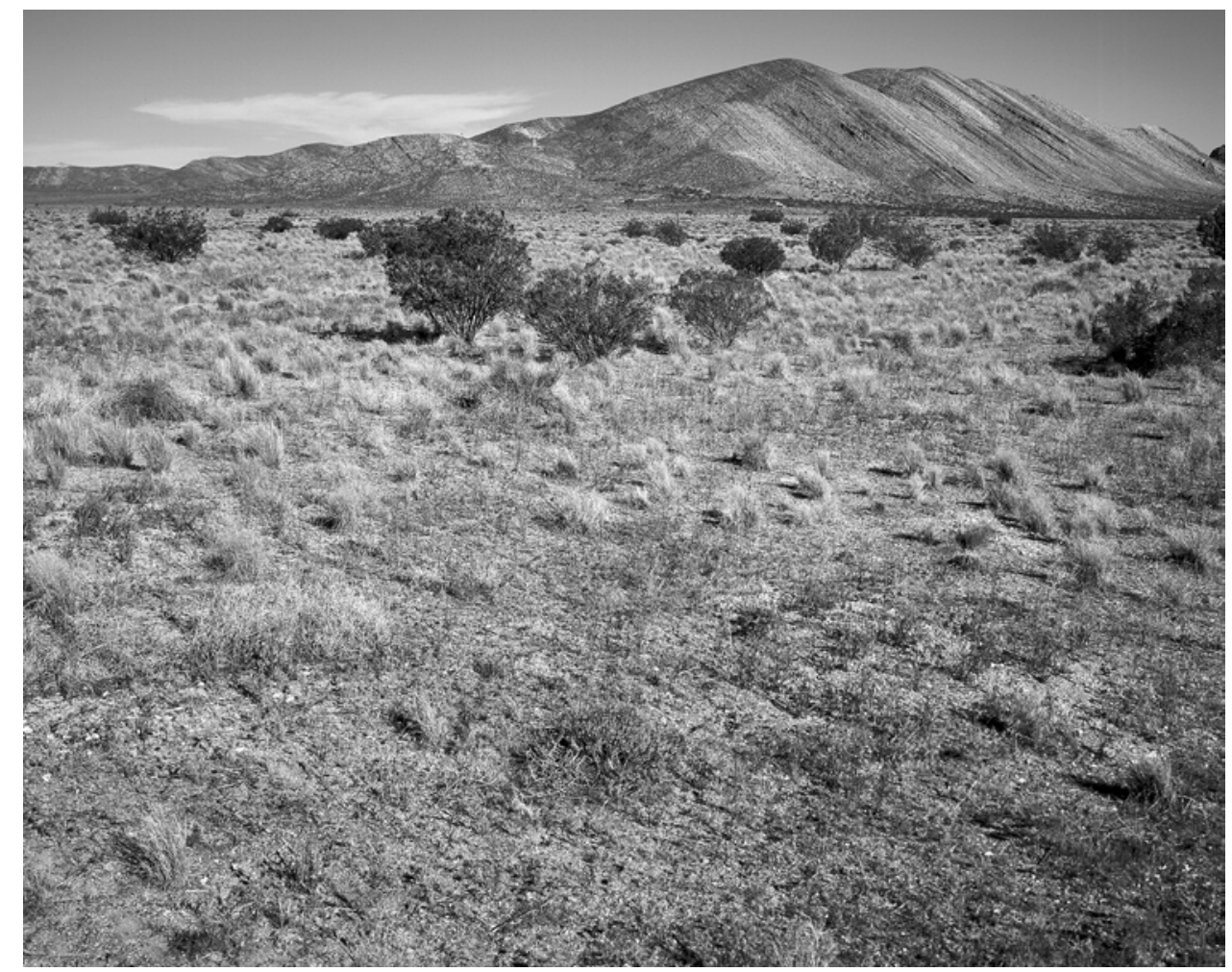

B. (June 9, 2001). Resprouting creosote bushes visually dominate the view. Needlegrass is now the dominant grass, although Indian rice grass is still present. Much of the biomass visible in this view is the dried leaves of bristly fiddleneck (Amsinckia tessellata), a native annual (Dominic Oldershaw, Stake 4073). 
Table A-53B. Summary plant data for Plot 53.

SPECIES
Acamptopappus shockleyi
Astragalus lentiginosus fremontii
Ceratoides lanata
Coleogyne ramosissima
Ephedra nevadensis
Ericameria cooperi
Grayia spinosa
Hymenoclea salsola
Larrea tridentata
Lycium andersonii
Oryzopsis hymenoides
Sitanion hystrix
Sphaeralcea ambigua
Stipa speciosa
Total Live
Dead Grass
Dead Shrubs
Total

\section{SPECIES}

Acamptopappus shockleyi

Astragalus lentiginosus fremontii

Ceratoides lanata

Coleogyne ramosissima

Ephedra nevadensis

Ericameria cooperi

Grayia spinosa

Hymenoclea salsola

Larrea tridentata

Lycium andersonii

Oryzopsis hymenoides

Sitanion hystrix

Sphaeralcea ambigua

Stipa speciosa

\section{Number of Plants}

$\begin{array}{cccccccc}\mathbf{1 9 6 3} & \mathbf{1 9 6 4} & \mathbf{1 9 6 5} & \mathbf{1 9 6 6} & \mathbf{1 9 6 7} & \mathbf{1 9 7 0} & \mathbf{1 9 7 5} & \mathbf{2 0 0 1} \\ 0 & 0 & 0 & 0 & 0 & 0 & 0 & 0 \\ 0 & 2 & 0 & 0 & 0 & 0 & 0 & 0 \\ 0 & 0 & 0 & 1 & 0 & 1 & 1 & 1 \\ 0 & 0 & 0 & 0 & 0 & 0 & 0 & 0 \\ 0 & 0 & 0 & 0 & 0 & 0 & 0 & 0 \\ 0 & 0 & 0 & 0 & 0 & 0 & 0 & 0 \\ 0 & 0 & 0 & 0 & 0 & 0 & 0 & 0 \\ 0 & 0 & 0 & 1 & 1 & 1 & 1 & 10 \\ 1 & 3 & 1 & 0 & 0 & 0 & 0 & 5 \\ 0 & 0 & 0 & 0 & 0 & 0 & 0 & 1 \\ 30 & 55 & 81 & 96 & 94 & 95 & 145 & 90 \\ 5 & 1 & 1 & 3 & 1 & 0 & 2 & 0 \\ 0 & 0 & 0 & 0 & 0 & 0 & 0 & 1 \\ 0 & 0 & 0 & 0 & 0 & 0 & 0 & 23 \\ 36 & 61 & 83 & 101 & 96 & 97 & 149 & 131 \\ 3 & 1 & 0 & 0 & 0 & 0 & 0 & 11 \\ 103 & 109 & 35 & 0 & 0 & 0 & 0 & 1 \\ 142 & 171 & 118 & 101 & 96 & 97 & 149 & 143\end{array}$

\section{Average Height (m)}

$\begin{array}{llllllll}\mathbf{1 9 6 3} & \mathbf{1 9 6 4} & \mathbf{1 9 6 5} & \mathbf{1 9 6 6} & \mathbf{1 9 6 7} & \mathbf{1 9 7 0} & \mathbf{1 9 7 5} & \mathbf{2 0 0 1} \\ 0.00 & 0.00 & 0.00 & 0.00 & 0.00 & 0.00 & 0.00 & 0.00 \\ 0.00 & 0.00 & 0.00 & 0.00 & 0.00 & 0.00 & 0.00 & 0.00 \\ 0.00 & 0.00 & 0.00 & 0.36 & 0.00 & 0.33 & 0.33 & 0.29 \\ 0.00 & 0.00 & 0.00 & 0.00 & 0.00 & 0.00 & 0.00 & 0.00 \\ 0.00 & 0.00 & 0.00 & 0.00 & 0.00 & 0.00 & 0.00 & 0.00 \\ 0.00 & 0.00 & 0.00 & 0.00 & 0.00 & 0.00 & 0.00 & 0.00 \\ 0.00 & 0.00 & 0.00 & 0.00 & 0.00 & 0.00 & 0.00 & 0.00 \\ 0.00 & 0.00 & 0.00 & 0.23 & 0.33 & 0.66 & 0.81 & 0.70 \\ 0.86 & 0.90 & 1.30 & 0.00 & 0.00 & 0.00 & 0.00 & 1.98 \\ 0.00 & 0.00 & 0.00 & 0.00 & 0.00 & 0.00 & 0.00 & 0.19 \\ 0.20 & 0.18 & 0.28 & 0.38 & 0.36 & 0.36 & 0.27 & 0.30 \\ 0.15 & 0.13 & 0.15 & 0.28 & 0.13 & 0.00 & 0.28 & 0.00 \\ 0.00 & 0.00 & 0.00 & 0.00 & 0.00 & 0.00 & 0.00 & 0.06 \\ 0.00 & 0.00 & 0.00 & 0.00 & 0.00 & 0.00 & 0.00 & 0.50\end{array}$


Table A-53B (continued). Summary plant data for Plot 53.

\begin{tabular}{|c|c|c|c|c|c|c|c|c|}
\hline & & & & & & & & \\
\hline SPECIES & 1963 & 1964 & 1965 & 1966 & 1967 & 1970 & 1975 & 2001 \\
\hline Acamptopappus shockleyi & 0.00 & 0.00 & 0.00 & 0.00 & 0.00 & 0.00 & 0.00 & 0.00 \\
\hline Astragalus lentiginosus fremontii & 0.00 & 0.00 & 0.00 & 0.00 & 0.00 & 0.00 & 0.00 & 0.00 \\
\hline Ceratoides lanata & 0.00 & 0.00 & 0.00 & 0.05 & 0.00 & 0.06 & 0.08 & 0.09 \\
\hline Coleogyne ramosissima & 0.00 & 0.00 & 0.00 & 0.00 & 0.00 & 0.00 & 0.00 & 0.00 \\
\hline Ephedra nevadensis & 0.00 & 0.00 & 0.00 & 0.00 & 0.00 & 0.00 & 0.00 & 0.00 \\
\hline Ericameria cooperi & 0.00 & 0.00 & 0.00 & 0.00 & 0.00 & 0.00 & 0.00 & 0.00 \\
\hline Grayia spinosa & 0.00 & 0.00 & 0.00 & 0.00 & 0.00 & 0.00 & 0.00 & 0.00 \\
\hline Hymenoclea salsola & 0.00 & 0.00 & 0.00 & 0.01 & 0.06 & 0.22 & 0.24 & 1.36 \\
\hline Larrea tridentata & 0.00 & 0.00 & 0.38 & 0.00 & 0.00 & 0.00 & 0.00 & 2.38 \\
\hline Lycium andersonii & 0.00 & 0.00 & 0.00 & 0.00 & 0.00 & 0.00 & 0.00 & 0.03 \\
\hline Oryzopsis hymenoides & 1.13 & 2.74 & 6.58 & 8.33 & 9.43 & 7.20 & 10.70 & 7.09 \\
\hline Sitanion hystrix & 0.24 & 0.03 & 0.04 & 0.10 & 0.01 & 0.00 & 0.10 & 0.00 \\
\hline Sphaeralcea ambigua & 0.00 & 0.00 & 0.00 & 0.00 & 0.00 & 0.00 & 0.00 & 0.03 \\
\hline Stipa speciosa & 0.00 & 0.00 & 0.00 & 0.00 & 0.00 & 0.00 & 0.00 & 2.89 \\
\hline Total Live & 1.36 & 2.76 & 7.00 & 8.48 & 9.50 & 7.48 & 11.12 & 13.87 \\
\hline Dead Grass & 0.15 & 0.01 & 0.00 & 0.00 & 0.00 & 0.00 & 0.00 & 0.68 \\
\hline Dead Shrubs & 8.64 & 9.59 & 4.26 & 0.00 & 0.00 & 0.00 & 0.00 & 0.32 \\
\hline \multirow[t]{2}{*}{ Total } & 10.15 & 12.36 & 11.26 & 8.48 & 9.50 & 7.48 & 11.12 & 14.87 \\
\hline & \multicolumn{8}{|c|}{ Biomass Index $\left(\mathrm{m}^{2}\right)$} \\
\hline SPECIES & 1963 & 1964 & 1965 & 1966 & 1967 & 1970 & 1975 & 2001 \\
\hline Acamptopappus shockleyi & 0.00 & 0.00 & 0.00 & 0.00 & 0.00 & 0.00 & 0.00 & 0.00 \\
\hline Astragalus lentiginosus fremontii & 0.00 & 2.00 & 0.00 & 0.00 & 0.00 & 0.00 & 0.00 & 0.00 \\
\hline Ceratoides lanata & 0.00 & 0.00 & 0.00 & 0.05 & 0.00 & 0.07 & 0.09 & 0.09 \\
\hline Coleogyne ramosissima & 0.00 & 0.00 & 0.00 & 0.00 & 0.00 & 0.00 & 0.00 & 0.00 \\
\hline Ephedra nevadensis & 0.00 & 0.00 & 0.00 & 0.00 & 0.00 & 0.00 & 0.00 & 0.00 \\
\hline Ericameria cooperi & 0.00 & 0.00 & 0.00 & 0.00 & 0.00 & 0.00 & 0.00 & 0.00 \\
\hline Grayia spinosa & 0.00 & 0.00 & 0.00 & 0.00 & 0.00 & 0.00 & 0.00 & 0.00 \\
\hline Hymenoclea salsola & 0.00 & 0.00 & 0.00 & 0.01 & 0.07 & 0.48 & 0.64 & 3.50 \\
\hline Larrea tridentata & 0.00 & 0.00 & 1.66 & 0.00 & 0.00 & 0.00 & 0.00 & 17.66 \\
\hline Lycium andersonii & 0.00 & 0.00 & 0.00 & 0.00 & 0.00 & 0.00 & 0.00 & 0.02 \\
\hline Oryzopsis hymenoides & 0.83 & 1.78 & 6.41 & 10.97 & 12.01 & 9.21 & 11.19 & 7.57 \\
\hline Sitanion hystrix & 0.14 & 0.01 & 0.02 & 0.10 & 0.00 & 0.00 & 0.09 & 0.00 \\
\hline Sphaeralcea ambigua & 0.00 & 0.00 & 0.00 & 0.00 & 0.00 & 0.00 & 0.00 & 0.01 \\
\hline Stipa speciosa & 0.00 & 0.00 & 0.00 & 0.00 & 0.00 & 0.00 & 0.00 & 5.05 \\
\hline Total Live & 0.97 & 3.79 & 8.09 & 11.13 & 12.09 & 9.76 & 12.01 & 33.90 \\
\hline
\end{tabular}


Table A-54A. Site characteristics for Plot 54.

Location: Yucca Flat

NTS Area: 10

NTS Grid: T-38

USGS 7.5’ Quadrangle Name: Oak Spring

\begin{tabular}{|c|c|c|c|c|}
\hline & & GPS Reac & Zone 11S & \\
\hline & Location & Northing & Easting & Condition of Original Corners \\
\hline Corner 1: & SW & 4112870 & 586768 & Standing \\
\hline Corner 2: & NW & 4112900 & 586779 & Standing \\
\hline Corner 3: & $\mathrm{NE}$ & 4112884 & 586807 & Standing \\
\hline Corner 4: & SE & 4112852 & 586793 & Standing \\
\hline DEM Plot & vation: 13 & & & Plot Aspect: $326^{\circ}$ \\
\hline Elevation & e Playa ( & Name): 1 & Yucca) & Plot Slope: $5^{\circ}$ \\
\hline
\end{tabular}

Annual Precipitation

Measured: $174 \mathrm{~mm}$

Modeled: $1670 \mathrm{~mm}$

Parent Material Type: alluvial fan

Substrate: alluvial fan, limestone dolomite, alluvium predominantly

Slate Geologic Unit: Qai

Beatley Plant Assemblage: Lycium-Larrea-Coleogyne

Ostler-Hanson Plant Association: NA

Ostler-Hanson Land Unit: 672

Abundance of Biological Soil Crusts: light

Type and Date of Disturbance: blast disturbance (Sedan 1962)

Plot Condition: good

Location of Transect 1: west side of plot

Direction Transects Are Read: north to south

Date(s) Plot Remeasured: 8 April 2001 
Figure A-54. Photographs showing Plot 54.

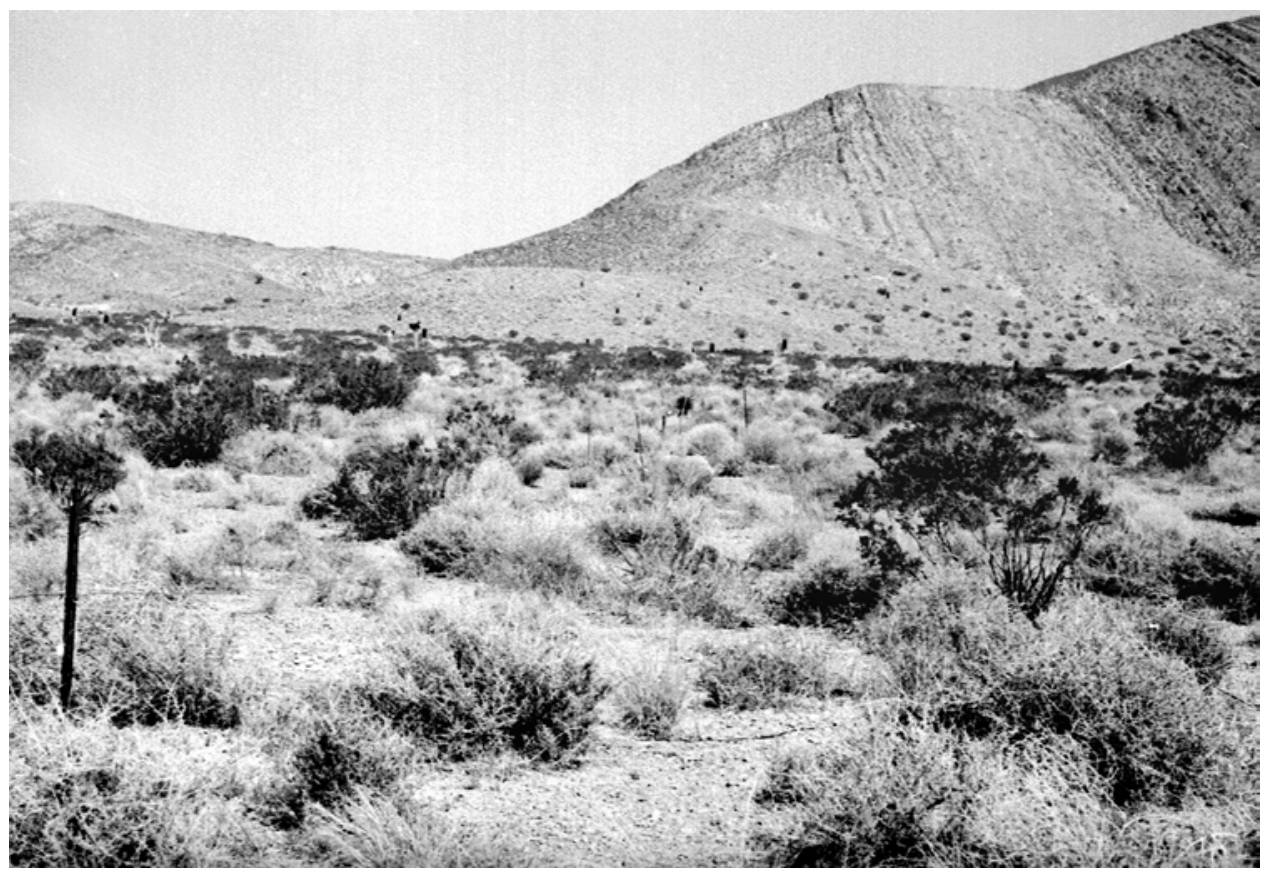

A. (June 1974). Plot 54 was only affected by the shockwave created by the Sedan Test on northern Yucca Flat. This southeasterly view across the plot shows Banded Mountain in the distance and a creosote bush assemblage in the foreground. Wolfberry is the dominant here, followed by creosote bush and blackbrush (Janice Beatley Collection, 19).

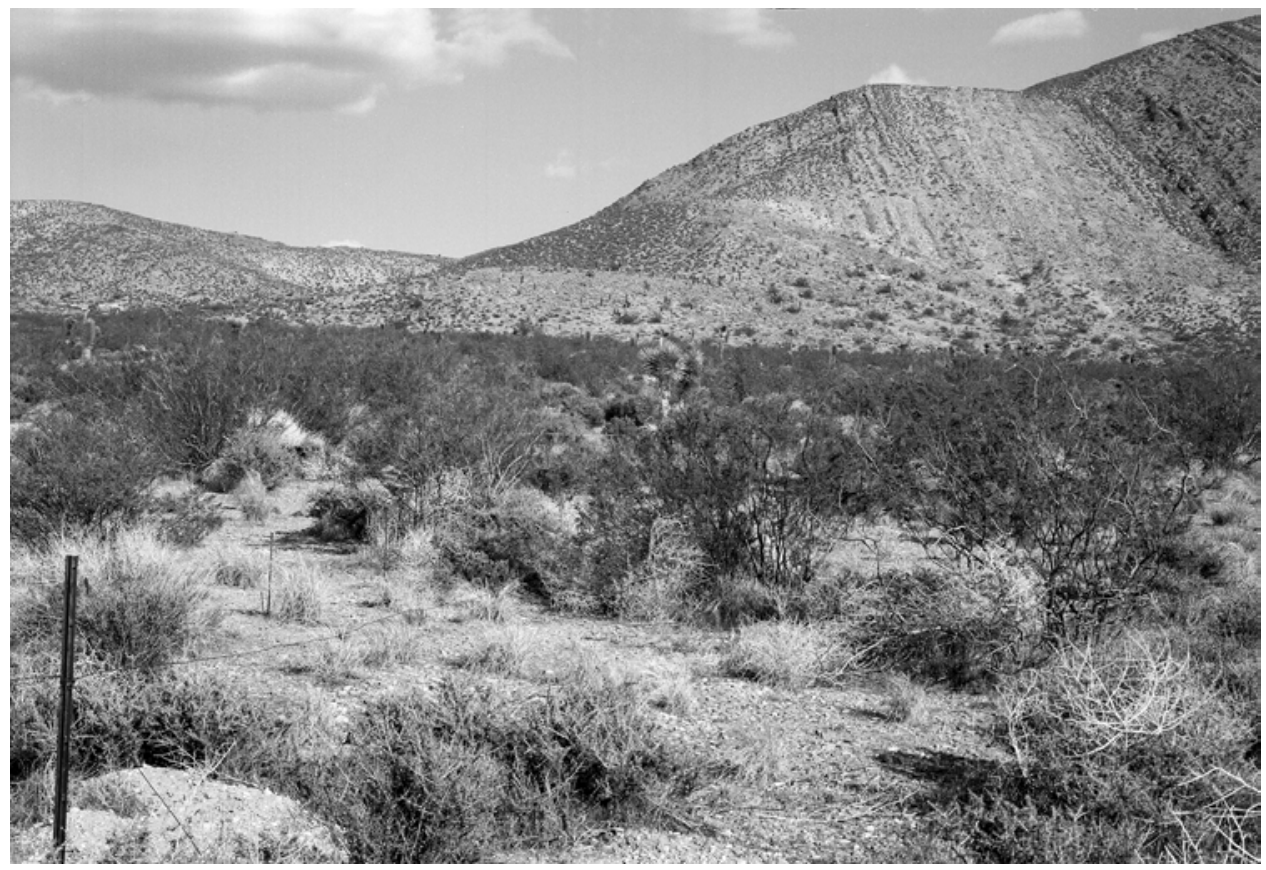

B. (April 8, 2001). Creosote bush has increased substantially here, blocking the view of the subshrubs in the midground. Joshua trees also appear to have increased (R.H. Webb, Stake 4143). 
Table A-54B. Summary plant data for Plot 54.

SPECIES
Acamptopappus shockleyi
Ceratoides lanata
Coleogyne ramosissima
Ephedra nevadensis
Ericameria cooperi
Eriogonum inflatum
Grayia spinosa
Hymenoclea salsola
Larrea tridentata
Lepidium fremontii
Lycium andersonii
Opuntia basilaris
Oryzopsis hymenoides
Sitanion hystrix
Sphaeralcea ambigua
Stanleya pinnata
Stipa speciosa
Unknown grass
Total Live
Dead grass
Dead shrubs
Total

SPECIES
Acamptopappus shockleyi
Ceratoides lanata
Coleogyne ramosissima
Ephedra nevadensis
Ericameria cooperi
Eriogonum inflatum
Grayia spinosa
Hymenoclea salsola
Larrea tridentata
Lepidium fremontii
Lycium andersonii
Opuntia basilaris
Oryzopsis hymenoides
Sitanion hystrix
Sphaeralcea ambigua
Stanleya pinnata
Stipa speciosa
Unknown grass

$\begin{array}{cc}\mathbf{1 9 6 3} & \mathbf{1 9 6 4} \\ 0 & 0 \\ 0 & 0 \\ 0 & 12 \\ 0 & 0 \\ 0 & 0 \\ 0 & 0 \\ 0 & 0 \\ 0 & 0 \\ 1 & 7 \\ 0 & 0 \\ 0 & 3 \\ 0 & 0 \\ 4 & 8 \\ 0 & 5 \\ 0 & 0 \\ 0 & 0 \\ 0 & 0 \\ 0 & 0 \\ 5 & 35 \\ 0 & 0 \\ 170 & 163 \\ 175 & 198 \\ 0 & \end{array}$

1965
0
0
9
0
0
0
0
0
10
1
21
0
23
7
0
0
0
0
71
0
107
178

\section{3}

0.00

0.00

0.00

0.00

0.00

0.00

0.00

0.00

0.48

0.00

0.00

0.00

0.37

0.00

0.00

0.00

0.00

0.00
Number of Plants

$1966 \quad 1967$

$\begin{array}{ccc}\mathbf{1 9 7 0} & \mathbf{1 9 7 5} & \mathbf{2 0 0 1} \\ 1 & 1 & 3 \\ 0 & 1 & 6 \\ 10 & 12 & 29 \\ 0 & 0 & 0 \\ 0 & 0 & 1 \\ 0 & 0 & 1 \\ 0 & 1 & 0 \\ 1 & 1 & 5 \\ 9 & 13 & 22 \\ 4 & 25 & 2 \\ 21 & 27 & 33 \\ 0 & 0 & 0 \\ 28 & 57 & 92 \\ 5 & 22 & 0 \\ 0 & 0 & 2 \\ 0 & 0 & 2 \\ 2 & 6 & 5 \\ 0 & 0 & 1 \\ 81 & 166 & 204 \\ 0 & 0 & 6 \\ 2 & 49 & 11 \\ 83 & 215 & 221\end{array}$

\section{Average Height (m)}

$\begin{array}{lllll}\mathbf{1 9 6 6} & \mathbf{1 9 6 7} & \mathbf{1 9 7 0} & \mathbf{1 9 7 5} & \mathbf{2 0 0 1} \\ 0.00 & 0.00 & 0.18 & 0.18 & 0.23 \\ 0.00 & 0.00 & 0.00 & 0.33 & 0.45 \\ 0.30 & 0.27 & 0.35 & 0.40 & 0.69 \\ 0.00 & 0.00 & 0.00 & 0.00 & 0.00 \\ 0.00 & 0.00 & 0.00 & 0.00 & 0.15 \\ 0.00 & 0.00 & 0.00 & 0.00 & 0.06 \\ 0.00 & 0.00 & 0.00 & 0.20 & 0.00 \\ 0.00 & 0.00 & 0.20 & 0.36 & 0.42 \\ 0.55 & 0.64 & 0.75 & 1.05 & 1.72 \\ 0.38 & 0.24 & 0.44 & 0.40 & 0.34 \\ 0.36 & 0.32 & 0.37 & 0.50 & 0.49 \\ 0.00 & 0.08 & 0.00 & 0.00 & 0.00 \\ 0.46 & 0.42 & 0.50 & 0.39 & 0.28 \\ 0.32 & 0.24 & 0.25 & 0.25 & 0.00 \\ 0.00 & 0.00 & 0.00 & 0.00 & 0.10 \\ 0.00 & 0.00 & 0.00 & 0.00 & 0.43 \\ 0.46 & 0.47 & 0.44 & 0.40 & 0.43 \\ 0.00 & 0.00 & 0.00 & 0.00 & 0.27\end{array}$


Table A-54B (continued). Summary plant data for Plot 54.

\begin{tabular}{|c|c|c|c|c|c|c|c|c|}
\hline SPECIES & 1963 & 1964 & 1965 & 1966 & 1967 & 1970 & 1975 & 2001 \\
\hline Acamptopappus shockleyi & 0.00 & 0.00 & 0.00 & 0.00 & 0.00 & 0.01 & 0.04 & 0.28 \\
\hline Ceratoides lanata & 0.00 & 0.00 & 0.00 & 0.00 & 0.00 & 0.00 & 0.01 & 0.54 \\
\hline Coleogyne ramosissima & 0.00 & 1.45 & 0.76 & 0.67 & 0.68 & 0.77 & 0.85 & 3.70 \\
\hline Ephedra nevadensis & 0.00 & 0.00 & 0.00 & 0.00 & 0.00 & 0.00 & 0.00 & 0.00 \\
\hline Ericameria cooperi & 0.00 & 0.00 & 0.00 & 0.00 & 0.00 & 0.00 & 0.00 & 0.08 \\
\hline Eriogonum inflatum & 0.00 & 0.00 & 0.00 & 0.00 & 0.00 & 0.00 & 0.00 & 0.03 \\
\hline Grayia spinosa & 0.00 & 0.00 & 0.00 & 0.00 & 0.00 & 0.00 & 0.04 & 0.00 \\
\hline Hymenoclea salsola & 0.00 & 0.00 & 0.00 & 0.00 & 0.00 & 0.02 & 0.09 & 0.50 \\
\hline Larrea tridentata & 0.19 & 2.00 & 2.95 & 0.74 & 2.27 & 1.76 & 4.05 & 11.20 \\
\hline Lepidium fremontii & 0.00 & 0.00 & 0.02 & 0.13 & 0.11 & 0.40 & 3.04 & 0.05 \\
\hline Lycium andersonii & 0.00 & 0.19 & 2.28 & 1.71 & 2.12 & 2.02 & 4.45 & 4.84 \\
\hline Opuntia basilaris & 0.00 & 0.00 & 0.00 & 0.00 & 0.01 & 0.00 & 0.00 & 0.00 \\
\hline Oryzopsis hymenoides & 2.20 & 0.29 & 1.85 & 2.41 & 2.75 & 2.21 & 5.00 & 5.98 \\
\hline Sitanion hystrix & 0.00 & 0.29 & 0.45 & 0.62 & 0.41 & 0.16 & 0.91 & 0.00 \\
\hline Sphaeralcea ambigua & 0.00 & 0.00 & 0.00 & 0.00 & 0.00 & 0.00 & 0.00 & 0.05 \\
\hline Stanleya pinnata & 0.00 & 0.00 & 0.00 & 0.00 & 0.00 & 0.00 & 0.00 & 0.23 \\
\hline Stipa speciosa & 0.00 & 0.00 & 0.00 & 0.05 & 0.12 & 0.05 & 0.40 & 0.57 \\
\hline Unknown grass & 0.00 & 0.00 & 0.00 & 0.00 & 0.00 & 0.00 & 0.00 & 0.05 \\
\hline Total Live & 2.39 & 4.23 & 8.31 & 6.32 & 8.47 & 7.41 & 18.85 & 28.10 \\
\hline Dead grass & 0.00 & 0.00 & 0.00 & 0.00 & 0.00 & 0.00 & 0.00 & 0.29 \\
\hline Dead shrubs & 17.45 & 15.43 & 10.91 & 0.00 & 0.00 & 0.20 & 4.92 & 1.66 \\
\hline Total & 19.84 & 19.65 & 19.22 & 6.32 & 8.47 & 7.61 & 23.77 & 30.05 \\
\hline & \multicolumn{8}{|c|}{ Biomass Index $\left(\mathrm{m}^{2}\right)$} \\
\hline SPECIES & 1963 & 1964 & 1965 & 1966 & 1967 & 1970 & 1975 & 2001 \\
\hline Acamptopappus shockleyi & 0.00 & 0.00 & 0.00 & 0.00 & 0.00 & 0.01 & 0.02 & 0.25 \\
\hline Ceratoides lanata & 0.00 & 0.00 & 0.00 & 0.00 & 0.00 & 0.00 & 0.01 & 0.80 \\
\hline Coleogyne ramosissima & 0.00 & 1.92 & 0.47 & 0.69 & 0.68 & 0.98 & 1.21 & 8.93 \\
\hline Ephedra nevadensis & 0.00 & 0.00 & 0.00 & 0.00 & 0.00 & 0.00 & 0.00 & 0.00 \\
\hline Ericameria cooperi & 0.00 & 0.00 & 0.00 & 0.00 & 0.00 & 0.00 & 0.00 & 0.04 \\
\hline Eriogonum inflatum & 0.00 & 0.00 & 0.00 & 0.00 & 0.00 & 0.00 & 0.00 & 0.01 \\
\hline Grayia spinosa & 0.00 & 0.00 & 0.00 & 0.00 & 0.00 & 0.00 & 0.02 & 0.00 \\
\hline Hymenoclea salsola & 0.00 & 0.00 & 0.00 & 0.00 & 0.00 & 0.01 & 0.11 & 0.61 \\
\hline Larrea tridentata & 0.31 & 7.50 & 5.07 & 1.57 & 5.21 & 5.32 & 15.11 & 71.26 \\
\hline Lepidium fremontii & 0.00 & 0.00 & 0.01 & 0.16 & 0.11 & 0.57 & 4.42 & 0.06 \\
\hline Lycium andersonii & 0.00 & 0.23 & 2.34 & 2.13 & 2.41 & 2.69 & 7.63 & 8.78 \\
\hline Opuntia basilaris & 0.00 & 0.00 & 0.00 & 0.00 & 0.00 & 0.00 & 0.00 & 0.00 \\
\hline Oryzopsis hymenoides & 0.26 & 0.30 & 2.98 & 3.82 & 4.20 & 3.78 & 6.90 & 5.81 \\
\hline Sitanion hystrix & 0.00 & 0.24 & 0.37 & 0.69 & 0.30 & 0.14 & 0.84 & 0.00 \\
\hline Sphaeralcea ambigua & 0.00 & 0.00 & 0.00 & 0.00 & 0.00 & 0.00 & 0.00 & 0.01 \\
\hline Stanleya pinnata & 0.00 & 0.00 & 0.00 & 0.00 & 0.00 & 0.00 & 0.00 & 0.36 \\
\hline Stipa speciosa & 0.00 & 0.00 & 0.00 & 0.07 & 0.19 & 0.08 & 0.59 & 0.91 \\
\hline Unknown grass & 0.00 & 0.00 & 0.00 & 0.00 & 0.00 & 0.00 & 0.00 & 0.05 \\
\hline Total Live & 0.57 & 10.19 & 11.24 & 9.14 & 13.11 & 13.58 & 36.86 & 97.89 \\
\hline
\end{tabular}


Table A-55A. Site characteristics for Plot 55.

Location: Yucca Flat

NTS Area: 9

NTS Grid: U-36

USGS 7.5’ Quadrangle Name: Yucca Flat

\begin{tabular}{|c|c|c|c|c|}
\hline & & GPS Reac & Zone 11S & \\
\hline & Location & Northing & Easting & Condition of Original Corners \\
\hline Corner 1: & SW & 4108942 & 586521 & Standing \\
\hline Corner 2: & NW & 4108957 & 586520 & Standing \\
\hline Corner 3: & $\mathrm{NE}$ & 4108956 & 586580 & Standing \\
\hline Corner 4: & SE & 4108939 & 586582 & Standing \\
\hline DEM Plo & vation: 13 & & & Plot Aspect: $315^{\circ}$ \\
\hline Elevation & re Playa & Name): & (ucca) & Plot Slope: $14^{\circ}$ \\
\hline
\end{tabular}

Annual Precipitation

Measured: $159 \mathrm{~mm}$

Modeled: $167 \mathrm{~mm}$

Parent Material Type: alluvial fan

Substrate: alluvial fan, drained limestone terrain

Slate Geologic Unit: Qai

Beatley Plant Assemblage: Larrea-Lycium-Grayia

Ostler-Hanson Plant Association: NA

Ostler-Hanson Land Unit: 765

Abundance of Biological Soil Crusts: NA

Type and Date of Disturbance: blast area associated with balloon testing

Plot Condition: good

Location of Transect 1: east side of plot

Direction Transects Are Read: south to north

Date(s) Plot Remeasured: 9 May 2001 
Figure A-55. Photographs showing Plot 55.

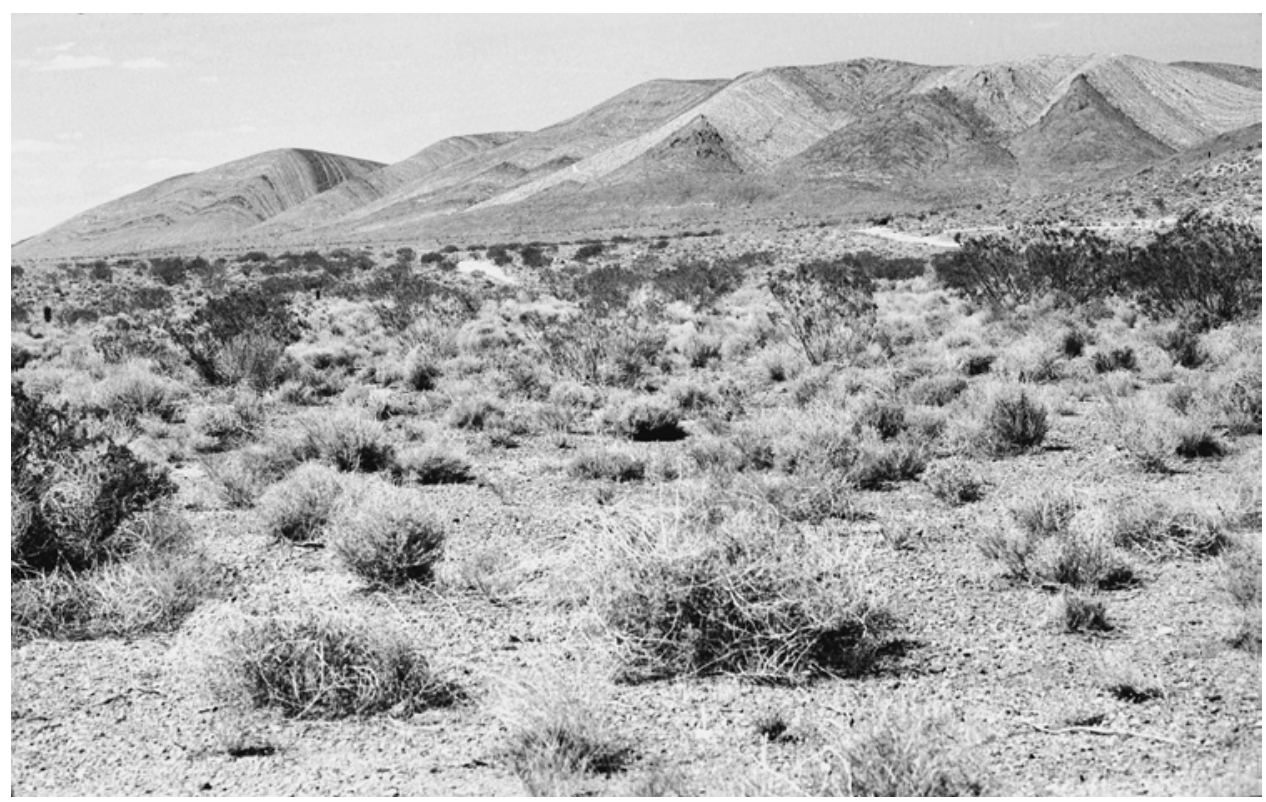

A. (June 25 or 26, 1974). This northeasterly view of Plot 55 shows Banded Mountain in the background and a mixed creosote bush scrub in the foreground. Plot 55 was the least disturbed of any of the plots Beatley established to look at the effects of the Sedan Test. Creosote bush dominates, followed by wolfberry and spiny hopsage. Tumbleweeds (Salsola iberica) that have blown in from disturbed sites to the west litter the shrubs in the foreground (Janice Beatley Collection, 17).

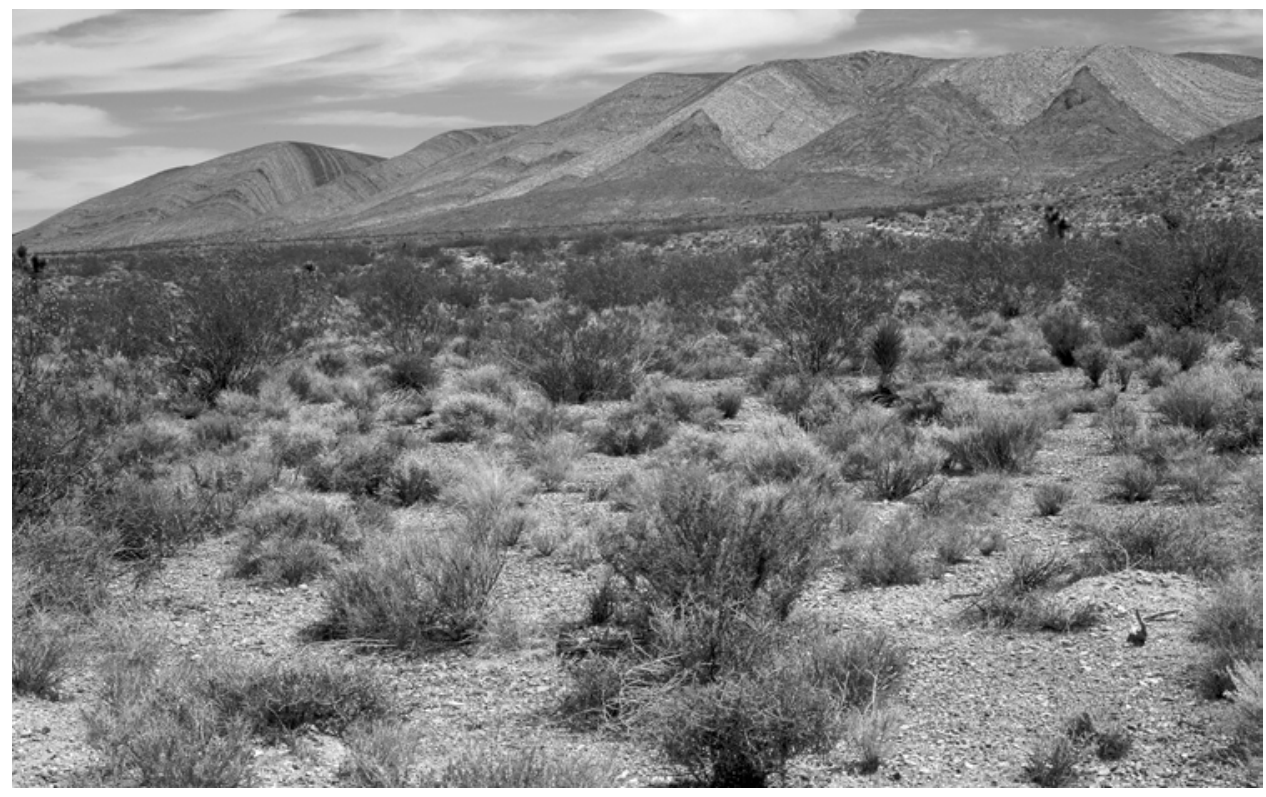

B. (June 10, 2001). Creosote bush dominates, and Joshua trees are once again becoming established in the area. Wolfberry and cheesebush are subdominants here, and spiny hopsage has greatly decreased. Once again, Joshua trees in the background have increased in size and density (Dominic Oldershaw, Stake 4076B). 
Table A-55B. Summary plant data for Plot 55.

SPECIES
Acamptopappus shockleyi
Ambrosia dumosa
Aristida glauca
Ceratoides lanata
Coleogyne ramosissima
Eriogonum inflatum
Ephedra nevadensis
Erioneuron pulchellum
Grayia spinosa
Hymenoclea salsola
Larrea tridentata
Lepidium fremontii
Lycium andersonii
Oryzopsis hymenoides
Sitanion hystrix
Sphaeralcea ambigua
Sphaeralcea grossulariae
Stanleya pinnata
Stipa speciosa
Yucca brevifolia
Total Live
Dead Grass
Dead Shrub
Total

\section{SPECIES}

Acamptopappus shockleyi

Ambrosia dumosa

Aristida glauca

Ceratoides lanata

Coleogyne ramosissima

Eriogonum inflatum

Ephedra nevadensis

Erioneuron pulchellum

Grayia spinosa

Hymenoclea salsola

Larrea tridentata

Lepidium fremontii

Lycium andersonii

Oryzopsis hymenoides

Sitanion hystrix

Sphaeralcea ambigua

Sphaeralcea grossulariae

Stanleya pinnata

Stipa speciosa

Yucca brevifolia

\section{3}

5

0

0

4

1

0

0

0

8

2

20

0

12

1

0

0

0

1

2

0

56

0

46

102

\section{3}

0.18

0.00

0.00

0.36

0.30

0.00

0.00

0.00

0.31

0.33

0.93

0.00

0.29

0.33

0.00

0.00

0.00

1.30

0.30

0.00
1964

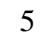

0

0

3$$
1
$$$$
\begin{aligned}
& 1 \\
& 0
\end{aligned}
$$$$
\begin{aligned}
& 0 \\
& 0
\end{aligned}
$$

0

6

4

24

0

7
0

0

0
0

0
0

0
1

1
1

1
0

0
52

52
0

27

79

1965

\title{
Number of Plants
}

$1966 \quad 1967$

$\begin{array}{ccc}\mathbf{1 9 7 0} & \mathbf{1 9 7 5} & \mathbf{2 0 0 1} \\ 4 & 15 & 17 \\ 1 & 1 & 3 \\ 0 & 0 & 0 \\ 7 & 18 & 8 \\ 0 & 2 & 1 \\ 0 & 6 & 2 \\ 0 & 0 & 0 \\ 0 & 0 & 0 \\ 5 & 12 & 0 \\ 5 & 9 & 18 \\ 18 & 27 & 37 \\ 0 & 3 & 0 \\ 11 & 19 & 24 \\ 0 & 14 & 24 \\ 0 & 2 & 0 \\ 0 & 1 & 1 \\ 0 & 0 & 0 \\ 0 & 2 & 1 \\ 1 & 3 & 6 \\ 0 & 0 & 0 \\ 52 & 134 & 142 \\ 0 & 0 & 0 \\ 0 & 4 & 19 \\ 52 & 138 & 161\end{array}$

\section{Average Height (m)}

1964

\section{5}

1966

1967

1970

1975

2001

$0.23 \quad 0.22$

\subsection{5}

0.21

0.28

$\begin{array}{ll}0.21 & 0.25 \\ 0.00 & 0.41\end{array}$

0.00

0.00

0.36

0.00

0.36

0.27

0.00

0.41
0.00

0.00

0.15

0.40

0.00

0.00

0.32

0.36

0.38

0.22

0.00

0.38

0.38

0.51

0.00

0.56

0.51

0.00

0.00

0.44

0.63

0.30

0.16

0.14

0.00

0.30

0.00

0.00

0.00

0.03

0.00

0.00

0.00

0.40

0.40

0.49

0.56

0.40

1.44

0.27

0.44

0.32

0.37

0.10

0.00

0.48

0.42

0.00

0.00

0.00

0.44

1.01

0.00

0.44

0.00

0.00

0.18

0.18

0.00

0.02

0.85

0.54

0.00

0.33

0.00

0.00

0.00

0.00

0.00

0.00

\begin{abstract}
0.00
\end{abstract}
0.00

0.56

0.46

0.00 
Table A-55B (continued). Summary plant data for Plot 55.

\begin{tabular}{|c|c|c|c|c|c|c|c|c|}
\hline \multirow[b]{2}{*}{ SPECIES } & \multicolumn{8}{|c|}{ Cover (\%) } \\
\hline & 1963 & 1964 & 1965 & 1966 & 1967 & 1970 & 1975 & 2001 \\
\hline Acamptopappus shockleyi & 0.33 & 0.30 & 0.20 & 0.33 & 0.27 & 0.29 & 0.78 & 1.25 \\
\hline Ambrosia dumosa & 0.00 & 0.00 & 0.00 & 0.00 & 0.00 & 0.03 & 0.07 & 0.29 \\
\hline Aristida glauca & 0.00 & 0.00 & 0.00 & 0.00 & 0.07 & 0.00 & 0.00 & 0.00 \\
\hline Ceratoides lanata & 0.20 & 0.27 & 0.26 & 0.46 & 0.41 & 0.41 & 1.41 & 0.66 \\
\hline Coleogyne ramosissima & 0.03 & 0.05 & 0.00 & 0.06 & 0.06 & 0.00 & 0.11 & 0.07 \\
\hline Eriogonum inflatum & 0.00 & 0.00 & 0.70 & 0.30 & 0.62 & 0.00 & 0.34 & 0.03 \\
\hline Ephedra nevadensis & 0.00 & 0.00 & 0.00 & 0.00 & 0.00 & 0.00 & 0.00 & 0.00 \\
\hline Erioneuron pulchellum & 0.00 & 0.00 & 0.01 & 0.02 & 0.01 & 0.00 & 0.00 & 0.00 \\
\hline Grayia spinosa & 0.36 & 0.42 & 0.59 & 0.74 & 0.40 & 0.78 & 1.49 & 0.00 \\
\hline Hymenoclea salsola & 0.17 & 0.36 & 0.31 & 0.48 & 0.37 & 0.62 & 0.97 & 1.48 \\
\hline Larrea tridentata & 4.36 & 4.72 & 5.50 & 4.79 & 5.30 & 6.10 & 7.82 & 19.40 \\
\hline Lepidium fremontii & 0.00 & 0.00 & 0.00 & 0.00 & 0.03 & 0.00 & 0.18 & 0.00 \\
\hline Lycium andersonii & 1.28 & 0.91 & 1.53 & 1.68 & 1.30 & 1.56 & 3.02 & 3.05 \\
\hline Oryzopsis hymenoides & 0.05 & 0.00 & 0.00 & 0.13 & 0.00 & 0.00 & 1.13 & 1.37 \\
\hline Sitanion hystrix & 0.00 & 0.00 & 0.00 & 0.00 & 0.00 & 0.00 & 0.04 & 0.00 \\
\hline Sphaeralcea ambigua & 0.00 & 0.00 & 0.07 & 0.09 & 0.01 & 0.00 & 0.03 & 0.02 \\
\hline Sphaeralcea grossulariae & 0.00 & 0.00 & 0.00 & 0.00 & 0.07 & 0.00 & 0.00 & 0.00 \\
\hline Stanleya pinnata & 0.30 & 0.32 & 0.28 & 0.26 & 0.25 & 0.00 & 0.29 & 0.13 \\
\hline Stipa speciosa & 0.07 & 0.04 & 0.14 & 0.19 & 0.11 & 0.07 & 0.25 & 0.34 \\
\hline Yucca brevifolia & 0.00 & 0.00 & 0.00 & 0.00 & 0.00 & 0.00 & 0.00 & 0.00 \\
\hline Total Live & 7.15 & 7.39 & 9.60 & 9.52 & 9.27 & 9.85 & 17.92 & 28.08 \\
\hline Dead Grass & 0.00 & 0.00 & 0.00 & 0.00 & 0.00 & 0.00 & 0.00 & 0.00 \\
\hline Dead Shrub & 2.90 & 1.78 & 0.23 & 0.00 & 0.32 & 0.00 & 0.39 & 3.28 \\
\hline \multirow[t]{2}{*}{ Total } & 10.05 & 9.17 & 9.83 & 9.52 & 9.58 & 9.85 & 18.31 & 31.35 \\
\hline & \multicolumn{8}{|c|}{ Biomass Index $\left(\mathrm{m}^{2}\right)$} \\
\hline SPECIES & 1963 & 1964 & 1965 & 1966 & 1967 & 1970 & 1975 & 2001 \\
\hline Acamptopappus shockleyi & 0.20 & 0.21 & 0.17 & 0.26 & 0.19 & 0.23 & 0.63 & 1.14 \\
\hline Ambrosia dumosa & 0.00 & 0.00 & 0.04 & 0.00 & 0.00 & 0.03 & 0.09 & 0.25 \\
\hline Aristida glauca & 0.00 & 0.00 & 0.00 & 0.00 & 0.03 & 0.00 & 0.00 & 0.00 \\
\hline Ceratoides lanata & 0.22 & 0.29 & 0.31 & 0.59 & 0.40 & 0.56 & 1.97 & 0.85 \\
\hline Coleogyne ramosissima & 0.03 & 0.08 & 0.00 & 0.10 & 0.09 & 0.00 & 0.17 & 0.13 \\
\hline Ephedra nevadensis & 0.00 & 0.00 & 0.67 & 0.33 & 0.33 & 0.00 & 0.17 & 0.00 \\
\hline Erioneuron pulchellum & 0.00 & 0.00 & 0.00 & 0.00 & 0.00 & 0.00 & 0.00 & 0.00 \\
\hline Grayia spinosa & 0.00 & 0.00 & 0.00 & 0.00 & 0.00 & 0.00 & 0.00 & 0.00 \\
\hline Eriogonum inflatum & 0.39 & 0.49 & 0.73 & 1.17 & 0.48 & 1.20 & 3.10 & 0.03 \\
\hline Hymenoclea salsola & 0.24 & 0.39 & 0.39 & 0.53 & 0.43 & 0.82 & 1.48 & 2.08 \\
\hline Larrea tridentata & 13.48 & 15.03 & 17.75 & 15.49 & 18.49 & 26.06 & 40.54 & 126.75 \\
\hline Lepidium fremontii & 0.00 & 0.00 & 0.00 & 0.00 & 0.01 & 0.00 & 0.21 & 0.00 \\
\hline Lycium andersonii & 1.41 & 1.18 & 1.92 & 1.95 & 1.60 & 2.34 & 4.92 & 2.96 \\
\hline Oryzopsis hymenoides & 0.05 & 0.00 & 0.00 & 0.15 & 0.00 & 0.00 & 1.27 & 1.81 \\
\hline Sitanion hystrix & 0.00 & 0.00 & 0.00 & 0.00 & 0.00 & 0.00 & 0.05 & 0.00 \\
\hline Sphaeralcea ambigua & 0.00 & 0.00 & 0.08 & 0.05 & 0.01 & 0.00 & 0.01 & 0.00 \\
\hline Sphaeralcea grossulariae & 0.00 & 0.00 & 0.00 & 0.00 & 0.03 & 0.00 & 0.00 & 0.00 \\
\hline Stanleya pinnata & 1.26 & 1.50 & 0.89 & 0.80 & 0.59 & 0.00 & 0.50 & 0.24 \\
\hline Stipa speciosa & 0.07 & 0.02 & 0.15 & 0.27 & 0.14 & 0.07 & 0.39 & 0.53 \\
\hline Yucca brevifolia & 0.00 & 0.00 & 0.00 & 0.00 & 0.00 & 0.00 & 0.00 & 0.00 \\
\hline Total Live & 17.35 & 19.19 & 23.09 & 21.69 & 22.83 & 31.32 & 55.49 & 136.77 \\
\hline
\end{tabular}


Table A-56A. Site characteristics for Plot 56.

Location: Yucca Flat

NTS Area: 7

NTS Grid: V-33

USGS 7.5’ Quadrangle Name: Paiute Ridge

\begin{tabular}{|c|c|c|c|c|}
\hline & & GPS Read & Zone 11S & \\
\hline & Location & Northing & Easting & Condition of Original Corners \\
\hline Corner 1: & SW & 4104427 & 589683 & Standing \\
\hline Corner 2: & NW & 4104448 & 589668 & Standing \\
\hline Corner 3: & $\mathrm{NE}$ & 4104467 & 589693 & Standing \\
\hline Corner 4: & SE & 4104444 & 589707 & Standing \\
\hline DEM Plo & vation: 13 & & & Plot Aspect: $330^{\circ}$ \\
\hline Elevation & e Playa ( & Name): 1 & Yucca) & Plot Slope: $15^{\circ}$ \\
\hline
\end{tabular}

Annual Precipitation

Measured: $165 \mathrm{~mm}$

Modeled: $169 \mathrm{~mm}$

Parent Material Type: alluvial fan

Substrate: moderately well developed desert pavement w/A-V horizon

Slate Geologic Unit: Qai

Beatley Plant Assemblage: Coleogyne/Larrea-Grayia-Lycium

Ostler-Hanson Plant Association: Coleogyne-Larrea-Lycium

Ostler-Hanson Land Unit: 709

Abundance of Biological Soil Crusts: NA

Type and Date of Disturbance: none

Plot Condition: good

Location of Transect 1: west side of plot

Direction Transects Are Read: north to south

Date(s) Plot Remeasured: 20 April 2002 
Figure A-56. Photographs showing Plot 56.

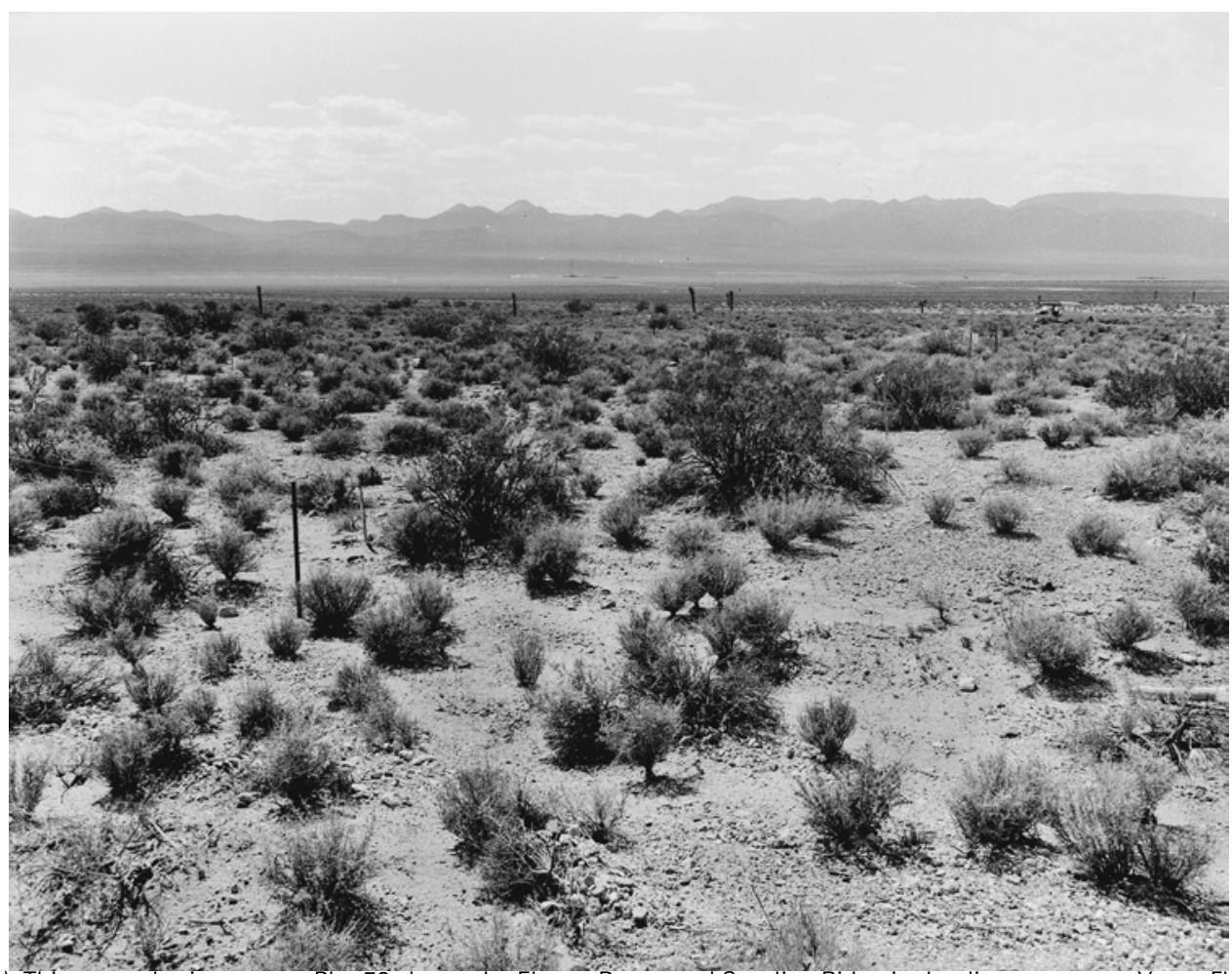

A. (May 9, 1964). This westerly view across Plot 56 shows the Eleana Range and Syncline Ridge in the distance across Yucca Flat. Shoshone Mountain juts above Syncline Ridge at left center. The vegetation here is dominated by blackbrush, creosote bush, spiny hopsage, and wolfberry (Janice Beatley Collection, 84-B).

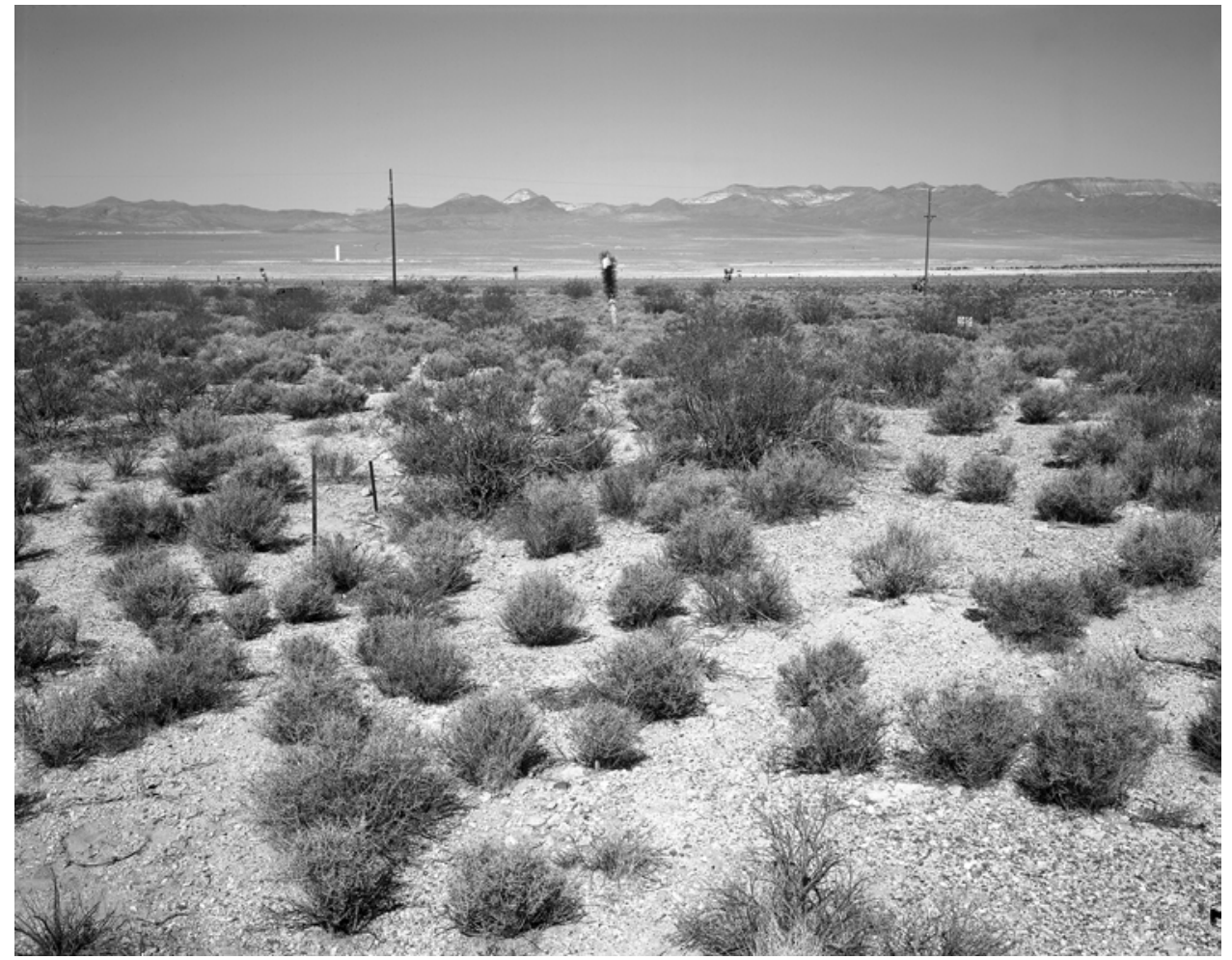

B. (April 21, 2002). In the foreground, many of the same blackbrush appear. The creosote bushes appear to be enlarged, and Joshua trees do not appear to have changed in abundance (Dominic Oldershaw, Stake 4187B). 
Table A-56B. Summary plant data for Plot 56.

$\begin{array}{lccc}\text { SPECIES } & \mathbf{1 9 6 3} & \mathbf{1 9 7 5} & \mathbf{2 0 0 2} \\ \text { Ceratoides lanata } & 0 & 0 & 1 \\ \text { Coleogyne ramosissima } & 122 & 135 & 138 \\ \text { Ephedra nevadensis } & 1 & 3 & 2 \\ \text { Grayia spinosa } & 25 & 35 & 7 \\ \text { Krameria parvifolia } & 0 & 0 & 1 \\ \text { Larrea tridentata } & 16 & 16 & 17 \\ \text { Lycium andersonii } & 23 & 34 & 10 \\ \text { Oryzopsis hymenoides } & 3 & 6 & 1 \\ \text { Sitanion hystrix } & 0 & 3 & 0 \\ \text { Stipa speciosa } & 0 & 1 & 4 \\ \text { Tetradymia axillaris } & 1 & 1 & 0 \\ \text { Xylorhiza tortifolia } & 1 & 1 & 1 \\ \text { Total Live } & 192 & 235 & 182 \\ \text { Dead Grass } & 0 & 1 & 0 \\ \text { Dead Shrub } & 18 & 4 & 44 \\ \text { Total } & 210 & 240 & 226 \\ & & & \\ \text { SPECIES } & \text { Average Height (m) } & \\ \text { Ceratoides lanata } & \mathbf{1 9 6 3} & \mathbf{1 9 7 5} & \mathbf{2 0 0 2} \\ \text { Coleogyne ramosissima } & 0.00 & 0.00 & 0.39 \\ \text { Ephedra nevadensis } & 0.45 & 0.44 & 0.56 \\ \text { Grayia spinosa } & 0.41 & 0.46 & 0.67 \\ \text { Krameria parvifolia } & 0.47 & 0.46 & 0.65 \\ \text { Larrea tridentata } & 0.00 & 0.00 & 0.51 \\ \text { Lycium andersonii } & 1.03 & 1.32 & 1.38 \\ \text { Oryzopsis hymenoides } & 0.38 & 0.45 & 0.36 \\ \text { Sitanion hystrix } & 0.40 & 0.29 & 0.22 \\ \text { Stipa speciosa } & 0.00 & 0.30 & 0.00 \\ \text { Tetradymia axillaris } & 0.00 & 0.25 & 0.35 \\ \text { Xylorhiza tortifolia } & 0.43 & 0.71 & 0.00 \\ & & 0.36 & 0.38\end{array}$

SPECIES
Ceratoides lanata
Coleogyne ramosissima
Ephedra nevadensis
Grayia spinosa
Krameria parvifolia
Larrea tridentata
Lycium andersonii
Oryzopsis hymenoides
Sitanion hystrix
Stipa speciosa
Tetradymia axillaris
Xylorhiza tortifolia
Total Live
Dead Grass
Dead Shrub
Total

$\begin{array}{ccc} & \text { Cover (\%) } \\ \mathbf{1 9 6 3} & \mathbf{1 9 7 5} & \mathbf{2 0 0 2} \\ 0.00 & 0.00 & 0.07 \\ 15.17 & 15.99 & 16.90 \\ 0.05 & 0.14 & 0.17 \\ 2.57 & 3.23 & 0.94 \\ 0.00 & 0.00 & 0.23 \\ 3.95 & 5.17 & 5.29 \\ 3.15 & 4.29 & 0.89 \\ 0.28 & 0.39 & 0.04 \\ 0.00 & 0.15 & 0.00 \\ 0.00 & 0.04 & 0.15 \\ 0.17 & 0.20 & 0.00 \\ 0.05 & 0.07 & 0.08 \\ 25.40 & 29.66 & 24.75 \\ 0.00 & 0.06 & 0.00 \\ 2.67 & 0.36 & 8.90 \\ 28.07 & 30.09 & 33.65\end{array}$

\section{SPECIES}

Ceratoides lanata

Coleogyne ramosissima

Ephedra nevadensis

Grayia spinosa

Krameria parvifolia

Larrea tridentata

Lycium andersonii

Oryzopsis hymenoides

Sitanion hystrix

Stipa speciosa

Tetradymia axillaris

Xylorhiza tortifolia

Total Live

\section{Biomass Index $\left(\mathrm{m}^{2}\right)$}

$\begin{array}{ccc}\mathbf{1 9 6 3} & \mathbf{1 9 7 5} & \mathbf{2 0 0 2} \\ 0.00 & 0.00 & 0.10 \\ 24.56 & 26.08 & 32.26 \\ 0.07 & 0.22 & 0.29 \\ 4.37 & 5.35 & 1.77 \\ 0.00 & 0.00 & 0.39 \\ 13.81 & 23.95 & 26.93 \\ 4.48 & 6.27 & 1.22 \\ 0.35 & 0.43 & 0.03 \\ 0.00 & 0.17 & 0.00 \\ 0.00 & 0.03 & 0.17 \\ 0.34 & 0.48 & 0.00 \\ 0.07 & 0.09 & 0.10 \\ 48.05 & 63.07 & 63.27\end{array}$


Table A-57A. Site characteristics for Plot 57A.

Location: Yucca Flat

NTS Area: 3

NTS Grid: V-31

USGS 7.5’ Quadrangle Name: Paiute Ridge

\begin{tabular}{llll} 
& & \multicolumn{2}{l}{ GPS Readings (Zone 11S) } \\
& Location & Northing & Easting \\
Corner 1: & SW & NA & NA \\
Corner 2: & NW & 4100073 & 589548 \\
Corner 3: & NE & 4100067 & 589579 \\
Corner 4: & SE & NA & NA
\end{tabular}

DEM Plot Elevation: 1254 m

Condition of Original Corners
On ground or missing
Standing
Standing
On ground or missing

Elevation Above Playa (Playa Name): 61 m (Yucca)

Plot Aspect: $335^{\circ}$

Plot Slope: $12^{\circ}$

Annual Precipitation

Measured: $177 \mathrm{~mm}$

Modeled: $162 \mathrm{~mm}$

Parent Material Type: alluvial fan

Substrate: mixed alluvium, weak desert pavement, limestone \& volcanic

Slate Geologic Unit: Qai

Beatley Plant Assemblage: Larrea-Grayia-Lycium

Ostler-Hanson Plant Association: NA

Ostler-Hanson Land Unit: 691

Abundance of Biological Soil Crusts: heavy

Type and Date of Disturbance: none

Plot Condition: about one-third of the plot was damaged in 1965 and abandoned by Beatley

Location of Transect 1: south side of plot

Direction Transects Are Read: east to west

Date(s) Plot Remeasured: 21 April 2002 
Figure A-57. Photographs showing Plot 57A.

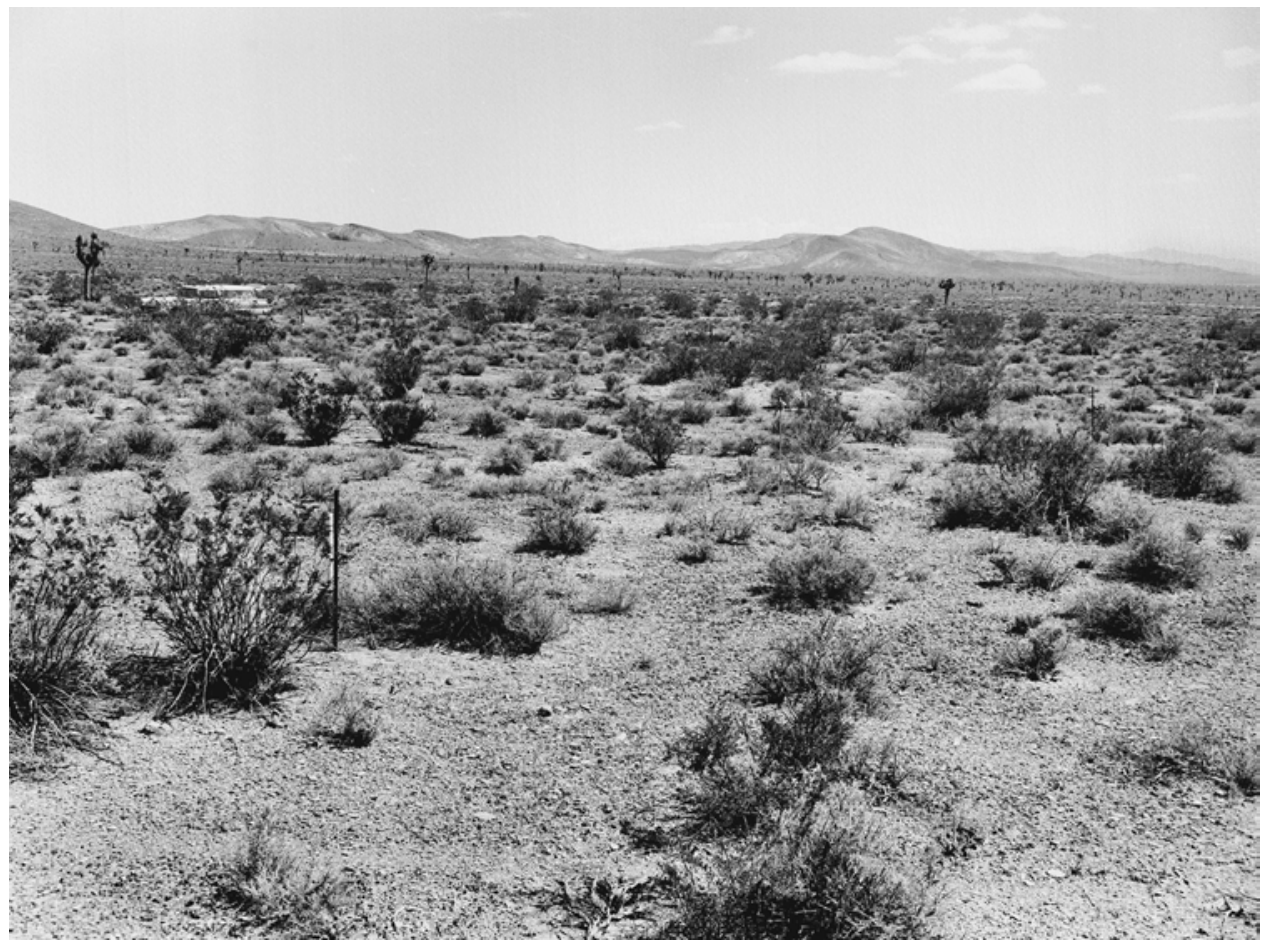

A. (May 9, 1964). This southeasterly view across Plot 57 shows a creosote bush assemblage on the eastern side of Yucca Flat. Unnamed hills appear in the center of the view, and the flank of Camera Station Butte appears at left. The assemblage is dominated by creosote bush, spiny hopsage, and wolfberry (Janice Beatley Collection, 86-B).

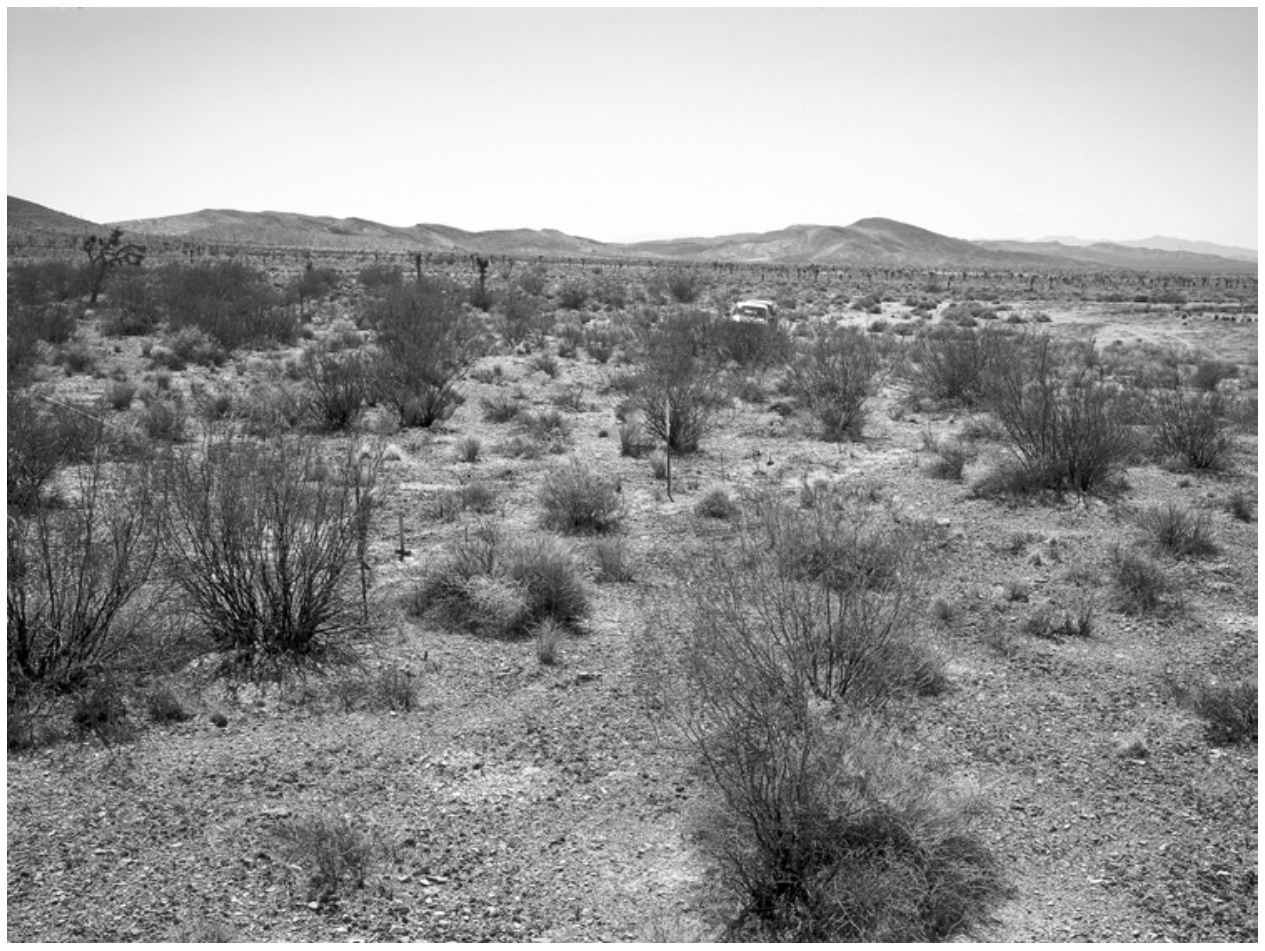

B. (April 21, 2002). Plot 57 was disturbed in 1966, and recovery from that disturbance dominates the midground. The plot was re-established behind this camera station as Plot 57A. The creosote bushes are greatly increased in size on the plot, and spiny hopsage has declined. The Joshua trees appear to have greatly increased in number in the background (Dominic Oldershaw, Stake 4189B). 
Table A-57B. Summary plant data for Plot 57A.

\section{SPECIES}

Acamptopappus shockleyi

Artemisia spinescens

Atriplex confertifolia

Ceratoides lanata

Chrysothamnus viscidiflorus

Ephedra nevadensis

Grayia spinosa

Hymenoclea salsola

Larrea tridentata

Lycium andersonii

Menodora spinescens

Mirabilis pudica

Oryzopsis hymenoides

Sitanion hystrix

Stanleya pinnata

Stipa speciosa

Tetradymia glabrata

Total Live

Dead Grass

Dead Shrub

Total

\section{SPECIES}

Acamptopappus shockleyi

Artemisia spinescens

Atriplex confertifolia

Ceratoides lanata

Chrysothamnus viscidiflorus

Ephedra nevadensis

Grayia spinosa

Hymenoclea salsola

Larrea tridentata

Lycium andersonii

Menodora spinescens

Mirabilis pudica

Oryzopsis hymenoides

Sitanion hystrix

Stanleya pinnata

Stipa speciosa

Tetradymia glabrata

\section{Number of Plants}

$\begin{array}{cc}\mathbf{1 9 6 3} & \mathbf{2 0 0 2} \\ 45 & 12 \\ 13 & 0 \\ 18 & 1 \\ 17 & 16 \\ 0 & 3 \\ 11 & 10 \\ 24 & 2 \\ 0 & 10 \\ 22 & 42 \\ 28 & 24 \\ 4 & 4 \\ 0 & 0 \\ 3 & 12 \\ 1 & 0 \\ 0 & 1 \\ 1 & 0 \\ 1 & 0 \\ 188 & 137 \\ 0 & 1 \\ 60 & 240 \\ 248 & \end{array}$

\section{Average Height (m)}

\section{3}

$2002^{*}$

0.19

0.23

0.35

0.36

0.00

0.32

0.43

0.00

0.93

0.40

0.13

0.00

0.25

0.20

0.00

0.20

0.43
0.21

0.00

0.35

0.36

0.30

0.41

0.68

0.44

1.44

0.42

0.16

0.00

0.20

0.00

0.26

0.00

0.00

*--plot was disturbed in 1965 to an unknown extent.

SPECIES

Acamptopappus shockleyi

Artemisia spinescens

Atriplex confertifolia

Ceratoides lanata

Chrysothamnus viscidiflorus

Ephedra nevadensis

Grayia spinosa

Hymenoclea salsola

Larrea tridentata

Lycium andersonii

Menodora spinescens

Mirabilis pudica

Oryzopsis hymenoides

Sitanion hystrix

Stanleya pinnata

Stipa speciosa

Tetradymia glabrata

Total Live

Dead Grass

Dead Shrub

Total

\section{SPECIES}

Acamptopappus shockleyi

Artemisia spinescens

Atriplex confertifolia

Ceratoides lanata

Chrysothamnus viscidiflorus

Ephedra nevadensis

Grayia spinosa

Hymenoclea salsola

Larrea tridentata

Lycium andersonii

Menodora spinescens

Mirabilis pudica

Oryzopsis hymenoides

Sitanion hystrix

Stanleya pinnata

Stipa speciosa

Tetradymia glabrata

Total Live
Cover (\%)

1963

$2002^{*}$

2.65

0.40

0.95

0.00

2.07

0.08

1.38

0.67

0.00

0.27

1.40

0.80

3.04

0.10

0.00

0.73

5.32

11.11

3.17

2.01

0.29

0.23

0.00

0.00

0.16

0.30

0.06

0.00

0.00

0.09

0.04

0.00

0.08

0.00

20.63

16.79

0.00

0.02

4.99

9.48

25.62

26.29

Biomass Index $\left(\mathrm{m}^{2}\right)$

1963

2002*

1.80

0.24

0.74

0.00

2.43

0.10

1.67

0.91

0.00

0.30

1.57

1.45

4.64

0.15

0.00

1.11

18.57

56.94

4.44

2.75

0.13

0.15

0.00

0.00

0.13

0.23

0.04

0.00

0.00

0.08

0.02

0.00

0.12

0.00

36.31

64.40 
Table A-57C. Site characteristics for Plot 57N.

Location: Yucca Flat

NTS Area: 3

NTS Grid: V-31

USGS 7.5’ Quadrangle Name: Paiute Ridge

\begin{tabular}{|c|c|c|c|c|}
\hline & & GPS Read & Zone 11S) & \\
\hline & Location & Northing & Easting & Condition of Original Corners \\
\hline Corner 1: & SW & NA & NA & NA \\
\hline Corner 2: & NW & 4100091 & 589681 & NA \\
\hline Corner 3: & $\mathrm{NE}$ & 4100072 & 589694 & NA \\
\hline Corner 4: & SE & 4100042 & 589665 & NA \\
\hline DEM Plo & vation: 12 & & & Plot Aspect: $335^{\circ}$ \\
\hline Elevation & e Playa ( & Name): & lсca) & Plot Slope: $12^{\circ}$ \\
\hline
\end{tabular}

Annual Precipitation

Measured: $177 \mathrm{~mm}$

Modeled: $162 \mathrm{~mm}$

Parent Material Type: alluvial fan

Substrate: mixed alluvium, weak desert pavement, limestone \& volcanic

Slate Geologic Unit: NA

Beatley Plant Assemblage: Larrea-Grayia-Lycium

Ostler-Hanson Plant Association: NA

Ostler-Hanson Land Unit: 691

Abundance of Biological Soil Crusts: heavy

Type and Date of Disturbance: none

Plot Condition: good

Location of Transect 1: south side of plot

Direction Transects Are Read: west to east

Date(s) Plot Remeasured: 21 April 2002 
This page is intentionally blank.

APPENDIX 204 
Table A-57D. Summary plant data for Plot 57N.

SPECIES
Acamptopappus shockleyi
Artemisia spinescens
Atriplex confertifolia
Ceratoides lanata
Chrysothamnus viscidiflorus stenophyllus
Ephedra nevadensis
Grayia spinosa
Larrea tridentata
Lepidium fremontii
Lycium andersonii
Menodora spinescens
Oryzopsis hymenoides
Sitanion hystrix
Sphaeralcea ambigua monticola
Stanleya pinnata pinnata
Stipa speciosa
Tetradymia axillaris
Tetradymia glabrata
Yucca brevifolia
Total Live
Dead grass
Dead shrubs
Total

SPECIES
Acamptopappus shockleyi
Artemisia spinescens
Atriplex confertifolia
Ceratoides lanata
Chrysothamnus viscidiflorus stenophyllus
Ephedra nevadensis
Grayia spinosa
Larrea tridentata
Lepidium fremontii
Lycium andersonii
Menodora spinescens
Oryzopsis hymenoides
Sitanion hystrix
Sphaeralcea ambigua monticola
Stanleya pinnata pinnata
Stipa speciosa
Tetradymia axillaris
Tetradymia glabrata
Yucca brevifolia

Number of Plants

$\begin{array}{ccc}\mathbf{1 9 6 6} & \mathbf{1 9 7 5} & \mathbf{2 0 0 2} \\ 49 & 78 & 3 \\ 3 & 8 & 1 \\ 32 & 31 & 5 \\ 33 & 52 & 34 \\ 0 & 1 & 0 \\ 7 & 11 & 14 \\ 18 & 18 & 7 \\ 20 & 21 & 24 \\ 0 & 1 & 0 \\ 23 & 28 & 29 \\ 2 & 2 & 4 \\ 12 & 15 & 19 \\ 0 & 3 & 1 \\ 2 & 0 & 0 \\ 0 & 2 & 0 \\ 0 & 0 & 2 \\ 4 & 3 & 0 \\ 3 & 3 & 0 \\ 0 & 1 & 2 \\ 208 & 278 & 145 \\ 1 & 1 & 2 \\ 86 & 37 & 123 \\ 295 & 316 & 270\end{array}$

\section{Average Height (m)}

$\begin{array}{lll}\mathbf{1 9 6 6} & \mathbf{1 9 7 5} & \mathbf{2 0 0 2} \\ 0.19 & 0.19 & 0.17 \\ 0.25 & 0.24 & 0.25 \\ 0.37 & 0.35 & 0.28 \\ 0.31 & 0.31 & 0.39 \\ 0.00 & 0.18 & 0.00 \\ 0.34 & 0.47 & 0.43 \\ 0.47 & 0.35 & 0.50 \\ 0.89 & 1.05 & 1.10 \\ 0.00 & 0.23 & 0.00 \\ 0.45 & 0.46 & 0.37 \\ 0.17 & 0.11 & 0.10 \\ 0.31 & 0.34 & 0.21 \\ 0.00 & 0.30 & 0.32 \\ 0.27 & 0.00 & 0.00 \\ 0.00 & 0.25 & 0.00 \\ 0.00 & 0.00 & 0.33 \\ 0.49 & 0.65 & 0.00 \\ 0.51 & 0.54 & 0.00 \\ 0.00 & 3.81 & 2.24\end{array}$

SPECIES

Acamptopappus shockleyi

Artemisia spinescens

Atriplex confertifolia

Ceratoides lanata

Chrysothamnus viscidiflorus stenophyllus

Ephedra nevadensis

Grayia spinosa

Larrea tridentata

Lepidium fremontii

Lycium andersonii

Menodora spinescens

Oryzopsis hymenoides

Sitanion hystrix

Sphaeralcea ambigua monticola

Stanleya pinnata pinnata

Stipa speciosa

Tetradymia axillaris

Tetradymia glabrata

Yucca brevifolia

Total Live

Dead grass

Dead shrubs

Total

\section{SPECIES}

Acamptopappus shockleyi

Artemisia spinescens

Atriplex confertifolia

Ceratoides lanata

Chrysothamnus viscidiflorus stenophyllus

Ephedra nevadensis

Grayia spinosa

Larrea tridentata

Lepidium fremontii

Lycium andersonii

Menodora spinescens

Oryzopsis hymenoides

Sitanion hystrix

Sphaeralcea ambigua monticola

Stanleya pinnata pinnata

Stipa speciosa

Tetradymia axillaris

Tetradymia glabrata

Yucca brevifolia

Total Live
Cover (\%)

$19661975 \quad 2002$

$\begin{array}{lll}2.32 & 3.51 \quad 0.08\end{array}$

$\begin{array}{lll}0.20 & 0.34 & 0.07\end{array}$

$\begin{array}{lll}3.17 & 3.25 & 0.28\end{array}$

$\begin{array}{lll}2.25 & 3.10 & 2.05\end{array}$

$\begin{array}{lll}0.00 & 0.01 & 0.00\end{array}$

$\begin{array}{lll}0.70 & 1.07 & 0.84\end{array}$

$\begin{array}{lll}1.85 & 1.80 \quad 0.65\end{array}$

$4.01 \quad 5.68 \quad 7.86$

$\begin{array}{lll}0.00 & 0.03 & 0.00\end{array}$

$2.84 \quad 4.20 \quad 2.70$

$\begin{array}{lll}0.17 & 0.09 & 0.28\end{array}$

$\begin{array}{lll}0.83 & 1.26 & 0.75\end{array}$

$\begin{array}{lll}0.00 & 0.15 & 0.01\end{array}$

$\begin{array}{lll}0.08 & 0.00 & 0.00\end{array}$

$\begin{array}{lll}0.00 & 0.05 & 0.00\end{array}$

$\begin{array}{lll}0.00 & 0.00 & 0.11\end{array}$

$\begin{array}{lll}0.36 & 0.37 & 0.00\end{array}$

$\begin{array}{lll}0.37 & 0.41 & 0.00\end{array}$

$\begin{array}{lll}0.00 & 0.18 & 0.32\end{array}$

$19.15 \quad 25.50 \quad 16.00$

$\begin{array}{lll}0.05 & 0.01 & 0.03\end{array}$

$\begin{array}{lll}6.27 & 2.80 & 8.94\end{array}$

$25.47 \quad 28.31 \quad 24.96$

Biomass Index $\left(\mathrm{m}^{2}\right)$

$\begin{array}{lll}1966 & 1975 & 2002\end{array}$

$\begin{array}{lll}1.57 & 2.43 \quad 0.05\end{array}$

$\begin{array}{lll}0.17 & 0.27 & 0.06\end{array}$

$\begin{array}{lll}4.23 & 4.19 & 0.31\end{array}$

$2.47 \quad 3.84 \quad 3.05$

$\begin{array}{lll}0.00 & 0.01 \quad 0.00\end{array}$

$\begin{array}{lll}0.81 & 1.68 & 1.44\end{array}$

$\begin{array}{lll}3.06 & 2.39 & 1.09\end{array}$

$\begin{array}{lll}12.82 & 21.49 \quad 34.54\end{array}$

$\begin{array}{lll}0.00 & 0.02 & 0.00\end{array}$

$\begin{array}{lll}4.39 & 6.93 & 3.98\end{array}$

$\begin{array}{lll}0.10 & 0.03 & 0.13\end{array}$

$\begin{array}{lll}0.87 & 1.43 & 0.74\end{array}$

$\begin{array}{lll}0.00 & 0.15 & 0.01\end{array}$

$\begin{array}{lll}0.07 & 0.00 & 0.00\end{array}$

$\begin{array}{lll}0.00 & 0.05 & 0.00\end{array}$

$\begin{array}{lll}0.00 & 0.00 \quad 0.11\end{array}$

$\begin{array}{lll}0.62 & 0.81 \quad 0.00\end{array}$

$\begin{array}{lll}0.63 & 0.73 \quad 0.00\end{array}$

$\begin{array}{lll}0.00 & 2.32 \quad 2.60\end{array}$

$31.81 \quad 48.77 \quad 48.11$ 
Table A-58A. Site characteristics for Plot 58.

Location: Yucca Flat

NTS Area: 3

NTS Grid: S-29

USGS 7.5’ Quadrangle Name: Yucca Flat

\begin{tabular}{llll} 
& & \multicolumn{2}{l}{ GPS Readings (Zone 11S) } \\
& Location & Northing & Easting \\
Corner 1: & SW & NA & NA \\
Corner 2: & NW & NA & NA \\
Corner 3: & NE & NA & NA \\
Corner 4: & SE & NA & NA
\end{tabular}

DEM Plot Elevation: 1207 m

Condition of Original Corners

Gone (destroyed by crater)

Gone (destroyed by crater)

Gone (destroyed by crater)

Gone (destroyed by crater)

Elevation Above Playa (Playa Name): 14 m (Yucca)

Plot Aspect: $288^{\circ}$

Plot Slope: $5^{\circ}$

Annual Precipitation

Measured: $178 \mathrm{~mm}$

Modeled: $162 \mathrm{~mm}$

Parent Material Type: alluvial fan

Substrate: alluvial fan

Slate Geologic Unit: Qai

Beatley Plant Assemblage: Atriplex-Ceratoides

Ostler-Hanson Plant Association: NA

Ostler-Hanson Land Unit: 9998

Abundance of Biological Soil Crusts: NA

Type and Date of Disturbance: none

Plot Condition: plot destroyed by below-ground testing between 1975 and 2000

Location of Transect 1: NA

Direction Transects Are Read: NA

Date(s) Plot Remeasured: NA 
Figure A-58. Photograph showing Plot 58.

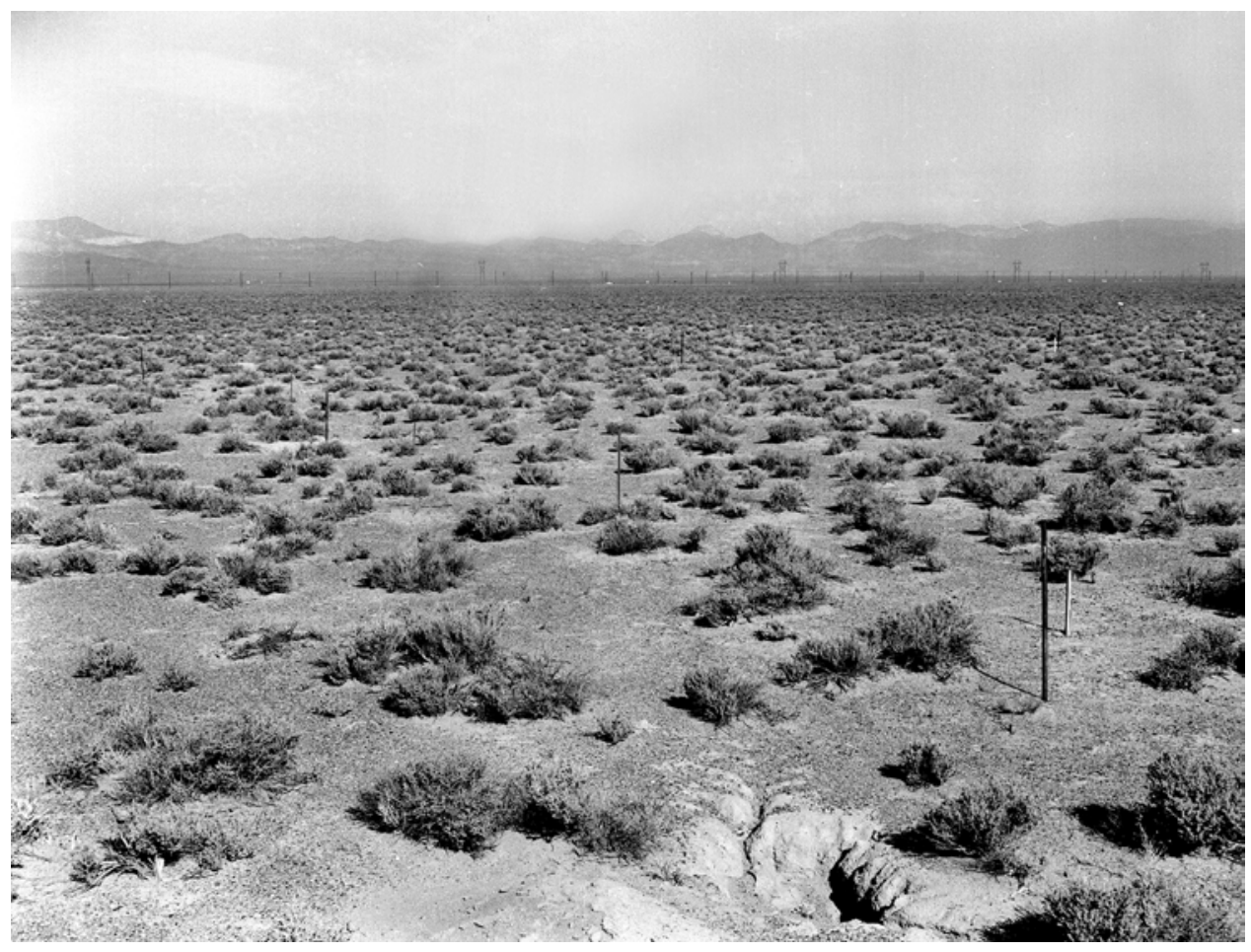

A. (May 13, 1964). This northwesterly view across Plot 58 shows a shadscale - winterfat association with various mountains ringing the northern end of Yucca Flat appearing in the distance. This plot was destroyed at an unknown time owing to underground testing and could not be remeasured (Janice Beatley Collection, 106-A, Stake 4190). 
Table A-58B. Summary plant data for Plot 58.

\begin{tabular}{|c|c|c|c|c|c|}
\hline \multicolumn{4}{|c|}{ Number of Plants } & \multicolumn{2}{|c|}{ Cover (\%) } \\
\hline SPECIES & 1963 & 1975 & SPECIES & 1963 & 1975 \\
\hline Artemisia spinescens & 33 & 88 & Artemisia spinescens & 2.67 & 4.80 \\
\hline Astragalus lentiginosus fremontii & 0 & 2 & Astragalus lentiginosus fremontii & 0.00 & 0.02 \\
\hline Atriplex confertifolia & 58 & 66 & Atriplex confertifolia & 7.29 & 8.55 \\
\hline Ceratoides lanata & 57 & 88 & Ceratoides lanata & 4.89 & 7.87 \\
\hline Grayia spinosa & 2 & 4 & Grayia spinosa & 0.34 & 0.74 \\
\hline Lycium andersonii & 5 & 5 & Lycium andersonii & 0.88 & 1.14 \\
\hline Mirabilis pudica & 1 & 0 & Mirabilis pudica & 0.03 & 0.00 \\
\hline Oryzopsis hymenoides & 5 & 27 & Oryzopsis hymenoides & 0.13 & 2.50 \\
\hline Sitanion hystrix & 1 & 0 & Sitanion hystrix & 0.03 & 0.00 \\
\hline Tetradymia axillaris & 1 & 1 & Tetradymia axillaris & 0.14 & 0.15 \\
\hline Total Live & 163 & 281 & Total Live & 16.39 & 25.76 \\
\hline Dead grass & 0 & 1 & Dead grass & 0.00 & 0.01 \\
\hline Dead shrubs & 16 & 19 & Dead shrubs & 1.29 & 2.32 \\
\hline Total & 179 & 301 & Total & 17.68 & 28.09 \\
\hline \multicolumn{4}{|c|}{ Average Height (m) } & \multicolumn{2}{|c|}{ Biomass Index (m²) } \\
\hline SPECIES & 1963 & 1975 & SPECIES & 1963 & 1975 \\
\hline Artemisia spinescens & 0.23 & 0.19 & Artemisia spinescens & 2.13 & 3.33 \\
\hline Astragalus lentiginosus fremontii & 0.00 & 0.03 & Astragalus lentiginosus fremontii & 0.00 & 0.00 \\
\hline Atriplex confertifolia & 0.34 & 0.37 & Atriplex confertifolia & 8.72 & 11.68 \\
\hline Ceratoides lanata & 0.30 & 0.39 & Ceratoides lanata & 5.34 & 11.84 \\
\hline Grayia spinosa & 0.52 & 0.59 & Grayia spinosa & 0.58 & 1.45 \\
\hline Lycium andersonii & 0.49 & 0.52 & Lycium andersonii & 1.47 & 2.09 \\
\hline Mirabilis pudica & 0.13 & 0.00 & Mirabilis pudica & 0.01 & 0.00 \\
\hline Oryzopsis hymenoides & 0.07 & 0.27 & Oryzopsis hymenoides & 0.03 & 2.32 \\
\hline Sitanion hystrix & 0.25 & 0.00 & Sitanion hystrix & 0.02 & 0.00 \\
\hline \multirow[t]{2}{*}{ Tetradymia axillaris } & 0.48 & 0.64 & Tetradymia axillaris & 0.22 & 0.31 \\
\hline & & & Total Live & 18.54 & 33.02 \\
\hline
\end{tabular}


Table A-59A. Site characteristics for Plot 59.

Location: Yucca Flat

NTS Area: 6

NTS Grid: S-27

USGS 7.5’ Quadrangle Name: Yucca Lake

\begin{tabular}{llll} 
& & \multicolumn{2}{l}{ GPS Readings (Zone 11S) } \\
& Location & Northing & Easting \\
Corner 1: & SW & NA & NA \\
Corner 2: & NW & NA & NA \\
Corner 3: & NE & NA & NA \\
Corner 4: & SE & NA & NA
\end{tabular}

DEM Plot Elevation: 1198 m

Elevation Above Playa (Playa Name): 3 m (Yucca)

Condition of Original Corners

One corner intact (specific corner not recorded)

One corner intact (specific corner not recorded)

One corner intact (specific corner not recorded)

One corner intact (specific corner not recorded)

Plot Aspect: $141^{\circ}$

Plot Slope: $9^{\circ}$

Annual Precipitation

Measured: $177 \mathrm{~mm}$

Modeled: $166 \mathrm{~mm}$

Parent Material Type: playa

Substrate: NA

Slate Geologic Unit: Qai

Beatley Plant Assemblage: Atriplex-Kochia

Ostler-Hanson Plant Association: NA

Ostler-Hanson Land Unit: 544

Abundance of Biological Soil Crusts: light

Type and Date of Disturbance: none

Plot Condition: about one-third of the plot was damaged by construction between 1975 and 2000

Location of Transect 1: NA

Direction Transects Are Read: NA

Date(s) Plot Remeasured: NA 
Figure A-59. Photographs showing Plot 59.

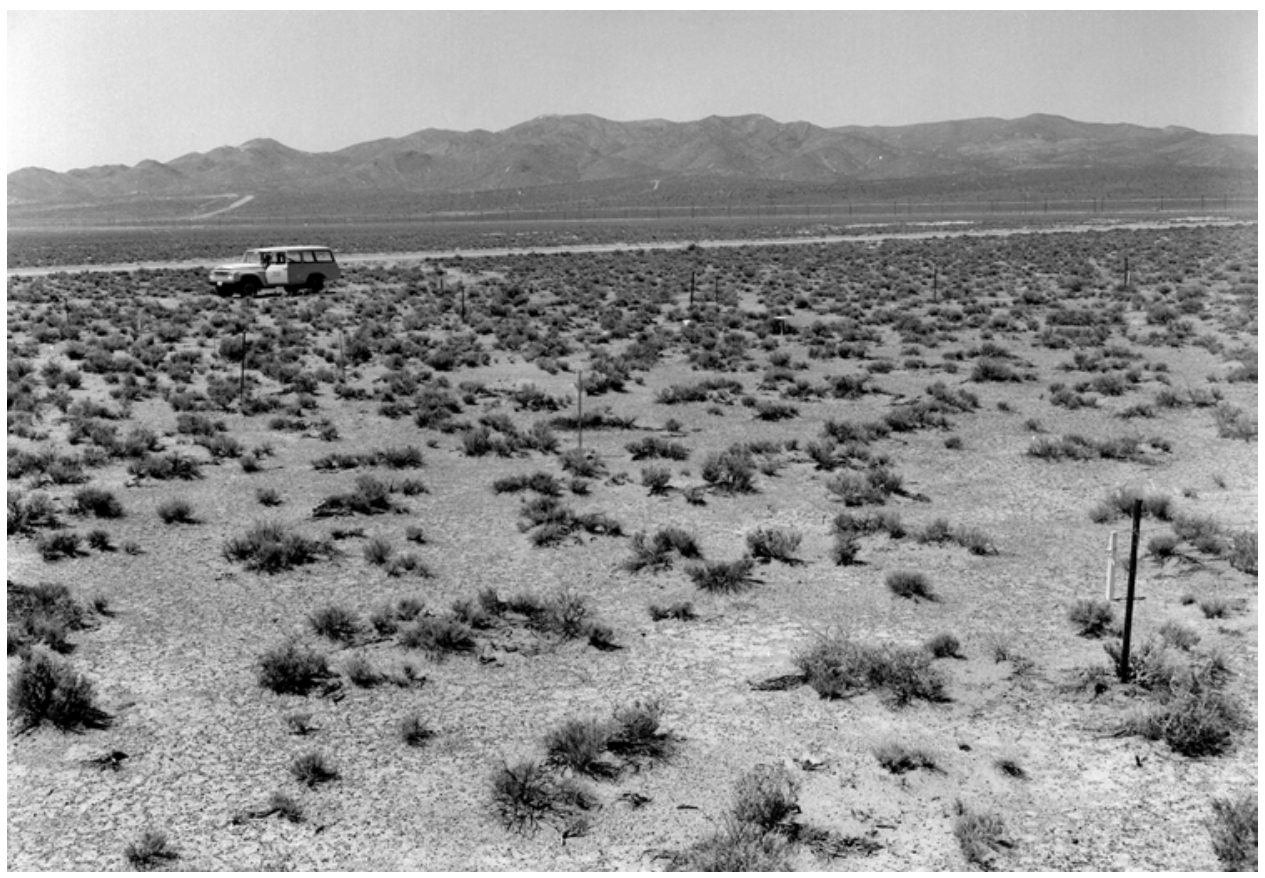

A. (1964). This southwesterly view across Plot 59 shows the north end of Yucca Lake in the midground and the CP Hills in the distance. The vegetation here was shadscale and greenmolly (Kochia americana) (Janice Beatley Collection, no number).

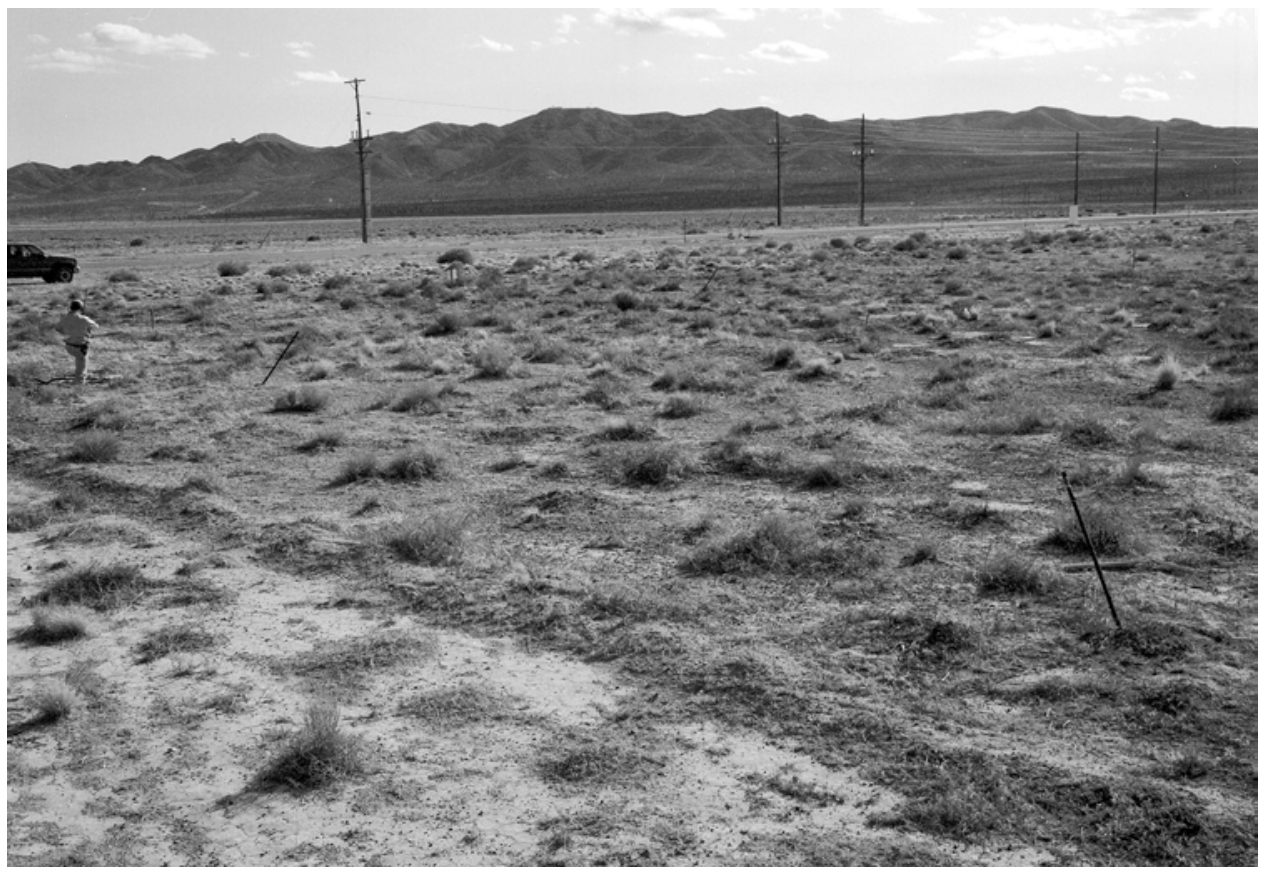

B. (April 20, 2002). Plot 59 was disturbed by construction activities in the vicinity. Tracks left by bulldozers are apparent at lower left. The Tweezer Road crosses the view at midground. Approximately one third of the plot was disturbed by these activities, so the plot was not remeasured (Dominic Oldershaw, Stake 4184A). 
Table A-59B. Summary plant data for Plot 59.

\section{SPECIES}

Atriplex confertifolia

Ceratoides lanata

Cymopterus globusus

Kochia americana vestita

Stipa speciosa

Total Live

Dead grass

Dead shrubs

Total

\section{SPECIES}

Atriplex confertifolia

Ceratoides lanata

Cymopterus globusus

Kochia americana vestita

Stipa speciosa

\section{SPECIES}

Atriplex confertifolia

Ceratoides lanata

Cymopterus globusus

Kochia americana vestita

Stipa speciosa

Total Live

Dead grass

Dead shrubs

Total

\section{SPECIES}

Atriplex confertifolia

Ceratoides lanata

Cymopterus globusus

Kochia americana vestita

Stipa speciosa

Total Live

*--plot affected by disturbance to unknown extent, 1975-2001.

\section{Number of Plants}

1963

50

0

0

120

0

170

0

16

186

1963

0.27

0.00

0.00

0.19

0.00

1963

5.31

0.00

0.00

7.81

0.00

13.12

0.00

1.75

14.86

1963

4.97

0.00

0.00

5.21

0.00

10.18

1975

84

0

3

131

0

218

0

13

231

$2003^{*}$

4

1

0

23

1

29

1

108

138

Average Height (m)

1975

2003*

0.31

0.23

0.64

0.00

0.22

0.20

\section{Cover (\%)}

1975

2003*

0.11

0.20

0.00

1.15

0.00

1.45

0.15

7.95

9.55

\section{Biomass Index $\left(\mathrm{m}^{2}\right)$}

$19752003^{*}$

11.54

0.06

0.00

0.43

0.00

0.89

0.00

1.38

0.00

20.57

0.00

9.02

2003*

43

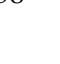


Table A-60A. Site characteristics for Plot 60.

Location: Yucca Flat

NTS Area: 6

NTS Grid: V-26

USGS 7.5’ Quadrangle Name: Plutonium Valley

\begin{tabular}{lllc} 
& & \multicolumn{2}{c}{ GPS Readings (Zone 11S) } \\
& Location & Northing & Easting \\
Corner 1: & SW & 4091133 & 589686 \\
Corner 2: & NW & 4091162 & 589693 \\
Corner 3: & NE & 4091158 & 589723 \\
Corner 4: & SE & 4091129 & 589716
\end{tabular}

DEM Plot Elevation: 1199 m

Condition of Original Corners
On ground or missing
On ground or missing
On ground or missing
Leaning

Plot Aspect: $207^{\circ}$

Elevation Above Playa (Playa Name): 9 m (Yucca)

Plot Slope: $6^{\circ}$

Annual Precipitation

Measured: $164 \mathrm{~mm}$

Modeled: $163 \mathrm{~mm}$

Parent Material Type: alluvial fan

Substrate: mixed alluvium, weak desert pavement, mostly volcanic

Slate Geologic Unit: Qai

Beatley Plant Assemblage: Grayia-Lycium

Ostler-Hanson Plant Association: Tetradymia-Lycium-Grayia

Ostler-Hanson Land Unit: 660

Abundance of Biological Soil Crusts: none

Type and Date of Disturbance: none

Plot Condition: good

Location of Transect 1: south side of plot

Direction Transects Are Read: west to east

Date(s) Plot Remeasured: 18 April 2002 
Figure A-60. Photographs showing Plot 60.

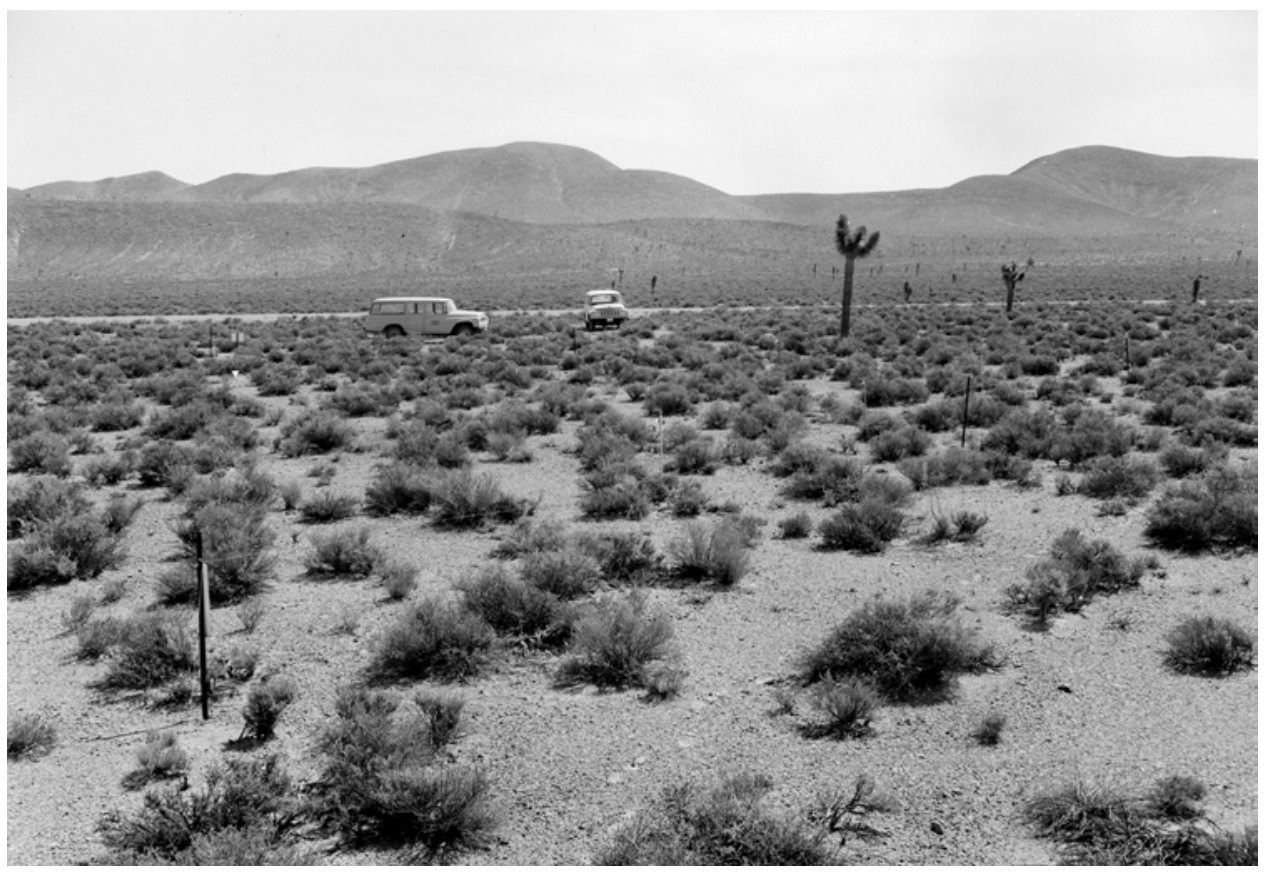

A. (May 13, 1964). This easterly view across Plot 60 shows unnamed hills on the eastern side of Yucca Flat in the distance. Only a few Joshua trees are visible in this view. Spiny hopsage and wolfberry dominate the vegetation on this plot (Janice Beatley Collection, 101-B).

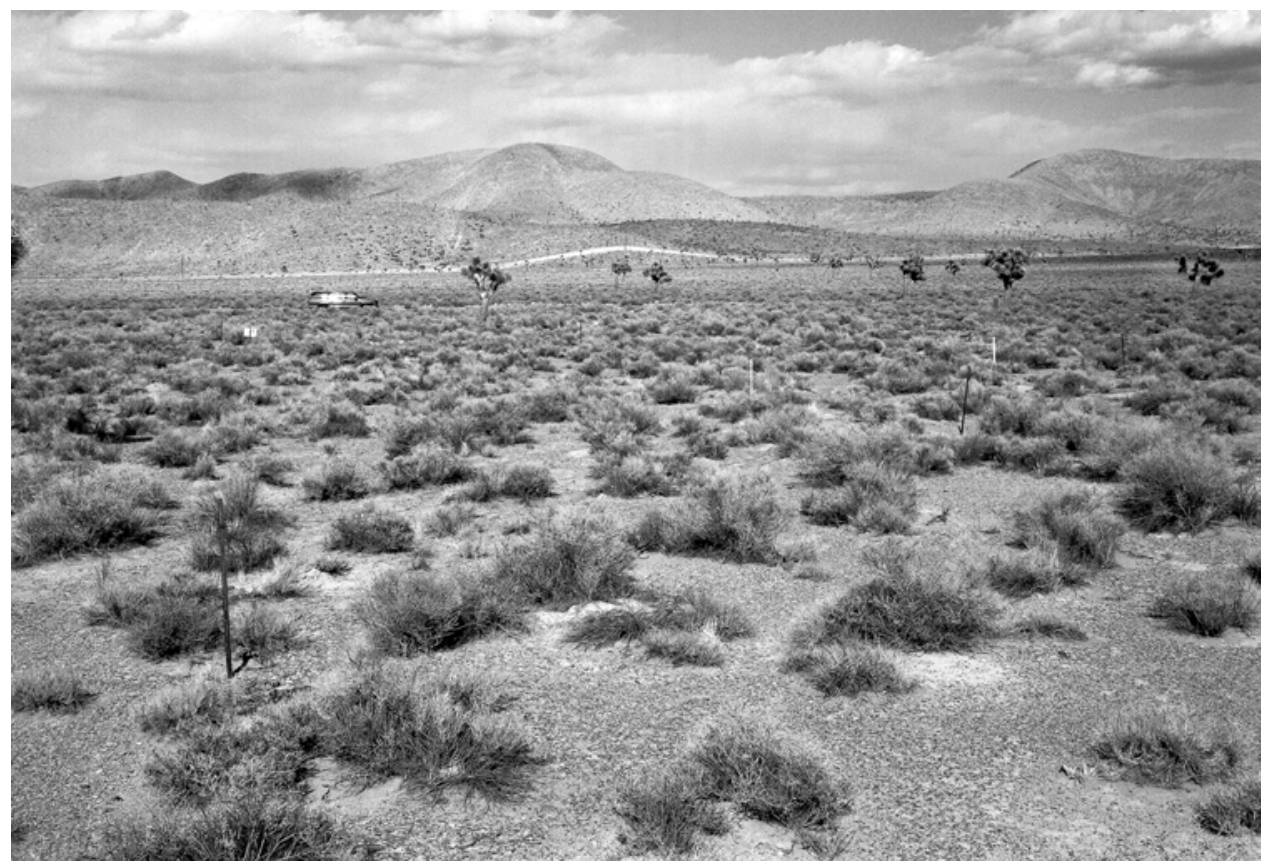

B. (April 20, 2002). The vegetation is now dominated by green rabbitbrush (Chrysothamnus viscidiflorus), wolfberry, and spiny hopsage. The latter has declined on this plot much as it has throughout the Nevada Test Site. The background Joshua trees have greatly increased in number and height. The Orange Blossom Road, built after 1964, creates the roadcut that is obvious at center (Dominic Oldershaw, Stake 4183B). 
Table A-60B. Summary plant data for Plot 60.

\begin{tabular}{|c|c|c|c|c|c|c|c|}
\hline \multicolumn{5}{|c|}{ Number of Plants } & \multicolumn{3}{|c|}{ Cover (\%) } \\
\hline SPECIES & 1963 & 1975 & 2002 & SPECIES & 1963 & 1975 & 2002 \\
\hline Artemisia spinescens & 30 & 21 & 5 & Artemisia spinescens & 1.86 & 1.24 & 0.20 \\
\hline Atriplex canescens & 1 & 2 & 13 & Atriplex canescens & 0.13 & 0.45 & 1.31 \\
\hline Atriplex confertifolia & 8 & 5 & 0 & Atriplex confertifolia & 0.54 & 0.67 & 0.00 \\
\hline Ceratoides lanata & 20 & 28 & 21 & Ceratoides lanata & 1.69 & 1.99 & 1.74 \\
\hline Chrysothamnus viscidiflorus & 39 & 39 & 77 & Chrysothamnus viscidiflorus & 3.27 & 2.93 & 6.14 \\
\hline Ephedra nevadensis & 2 & 3 & 5 & Ephedra nevadensis & 0.41 & 0.73 & 0.58 \\
\hline Grayia spinosa & 36 & 54 & 28 & Grayia spinosa & 6.18 & 6.04 & 2.41 \\
\hline Hymenoclea salsola & 0 & 1 & 0 & Hymenoclea salsola & 0.00 & 0.18 & 0.00 \\
\hline Lycium andersonii & 41 & 58 & 43 & Lycium andersonii & 5.71 & 6.71 & 4.28 \\
\hline Oryzopsis hymenoides & 1 & 1 & 7 & Oryzopsis hymenoides & 0.04 & 0.07 & 0.35 \\
\hline Sitanion hystrix & 0 & 3 & 16 & Sitanion hystrix & 0.00 & 0.18 & 0.99 \\
\hline Sphaeralcea ambigua monticola & 0 & 4 & 0 & Sphaeralcea ambigua monticola & 0.00 & 0.11 & 0.00 \\
\hline Tetradymia glabrata & 48 & 59 & 0 & Tetradymia glabrata & 6.92 & 9.32 & 0.00 \\
\hline Yucca brevifolia & 0 & 0 & 1 & Yucca brevifolia & 0.00 & 0.00 & 0.04 \\
\hline Total Live & 226 & 278 & 216 & Total Live & 26.75 & 30.61 & 18.03 \\
\hline Dead Grass & 0 & 0 & 0 & Dead Grass & 0.00 & 0.00 & 0.00 \\
\hline Dead Shrub & 12 & 20 & 60 & Dead Shrub & 1.09 & 1.45 & 8.39 \\
\hline Total & 238 & 298 & 276 & Total & 27.84 & 32.06 & 26.42 \\
\hline \multicolumn{5}{|c|}{ Average Height (m) } & \multicolumn{3}{|c|}{ Biomass Index $\left(\mathrm{m}^{2}\right)$} \\
\hline SPECIES & 1963 & 1975 & 2002 & SPECIES & 1963 & 1975 & 2002 \\
\hline Artemisia spinescens & 0.17 & 0.17 & 0.18 & Artemisia spinescens & 1.14 & 0.74 & 0.13 \\
\hline Atriplex canescens & 0.66 & 0.72 & 0.65 & Atriplex canescens & 0.28 & 1.32 & 2.82 \\
\hline Atriplex confertifolia & 0.31 & 0.40 & 0.00 & Atriplex confertifolia & 0.57 & 0.89 & 0.00 \\
\hline Ceratoides lanata & 0.30 & 0.38 & 0.49 & Ceratoides lanata & 1.85 & 2.79 & 2.97 \\
\hline Chrysothamnus viscidiflorus & 0.25 & 0.28 & 0.31 & Chrysothamnus viscidiflorus & 2.84 & 2.77 & 6.54 \\
\hline Ephedra nevadensis & 0.41 & 0.65 & 0.68 & Ephedra nevadensis & 0.93 & 1.91 & 1.36 \\
\hline Grayia spinosa & 0.50 & 0.49 & 0.51 & Grayia spinosa & 10.58 & 10.58 & 4.31 \\
\hline Hymenoclea salsola & 0.00 & 0.58 & 0.00 & Hymenoclea salsola & 0.00 & 0.36 & 0.00 \\
\hline Lycium andersonii & 0.35 & 0.42 & 0.45 & Lycium andersonii & 6.78 & 9.82 & 7.20 \\
\hline Oryzopsis hymenoides & 0.23 & 0.20 & 0.29 & Oryzopsis hymenoides & 0.03 & 0.05 & 0.34 \\
\hline Sitanion hystrix & 0.00 & 0.39 & 0.28 & Sitanion hystrix & 0.00 & 0.26 & 0.98 \\
\hline Sphaeralcea ambigua monticola & 0.00 & 0.14 & 0.00 & Sphaeralcea ambigua monticola & 0.00 & 0.06 & 0.00 \\
\hline Tetradymia glabrata & 0.43 & 0.50 & 0.00 & Tetradymia glabrata & 10.41 & 16.42 & 0.00 \\
\hline \multirow[t]{2}{*}{ Yucca brevifolia } & 0.00 & 0.00 & 0.25 & Yucca brevifolia & 0.00 & 0.00 & 0.03 \\
\hline & & & & Total Live & 35.41 & 47.96 & 26.68 \\
\hline
\end{tabular}


Table A-61A. Site characteristics for Plot 61.

Location:

NTS Area: 18

NTS Grid: F-37

USGS 7.5' Quadrangle Name: Ammonia Tanks

\begin{tabular}{|c|c|c|c|c|}
\hline & & GPS Reac & Zone 11S & \\
\hline & Location & Northing & Easting & Condition of Original Corners \\
\hline Corner 1: & SW & 4110123 & 565956 & NA \\
\hline Corner 2: & NW & 4110154 & 565959 & NA \\
\hline Corner 3: & $\mathrm{NE}$ & 4110149 & 565988 & NA \\
\hline Corner 4: & $\mathrm{SE}$ & 4110119 & 565986 & NA \\
\hline DEM Plot & vation: 17 & & & Plot Aspect: $328^{\circ}$ \\
\hline Elevation & e Playa & Name): & & Plot Slope: $59^{\circ}$ \\
\hline
\end{tabular}

Annual Precipitation

Measured: $204 \mathrm{~mm}$

Modeled: $222 \mathrm{~mm}$

Parent Material Type: broken terrain

Substrate: silt w/pebbles \& some stones on surface

Slate Geologic Unit: Tgc

Beatley Plant Assemblage: Artemisia nova

Ostler-Hanson Plant Association: NA

Ostler-Hanson Land Unit: 1032

Abundance of Biological Soil Crusts: none

Type and Date of Disturbance: none

Plot Condition: good; road construction near west side

Location of Transect 1: west side of plot

Direction Transects Are Read: south to north

Date(s) Plot Remeasured: 28 May 2002 
Figure A-61. Photographs showing Plot 61.

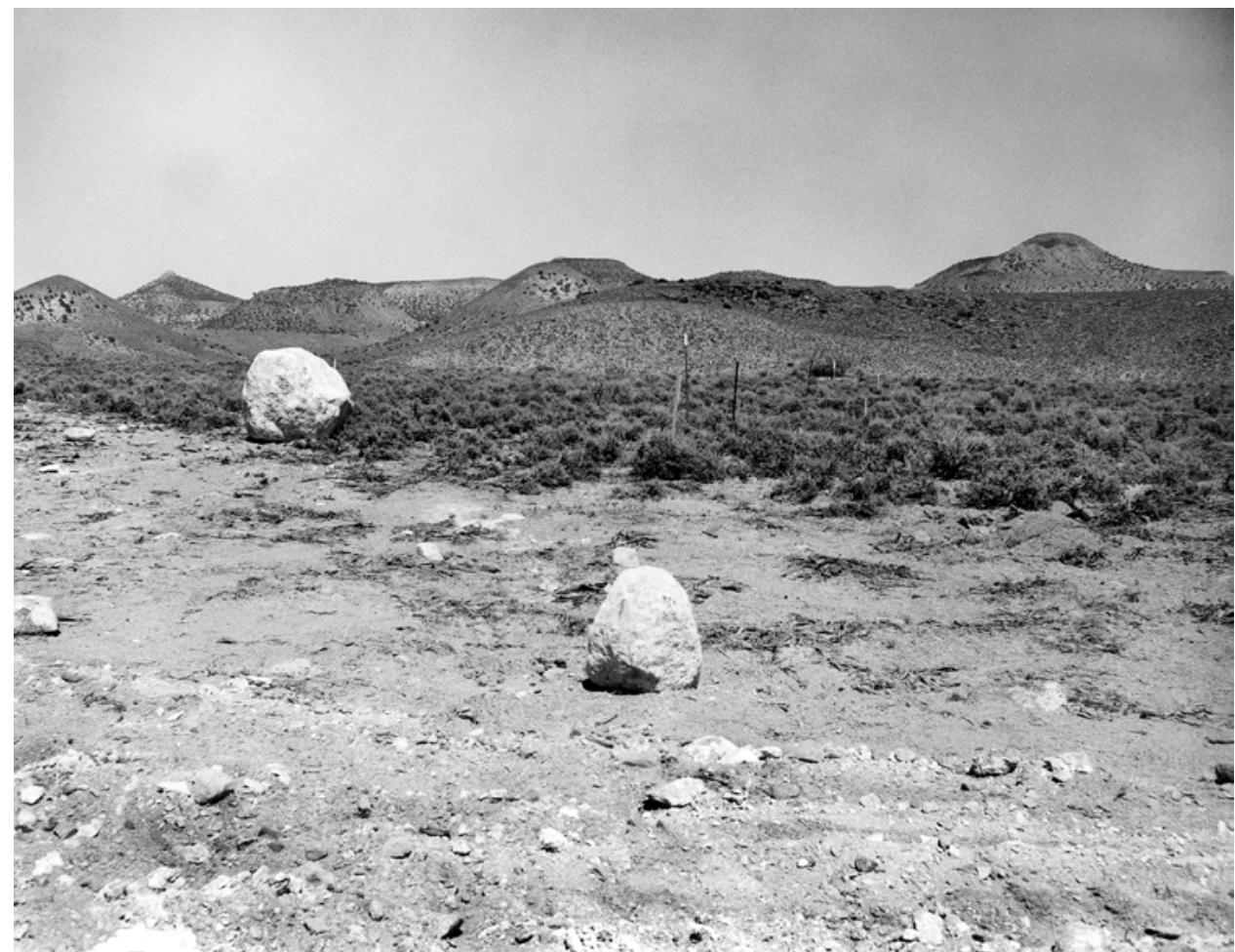

A. (1964). This easterly view across Plot 61 shows damage adjacent to the plot owing to construction of the Pahute Airport Road. The northwestern corner of the plot appears at center, indicating that the disturbance did not affect the center of the plot. The hills in the background are volcanic cones associated with the west side of the Eleana Range. The vegetation in the plot is dominated by black sagebrush (Artemisia nova) (Janice Beatley Collection, no number).

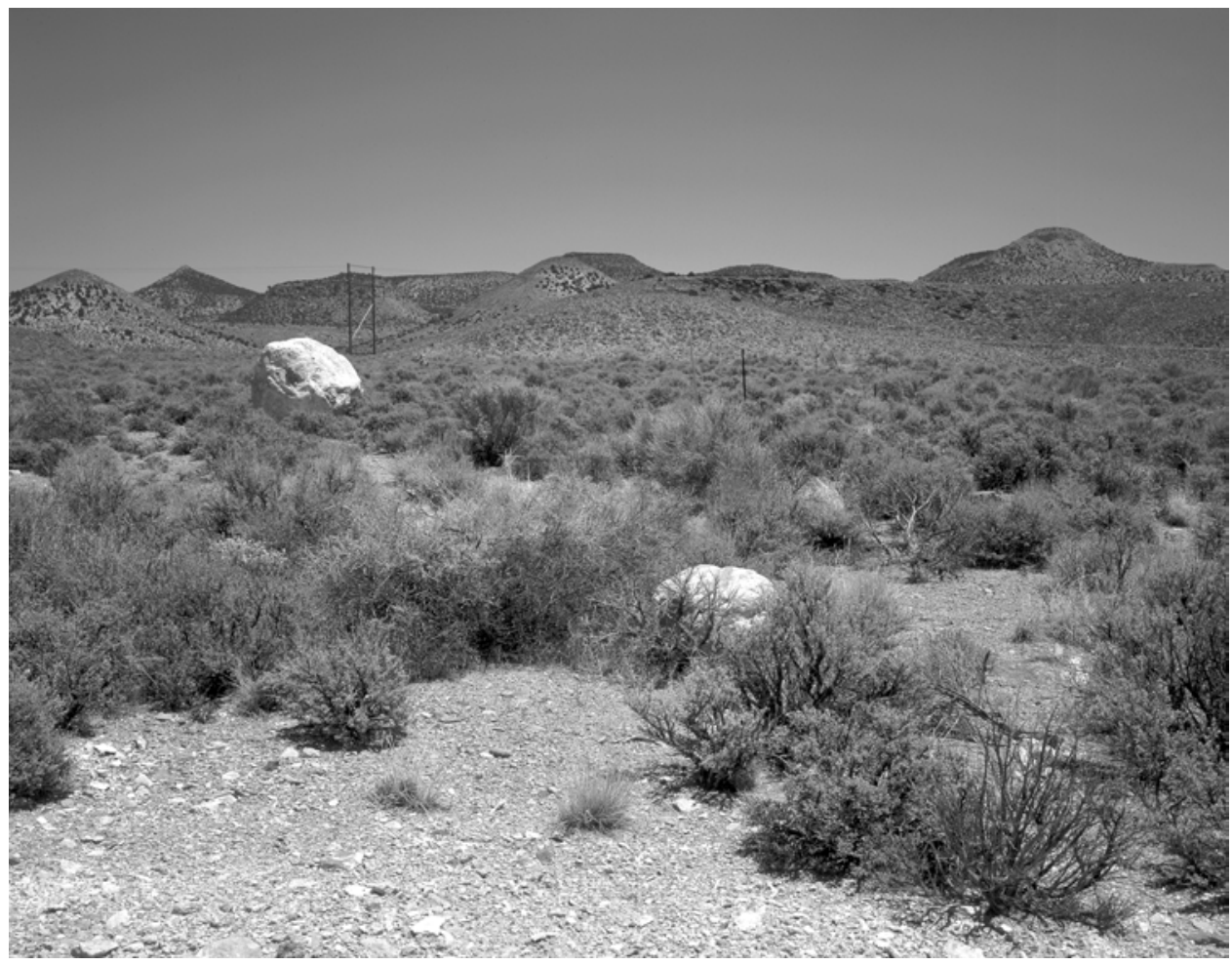

B. (April 30, 2002). Considerable recovery has occurred in the disturbed foreground, as sagebrush and other species have colonized the formerly barren area. The plot is still dominated by black sagebrush (Dominic Oldershaw, Stake 3643). 
Table A-61B. Summary plant data for Plot 61.

\begin{tabular}{|c|c|c|c|c|c|c|c|}
\hline \multicolumn{5}{|c|}{ Number of Plants } & \multicolumn{3}{|c|}{ Cover (\%) } \\
\hline SPECIES & 1963 & 1975 & 2002 & SPECIES & 1963 & 1975 & 2002 \\
\hline Arabis pulchra munciensis & 0 & 2 & 0 & Arabis pulchra munciensis & 0.00 & 0.03 & 0.00 \\
\hline Artemisia nova & 236 & 280 & 233 & Artemisia nova & 27.38 & 36.06 & 24.96 \\
\hline Astragalus lentiginosus & 0 & 1 & 0 & Astragalus lentiginosus & 0.00 & 0.01 & 0.00 \\
\hline Dichelostemma pulchella & 0 & 17 & 0 & Dichelostemma pulchella & 0.00 & 0.16 & 0.00 \\
\hline Ephedra nevadensis & 13 & 13 & 10 & Ephedra nevadensis & 2.12 & 1.72 & 0.61 \\
\hline Grayia spinosa & 14 & 15 & 5 & Grayia spinosa & 1.30 & 1.79 & 0.60 \\
\hline Lycium andersonii & 1 & 0 & 0 & Lycium andersonii & 0.04 & 0.00 & 0.00 \\
\hline Oryzopsis hymenoides & 2 & 0 & 3 & Oryzopsis hymenoides & 0.09 & 0.00 & 0.14 \\
\hline Sitanion hystrix & 27 & 20 & 20 & Sitanion hystrix & 1.41 & 0.72 & 0.90 \\
\hline Sphaeralcea ambigua & 0 & 1 & 0 & Sphaeralcea ambigua & 0.00 & 0.01 & 0.00 \\
\hline Stipa comata & 0 & 0 & 1 & Stipa comata & 0.00 & 0.00 & 0.04 \\
\hline Stipa speciosa & 0 & 1 & 1 & Stipa speciosa & 0.00 & 0.04 & 0.11 \\
\hline Total Live & 293 & 350 & 273 & Total Live & 32.34 & 40.54 & 27.35 \\
\hline Dead Grass & 0 & 0 & 0 & Dead Grass & 0.00 & 0.00 & 0.00 \\
\hline Dead Shrub & 68 & 29 & 36 & Dead Shrub & 6.51 & 2.23 & 8.12 \\
\hline Total & 361 & 379 & 309 & Total & 38.85 & 42.76 & 35.47 \\
\hline \multicolumn{5}{|c|}{ Average Height (m) } & \multicolumn{3}{|c|}{ Biomass Index (m²) } \\
\hline SPECIES & 1963 & 1975 & 2002 & SPECIES & 1963 & 1975 & 2002 \\
\hline Arabis pulchra munciensis & 0.00 & 0.14 & 0.00 & Arabis pulchra munciensis & 0.00 & 0.01 & 0.00 \\
\hline Artemisia nova & 0.36 & 0.39 & 0.37 & Artemisia nova & 35.54 & 50.86 & 34.56 \\
\hline Astragalus lentiginosus & 0.00 & 0.03 & 0.00 & Astragalus lentiginosus & 0.00 & 0.00 & 0.00 \\
\hline Dichelostemma pulchella & 0.00 & 0.08 & 0.00 & Dichelostemma pulchella & 0.00 & 0.05 & 0.00 \\
\hline Ephedra nevadensis & 0.45 & 0.47 & 0.54 & Ephedra nevadensis & 3.31 & 2.83 & 1.24 \\
\hline Grayia spinosa & 0.44 & 0.40 & 0.40 & Grayia spinosa & 2.03 & 2.75 & 0.99 \\
\hline Lycium andersonii & 0.38 & 0.00 & 0.00 & Lycium andersonii & 0.05 & 0.00 & 0.00 \\
\hline Oryzopsis hymenoides & 0.23 & 0.00 & 0.38 & Oryzopsis hymenoides & 0.07 & 0.00 & 0.18 \\
\hline Sitanion hystrix & 0.25 & 0.29 & 0.30 & Sitanion hystrix & 1.23 & 0.71 & 0.95 \\
\hline Sphaeralcea ambigua & 0.00 & 0.03 & 0.00 & Sphaeralcea ambigua & 0.00 & 0.00 & 0.00 \\
\hline Stipa comata & 0.00 & 0.00 & 0.48 & Stipa comata & 0.00 & 0.00 & 0.06 \\
\hline \multirow[t]{2}{*}{ Stipa speciosa } & 0.00 & 0.30 & 0.55 & Stipa speciosa & 0.00 & 0.04 & 0.20 \\
\hline & & & & Total Live & 42.21 & 57.25 & 38.17 \\
\hline
\end{tabular}


Table A-62A. Site characteristics for Plot 62.

Location:

NTS Area: 18

NTS Grid: F-37

USGS 7.5’ Quadrangle Name: Ammonia Tanks

\begin{tabular}{|c|c|c|c|c|}
\hline & & GPS Read & Zone 11S) & \\
\hline & Location & Northing & Easting & Condition of Original Corners \\
\hline Corner 1: & SW & 4110796 & 565892 & NA \\
\hline Corner 2: & NW & 4110796 & 565878 & NA \\
\hline Corner 3: & $\mathrm{NE}$ & 4110810 & $056902 ?$ & NA \\
\hline Corner 4: & SE & 4110785 & 565918 & NA \\
\hline DEM Plo & vation: 17 & & & Plot Aspect: $297^{\circ}$ \\
\hline Elevation & ze Playa ( & Name): I & & Plot Slope: $3^{\circ}$ \\
\hline
\end{tabular}

Annual Precipitation

Measured: $203 \mathrm{~mm}$

Modeled: $226.0 \mathrm{~mm}$

Parent Material Type: broken terrain

Substrate: silty sand in depression

Slate Geologic Unit: QTc

Beatley Plant Assemblage: Artemisia tridentata

Ostler-Hanson Plant Association: NA

Ostler-Hanson Land Unit: 948

Abundance of Biological Soil Crusts: none

Type and Date of Disturbance: none

Plot Condition: good, but plot is surrounded by disturbance on all 4 sides

Location of Transect 1: southwest side of plot

Direction Transects Are Read: southeast to northwest

Date(s) Plot Remeasured: 28 May 2002 
Figure A-62. Photographs showing Plot 62.

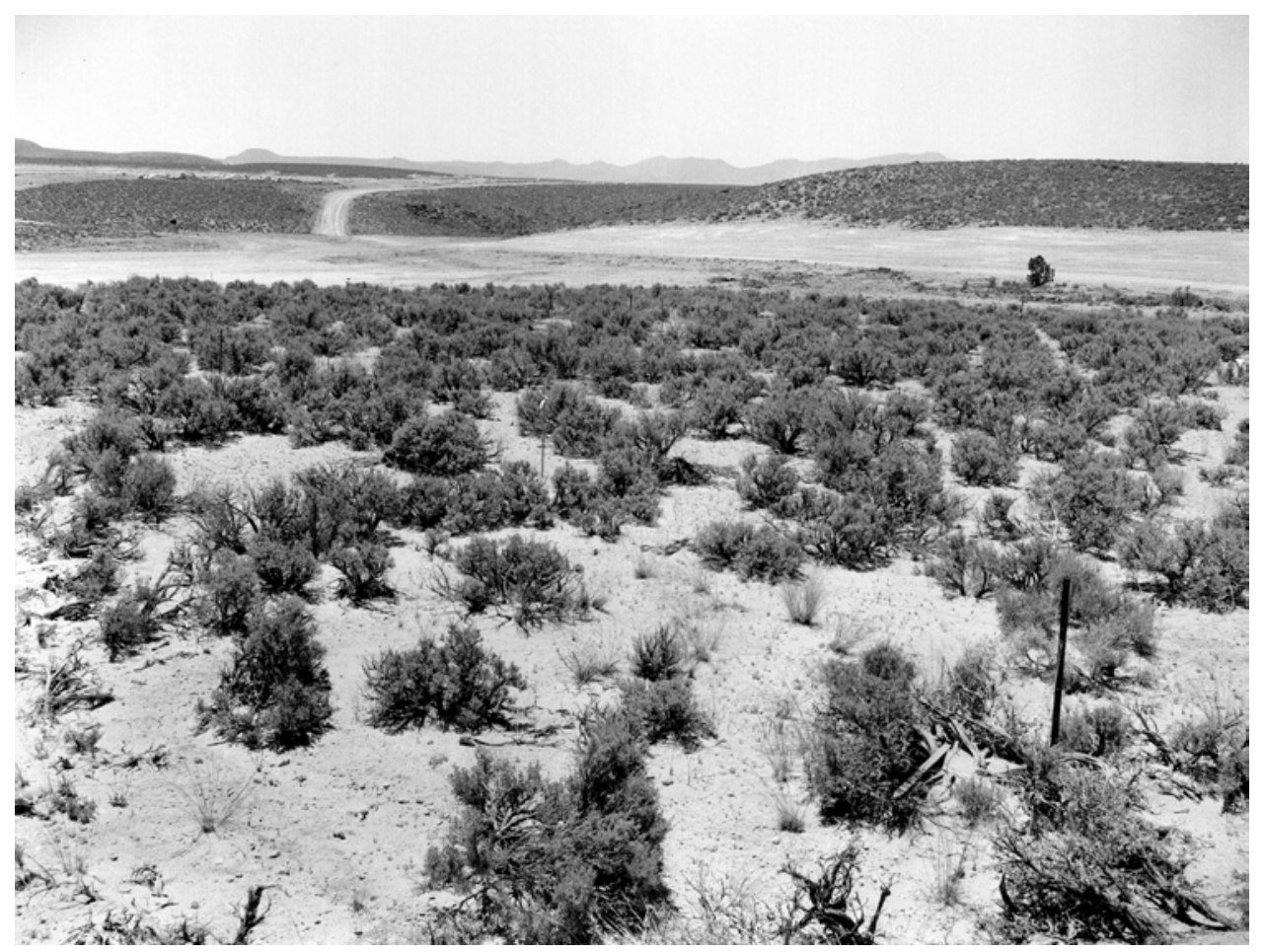

A. (Spring, 1964). This southerly view across Plot 62 shows a Great Basin sagebrush assemblage with considerable disturbance owing to road construction in the background. The mountains in the distance are south of Buckboard Mesa. The solitary tree at right midground is a Utah juniper (Juniperus osteosperma) (Janice Beatley Collection, no number).

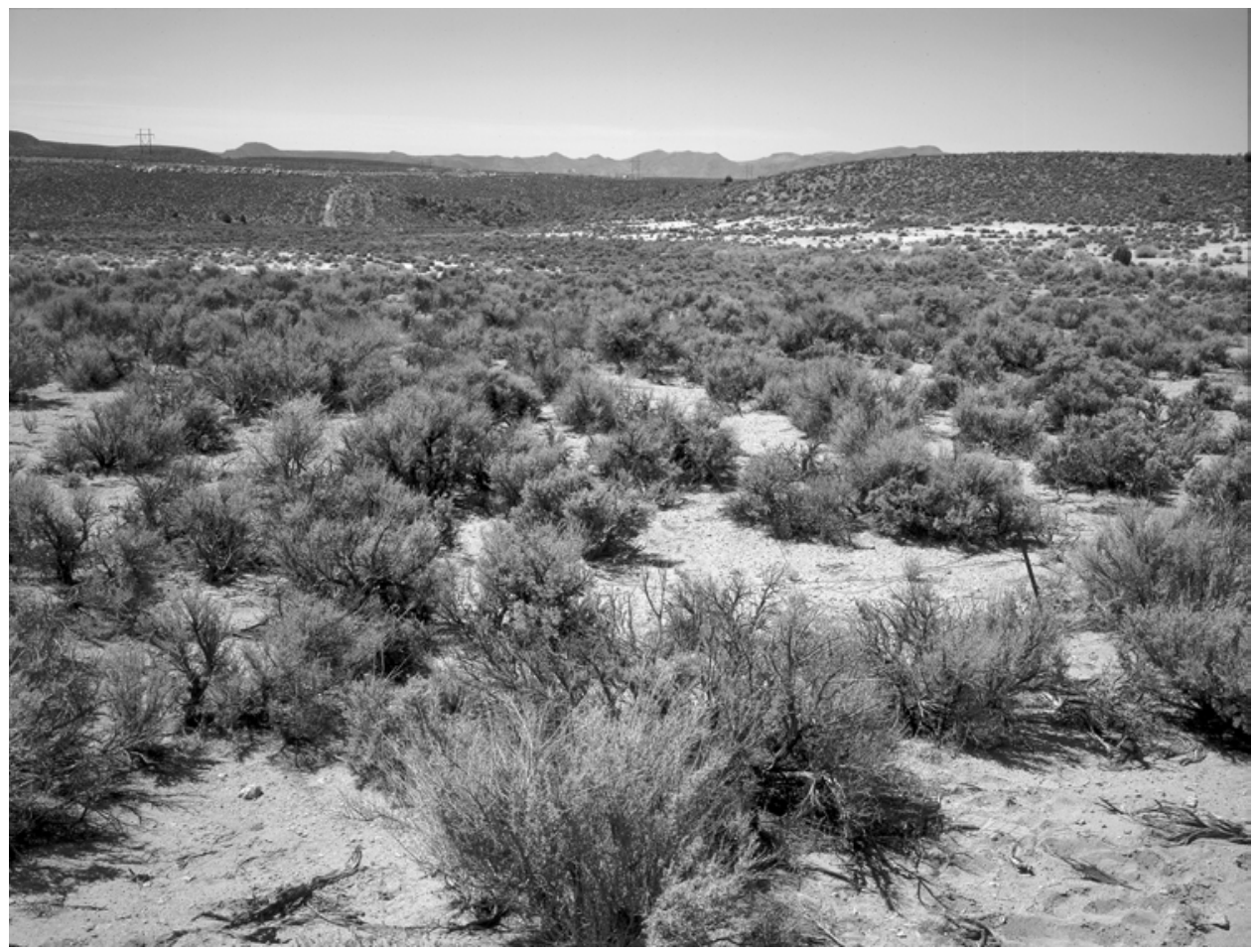

B. (May 28, 2002). Many of the Great Basin sagebrush individuals are larger now. The disturbance scars in the background have largely healed from a visual perspective. The number and size of Utah junipers on the slopes at right midground have increased, as they have regionally (Dominic Oldershaw, Stake 4196A). 
Table A-62B. Summary plant data for Plot 62.

\begin{tabular}{|c|c|c|c|c|c|c|c|}
\hline \multicolumn{5}{|c|}{ Number of Plants } & \multicolumn{3}{|c|}{ Cover (\%) } \\
\hline SPECIES & 1963 & 1975 & 2002 & SPECIES & 1963 & 1975 & 2002 \\
\hline Artemisia tridentata & 158 & 179 & 169 & Artemisia tridentata & 27.52 & 32.83 & 21.21 \\
\hline Atriplex canescens & 2 & 3 & 4 & Atriplex canescens & 0.26 & 0.36 & 0.54 \\
\hline Chrysothamnus viscidiflorus & 1 & 1 & 1 & Chrysothamnus viscidiflorus & 0.05 & 0.04 & 0.01 \\
\hline Cymopterus ripleyi & 0 & 1 & 0 & Cymopterus ripleyi & 0.00 & 0.01 & 0.00 \\
\hline Ephedra nevadensis & 1 & 2 & 2 & Ephedra nevadensis & 0.35 & 0.39 & 0.85 \\
\hline Eriogonum kearneyi & 5 & 8 & 9 & Eriogonum kearneyi & 0.43 & 0.35 & 1.01 \\
\hline Oryzopsis hymenoides & 7 & 1 & 3 & Oryzopsis hymenoides & 0.50 & 0.05 & 0.22 \\
\hline Sitanion hystrix & 8 & 4 & 6 & Sitanion hystrix & 0.52 & 0.31 & 0.35 \\
\hline Stipa comata & 27 & 5 & 3 & Stipa comata & 1.45 & 0.27 & 0.04 \\
\hline Stipa speciosa & 0 & 0 & 2 & Stipa speciosa & 0.00 & 0.00 & 0.11 \\
\hline Total Live & 209 & 204 & 199 & Total Live & 31.07 & 34.62 & 24.33 \\
\hline Dead Grass & 0 & 0 & 0 & Dead Grass & 0.00 & 0.00 & 0.00 \\
\hline Dead Shrub & 26 & 32 & 46 & Dead Shrub & 3.98 & 4.42 & 12.91 \\
\hline Total & 235 & 236 & 245 & Total & 35.05 & 39.04 & 37.24 \\
\hline \multicolumn{5}{|c|}{ Average Height (m) } & \multicolumn{3}{|c|}{ Biomass Index $\left(\mathrm{m}^{2}\right)$} \\
\hline SPECIES & 1963 & 1975 & 2002 & SPECIES & 1963 & 1975 & 2002 \\
\hline Artemisia tridentata & 0.60 & 0.65 & 0.62 & Artemisia tridentata & 61.60 & 77.07 & 50.03 \\
\hline Atriplex canescens & 0.62 & 0.64 & 0.75 & Atriplex canescens & 0.69 & 0.85 & 1.60 \\
\hline Chrysothamnus viscidiflorus & 0.18 & 0.25 & 0.49 & Chrysothamnus viscidiflorus & 0.03 & 0.03 & 0.01 \\
\hline Cymopterus ripleyi & 0.00 & 0.05 & 0.00 & Cymopterus ripleyi & 0.00 & 0.00 & 0.00 \\
\hline Ephedra nevadensis & 0.81 & 0.69 & 1.01 & Ephedra nevadensis & 0.94 & 1.07 & 2.53 \\
\hline Eriogonum kearneyi & 0.45 & 0.27 & 0.60 & Eriogonum kearneyi & 0.65 & 0.54 & 1.86 \\
\hline Oryzopsis hymenoides & 0.34 & 0.30 & 0.41 & Oryzopsis hymenoides & 0.61 & 0.06 & 0.31 \\
\hline Sitanion hystrix & 0.33 & 0.34 & 0.39 & Sitanion hystrix & 0.63 & 0.42 & 0.46 \\
\hline Stipa comata & 0.26 & 0.22 & 0.17 & Stipa comata & 1.40 & 0.22 & 0.02 \\
\hline \multirow[t]{2}{*}{ Stipa speciosa } & 0.00 & 0.00 & 0.32 & Stipa speciosa & 0.00 & 0.00 & 0.13 \\
\hline & & & & Total Live & 66.54 & 80.26 & 56.97 \\
\hline
\end{tabular}


Table A-63A. Site characteristics for Plot 63.

Location: Rainier Mesa

NTS Area: 19

NTS Grid: F-40

USGS 7.5’ Quadrangle Name: Ammonia Tanks

\begin{tabular}{lllc} 
& & \multicolumn{2}{c}{ GPS Readings (Zone 11S) } \\
& Location & Northing & Easting \\
Corner 1: & SW & 4117437 & 566326 \\
Corner 2: & NW & 4117466 & 566330 \\
Corner 3: & NE & 4117467 & 566361 \\
Corner 4: & SE & 4117436 & 566357
\end{tabular}

DEM Plot Elevation: 2000 m

Condition of Original Corners
Standing
On ground
On ground
Standing

Plot Aspect: $170^{\circ}$

Elevation Above Playa (Playa Name): NA

Plot Slope: $12^{\circ}$

Annual Precipitation

Measured: $270 \mathrm{~mm}$

Modeled: $265 \mathrm{~mm}$

Parent Material Type: alluvial fan

Substrate: old volcanic, rocky

Slate Geologic Unit: Tmr

Beatley Plant Assemblage: Artemisia nova-Pinyon-Juniper

Ostler-Hanson Plant Association: NA

Ostler-Hanson Land Unit: 1502

Abundance of Biological Soil Crusts: none

Type and Date of Disturbance: none

Plot Condition: good

Location of Transect 1: north side of plot

Direction Transects Are Read: west to east

Date(s) Plot Remeasured: 29 May 2002 
Figure A-63. Photographs showing Plot 63.

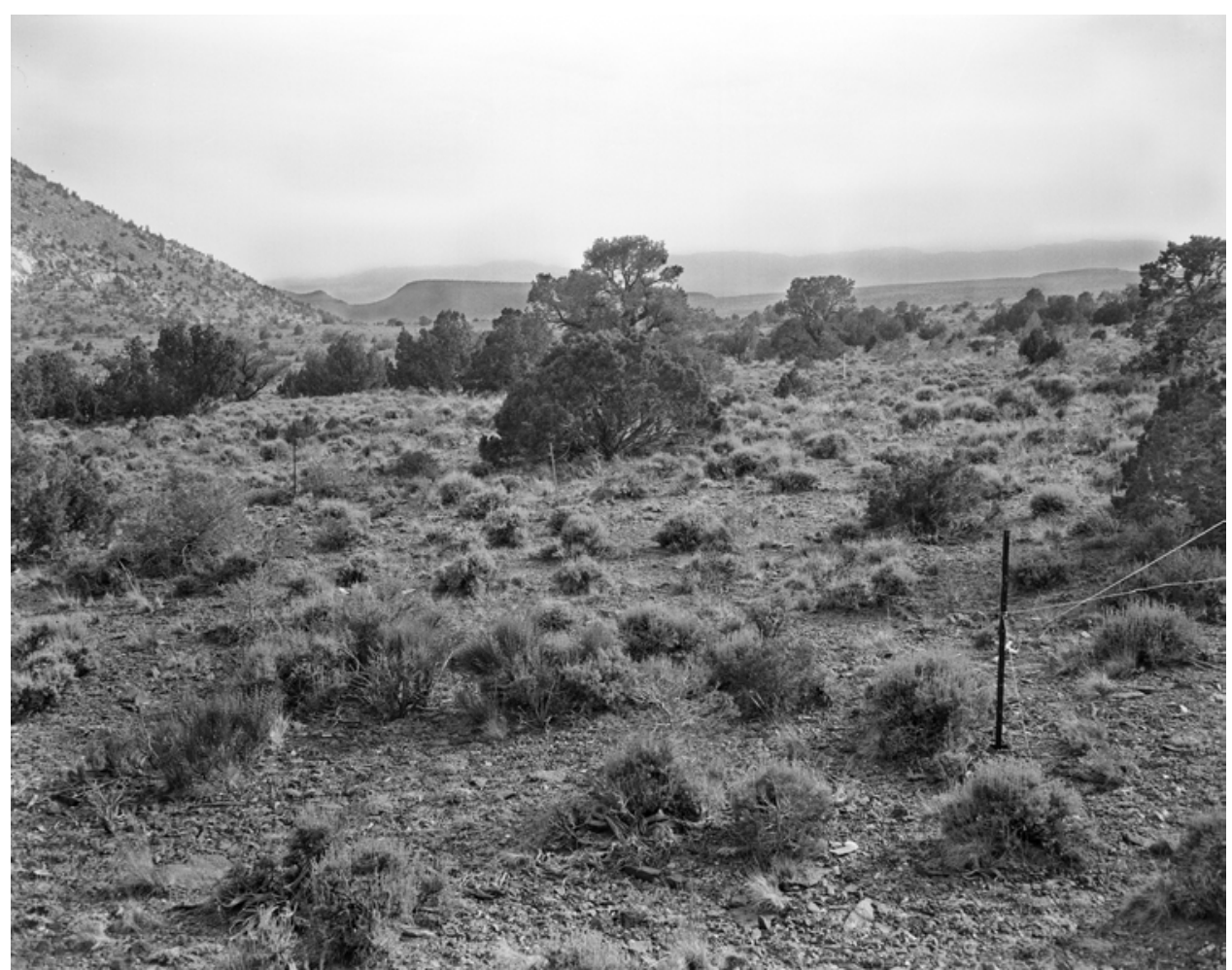

A. (1964). This southwesterly view across Plot 63 shows a pinyon-juniper assemblage with black sagebrush as the dominant shrub. Singleleaf pinyon (Pinus monophylla) is the most visually dominant tree in this view. The west flank of Rattlesnake Ridge appears at left, and Timber Mountain appears in the distance.(Janice Beatley Collection, no number).

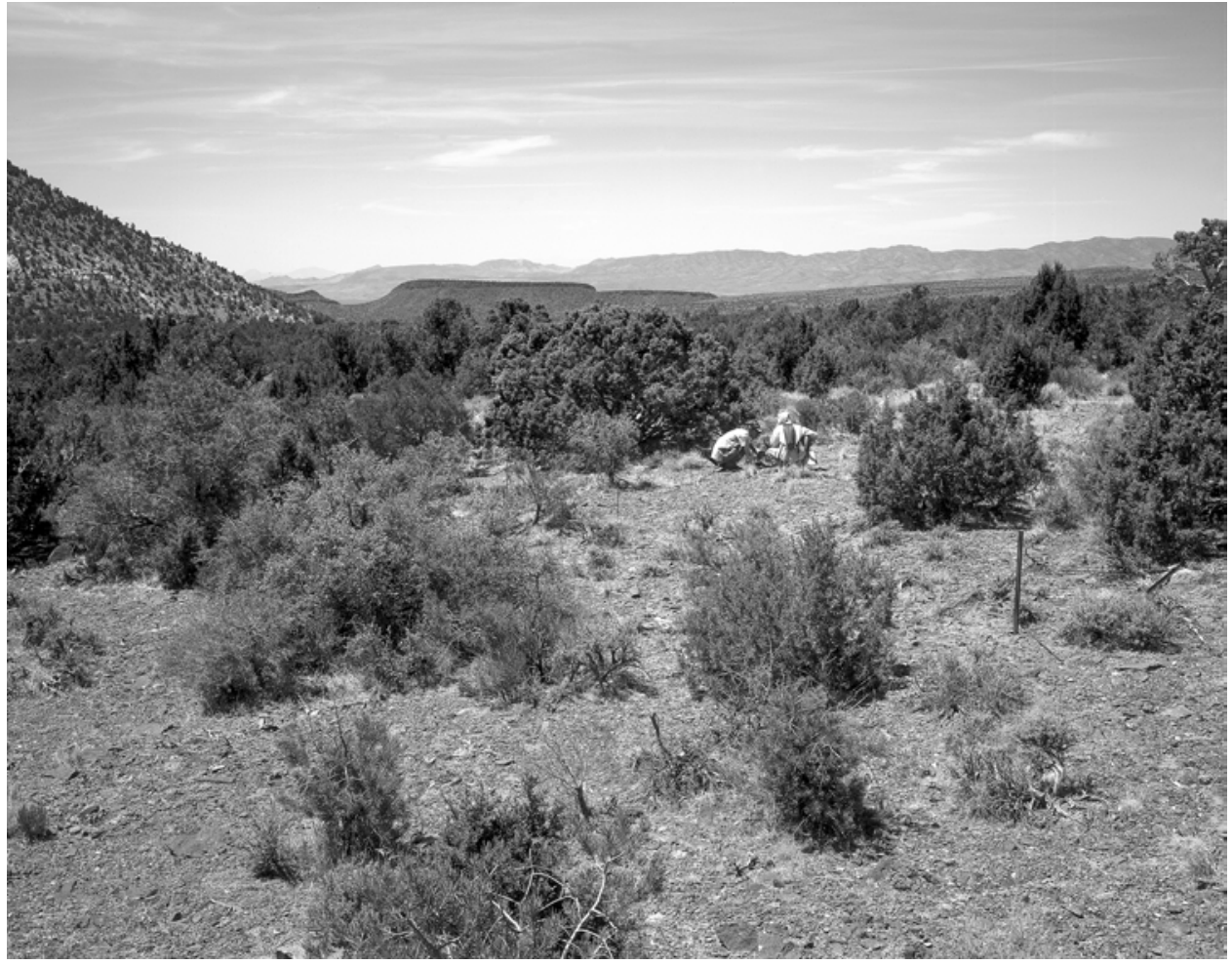

B. (May 29, 2002). The sizes of the individual black sagebrush have increased significantly. Both singleleaf pinyon and Utah juniper have increased in density and biomass (Dominic Oldershaw, Stake 3638A). 
Table A-63B. Summary plant data for Plot 63.

\section{SPECIES}

Arabis holboellii pinetorum

Artemisia nova

Astragalus purshii tinctus

Astragalus species

Brassicaceae species

Calochortus flexuosus

Chrysothamnus viscidiflorus puberulus

Crepis intermedia

Dichelostemma pulchella

Ephedra nevadensis

Ephedra viridis

Eriogonum cespitosum

Hymenoxys cooperi

Juniperus monosperma

Juniperus osteosperma

Lomatium nevadense nevadense

Oryzopsis hymenoides

Perennial grass

Phlox stansburyi

Pinus monophylla

Poa fendleriana

Poa sandbergii

Poa species

Purshia tridentata

Senecio multilobatus

Sitanion hystrix

Sphaeralcea ambigua monticola

Stipa thurberiana

Streptanthus cordatus

Unknown herbaceous perennial

Unknown shrub

Viguiera multiform nevadensis

Total Live

Dead Grass

Dead Shrub

Total Live

\section{Number of Plants}

1963

19752002

$$
0
$$

$163 \quad 191 \quad 102$

$\begin{array}{lll}0 & 5 & 0\end{array}$

$\begin{array}{lll}0 & 0 & 1\end{array}$

$\begin{array}{lll}0 & 0 & 1\end{array}$

$\begin{array}{lll}0 & 40 & 0\end{array}$

$\begin{array}{lll}0 & 18 & 3\end{array}$

$\begin{array}{lll}0 & 13 & 0\end{array}$

$\begin{array}{lll}0 & 224 & 0\end{array}$

$0 \quad 0 \quad 2$

$\begin{array}{lll}4 & 6 & 3\end{array}$

$\begin{array}{lll}437 & 238 & 48\end{array}$

$\begin{array}{lll}0 & 3 & 0\end{array}$

$\begin{array}{lll}0 & 0 & 4\end{array}$

$\begin{array}{lll}13 & 13 & 18\end{array}$

$\begin{array}{lll}0 & 17 & 0\end{array}$

$\begin{array}{lll}15 & 2 & 2\end{array}$

$\begin{array}{ccc}15 & 2 & 2 \\ 0 & 0 & 30\end{array}$

$\begin{array}{lll}0 & 32 & 0\end{array}$

$\begin{array}{lll}26 & 35 \quad 33\end{array}$

$1 \quad 6 \quad 0$

$14 \quad 27 \quad 0$

$\begin{array}{lll}0 & 0 & 29\end{array}$

$2 \quad 3 \quad 4$

$\begin{array}{lll}2 & 3 & 4 \\ 0 & 1 & 0\end{array}$

$\begin{array}{ccc}0 & 1 & 0 \\ 51 & 103 & 31\end{array}$

$\begin{array}{lll}0 & 1 & 0\end{array}$

$119 \quad 109 \quad 35$

$\begin{array}{lll}0 & 15 & 0\end{array}$

$\begin{array}{lll}0 & 0 & 7\end{array}$

$\begin{array}{lll}0 & 0 & 1\end{array}$

$0 \quad 33 \quad 0$

$561 \quad 1142 \quad 354$

$1 \quad 0 \quad 14$

$13 \quad 13 \quad 64$

$575 \quad 1155 \quad 432$

\section{SPECIES}

Arabis holboellii pinetorum

Artemisia nova

Astragalus purshii tinctus

Astragalus species

Brassicaceae species

Calochortus flexuosus

Chrysothamnus viscidiflorus puberulus

Crepis intermedia

Dichelostemma pulchella

Ephedra nevadensis

Ephedra viridis

Eriogonum cespitosum

Hymenoxys cooperi

Juniperus monosperma

Juniperus osteosperma

Lomatium nevadense nevadense

Oryzopsis hymenoides

Perennial grass

Phlox stansburyi

Pinus monophylla

Poa fendleriana

Poa sandbergii

Poa species

Purshia tridentata

Senecio multilobatus

Sitanion hystrix

Sphaeralcea ambigua monticola

Stipa thurberiana

Streptanthus cordatus

Unknown herbaceous perennial

Unknown shrub

Viguiera multiform nevadensis
Average Height (m)

$1963 \quad 19752002$

$\begin{array}{lll}0.00 & 0.29 & 0.00\end{array}$

$\begin{array}{lll}0.36 & 0.33 & 0.30\end{array}$

$\begin{array}{lll}0.00 & 0.04 & 0.00\end{array}$

$\begin{array}{lll}0.00 & 0.00 & 0.04\end{array}$

$\begin{array}{lll}0.00 & 0.00 & 0.04\end{array}$

$\begin{array}{lll}0.00 & 0.10 & 0.00\end{array}$

$\begin{array}{lll}0.27 & 0.25 & 0.26\end{array}$

$\begin{array}{lll}0.00 & 0.09 & 0.00\end{array}$

$\begin{array}{lll}0.00 & 0.08 & 0.00\end{array}$

$\begin{array}{lll}0.00 & 0.00 & 0.47\end{array}$

$\begin{array}{lll}0.60 & 0.52 & 0.40\end{array}$

$\begin{array}{lll}0.05 & 0.06 & 0.04\end{array}$

$\begin{array}{lll}0.00 & 0.07 & 0.00\end{array}$

$\begin{array}{lll}0.00 & 0.00 & 1.08\end{array}$

$\begin{array}{lll}1.23 & 1.41 & 1.94\end{array}$

$\begin{array}{lll}0.00 & 0.08 & 0.00\end{array}$

$\begin{array}{lll}0.29 & 0.23 \quad 0.30\end{array}$

$\begin{array}{lll}0.00 & 0.00 & 0.15\end{array}$

$\begin{array}{lll}0.00 & 0.13 & 0.00\end{array}$

$\begin{array}{lll}1.12 & 1.04 & 1.02\end{array}$

$\begin{array}{lll}0.08 & 0.34 & 0.00\end{array}$

$\begin{array}{lll}0.09 & 0.28 & 0.00\end{array}$

$\begin{array}{lll}0.00 & 0.00 & 0.27\end{array}$

$\begin{array}{lll}0.47 & 0.51 \quad 0.62\end{array}$

$\begin{array}{lll}0.00 & 0.03 & 0.00\end{array}$

$\begin{array}{lll}0.24 & 0.25 & 0.24\end{array}$

$\begin{array}{lll}0.00 & 0.08 & 0.00\end{array}$

$\begin{array}{lll}0.20 & 0.23 & 0.32\end{array}$

$\begin{array}{lll}0.00 & 0.21 \quad 0.00\end{array}$

$\begin{array}{lll}0.00 & 0.00 & 0.07\end{array}$

$\begin{array}{lll}0.00 & 0.00 & 0.21\end{array}$

$\begin{array}{lll}0.00 & 0.05 & 0.00\end{array}$ 
Table A-63B (continued). Summary plant data for Plot 63.

\begin{tabular}{|c|c|c|c|c|c|c|c|}
\hline \multicolumn{5}{|c|}{ Cover (\%) } & \multicolumn{3}{|c|}{ Biomass Index $\left(\mathrm{m}^{2}\right)$} \\
\hline SPECIES & 1963 & 1975 & 2002 & SPECIES & 1963 & 1975 & 2002 \\
\hline Arabis holboellii pinetorum & 0.00 & 0.12 & 0.00 & Arabis holboellii pinetorum & 0.00 & 0.11 & 0.00 \\
\hline Artemisia nova & 18.22 & 20.40 & 6.18 & Artemisia nova & 24.27 & 26.03 & 6.99 \\
\hline Astragalus purshii tinctus & 0.00 & 0.08 & 0.00 & Astragalus purshii tinctus & 0.00 & 0.01 & 0.00 \\
\hline Astragalus species & 0.00 & 0.00 & 0.01 & Astragalus species & 0.00 & 0.00 & 0.00 \\
\hline Brassicaceae species & 0.00 & 0.00 & 0.01 & Brassicaceae species & 0.00 & 0.00 & 0.00 \\
\hline Calochortus flexuosus & 0.00 & 0.45 & 0.00 & Calochortus flexuosus & 0.00 & 0.16 & 0.00 \\
\hline Chrysothamnus viscidiflorus puberulus & 1.06 & 1.09 & 0.22 & Chrysothamnus viscidiflorus puberulus & 0.99 & 1.05 & 0.25 \\
\hline Crepis intermedia & 0.00 & 0.22 & 0.00 & Crepis intermedia & 0.00 & 0.07 & 0.00 \\
\hline Dichelostemma pulchella & 0.00 & 1.88 & 0.00 & Dichelostemma pulchella & 0.00 & 0.54 & 0.00 \\
\hline Ephedra nevadensis & 0.00 & 0.00 & 0.13 & Ephedra nevadensis & 0.00 & 0.00 & 0.20 \\
\hline Ephedra viridis & 0.78 & 0.59 & 0.34 & Ephedra viridis & 2.01 & 1.43 & 0.45 \\
\hline Eriogonum cespitosum & 3.16 & 4.92 & 1.08 & Eriogonum cespitosum & 0.59 & 1.28 & 0.13 \\
\hline Hymenoxys cooperi & 0.00 & 0.03 & 0.00 & Hymenoxys cooperi & 0.00 & 0.01 & 0.00 \\
\hline Juniperus monosperma & 0.00 & 0.00 & 0.82 & Juniperus monosperma & 0.00 & 0.00 & 3.60 \\
\hline Juniperus osteosperma & 4.39 & 5.17 & 7.98 & Juniperus osteosperma & 30.19 & 36.02 & 62.15 \\
\hline Lomatium nevadense nevadense & 0.00 & 0.22 & 0.00 & Lomatium nevadense nevadense & 0.00 & 0.06 & 0.00 \\
\hline Oryzopsis hymenoides & 0.77 & 0.07 & 0.07 & Oryzopsis hymenoides & 0.81 & 0.06 & 0.07 \\
\hline Perennial grass & 0.00 & 0.00 & 0.67 & Perennial grass & 0.00 & 0.00 & 0.39 \\
\hline Phlox stansburyi & 0.00 & 0.60 & 0.00 & Phlox stansburyi & 0.00 & 0.33 & 0.00 \\
\hline Pinus monophylla & 5.18 & 7.82 & 6.27 & Pinus monophylla & 48.45 & 57.20 & 24.70 \\
\hline Poa fendleriana & 0.02 & 0.19 & 0.00 & Poa fendleriana & 0.00 & 0.21 & 0.00 \\
\hline Poa sandbergii & 0.41 & 0.71 & 0.00 & Poa sandbergii & 0.14 & 0.71 & 0.00 \\
\hline Poa species & 0.00 & 0.00 & 0.88 & Poa species & 0.00 & 0.00 & 0.98 \\
\hline Purshia tridentata & 0.55 & 0.82 & 0.85 & Purshia tridentata & 0.86 & 1.51 & 2.03 \\
\hline Senecio multilobatus & 0.00 & 0.01 & 0.00 & Senecio multilobatus & 0.00 & 0.00 & 0.00 \\
\hline Sitanion hystrix & 2.45 & 4.44 & 2.34 & Sitanion hystrix & 2.11 & 3.85 & 2.23 \\
\hline Sphaeralcea ambigua monticola & 0.00 & 0.02 & 0.00 & Sphaeralcea ambigua monticola & 0.00 & 0.00 & 0.00 \\
\hline Stipa thurberiana & 5.33 & 4.35 & 1.21 & Stipa thurberiana & 3.87 & 3.66 & 1.39 \\
\hline Streptanthus cordatus & 0.00 & 0.26 & 0.00 & Streptanthus cordatus & 0.00 & 0.22 & 0.00 \\
\hline Unknown herbaceous perennial & 0.00 & 0.00 & 0.13 & Unknown herbaceous perennial & 0.00 & 0.00 & 0.04 \\
\hline Unknown shrub & 0.00 & 0.00 & 0.02 & Unknown shrub & 0.00 & 0.00 & 0.01 \\
\hline Viguiera multiform nevadensis & 0.00 & 0.48 & 0.00 & Viguiera multiform nevadensis & 0.00 & 0.10 & 0.00 \\
\hline Total Live & 42.33 & 54.94 & 29.21 & Total Live & 114.30 & 134.64 & 105.61 \\
\hline Dead Grass & 0.40 & 0.00 & 0.48 & & & & \\
\hline Dead Shrub & 10.10 & 1.84 & 6.45 & & & & \\
\hline Total Live & 52.83 & 56.77 & 36.14 & & & & \\
\hline
\end{tabular}


Table A-64A. Site characteristics for Plot 64.

Location: Rainier Mesa

NTS Area: 12

NTS Grid: I-40

USGS 7.5’ Quadrangle Name: Rainier Mesa

\begin{tabular}{lllll} 
& & \multicolumn{2}{l}{ GPS Readings (Zone 11S) } \\
& Location & Northing & Easting & Condition of Original Corners \\
Corner 1: & SW & 4117113 & 570052 & On ground \\
Corner 2: & NW & 4117144 & 570051 & Standing \\
Corner 3: & NE & NA & NA & Missing \\
Corner 4: & SE & NA & NA & On ground
\end{tabular}

DEM Plot Elevation: 2252 m

Plot Aspect: $83^{\circ}$

Elevation Above Playa (Playa Name): NA

Plot Slope: $22^{\circ}$

Annual Precipitation

Measured: $270 \mathrm{~mm}$

Modeled: $321 \mathrm{~mm}$

Parent Material Type: mesa

Substrate: NA

Slate Geologic Unit: Tmr

Beatley Plant Assemblage: Artemisia nova-Pinyon-Juniper

Ostler-Hanson Plant Association: NA

Ostler-Hanson Land Unit: 860

Abundance of Biological Soil Crusts: NA

Type and Date of Disturbance: none

Plot Condition: road was constructed through southeast corner of plot between 1975 and 2000

Location of Transect 1: north side of plot

Direction Transects Are Read: east to west

Date(s) Plot Remeasured: 30 May 2002 
Figure A-64. Photograph showing Plot 64.

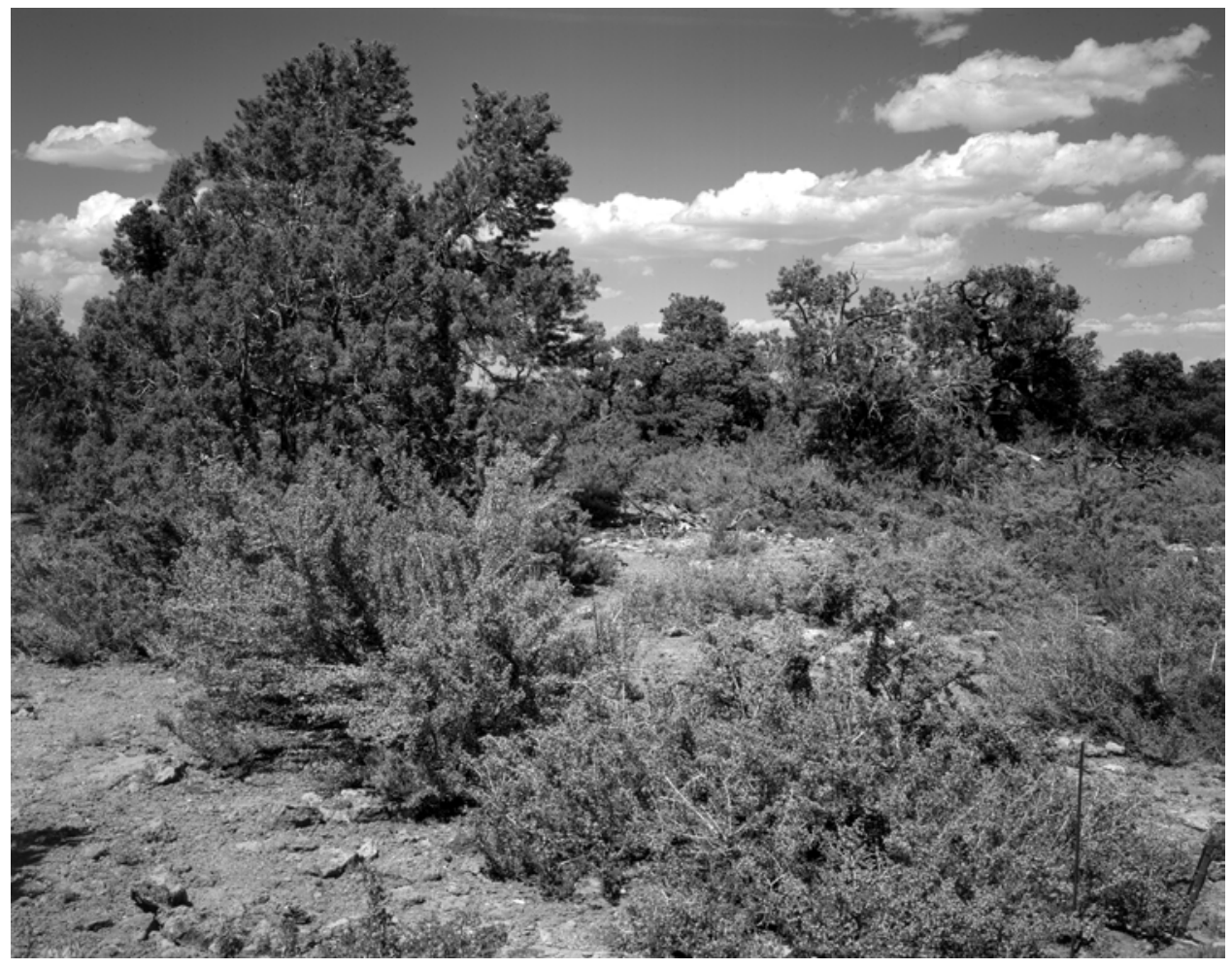

(June 7, 2003). This northeasterly view across Plot 64 shows the pinyon-juniper community that covers the top of Rainier Mesa. The foreground shrubs are bitterbrush (Purshia tridentata) (Dustin Haines and Todd Esque, Stake 4660). 
Table A-64B. Summary plant data for Plot 64.

\begin{tabular}{|c|c|c|c|c|c|c|c|}
\hline \multirow[b]{2}{*}{ SPECIES } & \multicolumn{3}{|c|}{ Number of Plants } & \multirow[b]{2}{*}{ SPECIES } & \multicolumn{3}{|c|}{ Average Height (m) } \\
\hline & 1964 & 1974 & 2002 & & 1964 & 1974 & 2002 \\
\hline Agoseris glauca & 3 & 0 & 0 & Agoseris glauca & 0.04 & 0.00 & 0.00 \\
\hline Antennaria dimorpha & 1 & 0 & 0 & Antennaria dimorpha & 0.03 & 0.00 & 0.00 \\
\hline Arenaria congesta subcongesta & 21 & 4 & 0 & Arenaria congesta subcongesta & 0.11 & 0.06 & 0.00 \\
\hline Artemisia nova & 59 & 75 & 83 & Artemisia nova & 0.42 & 0.49 & 0.36 \\
\hline Astragalus lentiginosus fremontii & 0 & 0 & 1 & Astragalus lentiginosus fremontii & 0.00 & 0.00 & 0.13 \\
\hline Chrysothamnus viscidiflorus & 2 & 4 & 10 & Chrysothamnus viscidiflorus & 0.33 & 0.23 & 0.19 \\
\hline Crepis intermedia & 1 & 0 & 0 & Crepis intermedia & 0.15 & 0.00 & 0.00 \\
\hline Cryptantha flavoculata & 0 & 1 & 0 & Cryptantha flavoculata & 0.00 & 0.05 & 0.00 \\
\hline Ephedra nevadensis & 0 & 0 & 1 & Ephedra nevadensis & 0.00 & 0.00 & 0.72 \\
\hline Ephedra viridis & 1 & 0 & 0 & Ephedra viridis & 0.18 & 0.00 & 0.00 \\
\hline Ericameria nana & 12 & 9 & 3 & Ericameria nana & 0.27 & 0.23 & 0.18 \\
\hline Eriogonum cespitosum & 2 & 3 & 0 & Eriogonum cespitosum & 0.08 & 0.04 & 0.00 \\
\hline Eriogonum microthecum lapidicola & 29 & 32 & 0 & Eriogonum microthecum lapidicola & 0.05 & 0.06 & 0.00 \\
\hline Eriogonum species & 0 & 0 & 10 & Eriogonum species & 0.00 & 0.00 & 0.05 \\
\hline Eriogonum umbellatum subaridum & 5 & 5 & 6 & Eriogonum umbellatum subaridum & 0.07 & 0.16 & 0.07 \\
\hline Hymenoxys cooperi & 0 & 11 & 0 & Hymenoxys cooperi & 0.00 & 0.03 & 0.00 \\
\hline Ipomopsis aggregata & 0 & 0 & 1 & Ipomopsis aggregata & 0.00 & 0.00 & 0.02 \\
\hline Ipomopsis congesta & 0 & 2 & 0 & Ipomopsis congesta & 0.00 & 0.08 & 0.00 \\
\hline Juniperus osteosperma & 1 & 1 & 1 & Juniperus osteosperma & 3.30 & 3.66 & 4.18 \\
\hline Koeleria cristata & 2 & 0 & 0 & Koeleria cristata & 0.28 & 0.00 & 0.00 \\
\hline Lesquerella kingii kingii & 4 & 0 & 2 & Lesquerella kingii kingii & 0.06 & 0.00 & 0.05 \\
\hline Linanthus nuttallii & 0 & 10 & 16 & Linanthus nuttallii & 0.00 & 0.15 & 0.09 \\
\hline $\begin{array}{l}\text { Lomatium foeniculaceum } \\
\text { fimbriatum }\end{array}$ & 2 & 0 & 0 & $\begin{array}{l}\text { Lomatium foeniculaceum } \\
\text { fimbriatum }\end{array}$ & 0.06 & 0.00 & 0.00 \\
\hline Machaeranthera canescens & 0 & 1 & 0 & Machaeranthera canescens & 0.00 & 0.03 & 0.00 \\
\hline Opuntia erinacea erinacea & 15 & 15 & 9 & Opuntia erinacea erinacea & 0.19 & 0.17 & 0.13 \\
\hline Oryzopsis hymenoides & 1 & 0 & 2 & Oryzopsis hymenoides & 0.33 & 0.00 & 0.26 \\
\hline Perennial grass & 0 & 70 & 46 & Perennial grass & 0.00 & 0.19 & 0.16 \\
\hline Petradoria pumila & 3 & 3 & 0 & Petradoria pumila & 0.19 & 0.15 & 0.00 \\
\hline Phlox stansburyi & 6 & 0 & 0 & Phlox stansburyi & 0.09 & 0.00 & 0.00 \\
\hline Pinus monophylla & 31 & 35 & 32 & Pinus monophylla & 2.76 & 2.74 & 2.61 \\
\hline Poa sandbergii & 70 & 0 & 0 & Poa sandbergii & 0.27 & 0.00 & 0.00 \\
\hline Purshia tridentata & 20 & 27 & 98 & Purshia tridentata & 0.52 & 0.50 & 0.66 \\
\hline Quercus gambelii & 11 & 12 & 3 & Quercus gambelii & 1.09 & 1.16 & 0.84 \\
\hline Ribes velutinum velutinum & 3 & 3 & 6 & Ribes velutinum velutinum & 1.10 & 1.05 & 0.53 \\
\hline Sitanion hystrix & 48 & 0 & 1 & Sitanion hystrix & 0.24 & 0.00 & 0.28 \\
\hline Stipa pinetorum & 65 & 0 & 4 & Stipa pinetorum & 0.22 & 0.00 & 0.26 \\
\hline Total Live & 418 & 323 & 335 & & & & \\
\hline Dead Grass & 0 & 1 & 0 & & & & \\
\hline Dead Shrub & 21 & 10 & 15 & & & & \\
\hline Total & 439 & 334 & 350 & & & & \\
\hline
\end{tabular}


Table A-64B (continued). Summary plant data for Plot 64.

\begin{tabular}{|c|c|c|c|c|c|c|c|}
\hline \multirow[b]{2}{*}{ SPECIES } & \multicolumn{3}{|c|}{ Cover (\%) } & \multirow[b]{2}{*}{ SPECIES } & \multicolumn{3}{|c|}{ Biomass Index $\left(\mathrm{m}^{2}\right)$} \\
\hline & 1964 & 1974 & 2002 & & 1964 & 1974 & 2002 \\
\hline Agoseris glauca & 0.12 & 0.00 & 0.00 & Agoseris glauca & 0.02 & 0.00 & 0.00 \\
\hline Antennaria dimorpha & 0.02 & 0.00 & 0.00 & Antennaria dimorpha & 0.00 & 0.00 & 0.00 \\
\hline Arenaria congesta subcongesta & 0.44 & 0.08 & 0.00 & Arenaria congesta subcongesta & 0.21 & 0.02 & 0.00 \\
\hline Artemisia nova & 7.16 & 9.65 & 8.55 & Artemisia nova & 11.40 & 17.73 & 12.61 \\
\hline Astragalus lentiginosus fremontii & 0.00 & 0.00 & 0.09 & Astragalus lentiginosus fremontii & 0.00 & 0.00 & 0.04 \\
\hline Chrysothamnus viscidiflorus & 0.12 & 0.31 & 0.49 & Chrysothamnus viscidiflorus & 0.13 & 0.36 & 0.50 \\
\hline Crepis intermedia & 0.05 & 0.00 & 0.00 & Crepis intermedia & 0.03 & 0.00 & 0.00 \\
\hline Cryptantha flavoculata & 0.00 & 0.02 & 0.00 & Cryptantha flavoculata & 0.00 & 0.00 & 0.00 \\
\hline Ephedra nevadensis & 0.00 & 0.00 & 0.05 & Ephedra nevadensis & 0.00 & 0.00 & 0.13 \\
\hline Ephedra viridis & 0.01 & 0.00 & 0.00 & Ephedra viridis & 0.01 & 0.00 & 0.00 \\
\hline Ericameria nana & 0.97 & 0.68 & 0.07 & Ericameria nana & 0.99 & 0.64 & 0.05 \\
\hline Eriogonum cespitosum & 0.06 & 0.05 & 0.00 & Eriogonum cespitosum & 0.02 & 0.01 & 0.00 \\
\hline Eriogonum microthecum lapidicola & 0.56 & 0.66 & 0.00 & Eriogonum microthecum lapidicola & 0.11 & 0.15 & 0.00 \\
\hline Eriogonum species & 0.00 & 0.00 & 0.04 & Eriogonum species & 0.00 & 0.00 & 0.01 \\
\hline Eriogonum umbellatum subaridum & 0.13 & 0.17 & 0.12 & Eriogonum umbellatum subaridum & 0.04 & 0.13 & 0.03 \\
\hline Hymenoxys cooperi & 0.00 & 0.11 & 0.00 & Hymenoxys cooperi & 0.00 & 0.01 & 0.00 \\
\hline Ipomopsis aggregata & 0.00 & 0.00 & 0.01 & Ipomopsis aggregata & 0.00 & 0.00 & 0.00 \\
\hline Ipomopsis congesta & 0.00 & 0.02 & 0.00 & Ipomopsis congesta & 0.00 & 0.00 & 0.00 \\
\hline Juniperus osteosperma & 0.38 & 0.43 & 0.46 & Juniperus osteosperma & 4.23 & 5.24 & 6.50 \\
\hline Koeleria cristata & 0.05 & 0.00 & 0.00 & Koeleria cristata & 0.04 & 0.00 & 0.00 \\
\hline Lesquerella kingii kingii & 0.07 & 0.00 & 0.02 & Lesquerella kingii kingii & 0.01 & 0.00 & 0.00 \\
\hline Linanthus nuttallii & 0.00 & 0.38 & 0.43 & Linanthus nuttallii & 0.00 & 0.22 & 0.16 \\
\hline $\begin{array}{l}\text { Lomatium foeniculaceum } \\
\text { fimbriatum }\end{array}$ & 0.02 & 0.00 & 0.00 & $\begin{array}{l}\text { Lomatium foeniculaceum } \\
\text { fimbriatum }\end{array}$ & 0.00 & 0.00 & 0.00 \\
\hline Machaeranthera canescens & 0.00 & 0.02 & 0.00 & Machaeranthera canescens & 0.00 & 0.00 & 0.00 \\
\hline Opuntia erinacea erinacea & 1.40 & 1.13 & 0.39 & Opuntia erinacea erinacea & 0.89 & 0.70 & 0.17 \\
\hline Oryzopsis hymenoides & 0.14 & 0.00 & 0.15 & Oryzopsis hymenoides & 0.15 & 0.00 & 0.13 \\
\hline Perennial grass & 0.00 & 2.52 & 1.29 & Perennial grass & 0.00 & 1.80 & 0.74 \\
\hline Petradoria pumila & 0.07 & 0.07 & 0.00 & Petradoria pumila & 0.05 & 0.04 & 0.00 \\
\hline Phlox stansburyi & 0.08 & 0.00 & 0.00 & Phlox stansburyi & 0.03 & 0.00 & 0.00 \\
\hline Pinus monophylla & 14.72 & 16.31 & 15.84 & Pinus monophylla & 160.03 & 187.30 & 168.69 \\
\hline Poa sandbergii & 2.35 & 0.00 & 0.00 & Poa sandbergii & 2.35 & 0.00 & 0.00 \\
\hline Purshia tridentata & 2.18 & 3.28 & 21.25 & Purshia tridentata & 5.14 & 6.38 & 62.06 \\
\hline Quercus gambelii & 2.78 & 1.50 & 0.33 & Quercus gambelii & 11.28 & 6.04 & 1.19 \\
\hline Ribes velutinum velutinum & 0.81 & 0.30 & 0.91 & Ribes velutinum velutinum & 3.39 & 1.21 & 2.29 \\
\hline Sitanion hystrix & 1.96 & 0.00 & 0.04 & Sitanion hystrix & 1.68 & 0.00 & 0.03 \\
\hline Stipa pinetorum & 2.85 & 0.00 & 0.11 & Stipa pinetorum & 2.33 & 0.00 & 0.09 \\
\hline Total Live & 39.51 & 37.69 & 50.64 & Total Live & 204.56 & 227.99 & 255.44 \\
\hline Dead Grass & 0.00 & 0.03 & 0.00 & & & & \\
\hline Dead Shrub & 3.54 & 1.69 & 4.01 & & & & \\
\hline Total & 43.05 & 39.41 & 54.65 & & & & \\
\hline
\end{tabular}


Table A-65A. Site characteristics for Plot 65.

Location: Rainier Mesa

NTS Area: 18

NTS Grid: G-34

USGS 7.5’ Quadrangle Name: Tippipah Spring

\begin{tabular}{lllll} 
& & \multicolumn{2}{l}{ GPS Readings (Zone 11S) } & \\
& Location & Northing & Easting & Condition of Original Corners \\
Corner 1: & SW & 4106120 & 566683 & Standing \\
Corner 2: & NW & 4106151 & 566680 & Standing \\
Corner 3: & NE & 4106154 & 566710 & Standing \\
Corner 4: & SE & 4106123 & 5660714 & Standing
\end{tabular}

DEM Plot Elevation: 1676 m

Plot Aspect: $123^{\circ}$

Elevation Above Playa (Playa Name): NA

Plot Slope: $35^{\circ}$

Annual Precipitation

Measured: $182 \mathrm{~mm}$

Modeled: $213 \mathrm{~mm}$

Parent Material Type: alluvial fan

Substrate: very sandy w/few pebbles

Slate Geologic Unit: Tgc

Beatley Plant Assemblage: Atriplex canescens

Ostler-Hanson Plant Association: NA

Ostler-Hanson Land Unit: 943

Abundance of Biological Soil Crusts: none

Type and Date of Disturbance: none

Plot Condition: good, but feral horses use this area

Location of Transect 1: south side of plot

Direction Transects Are Read: east to west

Date(s) Plot Remeasured: 27 May 2002 
Figure A-65. Photographs showing Plot 65.

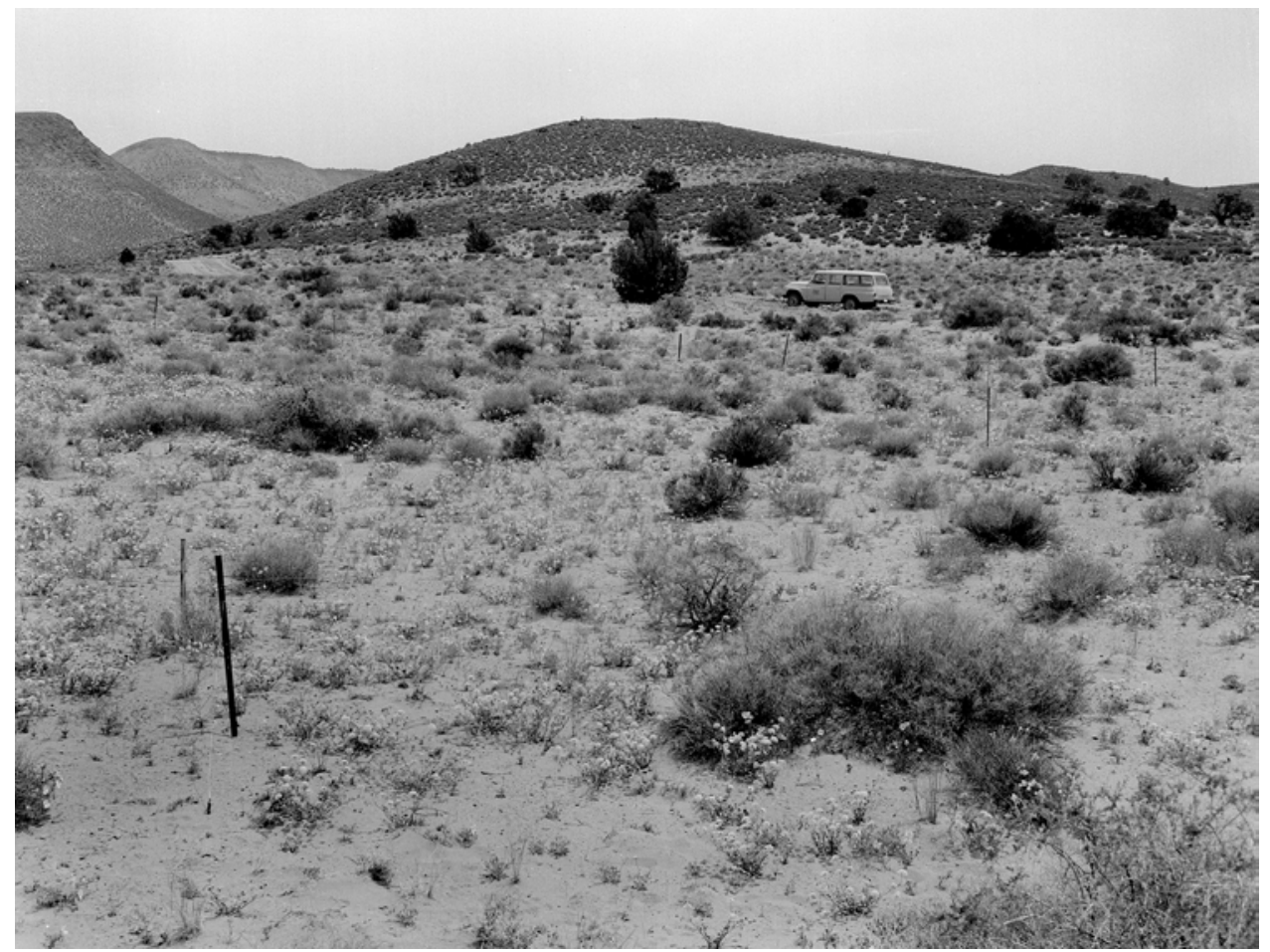

A. (1964). In this east-northeasterly view across Plot 2, unnamed hills of the Eleana Range and Big Butte (left rear) appear in the background. Four-wing saltbush is the dominant shrub, Indian rice grass is the dominant grass, and pale evening primrose (Oenothera pallida) is a significant herbaceous perennial. Utah junipers appear in the midground. This plot lies in an unusually sandy place that results from the weathering of volcanic ash (Janice Beatley Collection, no number).

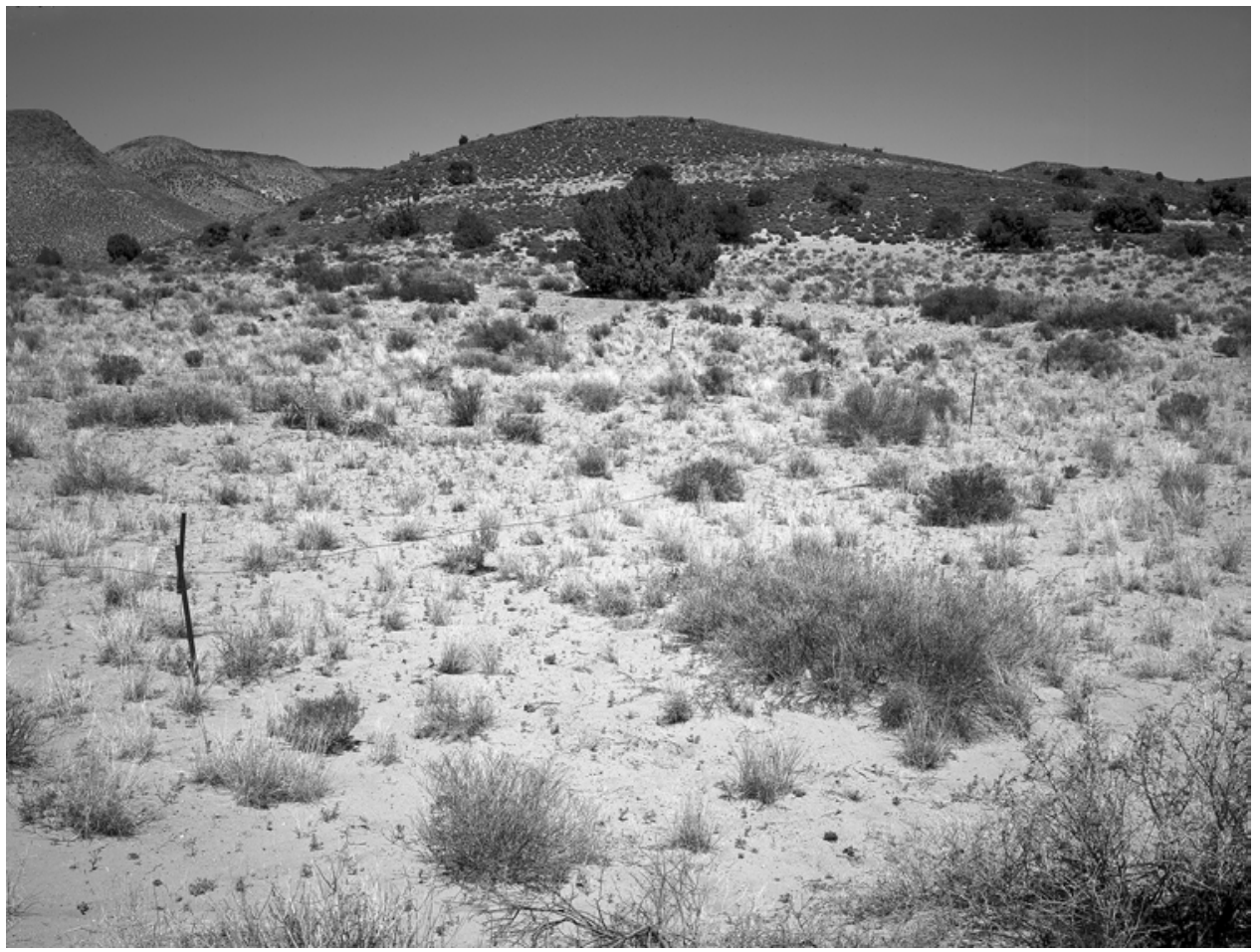

B. (May 27, 2002). Many of the same individual shrubs persist but appear to be slightly larger now. Owing to drought at the time of measurement, the pale evening primrose individuals were mostly small with few in bloom. The number of Utah junipers is about the same, but most of the individuals are much larger now (Dominic Oldershaw, Stake 4230B). 
Table A-65B. Summary plant data for Plot 65.

\begin{tabular}{|c|c|c|c|}
\hline \multirow[b]{2}{*}{ SPECIES } & \multicolumn{3}{|c|}{ 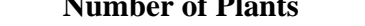 } \\
\hline & 1963 & 1975 & 2002 \\
\hline Atriplex canescens & 3 & 4 & 0 \\
\hline Chrysothamnus viscidiflorus & 15 & 8 & 14 \\
\hline Dalea searlsiae & 0 & 5 & 0 \\
\hline Ephedra nevadensis & 0 & 0 & 1 \\
\hline Ephedra viridis & 2 & 2 & 0 \\
\hline Eriogonum kearneyi & 58 & 62 & 44 \\
\hline Fabaceae species & 0 & 0 & 1 \\
\hline Oenothera pallida & 0 & 5 & 229 \\
\hline Oryzopsis hymenoides & 77 & 420 & 215 \\
\hline Psorothamnus polydenius & 15 & 9 & 12 \\
\hline Stipa comata & 0 & 0 & 10 \\
\hline Unknown herbaceous perennial & 0 & 0 & 2 \\
\hline Total Live & 170 & 515 & 528 \\
\hline Dead grass & 0 & 12 & 25 \\
\hline Dead shrub & 4 & 8 & 36 \\
\hline \multirow[t]{2}{*}{ Total } & 174 & 535 & 589 \\
\hline & \multicolumn{3}{|c|}{ Average Height (m) } \\
\hline SPECIES & 1963 & 1975 & 2002 \\
\hline Atriplex canescens & 0.73 & 0.84 & 0.00 \\
\hline Chrysothamnus viscidiflorus & 0.50 & 0.54 & 0.45 \\
\hline Dalea searlsiae & 0.00 & 0.13 & 0.00 \\
\hline Ephedra nevadensis & 0.00 & 0.00 & 0.83 \\
\hline Ephedra viridis & 0.71 & 0.71 & 0.00 \\
\hline Eriogonum kearneyi & 0.42 & 0.41 & 0.24 \\
\hline Fabaceae species & 0.00 & 0.00 & 0.00 \\
\hline Oenothera pallida & 0.00 & 0.07 & 0.00 \\
\hline Oryzopsis hymenoides & 0.21 & 0.27 & 0.32 \\
\hline Psorothamnus polydenius & 0.36 & 0.39 & 0.37 \\
\hline Stipa comata & 0.00 & 0.00 & 0.24 \\
\hline Unknown herbaceous perennial & 0.00 & 0.00 & 0.10 \\
\hline
\end{tabular}

SPECIES
Atriplex canescens
Chrysothamnus viscidiflorus
Dalea searlsiae
Ephedra nevadensis
Ephedra viridis
Eriogonum kearneyi
Fabaceae species
Oenothera pallida
Oryzopsis hymenoides
Psorothamnus polydenius
Stipa comata
Unknown herbaceous perennial
Total Live
Dead grass
Dead shrub
Total

\section{SPECIES}

Atriplex canescens

Chrysothamnus viscidiflorus

Dalea searlsiae

Ephedra nevadensis

Ephedra viridis

Eriogonum kearneyi

Fabaceae species

Oenothera pallida

Oryzopsis hymenoides

Psorothamnus polydenius

Stipa comata

Unknown herbaceous perennial

Total Live

\begin{tabular}{ccc}
\multicolumn{3}{c}{ Cover (\%) } \\
$\mathbf{1 9 6 3}$ & $\mathbf{1 9 7 5}$ & $\mathbf{2 0 0 2}$ \\
0.40 & 0.55 & 0.00 \\
3.09 & 1.81 & 1.33 \\
0.00 & 0.18 & 0.00 \\
0.00 & 0.00 & 0.23 \\
0.41 & 0.30 & 0.00 \\
10.39 & 9.64 & 3.12 \\
0.00 & 0.00 & 0.01 \\
0.00 & 0.07 & 2.67 \\
3.65 & 22.24 & 11.61 \\
1.18 & 1.03 & 1.18 \\
0.00 & 0.00 & 0.45 \\
0.00 & 0.00 & 0.40 \\
19.13 & 35.82 & 20.99 \\
0.00 & 0.75 & 0.51 \\
0.25 & 0.98 & 2.55 \\
19.37 & 37.55 & 24.05
\end{tabular}

\section{Biomass Index $\left(\mathrm{m}^{2}\right)$}

$1963 \quad 1975 \quad 2002$

$\begin{array}{lll}1.11 & 1.50 \quad 0.00\end{array}$

$\begin{array}{lll}5.36 & 3.45 & 2.20\end{array}$

$\begin{array}{lll}0.00 & 0.10 & 0.00\end{array}$

$\begin{array}{lll}0.00 & 0.00 & 0.63\end{array}$

$\begin{array}{lll}1.02 & 0.76 \quad 0.00\end{array}$

$15.47 \quad 14.98 \quad 2.70$

$\begin{array}{lll}0.00 & 0.00 \quad 0.00\end{array}$

$\begin{array}{lll}0.00 & 0.02 & 0.01\end{array}$

$2.78 \quad 21.74 \quad 13.19$

$\begin{array}{lll}1.92 & 1.54 & 1.86\end{array}$

$\begin{array}{lll}0.00 & 0.00 & 0.39\end{array}$

$\begin{array}{lll}0.00 & 0.00 \quad 0.02\end{array}$

$\begin{array}{lll}27.67 & 44.08 \quad 21.02\end{array}$ 
Table A-66A. Site characteristics for Plot 66.

Location: Jackass Flats

NTS Area: 26

NTS Grid: L-17

USGS 7.5’ Quadrangle Name: Skull Mountain

\begin{tabular}{lllll} 
& & \multicolumn{2}{l}{ GPS Readings (Zone 11S) } \\
& Location & Northing & Easting & Condition of Original Corners \\
Corner 1: & SW & NA & NA & NA \\
Corner 2: & NW & NA & NA & NA \\
Corner 3: & NE & NA & NA & NA \\
Corner 4: & SE & NA & NA & NA
\end{tabular}

DEM Plot Elevation: 1334 m

Plot Aspect: $309^{\circ}$

Elevation Above Playa (Playa Name): NA

Plot Slope: $18^{\circ}$

Annual Precipitation

Measured: $177 \mathrm{~mm}$

Modeled: $173 \mathrm{~mm}$

Parent Material Type: alluvial fan

Substrate: NA

Slate Geologic Unit: Qay

Beatley Plant Assemblage: Larrea-Grayia-Lycium

Ostler-Hanson Plant Association: NA

Ostler-Hanson Land Unit: 187

Abundance of Biological Soil Crusts: NA

Type and Date of Disturbance: none (control for Plot 67)

Plot Condition: good but one edge is historical disturbance

Location of Transect 1: east side

Direction Transects Are Read: north to south

Date(s) Plot Remeasured: 25 April 1999 (incorrectly), 10 April 2003 (correctly) 
Figure A-66. Photographs showing Plots 66 and 67.

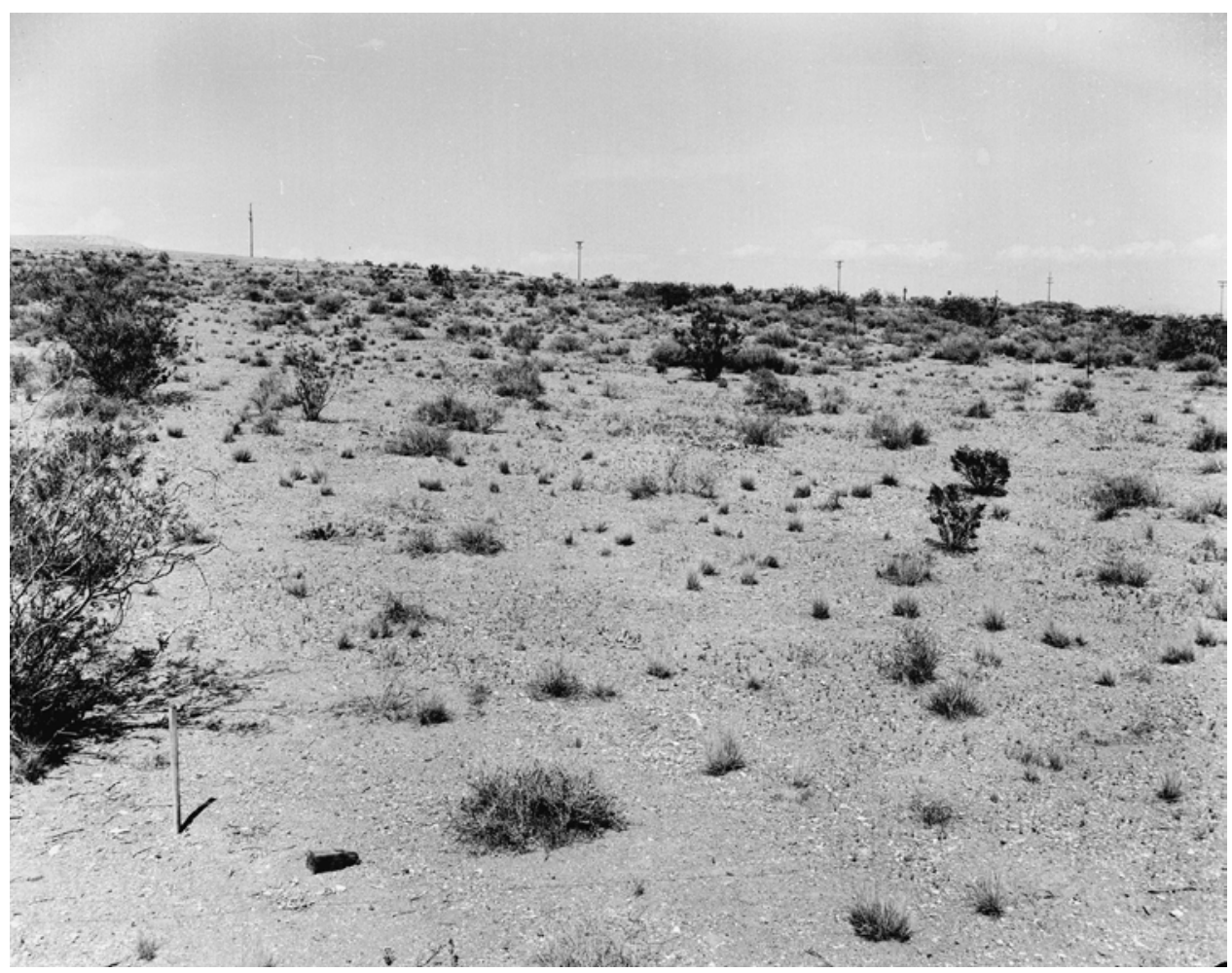

A. (May 1964). Plots 66 and 67 represent the recovery from the disturbance associated with Wahmonie townsite, constructed and abandoned in 1928. This northeasterly view shows the dividing line between the disturbed plot 67 (right) and part of control plot 66 (extreme left). Mormon tea and wolfberry were the dominants in the disturbed site here in 1964 (Janice Beatley Collection, 49-A).

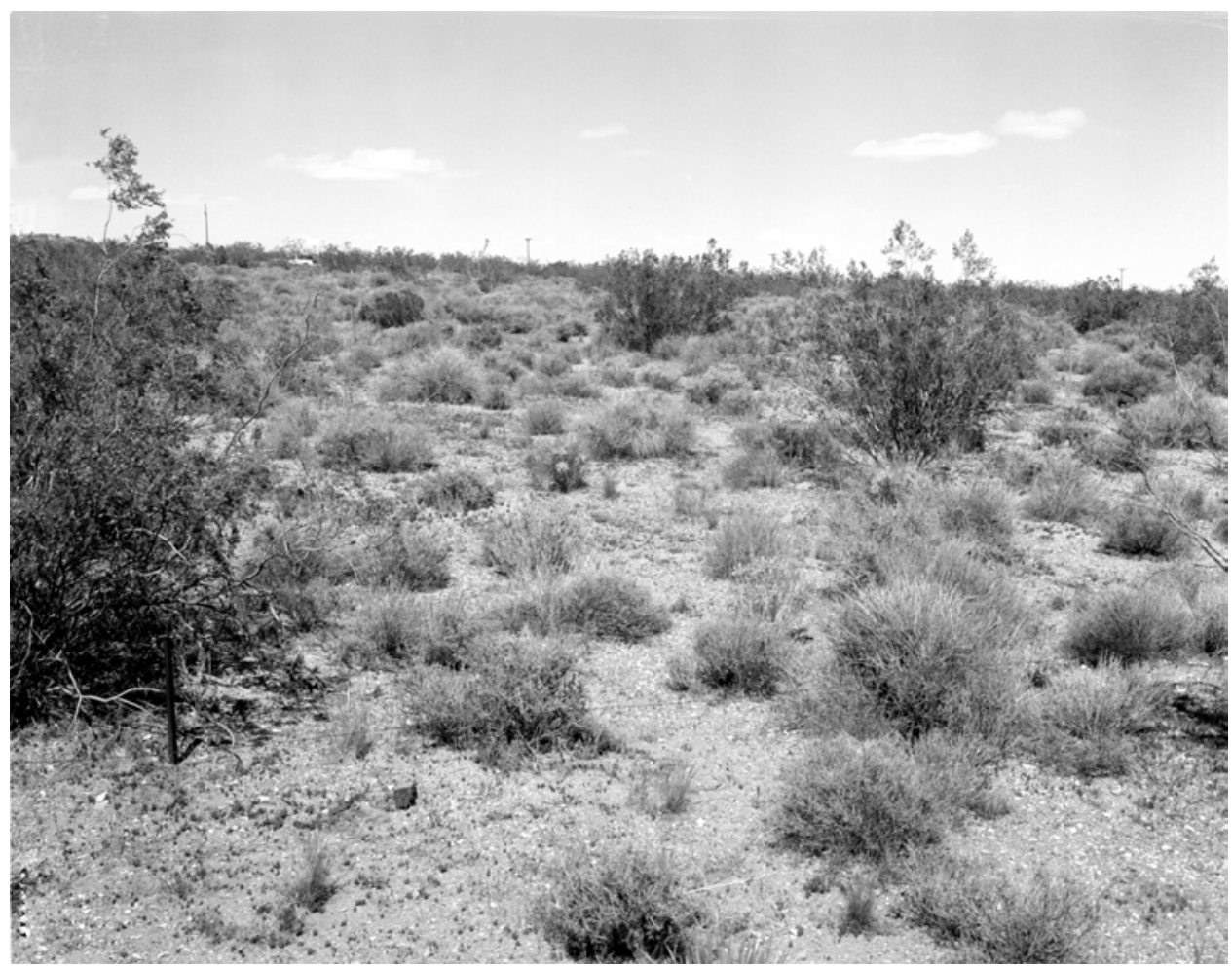

B. (May 11, 2000). The density, cover, and biomass have increased dramatically on both plots 66 and 67 . The recovery of the formerly barren area associated with the Wahmonie townsite is very striking (R.H. Webb, Stake 3822B). 
Table A-66B. Summary plant data for Plot 66.

\begin{tabular}{|c|c|c|c|c|c|c|c|}
\hline \multicolumn{5}{|c|}{ Number of Plants } & \multicolumn{3}{|c|}{ Cover (\%) } \\
\hline SPECIES & 1963 & 1975 & 2003 & SPECIES & 1963 & 1975 & 2003 \\
\hline Acamptopappus shockleyi & 1 & 1 & 0 & Acamptopappus shockleyi & 0.18 & 0.18 & 0.00 \\
\hline Coleogyne ramosissima & 1 & 1 & 3 & Coleogyne ramosissima & 0.24 & 0.26 & 0.55 \\
\hline Ephedra nevadensis & 22 & 26 & 46 & Ephedra nevadensis & 3.49 & 4.02 & 6.89 \\
\hline Ericameria cooperi & 1 & 1 & 0 & Ericameria cooperi & 0.16 & 0.15 & 0.00 \\
\hline Grayia spinosa & 48 & 43 & 9 & Grayia spinosa & 10.39 & 8.37 & 0.97 \\
\hline Hymenoclea salsola & 0 & 0 & 0 & Hymenoclea salsola & 0.00 & 0.00 & 0.00 \\
\hline Larrea tridentata & 24 & 29 & 42 & Larrea tridentata & 7.51 & 11.30 & 13.60 \\
\hline Lycium andersonii & 25 & 32 & 47 & Lycium andersonii & 4.86 & 5.06 & 4.10 \\
\hline Salazaria mexicana & 3 & 4 & 7 & Salazaria mexicana & 0.22 & 0.62 & 0.81 \\
\hline Stipa speciosa & 18 & 59 & 5 & Stipa speciosa & 1.01 & 4.69 & 0.22 \\
\hline Thamnosma montana & 3 & 3 & 37 & Thamnosma montana & 0.23 & 0.45 & 3.69 \\
\hline Yucca brevifolia & 1 & 1 & 0 & Yucca brevifolia & 0.52 & 0.07 & 0.00 \\
\hline Total Live & 147 & 200 & 196 & Total Live & 28.83 & 35.19 & 30.83 \\
\hline Dead Grass & 0 & 0 & 10 & Dead Grass & 0.00 & 0.00 & 0.29 \\
\hline Dead Shrub & 11 & 12 & 76 & Dead Shrub & 1.94 & 5.00 & 13.15 \\
\hline Total & 158 & 212 & 282 & Total & 30.76 & 40.19 & 44.27 \\
\hline \multicolumn{5}{|c|}{ Average Height (m) } & \multicolumn{3}{|c|}{ Biomass Index $\left(\mathrm{m}^{2}\right)$} \\
\hline SPECIES & 1963 & 1975 & 2003 & SPECIES & 1963 & 1975 & 2003 \\
\hline Acamptopappus shockleyi & 0.25 & 0.30 & 0.00 & Acamptopappus shockleyi & 0.15 & 0.19 & 0.00 \\
\hline Coleogyne ramosissima & 0.81 & 0.71 & 0.89 & Coleogyne ramosissima & 0.67 & 0.63 & 1.65 \\
\hline Ephedra nevadensis & 0.41 & 0.52 & 0.57 & Ephedra nevadensis & 5.69 & 7.48 & 15.10 \\
\hline Ericameria cooperi & 0.30 & 0.38 & 0.00 & Ericameria cooperi & 0.17 & 0.20 & 0.00 \\
\hline Grayia spinosa & 0.62 & 0.66 & 0.78 & Grayia spinosa & 22.80 & 19.46 & 2.69 \\
\hline Hymenoclea salsola & 0.00 & 0.00 & 0.00 & Hymenoclea salsola & 0.00 & 0.00 & 0.00 \\
\hline Larrea tridentata & 1.18 & 1.27 & 1.63 & Larrea tridentata & 33.41 & 52.88 & 85.45 \\
\hline Lycium andersonii & 0.48 & 0.45 & 0.42 & Lycium andersonii & 8.56 & 8.11 & 6.57 \\
\hline Salazaria mexicana & 0.29 & 0.40 & 0.46 & Salazaria mexicana & 0.23 & 0.95 & 1.44 \\
\hline Stipa speciosa & 0.33 & 0.36 & 0.26 & Stipa speciosa & 1.24 & 5.94 & 0.20 \\
\hline Thamnosma montana & 0.26 & 0.38 & 0.37 & Thamnosma montana & 0.21 & 0.60 & 4.70 \\
\hline \multirow[t]{2}{*}{ Yucca brevifolia } & 2.82 & 0.30 & 0.00 & Yucca brevifolia & 5.07 & 0.07 & 0.00 \\
\hline & & & & Total Live & 78.21 & 96.50 & 117.81 \\
\hline
\end{tabular}


Table A-67A. Site characteristics for Plot 67.

Location: Jackass Flats

NTS Area: 26

NTS Grid: L-17

USGS 7.5’ Quadrangle Name: Skull Mountain

\begin{tabular}{lllll} 
& & \multicolumn{2}{l}{ GPS Readings (Zone 11S) } \\
& Location & Northing & Easting & Condition of Original Corners \\
Corner 1: & SW & NA & NA & NA \\
Corner 2: & NW & NA & NA & NA \\
Corner 3: & NE & NA & NA & NA \\
Corner 4: & SE & NA & NA & NA
\end{tabular}

DEM Plot Elevation: 1328 m

Plot Aspect: $79^{\circ}$

Elevation Above Playa (Playa Name): NA

Plot Slope: $4^{\circ}$

Annual Precipitation

Measured: $177 \mathrm{~mm}$

Modeled: $173 \mathrm{~mm}$

Parent Material Type: alluvial fan

Substrate: NA

Slate Geologic Unit: Qay

Beatley Plant Assemblage: Ephedra-Lycium

Ostler-Hanson Plant Association: NA

Ostler-Hanson Land Unit: 187

Abundance of Biological Soil Crusts: NA

Type and Date of Disturbance: Wahmonie ghost town, abandoned in 1928

Plot Condition: good

Location of Transect 1: east side of plot

Direction Transects Are Read: north to south

Date(s) Plot Remeasured: 25 April 1999 (incorrectly), 10 April 2003 (correctly) 
Figure A-67. Photographs showing Plots 66 and 67.

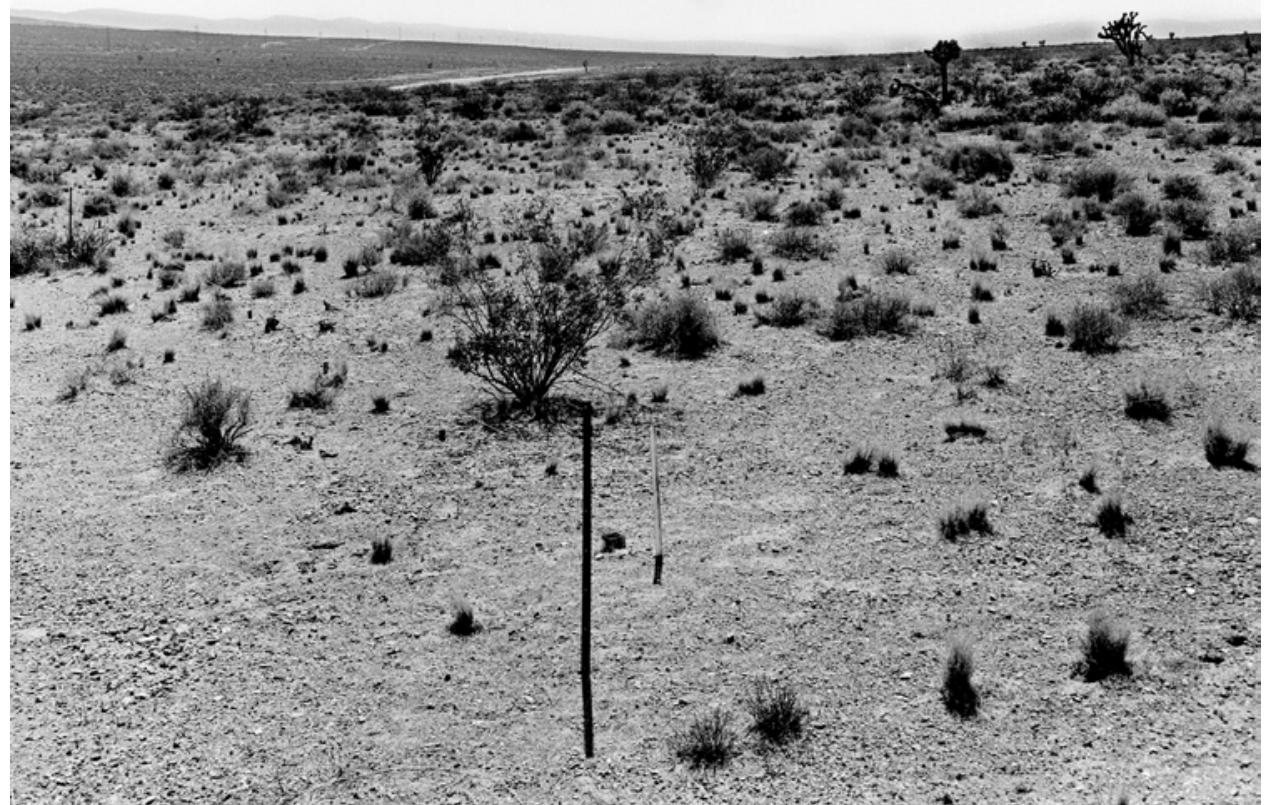

A. (May 1964). This southwesterly view of Plot 67 shows Plot 66 in the midground. The background is Little Skull Mountain (at left) and Yucca Mountain (at right). The original vegetation in Plot 67 is represented by Plot 66, which is dominated by creosote bush, spiny hopsage, and wolfberry (Janice Beatley Collection, 50-B).

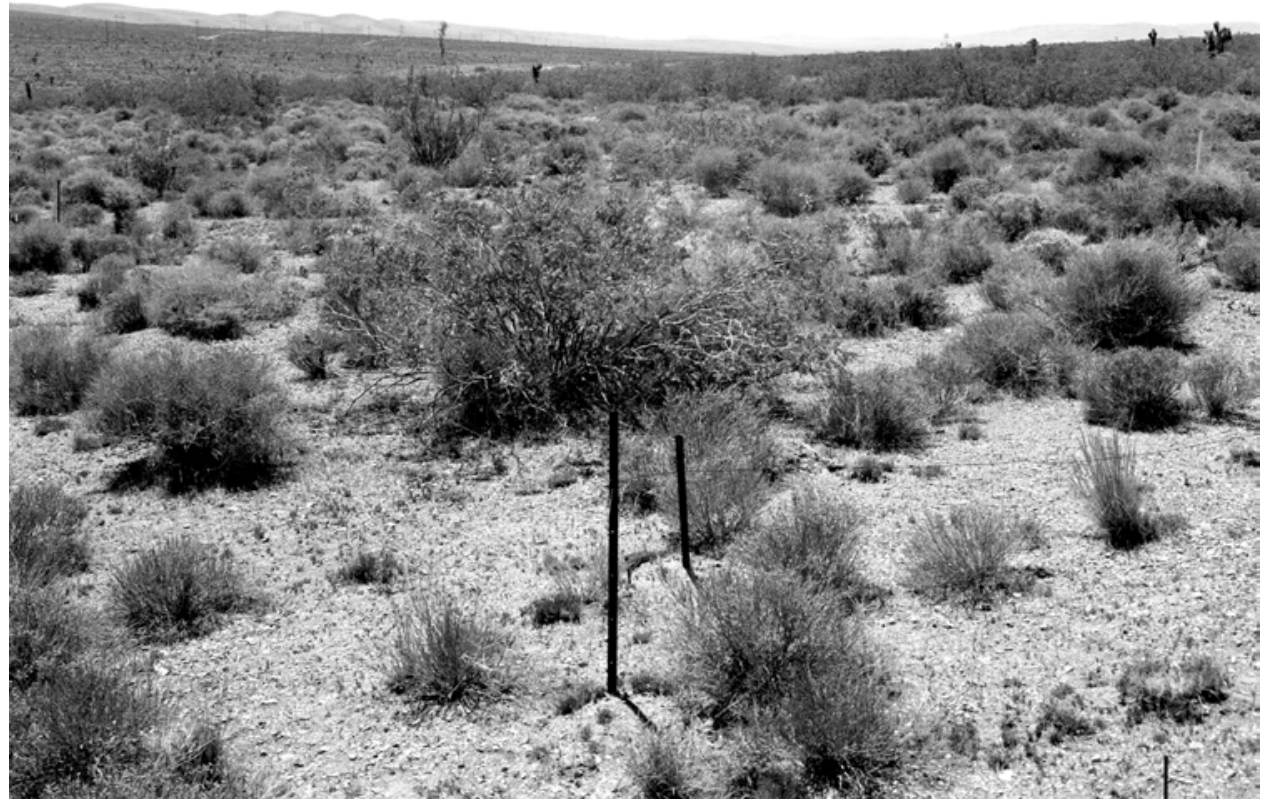

B. (May 11, 2000). The biomass of vegetation in the disturbed area has greatly increased. In the undisturbed vegetation of Plot 66, creosote bush, Mormon tea, and turpentinebroom now dominate. The number of Joshua trees appears to be more or less unchanged (R.H. Webb, Stake 3820). 
Table A-67B. Summary plant data for Plot 67.

\author{
SPECIES \\ Acamptopappus shockleyi \\ Astragalus lentiginosus \\ Coleogyne ramosissima \\ Dyssodia cooperi \\ Ephedra nevadensis \\ Grayia spinosa \\ Hymenoclea salsola \\ Larrea tridentata \\ Lycium andersonii \\ Salazaria mexicana \\ Stipa speciosa \\ Thamnosma montana \\ Total Live \\ Dead Grass \\ Dead Shrub \\ Total
}

\section{SPECIES}

Acamptopappus shockleyi Astragalus lentiginosus

Coleogyne ramosissima

Dyssodia cooperi

Ephedra nevadensis

Grayia spinosa

Hymenoclea salsola

Larrea tridentata

Lycium andersonii

Salazaria mexicana

Stipa speciosa

Thamnosma montana

1963
5
0
0
0
25
7
6
0
12
5
41
4
105
0
8
113

\section{3}

0.22

0.00

0.00

0.00

0.42

0.48

0.38

0.00

0.39

0.34

0.37

0.30

\begin{tabular}{cccc}
\multicolumn{2}{c}{ Number of Plants } & & \\
$\mathbf{1 9 6 7}$ & $\mathbf{1 9 7 0}$ & $\mathbf{1 9 7 5}$ & $\mathbf{2 0 0 3}$ \\
3 & 4 & 5 & 5 \\
54 & 0 & 0 & 0 \\
0 & 0 & 0 & 2 \\
1 & 0 & 0 & 1 \\
22 & 29 & 32 & 56 \\
3 & 3 & 2 & 2 \\
12 & 17 & 18 & 5 \\
0 & 0 & 0 & 6 \\
14 & 16 & 17 & 23 \\
5 & 5 & 5 & 7 \\
69 & 74 & 100 & 22 \\
5 & 5 & 6 & 42 \\
188 & 153 & 185 & 171 \\
0 & 0 & 0 & 18 \\
89 & 11 & 8 & 61 \\
277 & 164 & 193 & 250
\end{tabular}

\section{Average Height (m)}

$\begin{array}{llll}\mathbf{1 9 6 7} & \mathbf{1 9 7 0} & \mathbf{1 9 7 5} & \mathbf{2 0 0 3} \\ 0.25 & 0.27 & 0.25 & 0.29 \\ 0.16 & 0.00 & 0.00 & 0.00 \\ 0.00 & 0.00 & 0.00 & 0.77 \\ 0.10 & 0.00 & 0.00 & 0.06 \\ 0.46 & 0.60 & 0.60 & 0.60 \\ 0.58 & 0.67 & 0.75 & 0.78 \\ 0.40 & 0.57 & 0.57 & 0.45 \\ 0.00 & 0.00 & 0.00 & 1.92 \\ 0.36 & 0.38 & 0.46 & 0.43 \\ 0.38 & 0.41 & 0.46 & 0.43 \\ 0.32 & 0.32 & 0.39 & 0.29 \\ 0.33 & 0.58 & 0.38 & 0.36\end{array}$


Table A-67B (continued). Summary plant data for Plot 67.

\begin{tabular}{|c|c|c|c|c|c|}
\hline SPECIES & 1963 & 1967 & 1970 & 1975 & 2003 \\
\hline Acamptopappus shockleyi & 0.45 & 0.37 & 0.47 & 0.48 & 0.44 \\
\hline Astragalus lentiginosus & 0.00 & 1.97 & 0.00 & 0.00 & 0.00 \\
\hline Coleogyne ramosissima & 0.00 & 0.00 & 0.00 & 0.00 & 0.34 \\
\hline Dyssodia cooperi & 0.00 & 0.03 & 0.00 & 0.00 & 0.02 \\
\hline Ephedra nevadensis & 3.27 & 3.11 & 4.85 & 6.71 & 9.81 \\
\hline Grayia spinosa & 0.68 & 0.36 & 0.41 & 0.26 & 0.22 \\
\hline Hymenoclea salsola & 0.70 & 1.00 & 3.18 & 3.07 & 0.28 \\
\hline Larrea tridentata & 0.00 & 0.00 & 0.00 & 0.00 & 2.21 \\
\hline Lycium andersonii & 2.03 & 1.94 & 2.56 & 2.62 & 2.24 \\
\hline Salazaria mexicana & 0.44 & 0.71 & 0.82 & 0.99 & 0.99 \\
\hline Stipa speciosa & 2.82 & 4.32 & 5.81 & 8.84 & 1.00 \\
\hline Thamnosma montana & 0.43 & 0.56 & 0.60 & 0.84 & 4.01 \\
\hline Total Live & 10.82 & 14.36 & 18.70 & 23.81 & 21.56 \\
\hline Dead Grass & 0.00 & 0.00 & 0.00 & 0.00 & 1.04 \\
\hline Dead Shrub & 1.04 & 3.56 & 1.10 & 0.57 & 13.75 \\
\hline Total & 11.86 & 17.93 & 19.80 & 24.38 & 36.36 \\
\hline & \multicolumn{5}{|c|}{ Biomass Index $\left(\mathrm{m}^{2}\right)$} \\
\hline SPECIES & 1963 & 1967 & 1970 & 1975 & 2003 \\
\hline Acamptopappus shockleyi & 0.35 & 0.33 & 0.43 & 0.48 & 0.45 \\
\hline Astragalus lentiginosus & 0.00 & 1.17 & 0.00 & 0.00 & 0.00 \\
\hline Coleogyne ramosissima & 0.00 & 0.00 & 0.00 & 0.00 & 0.90 \\
\hline Dyssodia cooperi & 0.00 & 0.01 & 0.00 & 0.00 & 0.00 \\
\hline Ephedra nevadensis & 5.36 & 5.46 & 10.69 & 14.44 & 23.22 \\
\hline Grayia spinosa & 1.20 & 0.74 & 0.98 & 0.68 & 0.59 \\
\hline Hymenoclea salsola & 1.06 & 1.36 & 6.45 & 6.46 & 0.39 \\
\hline Larrea tridentata & 0.00 & 0.00 & 0.00 & 0.00 & 17.50 \\
\hline Lycium andersonii & 2.82 & 2.54 & 3.33 & 4.55 & 3.68 \\
\hline Salazaria mexicana & 0.54 & 1.03 & 1.20 & 1.67 & 1.58 \\
\hline Stipa speciosa & 3.72 & 5.21 & 7.11 & 12.31 & 1.01 \\
\hline Thamnosma montana & 0.47 & 0.64 & 1.32 & 1.12 & 5.40 \\
\hline Total Live & 15.52 & 18.48 & 31.51 & 41.71 & 54.74 \\
\hline
\end{tabular}


Table A-68A. Site characteristics for Plot 68.

Location: Rainier Mesa

NTS Area: 12

NTS Grid: G-39

USGS 7.5’ Quadrangle Name: Rainier Mesa

\begin{tabular}{lllll} 
& & \multicolumn{2}{l}{ GPS Readings (Zone 11S) } & \\
& Location & Northing & Easting & Condition of Original Corners \\
Corner 1: & SW & 4114400 & 566765 & Found washed away (debris flow) \\
Corner 2: & NW & 4114431 & 566783 & On ground \\
Corner 3: & NE & 4114414 & 566807 & Standing \\
Corner 4: & SE & 4114393 & 566791 & Missing
\end{tabular}

DEM Plot Elevation: 1825 m

Plot Aspect: $322^{\circ}$

Elevation Above Playa (Playa Name): NA

Plot Slope: $22^{\circ}$

Annual Precipitation

Measured: $261 \mathrm{~mm}$

Modeled: $240 \mathrm{~mm}$

Parent Material Type: broken terrain

Substrate: sandy w/debris flow activity

Slate Geologic Unit: Qay

Beatley Plant Assemblage: Artemisia tridentata-Pinyon-Juniper

Ostler-Hanson Plant Association: NA

Ostler-Hanson Land Unit: 952

Abundance of Biological Soil Crusts: none

Type and Date of Disturbance: none

Plot Condition: debris flow activity pre-and post-Beatley measurements had minimal impact on plot. Feral horses are abundant in this valley

Location of Transect 1: west side of plot

Direction Transects Are Read: south to north

Date(s) Plot Remeasured: 28, 30 \& 31 May 2002 
Figure A-68. Photographs showing Plot 68.

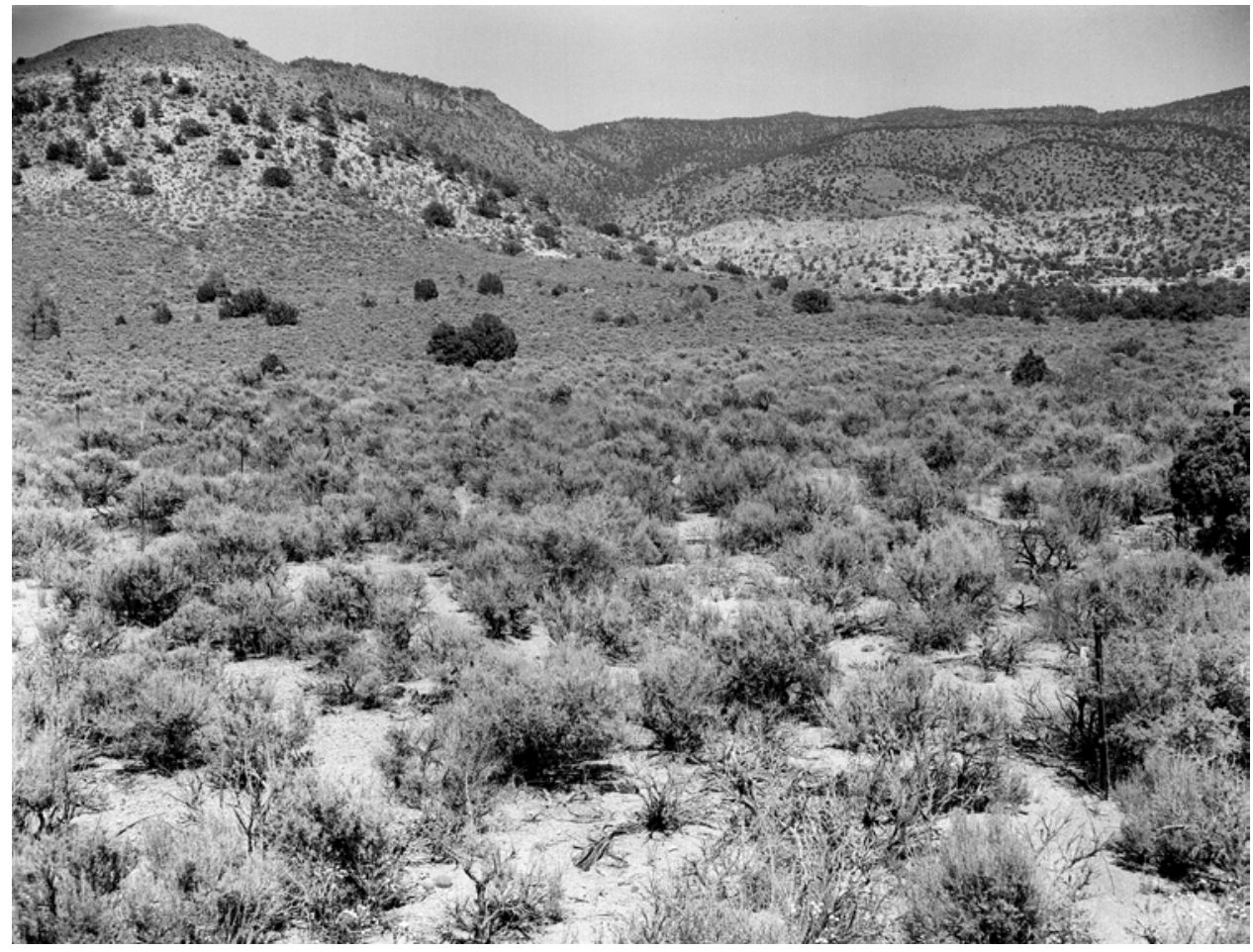

A. (1964). In this easterly view across Plot 68, the western flank of Rainier Mesa dominates the skyline. The vegetation is Great Basin sagebrush with singleleaf pinyon and Utah juniper (Janice Beatley Collection, no number).

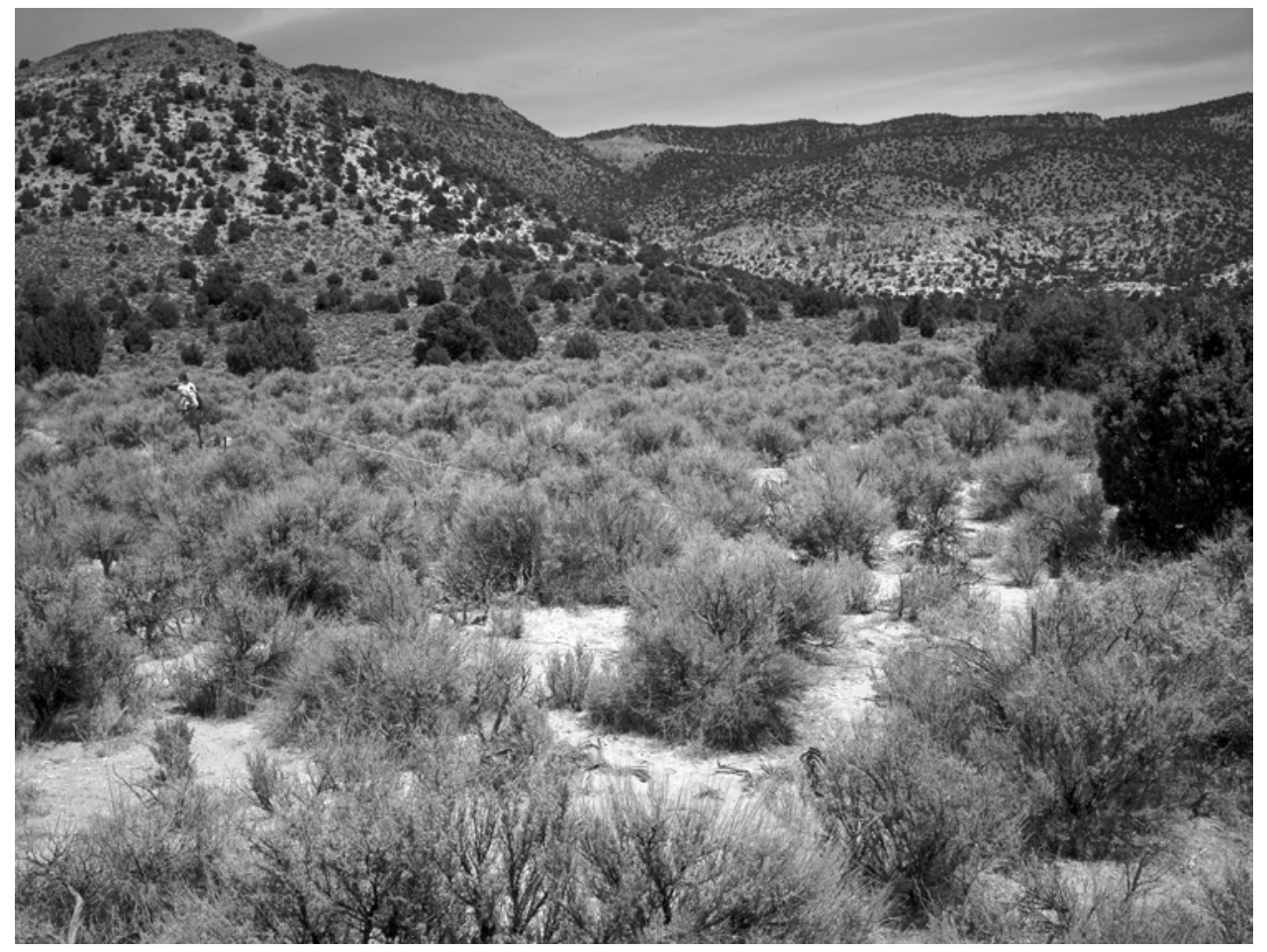

B. (April 28, 2002). The increase in density and biomass of singleleaf pinyon and Utah juniper, and particularly juniper, is striking. Needlegrass has declined significantly in the view, possibly owing to the wild horse herd that inhabits this little valley (Dominic Oldershaw, Stake 4198A). 
Table A-68B. Summary plant data for Plot 68.

\begin{tabular}{|c|c|c|c|c|c|c|c|}
\hline \multicolumn{5}{|c|}{ Number of Plants } & \multicolumn{3}{|c|}{ Cover (\%) } \\
\hline SPECIES & 1964 & 1975 & 2002 & SPECIES & 1964 & 1975 & 2002 \\
\hline Artemisia tridentata & 153 & 154 & 168 & Artemisia tridentata & 24.15 & 25.06 & 24.37 \\
\hline Astragalus lentiginosus & 0 & 1 & 0 & Astragalus lentiginosus & 0.00 & 0.03 & 0.00 \\
\hline Atriplex canescens & 8 & 14 & 7 & Atriplex canescens & 1.57 & 3.36 & 0.52 \\
\hline Chrysothamnus viscidiflorus & 11 & 23 & 31 & Chrysothamnus viscidiflorus & 1.64 & 2.71 & 2.45 \\
\hline Ephedra viridis & 0 & 0 & 1 & Ephedra viridis & 0.00 & 0.00 & 0.15 \\
\hline Eriogonum kearneyi & 0 & 0 & 2 & Eriogonum kearneyi & 0.00 & 0.00 & 0.33 \\
\hline Eriogonum ovalifolium & 0 & 1 & 0 & Eriogonum ovalifolium & 0.00 & 0.04 & 0.00 \\
\hline Juniperus osteosperma & 1 & 1 & 3 & Juniperus osteosperma & 0.25 & 0.60 & 1.65 \\
\hline Oryzopsis hymenoides & 16 & 8 & 7 & Oryzopsis hymenoides & 1.07 & 0.45 & 0.15 \\
\hline Pinus monophylla & 0 & 0 & 2 & Pinus monophylla & 0.00 & 0.00 & 1.30 \\
\hline Poa fendleriana & 0 & 1 & 0 & Poa fendleriana & 0.00 & 0.04 & 0.00 \\
\hline Sitanion hystrix & 25 & 28 & 3 & Sitanion hystrix & 1.76 & 1.52 & 0.08 \\
\hline Sphaeralcea ambigua & 1 & 1 & 0 & Sphaeralcea ambigua & 0.01 & 0.01 & 0.00 \\
\hline Stipa comata & 168 & 334 & 12 & Stipa comata & 16.34 & 17.55 & 0.75 \\
\hline Total Live & 383 & 566 & 236 & Total Live & 46.78 & 51.37 & 31.75 \\
\hline Dead Grass & 1 & 2 & 0 & Dead Grass & 0.08 & 0.05 & 0.00 \\
\hline Dead Shrub & 91 & 81 & 87 & Dead Shrub & 13.15 & 12.12 & 12.80 \\
\hline Total & 475 & 649 & 323 & Total & 60.02 & 63.55 & 44.55 \\
\hline \multicolumn{5}{|c|}{ Average Height (m) } & \multicolumn{3}{|c|}{ Biomass Index (m²) } \\
\hline SPECIES & 1964 & 1975 & 2002 & SPECIES & 1964 & 1975 & 2002 \\
\hline Artemisia tridentata & 0.71 & 0.70 & 0.78 & Artemisia tridentata & 61.02 & 68.48 & 73.01 \\
\hline Astragalus lentiginosus & 0.00 & 0.18 & 0.00 & Astragalus lentiginosus & 0.00 & 0.02 & 0.00 \\
\hline Atriplex canescens & 0.76 & 0.87 & 0.76 & Atriplex canescens & 4.41 & 10.41 & 1.28 \\
\hline Chrysothamnus viscidiflorus & 0.49 & 0.49 & 0.47 & Chrysothamnus viscidiflorus & 2.95 & 5.19 & 4.21 \\
\hline Ephedra viridis & 0.00 & 0.00 & 0.75 & Ephedra viridis & 0.00 & 0.00 & 0.37 \\
\hline Eriogonum kearneyi & 0.00 & 0.00 & 0.56 & Eriogonum kearneyi & 0.00 & 0.00 & 0.62 \\
\hline Eriogonum ovalifolium & 0.00 & 0.08 & 0.00 & Eriogonum ovalifolium & 0.00 & 0.01 & 0.00 \\
\hline Juniperus osteosperma & 1.57 & 2.18 & 2.73 & Juniperus osteosperma & 1.30 & 4.39 & 17.20 \\
\hline Oryzopsis hymenoides & 0.40 & 0.31 & 0.24 & Oryzopsis hymenoides & 1.63 & 0.49 & 0.15 \\
\hline Pinus monophylla & 0.00 & 0.00 & 3.06 & Pinus monophylla & 0.00 & 0.00 & 11.31 \\
\hline Poa fendleriana & 0.00 & 0.25 & 0.00 & Poa fendleriana & 0.00 & 0.03 & 0.00 \\
\hline Sitanion hystrix & 0.42 & 0.30 & 0.37 & Sitanion hystrix & 2.81 & 1.61 & 0.10 \\
\hline Sphaeralcea ambigua & 0.33 & 0.18 & 0.00 & Sphaeralcea ambigua & 0.01 & 0.01 & 0.00 \\
\hline \multirow[t]{2}{*}{ Stipa comata } & 0.56 & 0.27 & 0.43 & Stipa comata & 32.60 & 16.71 & 1.07 \\
\hline & & & & Total Live & 106.72 & 107.35 & 109.32 \\
\hline
\end{tabular}


Table A-69. Summary information on photographs of Beatley plots on the Nevada Test Site, Nye County, Nevada.

\begin{tabular}{|c|c|c|c|c|c|c|c|}
\hline $\begin{array}{c}\text { Plot } \\
\text { Number }\end{array}$ & $\begin{array}{c}\text { Stake }^{1} \\
\text { Number }\end{array}$ & $\begin{array}{l}\text { Latitude } \\
\text { (Degrees) }\end{array}$ & $\begin{array}{l}\text { Longitude } \\
\text { (Degrees) }\end{array}$ & $\begin{array}{c}\text { Elevation } \\
\text { (m) }\end{array}$ & $\begin{array}{c}\text { Date of Original } \\
\text { Photograph }\end{array}$ & $\begin{array}{l}\text { Original Photo } \\
\text { Number }\end{array}$ & $\begin{array}{l}\text { Date of } \\
\text { Match }\end{array}$ \\
\hline Plot 1 & $4032 a$ & 36.63988 & 116.03702 & 1022 & 04/19/1964 & $14-\mathrm{B}$ & $05 / 11 / 2000$ \\
\hline Plot 1 & $4032 b$ & 36.63988 & 116.03702 & 1022 & 04/19/1964 & $14-\mathrm{A}$ & $05 / 11 / 2000$ \\
\hline Plot 1 & 4033a & 36.63938 & 116.03673 & 1021 & 04/19/1964 & $13-\mathrm{A}$ & $05 / 12 / 2000$ \\
\hline Plot 1 & $4033 b$ & 36.63938 & 116.03673 & 1021 & 04/19/1964 & $13-B$ & $05 / 12 / 2000$ \\
\hline Plot 2 & $4034 a$ & 36.66955 & 116.12887 & 1087 & 05/14/1964 & $110-\mathrm{A}$ & $05 / 12 / 2000$ \\
\hline Plot 2 & $4034 b$ & 36.66955 & 116.12887 & 1087 & 05/14/1964 & $110-B$ & $05 / 12 / 2000$ \\
\hline Plot 2 & $4035 a$ & 36.66923 & 116.12948 & 1087 & 05/14/1964 & $109-B$ & $05 / 12 / 2000$ \\
\hline Plot 2 & $4035 b$ & 36.66923 & 116.12948 & 1087 & 05/14/1964 & $109-\mathrm{A}$ & $05 / 12 / 2000$ \\
\hline Plot 3 & 4019a & 36.69008 & 116.18902 & 1029 & 04/19/1964 & $16-\mathrm{A}$ & 05/09/2000 \\
\hline Plot 3 & $4019 b$ & 36.69008 & 116.18902 & 1029 & 04/19/1964 & $16-\mathrm{B}$ & 05/09/2000 \\
\hline Plot 3 & $4020 \mathrm{a}$ & 36.69055 & 116.18935 & 1028 & 04/19/1964 & $15-\mathrm{B}$ & 05/09/2000 \\
\hline Plot 3 & $4020 b$ & 36.69055 & 116.18935 & 1028 & 04/19/1964 & $15-\mathrm{A}$ & $05 / 09 / 2000$ \\
\hline Plot 4 & $4021 \mathrm{a}$ & 36.69767 & 116.17605 & 1055 & $04 / 22 / 1964$ & $22-B$ & 05/09/2000 \\
\hline Plot 4 & $4021 b$ & 36.69767 & 116.17605 & 1055 & $04 / 22 / 1964$ & $22-A$ & 05/09/2000 \\
\hline Plot 4 & $4022 \mathrm{a}$ & 36.69810 & 116.17637 & 1050 & $04 / 22 / 1964$ & $21-\mathrm{A}$ & 05/09/2000 \\
\hline Plot 4 & $4022 b$ & 36.69810 & 116.17637 & 1050 & $04 / 22 / 1964$ & $21-B$ & 05/09/2000 \\
\hline Plot 5 & $4036 a$ & 36.74867 & 116.24753 & 1112 & 05/07/1964 & $62-A$ & $05 / 12 / 2000$ \\
\hline Plot 5 & $4036 b$ & 36.74867 & 116.24753 & 1112 & 05/07/1964 & $62-B$ & $05 / 12 / 2000$ \\
\hline Plot 5 & $4037 a$ & 36.74930 & 116.24752 & 1117 & 05/07/1964 & $61-B$ & $05 / 12 / 2000$ \\
\hline Plot 5 & $4037 b$ & 36.74930 & 116.24752 & 1117 & 05/07/1964 & $61-A$ & $05 / 12 / 2000$ \\
\hline Plot 6 & $4102 a$ & 36.77033 & 116.26387 & 1079 & 05/14/1964 & $111-B$ & $04 / 05 / 2001$ \\
\hline Plot 6 & $4102 b$ & 36.77033 & 116.26387 & 1079 & 05/14/1964 & $111-A$ & $04 / 05 / 2001$ \\
\hline Plot 6 & 4103a & 36.77074 & 116.26336 & 1088 & 05/14/1964 & $112-\mathrm{A}$ & $04 / 05 / 2001$ \\
\hline Plot 6 & 4103b & 36.77074 & 116.26336 & 1088 & 05/14/1964 & $112-\mathrm{B}$ & $04 / 05 / 2001$ \\
\hline Plot 7 & $4109 a$ & 36.77135 & 116.35682 & 981 & 05/14/1964 & $107-A$ & $04 / 05 / 2001$ \\
\hline Plot 7 & $4109 b$ & 36.77135 & 116.35682 & 981 & 05/14/1964 & $107-B$ & $04 / 05 / 2001$ \\
\hline Plot 7 & $4110 a$ & 36.77177 & 116.35741 & 980 & 05/14/1964 & $108-A$ & $04 / 05 / 2001$ \\
\hline Plot 7 & $4110 \mathrm{~b}$ & 36.77177 & 116.35741 & 980 & 05/14/1964 & $108-B$ & $04 / 05 / 2001$ \\
\hline Plot 8 & $4107 a$ & 36.75388 & 116.37624 & 966 & 04/17/1964 & $8-B$ & $04 / 05 / 2001$ \\
\hline Plot 8 & $4107 \mathrm{~b}$ & 36.75388 & 116.37624 & 966 & 04/17/1964 & 8-A & $04 / 05 / 2001$ \\
\hline Plot 8 & $4108 a$ & 36.75427 & 116.37574 & 961 & 04/17/1964 & $7-B$ & $04 / 05 / 2001$ \\
\hline Plot 8 & $4108 b$ & 36.75427 & 116.37574 & 961 & 04/17/1964 & 7-A & $04 / 05 / 2001$ \\
\hline Plot 9 & $4125 a$ & 36.79382 & 116.36501 & 1010 & 04/18/1964 & $10-B$ & 04/07/2001 \\
\hline Plot 9 & $4125 b$ & 36.79382 & 116.36501 & 1010 & 04/18/1964 & $10-\mathrm{A}$ & 04/07/2001 \\
\hline Plot 9 & $4126 a$ & 36.79341 & 116.36461 & 1019 & 04/18/1964 & $9-A$ & $04 / 07 / 2001$ \\
\hline
\end{tabular}


Table A-69 (continued). Summary information on photographs of Beatley plots on the Nevada Test Site, Nye County, Nevada.

\begin{tabular}{|c|c|c|c|c|c|c|c|}
\hline $\begin{array}{c}\text { Plot } \\
\text { Number } \\
\end{array}$ & $\begin{array}{c}\text { Stake }^{1} \\
\text { Number } \\
\end{array}$ & $\begin{array}{c}\text { Latitude } \\
\text { (Degrees) }\end{array}$ & $\begin{array}{l}\begin{array}{l}\text { Longitude } \\
\text { (Degrees) }\end{array} \\
\end{array}$ & $\begin{array}{c}\text { Elevation } \\
(\mathbf{m})\end{array}$ & $\begin{array}{c}\text { Date of Original } \\
\text { Photograph }\end{array}$ & $\begin{array}{c}\text { Original Photo } \\
\text { Number }\end{array}$ & $\begin{array}{l}\text { Date of } \\
\text { Match }\end{array}$ \\
\hline Plot 9 & 4126b & 36.79341 & 116.36461 & 1019 & 04/18/1964 & 9-B & $04 / 07 / 2001$ \\
\hline Plot 10 & 4123a & 36.79470 & 116.31823 & 1059 & 04/18/1964 & $12-\mathrm{B}$ & 04/06/2001 \\
\hline Plot 10 & 4123b & 36.79470 & 116.31823 & 1059 & 04/18/1964 & $12-\mathrm{A}$ & $04 / 06 / 2001$ \\
\hline Plot 10 & $4124 a$ & 36.79458 & 116.31904 & 1031 & 04/18/1964 & $11-\mathrm{A}$ & 04/06/2001 \\
\hline Plot 10 & $4124 b$ & 36.79458 & 116.31904 & 1031 & 04/18/1964 & $11-B$ & 04/06/2001 \\
\hline Plot 11 & $4050 a$ & 36.83767 & 116.26100 & 1193 & 04/30/1964 & $45 \mathrm{~A}$ & 06/08/2001 \\
\hline Plot 11 & 4050b & 36.83767 & 116.26100 & 1193 & 04/30/1964 & $45 \mathrm{~B}$ & 06/08/2001 \\
\hline Plot 11 & $4051 \mathrm{a}$ & 36.83739 & 116.26161 & 1191 & 04/30/1964 & $46 \mathrm{~A}$ & 06/08/2001 \\
\hline Plot 11 & 4051b & 36.83739 & 116.26161 & 1191 & 04/30/1964 & $46 \mathrm{~B}$ & 06/08/2001 \\
\hline Plot 12 & $4052 a$ & 36.84343 & 116.25986 & 1220 & 05/06/1964 & $51 \mathrm{~A}$ & 06/08/2001 \\
\hline Plot 12 & $4052 b$ & 36.84343 & 116.25986 & 1220 & 05/06/1964 & $51 \mathrm{~B}$ & 06/08/2001 \\
\hline Plot 12 & 4053a & 36.84289 & 116.25964 & 1222 & 05/06/1964 & $52 \mathrm{~A}$ & $06 / 08 / 2001$ \\
\hline Plot 12 & 4053b & 36.84289 & 116.25964 & 1222 & 05/06/1964 & $52 \mathrm{~B}$ & 06/08/2001 \\
\hline Plot 13 & $4054 a$ & 36.85429 & 116.23701 & 1308 & 05/06/1964 & $54 \mathrm{~A}$ & 06/08/2001 \\
\hline Plot 13 & 4054b & 36.85429 & 116.23701 & 1308 & 05/06/1964 & $54 \mathrm{~B}$ & 06/08/2001 \\
\hline Plot 13 & $4055 a$ & 36.85435 & 116.23761 & 1301 & 05/06/1964 & $53 \mathrm{~A}$ & 06/08/2001 \\
\hline Plot 13 & 4055b & 36.85435 & 116.23761 & 1301 & 05/06/1964 & $53 \mathrm{~B}$ & 06/08/2001 \\
\hline Plot 14 & $4056 a$ & 36.88280 & 116.21070 & 1457 & 05/06/1964 & $55 \mathrm{~A}$ & 06/08/2001 \\
\hline Plot 14 & 4056b & 36.88280 & 116.21070 & 1457 & 05/06/1964 & $55 B$ & 06/08/2001 \\
\hline Plot 14 & $4057 a$ & 36.88230 & 116.21032 & 1460 & 05/06/1964 & $56 \mathrm{~A}$ & 06/08/2001 \\
\hline Plot 14 & $4057 b$ & 36.88230 & 116.21032 & 1460 & 05/06/1964 & $56 \mathrm{~B}$ & 06/08/2001 \\
\hline Plot 15 & $4121 \mathrm{a}$ & 36.78192 & 116.25987 & 1103 & 04/28/1964 & $37-A$ & 04/06/2001 \\
\hline Plot 15 & $4121 b$ & 36.78192 & 116.25987 & 1103 & 04/28/1964 & 38-B & 04/06/2001 \\
\hline Plot 15 & $4122 a$ & 36.78226 & 116.25927 & 1115 & 04/28/1964 & 38-A & 04/06/2001 \\
\hline Plot 15 & $4122 b$ & 36.78226 & 116.25927 & 1115 & 04/28/1964 & 37-B & $04 / 06 / 2001$ \\
\hline Plot 16 & $4101 \mathrm{a}$ & 36.79662 & 116.22780 & 1184 & 04/28/1964 & 39-B & $04 / 05 / 2001$ \\
\hline Plot 16 & 4101b & 36.79662 & 116.22780 & 1184 & 04/28/1964 & 39-A & $04 / 05 / 2001$ \\
\hline Plot 16 & $4104 a$ & 36.79621 & 116.22746 & 1182 & 04/28/1964 & $40-B$ & $04 / 05 / 2001$ \\
\hline Plot 16 & $4104 b$ & 36.79621 & 116.22746 & 1182 & 04/28/1964 & $40-\mathrm{A}$ & 04/05/2001 \\
\hline Plot 17 & $4105 a$ & 36.80790 & 116.20247 & 1272 & 04/28/1964 & $41-B$ & $04 / 05 / 2001$ \\
\hline Plot 17 & $4105 b$ & 36.80790 & 116.20247 & 1272 & 04/28/1964 & $41-\mathrm{A}$ & $04 / 05 / 2001$ \\
\hline Plot 17 & 4106a & 36.80757 & 116.20196 & 1266 & 04/28/1964 & $42-\mathrm{A}$ & $04 / 05 / 2001$ \\
\hline Plot 17 & 4106b & 36.80757 & 116.20196 & 1266 & 04/28/1964 & $42-B$ & $04 / 05 / 2001$ \\
\hline Plot 18 & $4042 a$ & 36.90130 & 116.27680 & 1457 & 05/07/1964 & 59-B & $06 / 20 / 2000$ \\
\hline Plot 18 & $4042 b$ & 36.90130 & 116.27680 & 1457 & 05/07/1964 & 59-A & 06/20/2000 \\
\hline Plot 18 & 4043a & 36.90158 & 116.27603 & 1462 & 05/07/1964 & $60-A$ & 06/20/2000 \\
\hline
\end{tabular}


Table A-69 (continued). Summary information on photographs of Beatley plots on the Nevada Test Site, Nye County, Nevada.

\begin{tabular}{|c|c|c|c|c|c|c|c|}
\hline $\begin{array}{c}\text { Plot } \\
\text { Number } \\
\end{array}$ & $\begin{array}{c}\text { Stake }^{1} \\
\text { Number } \\
\end{array}$ & $\begin{array}{c}\text { Latitude } \\
\text { (Degrees) }\end{array}$ & $\begin{array}{l}\begin{array}{c}\text { Longitude } \\
\text { (Degrees) }\end{array} \\
\end{array}$ & $\begin{array}{c}\text { Elevation } \\
(\mathbf{m})\end{array}$ & $\begin{array}{c}\text { Date of Original } \\
\text { Photograph }\end{array}$ & $\begin{array}{c}\begin{array}{c}\text { Original Photo } \\
\text { Number }\end{array} \\
\end{array}$ & $\begin{array}{l}\text { Date of } \\
\text { Match }\end{array}$ \\
\hline Plot 18 & 4043b & 36.90158 & 116.27603 & 1462 & 05/07/1964 & $60-B$ & $06 / 20 / 2000$ \\
\hline Plot 19 & 4038a & 36.90178 & 116.27710 & 1448 & 05/07/1964 & $58-B$ & $06 / 20 / 2000$ \\
\hline Plot 19 & 4038b & 36.90178 & 116.27710 & 1448 & 05/07/1964 & $58-\mathrm{A}$ & 06/20/2000 \\
\hline Plot 19 & 4039a & 36.90150 & 116.27773 & 1444 & 05/07/1964 & $57-\mathrm{A}$ & $06 / 20 / 2000$ \\
\hline Plot 19 & 4039b & 36.90150 & 116.27773 & 1444 & 05/07/1964 & 57-B & $06 / 20 / 2000$ \\
\hline Plot 19 & 4040 & 36.90147 & 116.27765 & 1447 & 05/07/1964 & 31,32 & $06 / 20 / 2000$ \\
\hline Plot 19 & 4041 & 36.90153 & 116.27745 & 1457 & 05/07/1964 & 35 & 06/20/2000 \\
\hline Plot 20 & $4087 a$ & 36.75238 & 116.02131 & 997 & 04/16/1964 & $3 \mathrm{~A}$ & 4/16/2002 \\
\hline Plot 20 & $4087 b$ & 36.75238 & 116.02131 & 997 & 04/16/1964 & $3 B$ & 4/16/2002 \\
\hline Plot 20 & $4100 a$ & 36.75238 & 116.02131 & 997 & 04/16/1964 & $4-\mathrm{A}$ & 4/16/2002 \\
\hline Plot 20 & 4100b & 36.75238 & 116.02131 & 997 & 04/16/1964 & $4-B$ & 4/16/2002 \\
\hline Plot 21 & $4146 a$ & 36.77795 & 116.01199 & 972 & 04/29/1964 & $43-A$ & 04/16/2002 \\
\hline Plot 21 & $4146 b$ & 36.77795 & 116.01199 & 972 & 04/29/1964 & 43-B & 04/16/2002 \\
\hline Plot 21 & 4213a & 36.77750 & 116.01161 & 970 & 04/29/1964 & 44-A & 04/16/2002 \\
\hline Plot 21 & $4213 b$ & 36.77750 & 116.01161 & 970 & 04/29/1964 & $44-\mathrm{A}$ & 04/16/2002 \\
\hline Plot 22 & $4158 a$ & 36.79441 & 116.01783 & 1005 & 04/16/1964 & $6-A$ & 04/18/2002 \\
\hline Plot 22 & $4158 b$ & 36.79441 & 116.01783 & 1005 & 04/16/1964 & $6-\mathrm{B}$ & 04/18/2002 \\
\hline Plot 22 & $4159 a$ & 36.79485 & 116.01797 & 987 & 04/16/1964 & $5-A$ & 04/18/2002 \\
\hline Plot 22 & 4159b & 36.79485 & 116.01797 & 987 & 04/16/1964 & $5-B$ & 04/18/2002 \\
\hline Plot 23 & $4149 a$ & 36.81146 & 115.98876 & 955 & 04/24/1964 & $27-B$ & 04/17/2002 \\
\hline Plot 23 & 4149b & 36.81146 & 115.98876 & 955 & 04/24/1964 & $27-A$ & $04 / 17 / 2002$ \\
\hline Plot 23 & $4176 a$ & 36.81121 & 115.98936 & 961 & 04/25/1964 & 30-A & 04/20/2002 \\
\hline Plot 23 & $4176 b$ & 36.81121 & 115.98936 & 961 & 04/25/1964 & 30-B & $04 / 20 / 2002$ \\
\hline Plot 24 & $4160 a$ & 36.85328 & 116.01126 & 1026 & 04/25/1964 & 29-A & 04/18/2002 \\
\hline Plot 24 & 4160b & 36.85328 & 116.01126 & 1026 & 04/25/1964 & 29-B & $04 / 18 / 2002$ \\
\hline Plot 24 & 4163a & 36.85320 & 116.01151 & 1026 & $\sim 1974$ & $27 a, 29 a$ & 04/19/2002 \\
\hline Plot 24 & $4163 b$ & 36.85320 & 116.01151 & 1026 & $\sim 1974$ & $28 a$ & 04/19/2002 \\
\hline Plot 24 & $4179 a$ & 36.85369 & 116.01165 & 1026 & 04/25/1964 & 28-B & $04 / 20 / 2002$ \\
\hline Plot 24 & 4179b & 36.85369 & 116.01165 & 1026 & 04/25/1964 & $28-A$ & $04 / 20 / 2002$ \\
\hline Plot 24 & 4191a & 38.85328 & 116.01203 & 1029 & $\sim 1974$ & $17 a$ & $04 / 21 / 2002$ \\
\hline Plot 24 & 4191b & 38.85328 & 116.01203 & 1029 & $\sim 1974$ & 19a & $04 / 21 / 2002$ \\
\hline Plot 24 & 4191c & 38.85328 & 116.01203 & 1029 & $\sim 1974$ & $16 a$ & $04 / 21 / 2002$ \\
\hline Plot 24 & 4191d & 38.85328 & 116.01203 & 1029 & $\sim 1974$ & $18 a$ & $04 / 21 / 2002$ \\
\hline Plot 25 & $4180 a$ & 36.83890 & 116.05725 & 1083 & 05/10/1964 & none & $04 / 20 / 2002$ \\
\hline Plot 25 & 4180b & 36.83890 & 116.05725 & 1083 & 05/10/1964 & none & $04 / 20 / 2002$ \\
\hline
\end{tabular}


Table A-69 (continued). Summary information on photographs of Beatley plots on the Nevada Test Site, Nye County, Nevada.

\begin{tabular}{|c|c|c|c|c|c|c|c|}
\hline $\begin{array}{c}\text { Plot } \\
\text { Number }\end{array}$ & $\begin{array}{c}\text { Stake }^{1} \\
\text { Number }\end{array}$ & $\begin{array}{c}\text { Latitude } \\
\text { (Degrees) }\end{array}$ & $\begin{array}{l}\begin{array}{l}\text { Longitude } \\
\text { (Degrees) }\end{array} \\
\end{array}$ & $\begin{array}{c}\text { Elevation } \\
(\mathbf{m})\end{array}$ & $\begin{array}{c}\text { Date of Original } \\
\text { Photograph }\end{array}$ & $\begin{array}{c}\text { Original Photo } \\
\text { Number }\end{array}$ & $\begin{array}{l}\text { Date of } \\
\text { Match }\end{array}$ \\
\hline Plot 25 & 4181a & 36.83889 & 116.05651 & 1086 & 05/10/1964 & none & $04 / 20 / 2002$ \\
\hline Plot 25 & 4181b & 36.83889 & 116.05651 & 1086 & 05/10/1964 & none & $04 / 20 / 2002$ \\
\hline Plot 26 & 4161a & 36.81033 & 116.10485 & 1208 & 05/10/1964 & 88-A & 04/19/2002 \\
\hline Plot 26 & 4161b & 36.81033 & 116.10485 & 1208 & 05/10/1964 & 88-B & 04/19/2002 \\
\hline Plot 26 & $4162 a$ & 36.81004 & 116.10538 & 1200 & 05/10/1964 & 87-A & 04/19/2002 \\
\hline Plot 26 & $4162 b$ & 36.81004 & 116.10538 & 1200 & 05/10/1964 & 87-B & 04/19/2002 \\
\hline Plot 27 & $4177 a$ & 36.89157 & 116.04849 & 1114 & 05/10/1964 & 12 & $04 / 20 / 2002$ \\
\hline Plot 27 & $4177 b$ & 36.89157 & 116.04849 & 1114 & 05/10/1964 & 11 & 04/20/2002 \\
\hline Plot 27 & $4178 \mathrm{a}$ & 36.89205 & 116.04896 & 1080 & 05/10/1964 & none & 04/20/2002 \\
\hline Plot 27 & $4178 b$ & 36.89205 & 116.04896 & 1080 & 05/10/1964 & 10 & 04/20/2002 \\
\hline Plot 28 & $4152 a$ & 36.85381 & 115.92033 & 985 & 04/22/1964 & 31-A & 04/18/2002 \\
\hline Plot 28 & $4152 b$ & 36.85381 & 115.92033 & 985 & 04/22/1964 & 31-B & $04 / 18 / 2002$ \\
\hline Plot 28 & $4153 a$ & 36.85412 & 115.91975 & 982 & 04/22/1964 & $32-A$ & 04/18/2002 \\
\hline Plot 28 & 4153b & 36.85412 & 115.91975 & 982 & 04/22/1964 & 32-B & 04/18/2002 \\
\hline Plot 29 & $4150 a$ & 36.85521 & 115.92362 & 987 & 04/22/1964 & 65-A & 04/18/2002 \\
\hline Plot 29 & 4150b & 36.85521 & 115.92362 & 987 & 04/22/1964 & $65-B$ & 04/18/2002 \\
\hline Plot 29 & 4151a & 36.85572 & 115.92402 & 987 & 04/22/1964 & 66-A & 04/18/2002 \\
\hline Plot 29 & 4151b & 36.85572 & 115.92402 & 987 & 04/22/1964 & $66-\mathrm{B}$ & 04/18/2002 \\
\hline Plot 30 & $4154 a$ & 36.83205 & 115.93901 & 942 & 05/11/1964 & 64-A & $04 / 18 / 2002$ \\
\hline Plot 30 & 4154b & 36.83205 & 115.93901 & 942 & 05/11/1964 & 64-B & $04 / 18 / 2002$ \\
\hline Plot 30 & 4155a & 36.83181 & 115.93968 & 940 & 05/11/1964 & 63-A & 04/18/2002 \\
\hline Plot 30 & 4155b & 36.83181 & 115.93968 & 940 & 05/11/1964 & 63-B & 04/18/2002 \\
\hline Plot 31 & 4156a & 36.82499 & 115.93374 & 938 & 05/11/1964 & $72-\mathrm{A}$ & 04/18/2002 \\
\hline Plot 31 & 4156b & 36.82499 & 115.93374 & 938 & 05/11/1964 & $72-B$ & $04 / 18 / 2002$ \\
\hline Plot 31 & $4157 a$ & 36.82460 & 115.93340 & 945 & 05/11/1964 & 71-A & $04 / 18 / 2002$ \\
\hline Plot 31 & $4157 b$ & 36.82460 & 115.93340 & 945 & 05/11/1964 & 71-B & $04 / 18 / 2002$ \\
\hline Plot 32 & $4164 a$ & 36.83640 & 115.86377 & 1037 & 05/11/1964 & $70-\mathrm{A}$ & $04 / 19 / 2002$ \\
\hline Plot 32 & $4164 b$ & 36.83640 & 115.86377 & 1037 & 05/11/1964 & $70-B$ & 04/19/2002 \\
\hline Plot 32 & $4165 a$ & 36.83675 & 115.86316 & 1026 & 05/11/1964 & 69-A & 04/19/2002 \\
\hline Plot 32 & $4165 b$ & 36.83675 & 115.86316 & 1026 & 05/11/1964 & $69-\mathrm{B}$ & $04 / 19 / 2002$ \\
\hline Plot 33 & 4166a & 36.82007 & 115.88552 & 983 & 04/24/1964 & $25-A$ & $04 / 19 / 2002$ \\
\hline Plot 33 & 4166b & 36.82007 & 115.88552 & 983 & 04/24/1964 & $25-\mathrm{B}$ & 04/19/2002 \\
\hline Plot 33 & $4167 a$ & 36.81976 & 115.88612 & 980 & 04/24/1964 & 26-A & 04/19/2002 \\
\hline Plot 33 & $4167 b$ & 36.81976 & 115.88612 & 980 & 04/24/1964 & $26-B$ & $04 / 19 / 2002$ \\
\hline Plot 34 & $4174 a$ & 36.79790 & 115.90508 & 933 & 05/11/1964 & 74-A & 04/20/2002 \\
\hline Plot 34 & $4174 b$ & 36.79790 & 115.90508 & 933 & 05/11/1964 & $74-B$ & 04/20/2002 \\
\hline
\end{tabular}


Table A-69 (continued). Summary information on photographs of Beatley plots on the Nevada Test Site, Nye County, Nevada.

\begin{tabular}{|c|c|c|c|c|c|c|c|}
\hline $\begin{array}{c}\text { Plot } \\
\text { Number }\end{array}$ & $\begin{array}{c}\text { Stake }^{1} \\
\text { Number }\end{array}$ & $\begin{array}{c}\text { Latitude } \\
\text { (Degrees) }\end{array}$ & $\begin{array}{l}\begin{array}{l}\text { Longitude } \\
\text { (Degrees) }\end{array} \\
\end{array}$ & $\begin{array}{c}\text { Elevation } \\
(\mathbf{m})\end{array}$ & $\begin{array}{c}\text { Date of Original } \\
\text { Photograph }\end{array}$ & $\begin{array}{c}\text { Original Photo } \\
\text { Number }\end{array}$ & $\begin{array}{l}\text { Date of } \\
\text { Match }\end{array}$ \\
\hline Plot 34 & $4175 a$ & 36.79823 & 115.90460 & 938 & 05/11/1964 & 73-A & $04 / 20 / 2002$ \\
\hline Plot 34 & $4175 b$ & 36.79823 & 115.90460 & 938 & 05/11/1964 & 73-B & $04 / 20 / 2002$ \\
\hline Plot 35 & $4172 \mathrm{a}$ & 36.77862 & 115.90589 & 997 & 04/24/1964 & $23-A$ & $04 / 20 / 2002$ \\
\hline Plot 35 & $4172 b$ & 36.77862 & 115.90589 & 997 & 04/24/1964 & $23-B$ & $04 / 20 / 2002$ \\
\hline Plot 35 & 4173a & 36.77896 & 115.90538 & 1006 & 04/24/1964 & $24-\mathrm{A}$ & $04 / 20 / 2002$ \\
\hline Plot 35 & $4173 b$ & 36.77896 & 115.90538 & 1006 & 04/24/1964 & $24-B$ & $04 / 20 / 2002$ \\
\hline Plot 36 & $4168 a$ & 36.78152 & 115.92319 & 924 & 04/15/1964 & $2-\mathrm{A}$ & $04 / 20 / 2002$ \\
\hline Plot 36 & $4168 b$ & 36.78152 & 115.92319 & 924 & 04/15/1964 & $2-B$ & 04/20/2002 \\
\hline Plot 36 & $4169 a$ & 36.78197 & 115.92361 & 936 & 04/15/1964 & 1-B & $04 / 20 / 2002$ \\
\hline Plot 36 & 4169b & 36.78197 & 115.92361 & 936 & 04/15/1964 & $1-\mathrm{A}$ & $04 / 20 / 2002$ \\
\hline Plot 37 & $4170 \mathrm{a}$ & 36.78362 & 115.92307 & 932 & 04/22/1964 & $35-A$ & $04 / 20 / 2002$ \\
\hline Plot 37 & $4170 \mathrm{~b}$ & 36.78362 & 115.92307 & 932 & 04/22/1964 & $35-B$ & $04 / 20 / 2002$ \\
\hline Plot 37 & $4171 \mathrm{a}$ & 36.78316 & 115.92272 & 938 & 04/22/1964 & 36-A & 04/20/2002 \\
\hline Plot 37 & $4171 b$ & 36.78316 & 115.92272 & 938 & 04/22/1964 & $36-B$ & $04 / 20 / 2002$ \\
\hline Plot 38 & $4147 a$ & 36.79361 & 115.9843 & 947 & 05/11/1964 & 68-A & $04 / 17 / 2002$ \\
\hline Plot 38 & $4147 b$ & 36.79361 & 115.9843 & 947 & 05/11/1964 & 68-B & $04 / 17 / 2002$ \\
\hline Plot 38 & $4148 \mathrm{a}$ & 36.79317 & 115.98390 & 936 & 05/11/1964 & $67-A$ & $04 / 17 / 2002$ \\
\hline Plot 38 & $4148 b$ & 36.79317 & 115.98390 & 936 & 05/11/1964 & $67-B$ & $04 / 17 / 2002$ \\
\hline Plot 39 & 4029 & 36.92493 & 116.16855 & 1378 & 05/10/2000 & 4029 & $05 / 10 / 2000$ \\
\hline Plot 40 & $4026 a$ & 36.92388 & 116.16888 & 1378 & 1964 & $40-3$ & $05 / 10 / 2000$ \\
\hline Plot 40 & $4026 b$ & 36.92388 & 116.16888 & 1378 & 1964 & $40-4$ & $05 / 10 / 2000$ \\
\hline Plot 40 & $4027 \mathrm{a}$ & 36.92427 & 116.16825 & 1372 & 1964 & $40-2$ & $05 / 10 / 2000$ \\
\hline Plot 40 & $4027 b$ & 36.92427 & 116.16825 & 1372 & 1964 & $40-1$ & $05 / 10 / 2000$ \\
\hline Plot 40 & $4028 a$ & 36.92408 & 116.16867 & 1374 & June 1974 & 6 & $05 / 10 / 2000$ \\
\hline Plot 40 & $4028 b$ & 36.92408 & 116.16867 & 1374 & June 1974 & 7 & $05 / 10 / 2000$ \\
\hline Plot 41 & 4023a & 36.96095 & 116.18352 & 1441 & 05/10/2000 & 4023a & $05 / 10 / 2000$ \\
\hline Plot 41 & 4023b & 36.96095 & 116.18352 & 1441 & 05/10/2000 & 4023b & $05 / 10 / 2000$ \\
\hline Plot 42 & 4024 & 36.96033 & 116.18393 & 1444 & June 1974 & 3,4 & $05 / 10 / 2000$ \\
\hline Plot 42 & 4025 & 36.96003 & 116.18395 & 1446 & 05/10/2000 & 4025 & $05 / 10 / 2000$ \\
\hline Plot 43 & $4044 a$ & 36.95977 & 116.07207 & 1273 & 05/12/1964 & $99 \mathrm{~A}$ & 06/07/2001 \\
\hline Plot 43 & $4044 b$ & 36.95977 & 116.07207 & 1273 & 05/12/1964 & $99 B$ & 06/07/2001 \\
\hline Plot 43 & $4045 a$ & 36.95937 & 116.07156 & 1266 & 05/12/1964 & $100 \mathrm{~A}$ & 06/07/2001 \\
\hline Plot 43 & $4045 b$ & 36.95937 & 116.07156 & 1266 & 05/12/1964 & $100 \mathrm{~B}$ & 06/07/2001 \\
\hline Plot 43 & 4228 & 36.95954 & 116.07211 & 1271 & $1959 ?$ & C59-647-7 & 05/31/02 \\
\hline $\begin{array}{c}\text { Near Plot } \\
\quad 43\end{array}$ & 4229 & 36.96172 & 116.06907 & 1250 & $1959 ?$ & none & 05/31/02 \\
\hline
\end{tabular}


Table A-69 (continued). Summary information on photographs of Beatley plots on the Nevada Test Site, Nye County, Nevada.

\begin{tabular}{|c|c|c|c|c|c|c|c|}
\hline $\begin{array}{c}\text { Plot } \\
\text { Number } \\
\end{array}$ & $\begin{array}{c}\text { Stake }^{1} \\
\text { Number }\end{array}$ & $\begin{array}{c}\text { Latitude } \\
\text { (Degrees) }\end{array}$ & $\begin{array}{l}\begin{array}{l}\text { Longitude } \\
\text { (Degrees) }\end{array} \\
\end{array}$ & $\begin{array}{c}\text { Elevation } \\
(\mathbf{m})\end{array}$ & $\begin{array}{c}\text { Date of Original } \\
\text { Photograph }\end{array}$ & $\begin{array}{c}\text { Original Photo } \\
\text { Number }\end{array}$ & $\begin{array}{l}\text { Date of } \\
\text { Match }\end{array}$ \\
\hline Plot 44 & $4046 a$ & 36.97936 & 116.08967 & 1267 & 05/12/1964 & $89 \mathrm{~A}$ & 06/07/2001 \\
\hline Plot 44 & 4046b & 36.97936 & 116.08967 & 1267 & 05/12/1964 & $89 B$ & 06/07/2001 \\
\hline Plot 44 & $4047 a$ & 36.97988 & 116.09007 & 1266 & 05/12/1964 & $90 \mathrm{~A}$ & 06/07/2001 \\
\hline Plot 44 & $4047 b$ & 36.97988 & 116.09007 & 1266 & 05/12/1964 & $90 \mathrm{~B}$ & 06/07/2001 \\
\hline Plot 45 & $4048 a$ & 36.98220 & 116.09941 & 1286 & 05/12/1964 & $92 \mathrm{~A}$ & 06/07/2001 \\
\hline Plot 45 & $4048 b$ & 36.98220 & 116.09941 & 1286 & 05/12/1964 & $92 B$ & 06/07/2001 \\
\hline Plot 45 & $4049 a$ & 36.98271 & 116.09984 & 1286 & 05/12/1964 & $91 \mathrm{~A}$ & 06/07/2001 \\
\hline Plot 45 & 4049b & 36.98271 & 116.09984 & 1286 & 05/12/1964 & 91B & 06/07/2001 \\
\hline Plot 46 & $4127 a$ & 37.00718 & 116.08487 & 1240 & 04/18/1964 & 98-A & 04/08/2001 \\
\hline Plot 46 & $4127 \mathrm{~b}$ & 37.00718 & 116.08487 & 1240 & 04/18/1964 & 98-B & 04/08/2001 \\
\hline Plot 46 & $4128 \mathrm{a}$ & 37.00687 & 116.08547 & 1245 & 04/18/1964 & 97-A & 04/08/2001 \\
\hline Plot 46 & $4128 b$ & 37.00687 & 116.08547 & 1245 & 04/18/1964 & $97-B$ & 04/08/2001 \\
\hline Plot 47 & $4059 a$ & 37.02193 & 116.17042 & 1472 & 04/21/1964 & $17 \mathrm{~A}$ & 06/08/2001 \\
\hline Plot 47 & 4059b & 37.02193 & 116.17042 & 1472 & 04/21/1964 & 17B & 06/08/2001 \\
\hline Plot 47 & 4060a & 37.02214 & 116.17109 & 1471 & 04/21/1964 & $18 \mathrm{~A}$ & 06/08/2001 \\
\hline Plot 47 & 4060b & 37.02214 & 116.17109 & 1471 & 04/21/1964 & 18B & 06/08/2001 \\
\hline Plot 48 & $4111 \mathrm{a}$ & 37.05432 & 116.09050 & 1285 & 05/12/1964 & 93-A & 04/06/2001 \\
\hline Plot 48 & $4111 b$ & 37.05432 & 116.09050 & 1285 & 05/12/1964 & 93-B & 04/06/2001 \\
\hline Plot 48 & $4112 a$ & 37.05480 & 116.09106 & 1303 & 05/12/1964 & 94-B & 04/06/2001 \\
\hline Plot 48 & $4112 b$ & 37.05480 & 116.09106 & 1303 & 05/12/1964 & 94-A & 04/06/2001 \\
\hline Plot 48 & 4113 & 37.05436 & 116.09072 & 1067 & 04/06/2001 & 4113 & 04/06/2001 \\
\hline Plot 48 & $4118 \mathrm{a}$ & 37.05448 & 116.09065 & 1299 & 06/1974 & $35 \mathrm{~mm} 8 \mathrm{~A}$ & 04/06/2001 \\
\hline Plot 48 & $4118 b$ & 37.05448 & 116.09065 & 1299 & 06/1974 & $35 \mathrm{~mm} \mathrm{10A}$ & 04/06/2001 \\
\hline Plot 48 & $4118 c$ & 37.05448 & 116.09065 & 1299 & 06/1974 & $35 \mathrm{~mm} 9 \mathrm{~A}$ & 04/06/2001 \\
\hline Plot 48 & 4119a & 37.05453 & 116.09071 & 1298 & 06/1974 & 35 mm 13 & 04/06/2001 \\
\hline Plot 48 & $4119 b$ & 37.05453 & 116.09071 & 1298 & 06/1974 & 35 mm 11A & 04/06/2001 \\
\hline Plot 48 & $4119 c$ & 37.05453 & 116.09071 & 1298 & 06/1974 & 35 mm 12 & 04/06/2001 \\
\hline Plot 48 & 4119d & 37.05453 & 116.09071 & 1298 & 06/1974 & 35 mm 11 & 04/06/2001 \\
\hline Plot 48 & $4119 e$ & 37.05453 & 116.09071 & 1298 & 06/1974 & 35 mm 10 & 04/06/2001 \\
\hline Plot 48 & 4120 & 37.05444 & 116.09060 & 1302 & 06/1974 & $35 \mathrm{~mm} 9$ & 04/06/2001 \\
\hline Plot 48 & 4226 & 37.05450 & 116.09023 & 1291 & $1959 ?$ & C59-647-8 & 05/31/02 \\
\hline Plot 49 & 4116a & 37.06201 & 116.07919 & 1279 & 05/12/1964 & $96-\mathrm{B}$ & 04/06/2001 \\
\hline Plot 49 & 4116b & 37.06201 & 116.07919 & 1279 & 05/12/1964 & 96-A & 04/06/2001 \\
\hline Plot 49 & $4117 \mathrm{a}$ & 37.06246 & 116.07869 & 1283 & 05/12/1964 & 95-A & 04/06/2001 \\
\hline Plot 49 & $4117 b$ & 37.06246 & 116.07869 & 1283 & 05/12/1964 & 95-B & 04/06/2001 \\
\hline Plot 50 & $4114 a$ & 37.07767 & 116.06135 & 1276 & 05/13/1964 & $104-A$ & 04/06/2001 \\
\hline
\end{tabular}


Table A-69 (continued). Summary information on photographs of Beatley plots on the Nevada Test Site, Nye County, Nevada.

\begin{tabular}{|c|c|c|c|c|c|c|c|}
\hline $\begin{array}{c}\text { Plot } \\
\text { Number } \\
\end{array}$ & $\begin{array}{c}\text { Stake }^{1} \\
\text { Number }\end{array}$ & $\begin{array}{l}\text { Latitude } \\
\text { (Degrees) }\end{array}$ & $\begin{array}{l}\begin{array}{l}\text { Longitude } \\
\text { (Degrees) }\end{array} \\
\end{array}$ & $\begin{array}{c}\text { Elevation } \\
(\mathbf{m})\end{array}$ & $\begin{array}{c}\text { Date of Original } \\
\text { Photograph }\end{array}$ & $\begin{array}{c}\begin{array}{c}\text { Original Photo } \\
\text { Number }\end{array} \\
\end{array}$ & $\begin{array}{l}\text { Date of } \\
\text { Match }\end{array}$ \\
\hline Plot 50 & 4114b & 37.07767 & 116.06135 & 1276 & 05/13/1964 & 104-B & 04/06/2001 \\
\hline Plot 50 & $4115 a$ & 37.07820 & 116.06173 & 1275 & 05/13/1964 & $103-A$ & 04/06/2001 \\
\hline Plot 50 & 4115b & 37.07820 & 116.06173 & 1275 & 05/13/1964 & 103-B & 04/06/2001 \\
\hline Plot 50 & 3641 & 37.07818 & 116.06133 & 1248 & 05/18/1964 & none & 05/30/02 \\
\hline Plot 50 & 3642 & 37.07772 & 116.06069 & 1268 & 05/13/1964 & none & 05/30/02 \\
\hline Plot 50 & 4222 & 37.07766 & 116.06097 & 1257 & 05/18/1964 & none & 05/31/02 \\
\hline Plot 50 & 4223 & 37.07816 & 116.06090 & 1257 & 05/18/1964 & 1 & 05/31/02 \\
\hline Plot 50 & 4224 & 37.07752 & 116.06090 & 1256 & 05/18/1964 & none & 05/31/02 \\
\hline Plot 50 & 4225 & 37.07756 & 116.06100 & 1258 & 05/13/1964 & none & 05/31/02 \\
\hline Plot 51 & $4074 a$ & 37.18024 & 116.14064 & 1509 & 04/21/1964 & $20 \mathrm{~A}$ & 06/10/2001 \\
\hline Plot 51 & $4074 b$ & 37.18024 & 116.14064 & 1509 & 04/21/1964 & $20 \mathrm{~B}$ & 06/10/2001 \\
\hline Plot 51 & $4075 a$ & 37.17991 & 116.14126 & 1509 & 04/21/1964 & $19 \mathrm{~A}$ & 06/10/2001 \\
\hline Plot 51 & $4075 b$ & 37.17991 & 116.14126 & 1509 & 04/21/1964 & 19B & 06/10/2001 \\
\hline Plot 52 & 4061 & 37.17886 & 116.02756 & 1331 & 07/16/1965 & $3074-13$ & 06/09/2001 \\
\hline Plot 52 & 4062 & 37.17841 & 116.02860 & 1341 & 07/16/1965 & 3074-12 & 06/09/2001 \\
\hline Plot 52 & 4129 & 37.17745 & 116.02814 & 1356 & 1964 & C64-1790-3 & 04/08/2001 \\
\hline Plot 52 & 4130 & 37.17741 & 116.02871 & 1354 & 1964 & C64-1790-4 & 04/08/2001 \\
\hline Plot 52 & 4131 & 37.17746 & 116.02871 & 1356 & 10/02/1962 & C62-1392-1 & 04/08/2001 \\
\hline Plot 52 & $4132 a$ & 37.17745 & 116.02863 & 1351 & 06/1974 & 35 mm 27 & 04/08/2001 \\
\hline Plot 52 & $4132 b$ & 37.17745 & 116.02863 & 1351 & 06/1974 & 35 mm 28 & 04/08/2001 \\
\hline Plot 52 & 4133 & 37.17747 & 116.02862 & 1351 & 06/1974 & 35 mm 29 & 04/08/2001 \\
\hline Plot 52 & 4134 & 37.17763 & 116.02865 & 1350 & 10/02/1962 & C62-1392-2 & 04/08/2001 \\
\hline Plot 52 & $4135 a$ & 37.17761 & 116.02848 & 1347 & 06/1974 & $35 \mathrm{~mm} 23 \mathrm{~A}$ & 04/08/2001 \\
\hline Plot 52 & $4135 b$ & 37.17761 & 116.02848 & 1347 & 06/1974 & $35 \mathrm{~mm} 24 \mathrm{~A}$ & 04/08/2001 \\
\hline Plot 52 & $4135 c$ & 37.17761 & 116.02848 & 1347 & 06/1974 & $35 \mathrm{~mm} 25 \mathrm{~A}$ & 04/08/2001 \\
\hline Plot 52 & $4136 a$ & 37.17769 & 116.02835 & 1351 & 05/08/1964 & 77-A & 04/08/2001 \\
\hline Plot 52 & 4136b & 37.17769 & 116.02835 & 1351 & 05/08/1964 & $77-B$ & 04/08/2001 \\
\hline Plot 52 & $4137 a$ & 37.17777 & 116.02823 & 1343 & 07/16/1965 & $3074-9$ & 04/08/2001 \\
\hline Plot 52 & $4137 b$ & 37.17777 & 116.02823 & 1343 & 07/16/1965 & $3074-10$ & 04/08/2001 \\
\hline Plot 52 & $4137 c$ & 37.17777 & 116.02823 & 1343 & 07/1976 & Roll 1 (\#6) & 04/08/2001 \\
\hline Plot 52 & 4137d & 37.17777 & 116.02823 & 1343 & 07/1976 & Roll 1 (\#5) & 04/08/2001 \\
\hline Plot 52 & 4138 & 37.17747 & 116.02850 & 1361 & 10/02/1962 & C62-1392-4 & 04/08/2001 \\
\hline Plot 52 & 4139a & 37.17733 & 116.02899 & 1360 & 05/08/1964 & 78-A & 04/08/2001 \\
\hline Plot 52 & 4139b & 37.17733 & 116.02899 & 1360 & 05/08/1964 & $78-B$ & 04/08/2001 \\
\hline $\begin{array}{l}\text { North of } \\
\text { Plot } 52\end{array}$ & 4140 & 37.17841 & 116.02874 & 1359 & 07/16/1965 & $3074-14$ & 04/08/2001 \\
\hline
\end{tabular}


Table A-69 (continued). Summary information on photographs of Beatley plots on the Nevada Test Site, Nye County, Nevada.

\begin{tabular}{|c|c|c|c|c|c|c|c|}
\hline $\begin{array}{c}\text { Plot } \\
\text { Number } \\
\end{array}$ & $\begin{array}{c}\text { Stake }^{1} \\
\text { Number }\end{array}$ & $\begin{array}{c}\text { Latitude } \\
\text { (Degrees) }\end{array}$ & $\begin{array}{l}\begin{array}{l}\text { Longitude } \\
\text { (Degrees) }\end{array} \\
\end{array}$ & $\begin{array}{c}\text { Elevation } \\
(\mathbf{m})\end{array}$ & $\begin{array}{c}\text { Date of Original } \\
\text { Photograph }\end{array}$ & $\begin{array}{c}\text { Original Photo } \\
\text { Number }\end{array}$ & $\begin{array}{l}\text { Date of } \\
\text { Match }\end{array}$ \\
\hline Plot 53 & 4063a & 37.16640 & 116.02908 & 1337 & 05/08/1964 & $76 \mathrm{~A}$ & 06/09/2001 \\
\hline Plot 53 & 4063b & 37.16640 & 116.02908 & 1337 & 05/08/1964 & $76 \mathrm{~B}$ & 06/09/2001 \\
\hline Plot 53 & $4064 a$ & 37.16683 & 116.02947 & 1352 & 05/08/1964 & $75 \mathrm{~A}$ & 06/09/2001 \\
\hline Plot 53 & 4064b & 37.16683 & 116.02947 & 1352 & 05/08/1964 & $75 B$ & 06/09/2001 \\
\hline Plot 53 & 4065 & 37.16668 & 116.02975 & 1339 & 07/1965 & $3074-2$ & 06/09/2001 \\
\hline Plot 53 & 4066 & 37.16665 & 116.02943 & 1340 & n.d. & C64-1786-11 & 06/09/2001 \\
\hline Plot 53 & 4067 & 37.16657 & 116.02912 & 1340 & n.d. & C64-1790-01 & 06/09/2001 \\
\hline Plot 53 & 4068 & 37.16657 & 116.02917 & 1342 & 10/02/1962 & C62-1391-9 & 06/09/2001 \\
\hline Plot 53 & $4069 a$ & 37.16643 & 116.02923 & 1342 & 06/1974 & 19A, 21A & 06/09/2001 \\
\hline Plot 53 & 4069b & 37.16643 & 116.02923 & 1342 & 06/1974 & $20 \mathrm{~A}$ & 06/09/2001 \\
\hline Plot 53 & 4070 & 37.16648 & 116.02931 & 1346 & n.d. & C64-1786-10 & 06/09/2001 \\
\hline Plot 53 & 4071 & 37.16632 & 116.02945 & 1345 & 07/16/1965 & $3074-3$ & 06/09/2001 \\
\hline Plot 53 & 4072 & 37.16630 & 116.02913 & 1349 & 06/1974 & 26 & 06/09/2001 \\
\hline Plot 53 & 4073 & 37.16632 & 116.02953 & 1344 & 07/16/1965 & $3074-6$ & 06/09/2001 \\
\hline Plot 54 & 4081 & 37.15833 & 116.02270 & 1350 & n.d. & C64-1786-7 & 06/10/2001 \\
\hline Plot 54 & 4082 & 37.15822 & 116.02243 & 1360 & n.d. & C64-1786-9 & 06/10/2001 \\
\hline Plot 54 & 4083 & 37.15814 & 116.02263 & 1360 & n.d. & C64-1786-6 & 06/10/2001 \\
\hline Plot 54 & 4084 & 37.15812 & 116.02251 & 1352 & 06/1974 & 20 & 06/10/2001 \\
\hline Plot 54 & $4141 \mathrm{a}$ & 37.15823 & 116.02286 & 1377 & 05/09/1964 & 79-B & $04 / 08 / 2001$ \\
\hline Plot 54 & 4141b & 37.15823 & 116.02286 & 1377 & 05/09/1964 & 79-A & $04 / 08 / 2001$ \\
\hline Plot 54 & $4142 a$ & 37.15843 & 116.02223 & 1382 & 05/09/1964 & 80-A & 04/08/2001 \\
\hline Plot 54 & $4142 b$ & 37.15843 & 116.02223 & 1382 & 05/09/1964 & 80-B & 04/08/2001 \\
\hline Plot 54 & 4143 & 37.15821 & 116.02284 & 1363 & 06/1974 & 35 mm \#19 & 04/08/2001 \\
\hline Plot 54 & 4144 & 37.15814 & 116.02250 & 1364 & 06/1974 & 35 mm \#21 & 04/08/2001 \\
\hline Plot 54 & $4145 a$ & 37.15827 & 116.02241 & 1360 & 06/1974 & $35 \mathrm{~mm} \# 16 \mathrm{~A}$ & $04 / 08 / 2001$ \\
\hline Plot 54 & $4145 b$ & 37.15827 & 116.02241 & 1360 & 06/1974 & 35 mm \#17A & 04/08/2001 \\
\hline Plot 55 & 4076a & 37.12279 & 116.02524 & 1319 & 06/1974 & 16,18 & 06/10/2001 \\
\hline Plot 55 & $4076 b$ & 37.12279 & 116.02524 & 1319 & 06/1974 & 17 & 06/10/2001 \\
\hline Plot 55 & 4077 & 37.12286 & 116.02517 & 1331 & 05/09/1964 & 81A-B & 06/10/2001 \\
\hline Plot 55 & 4078 & 37.12281 & 116.02528 & 1329 & 06/1974 & $12 \mathrm{~A}, 14 \mathrm{~A}, 13 \mathrm{~A}, 15 \mathrm{~A}$ & 06/10/2001 \\
\hline Plot 55 & 4079 & 37.12287 & 116.02592 & 1323 & 06/1974 & 15,14 & 06/10/2001 \\
\hline Plot 55 & 4080 & 37.12293 & 116.02616 & 1319 & 05/09/1964 & 82A-B & 06/10/2001 \\
\hline Plot 56 & 4186a & 37.08208 & 115.99123 & 1323 & 05/09/1964 & 83-A & $04 / 20 / 2002$ \\
\hline Plot 56 & 4186b & 37.08208 & 115.99123 & 1323 & 05/09/1964 & 83-B & $04 / 20 / 2002$ \\
\hline Plot 56 & $4187 a$ & 37.08196 & 115.99049 & 1323 & 05/09/1964 & 84-A & $04 / 21 / 2002$ \\
\hline Plot 56 & $4187 b$ & 37.08196 & 115.99049 & 1323 & 05/09/1964 & 84-B & $04 / 21 / 2002$ \\
\hline
\end{tabular}


Table A-69 (continued). Summary information on photographs of Beatley plots on the Nevada Test Site, Nye County, Nevada.

\begin{tabular}{|c|c|c|c|c|c|c|c|}
\hline $\begin{array}{c}\text { Plot } \\
\text { Number }\end{array}$ & $\begin{array}{c}\text { Stake }^{1} \\
\text { Number }\end{array}$ & $\begin{array}{c}\text { Latitude } \\
\text { (Degrees) }\end{array}$ & $\begin{array}{l}\begin{array}{l}\text { Longitude } \\
\text { (Degrees) }\end{array} \\
\end{array}$ & $\begin{array}{c}\text { Elevation } \\
(\mathbf{m})\end{array}$ & $\begin{array}{c}\text { Date of Original } \\
\text { Photograph }\end{array}$ & $\begin{array}{c}\begin{array}{c}\text { Original Photo } \\
\text { Number }\end{array} \\
\end{array}$ & $\begin{array}{l}\text { Date of } \\
\text { Match }\end{array}$ \\
\hline Plot 57 & 4188a & 37.04227 & 115.99268 & 1254 & 05/09/1964 & 85-A & 04/21/2002 \\
\hline Plot 57 & $4188 \mathrm{~b}$ & 37.04227 & 115.99268 & 1254 & 05/09/1964 & 85-B & $04 / 21 / 2002$ \\
\hline Plot 57 & 4189a & 37.04272 & 115.99311 & 1262 & 05/09/1964 & 86-A & $04 / 21 / 2002$ \\
\hline Plot 57 & 4189b & 37.04272 & 115.99311 & 1262 & 05/09/1964 & 86-B & 04/21/2002 \\
\hline Plot 58 & 4190 & 37.00190 & 116.04240 & 1200 & 05/13/1964 & $106-A$ & $04 / 21 / 2002$ \\
\hline Plot 59 & $4184 a$ & 36.97941 & 116.04143 & 1198 & 05/13/1964 & none & $04 / 20 / 2002$ \\
\hline Plot 59 & $4184 b$ & 36.97941 & 116.04143 & 1198 & 05/13/1964 & none & $04 / 20 / 2002$ \\
\hline Plot 59 & $4185 a$ & 36.97912 & 116.04210 & 1192 & 05/13/1964 & none & 04/20/2002 \\
\hline Plot 59 & $4185 b$ & 36.97912 & 116.04210 & 1192 & 05/13/1964 & none & 04/20/2002 \\
\hline Plot 60 & $4182 a$ & 36.96227 & 115.99216 & 1168 & 05/13/1964 & $102-\mathrm{A}$ & 04/20/2002 \\
\hline Plot 60 & $4182 b$ & 36.96227 & 115.99216 & 1168 & 05/13/1964 & $102-B$ & 04/20/2002 \\
\hline Plot 60 & 4183a & 36.96196 & 115.99265 & 1202 & 05/13/1964 & $101-\mathrm{A}$ & $04 / 20 / 2002$ \\
\hline Plot 60 & 4183b & 36.96196 & 115.99265 & 1202 & 05/13/1964 & 101-B & 04/20/2002 \\
\hline Plot 61 & 3643 & 37.13544 & 116.25764 & 1755 & 05/18/1964 & 2 & $05 / 30 / 2002$ \\
\hline Plot 61 & 4194a & 37.13511 & 116.25748 & 1746 & 1964 & none & $05 / 28 / 2002$ \\
\hline Plot 61 & 4194b & 37.13511 & 116.25748 & 1746 & 1964 & none & 05/28/2002 \\
\hline Plot 61 & 4195a & 37.13550 & 116.25691 & 1759 & 1964 & none & 05/28/2002 \\
\hline Plot 61 & 4195b & 37.13550 & 116.25691 & 1759 & 1964 & none & $05 / 28 / 2002$ \\
\hline Plot 61 & 4218 & 37.13548 & 116.25762 & 1761 & 05/18/1964 & none & 05/30/2002 \\
\hline Plot 61 & 4219 & 37.13531 & 116.25774 & 1749 & 05/18/1964 & 3 & 05/30/2002 \\
\hline Plot 61 & 4220 & 37.13578 & 116.25725 & 1762 & 05/18/1964 & C64-1805-8 & 05/30/2002 \\
\hline Plot 61 & 4221 & 37.13552 & 116.25719 & 1763 & 05/18/1964 & C64-1805-7 & 05/30/2002 \\
\hline Plot 61 & 4227 & 37.13558 & 116.25807 & 1758 & 05/18/1964 & none & $05 / 31 / 2002$ \\
\hline Plot 62 & 3634 & 37.14091 & 116.25766 & 1751 & 1964 & C64-1806-1 & $05 / 28 / 2002$ \\
\hline Plot 62 & 3635 & 37.14095 & 116.25787 & 1746 & 1964 & C64-1805-10 & $05 / 28 / 2002$ \\
\hline Plot 62 & 3636 & 37.14108 & 116.25807 & 1752 & 1964 & C64-1805-11 & $05 / 28 / 2002$ \\
\hline Plot 62 & 4196a & 37.14140 & 116.25792 & 1749 & 1964 & none & $05 / 28 / 2002$ \\
\hline Plot 62 & 4196b & 37.14140 & 116.25792 & 1749 & 1964 & none & $05 / 28 / 2002$ \\
\hline Plot 62 & $4197 \mathrm{a}$ & 37.14092 & 116.25806 & 1742 & 1964 & none & $05 / 28 / 2002$ \\
\hline Plot 62 & $4197 b$ & 37.14092 & 116.25806 & 1742 & 1964 & none & $05 / 28 / 2002$ \\
\hline $\begin{array}{l}\text { Near Plot } \\
\quad 62\end{array}$ & 3306 & 37.14052 & 116.25581 & 1817 & 1964 & C64-1805-12 & 05/28/2002 \\
\hline Plot 63 & $3637 a$ & 37.20105 & 116.25272 & 1978 & 1964 & none & $05 / 29 / 2002$ \\
\hline Plot 63 & $3637 b$ & 37.20105 & 116.25272 & 1978 & 1964 & none & 05/29/2002 \\
\hline Plot 63 & $3638 a$ & 37.20137 & 116.25214 & 1985 & 1964 & none & 05/29/2002 \\
\hline Plot 63 & $3638 b$ & 37.20137 & 116.25214 & 1985 & 1964 & none & 05/29/2002 \\
\hline
\end{tabular}


Table A-69 (continued). Summary information on photographs of Beatley plots on the Nevada Test Site, Nye County, Nevada.

\begin{tabular}{|c|c|c|c|c|c|c|c|}
\hline $\begin{array}{c}\text { Plot } \\
\text { Number }\end{array}$ & $\begin{array}{c}\text { Stake }^{1} \\
\text { Number }\end{array}$ & $\begin{array}{c}\text { Latitude } \\
\text { (Degrees) }\end{array}$ & $\begin{array}{l}\text { Longitude } \\
\text { (Degrees) }\end{array}$ & $\begin{array}{c}\text { Elevation } \\
(\mathbf{m})\end{array}$ & $\begin{array}{c}\text { Date of Original } \\
\text { Photograph } \\
\end{array}$ & $\begin{array}{c}\begin{array}{c}\text { Original Photo } \\
\text { Number }\end{array} \\
\end{array}$ & $\begin{array}{l}\text { Date of } \\
\text { Match }\end{array}$ \\
\hline Plot 65 & $4192 \mathrm{a}$ & 37.99370 & 116.24914 & 1675 & 1964 & none & 05/27/2002 \\
\hline Plot 65 & $4192 b$ & 37.99370 & 116.24914 & 1675 & 1964 & none & 05/27/2002 \\
\hline Plot 65 & 4230a & 37.99370 & 116.24914 & 1675 & 1964 & none & 05/27/2002 \\
\hline Plot 65 & 4230b & 37.99370 & 116.24914 & 1675 & 1964 & none & 05/27/2002 \\
\hline Plot 66 & 3817 & 36.81368 & 116.16436 & 1314 & 05/01/1964 & $48-B$ & 04/28/1999 \\
\hline Plot 66 & 3818 & 36.81363 & 116.16398 & 1314 & 05/01/1964 & $48-\mathrm{A}$ & 04/28/1999 \\
\hline Plot 66 & $3822 a$ & 36.81288 & 116.16486 & 1314 & 05/01/1964 & $47-B$ & 04/28/1999 \\
\hline Plot 66 & 3823 & 36.81285 & 116.16508 & 1314 & 05/01/1964 & 47-A & 04/28/1999 \\
\hline Plot 66-67 & 3819 & 36.81363 & 116.16398 & 1314 & 05/01/1964 & $50-B$ & 04/28/1999 \\
\hline Plot 67 & 3820 & 36.81352 & 116.16365 & 1314 & 05/01/1964 & 50-A & 04/28/1999 \\
\hline Plot 67 & 3821 & 36.81282 & 116.16461 & 1314 & 05/01/1964 & 49-B & 04/28/1999 \\
\hline Plot 67 & $3822 b$ & 36.81288 & 116.16486 & 1314 & 05/01/1964 & 49-A & 04/28/1999 \\
\hline Plot 67 & 4030 & 36.81288 & 116.16486 & 1314 & $6 / 25-26 / 1974$ & $6-A$ & $05 / 11 / 2000$ \\
\hline Plot 67 & 4031 & 36.81288 & 116.16486 & 1314 & 6/25-26/1974 & $3-A$ & $05 / 11 / 2000$ \\
\hline Plot 68 & 4198 & 37.17365 & 116.24800 & 1818 & 1964 & none & 05/28/2002 \\
\hline Plot 68 & 4199 & 37.17376 & 116.24731 & 1822 & 1964 & none & 05/28/2002 \\
\hline
\end{tabular}

1. Stake number is a unique number to identify photographic matches within the Desert Laboratory Repeat Photography Collection in Tucson, Arizona. 